\title{
Fast and Efficient Methods for Circuit-based Automotive EMC Simulation
}

Der Technischen Fakultät der Universität Erlangen-Nürnberg zur Erlangung des Grades

D O K T O R - I N G E N I E U R

vorgelegt von

Martin Ludwig Zitzmann

Erlangen - 2007 


\section{Als Dissertation genehmigt von der Technischen Fakultät der Universität Erlangen-Nürnberg}

Tag der Einreichung: 21. November 2006

Tag der Promotion: $\quad$ 09. Februar 2007

Dekan:

Prof. Dr. Alfred Leipertz

Berichterstatter: $\quad$ Prof. Dr. Robert Weigel

Prof. Dr. Rüdiger Vahldieck 


\section{... dedicated to my parents}

"The ensemble is more than the sum of its parts." Aristoteles, Greek philosopher, 384 - 322 B.C. 



\section{Acknowledgements}

The presented thesis is the result of my activities within the scope of a scholarship at the Research and Innovation Center (Forschungs- und Innovationszentrum, FIZ) of the BMW Group in Munich, Germany.

I am very grateful to my supervisor Professor Dr. Robert Weigel for the opportunity to work in an interesting field of scientific research and for motivating and supporting me during this time. Special thanks also go to Professor Dr. Rüdiger Vahldieck from the field theory group of the IFH at the ETH Zurich for the second opinion.

I wish to thank the head of the department EG-74 of the BMW Group, Mr. Heinz Oswald, for the chance to work in a very interesting industrial field and for offering the access to many companyinternal facilities. Thanks also go to my colleagues of the EMC team and my team leader $\mathrm{Mr}$. Wolfgang Kühn, for the cooperativeness, encouragement and support during all stages of this thesis. In this context, special considerations go to the experts in the field of automotive EMC simulation for many fruitful discussions and for concrete supports. The convenient working environment enormously contributed to the success of my work.

Very special thanks go to my colleagues Dr. Armin Englmaier and Dr. Gernot Steinmair, for their valuable and constructive criticism. Their interest concerning my work, their support and many precious ideas and discussions turned out to be indispensible for the success of this thesis.

I thank Mr. Robert Grillmair and Mr. Richard Varga for supporting my work within the scope of their diploma theses, internships and working student activities.

Thanks go to the associates of the SimLab Software GmbH, Mr. Florian Glaser, Dr. Matthias Tröscher, Mr. Heiko Grubrich and Mr. Hans-Peter Gerl for their support concerning various theoretical and programming issues.

The Fraunhofer Institute for Algorithms and Scientific Computing (SCAI) provided the software library SAMG for the implementation into the existing EMC simulation environment. I am deeply grateful to Dr. Tanja Clees for her kind help, for her tireless support and for so many fruitful discussions and explanations in the field of electrical engineering and mathematics.

The Max Planck Institute for Mathematics in the Sciences (MPI) provided the software library HLIB for research purposes and many helpful suggestions to accelerate the existing EMC simulation. I would like to thank, in particular Dr. Steffen Börm and Dr. Lars Grasedyk in the group of Professor Dr. Wolfgang Hackbusch, for their interest concerning my research topic.

Finally, I would like to thank my parents Johanna and Rudi, my sister Susanne and all my friends for so much more than words can express. 



\section{Abstract}

The numerical simulation of physical effects gains an enormous significance in today's automotive development process. Particularly the computational analysis of the electromagnetic compatibility (EMC) can help to detect problems already before a first prototype exists. The EMC is increasingly important due to a growing electrification of mechanical components and increased data rates in modern car electronics. As an important extension to the verification by measurement, simulation techniques have to be accurate and robust. A suitable modeling of 3D electromagnetic effects by an equivalent circuit representation can be accomplished by the well-established PEEC method. Typically, the resulting system matrices can be very large, dense and ill-conditioned. To enable the analysis of real-life problems, an acceleration has to be applied to the modeling as well as the solving process. Within the scope of this work, a methodology was developed to realize an overall EMC simulation process with significantly reduced complexity in terms of CPU time and storage demands for the underlying equation system. The latter is assumed to be the most serious bottleneck in EMC simulation. Various methods for model extraction and solving were investigated. Direct solvers usually are not efficient enough to be applicable to industrially relevant problems. Hierarchical matrices ( $\mathcal{H}$-matrices) and the well-known algebraic multigrid (AMG) approach are the most promising techniques, allowing simulations with almost optimal complexity, in principle. Moreover, they fulfill the accuracy demands and are suitable for parallel implementation. The generality, robustness, flexibility and efficiency of the proposed methods are shown by means of problems from the automotive environment. Numerical results demonstrate a significant speed-up of the modeling as well as the solution process. The proposed strategies work well in time domain and in frequency domain simulations and are regarded to be promising to enable an automotive EMC simulation on system level. This requires the combination of models of various types to capture the physical effects from components, the cable harnesses and the car chassis. 


\section{Zusammenfassung}

Die numerische Simulation physikalischer Effekte gewinnt in der heutigen Automobilentwicklung zunehmend an Bedeutung. Insbesondere die rechentechnische Analyse der elektromagnetischen Verträglichkeit (EMV) kann dazu dienen, Probleme bereits vor der Existenz eines ersten Prototypen zu detektieren. Die EMV stellt aufgrund einer zunehmenden Elektrifizierung mechanischer Komponenten und durch stetig anwachsende Datenraten in der modernen Automobilelektronik eine zunehmende Herausforderung dar. Als eine wichtige Ergänzung zur messtechnischen Verifikation müssen Simulationstechniken genau und robust sein. Eine geeignete Modellierung elektromagnetischer Effekte in 3D durch eine äquivalente Schaltkreisdarstellung kann durch die PEECMethode erfolgen. In der Regel können die resultierenden Systemmatrizen sehr groß, dicht besetzt und schlecht konditioniert sein. Um die Analyse realistischer Problemstellungen zu ermöglichen, müssen sowohl die Modellierung als auch der Lösungsprozess beschleunigt werden. Im Rahmen dieser Arbeit wurde eine Methodologie entwickelt, um eine EMV-Gesamtsimulation mit bedeutend reduzierter Komplexität in Bezug auf CPU-Zeit und Speicherbedarf für die Berechnung von Matrizen zu realisieren. Letzterer Aspekt wird als der kritischste Engpaß in der EMV-Simulation angesehen. Verschiedene Methoden der Modellextraktion und des Lösens wurden untersucht. Direkte Löser sind für einen Einsatz in industriell relevanten Problemstellungen in der Regel nicht effizient genug. Hierarchische Matrizen ( $\mathcal{H}$-Matrizen) und das bekannte algebraische Mehrgitterverfahren (AMG) stellen die vielversprechensten Techniken dar, um prinzipiell Simulationen mit annähernd optimaler Komplexität zu ermöglichen. Darüberhinaus erfüllen sie die Genauigkeitsanforderungen und eignen sich für eine parallele Implementierung. Die Allgemeingültigkeit, Robustheit, Flexibilität und Effizienz der vorgestellten Methoden wurden anhand von Problemen aus dem automobilen Umfeld verdeutlicht. Numerische Ergebnisse demonstrieren eine drastische Beschleunigung sowohl der Modellierung als auch des Lösungsprozesses. Die vorgestellten Strategien funktionieren gut in Zeitbereichs- und Frequenzbereichssimulationen und werden als vielversprechend angesehen, um eine EMV-Simulation auf Systemebene zu ermöglichen. Dies macht eine Verknüpfung verschiedenartiger Modelle notwendig, um die physikalischen Effekte von Steuergeräten, Kabelbäumen und der Karosserie zu berïcksichtigen. 


\section{Contents}

1 Introduction 1

1.1 Automotive EMC Simulation . . . . . . . . . . . . . . . . 1

1.2 State of the Art . . . . . . . . . . . . . . . . 3

1.3 Modern Solution Approaches . . . . . . . . . . . . . . . . . 4

1.4 Contents and Contributions of this Thesis . . . . . . . . . . . . . 5

2 Simulation Basics of Electromagnetic Effects 9

2.1 Maxwell's Equations . . . . . . . . . . . . . . . . . . 9 9

2.2 The Electromagnetic Scattering Problem . . . . . . . . . . . . . . . . . . . 11

2.3 The Retarded Electrodynamic Potentials . . . . . . . . . . . . . . . . . . . . 12

2.4 The Electric Field Integral Equation . . . . . . . . . . . . . . . . 13

2.5 Numerical Field Computation Basics . . . . . . . . . . . . . . . . . . . . 14

3 Equivalent Circuit Modeling $\quad 17$

3.1 The Petrov-Galerkin Approach . . . . . . . . . . . . . . . . . . . 18

3.2 Partial Elements for a Manhattan Discretization . . . . . . . . . . . . . . 19

3.2.1 Discretization of the EFIE . . . . . . . . . . . . . . . 19

3.2.2 Partial Element Extraction . . . . . . . . . . . . . . . . 20

3.3 Partial Elements for a Non-orthogonal Discretization . . . . . . . . . . . . 21

3.3 .1 Discretization of the EFIE . . . . . . . . . . . . . 22

3.3.2 Partial Element Extraction . . . . . . . . . . . . . 23

3.4 Equivalent Circuit Formulation . . . . . . . . . . . . . . . . 25

3.5 Dielectric Effects in PEEC Modeling . . . . . . . . . . . . . . . 26

3.5.1 Volume-Based PEEC Formulation . . . . . . . . . . . . . . . . . 27

3.5.2 Surface-Based PEEC Formulation . . . . . . . . . . . . . . 30

3.6 Structure of PEEC-Based Circuit-Level Simulation . . . . . . . . . . . . . . 32

3.7 Accuracy and Validity Aspects . . . . . . . . . . . . . . . . . . 34

3.7.1 Electrical Length of Interconnect Structures . . . . . . . . . . . . . . . . 34

3.7 .2 Accuracy Aspects . . . . . . . . . . . . . . . . 36 
3.7 .3 Validity Aspects . . . . . . . . . . . . . . . 36

3.7 .4 Simulation Artifacts $\ldots \ldots \ldots \ldots$

3.8 Numerical Integration by Cubature Techniques . . . . . . . . . . . . . . . . . . . . . .

3.8.1 Cubature Techniques According to Sauter . . . . . . . . . . . . . . 38

3.8.2 Hierarchical Quadrature . . . . . . . . . . . . . . . 40

3.9 Sparse Matrix Formulation via Partial Reluctances . . . . . . . . . . . . . . 42

3.9.1 Motivation ........................ 42

3.9 .2 Partial Reluctance $K \ldots \ldots \ldots \ldots$. . . . . . . . . . 43

3.9.3 Reluctance Matrix Properties . . . . . . . . . . . . . . . . . . 44

3.9.4 Truncation Process . . . . . . . . . . . . . . . . . 45

3.9.5 Reluctance-Based Circuit Representation . . . . . . . . . . . . . 45

3.9 .6 Numerical Examples . . . . . . . . . . . . . . . . . . . 48

3.10 Retardation Effects . . . . . . . . . . . . . . . . . . 50

3.10 .1 Time Domain . . . . . . . . . . . . . . . . . 51

3.10 .2 Frequency Domain . . . . . . . . . . . . . . 51

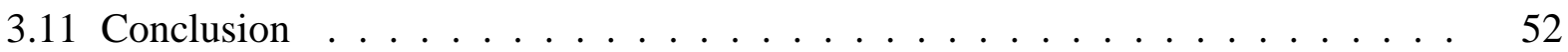

4 Fast and Efficient Extraction of Parasitic Effects

4.1 Historical Background and Motivation . . . . . . . . . . . . . . . . . 53

4.2 The Fast Multipole Method . . . . . . . . . . . . . . . . . 54

4.2.1 Theoretical Aspects . . . . . . . . . . . . . . . . 54

4.2.2 The Element Evaluation Process . . . . . . . . . . . . . 58

4.2.3 The Multilevel Fast Multipole Method . . . . . . . . . . . . . . . 59

4.2 .4 Implementation Details . . . . . . . . . . . . . . . . . . . . 59

4.3 Hierarchical Matrices . . . . . . . . . . . . . . . . . . . . . . . . . . . . . . . . . . 59

4.3.1 Theoretical Aspects . . . . . . . . . . . . . . . 60

4.3.2 The Element Evaluation Process . . . . . . . . . . . . . . . . 68

4.3 .3 Implementation Details . . . . . . . . . . . . . . . . . . 70

4.3 .4 Numerical Examples . . . . . . . . . . . . . . . . 71

4.3 .5 Further Improvements . . . . . . . . . . . . . . . 73

5 Iterative Methods in Circuit-Level Analysis $\quad 77$

5.1 Problem Description and Motivation . . . . . . . . . . . . . 78

5.1 .1 Direct Strategies . . . . . . . . . . . . . . . 81

5.1 .2 Iterative Strategies . . . . . . . . . . . . . . 83

5.2 Iterative Solution of Linear Systems of Equations . . . . . . . . . . . . . 83

5.2 .1 Basic Iterative Methods _ . . . . . . . . . . . . . . 83

5.2 .2 Subspace Methods . . . . . . . . . . . . . . 85

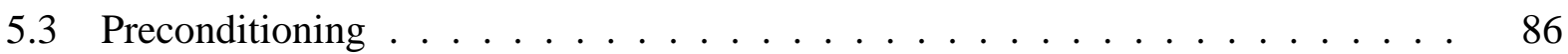

5.4 Implementation Issues $\ldots \ldots \ldots$. . . . . . . . . . . . . . . . . . . . . . . . . . . . . .

5.5 Sparse Matrix Storage Formats . . . . . . . . . . . . . . . . . . . 87

5.6 Further Improvements . . . . . . . . . . . . . . . . . . . . . . . . 90 
6 Hierarchical Matrices Based Solvers for PEEC $\quad 91$

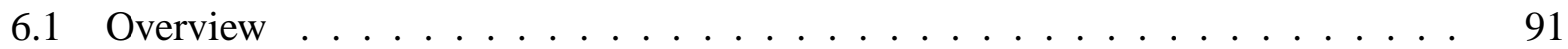

6.1 .1 Direct Strategy . . . . . . . . . . . . . . . . 92

6.1 .2 Iterative Strategy . . . . . . . . . . . . . . . . 93

6.2 Implementation Details for PEEC-Based Circuit Simulation . . . . . . . . . . . 93

$6.2 .1 \quad \mathcal{H}$-Stamping . . . . . . . . . . . . . . . . . . . . . . 94

$6.2 .2 \mathcal{H}$-Preconditioning . . . . . . . . . . . . . . . . 96

6.3 Numerical Results . . . . . . . . . . . . . . . . . . . . . . . . . . 97

6.3 .1 Convergence Behavior Analysis . . . . . . . . . . . . . . 97

6.3.2 Adaptive Preconditioning . . . . . . . . . . . . . . . . 99

$6.4 \mathcal{H}$-Matrices for the Retarded Case . . . . . . . . . . . . . . . . . . . 102

7 Multigrid Solvers for PEEC $\quad 105$

7.1 Motivation . . . . . . . . . . . . . . . . . 106

7.2 Basic Multigrid Strategies _ . . . . . . . . . . . . . . . . . . 107

7.2.1 Geometric Multigrid . . . . . . . . . . . . . . . . . . 107

7.2 .2 Algebraic Multigrid _. . . . . . . . . . . . . . . 107

7.3 AMG Components . . . . . . . . . . . . . . . . . . . 108

7.3.1 The Grid-Coarsening . . . . . . . . . . . . . . . . . . . 108

7.3.2 The Smoothing Process . . . . . . . . . . . . . . . . . . . 110

7.3.3 The Coarse-Level Correction Process _ . . . . . . . . . . . . . . . 110

7.3.4 The Multigrid Algorithm . . . . . . . . . . . . . . . . . . . 111

7.4 Multigrid Preconditioning _ . . . . . . . . . . . . . . . . . . . 112

7.5 AMG Preconditioners for PEEC Matrices . . . . . . . . . . . . . . . . . 113

7.5.1 AMG Preconditioners for Truncated PEEC Matrices . . . . . . . . . . 113

7.5.2 AMG Preconditioners for Data-Sparse PEEC Matrices . . . . . . . . . . 114

7.5 .3 Complexity Analysis . . . . . . . . . . . . . . . . . . . 116

7.6 Implementation Details for PEEC-Based Circuit Simulation ～. . . . . . . . . 116

7.7 Numerical Results . . . . . . . . . . . . . . . . . . . . . . . . . . . . . . . . 118

8 Parallelization Aspects for Hierarchical Methods $\quad 123$

8.1 Overview . . . . . . . . . . . . . . . . . . . . . 123

8.2 Parallel Performance Basics . . . . . . . . . . . . . . . . . . . . 124

8.3 Parallelization Architectures . . . . . . . . . . . . . . . . . . . . 125

8.3.1 Shared Memory Systems . . . . . . . . . . . . . . . . . 125

8.3.2 Distributed Memory Systems _. . . . . . . . . . . . . . 126

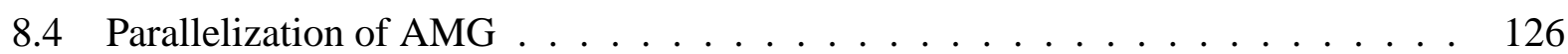

8.4.1 Implementation Issues . . . . . . . . . . . . . . . . . . . . . 127

8.5 Parallelization of $\mathcal{H}$-Matrices . . . . . . . . . . . . . . . . . . . . . . . . . 127 
8.5.1 Parallel $\mathcal{H}$-Matrix Approximation . . . . . . . . . . . . . . . . 127

8.5.2 Parallel $\mathcal{H}$-Matrix-Vector Multiplication . . . . . . . . . . . . . . . . . . 128

8.5.3 Parallel $\mathcal{H}$-Matrix Inversion . . . . . . . . . . . . . . . . . . 128

8.5 .4 Implementation Issues ． . . . . . . . . . . . . . . . . . . . . . 129

8.6 Parallelized Circuit Simulation . . . . . . . . . . . . . . . . . . . . . . 129

8.6.1 Model Extraction Process . . . . . . . . . . . . . . . . . . . . . 129

8.6.2 Simulation Process . . . . . . . . . . . . . . . . . . . 130

8.6 .3 Implementation Details . . . . . . . . . . . . . . . . . . . 130

9 Industrial Applications $\quad 133$

9.1 Motivation and Overview . . . . . . . . . . . . . . . . . . . . 133

9.2 Demonstrator Board Description . . . . . . . . . . . . . . . . . . . . . . 134

9.3 Experimental Verification . . . . . . . . . . . . . . . . . . . 135

9.4 Simulation Verification . . . . . . . . . . . . . . . . . . 136

9.5 Hybrid Solver Strategy $\ldots \ldots \ldots$. . . . . . . . . . . . . . . . . . 139

10 Conclusion $\quad 143$

$\begin{array}{ll}\text { Bibliography } & 147\end{array}$

$\begin{array}{ll}\text { List of Figures } & 163\end{array}$

$\begin{array}{ll}\text { List of Tables } & 165\end{array}$

A Mathematical Basics, Definitions and Notations $\quad 167$

A.1 Basic Matrix Types . . . . . . . . . . . . . . . . . . . . . . . . 167

A.2 Inner Products and Norms _ . . . . . . . . . . . . . . . 168

A.2.1 Vector Norms . . . . . . . . . . . . . . . . . . . 168

A.2.2 Matrix Norms . . . . . . . . . . . . . . . . . . . 168

A.3 Truncated Singular Value Decomposition _ . . . . . . . . . . . . . . 170

A.4 Gershgorin's Circle Theorem . . . . . . . . . . . . . . . . . . . . 171

A.5 Method of Moments . . . . . . . . . . . . . . . . . . 173

A.6 Numerical Integration by Quadrature . . . . . . . . . . . . . . . . . 176

B Green's Function $\quad 179$

$\begin{array}{lr}\text { C Coordinate Systems } & 183\end{array}$

C.1 Orthogonal Right-Handed Coordinate Systems . . . . . . . . . . . . . . . 183

C.2 Cartesian Coordinates . . . . . . . . . . . . . . . . . . . . . . . . . 184

C.3 Curvilinear Coordinates . . . . . . . . . . . . . . . . . . . . . . . . . . 184

C.4 Integration in Curvilinear Coordinates . . . . . . . . . . . . . . . . 186

C.4.1 The Functional Determinant . . . . . . . . . . . . . . . . . 186

C.4.2 Surface Integration . . . . . . . . . . . . . . . . . 188

C.4.3 Volume Integration . . . . . . . . . . . . . . . . . . 190 
$\begin{array}{ll}\text { D Circuit Simulation Basics } & 191\end{array}$

D.1 Fundamental Network Analysis _ . . . . . . . . . . . . . . . . . . . . . . . 191

D.2 The Nodal Analysis Formulation ～. . . . . . . . . . . . . . . . . . . 192

D.3 The Modified Nodal Analysis Formulation . . . . . . . . . . . . . . . . . . 193

D.4 Time Domain Analysis . . . . . . . . . . . . . . . . . . . . . . . . . 194

D.5 Frequency Domain Analysis . . . . . . . . . . . . . . . . 195

$\begin{array}{lll}\text { E } & \text { List of Symbols, Variables and Acronyms } & 197\end{array}$

E.1 Important Sets of Numbers and Constants . . . . . . . . . . . . . . . . . . . . . 197

E.2 Important Variables . . . . . . . . . . . . . . . . . . . . . . . 198

E.2.1 Electromagnetic Basics . . . . . . . . . . . . . . . . . 198

E.2.2 PEEC Modeling . . . . . . . . . . . . . . . . . . . . . . 198

E.2.3 Hierarchical/Multilevel Methods . . . . . . . . . . . . . . . . . . 200

E.2.4 Iterative Methods . . . . . . . . . . . . . . . . . . . . . 201

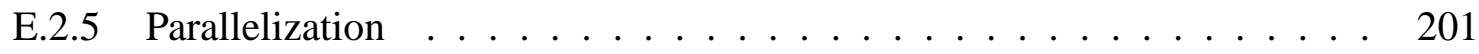

E.3 Mathematical Notations, Transforms and Operators . . . . . . . . . . . . 201

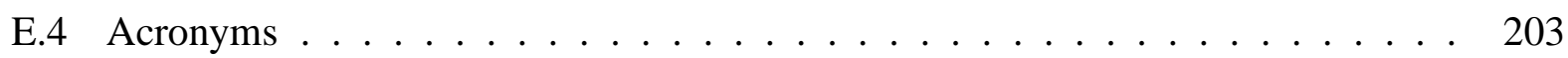

$\begin{array}{ll}\text { Index } & 207\end{array}$

$\begin{array}{lr}\text { Curriculum vitae } & 217\end{array}$ 


\section{1}

\section{Introduction}

$\boldsymbol{E}$

lectromagnetic compatibility (EMC) deals with the interaction between electronic systems and their electromagnetic (EM) environment. Developing electronic components, some EMC aspects have to be taken into consideration to guarantee overall functionality in a complex electromagnetic environment. The two basic requirements of EMC include aspects concerning electromagnetic emission (EMI, electromagnetic interference) as well as electromagnetic susceptibility (EMS), which are contained in the regulatory standards [1,2]. The sensitivity of a device to EMI is described by either susceptibility or immunity. In general, standards distinguish between domestic and industrial use [3] and are based on international or national recommendations from standardization institutes. Increasing data rates of communication systems, decreasing logic levels, decreasing design densities, mixed-signal analog/digital designs [4] within the same package and higher frequency switching power supplies represent challenges in today's electronic design. Therefore it is increasingly important to take EMC requirements into account [5]. For designing high-speed digital and mixed-signal systems it is necessary to include aspects concerning EMI, immunity (including electrostatic discharge (ESD)) as well as signal integrity (SI). These issues have to be captured by suitable modeling of parasitic effects. Many SI problems are electromagnetic phenomena in nature and hence related to EMC. The term signal integrity addresses two concerns in electrical design aspects - the timing and the quality of the signal. A digital system can be examined at three levels of abstraction - logic, circuit theory and electromagnetic fields. Numerical simulation techniques can be applied to evaluate the electromagnetic behavior of a system in a very early design stage [3]. The demand of high-frequency 3D EM modeling and package electrical modeling and the growing importance over the next decades are stressed in [6].

\subsection{Automotive EMC Simulation}

The aspects mentioned so far are also important in the field of automotive EMC, where the growing number of electrical equipment (electronic components concerning safety, reliability and comfort) lead to an increasing electromagnetic level of noise emission and interference in modern cars. For 
recommendations concerning emission and immunity levels refer to [7]. A car is a very complex three-dimensional electronic system, as depicted in principle in Figure 1.1, consisting of a variety of electronic sub-systems. It has to be noticed that, even if all sub-systems were developed

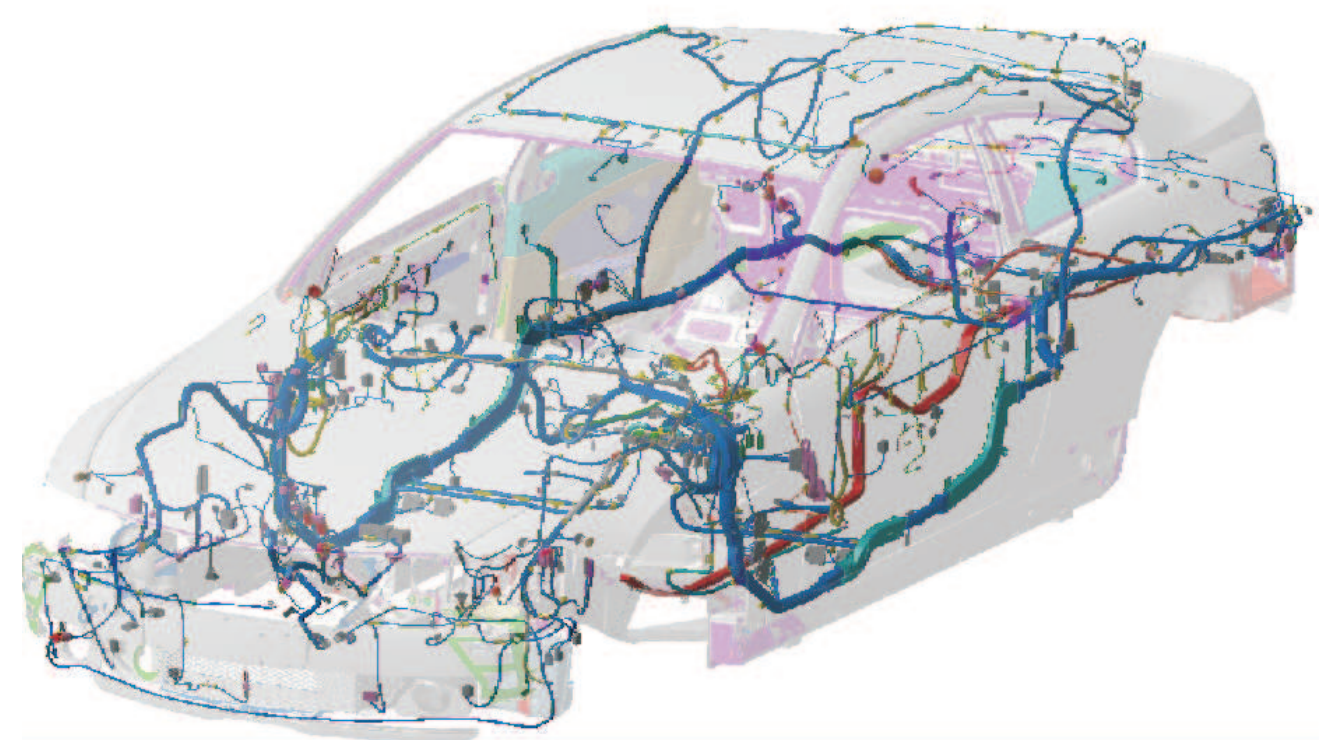

Fig. 1.1: Car body with cable harnesses and control systems, building the main electronic parts of a modern car.

regarding all EMC relevant issues, combining all sub-systems can lead to sources of potential hazards for the overall electromagnetic behavior [8]. Most important electromagnetic phenomena in automotive applications are crosstalk and switching-induced noise. These effects have to be well understood for developing high-performance electronic devices. Numerical simulation techniques are an important key to master EMC-related problems in the automotive design process at an early stage [9]. So, late and cost-intensive re-design cycles can be avoided. A continuous and concurrent EMC simulation process, based on the exchange of EMC models between car manufacturers, electronic suppliers and IC developers, is necessary for an accurate analysis [8, 9]. While the EM behavior of single traces on printed circuit boards (PCBs) can already be simulated efficiently, system simulations on car level represent a challenge. The main reason for this is the difficulty to handle very different geometric scales, related to the three main electronic parts of the system - the car body, the cable harnesses and the components. The different parts can be modeled and analyzed using full-wave 3D-, transmission line (TL) 2D- or circuit formulation approaches, leading to high demands on modern simulation techniques. An accurate simulation of electromagnetic effects up to high frequencies requires a very fine discretization with respect to (w.r.t.) the geometrical size [10]. This can lead to very large dimensions of the resulting systems of equations.

An integral equation (IE) based method, suitable for modeling of PCBs, VLSI (very large scale integration) circuit interconnects [11] and power systems, represents the partial element equivalent circuit (PEEC) method. This method enables a circuit representation of the electric field integral equation (EFIE) and therefore a connection between the field domain and the circuit domain [12]. Circuit simulators compute voltages and currents in lumped circuit elements as resistors, capacitors and inductors. A quasi-stationary consideration can often be sufficient performing SI analysis. Therefore the interconnects can be modeled using lumped circuit elements. If the signal rise time is 
comparable to the round-trip propagation time, however, a lumped model is not sufficient. Including so-called retardation effects, 2D and 3D effects have to be distinguished. While a distributed circuit model in 2D bases upon TL theory (Telegrapher's equations), 3D effects can only be captured applying a full-wave PEEC approach. The SPICE format is a well-established quasi-standard to formulate network equations from a netlist description. It enables a tool-independent exchange of EMC models of different modeling levels among different partners involved in the design process. The circuit description of behavioral models for control modules and ICs, together with TL models for the cable harnesses simplify the integration process and provide the possibility for further extensions. Details can be found in [9].

\subsection{State of the Art}

As described in the previous section, no single modeling tool is able to capture the effects from different parts in automobile electronics satisfactory. A functional decomposition can help to take the characteristic properties into account and enables accurate EMC performance prediction [9, 13]. The multilevel modeling approach proposed in [8] addresses all relevant issues to realize a simulation on system level. The matrices resulting from circuit-based approaches can be very large, dense and ill-conditioned, in general. Therefore, the memory requirements as well as the total simulation times severely limit the performance and the solvable problem size together with conventional techniques. By applying direct methods usually just small problem sizes can be handeled efficiently. These facts make numerical EMC analysis challenging and are the reason for ongoing research activities in this field. The two main bottlenecks in numerical EMC analysis are the modeling and the solving processes. Both tasks have to be optimized to realize a high-performance simulation. As a well-established methodology, model order reduction (MOR) techniques can be applied to large linear electrical networks resulting from PEEC models. A complexity reduction for simulation can be achieved in form of models with reduced dimensions with very similar input-output behavior, compared to the original model $[14,13]$. However, MOR techniques show some important shortcomings. The main disadvantage represents the memory bottleneck for assembling the full parasitics matrices before a model reduction can be applied. Moreover, important effects concerning electric and magnetic field couplings and geometrical mesh data can be lost during reduction and existing simulation artifacts in the results can be intensified [15]. Therefore, it is better to optimize modeling as well as solving processes in the simulator [16]. In combination with highly-sophisticated mathematical techniques, the PEEC-based EM modeling can be improved to realize a very efficient and accurate overall simulation process.

The PEEC method enables the extraction of circuit parameters from a physical design. Similar to other IE-based techniques the parasitic elements form dense matrices, resulting from the BEMbased discretization approach applied to the EFIE. The complexity is quadratic w.r.t. the matrix dimensions concerning the extraction time as well as storage requirements. To enable the analysis of large and dense systems, it is desirable to get matrices in a "storage-sparse" manner.

Hierarchical techniques allow to accelerate the simulation process tremendously. The fast multipole method (FMM) and the closely related hierarchical matrix $(\mathcal{H}$-matrix) technique provide a possibility to extract parasitic effects in an efficient way. Particularly $\mathcal{H}$-matrices provide a "blackbox"-like technique to produce a so-called data-sparse matrix representing an approximation for 
the dense matrix. A specific $\mathcal{H}$-matrix arithmetic enables matrix operations with almost linear complexity, which is essential for an efficient application together with iterative methods. Moreover, $\mathcal{H}$-matrices allow subsequent matrix recompression.

\subsection{Modern Solution Approaches}

In numerical circuit-based EMC simulation an efficient successive solution of a series of large linear systems of equations has to be realized [17]. In some cases, factorizations of the system matrix can be reused for following simulation steps (e.g. a problem of "many right-hand sides"), which can lead to an enhanced efficiency. Nevertheless, a high-performance system simulation only can be realized by applying highly-sophisticated modern numerical solving algorithms. For instance multigrid methods [18] and hierarchical matrices $[19,20]$ are investigated. In the field of automotive applications different modeling strategies represent an additional challenge for the solver. The different characters of the models directly influence the character of the resulting system matrix. The sparsity patterns as well as the dimensions of the matrices can considerably vary. Where the modeling of cable harnesses leads to sparse matrices, in general, matrices resulting from a circuit-level modeling of parts on a PCB are dense.

Direct solution methods are reliable, robust and predictable, both in terms of accuracy and cost [21]. For this reason, they are often applied for solving systems in an industrial environment. Such methods comprise cubic complexity concerning the matrix dimension w.r.t. CPU time and square complexity w.r.t. memory requirements for storing the matrix in the worst case. Although various innovations in this field lead to further improvements, direct methods are not efficient enough for large systems. In automotive EMC simulations, the storage requirements are often assumed to be more critical than time cost. While long simulation times could be tolerated under some circumstances, the lack of storage rapidly limits the problem size to be solved and consequently the achievable accuracy [22]. Standard one-level iterative methods are not able to achieve solutions with optimal complexity, in principle. Nevertheless, they perform better than direct methods. The iteration steps are of linear complexity for the typical systems to be solved, but the convergence behavior deteriorates with increasing problem sizes. For a given accuracy the number of required iteration steps grows linearly with the number of unknowns. This can be observed particularly in the field of circuit simulation approaches, where matrices show very large condition numbers. Although various enhancements have been made to improve the performance of iterative methods, large problems can not be solved efficiently enough using direct or conventional preconditioned iterative solution methods for sparse matrices. A breakthrough for an efficient solution of large industrial real-life problems from computational EMC analysis was achieved by introducing hierarchical solution algorithms. For more detailed information refer to [23, 24, 25, 26, 18]. At least for special problems, such hierarchical methods can achieve optimal complexity with linear order concerning the matrix dimension, referring to computational time as well as storage requirements. A further enhancement of such techniques can only be achieved using parallelization techniques in the most cases. A parallel implementation, however, is often difficult to realize. To achieve full computational power on parallel machines, the algorithms have to be tested w.r.t. their suitability for parallel processing and adapted accordingly. Hierarchical algorithms as FMM, $\mathcal{H}$-matrices or wavelet-based approaches are well parallelizable. The proposed hierarchical techniques are 
promising to enable efficient numerical simulations on system level. In [8] such simulation techniques are employed to regard the different levels of abstraction. The model accuracies on individual abstraction levels, however, have a strong influence to the accuracy of the system simulation.

\subsection{Contents and Contributions of this Thesis}

This thesis addresses the main shortcomings and bottlenecks in PEEC-based circuit simulation and proposes a methodology for a generally applicable simulation strategy, suitable for automotive EMC simulations on system level. An evaluation of specific properties of both models as well as simulation techniques and limitations in the simulation process shall be demonstrated and remedy methods are discussed. Although the given aspects are rather universal in nature, the relation to automotive applications is kept in mind. The presented work combines topics from mathematics as well as from electrical engineering, which are absolutely necessary for the development of high-performance algorithms and their efficient implementation in industrial codes. The proposed algorithms shall work well with general circuit-based approaches, and especially together with PEEC-based approaches for cartesian as well as curvilinear coordinates and TL models. As described in the following chapters, especially the algebraic multigrid method and hierarchical matrices have proven to be useful for circuit-based simulation. For historical reasons, a sparsification approach based on partial reluctances was also considered, ensuring robust and stable simulations. The combination of $\mathcal{H}$-matrices, AMG and PEEC models represents a novel approach to enable system simulations with significantly reduced complexity. In the course of this thesis, a complete framework of the mentioned modern hierarchical techniques will be introduced in detail to realize accelerated modeling as well as solving processes. In the past multigrid approaches were already discussed in a PEEC-modeling context in [3]. To enable effective preconditioning for relatively low frequencies, M. VERBEEK proposed a basis transformation that separates capacitive and inductive effects. A more flexible approach for the treatment of PEEC-based circuit simulation matrices, which moreover can be expected to perform more efficient and more accurate is proposed in the scope of this thesis together with a standard AMG solver. Due to the specific properties of circuit simulation matrices, however, care has to be taken realizing an efficient implementation. The investigations presented in this thesis demonstrate the requirements as well as occuring problems, concrete implementation guidelines and numerical results. Efficient matrix storage formats are applied, which are well-established in existing professional industrial codes. Each method is evaluated by means of complexity analysis, taking into consideration the required accuracy. Completing each chapter, implemention issues for an efficient practical implementation are given. The work is completed by detailed analysis of an industrially relevant automotive EMC problem. For the described example, simulation results are compared to measurement results to show accordance with reality. The presented hierarchical strategies are already well-established in other application fields.

The thesis is organized as depicted in Figure 1.2. Following this introduction, Chapter 2 shortly recapitulates some physical basics, which are important for the numerical analysis of electromagnetic effects. Chapter 3 presents details concerning the PEEC method for orthogonal as well as for non-orthogonal discretizations. W.r.t. their importance for the remaining chapters, the equivalent circuit formulation and the principle structure of a circuit-level simulator are shortly explained. To 


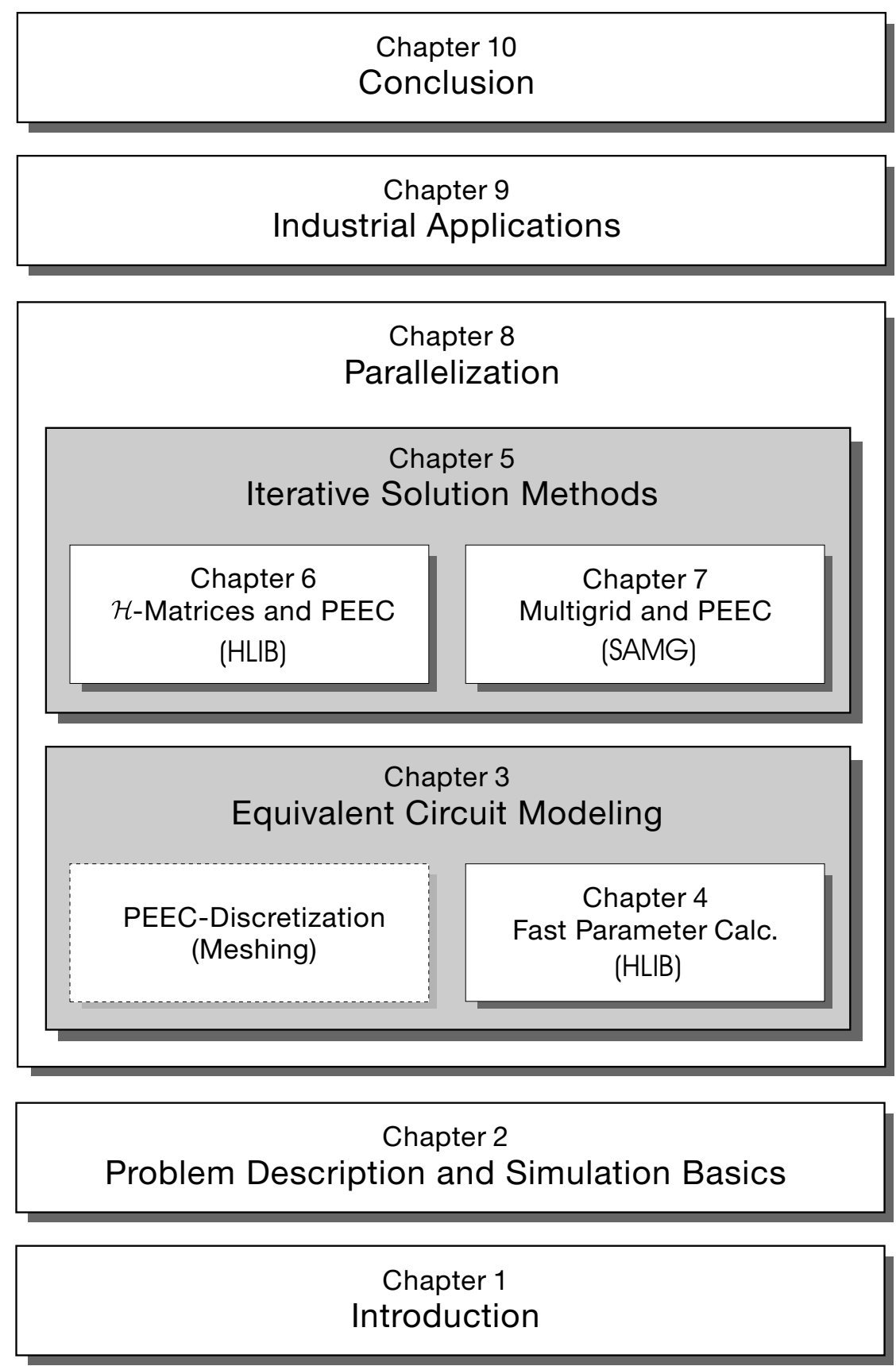

Fig. 1.2: Organization of the thesis.

avoid simulation artifacts and to ensure high accuracy, some guidelines concerning mesh generation and numerical integration are given. A sparse and positive definite system matrix, suitable for iterative solution methods, can be obtained using a reluctance-based approach together with a truncation, enabling stable simulations in the TD and the FD with high accuracy. To take into account dielectric as well as retardation effects, fundamental details are presented with regard to circuit implementations. Chapter 4 starts with a short review of the fast multipole principle to illustrate the benefits of hierarchical matrices in fast model extraction. After a detailed explanation of the foundation of $\mathcal{H}$-matrices and complexity considerations, the element evaluation process based on the cross-approximation principle is described. The proposed strategies are based on techniques from the $\mathcal{H}$-matrix library HLIB [27]. The functionality of $\mathcal{H}$-matrices will be used to increase 
the efficiency w.r.t. time and memory requirements as well as the modeling accuracy. Implementation details and numerical examples are given to make the workflow clear and to validate the functionality by means of concrete problems. Moreover, additional improvements are addressed to further increase the performance. An efficient overall simulation process requires an analysis of circuit-specific matrix properties. The fundamental investigations w.r.t. suitable solver strategies are presented in Chapter 5. Basic iterative methods for the solution of linear systems of equations from a circuit approach as well as preconditioning techniques are explained. In order to take advantages of the sparsity of the system matrices, efficient matrix storage formats are presented, suitable for the proposed methods. Especially for BEM-based approaches a useful treatment can be obtained using a data-sparse representation of the circuit simulation matrices, which is explained in Chapter 6. Although $\mathcal{H}$-matrices can efficiently be solved using direct strategies based on $\mathcal{H}$-matrix arithmetics, applying iterative one-level methods can lead to an increased performance. Implementation issues concerning specific $\mathcal{H}$-stamping for circuit simulation matrices and suitable $\mathcal{H}$-preconditioning are presented. Numerical results for real-life problems applying adaptive $\mathcal{H}$-preconditioned Krylov-subspace simulations are given. Chapter 7 presents an algebraic multigrid framework for common circuit-based simulation approaches. After a short summary of basic multigrid theory and multigrid preconditioning, details for the treatment of truncated PEEC matrices as well as for data-sparse PEEC matrices are presented. The proposed strategies are based on techniques from the standard algebraic multigrid library SAMG [28]. The introduced functions connecting multigrid-based strategies and $\mathcal{H}$-matrices together with local $\mathcal{H}$-matrix-based mesh-refinement, can help to further improve the overall efficiency and accuracy. The individual approaches are compared, referring to their complexity w.r.t. time and storage requirements. Implementation issues concerning the software library SAMG are given for reluctance-based circuit models in the TD and the FD. A validation of the efficiency of the proposed strategy is presented for industrially relevant problems. Comparisons to a direct solver are given. Due to the almost optimal complexity of hierarchical approaches, a further efficiency enhancement of hierarchical methods often can only be achieved by applying parallelization techniques. Chapter 8 points out some important aspects of parallelization strategies for AMG as well as for $\mathcal{H}$-matrices and is presented for completion. Implementation details for the integration into an existing EMC simulation environment are discussed. In Chapter 9, the efficiency, robustness and accuracy of the proposed methodology are demonstrated. Validation of the received results is performed by means of measurements of an existing equipment. A conclusion and outlook on future research is given in Chapter 10. 


\section{2 \\ Simulation Basics of Electromagnetic Effects}

$\boldsymbol{A}$

ny time-dependent electrodynamic problem can be described in terms of MAXWELL's equations which are given in Section 2.1. These four equations represent a system of coupled partial differential equations (PDE), inducing a connection between electromagnetic (EM) field components, current and charge densities and material properties. Starting from the electromagnetic scattering problem in Section 2.2, including retarded electrodynamic potentials from Section 2.3, an integral equation formulation (Section 2.4) can be obtained which describes the electromagnetic effects in total space completely.

To realize a fast and efficient numerical simulation of parasitic effects, the $\underline{\text { method } o f} \underline{\text { moments }}$ (MoM) (Section 2.5, Appendix A.5) is a suitable technique to transform a linear functional equation into a matrix equation [29]. For this purpose, a domain discretization is applied to approximate the continuous quantities. A solution can be obtained applying conventional solvers for linear equations $[24,30,31]$.

\subsection{Maxwell's Equations}

For a mathematical description of electrodynamic effects, a domain in space free of sources is considered, containing an inhomogeneity $\mathcal{X} \in \mathbb{R}^{3}$. The inhomogeneity with surface $S$ and volume $V$ (refer to Figure 2.1 on page 12) is characterized using relative permittivity $\varepsilon_{\mathrm{r} 1}$ and relative permeability $\mu_{\mathrm{r} 1}$, which are both assumed to be location-independent. An applied incident and time-dependent wave field $\left(\vec{E}^{i}(\vec{r}, t), \vec{H}^{i}(\vec{r}, t)\right)$, generated by sources at infinity, causes a surface current density ${ }^{1} \vec{J}$ and a surface charge density $\rho$ at the surface of the scattering object [32]. Electromagnetic waves are characterized by the coupling of electric $(\vec{E})$ and magnetic $(\vec{H})$ field

\footnotetext{
${ }^{1}$ In the literature, the term vector current density demonstrates the vectorial character of the current. For simplification reasons, this auxiliary expression is dropped in the remainder.
} 
quantities. The propagation direction of the electromagnetic wave is given by the Poynting vector $\vec{S}=\vec{E}^{i} \times \vec{H}^{i}$, which is also a measure for the power density [33]. With ideal conducting scattering objects, the resulting current and charge densities are restricted to the object's surface due to the material properties. This leads to the fact, that the object's interior is free of field. In the absence of a scatterer, the incident field quantities have to be a solution of Maxwell's equations ${ }^{2}$ [32], which can be written in differential equation formulation or integral equation formulation [34]:

\begin{tabular}{|cc|c|}
\hline \multicolumn{2}{|c|}{ Differential formulation } & \multicolumn{2}{c|}{ Integral formulation } \\
of Maxwell's equations \\
\hline \hline $\operatorname{rot} \vec{H}(\vec{r}, t)=\vec{J}(\vec{r}, t)+\frac{\partial \vec{D}(\vec{r}, t)}{\partial t}$ & $(2.1)$ & $\oint_{C} \vec{H}(\vec{r}, t) \mathrm{d} \vec{s}=\iint_{S}\left(\vec{J}(\vec{r}, t)+\frac{\partial \vec{D}(\vec{r}, t)}{\partial t}\right) \mathrm{d} \vec{S}$ (2.2) \\
\hline $\operatorname{rot} \vec{E}(\vec{r}, t)=-\frac{\partial \vec{B}(\vec{r}, t)}{\partial t}$ & $(2.3)$ & $\oint_{C} \vec{E}(\vec{r}, t) \mathrm{d} \vec{s}=-\iint_{S} \frac{\partial \vec{B}(\vec{r}, t)}{\partial t} \mathrm{~d} \vec{S}$ \\
\hline $\operatorname{div} \vec{D}(\vec{r}, t)=\rho(\vec{r}, t)$ & $(2.5)$ & $\oiint_{S} \vec{D} \mathrm{~d} \vec{S}=\iiint_{V} \rho(\vec{r}, t) \mathrm{d} V$ \\
\hline $\operatorname{div} \vec{B}(\vec{r}, t)=0$ & $(2.7)$ & $\oiint_{S} \vec{B}(\vec{r}, t) \mathrm{d} \vec{S}=0$ \\
\hline
\end{tabular}

The material equations

$$
\begin{aligned}
& \vec{D}(\vec{r}, t)=\varepsilon \vec{E}(\vec{r}, t) \\
& \vec{B}(\vec{r}, t)=\mu \vec{H}(\vec{r}, t) \\
& \vec{J}(\vec{r}, t)=\sigma \vec{E}(\vec{r}, t)
\end{aligned}
$$

establish a combination between field strengths, flux densities and current densities via material impacts. The following investigations are restricted to isotropic, linear and homogeneous materials. In addition to the electric field strength $\vec{E}$ and the magnetic field strength $\vec{H}$, electric flux density $\vec{D}$, magnetic flux density $\vec{B}$, the current density $\vec{J}$, the volume charge density $\rho$, the dielectric constant $\varepsilon=\varepsilon_{0} \varepsilon_{\mathrm{r}}$, the permeability constant $\mu=\mu_{0} \mu_{\mathrm{r}}$ and the electric conductance $\sigma$ are involved. In the following, the material parameters are assumed to be local, scalar and constant over time. The first Maxwell's equation (Equations (2.1) and (2.2)), which is also known as AMPERE's Law, implies that the magnetic flux density $\vec{B}$ exhibits curls at locations where currents flow and the electric fields vary with time [35]. As it can be observed from (2.1) or (2.2), the displacement current density $\partial \vec{D} / \partial t$ also contributes to a magnetic field [36]. The first Maxwell's equation incorporates the basics of charge conservation. The continuity equation relates the current

\footnotetext{
${ }^{2}$ James Clerk Maxwell, Scottish mathematician and physicist, 1831-1879.
} 
density $\vec{J}$ with the charge density $\rho$ [32], which is apparent from applying the divergence operation to both sides of (2.1) or (2.2), respectively. Equation (2.1) can be written as

$$
\operatorname{div} \vec{J}(\vec{r}, t)+\operatorname{div} \frac{\partial \vec{D}(\vec{r}, t)}{\partial t}=\operatorname{div} \vec{J}(\vec{r}, t)+\frac{\partial \rho(\vec{r}, t)}{\partial t}=0
$$

regarding that $\operatorname{div}(\operatorname{rot} \vec{H})=0$ holds. The second Maxwell's equation (2.3) and (2.4), which is also known as the FARADAY's Law, states that the electric field strength contains curls at locations where magnetic fields vary with time [35]. Time dependent and quasistationary Maxwell's equations differ in the existence of the displacement current density and the induction term $-\partial \vec{B} / \partial t$ [37]. In various cases the displacement current term can be neglected, while Faraday's law has to be fully regarded [38]. A description of phenomena based on wave propagation, disregarding the displacement current, is not possible in general. The skin effect and eddy current effects can be included independently in a straightforward manner. If time variations can be assumed to occur slowly, such a quasi-stationary approximation by neglecting the displacement current term can be applied. The quasi-stationary approximation implies that

$$
\beta r=\frac{\omega r}{c} \ll 1 \quad \rightarrow \quad \frac{r}{c} \ll \frac{1}{\omega}
$$

with the wave number $\beta=\omega \sqrt{\varepsilon \mu}=\frac{\omega}{c}$ [36] in the inhomogeneous wave equation, the propagation distance $^{3} r \triangleq\left\|\vec{r}^{\prime}-\vec{r}\right\|$ between source and sink points and the angular frequency $\omega$. Electric charges are assumed to represent sources of electric fields (refer to (2.5) and (2.6), GAUSS's Law). The magnetic field is assumed to be free of sources (refer to (2.7) and (2.8)) in the following. Magnetic sources as referred in the literature [39, 40, 34] are neglected.

\subsection{The Electromagnetic Scattering Problem}

For a solution of the electromagnetic field problem in automotive EMC simulation, Maxwell's equations often have to be applied to arbitrarily shaped complex geometric objects. Objects containing dielectric or permeable materials often build up boundary layers togehter with materials implying conductance $\sigma$. They are termed perfect electric conductors (assuming $\sigma \rightarrow \infty$ ) in the following. Boundary conditions will be enforced by the object, leading to a boundary value problem. The whole three-dimensional space is assumed to be separated into two domains called scatterer and surrounding with finite conductances $\sigma_{1}$ and $\sigma_{2}$, respectively. For an exact formulation of the boundary conditions, the reader is referred to [41]. The sources, causing these field quantities, are supposed to be located at infinity and are not specified further on. They generate an incident, time dependent electromagnetic wave field $\left(\vec{E}^{i}(\vec{r}, t), \vec{H}^{i}(\vec{r}, t)\right)$, exciting surface current density $\vec{J}$ and surface charge density $\rho$ at the scatterer's surface. The presence of the scatterer causes a scattered electromagnetic field $\left(\vec{E}^{s}(\vec{r}, t), \overrightarrow{H^{s}}(\vec{r}, t)\right)$ from the incident field components, which has to fulfill Maxwell's equations in the exterior. Due to the linearity of Maxwell's equations, the total field in space can be calculated by superposition of incident and scattered electromagnetic field quantities $[42,41]$ as

$$
\vec{E}(\vec{r}, t)=\vec{E}^{i}(\vec{r}, t)+\vec{E}^{s}(\vec{r}, t)
$$

\footnotetext{
${ }^{3}$ Without any specification $\|\cdot\|$ denotes the Euclidean norm $\|\cdot\|_{2}$ (refer to Appendix A.2).
} 


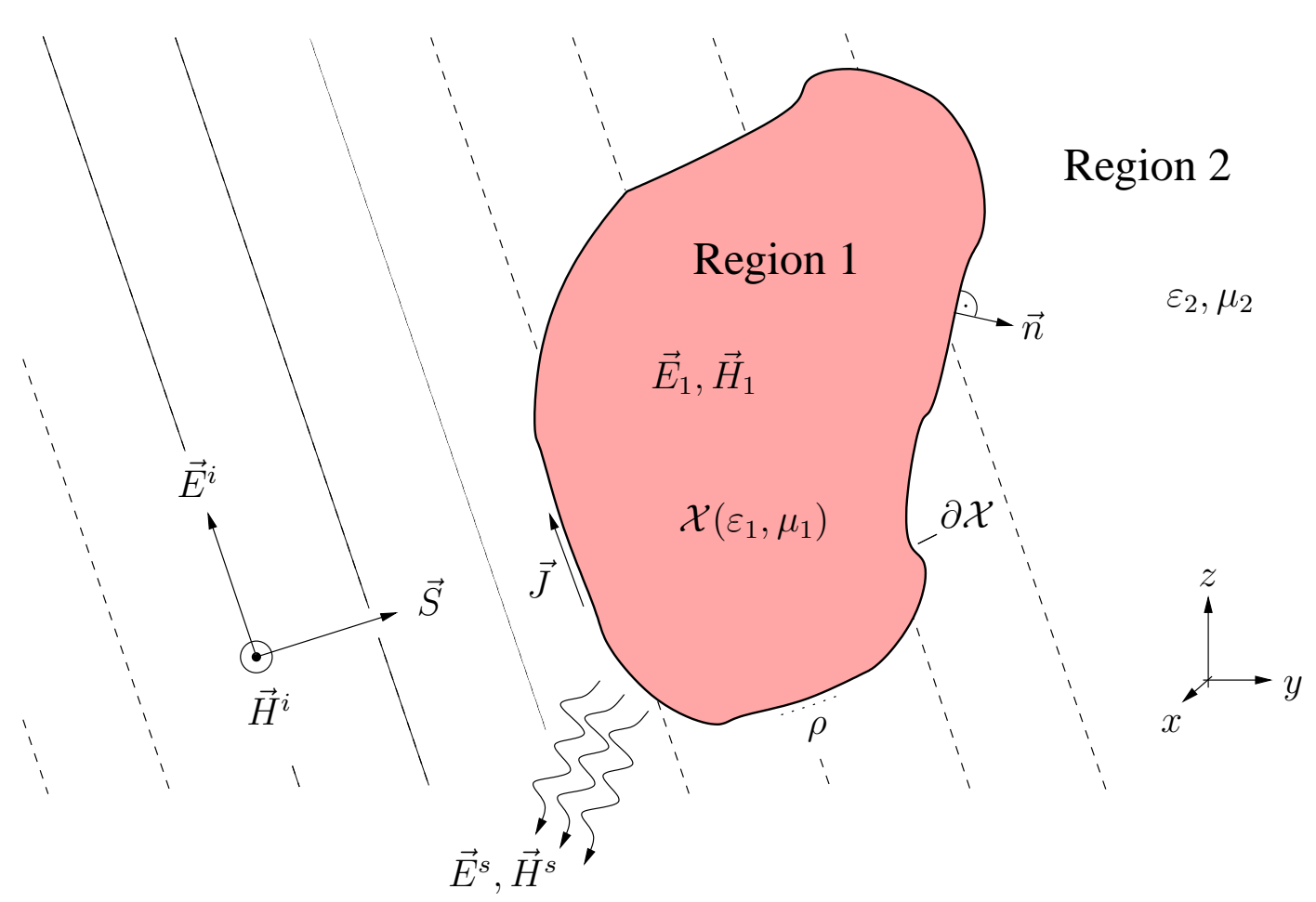

Fig. 2.1: The electromagnetic scattering problem. An arbitrarily shaped scattering object $\mathcal{X}$ with boundary $\Gamma \triangleq \partial \mathcal{X}$ is illuminated by a plane wave causing a scattered electromagnetic field.

and

$$
\vec{H}(\vec{r}, t)=\vec{H}^{i}(\vec{r}, t)+\vec{H}^{s}(\vec{r}, t) .
$$

A solution of the scattering problem can be achieved by applying the so-called equivalence principle [41]. This theorem states, that inhomogeneous materials are equivalent to incident sources in space, concerning their impact on the electromagnetic field. Based on this fact, the electromagnetic action of a scatterer can be emulated by "fictitious" currents on the scatterer's surface (refer to Figure 2.1). These currents can be considered as sources of the scattered field [32]. The field quantities inside and outside the object thus can be computed separately, regarding the underlying medium. Together with dielectric objects, equivalent surface and volume currents have to be applied $[39,40]$. For applications as considered in this thesis, the scatterer's surface is assumed to be perfect conducting. Inside the conductor, OHM's Law (2.11) must hold [3].

\subsection{The Retarded Electrodynamic Potentials}

A solution of the system of Maxwell's equations turns out to be very complicated for industrially relevant (real-life) problems. The introduction of so-called electrodynamic potentials enables a transformation of the system of coupled partial differential equations of the first order into decoupled differential equations of higher order $[32,36]$, which are easier to solve. The field strengths can be computed as derivatives from the electrodynamic potentials, which have to fulfill Maxwell's equations [33]. From a system-theoretic point of view, the current density $\vec{J}$ represents the input and the unknown electric field strength $\vec{E}$ represents the output of the system. Similar to linear systems, a system derived from linear partial differential equations can be characterized by the impulse response or transfer function completely [43, 34]. A solution of the considered problem 
can be obtained by superposition of the impulse responses, where excitations with impulse current sources are applied at different source locations. Green's function $G\left(\vec{r}_{p}, \vec{r}_{q}\right)$, connecting the observation point $\vec{r}_{p}$ to the source point $\vec{r}_{q}$ (refer to Appendix B), embodies these impulse responses and represents the electric field as a response to the excitation. Thus, for a one-conductor system the time-dependent magnetic vector potential

$$
\vec{A}\left(\vec{r}_{p}, t\right)=\mu \iiint_{V_{q}} G\left(\vec{r}_{p}, \vec{r}_{q}\right) \vec{J}\left(\vec{r}_{q}, t_{r}\right) \mathrm{d} V
$$

and the time-dependent electric scalar potential

$$
\Phi\left(\vec{r}_{p}, t\right)=\frac{1}{\varepsilon} \iiint_{V_{q}} G\left(\vec{r}_{p}, \vec{r}_{q}\right) \rho\left(\vec{r}_{q}, t_{r}\right) \mathrm{d} V
$$

at the observation point can be defined. For an exact mathematical derivation, the reader is referred to [38]. The propagation time of the electromagnetic wave between source point and observation point in (2.16) and (2.17) can be given as

$$
t_{r}=t-\frac{\left\|\vec{r}_{p}-\vec{r}_{q}\right\|}{c}
$$

with $c=2.998 \cdot 10^{8} \mathrm{~m} / \mathrm{s}$ representing the speed of light; $t_{r}$ is also called retardation time of the electromagnetic wave.

\subsection{The Electric Field Integral Equation}

Maxwell's equations together with the electrodynamic potentials enable a computation of electric and magnetic field quantities if the field sources are known. The current and charge densities on the scatterer's surface represent the scattering sources. These are unknown in general, which makes additional equations necessary $[41,12,44]$. Integral or integro-differential equations ${ }^{4}$ can be formulated for the unknown sources by regarding the continuity and the boundary conditions of the source-field-relation. For that purpose, equivalent sources are placed on the scatterer's surface. The currents thus only exist as surface currents. Formulating the homogeneous linear boundary value problem as boundary integral equation (BIE) with suitable boundary conditions, a solution of the IE can be obtained $[45,46,3]$. The so-called boundary element $\underline{\text { method }}$ (BEM) ${ }^{5}$ is a flexible discretization technique to solve these equations numerically. Whereas applying the BEM results in a dense matrix, the matrices resulting from a finite element method (FEM) approach are sparse, in general. The number of degrees of freedom (DOF), however, can be drastically reduced by

\footnotetext{
${ }^{4}$ In accordance with the literature, equations are termed integral equations instead of the more exact mathematical expression "integro-differential equations" if the derivative is only applied to the integrand [32, 40].

${ }^{5}$ Where the finite element method (FEM) assumes a bounded computational domain, the modeling of such a problem class always demands some restriction implying less accuracy. Using the BEM, we are able to obtain exact results on arbitrary points in the unbounded domain. In this case only the surface of the geometric body needs to be discretized and therefore the dimension of the computational domain is of order one less than the dimension of an equal FEM discretization. Moreover, the BEM allows the treatment of exterior domain problems in a natural way [47].
} 
applying BEM approaches. Based on the retarded electrodynamic potentials and regarding (2.14), the total electric field at an arbitrary location $\vec{r}$ can be computed as

$$
\vec{E}(\vec{r}, t)=\vec{E}^{i}(\vec{r}, t)+\vec{E}^{s}(\vec{r}, t)=\vec{E}^{i}(\vec{r}, t)-\operatorname{grad} \Phi(\vec{r}, t)-\frac{\partial \vec{A}(\vec{r}, t)}{\partial t} .
$$

If the observation point $\vec{r}$ is located on the object's surface, the total electric field can be written as

$$
\vec{E}(\vec{r}, t)=\frac{\vec{J}(\vec{r}, t)}{\sigma} \quad \vec{r}=\vec{r}_{p}=\vec{r}_{q} \in \Gamma \triangleq \partial \mathcal{X},
$$

resulting in

$$
\vec{E}^{i}(\vec{r}, t)=\frac{\vec{J}(\vec{r}, t)}{\sigma}+\operatorname{grad} \Phi(\vec{r}, t)+\frac{\partial \vec{A}(\vec{r}, t)}{\partial t}
$$

for the incident electric field $\vec{E}^{i}$. A perfect conducting scattering object is assumed in the following, implying $\vec{E}_{1}=\overrightarrow{0}$ inside the scatterer. Inserting (2.16) and (2.17) into (2.21) and due to the fact that only observation points on the conductors surface are considered (vanishing tangential component of the total electric field), the so-called electric field integral equation (EFIE) in the TD

$$
\begin{aligned}
\vec{n}\left(\vec{r}_{p}\right) \times \vec{E}^{i}\left(\vec{r}_{p}, t\right) & =\vec{n}\left(\vec{r}_{p}\right) \times\left[\frac{\vec{J}\left(\vec{r}_{p}, t\right)}{\sigma}\right] \\
& +\vec{n}\left(\vec{r}_{p}\right) \times\left[\mu \iiint_{V_{q}} G\left(\vec{r}_{p}, \vec{r}_{q}\right) \frac{\partial \vec{J}\left(\vec{r}_{q}, t_{r}\right)}{\partial t} \mathrm{~d} V\right] \\
& +\vec{n}\left(\vec{r}_{p}\right) \times\left[\frac{1}{\varepsilon} \operatorname{grad} \iiint_{V_{q}} G\left(\vec{r}_{p}, \vec{r}_{q}\right) \rho\left(\vec{r}_{q}, t_{r}\right) \mathrm{d} V\right]
\end{aligned}
$$

can be offered with the surface normal vector $\vec{n}$ pointing away from the scattering object. The EFIE is often termed mixed potential integral equation (MPIE) in the literature [48]. It is an important equation for the solution of boundary value problems of time dependent processes and enables a relation between the electric field and the sources. For a calculation of unknown current and charge distributions on ideal conducting objects, the so-called magnetic field integral equation (MFIE) can be used equivalently [32, 40,43]. The MFIE often shows a higher computational complexity compared to the EFIE. The EFIE is better suited for the modeling of arbitrary shaped, thin, open and closed objects, in general. Therefore it is advantageous for general EM problems, especially for the PEEC method (refer to Chapter 3). While the MFIE is restricted to an efficient modeling of closed structures [49, 43, 40, 32], applying a combination of both, the so-called combined field integral equation (CFIE) can be advantageous. Internal resonances, occuring from the EFIE and MFIE, can cause problems [3]. In the following the EFIE is used exclusively.

\subsection{Numerical Field Computation Basics}

Regarding the underlying geometry, material parameters and excitation, the EFIE describes the electromagnetic scattering problem completely. The unknown electric and magnetic field quantities can be determined indirectly by using equivalent currents and charges, referring to the equivalence principle as described in Section 2.2. From a physical point of view, one has to find the 
surface current whose resulting electric field meets the boundary conditions by taking the external electric field into consideration. Due to the enormous complexity of an analytic solution of reallife problems, it is advantageous to transform a linear functional equation as the EFIE into a matrix equation [32, 44, 50]. Applying common numerical solving techniques an approximative solution can be obtained, adequate to predict the continuous behavior. For a more detailed consideration of numerical 3D field calculation techniques, the reader is referred to [43, 51]. The $\underline{\text { method } o f}$ moments (MoM), which was published by R. F. HARRINGTON [29] the first time, belongs to the so-called matrix methods and is very suited for the solution of field problems. With regard to the close relation to the PEEC method [52] and due to its importance for iterative solution techniques, the theoretical basics and characteristics of MoM are shortly described in Appendix A.5. The $\mathrm{BEM}$, as a weighted residual technique, is a variant of the method of moments where basis and test functions are only defined on the boundary surface. For that purpose, an arbitrary discretization of the underlying structure into suitable surface segments or cells is assumed. Neighbouring segments are connected through so-called elementary basis functions. Applying a superposition, the basis functions enable to approximate a continuous current or charge distribution. Together with the BEM approach only the conducting or dielectric medium has to be discretized. A modeling technique incorporating these advantages represents the already mentioned PEEC method which is described in detail in the following chapter. 


\section{3 \\ Equivalent Circuit Modeling}

$\boldsymbol{A}$

tremendous increase in clock frequencies and an enormous rise in packing densities of modern high-speed electronic circuits make it necessary to capture the parasitic effects in electromagnetic modeling very accurately. Simple TL models in 2D are often not sufficient to simulate complex irradiation effects of modern mixed EM and circuit problems [3]. For accurate SI analysis as well as EMC issues of PCBs, interconnects and packaging, the $3 D$ partial element equivalent circuit (PEEC) method $[12,53,54,55]$ proved to be useful. The PEEC method, which was published by A. E. RUEHLI in 1974 the first time, enables a circuit interpretation of the EFIE full-wave formulation in 3D for a combined analysis of electromagnetic and circuit problems. Power systems, VLSI systems, EMI and ESD are typical problems, which are studied by means of circuit simulators based on the solution of ordinary differential equations (ODE). The PEEC formulation proved to be equivalent to a MoM formulation but it exhibits some important advantages. The resulting circuit representation can be analyzed in the TD as well as in the FD using conventional SPICE simulators [56]. It allows to include dielectrics and retardation effects, it is compatible with non-orthogonal discretizations [57, 48], it enables the application of various methods of model order reduction and it is very flexible for extensions by additional circuit elements. In contrast with MoM, PEEC is a full-spectrum method, valid from DC to the maximum admissible frequency defined by the discretization.

After a short recapitulation of the Petrov-Galerkin approach in Section 3.1, the derivation of the PEEC method is described for orthogonal as well as for non-orthogonal discretizations in Section 3.2 and 3.3, respectively. The equivalent network can be constructed as shown in Section 3.4 by including dielectric effects (Section 3.5) and suitable for a circuit-level simulation (Section 3.6). Taking accuracy and validity aspects from Section 3.7 into consideration, together with the basics of modern cubature techniques (Section 3.8) a suitable EM modeling and simulation strategy can be realized. With regard to iterative methods, Section 3.9 describes a matrix sparsification approach for parasitics, preserving model passivity. For completeness, retarded PEEC models are described shortly in Section 3.10. 


\subsection{The Petrov-Galerkin Approach}

Based on the so-called Petrov-Galerkin discretization, the EFIE (2.22) can efficiently be solved numerically, as mentioned in Section 2.5. The approximative solution can be found by applying a Galerkin projection, leading to a minimization problem. The Petrov-Galerkin approach represents a weighted residual method with basis and test functions originating from different "ansatz spaces". Together with PEEC a $3 D$ volume discretization of current densities and a $2 D$ surface discretization of charge densities are applied to enable the mapping to an equivalent circuit representation. The refinement of the discretization is specified by the dimensions of the cells and represents a tradeoff, adjusted to the problem. An upper bound for the meshsize is affected by the required accuracy as well as the wavelength of the highest occuring frequency. For further details refer to Section 3.7. The Petrov-Galerkin approach approximates the current and charge density distributions by accomplishing linear combinations of the form

$$
\vec{J}(\vec{r}, t)=\sum_{m=1}^{N_{V}} J_{m}\left(t_{m}\right) \vec{f}_{b m}^{(\vec{J})}\left(\vec{r}_{m}\right)
$$

and

$$
\rho(\vec{r}, t)=\sum_{n=1}^{N_{S}} \rho_{n}\left(t_{n}\right) f_{b n}^{(\rho)}\left(\vec{r}_{n}\right),
$$

using vectorial current basis functions $\vec{f}_{b}^{(\vec{J})}$ and scalar charge basis functions $f_{b}^{(\rho)}$. The basis functions are weighted by means of current density amplitudes $J_{m}$ and charge density amplitudes $\rho_{n}$ for $N_{V}$ volume cells and $N_{S}$ surface cells. With the position vectors of the cell locations $\vec{r}_{m}$ and $\vec{r}_{n}$, the discrete times for summation result in $t_{m / n}=t-\left\|\vec{r}_{q}-\vec{r}_{m / n}\right\| / c$. The application of the Petrov-Galerkin method requires the definition of a suitable inner product of the form

$$
\langle f, b\rangle=\iiint_{V} f(\vec{r}) b(\vec{r}) \mathrm{d} V,
$$

leading to an equation system [58]

$$
\vec{u}^{i}=\boldsymbol{R} \cdot \vec{i}+\boldsymbol{L} \cdot \dot{\vec{i}}+\boldsymbol{P} \cdot \vec{q}
$$

if applied to (2.22). At the moment, dielectric effects are neglected. The individual terms can be interpreted as voltage drops at partial elements of an equivalent circuit. Different from electromagnetic field problems, the driving force in an electrical device is modeled as a voltage drop located at the connection between wires and the board [3]. Among the vectors $\vec{i}$ and $\vec{q}$ of the unknown currents and charges, matrices $\boldsymbol{R}, \boldsymbol{L}$ and $\boldsymbol{P}$ represent ohmic losses, partial inductances and partial coefficients of potential. The derivative w.r.t. time is denoted by $i=\partial i / \partial t$. A discretization of the continuity equation (2.12) leads to the expression

$$
\boldsymbol{D} \cdot \vec{i}+\dot{\vec{q}}=\overrightarrow{0}
$$

Orthogonal cells proved to be appropriate for the PEEC method w.r.t. a tradeoff between complexity and accuracy [3]. This leads to the so-called Manhattan discretization. To ensure a physical feasible modeling the so-called RITZ-GALERKIN approach, as a specialization of the Petrov-Galerkin 
approach with identical basis and test functions, is applied in the context of PEEC ${ }^{1}$. The identical basis and test functions guarantee constant current and charge densities in each cell which is necessary to transform the electric field problem to discrete and constant circuit primitives.

\subsection{Partial Elements for a Manhattan Discretization}

A very common kind of discretization for a simple implementation is based on the partitioning of conducting (or dielectric) structures into orthogonal (rectangular) elements, a so-called Manhattan discretization. The three coordinate directions of the cartesian coordinate system are treated independently. For a detailed consideration of orthogonal "right-handed" coordinate systems, refer to Appendix C.

\subsubsection{Discretization of the EFIE}

In order to apply an orthogonal discretization w.r.t. current and charge densities, a partitioning of the conductor's surface and volume into orthogonal cells is necessary. Constant pulse functions are used as basis and test functions for each cell. The current density $\vec{J}$ can be defined as

$$
\vec{J}=J_{x} \vec{e}_{x}+J_{y} \vec{e}_{y}+J_{z} \vec{e}_{z}
$$

together with orthogonal global cartesian coordinates. A volume cell therefore shows a concrete direction, with $\vec{e}_{\gamma}$ representing the unit vector in the specific coordinate direction $\gamma=x, y, z$. Each volume cell connects two nodes. The current density distribution (3.1) thus decomposes into

$$
J_{\gamma}(\vec{r}, t)=\sum_{m=1}^{N_{V \gamma}} J_{\gamma m}\left(t_{m}\right) f_{b \gamma m}^{(\vec{J})}\left(\vec{r}_{m}\right), \quad \gamma=x, y, z,
$$

while the charge density distribution is identical to (3.2). A graphical illustration of an orthogonal discretization of the part of a conducting structure into suface and volume cells is depicted in Figure 3.1. The surface cells, representing the surface charges, are denoted by $q_{n}$ and are placed around the respective node $n$. The volume cells, representing the volume currents $i_{n_{1}, n_{2}}$, are arranged between the corresponding nodes $n_{1}$ and $n_{2}$ and carry charges from one node to another. For validity reasons the meshsize for the discretization has to be chosen w.r.t. Section 3.7. Consequently, a mapping of the vector-valued EFIE to a function $b(\vec{r})$ can be realized for cartesian coordinates using the inner product

$$
\frac{1}{A_{k}} \iiint_{V_{k}} b(\vec{r}) \mathrm{d} V=\frac{1}{A_{k}} \iint_{A_{k}} \int_{l_{k}} b(\vec{r}) \mathrm{d} l \mathrm{~d} A
$$

with cross section $A_{k}$ and length $l_{k}$ of a representative cell $k$ (refer to Figure 3.1). The three resulting equations can be understood as decoupled from each other. Assuming constant conductance

\footnotetext{
${ }^{1}$ In accordance with the literature and for reasons of simplification, the term Galerkin instead of Ritz-Galerkin is used in the following.
} 


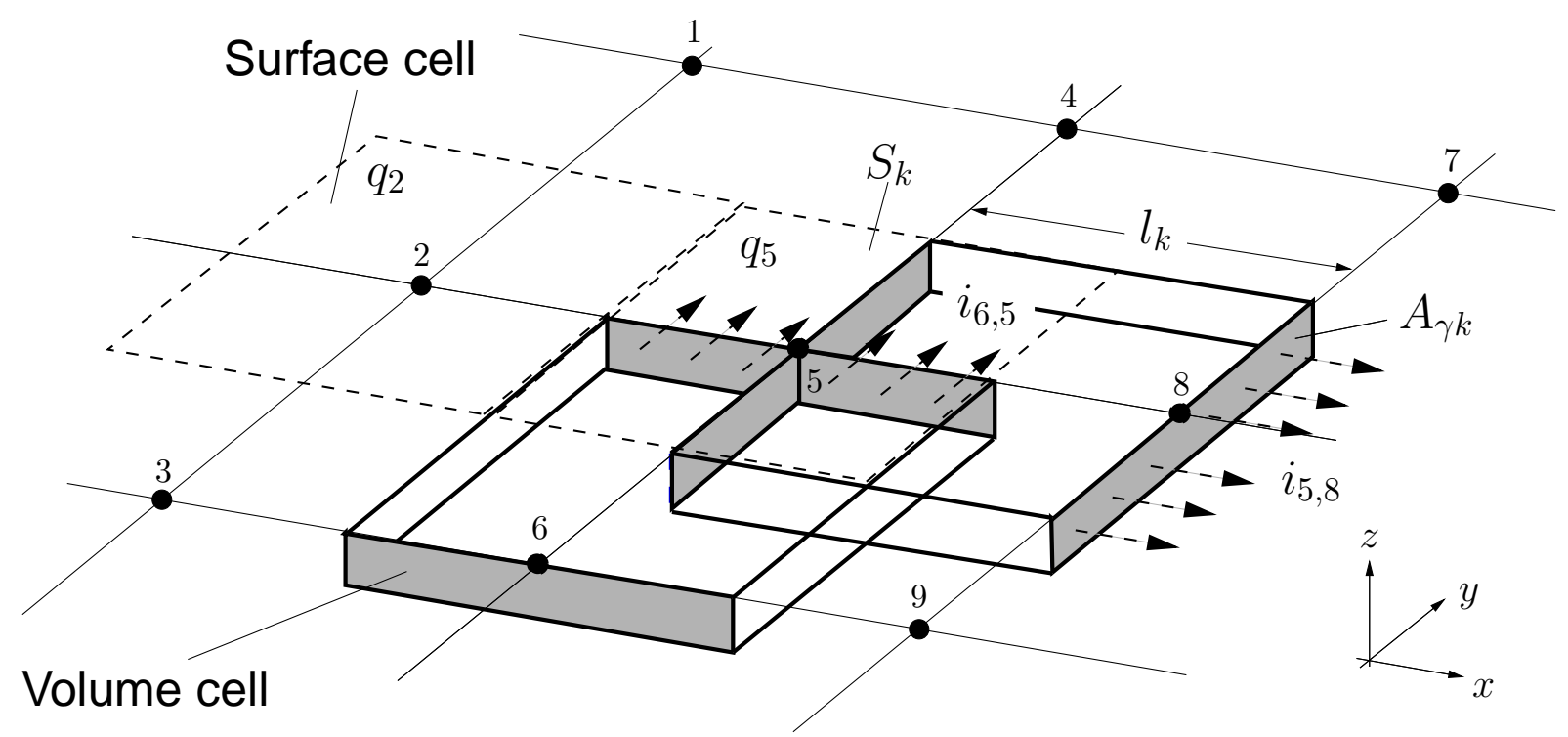

Fig. 3.1: PEEC volume and surface discretization principle for conducting medium in cartesian coordinates. The surface cells, supporting charge amplitudes $q_{n}$, are placed around the corresponding nodes $n$ (represented by $\bullet$ ). The volume cells, exhibiting current amplitudes $i_{n_{1}, n_{2}}$, are placed between two nodes $n_{1}$ and $n_{2}$.

$\sigma$ in each volume cell and regarding the accumulation of the charge density at the surface, the application of the inner product to the discretized EFIE results in

$$
\begin{aligned}
\frac{1}{A_{k}} \iiint_{V_{k}} E_{\gamma}^{i}\left(\vec{r}_{p}, t\right) \mathrm{d} V & =\frac{1}{A_{k} \sigma} \iiint_{V_{k}} J_{\gamma k}(t) \mathrm{d} V \\
& +\frac{\mu}{A_{k}} \sum_{m=1}^{N_{V \gamma}} \iiint_{V_{k}} \iiint_{V_{m}^{\prime}} G\left(\vec{r}_{p}, \vec{r}_{q}\right) \frac{\partial}{\partial t} J_{\gamma m}\left(t_{m}\right) \mathrm{d} V^{\prime} \mathrm{d} V \\
& +\frac{1}{A_{k} \varepsilon} \sum_{n=1}^{N_{S}} \iiint_{V_{k}}\left[\frac{\partial}{\partial \gamma} \iint_{S_{n}} G\left(\vec{r}_{p}, \vec{r}_{q}\right) \rho_{n}\left(t_{n}\right) \mathrm{d} S\right] \mathrm{d} V .
\end{aligned}
$$

For a consideration of dielectric effects, the dielectric medium has to be discretized in a similar manner. The individual terms in (3.9) can be interpreted as voltage drops at circuit primitives and incident field sources of the form

$$
u_{k}^{i}=u_{R_{k}}+u_{L_{k}}+u_{C_{k}}
$$

\subsubsection{Partial Element Extraction}

A detailed formulation of partial elements for the orthogonal case can be found in $[12,48,13,3]$. The results are shortly summarized for completeness in the following.

\subsubsection{Partial Inductances}

The partial inductance between volume cells (segments) $k$ and $m$ is defined as

$$
L_{p_{k m}}=\frac{\mu}{A_{k} A_{m}} \iiint_{V_{k}} \iiint_{V_{m}^{\prime}} G\left(\vec{r}_{k}, \vec{r}_{m}\right) \cos \varphi\left(\vec{e}_{k}, \vec{e}_{m}\right) \mathrm{d} V^{\prime} \mathrm{d} V
$$


with $\cos \varphi$ regarding the current direction between the corresponding cells and $\vec{r}_{m}, \vec{r}_{k}$ denoting the corresponding source and observation points in the cells.

\subsubsection{Partial Coefficients of Potential}

The partial coefficient of potential between surface cells $k$ and $m$ is defined as

$$
P_{p_{k m}}=\frac{1}{\varepsilon S_{k} S_{m}} \iint_{S_{k}} \iint_{S_{m}^{\prime}} G\left(\vec{r}_{k}, \vec{r}_{m}\right) \mathrm{d} S^{\prime} \mathrm{d} S .
$$

In the quasi-stationary case the capacitance matrix can be computed by combining free surface charges and potentials of the conductor segments [49]. The relation between the electric charges of the surface cells and the electric potentials of the corresponding nodes can be described in terms of a linear system of equations [59]

$$
\vec{\phi}_{s}=\boldsymbol{P} \cdot \vec{q}_{s}
$$

with the vector of cell potentials $\vec{\phi}_{s}$, the matrix of coefficients of potential $\boldsymbol{P}=\left[P_{p_{k m}}\right]$ and the vector of cell charges $\vec{q}_{s}$. A transformation from the coefficients of potential matrix to the socalled short circuit capacitance matrix $\boldsymbol{C}_{s}$ can be accomplished by

$$
\boldsymbol{C}_{s}=\boldsymbol{P}^{-1}
$$

where $C_{s}$ represents a M-matrix (refer to Appendix A.1) with positive diagonal and negative offdiagonal elements. The positive diagonal entries of $\boldsymbol{C}_{s}$ are called coefficients of capacitance while the negative off-diagonal entries are called coefficients of electrostatic induction. A relationship between the elements in $\boldsymbol{C}_{s}$ and a square capacitance matrix $\boldsymbol{C}$ of dimension $N \times N$ for a $N$ conductor system can be formulated as

$$
\boldsymbol{C}=\left[\begin{array}{ccccc}
\sum_{j=1}^{N} C_{s_{1 j}} & -C_{s_{12}} & -C_{s_{13}} & \ldots & -C_{s_{1 N}} \\
-C_{s_{21}} & \sum_{j=1}^{N} C_{s_{2 j}} & -C_{s_{23}} & \ldots & -C_{s_{2 N}} \\
-C_{s_{31}} & -C_{s_{32}} & \sum_{j=1}^{N} C_{s_{3 j}} & \ldots & -C_{s_{3 N}} \\
\vdots & \vdots & & \ddots & \\
-C_{s_{N 1}} & -C_{s_{N 2}} & -C_{s_{N 3}} & \ldots & \sum_{j=1}^{N} C_{s_{N j}}
\end{array}\right]
$$

with the diagonal terms (so-called self-capacitances) and the off-digonal terms (so-called mutual capacitances). Dealing with segmented conductors these capacitances are also called partial capacitances $C_{p}$ [59]. For the two-terminal case diagonal capacitances can be obtained by summing each row in the short circuit capacitance matrix $C_{p_{i i}}=\sum_{\forall j} C_{s_{i j}}$ and the off-diagonal capacitances by negating the corresponding off-diagonal elements from matrix $C_{s}$, i.e. $C_{p_{i j}}=-C_{s_{i j}}$.

\subsection{Partial Elements for a Non-orthogonal Discretization}

For various complex problems as the modeling of vias and interconnects on a PCB, a Manhattan discretization in 2D and 3D is not efficient enough. Using quadrilateral (2D) and hexahedral (3D) shaped cells, it is possible to increase the modeling accuracy while simultaneously reducing the model size [57, 60]. Curvilinear coordinates are advantageous to formulate the computation rules efficiently. For that purpose, it is necessary to apply a coordinate transformation which is explained in detail in Appendix C. 


\subsubsection{Discretization of the EFIE}

\subsubsection{Local Coordinate System}

To accomplish a suitable coordinate transformation, a volume cell is considered as depicted in Figure 3.2, which can consist of dielectric or conducting material. A mathematical treatment of quadrilateral or hexahedral shaped cells can be performed analogously to the cartesian case. Each cell position can be described equivalently using global cartesian coordinates $\vec{r}_{g}$ or $\underline{l}$ ocal coordinates $\vec{r}_{l}$. For a detailed view to the treatment of hexahedral shaped cells with quadrilateral sides refer to [60]. Each arbitrarily chosen cell location can be specified by the local position vector $\vec{r}_{l}=\left(a \vec{e}_{a}+b \vec{e}_{b}+c \vec{e}_{c}\right)$ with $a, b, c \in[-1,+1]$. An unambiguous mapping of a local position vector

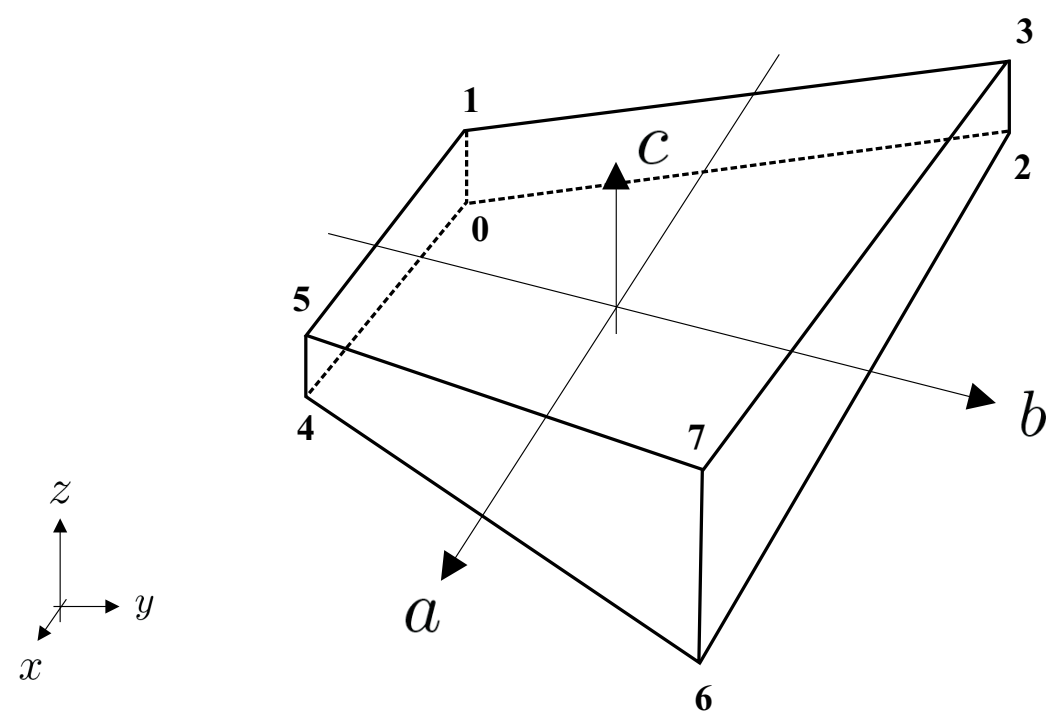

Fig. 3.2: Hexahedral shaped volume cell with quadrilateral shaped surface cells, enabling the connection between local curvilinear coordinates $(a, b, c)$ and cartesian coordinates $(x, y, z)$.

to a global one is possible by specifying the global positions of the eight corners of the hexahedron corresponding to the local extremal values $a, b, c= \pm 1$. To realize a definite and mathematical proper conversion in both directions (global to local and vice versa) a fixed indexing of the corners is introduced.

\subsubsection{Inner Product}

Similar to (3.8), the transformation of the terms of the EFIE into voltage drops makes it necessary to apply a suitable inner product, regarding the accomplished coordinate transformation. For an integration w.r.t. the "new" coordinates $a, b, c$, the Jacobian det $\Delta$ has to be included as described in Appendix C. Based on this fact, the modified inner product for a single coordinate $a$ with cell cross section $A_{a}$ perpendicular to the coordinate direction and regarding the metric coefficient $\hbar$ can be written as

$$
\begin{aligned}
u_{a} & =\iiint_{a b c} \frac{1}{A_{a}} \vec{e}_{a} \vec{E}(\vec{r}(a, b, c)) \operatorname{det} \Delta \mathrm{d} a \mathrm{~d} b \mathrm{~d} c \\
& =\iiint_{a b c} \frac{\hbar_{a}}{\left|\frac{\partial \vec{r}}{\partial a}\left(\frac{\partial \vec{r}}{\partial b} \times \frac{\partial \vec{r}}{\partial c}\right)\right|} \overrightarrow{e_{a}} \vec{E}(\vec{r}(a, b, c)) \operatorname{det} \Delta \mathrm{d} a \mathrm{~d} b \mathrm{~d} c
\end{aligned}
$$


and alternatively as

$$
u_{a}=\iiint_{a b c} \hbar_{a} \vec{e}_{a} \vec{E}(\vec{r}(a, b, c)) \mathrm{d} a \mathrm{~d} b \mathrm{~d} c
$$

regarding the Jacobian

$$
\operatorname{det} \Delta \triangleq\left|\frac{\partial \vec{r}}{\partial a}\left(\frac{\partial \vec{r}}{\partial b} \times \frac{\partial \vec{r}}{\partial c}\right)\right|
$$

The voltage components for the remaining coordinates can be computed in a similar manner. Applying the inner product to the terms of the EFIE leads to the discrete circuit primitives for curvilinear coordinates. A graphical illustration of the PEEC discretization using curvilinear coordinates similar to Figure 3.1 is given in Figure 3.3. Different from Section 3.2, while global (cartesian) coordinates can be used to locate an arbitrary position independently from the chosen cell, the local (curvilinear) coordinate systems have to be computed cell-specifically. The compatibility of orthogonal discretization and non-orthogonal discretization is obvious as can be seen from Figure 3.3 .

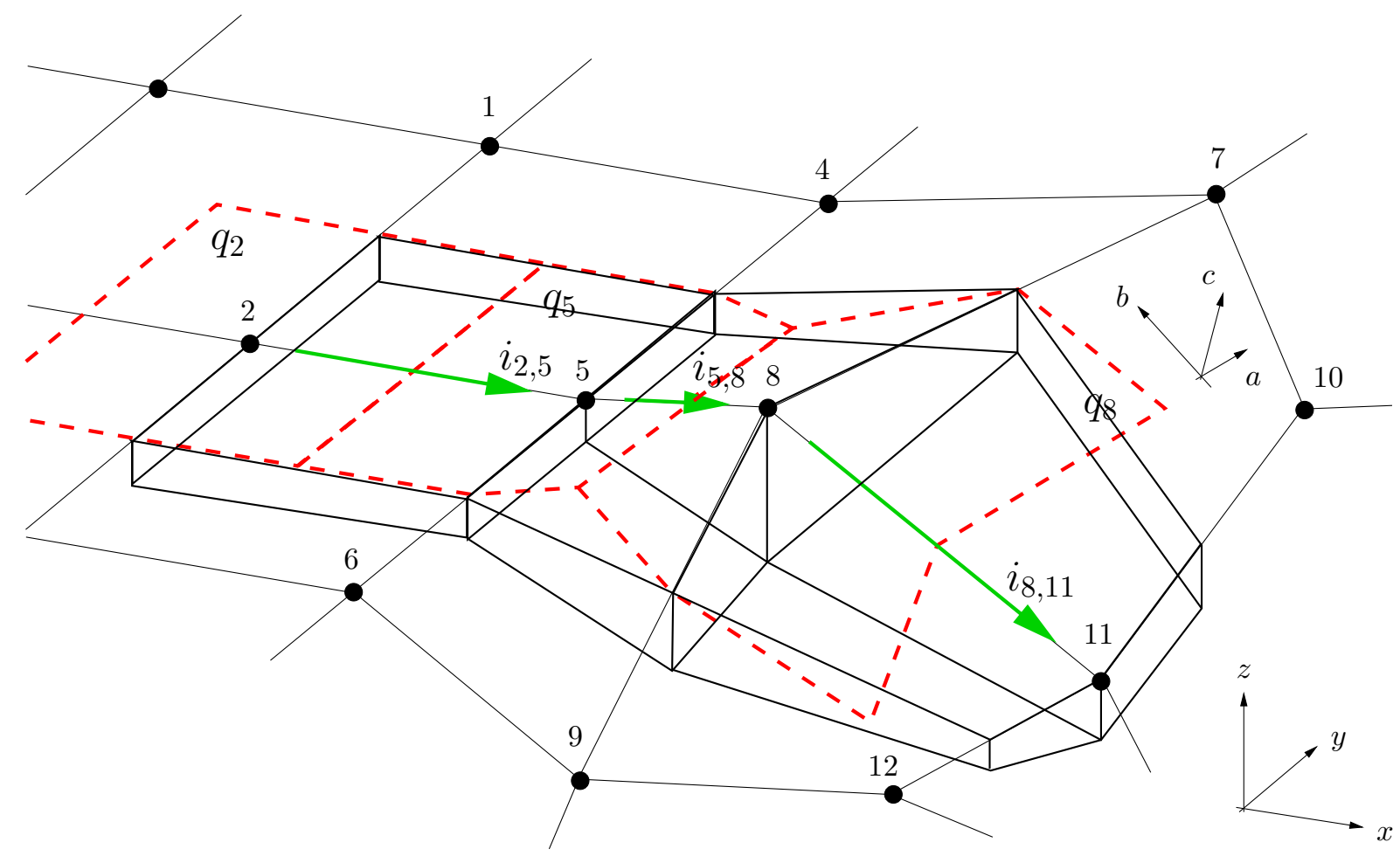

Fig. 3.3: PEEC volume and surface discretization principle for conducting medium in curvilinear coordinates. The surface and volume cells are quadrilateral or hexahedral shaped, carrying charge amplitudes $q_{n}$ and current amplitudes $i_{n_{1}, n_{2}}$. Compatibility to cartesian coordinates is warranted.

\subsubsection{Partial Element Extraction}

Similar to Section 3.2.2, the formulation of parasitic elements for curvilinear coordinates can be accomplished as described in the following. 


\subsubsection{Partial Ohmic Losses}

The application of the inner product (3.17) to the first term on the right hand side of (3.9) leads to the ohmic voltage drop

$$
\begin{aligned}
u_{R_{a}} & =\iiint_{a b c} \hbar_{a} \vec{e}_{a} \frac{\vec{J}\left(\vec{r}_{p}, t_{r}\right)}{\sigma} \mathrm{d} a \mathrm{~d} b \mathrm{~d} c \\
& =\frac{1}{\sigma} \iiint_{a b c} \hbar_{a} \frac{i_{a}\left(\vec{r}_{p}, t_{r}\right)}{A_{a}} \mathrm{~d} a \mathrm{~d} b \mathrm{~d} c
\end{aligned}
$$

which can be written as

$$
u_{R_{a}}=\frac{1}{\sigma} \iiint_{a b c} \hbar_{a} \frac{\hbar_{a}^{\prime} i_{a}\left(\vec{r}_{p}, t_{r}\right)}{\left|\frac{\partial \vec{r}}{\partial a}\left(\frac{\partial \vec{r}}{\partial b} \times \frac{\partial \vec{r}}{\partial c}\right)\right|} \mathrm{d} a \mathrm{~d} b \mathrm{~d} c
$$

regarding the Jacobian and the cell cross section $A_{a}=\left|\frac{\partial \vec{r}}{\partial b} \times \frac{\partial \vec{r}}{\partial c}\right|$. In combination with Ohm's law $R_{a}=u_{a} / i_{a}$, the ohmic resistance for a curvilinear cell in $a$-direction can be given as

$$
R_{a}=\frac{1}{\sigma} \iiint_{a b c} \frac{\hbar_{a}^{2}}{\left|\frac{\partial \vec{r}}{\partial a}\left(\frac{\partial \vec{r}}{\partial b} \times \frac{\partial \vec{r}}{\partial c}\right)\right|} \mathrm{d} a \mathrm{~d} b \mathrm{~d} c .
$$

\subsubsection{Partial Inductances}

Partial inductances for curvilinear coordinate systems can be derived from applying the inner product (3.17) to the second term on the right-hand side of (3.9), resulting in

$$
\begin{aligned}
u_{L_{a}}=\iiint_{a b c} \hbar_{a} \vec{e}_{a} & {\left[\mu \iiint_{a^{\prime} b^{\prime} c^{\prime}} G\left(\vec{r}_{p}(a, b, c), \vec{r}_{q}\left(a^{\prime}, b^{\prime}, c^{\prime}\right)\right) \cdot\right.} \\
& \left.\cdot \vec{e}_{a}^{\prime} \frac{\partial J_{a}\left(\vec{r}_{q}, t_{r}\right)}{\partial t} \operatorname{det} \Delta^{\prime} \mathrm{d} a^{\prime} \mathrm{d} b^{\prime} \mathrm{d} c^{\prime}\right] \mathrm{d} a \mathrm{~d} b \mathrm{~d} c
\end{aligned}
$$

with primed coordinates $\gamma^{\prime}$ corresponding to the source locations $\left(\vec{r}_{q}\right)$ and non-primed coordinates $\gamma$ corresponding to the observation point locations $\left(\vec{r}_{p}\right)$. Together with $J_{a}=i_{a} / A_{a}$, the expression

$$
\begin{aligned}
u_{L_{a}}=\iiint_{a b c} \hbar_{a} \vec{e}_{a} \mu \iiint_{a^{\prime} b^{\prime} c^{\prime}} G\left(\vec{r}_{p}, \vec{r}_{q}\right) \cdot \\
\cdot \vec{e}_{a}{ }^{\prime} \frac{\hbar_{a}^{\prime}}{\left|\frac{\partial \vec{r}}{\partial a}\left(\frac{\partial \vec{r}}{\partial b} \times \frac{\partial \vec{r}}{\partial c}\right)\right|^{\prime}} \frac{\partial i_{a}\left(\vec{r}_{q}, t_{r}\right)}{\partial t} \operatorname{det} \Delta^{\prime} \mathrm{d} a^{\prime} \mathrm{d} b^{\prime} \mathrm{d} c^{\prime} \mathrm{d} a \mathrm{~d} b \mathrm{~d} c
\end{aligned}
$$

follows, which can be simplified as

$$
\begin{aligned}
u_{L_{a}} & =\iiint_{a b c} \hbar_{a} \vec{e}_{a} \mu \iiint_{a^{\prime} b^{\prime} c^{\prime}} G\left(\vec{r}_{p}, \vec{r}_{q}\right) \vec{e}_{a}^{\prime} \hbar_{a}^{\prime} \frac{\partial i_{a}\left(\vec{r}_{q}, t_{r}\right)}{\partial t} \mathrm{~d} a^{\prime} \mathrm{d} b^{\prime} \mathrm{d} c^{\prime} \mathrm{d} a \mathrm{~d} b \mathrm{~d} c \\
& =\mu \iiint_{a b c} \iiint_{a^{\prime} b^{\prime} c^{\prime}} G\left(\vec{r}_{p}, \vec{r}_{q}\right) \hbar_{a} \hbar_{a}^{\prime} \frac{\partial i_{a}\left(\vec{r}_{q}, t_{r}\right)}{\partial t}\left(\vec{e}_{a} \cdot \vec{e}_{a}^{\prime}\right) \mathrm{d} a^{\prime} \mathrm{d} b^{\prime} \mathrm{d} c^{\prime} \mathrm{d} a \mathrm{~d} b \mathrm{~d} c
\end{aligned}
$$


using (3.18). Regarding the relationship $L_{p_{a a^{\prime}}}=\frac{u_{a}}{\mathrm{~d} i_{a^{\prime}} / \mathrm{d} t}$, accomplishing the connection between current and voltage at an inductance, the partial inductance for curvilinear cells can be formulated as

$$
L_{p_{a a^{\prime}}}=\mu \iiint_{a b c} \iiint_{a^{\prime} b^{\prime} c^{\prime}} G\left(\vec{r}_{p}, \vec{r}_{q}\right) \hbar_{a} \hbar_{a}^{\prime}\left(\vec{e}_{a} \cdot \vec{e}_{a}^{\prime}\right) \mathrm{d} a^{\prime} \mathrm{d} b^{\prime} \mathrm{d} c^{\prime} \mathrm{d} a \mathrm{~d} b \mathrm{~d} c .
$$

\subsubsection{Partial Coefficients of Potential}

Regarding the curvilinear coordinate transformation, the volume charge density can be written as

$$
\rho\left(a^{\prime}, b^{\prime}, c^{\prime}\right)=\frac{q}{\left|\frac{\partial \vec{r}}{\partial a} \cdot\left(\frac{\partial \vec{r}}{\partial b} \times \frac{\partial \vec{r}}{\partial c}\right)\right|} .
$$

Starting from the influence term of the EFIE

$$
\vec{E}_{a}^{C}=\frac{1}{\varepsilon} \frac{\partial}{\partial a} \iint_{S^{\prime}} G\left(\vec{r}_{p}, \vec{r}_{q}\right) \rho\left(\vec{r}_{q}, t_{r}\right) \mathrm{d} S^{\prime}
$$

and applying the inner product leads to the capacitive voltage drop

$$
u_{C_{a}}=\iint_{a b} \hbar_{a}\left[\frac{1}{\varepsilon} \frac{\partial}{\partial a} \iint_{S^{\prime}} G\left(\vec{r}_{p}, \vec{r}_{q}\right) \rho\left(\vec{r}_{q}, t_{r}\right) \operatorname{det} \Delta \mathrm{d} S^{\prime}\right] \mathrm{d} a \mathrm{~d} b,
$$

by taking into consideration that charges are only placed on the objects surface. Inserting (3.27) into (3.29),

$$
\begin{aligned}
u_{C_{a}} & =\iint_{a b} \hbar_{a}\left[\frac{1}{\varepsilon} \frac{\partial}{\partial a} \iint_{a^{\prime} b^{\prime}} G\left(\vec{r}_{p}, \vec{r}_{q}\right) \frac{q\left(\vec{r}_{q}, t_{r}\right)}{\left|\frac{\partial \vec{r}}{\partial a} \cdot\left(\frac{\partial \vec{r}}{\partial b} \times \frac{\partial \vec{r}}{\partial c}\right)\right|} \operatorname{det} \Delta \mathrm{d} S^{\prime}\right] \mathrm{d} a \mathrm{~d} b \\
& =\frac{1}{\varepsilon} \iint_{a b} \frac{\partial}{\partial a} \iint_{a^{\prime} b^{\prime}} G\left(\vec{r}_{p}, \vec{r}_{q}\right) q\left(\vec{r}_{q}, t_{r}\right) \mathrm{d} a^{\prime} \mathrm{d} b^{\prime} \mathrm{d} a \mathrm{~d} b,
\end{aligned}
$$

can be obtained with the spatial derivative represented as a separation of the form

$$
u_{C_{a}}=\sum_{m=1}^{N_{S}}\left(P_{p_{a+m}}-P_{p_{a^{-}}}\right) q_{m}\left(t_{m}\right),
$$

together with $P_{p}=u / q$, resulting in the partial coefficients of potential

$$
P_{p_{k m}}=\frac{1}{\varepsilon} \iint_{a b} \iint_{a^{\prime} b^{\prime}} G\left(\vec{r}_{p}, \vec{r}_{q}\right) \mathrm{d} a^{\prime} \mathrm{d} b^{\prime} \mathrm{d} a \mathrm{~d} b
$$

in curvilinear coordinates.

\subsection{Equivalent Circuit Formulation}

The spatially discretized system (3.4) and (3.5), providing the basis for the partial element computation, enables an equivalent circuit formulation. Where each charge basis function is represented 
by a circuit node, each current basis function embodies a circuit branch [58]. The charges supplied from the charge basis functions are stored in capacitors between one node and ground. The implementation of capacitive, inductive and incident field couplings can not be realized by circuit elements, but using controlled and independent sources. The resulting linear network can be analyzed using SPICE by means of two possible kinds of models to realize capacitive couplings. While the THEVENIN model realizes the couplings with voltage controlled voltage sources (VCVS), the NORTON model uses current controlled current sources (CCCS) [61]. For two nodes and a connecting branch, the two equivalent models are depicted in Figure 3.4. The voltages and

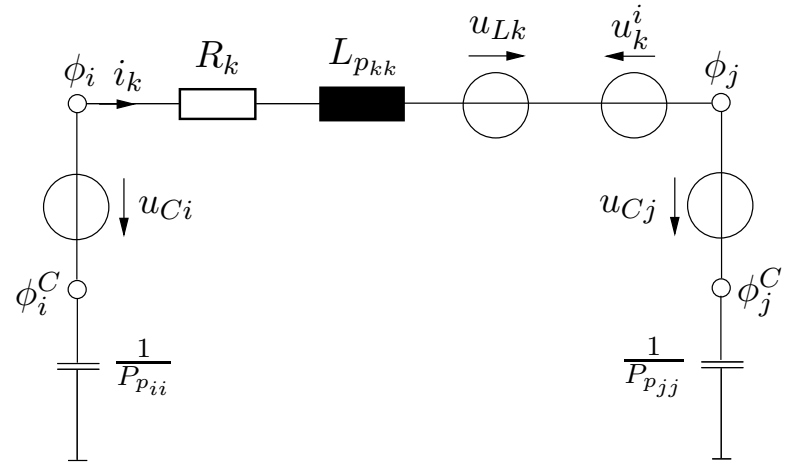

a)

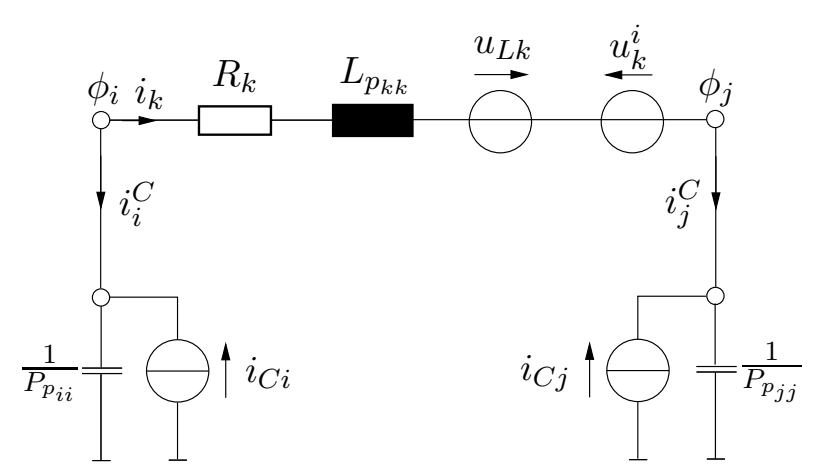

b)

Fig. 3.4: Equivalent circuit representation of PEEC models according to [61]. a) Realization of capacitive couplings with controlled voltage sources (Thevenin model). b) Realization of capacitive couplings with controlled current sources (Norton model).

currents from controlled sources can be computed as

$$
\begin{gathered}
u_{L k}(t)=\sum_{\substack{m=1 \\
m \neq k}}^{N_{V}} L_{p_{k m}} \frac{\mathrm{d} i_{m}\left(t-\tau_{k m}\right)}{\mathrm{d} t}=\sum_{\substack{m=1 \\
m \neq k}}^{N_{V}} \frac{L_{p_{k m}}}{L_{p_{m m}}} u_{L m}\left(t-\tau_{k m}\right) \\
u_{C \ell}(t)=\sum_{\substack{n=1 \\
n \neq \ell}}^{N_{S}} \frac{P_{p_{\ell n}}}{P_{p_{n n}}} \phi_{n}^{C}\left(t-\tau_{\ell n}\right) \\
i_{C \ell}(t)=\sum_{\substack{n=1 \\
n \neq \ell}}^{N_{S}} \frac{P_{p_{\ell n}}}{P_{p_{n n}}} i_{n}^{C}\left(t-\tau_{\ell n}\right),
\end{gathered}
$$

with $N_{V}$ inductive and $N_{S}$ capacitive couplings and retardation delay $\tau$. The equivalent circuit model is connectable to an external SPICE network via terminals. A separate analysis of the PEEC model by applying external sources and loadings is possible [58]. For a more profound understandig of retardation effects and implementation issues the reader is referred to Section 3.10 or $[61,58]$.

\subsection{Dielectric Effects in PEEC Modeling}

To take dielectric effects into account, volume- and surface-based formulations have to be distinguished w.r.t. the geometric properties of the underlying structure. Surface integral equation 
formulations pointed out to be advantageous especially for simulating packaging and interconnect structures at high frequencies ${ }^{2}$. The volume-based formulation can be suitable for small or flat arrangements. In the following, a suitable discretization into surface or volume cells is assumed, which guarantees a valid approximation with constant $\varepsilon$ in each cell.

\subsubsection{Volume-Based PEEC Formulation}

The purpose of the volume equivalence principle $[41,39]$ is, to transform the material problem into a free space problem with equivalent sources. If the point of observation is located inside a voluminous dielectric region with relative permittivity $\varepsilon_{\mathrm{r}}$, a discretization into dielectric volume cells is necessary similar to the case of perfect conductors. To extend the equivalent circuit model by dielectric effects, the EFIE (2.22) has to be updated. Analyzing the electromagnetic scattering problem from Section 2.2 and regarding the equivalence principle, the first of Maxwell's equations for the specific domains can be formulated as

$$
\begin{aligned}
\operatorname{rot} \vec{H}_{1}=\left(\sigma_{1}+\mathrm{j} \omega \varepsilon_{1}\right) \vec{E}_{1} & \text { in the interior region } 1 \\
\operatorname{rot} \vec{H}_{2}=\vec{J}^{\imath}+\mathrm{j} \omega \varepsilon_{2} \vec{E}_{2} & \text { in the exterior region } 2,
\end{aligned}
$$

formulated in the FD. Originating from the presence of the dielectric object, the scattered field corresponds to the difference between both fields

$$
\begin{aligned}
\operatorname{rot} \vec{H}^{s} & =\operatorname{rot}\left(\vec{H}_{1}-\vec{H}_{2}\right) \\
& =\left(\sigma_{1}+\mathrm{j} \omega \varepsilon_{1}\right) \vec{E}_{1}-\mathrm{j} \omega \varepsilon_{2} \vec{E}_{2}-\vec{J}^{\imath}
\end{aligned}
$$

in accordance with (2.15). Exploiting (2.14), it follows

$$
\operatorname{rot} \vec{H}^{s}=\left(\sigma_{1}+\mathrm{j} \omega \varepsilon_{1}\right) \vec{E}_{1}-\mathrm{j} \omega \varepsilon_{2}\left(\vec{E}_{1}-\vec{E}^{s}\right)-\vec{J}^{\imath}
$$

or

$$
\operatorname{rot} \vec{H}^{s}=\left(\sigma_{1}+\mathrm{j} \omega \varepsilon_{0}\left(\varepsilon_{\mathrm{r} 1}-1\right)\right) \vec{E}_{1}+\mathrm{j} \omega \varepsilon_{0} \vec{E}^{s}-\vec{J}^{i},
$$

respectively, assuming $\varepsilon_{1}=\varepsilon_{0} \varepsilon_{\mathrm{r} 1}$ and $\varepsilon_{2}=\varepsilon_{0}$ (refer to Figure 2.1). Assuming the incident electric field to be zero inside the dielectric object $\left(\vec{J}^{i}=\overrightarrow{0}\right)$, Maxwell's equation can be formulated as

$$
\begin{aligned}
\operatorname{rot} \vec{H}^{s} & =\left(\sigma_{1}+\mathrm{j} \omega \varepsilon_{0}\left(\varepsilon_{\mathrm{r} 1}-1\right)\right) \vec{E}_{1}+\mathrm{j} \omega \varepsilon_{0} \vec{E}^{s} \\
& =\vec{J}^{C}+\vec{J}^{P}+\mathrm{j} \omega \varepsilon_{0} \vec{E}^{s}
\end{aligned}
$$

with the conducting current density $\vec{J}^{C}$ given by (2.11) and the polarization current density

$$
\vec{J}^{P} \triangleq \mathrm{j} \omega \varepsilon_{0}\left(\varepsilon_{\mathrm{r} 1}-1\right) \vec{E}_{1} .
$$

Introducing equivalent (electric and magnetic volume polarization current) sources, a transformation from the original problem to an equivalent free space problem can be realized, as illustrated in Figure 3.5. Using equivalent sources $\vec{J}^{C}$ and $\vec{J}^{P}$, the dielectric object in presence of an electric field can be replaced by the free space permittivity $\varepsilon=\varepsilon_{0}$ [62]. To extend the EFIE by dielectric

\footnotetext{
${ }^{2}$ At low frequencies the surface impedance approximation can be insufficient to model skin effects accurately.
} 


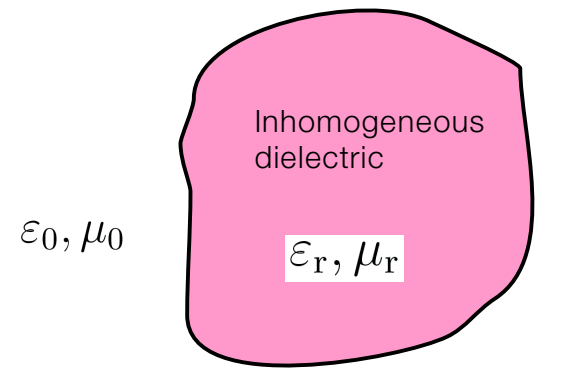

a)

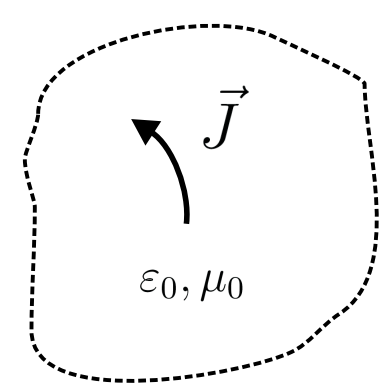

b)

Fig. 3.5: Volume equivalence principle for dielectric objects. a) original problem; b) equivalent free space problem.

effects, it is necessary to incorporate the influence of the dielectric displacement current in current densities as well as charge densities. The total current density can be written as

$$
\vec{J}=\vec{J}^{C}+\vec{J}^{P}
$$

which reduces to $\vec{J}=\vec{J}^{C}$ for perfect conducting objects and $\vec{J}=\vec{J}^{P}$ for perfect dielectrics. In the same way, the charge density incorporates free as well as bound charges

$$
\rho=\rho^{F}+\rho^{B}
$$

which reduces to $\rho=\rho^{F}$ for perfect conducting objects and $\rho=\rho^{B}$ for perfect dielectrics. Introducing the total current density (3.43) into (2.22), the extended EFIE in the FD can be obtained as

$$
\begin{aligned}
\vec{n}\left(\vec{r}_{p}\right) \times \vec{E}^{i}\left(\vec{r}_{p}, t\right) & =\vec{n}\left(\vec{r}_{p}\right) \times\left[\frac{\vec{J}\left(\vec{r}_{p}, t\right)}{\sigma}\right] \\
& +\vec{n}\left(\vec{r}_{p}\right) \times\left[\mu \mathrm{j} \omega \iiint_{V_{q}} G\left(\vec{r}_{p}, \vec{r}_{q}\right) \vec{J}^{C}\left(\vec{r}_{q}, t_{r}\right) \mathrm{d} V\right] \\
& -\vec{n}\left(\vec{r}_{p}\right) \times\left[\varepsilon_{0}\left(\varepsilon_{\mathrm{r}}-1\right) \mu(\mathrm{j} \omega)^{2} \iiint_{V_{q}} G\left(\vec{r}_{p}, \overrightarrow{r_{q}}\right) \vec{E}\left(\vec{r}_{q}, t_{r}\right) \mathrm{d} V\right] \\
& +\vec{n}\left(\vec{r}_{p}\right) \times\left[\frac{1}{\varepsilon_{0}} \operatorname{grad} \iiint_{V_{q}} G\left(\vec{r}_{p}, \vec{r}_{q}\right) \rho\left(\vec{r}_{q}, t_{r}\right) \mathrm{d} V\right] .
\end{aligned}
$$

Together with $\vec{E}=\frac{\vec{J}^{C}}{\sigma_{1}}$, the most common form of the EFIE for a PEEC formulation can be obtained.

Equivalent Circuit Formulation Similar to conducting domains, the dielectric discretization of the EFIE into volume cells enables an equivalent circuit representation. Applying the inner product from (3.3) enables a transformation of the electric field strength into a voltage drop at a discrete electrical component. Two dielectric cells $\gamma$ and $\gamma^{\prime}$ are considered. The resulting voltage drop

$$
u_{C}=\varepsilon_{0}\left(\varepsilon_{\mathrm{r}}-1\right) \mu(\mathrm{j} \omega)^{2} \iiint_{V_{\gamma}} \iiint_{V_{\gamma}^{\prime}} G\left(\vec{r}_{p}, \vec{r}_{q}\right) \vec{E}\left(\vec{r}_{q}, t_{r}\right) \mathrm{d} V^{\prime} \mathrm{d} V
$$


resulting from the third term on the right hand side of (3.45), describes the dielectric effects and has the form of a partial inductance [62]. Applying Ohm's law (2.11) for domain 1, the electric field strength $\vec{E}$ can be eliminated. Assuming constant current and charge density distributions inside the cells and regarding the relation $i=J / A$ with cross section $A$ of the volume cell and current $i$ penetrating the cell, the voltage drop can be computed as

$$
u_{C}=(\mathrm{j} \omega)^{2} L_{p_{\gamma \gamma^{\prime}}} \frac{i_{\gamma}}{\sigma} \varepsilon_{0}\left(\varepsilon_{\mathrm{r}_{\gamma}}-1\right)
$$

in the FD. Incorporating the electric conductivity $\sigma$ to represent the ohmic losses (referring to $[48,13])$ as ohmic resistors, leads to the voltage drop

$$
u_{C}=(\mathrm{j} \omega)^{2} L_{p_{\gamma \gamma^{\prime}}} R_{\gamma} i_{\gamma} \frac{\varepsilon_{0}\left(\varepsilon_{\mathrm{r}_{\gamma}}-1\right)}{l_{\gamma}} A_{\gamma}
$$

Defining the so-called "excess capacitance"

$$
C_{\gamma}^{+} \triangleq \frac{\varepsilon_{0}\left(\varepsilon_{\mathrm{r}_{\gamma}}-1\right)}{l_{\gamma}} A_{\gamma}
$$

for dielectric cell $\gamma$ leads to the expression

$$
u_{C}=(\mathrm{j} \omega)^{2} L_{p_{\gamma \gamma^{\prime}}} R_{\gamma} C_{\gamma}^{+} i_{\gamma},
$$

enabling a circuit representation of the dielectric cell, as depicted in Figure 3.6, without modifying the existing PEEC model for conductors.

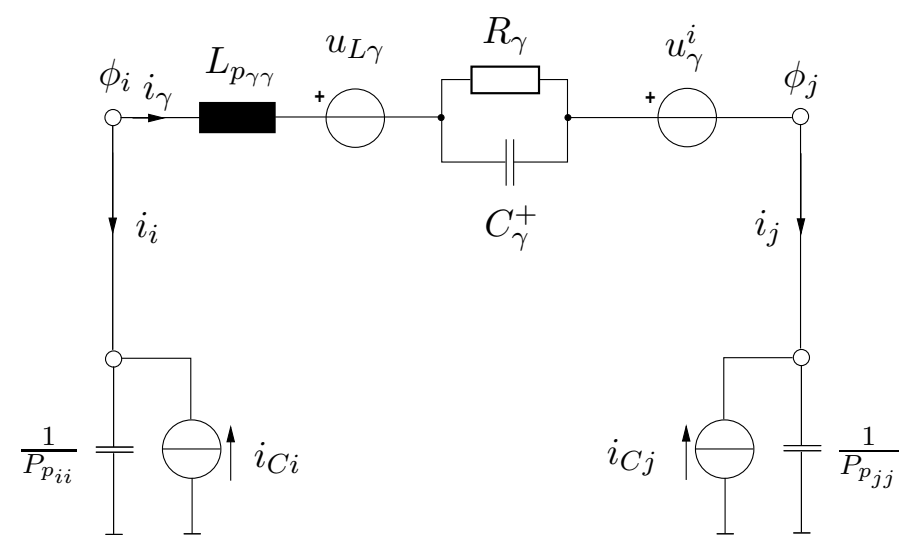

Fig. 3.6: Equivalent circuit representation of a dielectric cell according to [62] (Norton model). The inductive and incident field couplings are denoted by $u_{L \gamma}$ and $u_{\gamma}^{i}$, respectively.

In curvilinear coordinates the excess capacitance can be expressed by ${ }^{3}$

$$
C_{\gamma}^{+\not} \triangleq \frac{\varepsilon_{0}\left(\varepsilon_{\mathrm{r}_{\gamma}}-1\right)}{l_{\gamma}} A_{\gamma} \frac{1}{\iiint_{V} \frac{\hbar_{a}^{2}}{\left|\frac{\partial \vec{r}}{\partial a} \cdot\left(\frac{\partial \vec{r}}{\partial b} \times \frac{\partial \vec{r}}{\partial c}\right)\right|} \mathrm{d} V} .
$$

A non-orthogonal PEEC case requires numerical space-integration techniques, whereas the computation of the partial elements in the classical (orthogonal) case can be accomplished by means of

\footnotetext{
${ }^{3}$ The notation $\not \perp$ indicates a non-orthogonal discretization.
} 
very accurate and fast analytic formulae, in general. W.r.t. implementation, both rectangular and non-orthogonal meshes are compatible and enable a flexible and efficient simulation [57]. For a dielectric-to-conductor coupling the equivalent circuit from the volume-based approach is depicted in Figure 3.7.

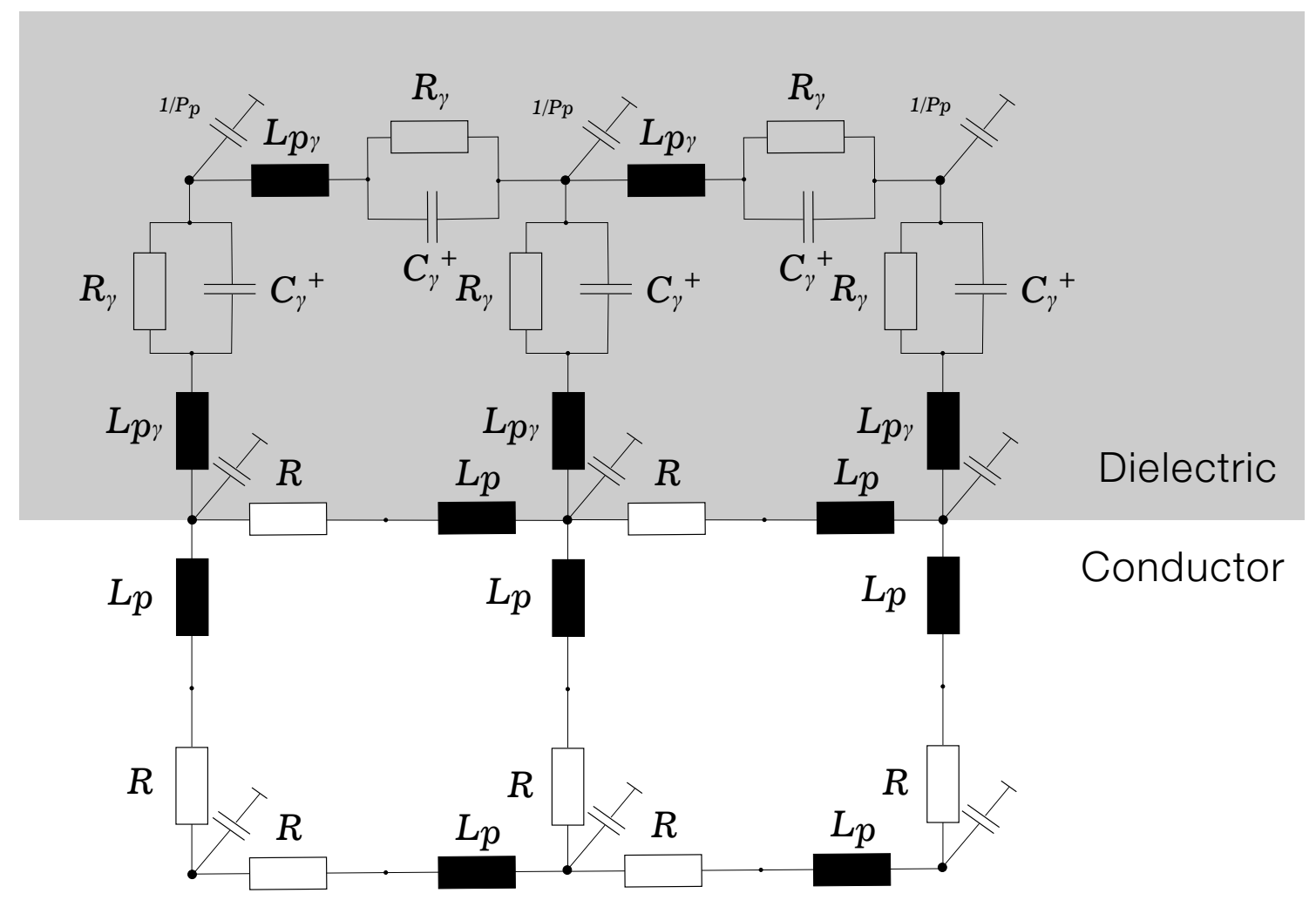

Fig. 3.7: Equivalent circuit representation of a dielectric-to-conductor coupling using the volume-based formulation. The partial elements for dielectric cells are denoted by $\gamma$, where the partial elements for the conducting medium are specified in Section 3.2 and 3.3, respectively. Remark: Couplings are neglected for simplification purposes.

\subsubsection{Surface-Based PEEC Formulation}

The surface-based PEEC modeling (“(S)PEEC”) has been developed for conductors with surface impedance approximation [63]. Based on the surface equivalence principle [41] and the PMCHWT formulation $[64,65]$, it represents the system by a separate equivalent electric circuit and an equivalent magnetic circuit ${ }^{4}$ (Figure 3.8). Both circuits can be interpreted by ordinary lumped circuit primitives in the form of partial inductances, capacitances and dependent sources. The electric and magnetic equivalent circuits are connected by mutual couplings. Applying the surface equivalence principle, the original problem can be splitted into an exterior and an interior equivalent problem. To guarantee the continuity of the tangential components of the electric and magnetic field quantities at the surface of the dielectric, it is necessary to introduce equivalent electric and magnetic surface currents [39]. Therefore, together with $\left.\vec{H}_{\tan }\right|_{\Gamma^{+}}=\left.\vec{H}_{\tan }\right|_{\Gamma^{-}}$

$$
\vec{J}_{s}^{\mathrm{int}}=-\vec{J}_{s}^{\mathrm{ext}}
$$

\footnotetext{
${ }^{4}$ Although two different equivalent circuits are necessary to capture the dielectric effects, a surface-based approach leads to a reduced number of unknowns compared to the volume-based approach.
} 
must hold for the surface current densities. To derive a surface-based PEEC formulation, it is

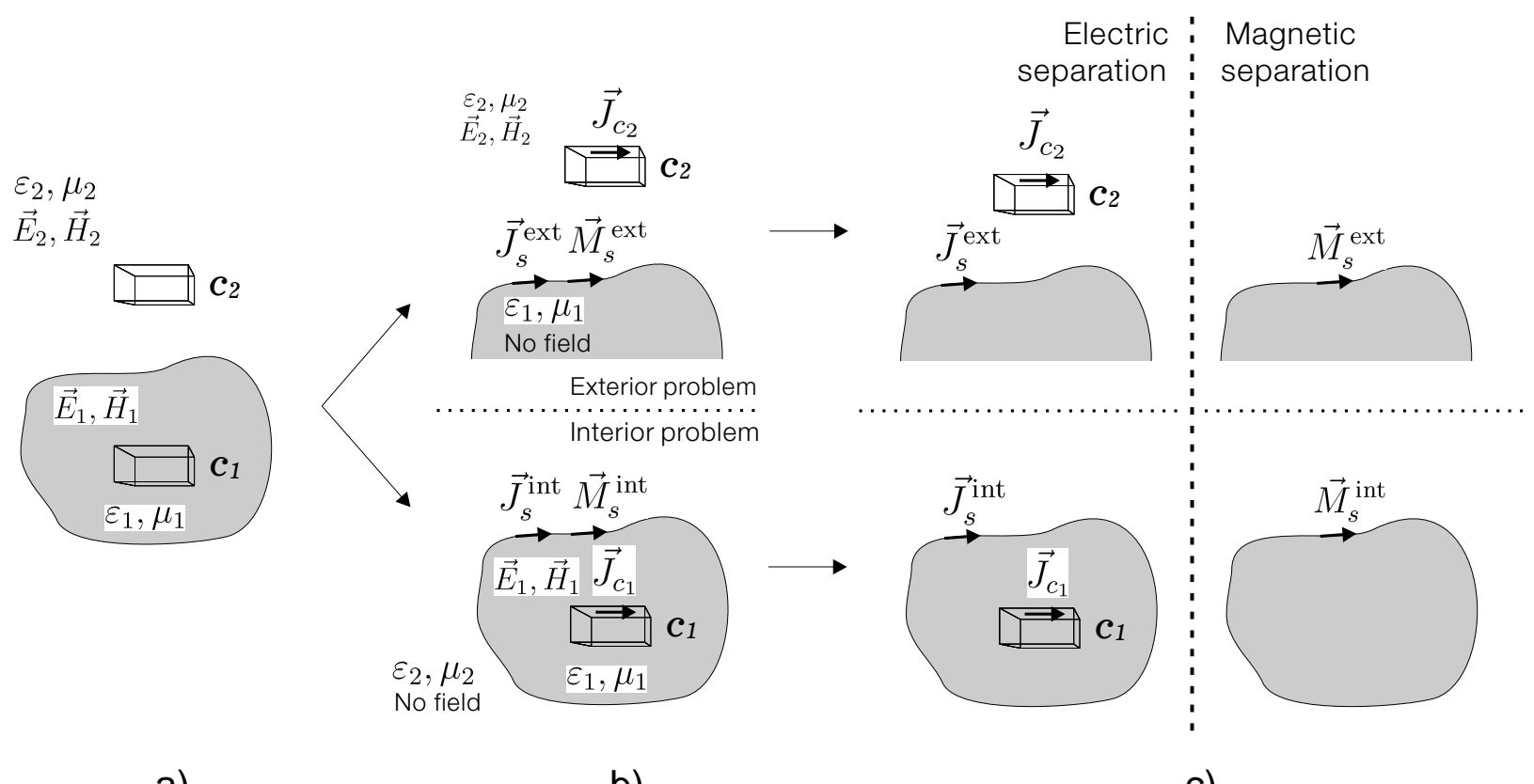

a)

b)

c)

Fig. 3.8: a) Original problem, b) surface equivalence principle, c) electric/magnetic separation of a dielectricconductor problem.

necessary to extend Maxwell's equations from Section 2.1 by the magnetic volume/surface current denstity $\vec{M}[39]$ with

$$
\vec{M}_{s}^{\text {int }}=-\vec{M}_{s}^{\text {ext }}
$$

In a second step, the electric and magnetic effects can be separated by means of two mutually coupled equivalent circuits with the nodal voltages and branch currents as unknowns. Taking the boundary conditions

$$
\begin{aligned}
\vec{n} \times\left(\vec{E}^{i}+\vec{E}_{2}\right) & =\vec{n} \times \vec{E}_{1} \\
\vec{n} \times\left(\vec{H}^{i}+\vec{H}_{2}\right) & =\vec{n} \times \vec{H}_{1},
\end{aligned}
$$

into account, the scattered fields at any point in space can be quantified as $[63,65]$

$$
\begin{aligned}
& \vec{E}_{1,2}(\vec{r})=-\mathrm{j} \omega \vec{A}_{1,2}(\vec{r})-\operatorname{grad} \phi_{1,2}^{e}(\vec{r})-\frac{1}{\varepsilon_{1,2}} \operatorname{rot} \vec{F}_{1,2}(\vec{r}) \\
& \vec{H}_{1,2}(\vec{r})=-\mathrm{j} \omega \vec{F}_{1,2}(\vec{r})-\operatorname{grad} \phi_{1,2}^{m}(\vec{r})+\frac{1}{\mu_{1,2}} \operatorname{rot} \vec{A}_{1,2}(\vec{r})
\end{aligned}
$$

together with

$$
\begin{gathered}
\vec{A}_{1,2}\left(\vec{r}_{p}\right)=\mu_{1,2} \iint_{S^{\prime}} G\left(\vec{r}_{p}, \vec{r}_{q}\right)( \pm) \vec{J}_{1}\left(\vec{r}_{q}\right) \mathrm{d} S^{\prime} \\
\vec{F}_{1,2}\left(\vec{r}_{p}\right)=\varepsilon_{1,2} \iint_{S^{\prime}} G\left(\vec{r}_{p}, \vec{r}_{q}\right)( \pm) \vec{M}_{1}\left(\vec{r}_{q}\right) \mathrm{d} S^{\prime} \\
\vec{\phi}_{1,2}^{e}\left(\vec{r}_{p}\right)=\frac{1}{\varepsilon_{1,2}} \iint_{S^{\prime}} G\left(\vec{r}_{p}, \vec{r}_{q}\right)( \pm) q_{1}^{e} \mathrm{~d} S^{\prime}
\end{gathered}
$$




$$
\vec{\phi}_{1,2}^{m}\left(\vec{r}_{p}\right)=\frac{1}{\mu_{1,2}} \iint_{S^{\prime}} G\left(\vec{r}_{p}, \vec{r}_{q}\right)( \pm) q_{1}^{m} \mathrm{~d} S^{\prime}
$$

and

$$
\begin{gathered}
q_{1}^{e}=\operatorname{div} \vec{J} \\
q_{1}^{m}=\operatorname{div} \vec{M} .
\end{gathered}
$$

Introducing (3.56) and (3.57) into (3.54) or (3.55), the integral equations

$$
\begin{aligned}
\vec{n} \times \vec{E}^{i}(\vec{r}) & =\vec{n} \times\left(j \omega\left(\vec{A}_{1}(\vec{r})+\vec{A}_{2}(\vec{r})\right)\right. \\
& +\operatorname{grad}\left(\phi_{1}^{e}(\vec{r})+\phi_{2}^{e}(\vec{r})\right) \\
& \left.+\left(\frac{1}{\varepsilon_{1}} \operatorname{rot} \vec{F}_{1}(\vec{r})+\frac{1}{\varepsilon_{2}} \operatorname{rot} \vec{F}_{2}(\vec{r})\right)\right) \\
\vec{n} \times \vec{H}^{i}(\vec{r}) & =\vec{n} \times\left(j \omega\left(\vec{F}_{1}(\vec{r})+\vec{F}_{2}(\vec{r})\right)\right. \\
& +\operatorname{grad}\left(\phi_{1}^{m}(\vec{r})+\phi_{2}^{m}(\vec{r})\right) \\
& \left.+\left(\frac{1}{\mu_{1}} \operatorname{rot} \vec{A}_{1}(\vec{r})+\frac{1}{\mu_{2}} \operatorname{rot} \vec{A}_{2}(\vec{r})\right)\right)
\end{aligned}
$$

for the electric and magnetic fields can be obtained. In curvilinear coordinates, the surface-based PEEC elements can be computed as [63]

$$
\begin{gathered}
L_{p_{a a^{\prime}}}=\mu \iint_{a b} \iint_{a^{\prime} b^{\prime}}\left(\vec{e}_{a} \cdot \vec{e}_{a}{ }^{\prime}\right) \hbar_{a}^{\prime} G\left(\vec{r}_{p}, \vec{r}_{q}\right) \mathrm{d} a^{\prime} \mathrm{d} b^{\prime} \hbar_{a} \mathrm{~d} a \mathrm{~d} b \\
P_{p_{a a^{\prime}}}=\frac{1}{\varepsilon} \iint_{a b} \iint_{a^{\prime} b^{\prime}} G\left(\vec{r}_{p}, \vec{r}_{q}\right) \mathrm{d} a^{\prime} \mathrm{d} b^{\prime} \mathrm{d} a \mathrm{~d} b
\end{gathered}
$$

for a characterization of the electric and magnetic equivalent circuit representation as depicted in Figure 3.9.

\subsection{Structure of PEEC-Based Circuit-Level Simulation}

For the analysis of PEEC models, a conventional SPICE-like circuit simulator can be used. The parasitic PEEC elements are transfered to the circuit simulator together with other subcircuits (e.g. TL models, PEEC models or subcircuit models) as depicted in Figure 3.10. The circuit simulator itself contains a read-in stage, supplying the required data in a suitable form to a following setup stage. In the setup stage, the network matrix is prepared prior to a network analysis block, which sets up the network equations and initiates the solution phase. If non-linear circuit elements as diodes or transistors exist, a linearization w.r.t. the operating point is necessary. The intermediate results for nodal voltages as well as branch currents are stored. For graphical representation, the data can be provided in a suitable form after passing the output processor. A control unit sets input signal waveforms, controls the type of analysis, accesses the paths to the sets of modeling 
a)

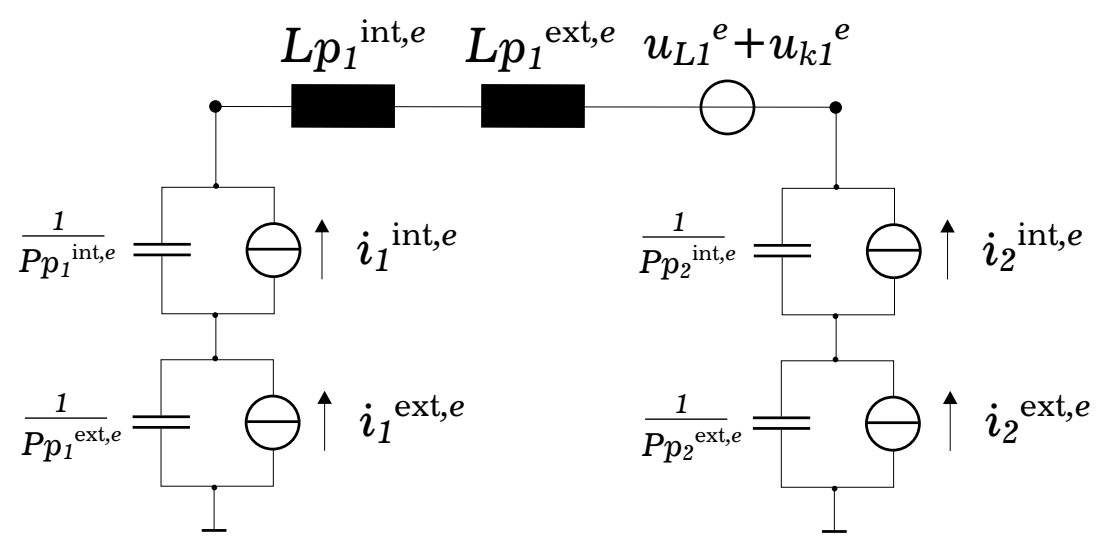

b)

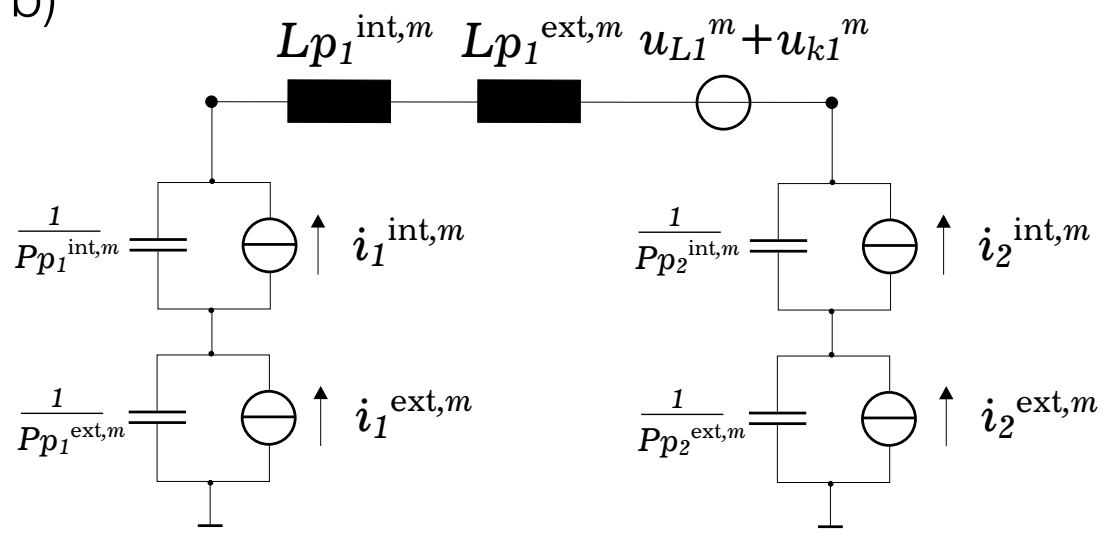

Fig. 3.9: (S)PEEC equivalent circuit representation for a single dielectric cell. a) electric cell, b) magnetic cell.

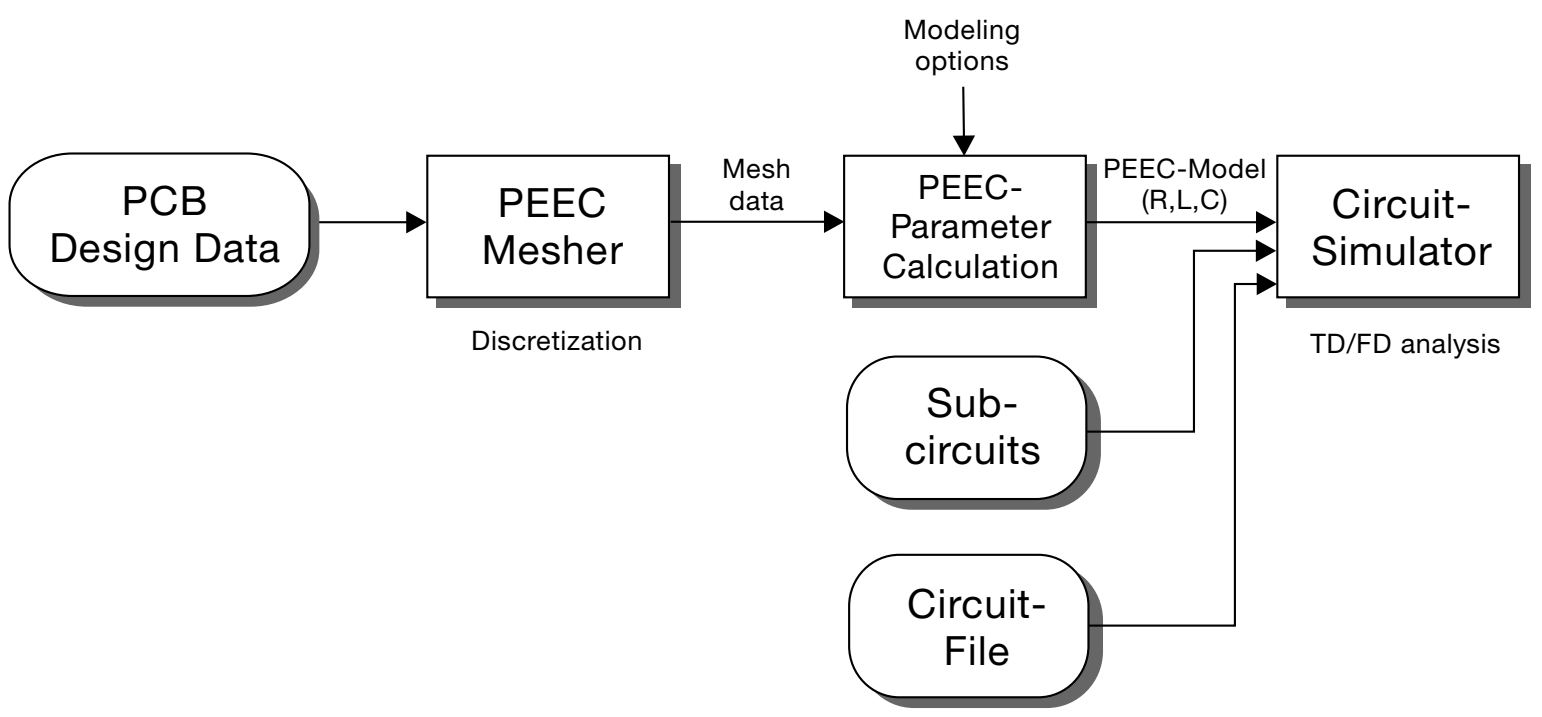

Fig. 3.10: Workflow of a PEEC-based modeling and circuit simulation.

parameters for the circuit elements, the settings for the result and supervises the simulation process. The type of the resulting equation system depends on the type of analysis. Where a transient 
analysis in the TD leads to a system of ODEs of the first order with the unknown current and voltage functions, a DC point analysis results in a non-linear equation system with the unknown current and voltage values in the operating point. With both kinds of analysis the numerical solution of the network equation system is the core component of the circuit-level simulation. W.r.t. Section 3.7.4, the numerical integration technique has to be chosen suitably to guarantee a stable solution process and accurate results.

\subsection{Accuracy and Validity Aspects}

Without violating general validity, a discretization of the considered structure into triangular surface panels is assumed. The set $\mathbb{T}\left\{\mathfrak{T}_{1}, \mathfrak{T}_{2}, \ldots, \mathfrak{T}_{n}\right\}$, consisting of disjoint, possibly curved triangles $\mathfrak{T}$ in $\mathbb{R}^{3}$ with extent $\operatorname{diam}(\mathfrak{T})$, enables a surface triangulation of the boundary $\Gamma$ as [66]

$$
\Gamma=\bigcup_{\mathfrak{T} \in \mathbb{T}} \mathfrak{T},
$$

with a stepsize or meshsize

$$
h=\max _{\forall \mathfrak{T} \in \mathbb{T}} \operatorname{diam}(\mathfrak{T})
$$

of the grid. For visualization purposes, a part of a triangular mesh for the PEEC modeling of partial coefficients of potential is illustrated in Figure 3.11. The meshsize has to be chosen suitably w.r.t. a variety of possible aspects. The discretization has to be fine enough, such that the current and charge densities can be assumed to be constant in each cell. On the other hand, the meshsize will be determined by the maximum frequency. If objects are very close next to each other, a further refinement of the volume and surface discretization can be necessary [68]. Various properties of a mesh can help to evaluate its quality w.r.t. accuracy and validity. Meshing gaps and overlaps have to be avoided. Moreover, so-called mesh conformity has to be guaranteed (e.g. every edge shared exactly 2 triangles) and high-quality elements have to be used (approximately equilateral triangles). Usually, mesh generation is based on heuristic methods and practical experience. The common types of meshes are structured grids, unstructured grids and hybrid grids. Hybrid grids 5 result from a partitioning of the total area into non-regular parts, which are splitted into regular subdomains in a second step. Whereas the theory behind structured meshes is well understood, they can only be applied to simple geometries with smooth variations. For more complex structures with fast varying solutions, unstructured meshes have to be prefered. Frequently, a mesh generator operates just with one following refinement stage. To avoid numerical problems in simulation and to achieve realistic results, some mesh generation guidelines have to be kept in mind. Furthermore, it is desirable to be able to predict the quality of the mesh in form of a quantitative parameter prior to simulation. If discretization rules are violated consciously, accuracy degradations and simulation artifacts can occur. Various geometric cell forms are possible w.r.t. the specific application, accuracy and flexibility aspects.

\subsubsection{Electrical Length of Interconnect Structures}

The ratio of meshsize and wavelength is decisive for the choice of a suitable discretization fineness. In general, the so-called "electrical length" of a conducting structure can be defined, taking the

\footnotetext{
${ }^{5}$ A general type of hybrid grids is also used together with PEEC extraction of partial coefficients of potential [69].
} 


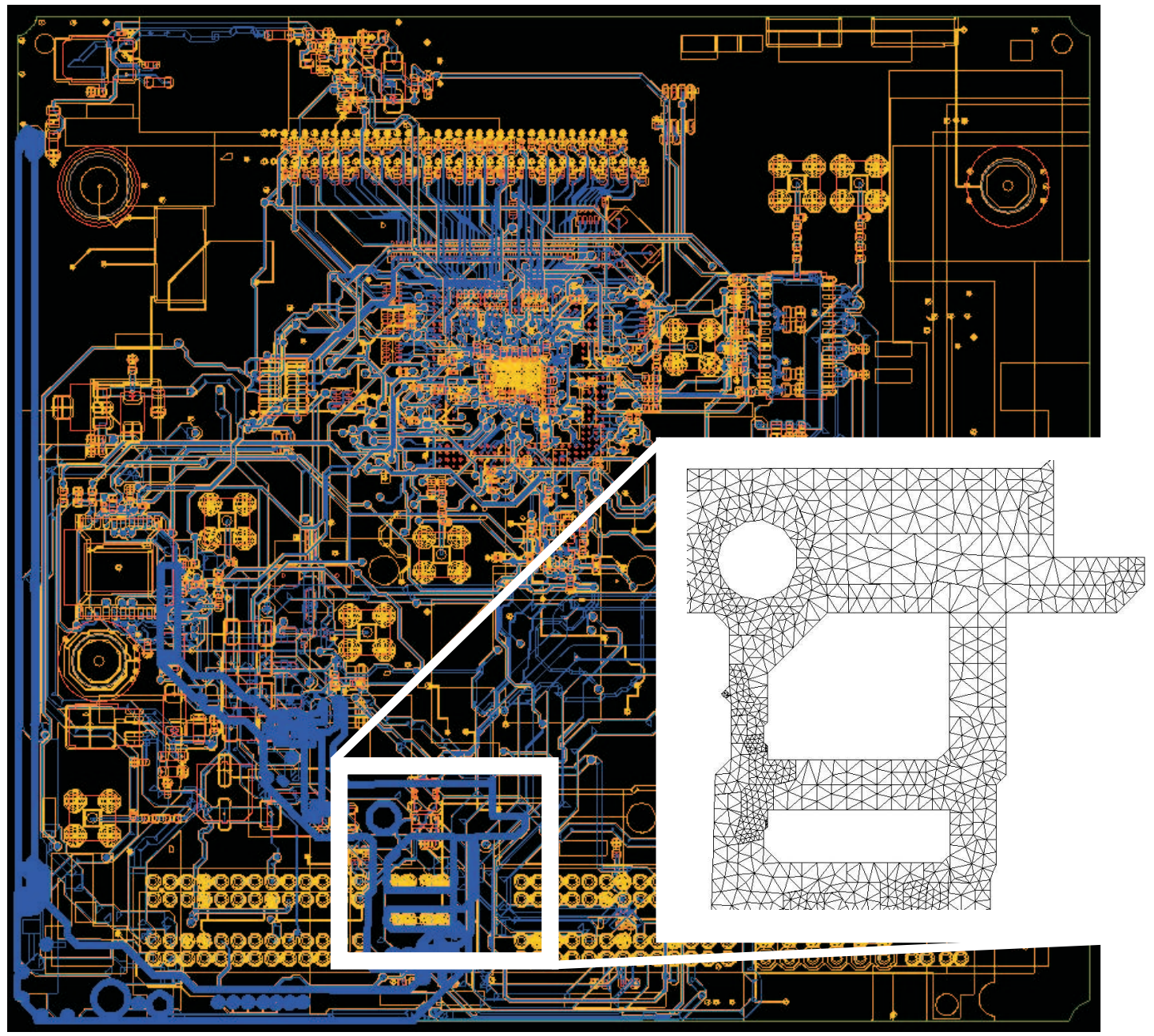

Fig. 3.11: Surface triangulation of a part of the net VB on the MISEA demonstrator board [67] (for a detailed description of the demonstrator board refer to Chapter 9) for the extraction of partial coefficients of potential.

maximum occuring signal frequency $f_{\max }$ (or the minimum wavelength $\lambda_{\min }$ ) [11] in a system into consideration. The conducting structure is called "electrically short" if the length $l$, with propagation speed $c$ of the signals, fulfills the condition

$$
l \leqslant \frac{c}{\chi f_{\max }}=\frac{\lambda_{\min }}{\chi}
$$

On the other hand, structures fulfilling the relation

$$
l>\frac{c}{\chi f_{\max }}
$$

are denoted "electrically long". Parameter $\chi$ follows heuristically from experimental analysis and is mainly based on experiences. It varies from $\chi=3$ [70], $\chi=5$ [71], $\chi=10$ [11, 72], $\chi=20$ $[68,69,73,74]$ to $\chi=30[75,76,77]$. Where originally $\chi=10$ was applied in EM modeling, $\chi=$ 20 proved to be most suitable for PEEC models [15]. Referring to [68] tests showed, that together with $\chi=20$ frequency analysis results for MoM and PEEC show good accordance. The electrical length directly has to be regarded in computing partial elements. While electrically short structures can be extracted accurately by (quasi-)stationary computation, electrically long structures require a dynamic computation. A graphical interpretation is depicted in Figure 3.12. According to [75, 78], 


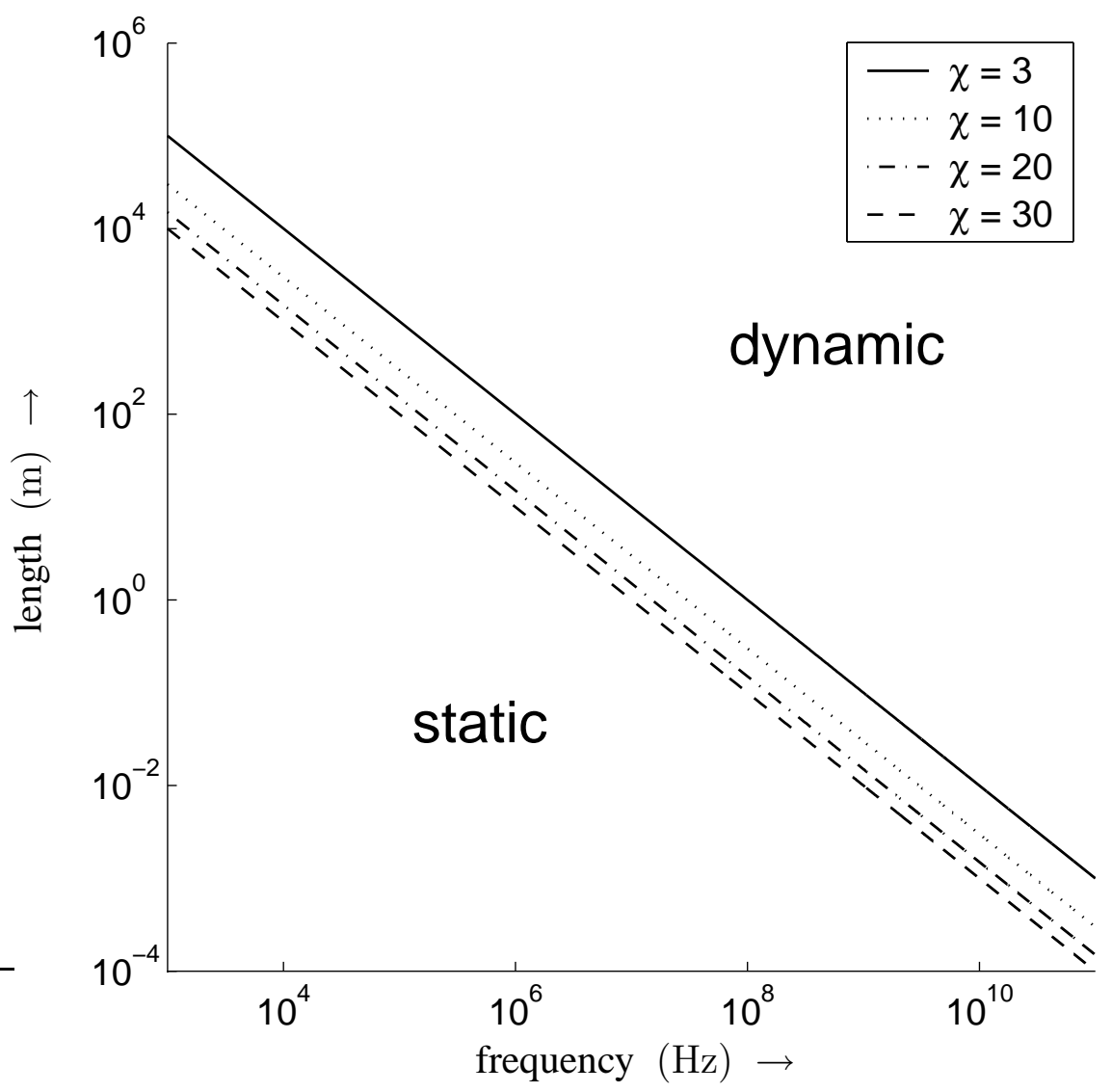

Fig. 3.12: Calculation domains for PEEC modeling according to [36].

quasi-stationary analysis only requires one static field computation in modeling of electric circuits for arbitrary frequencies below the maximum allowed frequency. Structural properties as structure length, cross section extensions, slew-rate behavior, clock rate, logic level, dielectrics and ohmic losses can help to evaluate the relevance of effects, resulting from fast signal transmission. While electrically short structures can efficiently be captured by lumped models, distributed or full-wave approaches are more suitable for electrically long structures [11].

\subsubsection{Accuracy Aspects}

To achieve high accuracy in partial element computation, the shape of the structure and thus the ratio of length to width and thickness of the cells resulting from discretization have to be taken into consideration. Both with analytical and numerical integration formulae, unqualified mesh generation can lead to accuracy degradations. If numerical integration techniques are involved, accuracy degradation can significantly affect the simulation stability. In the case of coplanar (plane) arrangements, the ratio of meshsize and distance between the plates determines the accuracy of the solution considerably. Moreover, inhomogeneous meshing often leads to accuracy and stability problems [79].

\subsubsection{Validity Aspects}

For the investigation of validity, a separate view to the TD and the FD is meaningful. 


\subsubsection{Frequency Domain}

The so-called "active" frequency range ${ }^{6}$ significantly influences the validity of PEEC models. Regarding (3.70) for electrically short structures, an accurate approximation of the waveform can be obtained if a wavelength was described with at least $\chi=20$ cells. This corresponds to an active frequency range $f=0 \ldots \frac{c}{\chi h} \mathrm{~Hz}$ for a maximum cell extension ${ }^{7} h$. Applying the quasi-stationary approximation, a so-called $\left(L_{p}, C_{p}, R\right) \mathrm{PEEC}$ model results with partial capacitances $C_{p}$ from electrostatic considerations. The electromagnetic wave in conducting medium is assumed to propagate with infinite speed. The frequency range $f=\frac{c}{\chi h} \ldots f_{e} \mathrm{~Hz}$ with the upper bound $f_{e} \approx 50 f_{\max }$, is called the "extended" frequency range $[15,68]$. To guarantee simulation accuracy and validity in the extended frequency range, the assumption of infinite propagation speed of the electromagnetic wave is no longer valid. For a full-wave approach, a model based on electrostatic partial capacitances is not sufficient. In this case a $\left(L_{p}, P_{p}, R, \tau\right)$ PEEC model is necessary, where capacitive effects are modeled by partial coefficients of potential. To guarantee a valid PEEC model computation for the retarded case (refer to Section 3.10), a subdivision of the cells into subcells with maximum cell extension

$$
h_{e}=\frac{c}{\alpha f_{e}}
$$

for a suitably chosen constant $\alpha$ can be useful. In accordance with the literatur $[68,15]$, the resulting models are called + PEEC models.

\subsubsection{Time Domain}

The bound of the active frequency range can be determined from rise/fall times of a transient signal. Even if the spectrum defined by the signal reaches against infinity, a maximum frequency can be defined representing an upper bound for relevant frequency components [11]. Assuming a finite frequency spectrum is reasonable because higher frequency components can be neglected without accuracy degradation. In practical applications with signal rise time $t_{\text {rise }}$, the expression

$$
f_{\text {max }} \approx \frac{0,35}{t_{\text {rise }}}
$$

holds. In extremal cases, the maximum frequency can amount up to $f_{\max } \approx \frac{1}{t_{\text {rise }}}$. The minimal rise time can be achieved combining (3.73) and (3.70), resulting in

$$
t_{\text {rise }} \geqslant \frac{0,35 \cdot h \cdot 20}{c}
$$

which directly impacts the minimum number of cells

$$
N_{\min }=\frac{l_{\max }}{h}=\frac{0,35 \cdot 20}{c} \cdot \frac{l_{\max }}{t_{\text {rise }}}=\frac{0,35 \cdot 20}{t_{\text {rise }}} \tau,
$$

with the maximum extension $l_{\max }$ of the structure. The time step for TD analysis has to be chosen so that it is small enough to enable an exact characterization of the fields, regarding the TD basis

\footnotetext{
${ }^{6}$ The active frequency range is specified by the largest extension of inductive and capacitive cells.

${ }^{7}$ In integrated circuits, the maximum frequency can be estimated by means of the fastest on-chip slew-rate time, which amounts approximately 50 to 100 times the clock frequency [80].
} 
functions. On the other hand, the time step has to be large enough to guarantee implicity of the equation system and stability [72]. Both very high clock frequencies and very short rise times result in very large models. Especially for TD simulations and together with MOR techniques, the analysis of the complex resonance behavior in the extended frequency range can be necessary [68].

\subsubsection{Simulation Artifacts}

Simulation artifacts in the TD and in the FD can occur as a result of the specific character of the PEEC model or from a violation of meshing-, modeling- and simulation guidelines [15]. So-called late-time instabilities, typical for IE-based EM modeling in TD analysis can occur from a discretization of the IE and/or the numerical integration technique [15]. In FD simulation, a separate view to both frequency ranges from Section 3.7.1 is meaningful. Simulation artifacts in the extended frequency range can appear as a kind of high-frequency instabilities and are often shown as negative real parts in the input impedance of a PEEC model analysis. For a treatment of such kind of artifacts, refer to [68]. The main focus of this thesis is on FD artifacts in the active frequency range. Such artifacts can occur from poor meshing, from inaccurate partial element computation and from PEEC model complexity reduction. Figure 3.13 shows an example of FD artifacts in quasi-stationary PEEC modeling. Applying a poor meshing strategy can increase already existing artifacts in the same way as the application of MOR techniques [15].

\subsection{Numerical Integration by Cubature Techniques}

Singular integrals, as arising from computations in Sections 3.2 and 3.3, make it necessary to apply efficient and accurate numerical integration methods. Based on quadrature theory ${ }^{8}$, cubature methods for the approximation of surface integrals arising from Galerkin discretizations of three-dimensional boundary integral equations, are the key. An efficient cubature is necessary to guarantee an accurate element extraction in PEEC modeling. This can help to avoid the occurance of simulation artifacts. A consideration of suitable quadrature techniques w.r.t. memory requirements for storing a matrix and time cost for the application of a matrix-vector multiplication is especially important in the context of hierarchical matrices in Section 4.3. The aim is, to approximate a system matrix from a Galerkin discretization with storage $\mathcal{O}(n)$ and to enable processing with $\mathcal{O}(f(n) n)$ [47]. The complexity $f(n)$ for the computation of $n$ entries of the system matrix incorporates the complexity of the numerical quadrature, which has to be accomplished more accurately the finer the discretization of the surface. The context $f(n)=n \log (n)^{\kappa}, \kappa>0$ holds in general, with the parameter $\kappa$ depending on the kernel of the integral operator and the kind of discretization. The influence of $f(n)$ originates from the nearfield and can not be avoided [47]. A detailed look at quadrature basics is presented in Appendix A.6.

\subsubsection{Cubature Techniques According to Sauter}

The cubature method is very flexible for a wide class of integral equations. The aim is, to realize an efficient implementation always choosing the minimum cubature order by ensuring a required

\footnotetext{
${ }^{8}$ The theory on numerical quadrature was already presented by GAUSS in 1814 [81].
} 

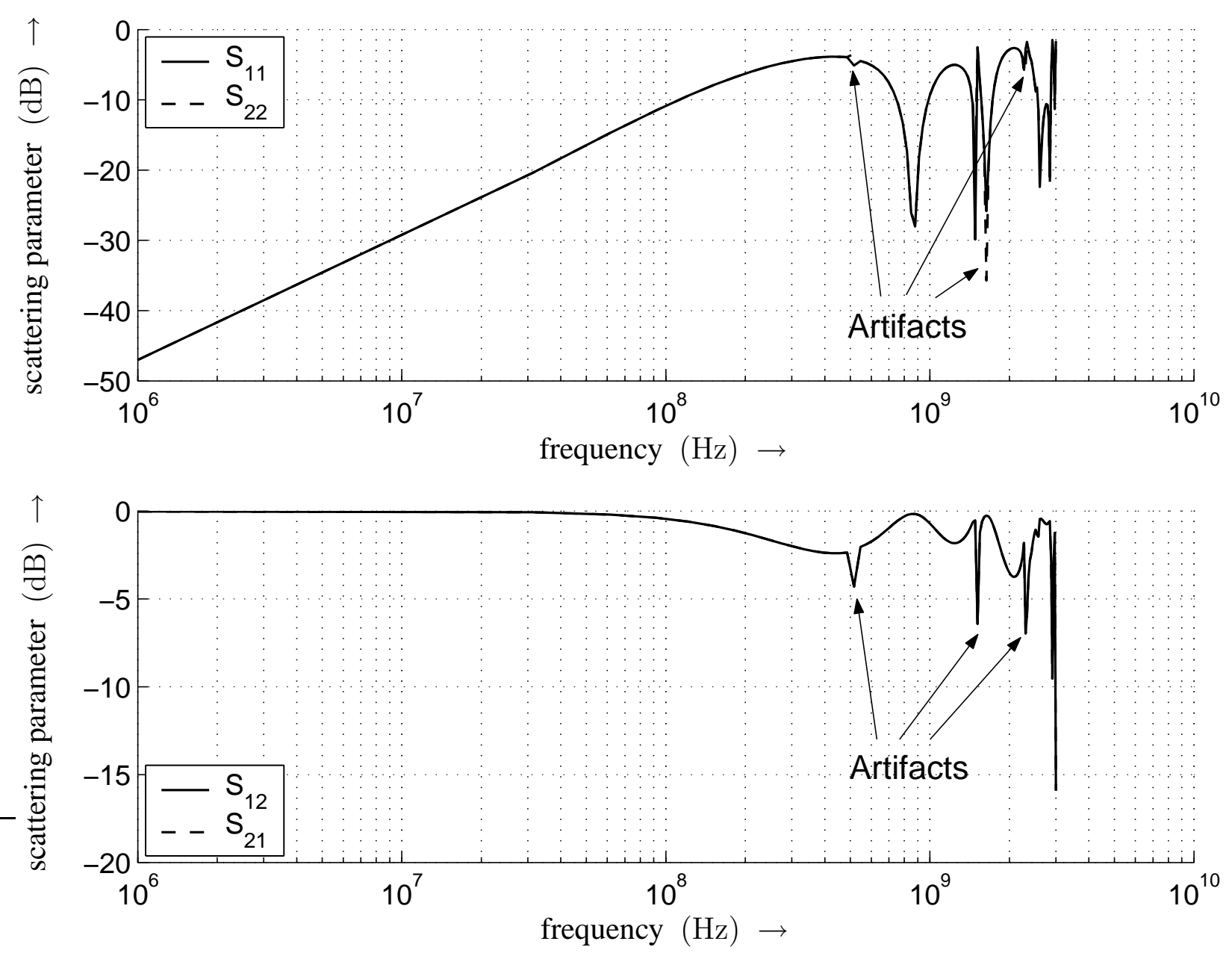

Fig. 3.13: Frequency domain simulation artifacts resulting from inaccurate model computation.

accuracy. The cubature method from SAUTER is fully discrete. For computation merely the kernel specific subroutine has to be replaced, not the numerical integrator itself. The idea is, to apply a regulization to the singular integrals such that the integrands are analytic and standard cubature formulae can be used. According to [82, 83], the four cases - identical panels, common edge, common vertex and regular case have to be distinguished. The cubature order and the number of cubature points have to be chosen, so that asymptotic convergence of the Galerkin method has to be ensured. The aim is a minimization of the cubature order by simultaneously ensuring proper consistency and stability. In the case of identical panels the location of the singularity is not fixed. Therefore, the singularity can not be cancelled out by degenerate coordinate transfroms, e.g. polar coordinates. To solve such problems, Sauter introduced a specific coordinate transform to fix the singularity by applying relative coordinates. The mathematical details can be found in [82]. For the cases of common edges and common vertices the transforms are similar. The quadrature point distributions in the reference triangle for the different cases are depicted in Figure 3.14 for a quadrature order of 7 . To validate the accuracy of the quadrature results, an arrangement of two triangles in 3D is considered, as depicted in Figure 3.15. While the location of one triangle is fixed, the second triangle is moved in $x$ - and $z$-direction. The interactions between the two triangles according to (3.12) are numerically computed, dependent on $\mathrm{d} x$ and $\mathrm{d} z$. The curves show continuous behavior and agree with analytic formulae. 


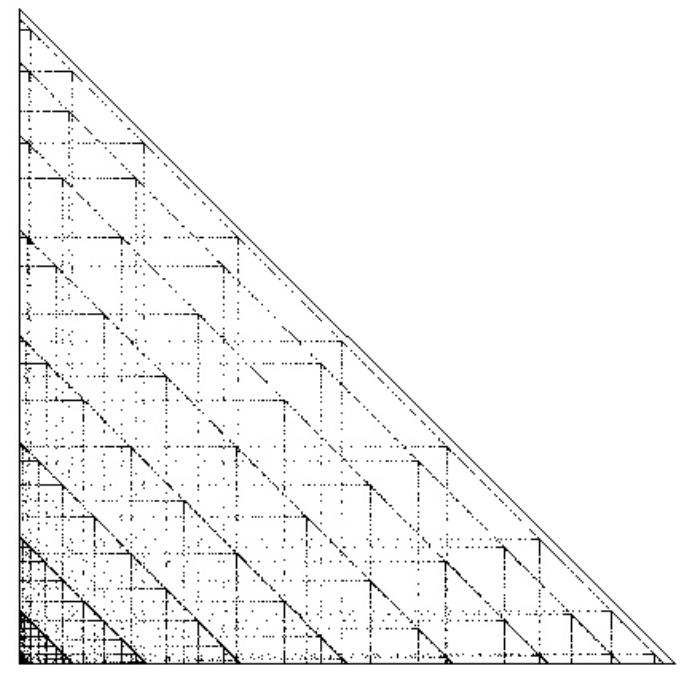

a)

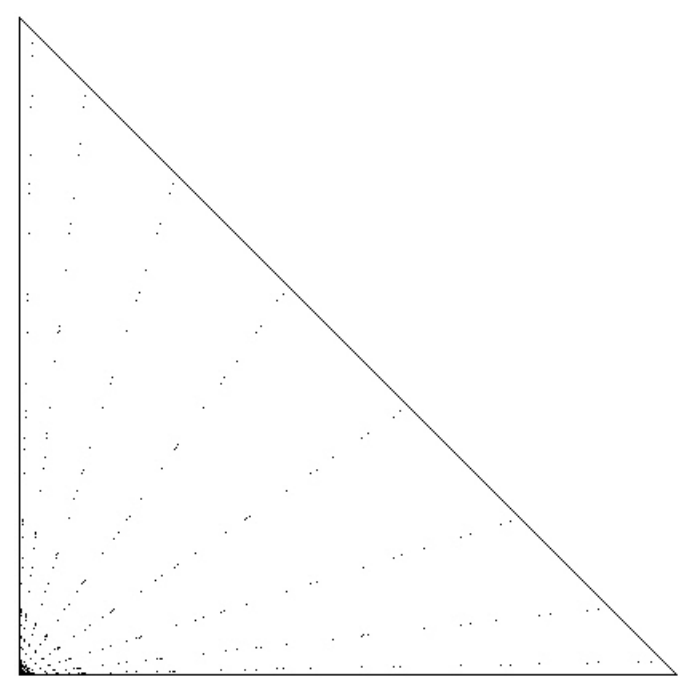

c)

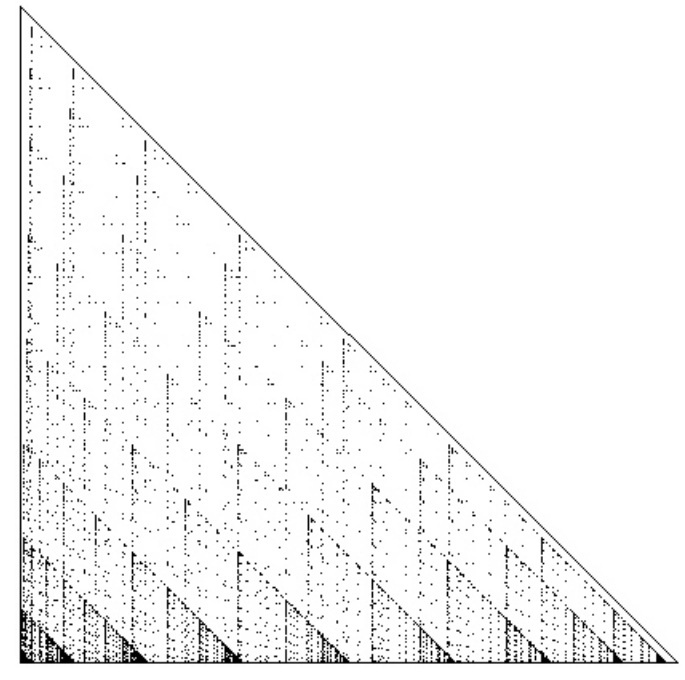

b)

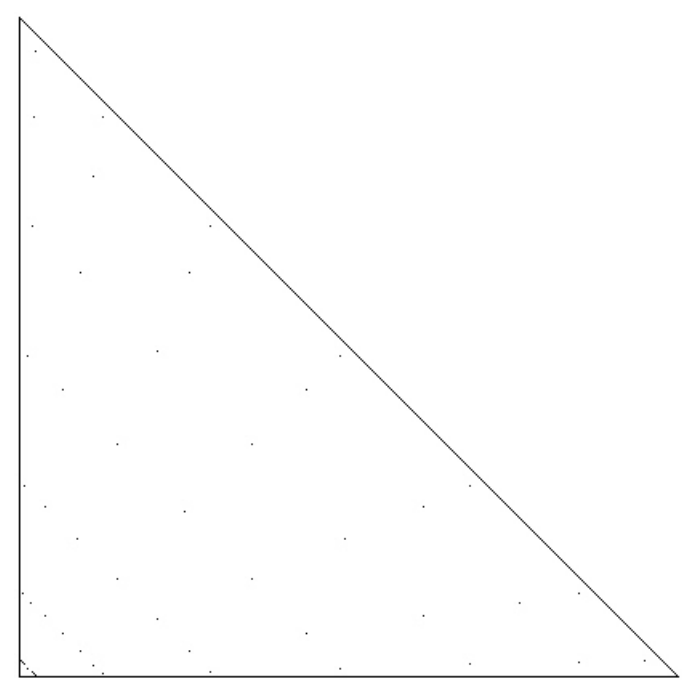

d)

Fig. 3.14: Distribution of Sauter quadrature points of order 7 in the reference triangle $(0,0),(1,0),(0,1)$ for the different cases a) identical triangles, b) triangles with common edge, c) triangles with common vertex and d) distant triangles.

\subsubsection{Hierarchical Quadrature}

The concept of a hierarchical quadrature bases on an admissible partitioning into subdomains, as known from the panel clustering [84] and hierarchical matrix techniques $[19,20]$. Similar to hierarchical methods as desribed in Chapter 4, a nearfield and farfield separation is applied. The hierarchical quadrature is used together with adaptive refinement techniques (refer to Section 4.3.5.1) implying a gradual splitting of the integration region into suitable subregions. If subregions contain no singularity, they can be treated by standard quadrature. The remaining subregions are split again [85, 66]. Adaptive refinement comprises the advantage to be generally applicable. Nevertheless, it is very costly because the number of levels of refinement can be very large. Based on the structural properties of the integral, which is essential for the efficiency of the method, 


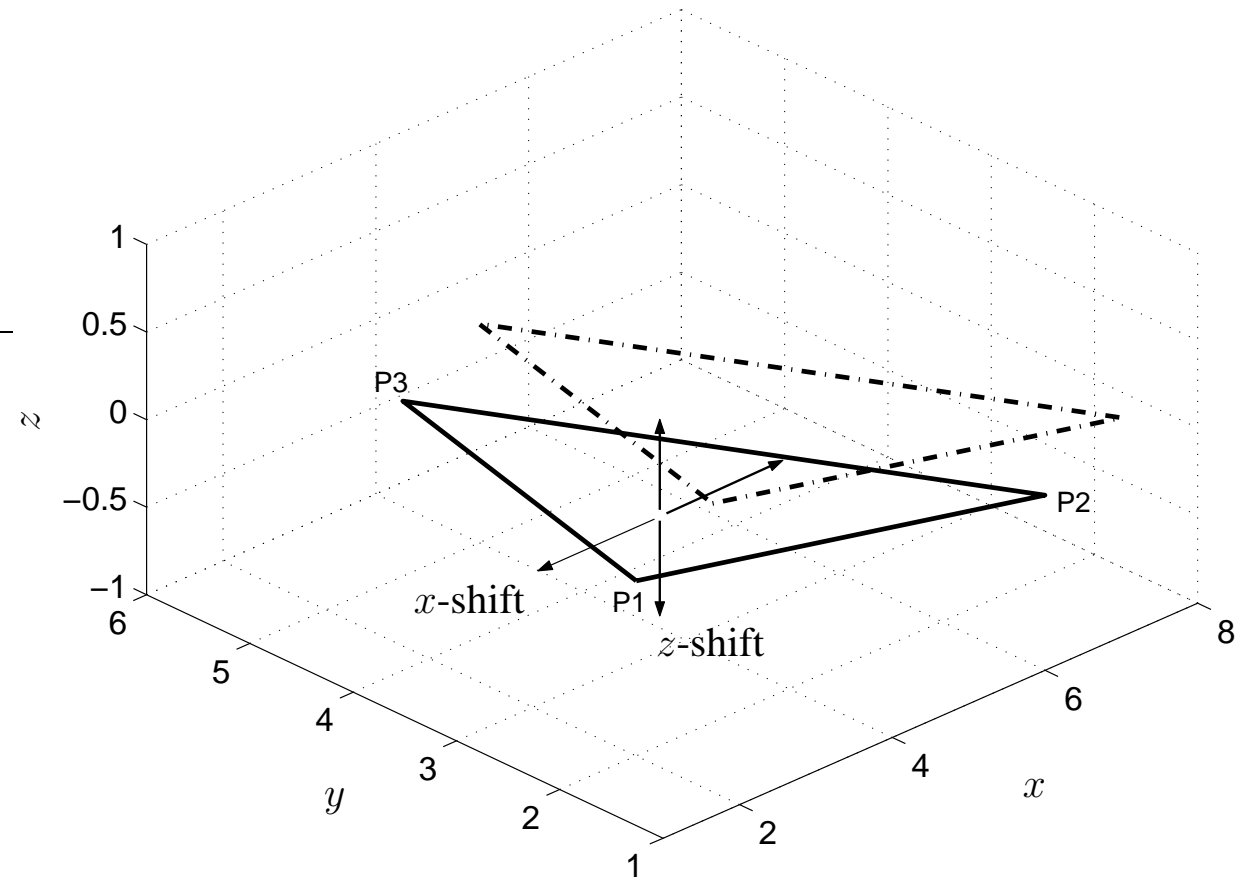

a)

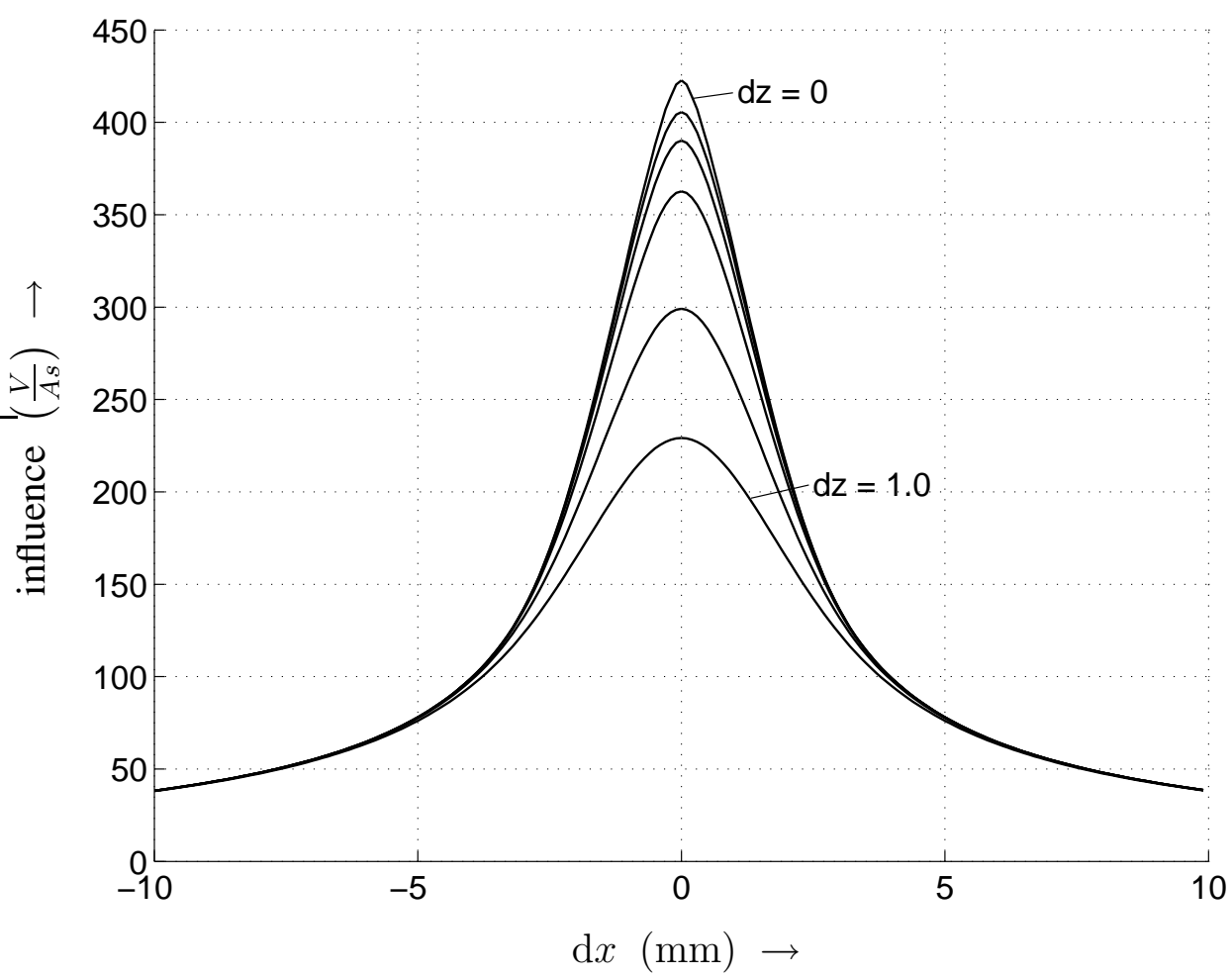

b)

Fig. 3.15: Convergence behavior and accuracy of the Sauter quadrature for the influence computation between two triangles. a) Triangle arrangement in $3 \mathrm{D}$ with triangle $1:(2,2,0)^{\mathrm{\top}},(6,1,0)^{\mathrm{\top}},(3,5,0)^{\mathrm{T}}$; triangle $2:(2+\mathrm{d} x, 2, \mathrm{~d} z)^{\mathrm{T}}$, $(6+\mathrm{d} x, 1, \mathrm{~d} z)^{\mathrm{\top}},(3+\mathrm{d} x, 5, \mathrm{~d} z)^{\top}$. Relative to the origin, increments $\mathrm{d} x$ in $x$-direction and discrete shifts $\mathrm{d} z=$ $0,0.05,0.1,0.2,0.5,1.0 \mathrm{~mm}$ in $z$-direction are applied. The axes scale in millimeter. $\mathrm{b}$ ) Resulting influence curves. 
the cases of weak singularities, integrals with polynomials and strong singularities have to be distinguished [85]. Exploiting such properties, the algorithmic complexity of the approach can be reduced significantly. The successive partitioning leads to the construction of a so-called splitting tree.

\subsection{Sparse Matrix Formulation via Partial Reluctances}

\subsubsection{Motivation}

Parasitics matrices resulting from a PEEC-based extraction can be very large and dense for real-life problems. This makes circuit simulation prohibitively costly. To avoid a disproportional increase in memory and time requirements for model extraction as well as for simulation, it is necessary to apply suitable matrix compression techniques. A very simple strategy bases on the truncation of small off-diagonal matrix elements. From an engineering-based point of view, this method is easy to implement and produces stable and accurate models at least for partial capacitances. The truncation of partial inductances, however, requires some additional investigations. Partial inductances purely depend on the geometric arrangement between conductor segments. This makes partial mutual inductances reciprocal and the constitutive branch equations for partial inductances are identical to that of closed-loop inductances [86]. With integrated circuits, current return paths often can not be determined prior to extraction or simulation. Assuming the return paths to be located at infinity directly leads to extremely large and dense inductance matrices because all couplings have to be taken into account. From a physical point of view, partial inductances can be interpreted as the drop of the normalized magnetic vector potential along a conductor segment, caused by a current in the considered or a neighbouring segment. Regarding the computation rule for partial inductances (refer to [54]), the partial inductance matrix $\boldsymbol{L}=\left[L_{i j}\right], i, j=1, \ldots, N$ for a system of $N$ conductors is dense, real-valued and symmetric positive definite (SPD). By multiplying $\boldsymbol{L}$ with a vector of $N$ branch currents, a vector containing the drops of the magnetic vector potential along the segments can be obtained, resulting in the equation system

$$
\left[\begin{array}{cccc}
L_{11} & L_{12} & \ldots & L_{1 N} \\
L_{21} & L_{22} & \ldots & L_{2 N} \\
\vdots & \vdots & \ddots & \vdots \\
L_{N 1} & L_{N 2} & \ldots & L_{N N}
\end{array}\right] \cdot\left[\begin{array}{c}
i_{1} \\
i_{2} \\
\vdots \\
i_{N}
\end{array}\right]=\left[\begin{array}{c}
\sum_{i=1}^{N}\left(\int A_{1 i} \mathrm{~d} \vec{l}_{1}\right) \\
\sum_{i=1}^{N}\left(\int A_{2 i} \mathrm{~d} \vec{l}_{2}\right) \\
\vdots \\
\sum_{i=1}^{N}\left(\int A_{N i} \mathrm{~d} \vec{l}_{N}\right)
\end{array}\right]
$$

From [87] it is apparent that coefficients $L_{i j}$ only depend on the relative position and length of the individual conductor segments. The coefficients are independent from the presence of neighbouring conductors. Different to capacitances, the off-diagonal elements of $\boldsymbol{L}$ decrease slightly with order of $\mathcal{O}\left(\log \left(\left\|\vec{r}_{p}-\vec{r}_{q}\right\|\right)\right)$ for increasing distance between source and observation point. A truncation of small off-diagonal elements of $\boldsymbol{L}$ can lead to a violation of the positive definiteness [86]. As a result simulation instabilities can occur. 


\subsubsection{Partial Reluctance $K$}

W.r.t. iterative solution methods (refer to Chapter 5) a sparse representation of the network matrix is desirable. This can be realized by applying a truncation process to small off-diagonal matrix entries. The sparsity of a matrix results from the ratio of the number of non-zero elements and the total number of coefficients of the matrix. As mentioned in Section 3.9.1, numerical problems can occur from truncation $[88,86]$. To preserve the positive definiteness of the partial inductance matrix after truncation, several truncation strategies were developed [87] as the shift-truncate potential method [86, 88, 89, 90], the return-limited loop inductance method [80] and the partial reluctance method $[87,91]$. The first two approaches often suffer from loosing accuracy because of unfit current return path assumptions. The third approach uses the partial reluctance $e^{9}$ matrix

$$
\boldsymbol{K} \triangleq \boldsymbol{L}^{-1}
$$

instead of the partial inductance matrix formulation. For physical explanations refer to [92]. Equation system (3.76) together with (3.77) result in

$$
\left[\begin{array}{cccc}
K_{11} & K_{12} & \ldots & K_{1 N} \\
K_{21} & K_{22} & \ldots & K_{2 N} \\
\vdots & \vdots & \ddots & \vdots \\
K_{N 1} & K_{N 2} & \ldots & K_{N N}
\end{array}\right] \cdot\left[\begin{array}{c}
\sum_{i=1}^{N}\left(\int A_{1 i} \mathrm{~d} \vec{l}_{1}\right) \\
\sum_{i=1}^{N}\left(\int A_{2 i} \mathrm{~d} \vec{l}_{2}\right) \\
\vdots \\
\sum_{i=1}^{N}\left(\int A_{N i} \mathrm{~d} \vec{l}_{N}\right)
\end{array}\right]=\left[\begin{array}{c}
i_{1} \\
i_{2} \\
\vdots \\
i_{N}
\end{array}\right]
$$

From (3.78) it is obvious that the partial reluctances can be computed in two different ways - from an elementwise extraction similar to the extraction of partial inductances or from an inversion of the partial inductance matrix. Both extraction approaches are equivalent if skin- and proximity-effects can be neglected and each conductor segment can be regarded as an individual conductor filament [93]. Formally, a physical interpretation of element $K$ can be given as described in Definition 3.1 $[89,92]$, which can be regarded as a rule for computation.

Definition 3.1: Element $K_{i j}$ represents the induced normalized current, flowing through conductor segment $i$, assuming that the magnetic vector potential drops along all other segments excepting segment $j$, is set to zero. The magnetic vector potential drop along segment $j$ then amounts to value one. From (3.78) it is obvious that the current induced in each individual segment corresponds to the elements in the $i$ th column of matrix $\boldsymbol{K}$ if the magnetic vector potential drop along segment $j$ is one.

For partial reluctances it is not possible to formulate a context similar to (3.11) or (3.26). The elements of $\boldsymbol{K}$ depend on the geometry of the conductors. A computation of the values makes it necessary to solve a linear equation system.

\footnotetext{
${ }^{9}$ Remark: The expression susceptance, which is often used in the literature for the inverse of a partial inductance, formally represents the imaginary part of a complex impedance. Due to this fact, susceptance can also represent a capacitance and therefore can be slightly misleading in this context.
} 


\subsubsection{Reluctance Matrix Properties}

The analysis of the reluctance matrix properties are historically based on bus structure arrangements [92]. Matrix $\boldsymbol{K}$ is unsymmetric in general because of $K_{i j}^{(i)} \neq K_{i j}^{(j)}$. A symmetric and stable reluctance model can be guaranteed by choosing the value with smaller magnitude [90].

\subsubsection{Screening Effect and Locality}

Regarding multiconductor arrangements it can be observed, that the magnetic field resulting from a current flow through the neighbouring conductors counteracts the field injected in the so-called aggressor. The fields of the neighbouring conductors therefore shield the field coupling into the aggressor. The shielding effect is the physical reason for the locality of matrix $\boldsymbol{K}$. Due to locality, only few conductors in small distance from the considered conductor have to be taken into consideration $[93,87]$. The shielding effect leads to the fact, that mutual reluctance terms drop off much faster than the mutual inductance terms. By applying a truncation to small coupling values in the partial reluctance matrix, the inductive effects can be captured more effectively compared to partial inductances. A mathematical explanation for the relationship between reluctances $K$ and capacitances $C$ and between inductances $L$ and influences $I$ can be derived from the assumption, that an existing left- or right-inverse $\mathcal{D}^{-1}$ of a linear partial differential operator $\mathcal{D}$ can be understood as an integral operator

$$
\mathcal{D}^{-1} u(\vec{x})=\int g(\vec{x}, \vec{y}) u(\vec{y}) \mathrm{d} \vec{y}
$$

using Green's function as SCHWARTZ kernel $g[94,95]$. Therefore, the reluctance as the inverse of an inductance matrix, can be computed as based on a differential approach similar to the capacitance matrix.

\subsubsection{Stability}

Assuming some normalized vector potential $\sum_{j=1}^{N}\left(\int \vec{A}_{i j} \mathrm{~d} \vec{l}_{j}\right)=1, \forall i=1,2, \ldots, N$, together with (3.78) it follows

$$
i_{i}=\sum_{j=1}^{N} K_{i j}>0, \quad i=1,2, \ldots, N
$$

resulting in

$$
K_{i i}>\sum_{\substack{j=1 \\ j \neq i}}^{N}\left|K_{i j}\right|
$$

for $K_{i i}>0$ und $K_{i j}<0, \forall i \neq j$. The property (3.81) is known as strict diagonal dominance of a matrix. By means of GERSHGORIN's circle theorem (refer to Appendix A.4) it can be shown [92], that a matrix is positive definite if it is strictly diagonally dominant and all entries on the main diagonal are positive ${ }^{10}$. The property to be strictly diagonally dominant holds for reluctance matrices for arbitrary conductor arrangements [92] and is preserved during truncation [89] because of $K_{i i}>0$. A stability check can be realized by observing the locations of eigenvalues of the

\footnotetext{
${ }^{10}$ Even if the off-diagonal elements of matrix $\boldsymbol{K}$ are non-negative, which can occur in improper chosen conductor arrangements [96], the positive definiteness of the reluctance matrix can be proven [89, 97].
} 
system in the complex plane. It is well known from circuit theory that lumped circuits without independent as well as dependent sources and with positive circuit elements are passive. Referring to Appendix A.1, a positive definite matrix is a symmetric MinKOWSKI-matrix. However, a sparsified reluctance matrix similar to the influence matrix of the electric field not inevitably has to be symmetric. Therefore, in this context it is recommended to speak about M-matrices rather than positive definite matrices. For the proof that a truncated reluctance matrix is again a M-matrix, the reader is referred to [89].

\subsubsection{Truncation Process}

A sparsification of the reluctance matrix $K$ can be realized by applying a truncation process

$$
\mathcal{T}_{K}: \boldsymbol{K} \longrightarrow \tilde{\boldsymbol{K}}
$$

to small off-diagonal couplings by means of the coupling factor

$$
k_{i j} \triangleq \sqrt{\frac{K_{i j}^{2}}{\left|K_{i i}\right| \cdot\left|K_{j j}\right|}}
$$

between the inductive elements $i$ and $j$. The truncation rule (Algorithm 1) cancels out off-diagonal entries if the coupling value is below a given threshold $\delta$. The density (sparsity) of matrix $\tilde{\boldsymbol{K}}$ of

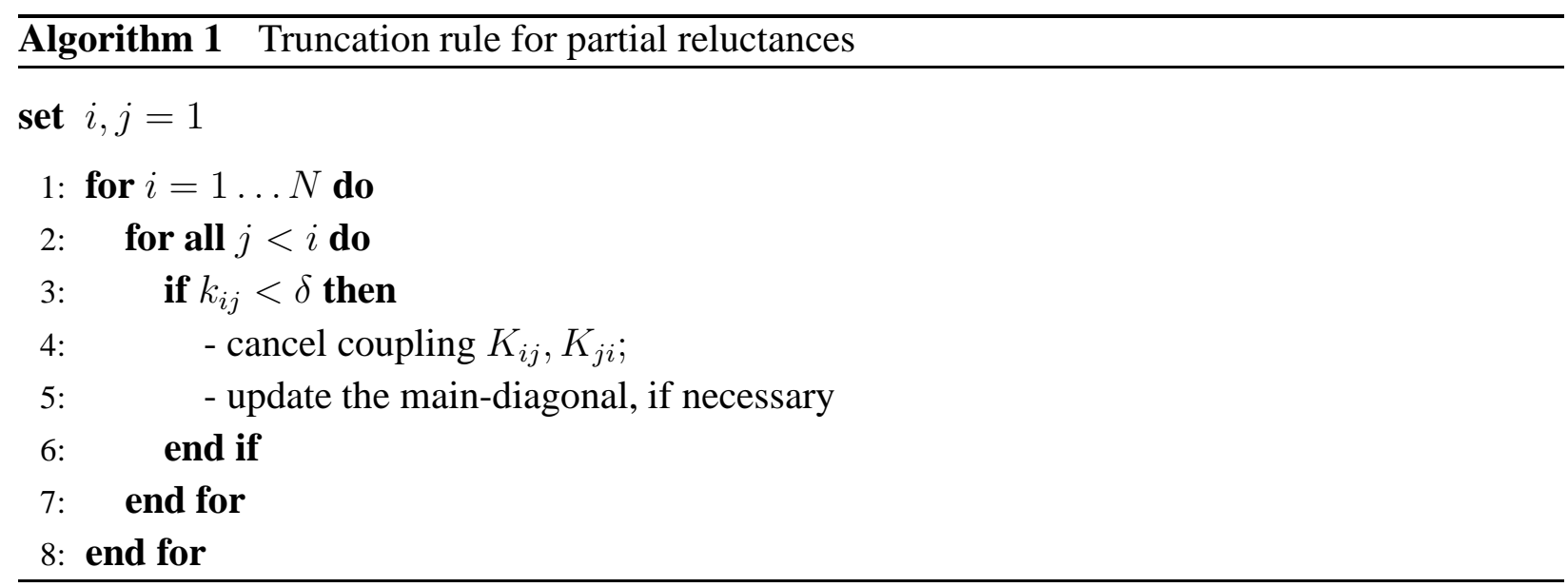

dimension $N \times N$ after truncation can be defined as

$$
\operatorname{dens}_{\delta}(\tilde{\boldsymbol{K}}) \triangleq \frac{n_{\mathrm{nz}}(\tilde{\boldsymbol{K}})}{N^{2}}
$$

with the number of non-zero elements $n_{\mathrm{nz}}(\tilde{\boldsymbol{K}})$.

\subsubsection{Reluctance-Based Circuit Representation}

To enable an efficient and accurate simulation based on sparsified reluctance and capacitance matrices, a SPICE compatible formulation is necessary based on the MNA formulation as described in Appendix D. From [91] it is evident, that linear networks merely containing resistances, capacitances, reluctances and current sources, represent symmetric positive definite systems. For a mathematical proof and further details, the reader is referred to [91, 98, 99, 100, 101]. 


\subsubsection{SPICE Implementation}

Based on the MNA formulation

$$
\left[\begin{array}{cc}
\boldsymbol{G} & \boldsymbol{A}_{l}^{\top} \\
-\boldsymbol{A}_{l} & \mathbf{0}
\end{array}\right] \cdot\left[\begin{array}{c}
\vec{u}_{n} \\
\vec{i}_{l}
\end{array}\right]+\left[\begin{array}{cc}
\boldsymbol{C} & \mathbf{0} \\
\mathbf{0} & \boldsymbol{L}
\end{array}\right] \cdot\left[\begin{array}{c}
\dot{\vec{u}}_{n} \\
\dot{\vec{i}}_{l}
\end{array}\right]=\left[\begin{array}{c}
-\boldsymbol{A}_{s}^{\top} \vec{i}_{q} \\
\overrightarrow{0}
\end{array}\right]
$$

including the partial inductance matrix $\boldsymbol{L}$ (refer to (D.18) on side 194), the reluctance-based MNA formulation

$$
\left[\begin{array}{cc}
\boldsymbol{G} & \boldsymbol{A}_{l}^{\top} \\
-\tilde{\boldsymbol{K}} \boldsymbol{A}_{l} & \mathbf{0}
\end{array}\right] \cdot\left[\begin{array}{c}
\vec{u}_{n} \\
\vec{i}_{l}
\end{array}\right]+\left[\begin{array}{cc}
\boldsymbol{C} & \mathbf{0} \\
\mathbf{0} & \mathbf{1}
\end{array}\right] \cdot\left[\begin{array}{c}
\dot{\vec{u}}_{n} \\
\dot{\vec{i}}_{l}
\end{array}\right]=\left[\begin{array}{c}
-\boldsymbol{A}_{s}^{\top} \vec{i}_{q} \\
\overrightarrow{0}
\end{array}\right],
$$

can be obtained by multiplying the second equation line in (3.85) by the sparsified reluctance matrix $\tilde{\boldsymbol{K}}$, assuming

$$
\tilde{K} L \approx 1
$$

Matrix $\tilde{\boldsymbol{K}}$ contributes to the unsymmetry of the system matrix which represents a challenge for common iterative solvers. A remedy can be achieved using the NA instead of the MNA formulation. For that purpose, the equation system (3.86) is split up into two parts

$$
\boldsymbol{G} \vec{u}_{n}+\boldsymbol{A}_{l}^{\top} \vec{i}_{l}+\boldsymbol{C} \dot{\vec{u}}_{n}=-\boldsymbol{A}_{s}^{\top} \vec{i}_{q}
$$

and

$$
-\tilde{\boldsymbol{K}} \boldsymbol{A}_{l} \vec{u}_{n}+\dot{\overrightarrow{i_{l}}}=\overrightarrow{0} .
$$

Applying the LAPLACE transform and inserting (3.89) into (3.88) result in

$$
\underbrace{\left(\boldsymbol{G}+s \boldsymbol{C}-\frac{1}{s} \boldsymbol{A}_{l}^{\top} \tilde{\boldsymbol{K}} \boldsymbol{A}_{l}\right)}_{\boldsymbol{Y}^{\mathrm{NA}-\mathrm{K}}} \vec{u}_{n}=\underbrace{-\boldsymbol{A}_{s}^{\top} \vec{i}_{q}}_{\vec{b}}
$$

with the diagonally dominant and SPD system matrix $\boldsymbol{Y}^{\mathrm{NA}-\mathrm{K}}$. In general, the density of the NA system matrix is slightly larger than the density in the MNA case since $\boldsymbol{A}_{l}^{\top} \tilde{\boldsymbol{K}} \boldsymbol{A}_{l}$ produces more matrix entries as originally existing in $\boldsymbol{L}$. This overhead, however, will be more than compensated through the advantageous of the improved matrix properties for iterative methods.

\subsubsection{Reluctance Stamping}

Similar to the stamping procedure for resistances, inductances and capacitances (refer to [102]), a stamping for partial reluctances can be applied in a straightforward manner. The stamp for the matrix entries of $\boldsymbol{A}_{l}^{\top} \tilde{\boldsymbol{K}} \boldsymbol{A}_{l}$ in the network matrix for the quasi-stationary case is given in Table 3.1. For each partial inductance this stamp contains eight terms, to be added at the corresponding locations in the network matrix. Where the partial self-reluctances are represented by the four upper entries of the stamp, the lower entries describe the mutual reluctance couplings to an inductivity located in branch $k$. Consequently, the lower part of the stamp has to be added for any additional inductive coupling. The reluctance stamping procedure for an example PEEC circuit with three nodes and two inductive branches (Figure 3.16) is described in the following. 


\begin{tabular}{|c||c|c|}
\hline node & $n+$ & $n-$ \\
\hline \hline$n+$ & $\frac{1}{\mathrm{j} \omega K_{n n}}$ & $-\frac{1}{\mathrm{j} \omega K_{n n}}$ \\
\hline$n-$ & $-\frac{1}{\mathrm{j} \omega K_{n n}}$ & $\frac{1}{\mathrm{j} \omega K_{n n}}$ \\
\hline$k+$ & $\frac{1}{\mathrm{j} \omega K_{n k}}$ & $-\frac{1}{\mathrm{j} \omega K_{n k}}$ \\
\hline$k+$ & $-\frac{1}{\mathrm{j} \omega K_{n k}}$ & $\frac{1}{\mathrm{j} \omega K_{n k}}$ \\
\hline
\end{tabular}

Table 3.1: Stamp for partial self-reluctance and mutual reluctance couplings for the quasi-stationary case. The involved inductive branches are denoted by $n$ and $k$ with nodes $n+, n-, k+$ and $k-$.

\section{Example $3.1 \quad K$-Stamping}

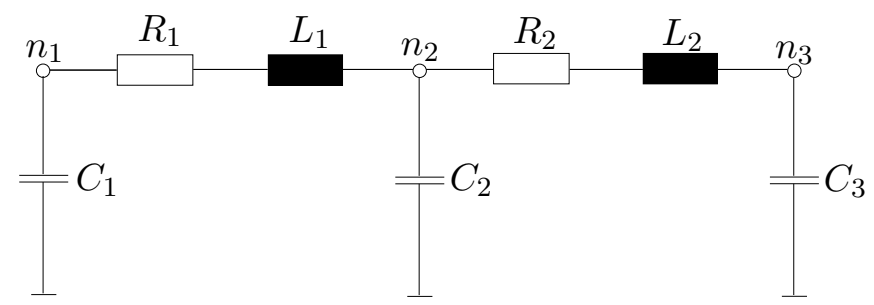

Fig. 3.16: Quasi-stationary PEEC model with three nodes and two branches.

According to the transformer equations [103] the connection

$$
\left[\begin{array}{l}
u_{1} \\
u_{2}
\end{array}\right]=\mathrm{j} \omega\left[\begin{array}{ll}
L_{11} & L_{12} \\
L_{21} & L_{22}
\end{array}\right] \cdot\left[\begin{array}{l}
i_{1} \\
i_{2}
\end{array}\right]
$$

between voltage and current can be defined, leading to

$$
\left[\begin{array}{c}
i_{1} \\
i_{2}
\end{array}\right] \stackrel{(3.77)}{=} \frac{1}{\mathrm{j} \omega}\left[\begin{array}{ll}
K_{11} & K_{12} \\
K_{21} & K_{22}
\end{array}\right] \cdot\left[\begin{array}{l}
u_{1} \\
u_{2}
\end{array}\right]
$$

including the reluctance matrix $\boldsymbol{K}$. This arrangement leads to the inductive part

$$
\boldsymbol{A}_{l}=\left[\begin{array}{ccc}
+1 & -1 & 0 \\
0 & +1 & -1
\end{array}\right]
$$

of the adjacency matrix $\boldsymbol{A}$. With the $2 \times 2$ partial inductance matrix and the corresponding inverse in (3.92), the reluctance part of the system matrix results in

$\boldsymbol{A}_{l}^{\top} \boldsymbol{K} \boldsymbol{A}_{l}=$

$$
\left[\begin{array}{ccc}
K_{11} & -K_{11}+K_{12} & -K_{12} \\
-K_{11}+K_{21} & K_{11}-K_{12}-K_{21}+K_{22} & K_{12}-K_{22} \\
-K_{21} & K_{21}-K_{22} & K_{22}
\end{array}\right]
$$

An agreement between the matrix entries and Table 3.1 is obvious. 


\subsubsection{Numerical Examples}

The following simulation results are obtained from selected traces and nets on the MISEA demonstrator board. Main attention for the investigations was focused on validity, accuracy and efficiency. For a detailed view concerning technical issues and the equipment under test, refer to Chapter 9 and [67], respectively.

\subsubsection{Validity and Accuracy Investigations}

To demonstrate the applicability of reluctance-based PEEC models, a $\left(K_{p}, C_{p}, R\right)$ PEEC-model of the net $\operatorname{HBUS}(0)$ on the MISEA demonstrator board is considered. The validity of the model in the FD up to $5 \mathrm{GHz}$ was assured by suitably choosing the meshsize, resulting in a network matrix of dimension $226 \times 226$. A sparsification of the resulting network matrix can be achieved by applying the truncation processes $\mathcal{T}_{C}$ and $\mathcal{T}_{K}$ to capacitances as well as reluctances, as described in Section 3.9.4. The resulting sparsity patterns of the network matrices for the three cases $\delta_{K}=0 \%, \delta_{C}=$ $0 \%, \delta_{K}=0.2 \%, \delta_{C}=0 \%$ and $\delta_{K}=0.2 \%, \delta_{C}=0.2 \%$ are illustrated in Figure 3.17 at a frequency of $1 \mathrm{MHz}$. As a result, a reduction of the matrix density from $\approx 73 \%$ down to $\approx 25 \%$ can be achieved. Consequently, a fast impedance analysis in the FD can be accomplished without worth mentioning loss in accuracy. From Figure 3.18 a) it is obvious that the impedance result curves for the proposed three cases show good accordance, while guaranteeing stability during simulation. The corresponding eigenvalue distributions in the complex plane are given in Figure $3.18 \mathrm{~b}$ ) also certifying good accordance.
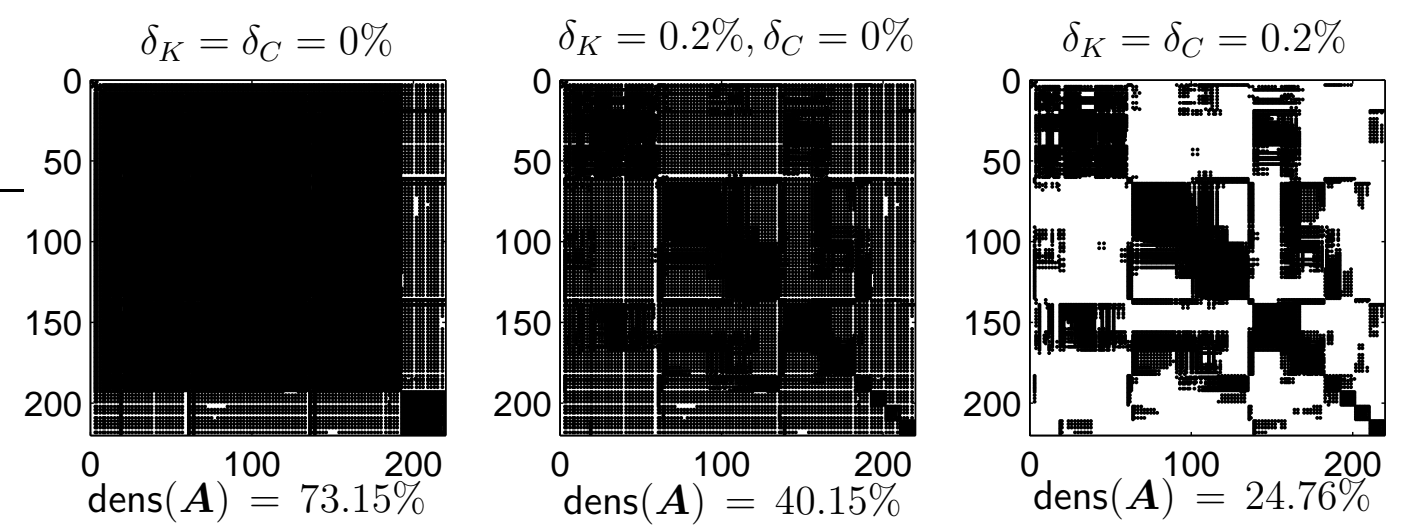

Fig. 3.17: Sparsity pattern of the resulting network matrices of dimension $226 \times 226$ for the three tolerance limits from reluctance-based PEEC modeling. The matrices were derived from a FD simulation at $1 \mathrm{MHz}$.

\subsubsection{Efficiency Investigation}

As shown in the previous section, the proposed sparsification approach enables an accurate and stable circuit simulation. Already small network matrices allow an enormous degree of sparsification compared to the conventional method. For investigations concerning efficiency a FD analysis was accomplished and the total simulation time was analyzed for different tolerance limits. The results were obtained using a direct solver (GMATRIX [73]). By applying an iterative solver, a higher gain compared to conventional methods can be expected. Again, a FD impedance analysis of a trace on the MISEA demonstrator board was considered, resulting in a network matrix of dimension $2026 \times 2026$. The tolerance limits for reluctances as well as for capacitances are varied such that 


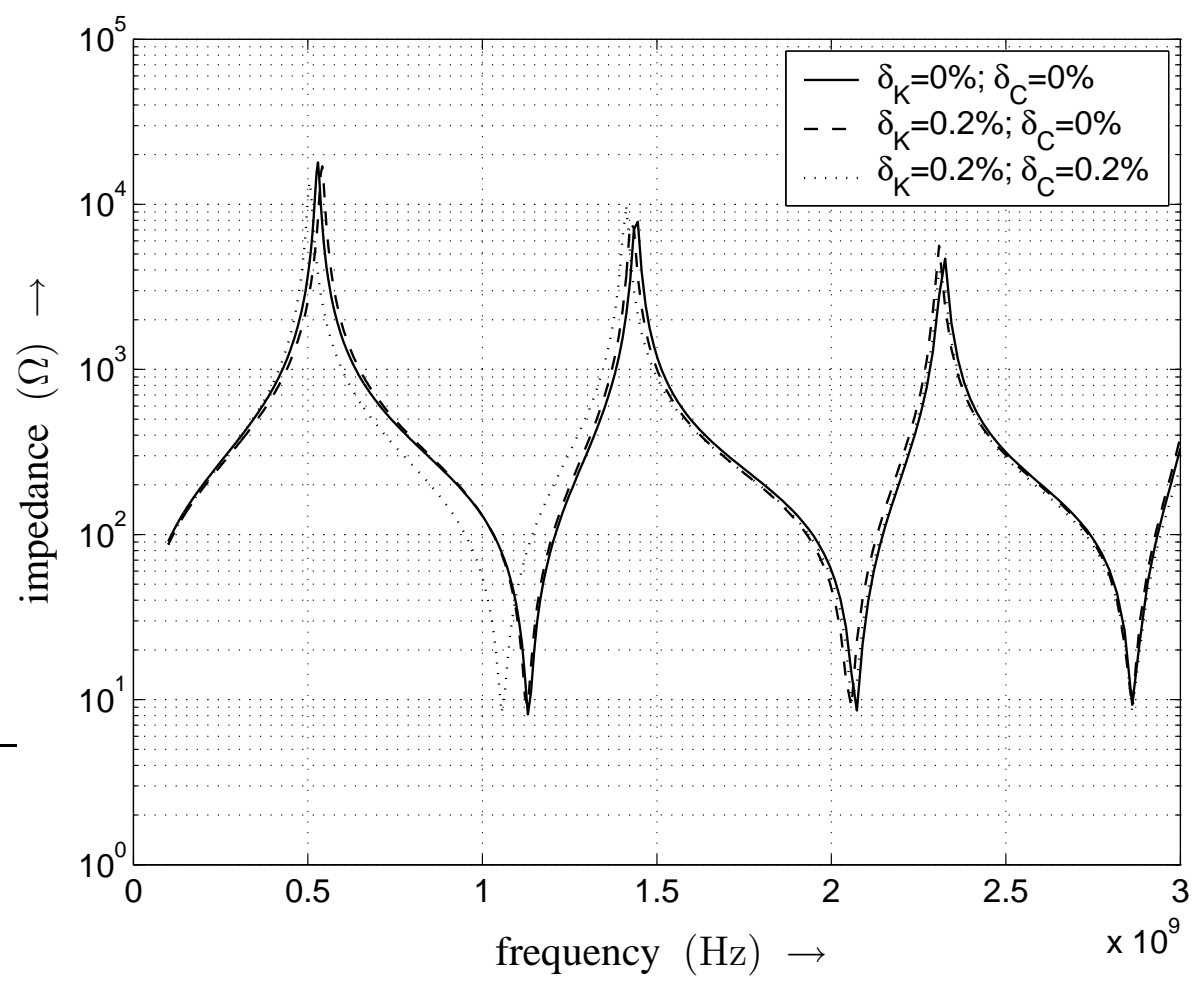

a)

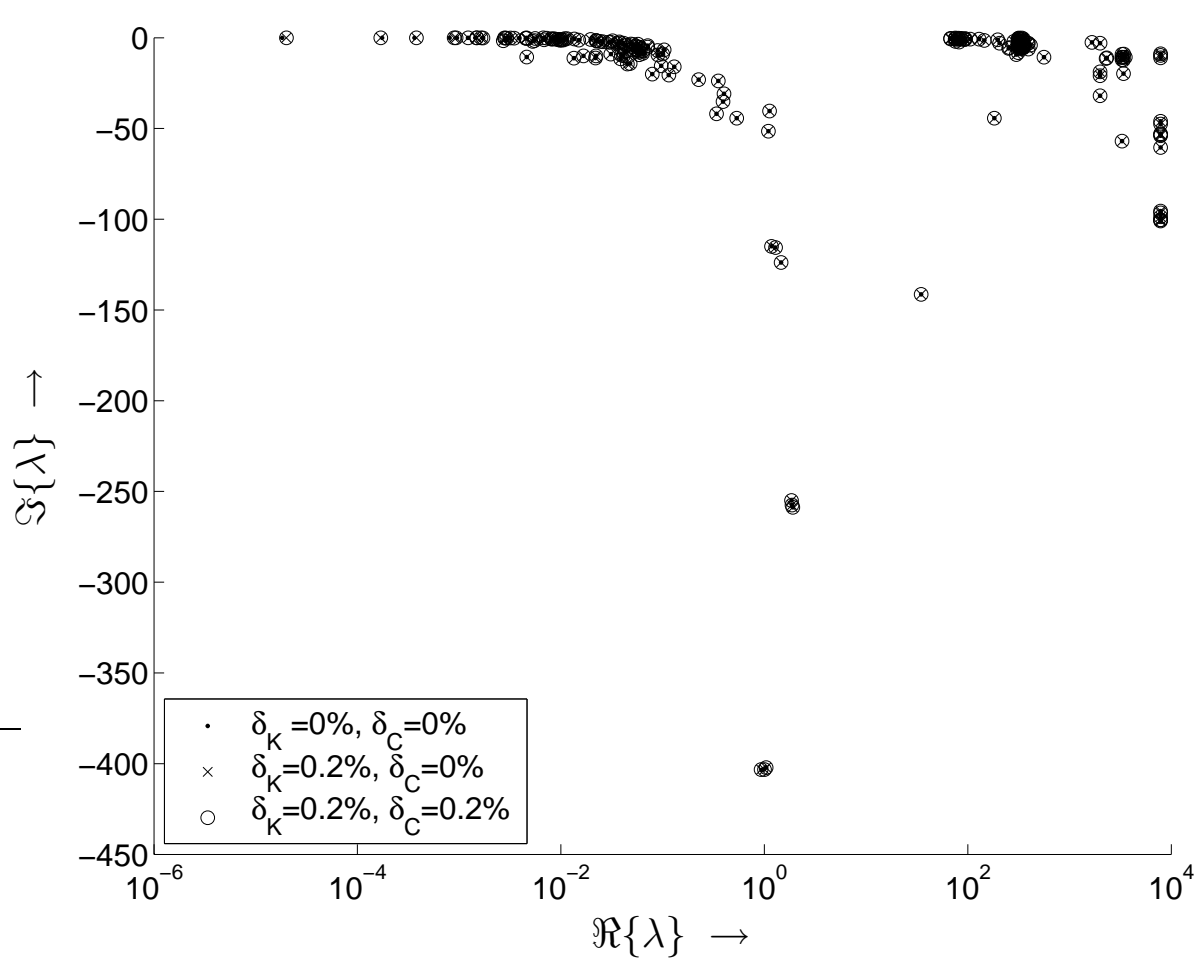

b)

Fig. 3.18: Impedance analysis comparison at different degrees of matrix sparsification. a) Input impedance analysis curves. b) Corresponding eigenvalue distributions in the complex plane. Remark: The eigenvalues show good accoradance also with increasing tolerance limits. 
in each step $\delta_{C}=\delta_{K}$ holds. A matrix sparsification from $\approx 93 \%$ down to $\approx 1 \%$ can be achieved. For the direct solver, a speed-up with a factor of $\approx 5$ results from varying $\delta_{C}=\delta_{K}=0 \ldots 1 \%$. As it is obvious from Figure 3.18 a), with $\delta_{C}=\delta_{K}>0.2 \%$ the loss in accuracy possibly can be unacceptable. The detailed results are presented in Table 3.2.

\begin{tabular}{|c||c|c|c|c|c|c|c|c|c|c|c|}
\hline \multicolumn{1}{|c||}{} & \multicolumn{10}{c|}{$\delta_{C}=\delta_{K}$ in $\%$} \\
& 0 & 0.1 & 0.2 & 0.3 & 0.4 & 0.5 & 0.6 & 0.7 & 0.8 & 0.9 & 1.0 \\
\hline \hline$t_{\text {sim }}$ & 574 & 547 & 531 & 516 & 287 & 240 & 210 & 165 & 145 & 127 & 116 \\
\hline
\end{tabular}

Table 3.2: Total simulation times $t_{\text {sim }}$ in seconds for a FD impedance analysis with varying tolerance limits for a network matrix of dimension $2026 \times 2026$.

\subsection{Retardation Effects}

Together with PEEC models in higher frequency ranges (exceeding the active frequency range) retardation effects can no longer be neglected and even 2D TL models become insufficient for the analysis due to dominating 3D electromagnetic effects [104]. This is especially important if the propagation time between the coupled elements violates condition (3.70) [105]. The limitation is less important for geometric arrangements where the propagation is on wires with nearby ground planes. In EMI analysis, however, these effects have to be considered if the distances are no longer small compared to the wavelength. As a consequence, the non-retarded partial mutual inductances and mutual capacitances may couple with the wrong phase ${ }^{11}$ if retardation effects are neglected. An efficient and accurate analysis of large meaningful problems requires the application of a full-wave partial element equivalent circuit method incorporating the delays among the partial elements. An extension of the PEEC approach to the so-called rPEEC approach enables the equivalence to the full-wave MoM approach. Different from the differential-equation-based finite difference time domain (FDTD) method, which is also a full-wave solution approach, the integral-equationbased PEEC method turns out to be more advantageous for a flexible modeling of wires [106]. A full-wave PEEC model with delays and losses is denoted a $\left(L_{p}, P_{p}, R, \tau\right)$ PEEC model with $\tau$ representing the time-of-flight

$$
\tau_{i j} \triangleq \frac{\left\|\vec{r}_{i}-\vec{r}_{j}\right\|}{c}
$$

in all coupling elements ${ }^{12}$. The derivation of the rPEEC formulation starts from the EFIE (Section 2.4) by setting

$$
t_{r, i j}=t-\tau_{i j}
$$

instead of $t_{r, i j}=t$ in the non-retarded case. The resulting equivalent circuit with retardation is identical to the case without retardation [58], in principle. In the non-retarded case, (3.13) can be inverted to achieve the familiar capacitance matrix. With retardation, however, the capacitance

\footnotetext{
${ }^{11}$ While for a differential mode excitation a quasi-stationary PEEC model without delays is sufficient, for a common mode excitation the retardation delays must be included to obtain accurate simulation results [106].

${ }^{12}$ If the maximum circuit element-to-element delay is much larger than the rise time of the fastest transition, false results will occur if the delay is not included [106].
} 
matrix can no longer be computed by inversion. Therefore, together with retarded PEEC models it is necessary to express the equations in terms of the partial coefficients of potential. This can be accomplished by replacing the partial self-capacitances by pseudo-capacitances $C_{p_{i i}}=P_{p_{i i}}^{-1}$ and the mutual capacitive couplings by controlled current sources. The inductive terms can be treated in a similar way, with couplings represented by a voltage source in series with the corresponding partial self-inductance $[107,59]$. For a detailed theoretical view to retarded PEEC models, refer to $[107,105,108,104,53,59]$. It is not meaningful to realize a time-delay for retarded models by using lossless transmission lines. Each retarded coupling would require an additional TL model which leads to an unaccaptable increase in computational complexity. The computational effort can be kept to a minimum by modifying only the independent voltage source since only one modified source per cell is required to realize all couplings to this cell [109].

\subsubsection{Time Domain}

The partial inductive and capacitive couplings can be realized by voltage drops

$$
\begin{array}{ll}
u_{L k}(t)=\sum_{\substack{m=1 \\
m \neq k}}^{N_{V}} \frac{L_{p_{k m}}}{L_{p_{m m}}} u_{L m}\left(t-\tau_{k m}\right), & k=1, \ldots, N_{V} \\
u_{C \ell}(t)=\sum_{\substack{n=1 \\
n \neq \ell}}^{N_{S}} \frac{P_{p_{\ell n}}}{P_{p_{n n}}} \phi_{n}^{C}\left(t-\tau_{\ell n}\right), & \ell=1, \ldots, N_{S} .
\end{array}
$$

The delay between two cells is quantified by the time-of-flight (3.95) with the center-to-center distance $\left\|\vec{r}_{i}-\vec{r}_{j}\right\|$ between two cells $i$ and $j$ and $c$ representing the velocity in medium. The corresponding MNA stamps for a circuit simulator implementation (according to Appendix D) can be applied in a straightforward manner. In the TD, all waveforms in the past are assumed to be known. A very large portion of the coupling elements can be shifted to the right-hand side of the equation system by choosing the time step smaller than most of the delays. Consequently, the resulting network matrix will be very sparse. A "history" (background memory) for the capacitive potentials and the inductive voltages has to be stored. This overhead reduces the simulation efficiency and bounds the solvable problem size. Moreover, an adaptive time step control can further decrease the performance. During preparation of a succeeding time step, the network matrix and the source vector have to be updated. Care has to be taken with retarded PEEC models in the TD, since numerical interpolation errors can lead to simulation instabilities [104, 109, 15].

\subsubsection{Frequency Domain}

The retardation can easily be implemented in the FD. The time delay converts to an exponential term

$$
t_{r, i j}=t-\frac{\left\|\vec{r}_{i}-\vec{r}_{j}\right\|}{c} \longrightarrow \mathrm{e}^{-\mathrm{j} \beta\left\|\vec{r}_{i}-\vec{r}_{j}\right\|}
$$

which represents a phase shift multiplied into the non-retarded expression. The corresponding Green's function in the FD states

$$
G\left(\vec{r}_{i}, \vec{r}_{j}\right)=\frac{e^{-\mathrm{j} \omega\left\|\vec{r}_{i}-\vec{r}_{j}\right\| / c}}{4 \pi\left\|\vec{r}_{i}-\vec{r}_{j}\right\|}
$$


for full-wave analysis [108]. This leads to

$$
\begin{array}{ll}
u_{L k}(\omega)=\sum_{\substack{m=1 \\
m \neq k}}^{N_{V}} \frac{L_{p_{k m}}}{L_{p_{m m}}} u_{L m}(\omega) \mathrm{e}^{-\mathrm{j} \beta\left\|\vec{r}_{k}-\vec{r}_{m}\right\|}, & k=1, \ldots, N_{V} \\
u_{C \ell}(\omega)=\sum_{\substack{n=1 \\
n \neq \ell}}^{N_{S}} \frac{P_{p_{\ell n}}}{P_{p_{n n}}} \phi_{n}^{C}(\omega) \mathrm{e}^{-\mathrm{j} \beta\left\|\vec{r}_{\ell}-\vec{r}_{n}\right\|}, & \ell=1, \ldots, N_{S}
\end{array}
$$

for the inductive and capacitive voltage drops, respectively. Only the voltages, currents and the parameter $\beta$ are variables, all other elements are constants, calculated in the setup phase. A socalled compressed MNA matrix stamp (refer to [58]) allows an efficient simulation of the retarded PEEC model in the FD with the order of the network matrix increasing proportionally with the number of cells. For a detailed circuit realization of retardation refer to [61, 58, 109, 59, 110].

\subsection{Conclusion}

The PEEC method as presented in Sections 3.2 and 3.3 for cartesian as well as for curvilinear coordinates, represents an efficient method to enable electrodynamic analysis in the circuit domain. By formulating large systems of network equations, simulations in the TD and the FD can be performed including incident field couplings, dielectrics and retardation effects. Some guidelines concerning modeling have to be regarded to ensure an accurate and valid system simulation. Based on a BEM discretization approach, PEEC matrices are dense, in general. W.r.t. fast solution methods, a sparsification of the resulting network matrices is desired. Although the reluctance PEEC method with truncation to off-diagonal elements represents an effective sparsification approach, it comprises some serious shortcomings. On the one hand, to realize a real-time system simulation, e.g. in automotive applications, very sparse network matrices are necessary leading to an unacceptable loss in accuracy, together with truncated PEEC models. On the other hand, prior to the trunction process the dense element matrices have to be calculated completely with square complexity. Consequently, a memory bottleneck results, limiting the problem size which can be analyzed. As a solution, the two described drawbacks can be avoided using hierarchical matrices ( $\mathcal{H}$-matrices) as explained in detail in the following Chapter. Based on block-partitioning of the original matrix and low-rank approximations of the farfield blocks, $\mathcal{H}$-matrices represent an effective method to handle BEM-based matrices with high accuracy. A so-called data-sparse representation of the matrices enables an efficient compression in combination with a suitable $\mathcal{H}$-matrix arithmetic, advantageous for applications in iterative solution methods. 


\section{4 \\ Fast and Efficient Extraction of Parasitic Effects}

$\boldsymbol{A}$

fast and accurate computation of matrix elements is one major issue to realize an efficient simulation process. This is especially important for parasitic matrices originating from electromagnetic boundary integral equation approaches as the PEEC method. BEMbased matrices as inductance matrices and potential coefficient matrices are dense in general, with $n=\mathcal{O}\left(h^{-2}\right)$ degrees of freedom for geometric meshing with meshsize $h$ thus exhibiting $\mathcal{O}\left(h^{-4}\right)$ matrix entries [3]. Both the TD and the FD analysis need to compute large numbers of interactions between basis functions for each and every simulation step, leading to enormous CPU time and memory requirements. This can form a severe limitation to the number of DOF in practical relevant problems. With regard to iterative methods in Chapter 5, fast methods are necessary to reduce the complexity of basic arithmetic operations.

After a short historical review and motivation in Section 4.1, a survey of the fast multipole method (FMM) w.r.t. PEEC models is outlined in Section 4.2. In Section 4.3, the fundamental theory of hierarchical matrices $(\mathcal{H}$-matrices) is described with regard to a fast extraction of parasitic effects in EM simulation. The fast element evaluation process based on the cross-approximation principle as well as implementation details, numerical examples and suggestions for further improvements are presented in the remainder of this chapter (Sections 4.3.2 to 4.3.5).

\subsection{Historical Background and Motivation}

The basic idea of fast computation methods for BEM-based matrices is, to take advantage of the smoothness of the integral operator's kernel function far away from the singularity. Smooth functions can be described by series expansions, in general. A good approximation can be obtained by applying a truncation to the series expansion. The FMM $[111,112]$ as a special case of the 
panel clustering technique [84] uses special multipole expansions instead of common Taylor series expansions. While a Taylor series expansion of order $m$ in 3D requires the computation of $\mathcal{O}\left(\mathrm{m}^{3}\right)$ terms, a multipole expansion only needs to compute $\mathcal{O}\left(\mathrm{m}^{2}\right)$ terms. As a result, the multipole expansion is more efficient with higher orders but it is more complex to implement. Panel clustering methods using an interpolation ${ }^{1}$ instead of a Taylor series expansion are easy to implement $[114,115,116]$. The FMM enables fast model computations with complexity of $\mathcal{O}\left(n^{1.5}\right)$ for matrix dimension $n$, in principle. In a multilevel context, the FMM allows to reduce the storage as well as the computational complexity to $\mathcal{O}\left(n \log ^{\kappa} n\right)$, where $\kappa=4$ holds for some special kernel functions and $\kappa \sim 6,7$ for general kernels [85]. A graphical illustration of the impact of the kernel function to the arithmetic complexity is depicted in Figure 4.1. The expansions can not be applied globally to the whole matrix but only to matrix subblocks belonging to smooth areas of the kernel function. This is the reason why the matrix is represented as a decomposition of matrix subblocks. Consequently, the approximation has to be applied locally in each subblock. A problem occurs with blocks containing a singularity. Where such nearfield blocks can effectively be treated by quadrature rules, the farfield blocks can be well approximated by matrices with low rank. Based on these facts, various kinds of methods can be developed. In multipole methods the farfield blocks are computed "on-the-fly", which makes it necessary only to store the nearfield. Using $\mathcal{H}$-matrices, the kernel function expansions enable a description of farfield blocks by matrices with low rank. It can be shown, that the rank for these low-rank blocks is bounded by the number of terms in the expansion. By storing the farfield subblocks as low-rank matrices, one can take advantage of the possibility to apply special optimization algorithms combined with minimal storage requirements [117]. Adaptive recompression techniques allow an approximation with "optimal" rank, comprising an optimization with regard to minimal storage requirements. The FMM shows the disadvantage that the implementation needs a complete reconding of the matrix-vector multiplication [118]. Moreover, $\mathcal{H}$-matrix techniques allow to apply a special $\mathcal{H}$-matrix arithmetic which makes it possible to realize efficient direct as well as iterative methods $[19,20]$.

\subsection{The Fast Multipole Method}

The FMM, which was originally introduced by GREENGARD and ROKHLIN in 1987 for accoustic wave scattering in two dimensions, was extended by COIFMAN, ROKHLIN and WANDZURA in 1993 to the three-dimensional wave equation. In the past, mainly applied to scattering problems that are usually solved together with the MoM, the FMM soon has been found to be very effective in speeding-up iterative solution methods [119].

\subsubsection{Theoretical Aspects}

With regard to the reliability in the field of circuit-based EM simulation and the suitability for BEM-based problems, in this section the theoretical basics of the FMM are shortly recapitulated in preparation for the understanding of hierarchical matrices.

\footnotetext{
${ }^{1}$ For details concerning low-rank approximation by interpolation refer to [113].
} 


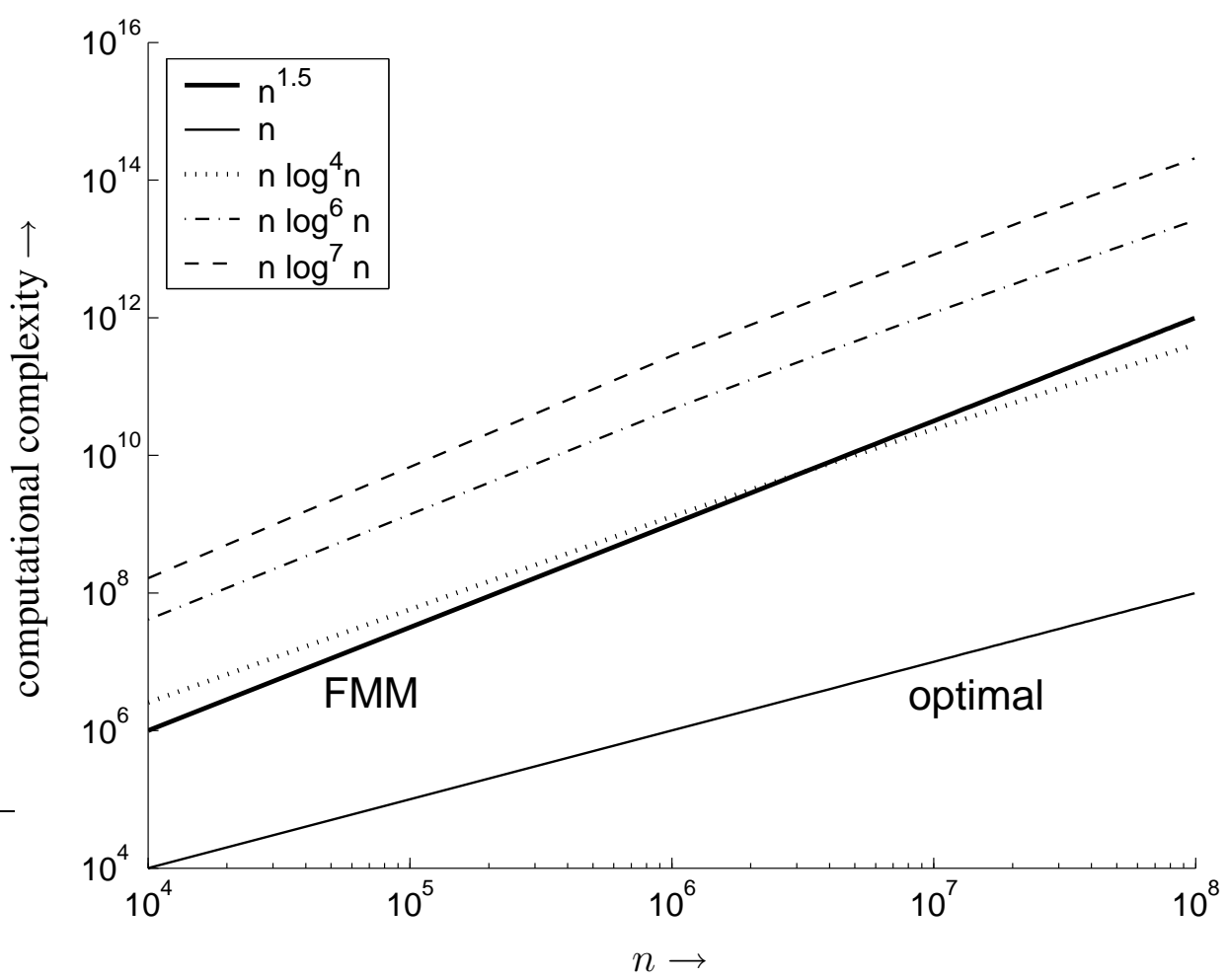

Fig. 4.1: Influence of the integral operator's kernel function to the arithmetic complexity of hierarchical methods.

\subsubsection{The Grouping}

The FMM bases on a hierarchical subdivision of all basis functions into clusters, enabling a socalled grouping, as depicted in Figure 4.2. The decomposition of a cluster stops at a certain level if it no longer contains any basis functions [120]. The resulting tree structure enables an efficient FMM-based preconditioning in iterative methods. The stopping criterions for the hierarchical partitioning together with PEEC are the cluster size, the frequency and the number of basis functions in one cluster. Thus, based on the resulting hierarchy of levels, a separation of the entries of interaction matrix $\boldsymbol{A}$ into nearfield and farfield $[120,76,77]$ as

$$
\boldsymbol{A}=\boldsymbol{A}^{\text {near }}+\boldsymbol{A}^{\text {far }}
$$

is possible. For further details concerning group theory refer to [23].

\subsubsection{The Farfield Approximation}

With so-called n-body problems, the function of interest $f$ can be described using sums of pairwise interactions

$$
f(\vec{y})=\sum_{i=1}^{n} q_{i} g\left(\vec{y}, \vec{x}_{i}\right)
$$

with a set of source points $\vec{x}_{i}$ [121], regardless of function's $f$ complexity in the nearfield. If function $g$ is smooth far away from zero, $f$ will be well-behaved in the farfield. One possible remedy to simplify the matrix-vector multiplication for iterative solvers, comprising an algorithmic complexity of $\mathcal{O}\left(n^{2}\right)$ for dense matrices is, to apply low-rank approximations. For low-rank matrices of dimension $n \times n$ and of rank $k$ a matrix-vector multiplication can be performed with complexity 


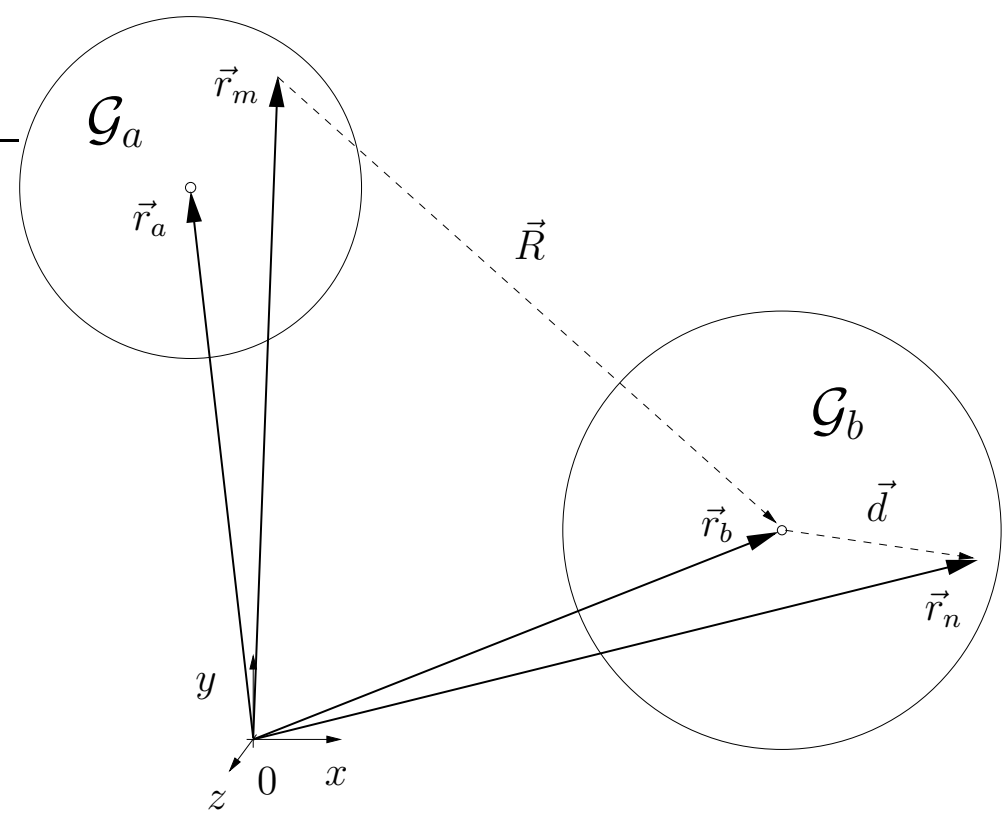

Fig. 4.2: Grouping of basis functions in FMM to realize a separation into nearfield and farfield. The considered groups $\mathcal{G}_{a}$ and $\mathcal{G}_{b}$ are spherically shaped with the centers $\vec{r}_{a}$ and $\vec{r}_{b}$ in $3 \mathrm{D}$.

$\mathcal{O}(k n)$. BEM-based matrices, however, usually exhibit full ranks. Therefore, the consideration of the so-called effective rank is reasonable. The effective rank $r_{\varepsilon}$ is defined in terms of some numerical tolerance $\varepsilon$ as the number of singular values which are larger than $\varepsilon \sigma_{1}$, with $\sigma_{1}$ denoting the largest singular value. On this note, a low-rank approximation with rank $k$ from a matrix can be obtained by considering singular values which are larger than $r_{\varepsilon}$ (refer also to the truncated singular value decomposition (SVD) in Appendix A.3). Exploiting the known analytic structure of the kernel function $g$, a low-rank approximation directly can be derived from the analytic series expansion of $g$ [121]. Assuming that the kernel function $g$ is in fact degenerate, i.e.

$$
\left|g\left(\vec{r}_{m}, \vec{r}_{n}\right)-\sum_{k=0}^{p-1} \Phi_{k}\left(\vec{r}_{m}\right) \Psi_{k}\left(\vec{r}_{n}\right)\right| \leqslant \varepsilon,
$$

the function of interest (4.2) can be specified as

$$
\begin{aligned}
f\left(\vec{r}_{n}\right) & =\sum_{i=1}^{n} \sum_{k=0}^{p-1} \Phi_{k}\left(\vec{r}_{m, i}\right) \Psi_{k}\left(\vec{r}_{n}\right) \\
& =\sum_{k=0}^{p-1}\left(\sum_{i=1}^{n} \Phi_{k}\left(\vec{r}_{m, i}\right)\right) \Psi_{k}\left(\vec{r}_{n}\right) .
\end{aligned}
$$

To find a unique series expansion of (4.3), valid for all possible source and observation points $\vec{r}_{m}, \vec{r}_{n}$, is easy in the most cases [76]. The multipole method represents a technique to compute a series expansion for well-separated source and observation points. An approximation of the retarded Green's function (refer to (B.9) in Appendix B)

$$
G\left(\vec{r}_{m}, \vec{r}_{n}\right)=\frac{e^{\mathrm{j} \beta\left\|\vec{r}_{m}-\vec{r}_{n}\right\|}}{4 \pi\left\|\vec{r}_{m}-\vec{r}_{n}\right\|},
$$


representing the fundamental solution of the electromagnetic field problem, can be accomplished as described in $[122,112]$ by an expansion

$$
\frac{e^{\mathrm{j} \beta\left\|\vec{r}_{m}-\vec{r}_{n}\right\|}}{\left\|\vec{r}_{m}-\vec{r}_{n}\right\|}=\frac{e^{\mathrm{j} \beta\|\vec{R}+\vec{d}\|}}{\|\vec{R}+\vec{d}\|}=\mathrm{j} \beta \sum_{\ell=0}^{\infty}(-1)^{\ell}(2 \ell+1) J_{\ell}(\beta\|\vec{d}\|) H_{\ell}^{(1)}(\beta\|\vec{R}\|) P_{\ell}\left(\vec{e}_{d} \cdot \vec{e}_{R}\right)
$$

of the kernel (GEGENBAUER's addition theorem $[112,23]$ ), with $\vec{e}_{d}=\frac{\vec{d}}{\|\vec{d}\|},\|\vec{R}\|>\|\vec{d}\|$. Equation (4.6) contains the spherical Bessel function of the first kind $J_{\ell}$, the spherical Hankel function of the first kind $H_{\ell}^{(1)}$ and the Legendre polynomial $P_{\ell}$. Exploiting the orthonormality of the Legendre polynomials on the unit sphere $\mathbb{S}^{2}$ and the expansion of spherical waves, we can write [123]

$$
4 \pi \mathrm{j}^{\ell} J_{\ell}(\beta\|\overrightarrow{d \|}\|) P_{\ell}\left(\vec{e}_{d} \cdot \vec{e}_{R}\right)=\iint_{\mathbb{S}^{2}} e^{\mathrm{j} \vec{\beta} \vec{d}} P_{\ell}\left(\vec{e}_{\beta} \cdot \vec{e}_{R}\right) \mathrm{d}^{2} \vec{e}_{\beta}
$$

with

$$
\iint_{\mathbb{S}^{2}} \mathrm{~d}^{2} \vec{e}_{\beta} \equiv \int_{\varphi=0}^{2 \pi} \int_{\vartheta=0}^{\pi} \sin \vartheta \mathrm{d} \vartheta \mathrm{d} \varphi
$$

describing the integration over the unit sphere with

$$
\vec{\beta}=\sin \vartheta \cos \varphi \vec{e}_{x}+\sin \vartheta \sin \varphi \vec{e}_{y}+\cos \vartheta \vec{e}_{z} .
$$

As a result, one gets a diagonal form

$$
\frac{e^{\mathrm{j} \beta\|\vec{R}+\vec{d}\|}}{\|\vec{R}+\vec{d}\|}=\frac{\mathrm{j} \beta}{4 \pi} \sum_{\ell=0}^{\infty} \mathrm{j}^{\ell}(2 \ell+1) H_{\ell}^{(1)}(\beta\|\vec{R}\|) \iint_{\mathbb{S}^{2}} e^{\mathrm{j} \vec{\beta} \vec{d}} P_{\ell}\left(\vec{e}_{\beta} \cdot \vec{e}_{R}\right) \mathrm{d}^{2} \vec{e}_{\beta}
$$

of the multipole expansion. The occuring summation has to be truncated since the Hankel function in (4.10) diverges for large $\ell$. For the truncated series, summation and integration can be interchanged and one defines the translation operator

$$
\Lambda\left(\vec{e}_{\beta} \cdot \vec{e}_{R}\right) \triangleq \sum_{\ell=0}^{L} \mathrm{j}^{\ell}(2 \ell+1) H_{\ell}^{(1)}(\beta\|\vec{R}\|) P_{\ell}\left(\vec{e}_{\beta} \cdot \vec{e}_{R}\right)
$$

which solely depends on the distance vector $\vec{R}$ and allows the implementation of an efficient multilevel scheme. While the nearfield part of the boundary integral operator can be evaluated directly, for the farfield part the multipole method is applied. As a result, it can be summarized that

$$
\frac{e^{\mathrm{j} \beta\|\vec{R}+\vec{d}\|}}{\|\vec{R}+\overrightarrow{d \|}\|} \simeq \frac{\mathrm{j} \beta}{4 \pi} \iint_{\mathbb{S}^{2}} e^{\mathrm{j} \vec{\beta} \vec{d}} \Lambda\left(\vec{e}_{\beta} \cdot \vec{e}_{R}\right) \mathrm{d}^{2} \vec{e}_{\beta}
$$

holds. A rule for the separation between nearfield and farfield can be given by [122]

$$
\left\|\vec{r}_{a}-\vec{r}_{b}\right\|<c \frac{\|\vec{d}\|}{2}
$$

with $\left\|\vec{r}_{a}-\vec{r}_{b}\right\|$ representing the distance between the center points of the corresponding groups and a suitably chosen constant $c$ [112]. 


\subsubsection{The Element Evaluation Process}

The complexity for the computation of the dense system matrix and the solution process dominate the overall expenditure in BEM-based circuit simulation. By introducing (4.12) into the original definitions of partial inductances and partial coefficients of potential from (3.11) and (3.12), the following FMM approximation can be obtained [77].

\subsubsection{Partial Inductances}

Partial Inductances can be computed by

$$
L_{p_{m n}}=\frac{\mathrm{j} \beta \mu \cos \varphi_{m n}}{(4 \pi)^{2} A_{m} A_{n}} \iint_{\mathbb{S}^{2}}\left[\iiint_{V_{m}} e^{\mathrm{j} \vec{\beta} \vec{r}_{m a}} \mathrm{~d} V\right] \Lambda_{L, a b}\left(\vec{e}_{\beta} \cdot \vec{e}_{a b}\right) \quad\left[\iiint_{V_{n}^{\prime}} e^{-\mathrm{j} \vec{\beta} \vec{r}_{n b}} \mathrm{~d} V^{\prime}\right] \mathrm{d}^{2} \vec{e}_{\beta}
$$

which can be described as

$$
L_{p_{m n}}=\frac{\mathrm{j} \beta \mu \cos \varphi_{m n}}{(4 \pi)^{2}} \iint_{\mathbb{S}^{2}} F_{L, m} \Lambda_{L, a b}\left(\vec{e}_{\beta} \cdot \vec{e}_{a b}\right) \tilde{F}_{L, n} \mathrm{~d}^{2} \vec{e}_{\beta}
$$

with

$$
\begin{aligned}
& F_{L, m} \triangleq \frac{1}{A_{m}} \iiint_{V_{m}} e^{\mathrm{j} \vec{\beta} \vec{r}_{m a}} \mathrm{~d} V \\
& \tilde{F}_{L, n} \triangleq \frac{1}{A_{n}} \iiint_{V_{n}^{\prime}} e^{-\mathrm{j} \vec{\beta} \vec{r}_{n b}} \mathrm{~d} V^{\prime}
\end{aligned}
$$

and $\vec{r}_{i j} \triangleq \vec{r}_{i}-\vec{r}_{j}$.

\subsubsection{Partial Coefficients of Potential}

A FMM representation of partial coefficients of potentials can be computed in a similar manner. For this, (4.12) will be introduced in the original definition (3.12), leading to

$$
P_{p_{m n}}=\frac{\mathrm{j} \beta}{(4 \pi)^{2} \varepsilon S_{m} S_{n}} \iint_{\mathbb{S}^{2}}\left[\iint_{S_{m}} e^{\mathrm{j} \vec{\beta}_{m a}} \mathrm{~d} S\right] \Lambda_{P, a b}\left(\vec{e}_{\beta} \cdot \vec{e}_{a b}\right)\left[\iint_{S_{n}^{\prime}} e^{-\mathrm{j} \vec{\beta} \vec{r}_{n b}} \mathrm{~d} S^{\prime}\right] \mathrm{d}^{2} \vec{e}_{\beta}
$$

which can be described more compactly as

$$
P_{p_{m n}}=\frac{\mathrm{j} \beta}{(4 \pi)^{2} \varepsilon} \iint_{\mathbb{S}^{2}} F_{P, m} \Lambda_{P, a b}\left(\vec{e}_{\beta} \cdot \vec{e}_{a b}\right) \tilde{F}_{P, n} \mathrm{~d}^{2} \vec{e}_{\beta}
$$

using

$$
\begin{gathered}
F_{P, m} \triangleq \frac{1}{S_{m}} \iint_{S_{m}} e^{\mathrm{j} \vec{\beta}_{m a}} \mathrm{~d} S \\
\tilde{F}_{P, n} \triangleq \frac{1}{S_{n}} \iint_{S_{n}^{\prime}} e^{-\mathrm{j} \vec{\beta} \vec{r}_{n b}} \mathrm{~d} S^{\prime} .
\end{gathered}
$$

The terms in $(4.16,4.17)$ and $(4.20,4.21)$ can be computed a priori. 


\subsubsection{The Multilevel Fast Multipole Method}

The FMM allows to perform a fast model extraction with complexity $\mathcal{O}\left(n^{1.5}\right)$, in principle. To further reduce the complexity of a matrix-vector multiplication to $\mathcal{O}(n \log n)$ w.r.t. time and memory requirements, it is necessary to extend the FMM to a multilevel method [23, 24, 124]. According to [23, 124], the so-called multilevel fast multipole algorithm (MLFMA) is a state-of-the-art technique for solving electromagnetic problems. This can be realized enclosing the considered object in a large cube, which will successively be partitioned into smaller subcubes. The partitioning stops if the edge length of the finest cube is about a quarter wavelength [23]. An indexing of all cubes at all levels is assumed. On the finest level only non-empty cubes are regarded and reordered by using tree-structured data at all levels. Level 0 contains only one non-empty cube. The MLFMA is used to speed up the matrix-vector multiplication in iterative methods by decomposing the matrix-vector multiplication into two stages [125, 23]. First, outgoing wave expansions for each non-empty cube are constructed during the hierarchy of levels and all sources are combined at the finest level. In a second stage, local incoming wave expansions are constructed from the coarse to the finest levels and translated to all cubes at the same level. Processing from a finer level to a coarser one, the cubes become larger and the number of plane waves for an accurate representation of the radiation pattern should increase. At the coarsest level, the local multipole expansions contributing from well-separated cubes are calculated using translation. By using shifting and anterpolation (adjoint interpolation) operations, the expansions for coarser level $\ell-1$ can be computed from contributions from level $\ell$. In [124], a preconditioning strategy based on the sparse approximate inverse ${ }^{2}$ (SAI) of the near part matrix in the MLFMA is described. The results show, that the SAI preconditioner constructed from the near part matrix improves the computational efficiency in terms of both memory requirements and CPU time and leads to a robust iterative Krylov method.

\subsubsection{Implementation Details}

For an efficient FMM implementation, it is necessary to take parallelization aspects, hardware architectures, algorithmic specifications as well as a suitable formulation of the problem into consideration [127]. A suitable problem analysis can help to improve the conditioning and the performance. It has to be noticed, that EFIE and MFIE lead to ill-conditioned systems, in general. In the context of PEEC, however, the CFIE is often not applicable and leads to bad parallelization properties of the preconditioner. It can be observed that the EFIE leads to bad convergence results together with preconditioned iterative solvers, while the MFIE performs slightly better and the CFIE shows the best results. The number of required iterations to achieve convergence is approximately independent of the matrix dimension, using the CFIE.

\subsection{Hierarchical Matrices}

Hierarchical matrices $(\mathcal{H}$-matrices) provide a purely mathematical approach to describe dense matrices by so-called data-sparse matrices. Data-sparse matrices are sparse in a sense, that only few data are necessary for representation [128]. Based on this kind of description it is possible to

\footnotetext{
${ }^{2}$ For further details on sparse approximate inverses refer to [126].
} 
apply approximative algorithms for matrix arithmetic with almost optimal complexity. Together with PEEC matrices one exploits the smoothness of Green's function far from the origin. This matter of fact makes it possible, to approximate the integral operator of the occuring terms up to a tolerance $\mathcal{O}\left(n^{-\kappa}\right), \kappa>0$ by a data-sparse $\mathcal{H}$-matrix of dimension $n \times n$ with almost linear complexity. Compared to the FMM, $\mathcal{H}$-matrices offer the advantage that the kernel function has not to be known explicitely. It is sufficient that the kernel proceeds smooth far away from the singularity. Furthermore, it is possible to store nearfield parts as well as farfield parts of the matrix in an efficient way with only low storage requirements and to apply modern recompression algorithms for a further optimization. $\mathcal{H}$-matrix techniques require slightly more memory compared to FMM-based methods in general, because the farfield part has to be stored. In contrast, $\mathcal{H}$-matrices are more efficient in the computation of matrix-vector multiplications since only elementary operations as multiplications and additions of floating point values have to be executed. The purely algebraic approach of $\mathcal{H}$-matrices, together with a suitably defined $\mathcal{H}$-matrix arithmetic, exhibits the possibility to construct very efficient preconditioners for iterative methods. This represents a bonus, leading to a maximum of flexibility. To enable an optimal asymptotic efficiency for very large systems, so-called $\mathcal{H}^{2}$-matrices can be useful. $\mathcal{H}^{2}$-matrices are a variant of $\mathcal{H}$-matrices and apply techniques from the field of multigrid methods (refer to Chapter 7 ). $\mathcal{H}^{2}$-matrices enable optimizations similar to $\mathcal{H}$-matrices, however, the securing of special structural properties leads to a higher algorithmic complexity. Basic algorithms and numerical results for verification are presented in [129].

\subsubsection{Theoretical Aspects}

\subsubsection{Objective}

Hierarchical matrices are based on the fact that integral operators, as occuring in the PEEC method, can efficiently be treated by using separable expansions of the corresponding kernel functions. In the case of BEM-based matrices, the integral operator

$$
\mathcal{L}[u](\vec{x})=\int_{\Gamma} g(\vec{x}, \vec{y}) u(\vec{y}) \mathrm{d} \vec{y} \quad \vec{x} \in \Gamma \triangleq \partial \mathcal{X}
$$

is defined on the domain $\mathcal{X} \in \mathbb{R}^{d}$ of dimension $d$, with kernel

$$
g: \mathbb{R}^{d} \times \mathbb{R}^{d} \rightarrow \mathbb{R}
$$

In EM modeling $g$ usually represents the non-local Green's function $G$, leading to a dense interaction matrix. As a direct result, the available memory restricts the dimension of possible problems. The handling of large dense matrices can be avoided by using techniques as the FMM, panel clustering, wavelet techniques or the fast fourier transform (FFT). The first two methods rely on an explicit knowledge of a series expansion of the non-local kernel function of the boundary integral operator. Both approximations help to achieve an efficient matrix-vector multiplication without explicit setup of the associated stiffness matrix ${ }^{3}$. This technique corresponds to a hierarchical partitioning of the matrix, where the truncated series expansion of the kernel function complies with

\footnotetext{
${ }^{3}$ The term "stiffness-matrix" historically originates from linear algebra approaches for solving the forces and displacements of a truss. In solving a truss, each piece can be thought of as a linear spring. By applying boundary conditions and a force, the displacement of each node can be computed by constructing a stiffness matrix which combines the stiffness of each element in the system and the nodes each connects.
} 
a low-rank approximation of the associated matrix-block. Green's function comprises the property of the singularity, solely occuring on the main diagonal and the function proceeding smooth otherwise. Already together with the panel clustering method, exploitation of the smoothness of the kernel function enables an approximation by truncated Taylor expansion [95]. In each admissible block Green's function can be approximated using separable functions. Due to the need of an explicit Taylor series expansion for the given kernel function in panel clustering, individual analytic derivations of the suitable recursion formulae have to be computed. This disadvantage can be avoided using general interpolation formulae $[113,114]$. Similar to $(4.3)$, the degenerate kernel approximation can be formulated as

$$
g(\vec{x}, \vec{y}) \approx \sum_{\ell=1}^{k} \Psi_{\ell}(\vec{x}) \Phi_{\ell}(\vec{y}) \quad \vec{x} \in \mathcal{G}_{1}, \vec{y} \in \mathcal{G}_{2}
$$

on a pair of domains $\left(\mathcal{G}_{1}, \mathcal{G}_{2}\right)$, located in a certain distance relative to their diameters. The kernel function can not globally be approximated by a degenerate kernel in the whole domain. Therefore, local approximations on subdomains are applied if the kernel function behaves smooth in the corresponding subdomain. Hierarchical matrices are the algebraic counterpart of the local degenerate approximations used in panel clustering and multipole techniques [113]. Looking at (4.24) from an algebraic point of view, this means nothing but a low-rank approximation of the associated matrixblock $\boldsymbol{A}^{t \times s}$ in the rows and columns $t, s \in\{1, \ldots, n\}$ of $\boldsymbol{A} \in \mathbb{R}^{n \times n}$. Due to the singular behavior of Green's function at $\vec{x}=\vec{y}$, such separable approximations can not be applied globally. Introducing a hierarchical decomposition of the product computation domain $\mathcal{X} \times \mathcal{X}$ this problem can be solved, where on each patch one can approximate the kernel function by a short sum of separable functions [130]. Applying a collocation scheme ${ }^{4}$, this is equivalent to a low-rank approximation of matrix-blocks, leading to a hierarchical matrix. The advantage of this purely algebraic approach is obvious for very complex kernel functions [95]. The intention of $\mathcal{H}$-matrices is, to approximate a given dense matrix $\boldsymbol{A}$, e.g. from a PEEC approach, by an $\mathcal{H}$-matrix $\boldsymbol{A}_{\mathcal{H}}$ with error

$$
\left\|\boldsymbol{A}-\boldsymbol{A}_{\mathcal{H}}\right\| \leqslant \varepsilon
$$

with linear complexity. These error has to be attainable for adequate block-wise ranks $k=$ $\mathcal{O}\left(\left|\log ^{q} \varepsilon\right|\right)$, where $q=\mathcal{O}(d)$. The basic steps for an $\mathcal{H}$-matrix construction are [130]:

- construction of an hierarchical tree (cluster tree) $T_{\mathcal{I}}$ of index set $\mathcal{I}$;

- admissible partitioning $\mathcal{P}$ of $\mathcal{I} \times \mathcal{I}$ based on a block cluster tree $T_{\mathcal{I} \times \mathcal{I}}$;

- low-rank representation of all admissible blocks in $\mathcal{P}$,

which are explained in detail in the remainder of this section.

\footnotetext{
${ }^{4}$ Refer to Appendix A.5.
} 


\subsubsection{Index Set Partitioning}

The entries of a PEEC matrix can be interpreted as entries of a so-called Ritz-Galerkin-matrix or stiffness-matrix (refer also to Section 2.5)

$$
\boldsymbol{A}=\left[A_{i j}\right]_{\substack{i=1, \ldots, n \\ j=1, \ldots, n}}, \quad A_{i j}=\left\langle\varphi_{i}, \mathcal{L} \varphi_{j}\right\rangle_{L^{2}}
$$

with the boundary element basis $\left(\varphi_{i}\right)_{i \in \mathcal{I}}$. Let $\mathcal{I}$ be the index set of unknowns, corresponding to

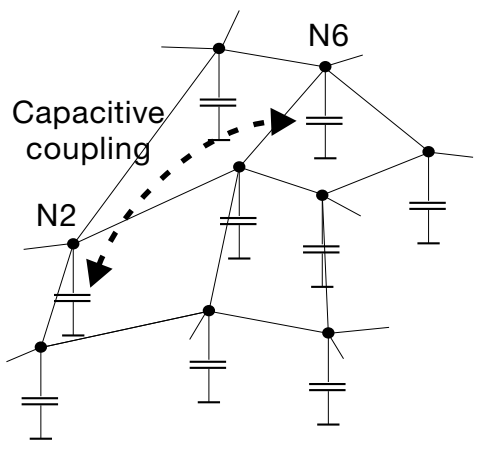

Nodal points (DOF)

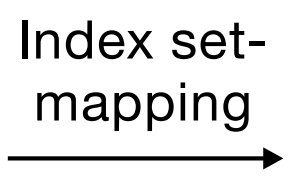

Matrix indices

Fig. 4.3: Index set mapping between nodal points (DOF) and matrix indices in the interactions matrix of partial coefficients of potential.

the nodal points from Ritz-Galerkin discretization [130] as depicted in Figure 4.3. To be able to uniquely localize an element $A_{i j}$ of matrix $\boldsymbol{A}$ in block-matrices on arbitrary levels, it is necessary to apply a partitioning to the index set

$$
\mathcal{I}=\{1,2,3, \ldots, n-1, n\}
$$

of the original matrix in suitably chosen subsets on indices. Each index $i \in \mathcal{I}$ is associated with the basis function $\varphi_{i}$ of the Galerkin ansatz space $\mathcal{F}_{\mathcal{I}} \triangleq \operatorname{span}\left\{\varphi_{i}\right\}_{i \in \mathcal{I}}$ so that the support of the basis functions is denoted by

$$
\mathfrak{S}_{i} \triangleq \operatorname{supp}\left(\varphi_{i}\right) \quad \text { for } i \in \mathcal{I} .
$$

To build up an hierarchical tree, geometry information to each index is necessary. Therefore, it is assumed that each index can be assigned to a nodal point $\vec{x} \in \mathbb{R}^{d}$. Each block-matrix at partitioning level $\lambda$ thereby can be represented by index subsets $\mathcal{I}_{i}, \mathcal{I}_{j} \subset \mathcal{I}$. The partitioning

$$
\mathcal{P}(\mathcal{I})=\left\{\mathcal{I}_{i}\right\}_{i=1}^{p}, \quad p \in \mathbb{N}
$$

of the index set $\mathcal{I}$ in pairwise disjoint index subsets $\mathcal{I}_{i}$ of dimension $n_{i}=\operatorname{dim}\left(\mathcal{I}_{i}\right)$ with $\mathcal{I}_{i} \cap \mathcal{I}_{j}=\{\}$ for $i \neq j$, allows the specification of a block-matrix $\boldsymbol{A}_{i j}$ uniquely emanating from matrix $\boldsymbol{A}$. Consequently, the partitioning represents a complete decomposition of the index set. For index set partitioning two different approaches are possible in principle. While the tensor-product partitioning with storage requirements $\mathcal{N}_{\text {st,TPP }}=\mathcal{O}\left(n^{3 / 2}\right)$ is not optimal, the hierarchical partitioning leads to an almost linear complexity $\mathcal{N}_{\text {st,HP }}=\mathcal{O}(n \log n)[131]$. 


\subsubsection{Cluster Tree}

The set of all clusters together with the corresponding index set partitioning is called the cluster tree $(\mathrm{CT}) T_{\mathcal{I}}$ of an index set $\mathcal{I}$. By defining a default value for the cardinality $n_{\min }$ of the cluster, a subdivision can be applied until only one index remains. In practice, one should stop the splitting if the cardinality of a node is less or equal to a threshold parameter $C_{\text {leaf }}{ }^{5}$. The depth of $T_{\mathcal{I}}$ will be denoted by $p \triangleq \operatorname{depth}\left(T_{\mathcal{I}}\right)$. For reasonable cluster trees one would always expect $p \sim \log n$. The complexity of building the cluster tree in the case of quasi-uniform grids can be estimated as $\mathcal{O}(n \log n)$. If each leaf has two sons, $T$ is also called binary tree (refer to Figure 4.4). The union

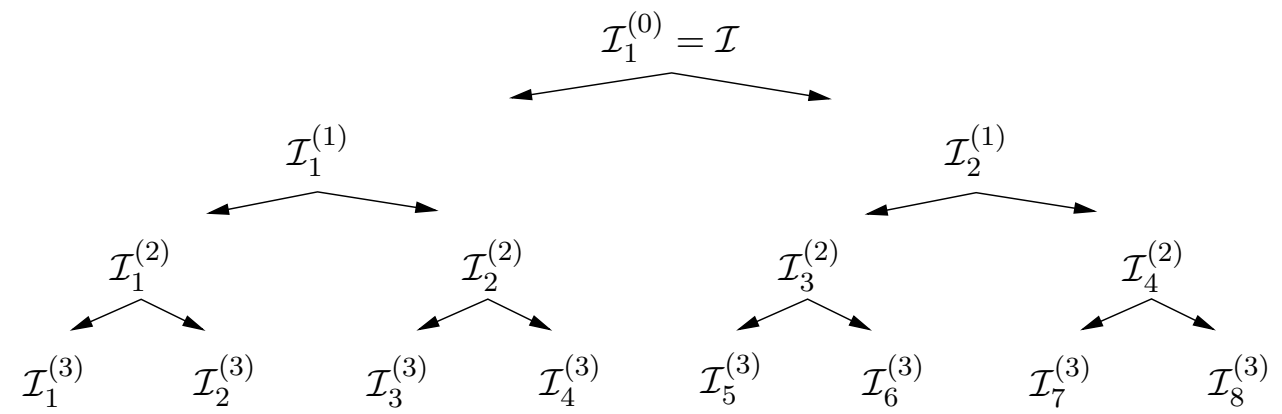

Fig. 4.4: The cluster tree $T_{\mathcal{I}}$ for index set $\mathcal{I}$ and $p=3$, implying a binary hierarchical partitionining of the index set.

of the supports of the basis functions corresponding to its elements, i.e.

$$
\mathfrak{S}_{t} \triangleq \bigcup_{i \in t} \mathfrak{S}_{i}, \quad t \in T_{\mathcal{I}}
$$

is also called the support of a cluster $t$, regarding (4.28). Based on this facts, the "diameter" of a cluster

$$
\operatorname{diam}(t) \triangleq \max _{\vec{x}, \vec{y} \in \mathfrak{S}_{t}}\|\vec{x}-\vec{y}\|, \quad t \in T_{\mathcal{I}}
$$

and the "distance" between two clusters

$$
\operatorname{dist}(t, s) \triangleq \min _{\substack{\vec{x} \in \mathfrak{S}_{t} \\ \vec{y} \in \mathfrak{S}_{s}}}\|\vec{x}-\vec{y}\|, \quad t, s \in T_{\mathcal{I}}
$$

can be defined with the Euclidean distance $\|\cdot\|$ in $\mathbb{R}^{d}$. A possible construction of the cluster tree can be performed by geometry-based splitting (bisection) of the index set, which is probably the most suitable one for PEEC matrices. For this purpose, one assigns a point $\vec{x}_{i} \in \mathbb{R}^{d}$, e.g. the center of the support of the corresponding basis functions to a DOF $i \in \mathcal{I}$, corresponding to the nodal unknowns of the PEEC network. Using bisection as depicted in Figure 4.5, a given cluster $t \subseteq \mathcal{I}$ splits into two sons such that the points $\vec{x}_{i}$ are separated by a hyper-plane [95].

\subsubsection{Block Cluster Tree}

A block cluster tree (BCT) arises from the grouping of pairs of clusters from the $\mathrm{CT}$, as depicted in Figure 4.6. The BCT is nothing else the $\mathrm{CT}$ for the index set $\mathcal{I} \times \mathcal{I}$ instead of $\mathcal{I}$. Considering the

\footnotetext{
${ }^{5} \mathrm{~A}$ parameter $C_{\text {leaf }}=32$ leads to good performance, in general.
} 


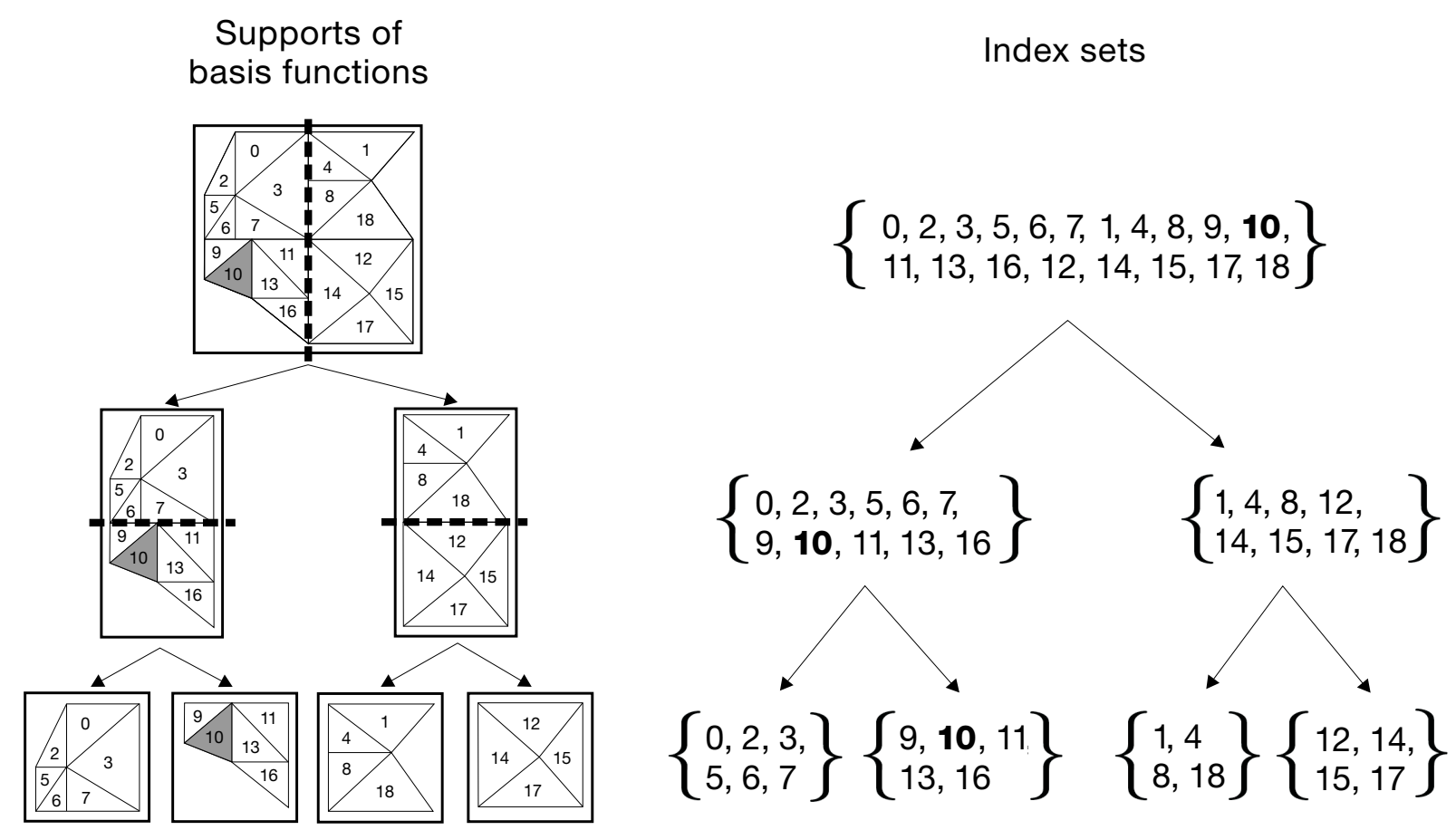

Fig. 4.5: Decomposition of a given triangular discretization (bisection) into clusters (left) and the corresponding index set partitioning (right). Remark: Each basis function appears at each level.

nodes of a mesh with clusters $t$ and $s$ of the index set fulfilling a specific "admissibility" condition (refer to Section 4.3.1.5), a low-rank matrix-block approximation is possible. The application of (rectangular) bounding boxes [130] can help to implement the admissible partitioning for different kinds of basis functions in industrial codes. For an arbitrary block $t \times s$, the minimal bounding boxes for $\mathfrak{S}_{t}, \mathfrak{S}_{s}$ are denoted by $\mathcal{B}_{t}, \mathcal{B}_{s}$. All blocks $b \in T_{\mathcal{I} \times \mathcal{I}}$ are of the form $b=t \times s$ with $t, s \in T_{\mathcal{I}}$. If $T_{\mathcal{I}}$ is a binary tree, then $T_{\mathcal{I} \times \mathcal{I}}$ is a quad-tree.
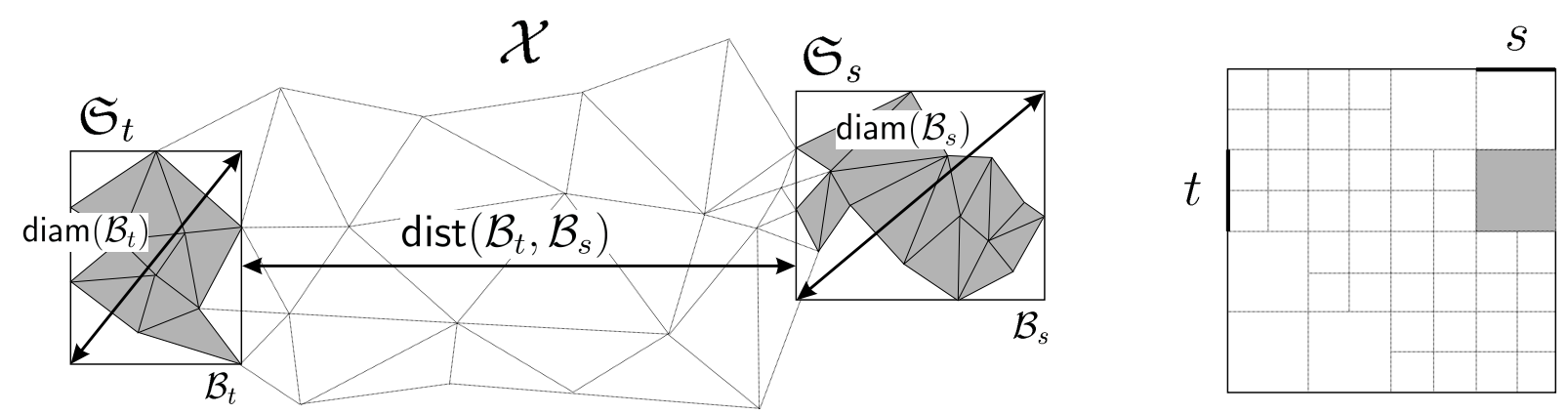

Fig. 4.6: Correspondence between the discretized geometric arrangement with subsets $\mathcal{X}_{t} \times \mathcal{X}_{s}$ of $\mathcal{X} \subseteq \mathbb{R}^{d}$ and the appropriate matrix-block $t \times s \subseteq \mathcal{I} \times \mathcal{I}$. Shown is a grouping of pairs of clusters to construct a BCT.

\subsubsection{Admissible Partitioning $\mathcal{P}$ of $\mathcal{I} \times \mathcal{I}$}

To be able to approximate a matrix by a block-wise low-rank approximation, the subblocks have to fulfill a so-called admissibility condition [132]. A partitioning $\mathcal{P}$ of $\mathcal{I} \times \mathcal{I}$ represents a disjoint decomposition of $\mathcal{I} \times \mathcal{I}$ into blocks $b_{i}^{\lambda}$ on the corresponding levels $\lambda$. To enable a good approximation 
of a given (dense) matrix with regard to (4.25), it is necessary to prove if a block $(t, s) \in T_{\mathcal{I}} \times T_{\mathcal{I}}$ allows a suitable low-rank approximation. Assuming a smooth kernel function,

$$
\min \left\{\operatorname{diam}\left(\mathcal{B}_{t}\right), \operatorname{diam}\left(\mathcal{B}_{s}\right)\right\} \leqslant \eta \operatorname{dist}\left(\mathcal{B}_{t}, \mathcal{B}_{s}\right)
$$

with $\eta \in \mathbb{R}^{+}$must hold. The choice of $\eta$ exhibits a tradeoff and has to be carried out w.r.t. the number of admissible blocks (algorithmic complexity) and the convergence speed (quality of the approximation). A partitioning $\mathcal{P}$ is called admissible, if all blocks $b \in \mathcal{P}$ are admissible. The convergence rate strongly depends on the choice of $\eta$. The larger $\eta$, the slower the convergence. A graphical illustration of an hierarchical partitioning of a matrix is depicted in Figure 4.7.

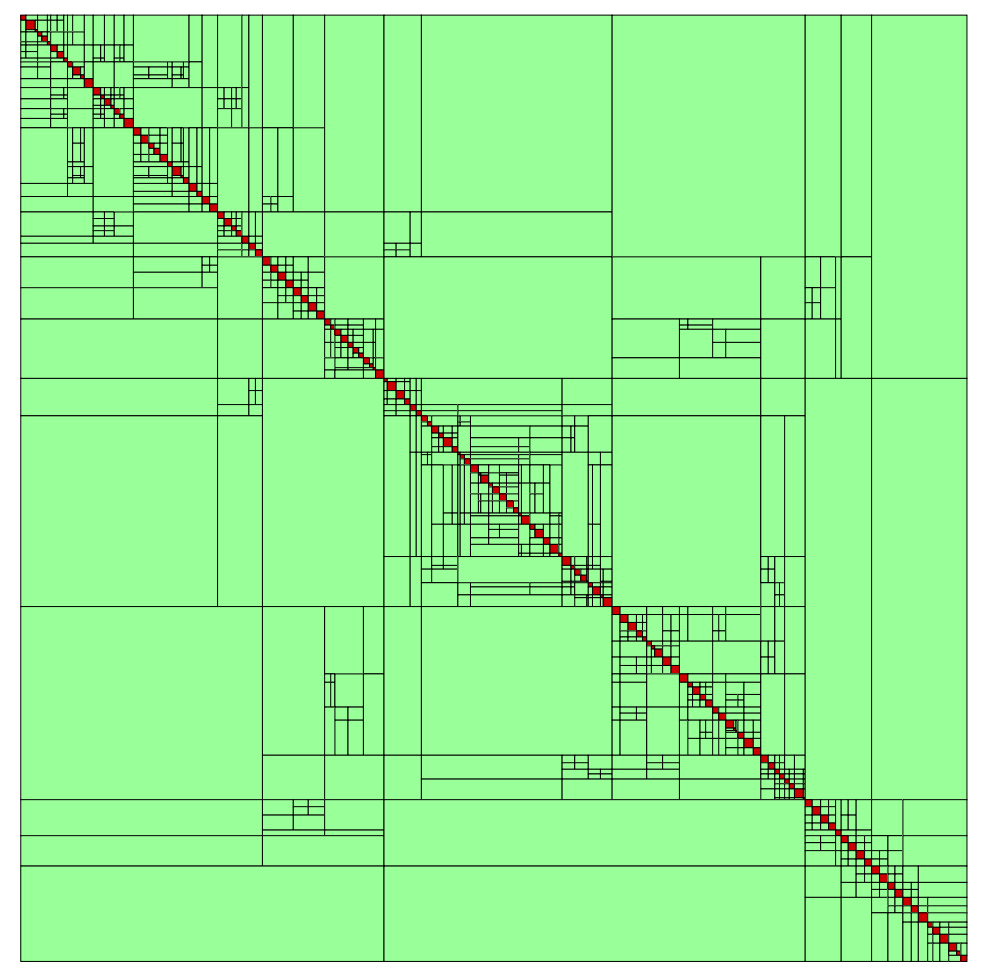

Fig. 4.7: Hierarchical admissible partitioning of a matrix. Admissible (farfield) blocks are colored in light green, where inadmissible (nearfield) blocks on the main diagonal are colored in red.

\subsubsection{6 $\mathcal{R}_{k}$-Matrices and Data-Sparse Formulation}

Based on an admissible partitioning of the original matrix, a low-rank approximation of each admissible matrix-block can be applied. A given block with disjoint bounding boxes can be approximated by low rank, separating the partitioning into

$$
\mathcal{P}=\mathcal{P}^{\text {near }} \cup \mathcal{P}^{\text {far }}
$$

with

$$
\mathcal{P}^{\text {far }} \triangleq\left\{t \times s \in \mathcal{P}: \operatorname{dist}\left(\mathcal{B}_{\tau}, \mathcal{B}_{\sigma}\right)>0\right\}
$$


similar to the FMM. All nearfield blocks are stored as full matrices. One possible kind of low-rank matrix representation exhibits the class of so-called $\mathcal{R}_{k}$-matrices. By this means, a matrix-block $\boldsymbol{A}^{t \times s}$ of dimension $t \times s$ and $\operatorname{rank}^{6} k$ can be represented in the form $[132,130]$

$$
\boldsymbol{A}_{\text {rank } k}=\boldsymbol{U} \boldsymbol{V}^{\boldsymbol{\top}}=\sum_{\ell=1}^{k} \vec{u}_{\ell}\left(\vec{v}_{\ell}\right)^{\top} \in \mathcal{R}_{k}
$$

where $\vec{u}_{\ell} \in \mathbb{R}^{t}, \vec{v}_{\ell} \in \mathbb{R}^{s}, \boldsymbol{U} \in \mathbb{R}^{t \times k}, \boldsymbol{V} \in \mathbb{R}^{s \times k}$ and $k$ is assumed to be small compared to $|t|$ and $|s|$. This is equivalent to a polynomial interpolation [114] of the kernel function $g$ of the integral operator. Each matrix with rank less than $k$ can be represented as a $\mathcal{R}_{k}$-matrix. From (4.36) it can be observed, that an $\mathcal{R}_{k}$-matrix offers a memory complexity of $\mathcal{N}_{\text {st }, \mathcal{R}_{k}}=k(|t|+|s|)$. In order to achieve a representation in $\mathcal{R}_{k}$ for an arbitrary matrix, a kind of truncated singular value decomposition [131, 133] (refer to Appendix A.3) is preferable, because it exhibits a "bestapproximation" in the Frobenius norm as well as in the spectral norm. The computation of a SVD of a $|t| \times|s|$ matrix needs $\mathcal{O}\left(|t|^{3}+|s|^{3}\right)$ operations in general. The intention is, to apply a method with less computational complexity, which directly computes the SVD tuncated to rank $k$. The SVD of a $\mathcal{R}_{k}$-matrix can be calculated with complexity $\mathcal{O}\left(k^{3}\right)+O\left(k^{2}(|t|+|s|)\right)$, in principle. Therefore $k \ll|t|,|s|$ is assumed, so that the term $\mathcal{O}\left(k^{2}(|t|+|s|)\right)$ dominates the cost [134]. The matrix-vector multiplication of a $\mathcal{R}_{k}$-matrix with a vector $\vec{x} \in \mathbb{R}^{s}$ results in

$$
\boldsymbol{A}_{\text {rank } k} \cdot \vec{x}=\sum_{\ell=1}^{k} \vec{u}_{\ell}\left(\vec{v}_{\ell}\right)^{\top} \cdot \vec{x}=\sum_{\ell=1}^{k}\left(\left(\vec{v}_{\ell}\right)^{\top} \cdot \vec{x}\right) \cdot \vec{u}_{\ell} .
$$

This can be accomplished with algorithmic complexity $\mathcal{N}_{\mathrm{MV}, \mathcal{R}_{k} \cdot \vec{x}}=\mathcal{O}(k(|t|+|s|))$. The summation of two $\mathcal{R}_{k}$-matrices result in a $\mathcal{R}_{2 k}$-matrix. The multiplication of a $\mathcal{R}_{k}$-matrix with an arbitrary matrix from left or right, respectively, again produces a $\mathcal{R}_{k}$-matrix.

\subsubsection{Set of Hierarchical Matrices}

Based on the $\mathrm{CT}$ and the BCT, the class of $\mathcal{H}$-matrices for an admissible partitioning $\mathcal{P}$ and a number $k \in \mathbb{N}$ of an arbitrary matrix $\boldsymbol{A} \in \mathbb{R}^{\mathcal{I} \times \mathcal{I}}$ can be defined as

$$
\mathcal{M}_{\mathcal{H}, k}(\mathcal{I} \times \mathcal{I}, \mathcal{P}) \triangleq\left\{\boldsymbol{A} \in \mathbb{R}^{\mathcal{I} \times \mathcal{I}}: \operatorname{rank}\left(\boldsymbol{A}_{b}\right) \leqslant k \forall b \in \mathcal{P}\right\}
$$

with matrix blocks $\boldsymbol{A}_{b}$ of $\boldsymbol{A}$. All admissible blocks $t \times s \in T_{\mathcal{I} \times \mathcal{I}}$ of the matrix can be represented as $\mathcal{R}_{k}$-matrices with blockwise rank $\leqslant k$, whereas non-admissible subblocks (leaves of the tree) show no specific structure and can be understood as full matrices. The set of hierarchical matrices represents no linear space since the sum of two rank- $k$-matrices exceeds rank $k$, in general [132]. The addition of two matrices from $\mathcal{M}_{\mathcal{H}, k}$ can be performed blockwise with linear complexity resulting in a matrix from $\mathcal{M}_{\mathcal{H}, 2 k}$. The approximate multiplication of two matrices again can be applied recursively and with linear complexity, regarding the $\mathcal{H}$-matrix structure of the partitioning $\mathcal{P}$. A recursive algorithm for realizing a matrix inversion with linear complexity can also be applied based on hierarchical matrix techniques. The inversion of the whole matrix in one step can be

\footnotetext{
${ }^{6} \mathrm{~A}$ suitable choice for the rank is $k \approx \log (n)$ [113].
} 
avoided using the SCHUR-complement. The storage requirements for an $\mathcal{H}$-matrix and a matrixvector multiplication amount to

$$
\mathcal{N}_{\mathrm{st}, \mathcal{H}}=\mathcal{N}_{\mathrm{MV}, \mathcal{H} \cdot \vec{x}}=\mathcal{O}(k n \log n)
$$

for the 1D and 2D BCT. The same bound can be achieved using $\mathcal{H}$-matrices based on the same BCT. Considering arbitrary smooth kernels, a blockwise rank $k=\mathcal{O}\left(\left|\log ^{d} \varepsilon\right|\right)$ can be achieved, in general. For illustration purposes, an $\mathcal{H}$-matrix approximation of a PEEC-based network matrix is depicted in Figure 4.8.

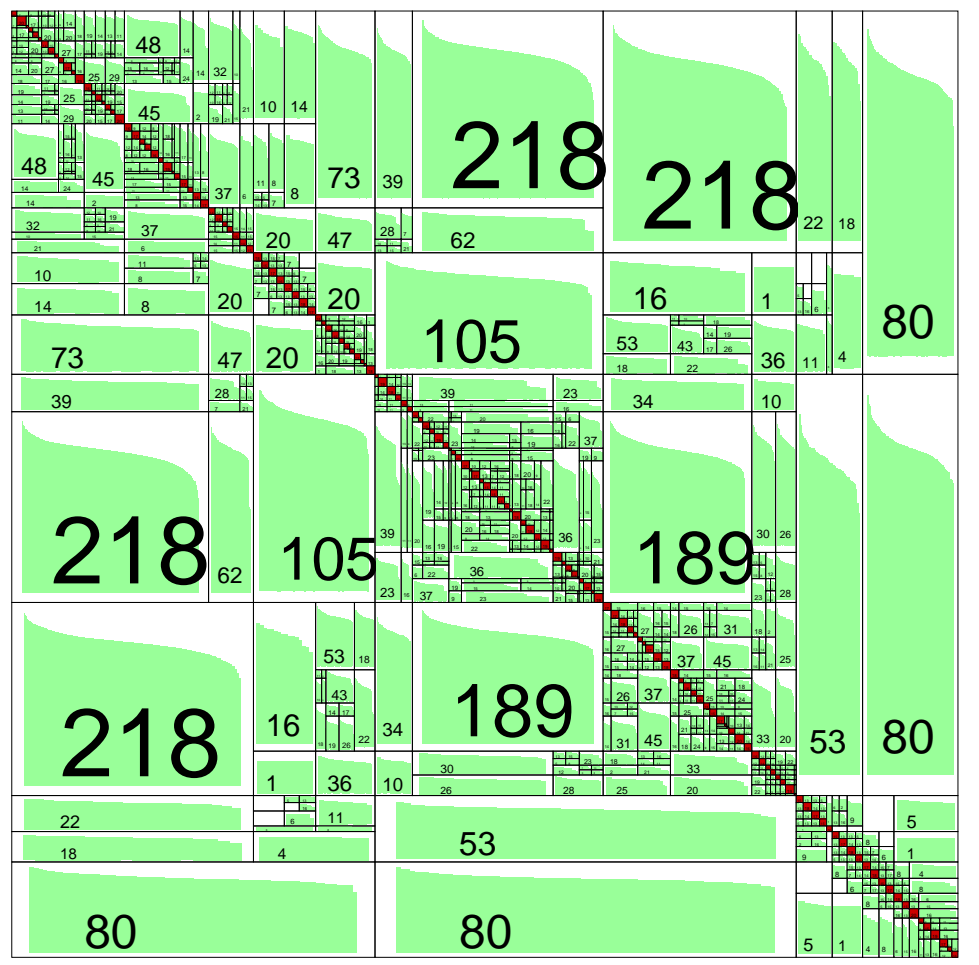

Fig. 4.8: $\mathcal{H}$-matrix approximation for a PEEC-based circuit simulation matrix. Off-diagonal blocks are respresented by low-rank matrices with rank indication by numbers in the blocks. The singular values of the blocks are plotted in a logarithmic scale. Blocks on the main diagonal represent full-rank matrices. Remark: It is desired, that the singular values of the matrix-block decay exponentially and ensure this by sufficient conditions (standard admissibility, weak admissibility) [117].

\subsubsection{Tree Structure Complexity}

A sparse BCT can be constructed with complexity $\mathcal{O}(n \log n)$, almost for arbitrary grids. Assuming local supports $\mathfrak{S}_{i}$ of the corresponding basis functions for quasi-uniform grids, the complexity of building the BCT can be estimated as $\mathcal{O}\left(\eta^{-\kappa} n \log n\right)$ [135] and even $\mathcal{O}(n)$ can be achieved [136].

\subsubsection{Formatted $\mathcal{H}$-Matrix Arithmetic}

Arithmetic operations between $\mathcal{H}$-matrices usually lead to a result not contained in the set $\mathcal{M}_{\mathcal{H}, k}$ (refer to Section 4.3.1.7). To realize an $\mathcal{H}$-matrix arithmetic that still works, the conventional 
matrix-operations have to be replaced by truncated matrix operations. It must be assured, that the operation results exhibit identical rank compared to the original operands. For the detailed theory and algorithmic operations refer to [19, 132]. Table 4.1 gives a short list, containing the most important operations w.r.t. iterative methods and their conventional counterparts for dense matrices.

\begin{tabular}{|l|c|c|}
\hline & \multicolumn{2}{|c|}{ computational complexity } \\
operation & $\mathcal{H}$-arithmetic & conventional arithmetic \\
\hline \hline matrix-vector multiplication & $\mathcal{O}(k n \log n)$ & $\mathcal{O}\left(n^{2}\right)$ \\
\hline matrix addition & $\mathcal{O}\left(k^{2} n \log (n)^{2}\right)$ & $\mathcal{O}\left(n^{2}\right)$ \\
\hline matrix multiplication & $\mathcal{O}\left(k^{2} n \log (n)^{2}\right)$ & $\mathcal{O}\left(n^{3}\right)$ \\
\hline matrix inversion & $\mathcal{O}\left(k^{2} n \log (n)^{2}\right)$ & $\mathcal{O}\left(n^{3}\right)$ \\
\hline$L U$-decomposition & $\mathcal{O}\left(n|\log \varepsilon|^{4} \log ^{2} n\right)$ & $\mathcal{O}\left(n^{3}\right)$ \\
\hline FW/BW-substitution & $\mathcal{O}(n \log n)$ & $\mathcal{O}\left(n^{2}\right)$ \\
\hline
\end{tabular}

Table 4.1: $\quad$ Complexities of formatted (truncated) $\mathcal{H}$-matrix operations. The rank is denoted by $k$, the matrix dimension by $n$.

\subsubsection{The Element Evaluation Process}

In $\mathcal{H}$-matrix-based model computation a fast and accurate approximation of a parasitics matrix is aspired, comparable to the FMM. Controlled by a suitable heuristics only required matrix entries have to be computed, leading to a complexity reduction from $\mathcal{O}\left(n^{2}\right)$ to $\mathcal{O}(n \log n)$ [137]. Furthermore, $\mathcal{H}$-matrices provide additional advantageous features to enhance efficiency and flexibility, compared to the FMM. First of all, a block clustering structure (empty $\mathcal{H}$-matrix) has to be arranged as described in Section 4.3.1.4, solely based on the geometric information concerning the grid nodes. A graphical illustration for an influence extraction is presented in Figure 4.9. The interactions between the clusters can be classified into nearfield and farfield, implying the admissibility condition. Such a separation leads to the fact, that for cluster pairs offering a certain distance to each other, the singularity in the boundary integral kernels not need to be considered [138]. In a second step, the farfield low-rank blocks representing the interactions have to be filled data-sparse. This can be realized intelligently using the ACA algorithm (adaptive cross approximation) $)^{7}$ [139] which needs no explicit knowledge about the kernel function ${ }^{8}$, unlike together with the FMM. The approximation purely bases on algebraic transformations where the heuristics only requests such entries for computation, which are necessary for filling the blocks. The so-called " $\eta$-admissibility" has to be checked for each matrix-block in the empty $\mathcal{H}$-matrix. If the admissibility condition is

\footnotetext{
${ }^{7}$ From literature [139] it is well known, that under suitable conditions for BEM matrices and applying the single layer potential (refer to Appendix B) for asymptotically smooth kernels an approximation of the truncated SVD can be obtained by ACA with time complexity $\mathcal{O}(n k)$, where $k$ denotes the reduced rank [140].

${ }^{8}$ Remark: While the FMM implementation for the Helmholtz equation (refer to (B.9)) becomes unstable even at low frequencies, in general, the construction of $\mathcal{H}$-matrices using ACA and $\mathcal{H}$-arithmetics is numerically stable, regardless of accuracy and frequency [140].
} 


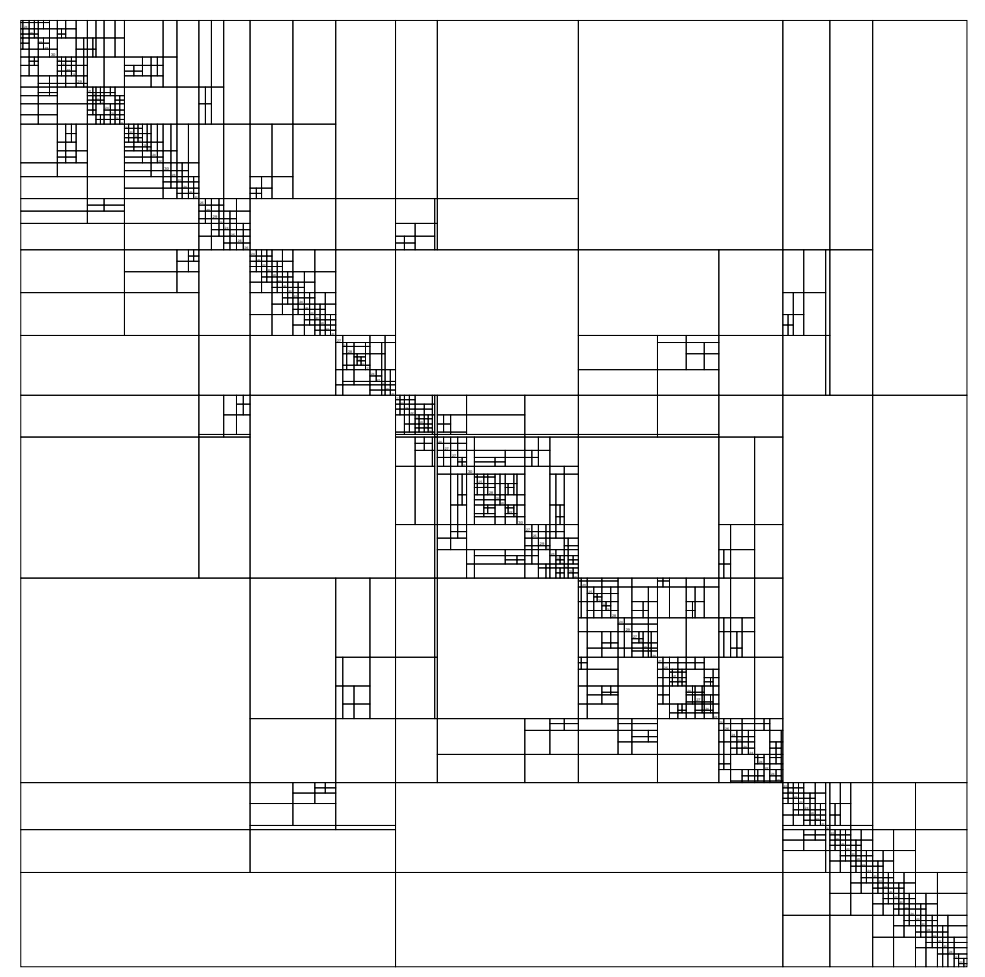

Fig. 4.9: Admissible partitioning for an empty influence matrix based on the nodal geometric data from the PEEC mesh (refer also to Figure 3.10).

fulfilled, only few entries have to be computed. In contrast, inadmissible blocks are computed as full matrices with complexity $\mathcal{O}\left(n^{2}\right)$. In principle, the ACA algorithm can be understood as a function that returns two rank- $k$ matrices $\boldsymbol{A}_{k}, \boldsymbol{B}_{k} \in \mathbb{C}^{n \times k}$ such that

$$
\left\|\boldsymbol{A}-\boldsymbol{A}_{k} \boldsymbol{B}_{k}^{\top}\right\|_{2}<\varepsilon
$$

for a given accuracy $\varepsilon>0$ and the matrix $\boldsymbol{A}$ corresponding to an admissible block. Only $\mathcal{O}(n \log n) \ll \mathcal{O}\left(n^{2}\right)$ matrix entries $A_{i j}$ for an index-pair $(i, j) \in t \times s$ have to be computed. According to [113] a relative approximation error of $\mathcal{O}(\varepsilon)$ can be achieved using $k=\log (\varepsilon)^{2}$. Although the ACA algorithm needs no expansion of the kernel function, it may malfunction together with not asymptotic smooth kernel functions also in the modified version ${ }^{9}$. While the ACA is absolutely adequate for the quasi-stationary PEEC case, problems can occur in the retarded case. A remedy can be achieved by applying an enhanced version of the ACA, the so-called HCA algorithm (hybrid cross approximation) [113].

Cross-Approximation Principle Based on the given problem description w.r.t. [113] it is approved and meaningful to assume, that the kernel function $g$ results from applying a partial differential operator to a sufficiently smooth generator function $\gamma: \mathbb{R}^{3} \times \mathbb{R}^{3} \rightarrow \mathbb{R}$ such that

$$
g=\mathcal{D}_{x} \mathcal{D}_{y} \gamma
$$

\footnotetext{
${ }^{9}$ Remark: The ACA is not an exact method, since it does not produce the optimal rank $k$. Compared to the truncated SVD which results in the optimal approximation, however, the ACA produces almost optimal results in practice with only a fraction of the cost.
} 
holds for some kind of differential operators $\mathcal{D}_{x}, \mathcal{D}_{y}$. Approximating the generator function $\gamma(\vec{x}, \vec{y})$ from (4.41) in an admissible bounding box $\mathcal{B}_{t} \times \mathcal{B}_{s}$ by a suitable functional, the final degenerate kernel function can be described as

$$
\begin{aligned}
\tilde{g} & \triangleq \mathcal{D}_{x} \mathcal{D}_{y} \tilde{\gamma} \\
& =\sum_{\ell=1}^{k}\left(\sum_{q=1}^{\ell} \mathcal{D}_{x} \gamma\left(\vec{x}, \vec{y}_{j q}\right) C_{\ell, q}\right)\left(\sum_{q=1}^{\ell} \mathcal{D}_{y} \gamma\left(\vec{x}_{i q}, \vec{y}\right) D_{\ell, q}\right)
\end{aligned}
$$

where $C_{\ell, q}, D_{\ell, q}$ are given by recursion formulae. Thus it is possible to separate the twofold integrals of the form $\iint \varphi_{i}(\vec{x}) g(\vec{x}, \vec{y}) \varphi_{j}(\vec{y}) \mathrm{d} \vec{x} \mathrm{~d} \vec{y}$ into two single integrals as

$$
\int_{\mathcal{X}} \varphi_{i}(\vec{x}) \mathcal{D}_{x} \gamma\left(\vec{x}, \vec{y}_{j \ell}\right) \mathrm{d} \vec{x} \int_{\mathcal{X}} \varphi_{j}(\vec{y}) \mathcal{D}_{y} \gamma\left(\vec{x}_{i \ell}, \vec{y}\right) \mathrm{d} \vec{y} .
$$

Consequently, the complexity of a $q$-point quadrature per basis function in the Galerkin discretization is reduced from $\left((|t|+|s|) k q^{2}\right)$ to linear cost $((|t|+|s|) k q)$. For the computation of the $k^{2}$ entries of $\boldsymbol{C}$ and $\boldsymbol{D}, \mathcal{O}\left(k^{3}\right)$ arithmetic operations are necessary. The total complexity for the HCA amounts to [113]

$$
\mathcal{O}\left((|t|+|s|) k^{2}+M k^{2}\right)
$$

with interpolation order $M$.

\subsubsection{Implementation Details}

A technical implementation of a fast parasitic extraction for partial elements from Sections 3.2 and 3.3 can be realized using the $\mathcal{H}$-matrix library HLiв [27]. Keeping the theoretical details from Section 4.3.1 in mind, the kernel functions from (3.11) and (3.12) as well as from (3.26) and (3.32) show the required properties for a possible degenerate approximation. Due to the similarity of the mathematical computation of inductances and influences we restrict ourself to the influence extraction process, which is describe in detail in the remainder. All aspects for an implementation of the partial inductance extraction can be concluded in a similar manner. For a technical realization and implementation of the required algorithms some important aspects are described in the following. In a first step, based on the geometric data of the mesh, a construction of an empty $\mathcal{H}$ Influence-matrix is necessary, as outlined in Figure 4.10. Starting from the nodal mesh data and a

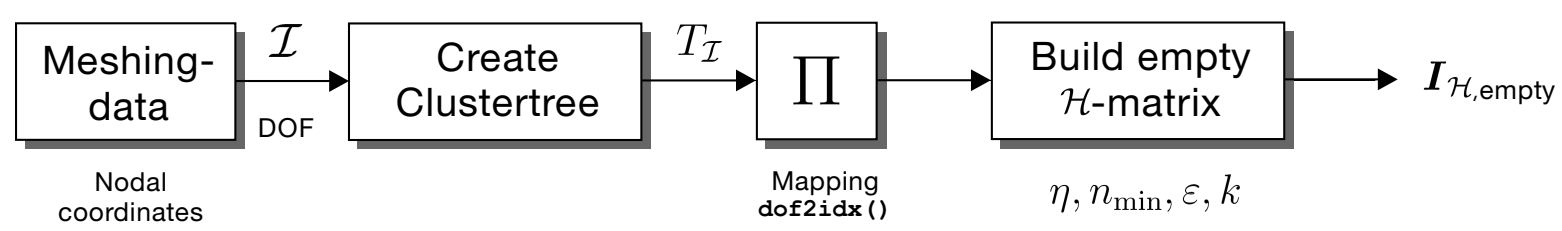

Fig. 4.10: Construction of an empty $\mathcal{H}$-influence-matrix from the nodal PEEC mesh data.

suitable indexing (index set $\mathcal{I}$ ), a cluster tree can be constructed regarding the admissibility condition from Section 4.3.1.5. In PEEC modeling the DOF correspond to the unknown nodal voltages or branch currents, respectively. The nodal coordinates in 3D are stored in a clusterfactory and suitable bounding boxes $\mathcal{B}_{t}, \mathcal{B}_{s}$ have to be defined. The CT can be constructed from the clusterfactory by applying geometrically-balanced clustering. A mapping $\Pi$ applies a sorting of all 
DOF to the corresponding indices. This realizes the geometric assignment which is necessary for the local separation. Regarding the admissibility condition, the empty $\mathcal{H}$-Influence matrix can be constructed using the standard way (HLIB_FEMNONSYM), as defined in the HLIB. In a second step, the empty $\mathcal{H}$-Influence-matrix can be filled recursively by ACA, ACA + or HCA, as depicted in Figure 4.11 The procedure recursive_filling_supermatrix () ${ }^{10}$, which was

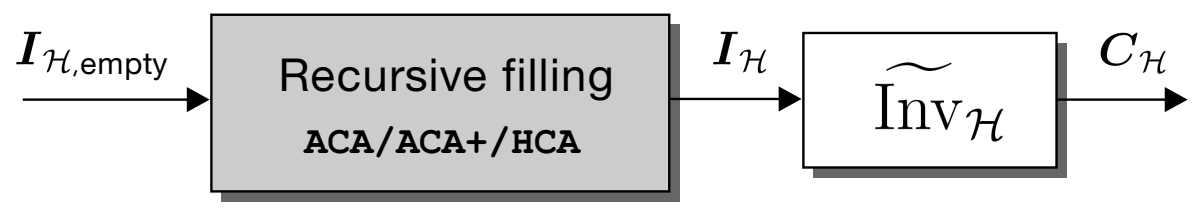

Fig. 4.11: Recursive filling of the $\mathcal{H}$-influence-matrix $\boldsymbol{I}_{\mathcal{H}}$ using the ACA algorithm and following $\mathcal{H}$-inversion to obtain the capacitance matrix $\boldsymbol{C}_{\mathcal{H}}$ in the $\mathcal{H}$-matrix format.

developed in the course of this thesis, enables a combination and information exchange between the $\mathcal{H}$-matrix library and the EM modeling software. It takes the three different matrix cases into account as described in detail in Algorithm 2. The ultimate influence computation is realized by a

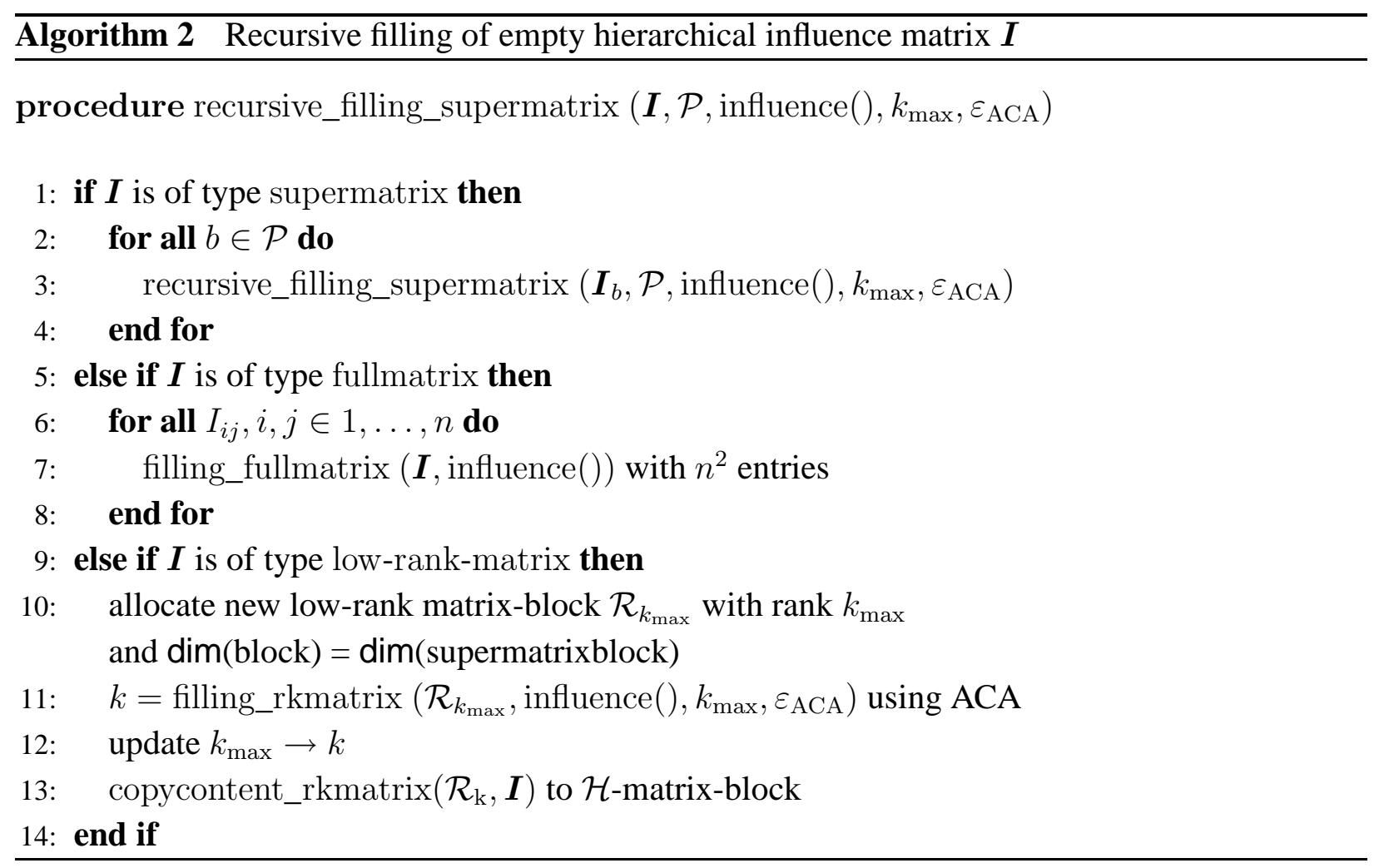

callback-function, requesting only those values addressed by their indices $i, j$ in the matrix which are required by the cross-approximation algorithm. An additional MOR scheme can be applied to the resulting matrix in a straightforward manner.

\subsubsection{Numerical Examples}

A PEEC-based fast influence extraction from net VB on the MISEA demonstrator board is considered in the following, as illustrated in Figure 4.12. Resulting from the computation rule (3.12),

\footnotetext{
${ }^{10}$ In the $\mathcal{H}$-matrix library HLIB, an $\mathcal{H}$-matrix is denoted "supermatrix".
} 
combined with a variation of the meshsize, five benchmark example matrices can be obtained with different matrix dimensions. For each case a comparsion between conventional and ACA-based recursive model extraction is accomplished. Three different parameter settings for ACA-based matrix computation are applied as specified in Table 4.2, to cover the spectrum of possible effects to the resulting matrix compression process. Setup times $t_{\text {set }}$ for filling the matrix, times for inverting the matrix $t_{\text {inv }}$ as well as storage requirements (mem) are acquired and compared. The results are presented in Table 4.3. Whereas the geometric properties of vias are taken into account, dielectric effects are neglected. For the case of matrix dimension $3623 \times 3623$ (case PEEC21) the $\mathcal{H}$-matrix structure, the filled $\mathcal{H}$-influence matrix and the $\mathcal{H}$-capacitance matrix are depicted in Figure 4.13. It can be verified by inspection that the dense $\mathcal{H}$-capactitance matrix is represented data-sparse. The numerical experiments were performed on a INTEL(R) Xeon(TM) 2.80 GHz Modell2 CPU with cache size $512 \mathrm{~KB}$ running Linux. The results show some important facts. On the one hand, it can be observed that the gain in computational speed as well as storage requirements strongly increases with increasing matrix dimension. On the other hand, for parameter setting 2 the achievable speed-up for the setup phase reaches a factor 3.18. The speed-up for the inverting phase varies between $40 \ldots 10000$ for the parameter range. It has to be noticed, that a larger $\varepsilon$ directly implies a higher loss in accuracy. The gain in memory requirements ranges between $8.42 \ldots 50$.

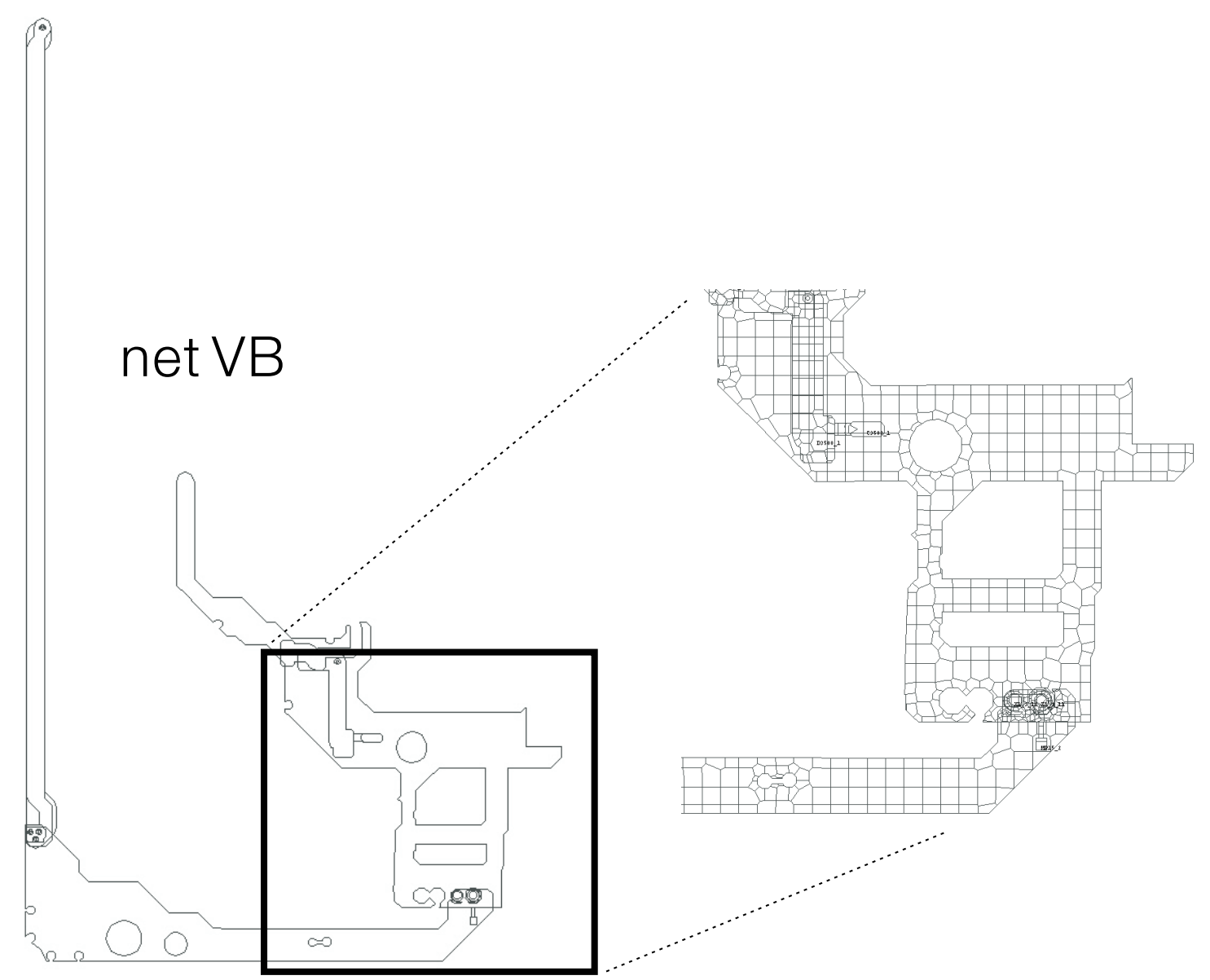

Fig. 4.12: Power supply net VB on the MISEA demonstrator board used for influence extraction with connected terminals. A small part of the capacitive mesh is depicted for visualization purposes. 


\begin{tabular}{|c||c|c|c|c|c|c|}
\hline \multicolumn{1}{|c||}{$\begin{array}{c}\text { parameter- } \\
\text { setting (ps) }\end{array}$} & \multicolumn{4}{c|}{$\boldsymbol{A}_{\mathcal{H}}$} & \multicolumn{2}{c|}{ ACA } \\
\hline \hline 1 & 2.0 & $n_{\min }$ & $\varepsilon$ & $k$ & $\varepsilon_{\mathrm{ACA}}$ & $k_{\max }$ \\
\hline 2 & 2.0 & 20 & $10^{-1}$ & adaptive & $10^{-1}$ & 1 \\
3 & 2.0 & 30 & $10^{-3}$ & adaptive & $10^{-2}$ & 5 \\
\hline
\end{tabular}

Table 4.2: Parameter settings (ps) for ACA-based recursive influence extraction. For a detailed description of the parameters refer to [27].

\begin{tabular}{|c||c|c|c|c|c|c|c|c||c|c|c|}
\hline \multicolumn{1}{|c||}{} & \multicolumn{3}{|c|}{} & \multicolumn{3}{c|}{$\begin{array}{c}\text { conventional } \\
\text { model extraction }\end{array}$} & \multicolumn{3}{c||}{$\begin{array}{c}\text { ACA-based } \\
\text { model extraction }\end{array}$} & \multicolumn{3}{c|}{ speed-up } \\
benchmark & dim & $t_{\text {set }}$ & $t_{\text {inv }}$ & mem & ps & $t_{\text {set }}$ & $t_{\text {inv }}$ & mem & $t_{\text {set }}$ & $t_{\text {inv }}$ & mem \\
\hline \hline PEEC19 & 214 & 3.68 & 0.23 & 0.35 & 1 & 2.34 & 0.01 & 0.06 & 1.57 & 23 & 5.83 \\
& & & & & 2 & 4.6 & 0.08 & 0.18 & 0.8 & 2.88 & 1.94 \\
& & & & & 3 & 4.87 & 0.09 & 0.26 & 0.76 & 2.56 & 1.35 \\
\hline PEEC20 & 396 & 4.56 & 1.24 & 1.2 & 1 & 2.39 & 0.01 & 0.13 & 1.91 & 124 & 9.23 \\
& & & & & 2 & 4.43 & 0.22 & 0.41 & 1.03 & 5.64 & 2.93 \\
& & & & & 3 & 5.70 & 0.31 & 0.68 & 0.8 & 4 & 1.76 \\
\hline PEEC9 & 796 & 16.15 & 8.08 & 4.8 & 1 & 4.31 & 0.01 & 0.31 & 3.75 & 808 & 15.48 \\
& & & & & 2 & 11.46 & 0.78 & 1.1 & 1.41 & 10.36 & 4.36 \\
& & & & 3 & 15.75 & 1.33 & 1.8 & 1.03 & 6.08 & 2.67 \\
\hline PEEC10 & 1345 & 26.82 & 34.53 & 14 & 1 & 4.94 & 0.01 & 0.6 & 5.43 & 3453 & 23.33 \\
& & & & & 2 & 14.77 & 1.86 & 2.1 & 1.82 & 18.56 & 6.67 \\
& & & & & 3 & 21.14 & 3.3 & 3.6 & 1.27 & 10.46 & 3.89 \\
\hline PEEC21 & 3623 & 44.35 & 583 & 101 & 1 & 13.93 & 0.04 & 1.9 & 3.18 & 14575 & 53.16 \\
& & & & & 2 & 38.1 & 7.17 & 7.0 & 1.16 & 81.31 & 14.43 \\
& & & & & 3 & 57.02 & 14.7 & 12 & 0.78 & 39.66 & 8.42 \\
\hline
\end{tabular}

Table 4.3: Performance of conventional and recursive ACA-based influence extraction for five benchmark models with varying matrix dimensions. Setup times $t_{\text {set }}$ and times for inversion $t_{\text {inv }}$ are given in seconds. Memory requirements for the influence matrices are given in MBytes. The parameter settings (ps) are specified in Table 4.2.

\subsubsection{Further Improvements}

Although the described properties of $\mathcal{H}$-matrices already enable an efficient and accurate EM modeling process, further improvements are still under investigation. The main topics, relevant for circuit simulation, are shortly explained in the following.

\subsubsection{Adaptive Refinement}

For accuracy and validity reasons mesh refinement of a given triangulation can be necessary. Often the refinement is only locally applied and consequently the boundary element matrices before and after the refinement coincide in most of the matrix entries. An overall matrix recomputation 


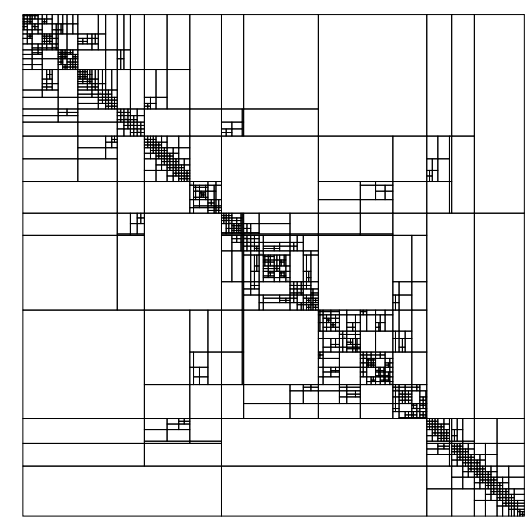

a)

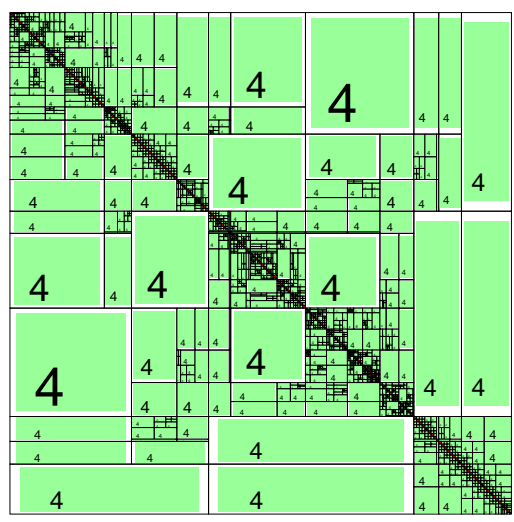

b)

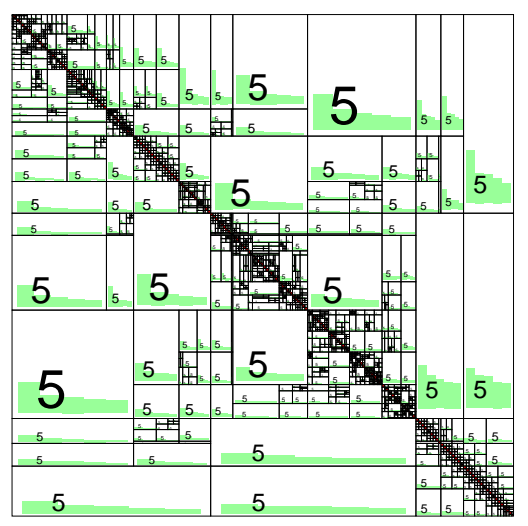

c)

Fig. 4.13: Benchmark example PEEC21. a) $\mathcal{H}$-influence-matrix structure; b) filled $\mathcal{H}$-influence-matrix; c) $\mathcal{H}$ capacitance-matrix.

therefore is not meaningful. The aim is, to achieve a predefined discretization error with as less unknowns as possible. For this, it is necessary to estimate the discretization error and to apply a suitable refinement strategy. Multigrid methods (refer to Section 7) can be combined with adaptive local grid refinement strategies in a simple and straightforward manner ${ }^{11}$. Concerning hierarchical matrices, the defined block partitioning has to be taken into consideration. A local refinement of an existing index set $\mathcal{I}$ with $n=|\mathcal{I}|$ directly leads to an enlarged index set $\mathcal{I}^{\prime}$ with $n^{\prime} \triangleq\left|\mathcal{I}^{\prime}\right|, n^{\prime}>n$ [141]. From the literature $[136,142]$ it is well-known that the internal format of $\mathcal{H}$-matrices requires only local changes. As a first step, the cluster tree has to be updated [136]. Based on an already existing cluster tree $T_{\mathcal{I}}$ an updated cluster tree $T_{\mathcal{I}^{\prime}}$ can be obtained by adding new basis functions. The cost for inserting an additional single index in the tree is $\mathcal{O}\left(\operatorname{depth}\left(T_{\mathcal{I}}\right)\right)$. In a second step, the block cluster tree $T_{\mathcal{I} \times \mathcal{I}}$ has to be updated. This goes along with the update of the matrix entries, where only entries corresponding to the row and column of the new index have to be updated. For implementation a modified admissibility condition and a separate consideration of the kind of block to be updated, is necessary.

\subsubsection{Adaptive Recompression}

Low-rank matrices can be (re-)compressed to further minimize the storage requirements. This can lead to a significant speed-up of arithmetic operations compared to the FMM [117]. We consider a BEM-based hierarchical matrix with blockwise rank $k$ or error $\varepsilon$, respectively. To choose $k^{\prime}<k$, implying $\varepsilon^{\prime}>\varepsilon, \varepsilon^{\prime}<1$, directly leads to smaller cost, but also to a degraded convergence behavior of the iterative solution method. An intelligent recompression procedure basically consists of two fundamental steps $[117,143]$. In a first step, a blockwise rank-recompression

$$
\boldsymbol{A}_{\mathcal{H}} \in \mathcal{M}_{\mathcal{H}, k}(\mathcal{I} \times \mathcal{I}, \mathcal{P}) \mapsto \tilde{\boldsymbol{A}}_{\mathcal{H}} \in \mathcal{M}_{\mathcal{H}, k^{\prime}}(\mathcal{I} \times \mathcal{I}, \mathcal{P})
$$

with $k^{\prime}<k, \varepsilon^{\prime}>\varepsilon$ can be applied, resulting in lower cost for the computation of $\tilde{\boldsymbol{A}}_{\mathcal{H}}^{-1}$ and $\widetilde{\boldsymbol{L}_{\mathcal{H}} \boldsymbol{U}_{\mathcal{H}}}$. The recompression can be applied "on-the-fly", i.e. constructing the low-rank blocks can directly

\footnotetext{
${ }^{11}$ It can be proven that such techniques work with optimal complexity.
} 
be combined with a following recompression stage. In a second step, admissible blocks $b=(t, s)$ consisting of subblocks $b_{i}, i=1,2,3,4$ and resulting from a permutation of the corresponding subindex sets $\left(t_{i}, s_{j}\right), i, j=1,2$, are understood as one single block. This procedure allows a further reduction of the required rank. For this purpose, an update of the existing cluster tree $\tilde{\mathcal{P}}$ is necessary. The resulting $\mathcal{H}$-matrix

$$
\tilde{\tilde{\boldsymbol{A}}}_{\mathcal{H}} \in \mathcal{M}_{\mathcal{H}, k^{\prime \prime}}(\mathcal{I} \times \mathcal{I}, \tilde{\mathcal{P}})
$$

helps to reduce the complexity concerning storage requirements as well as arithmetic operations. For further details refer to [144].

\subsubsection{3 $\mathcal{H}^{2}$-Matrices}

By introducing a further hierarchy stage in addition to the hierarchy already impressed by the cluster tree, a further specialization of $\mathcal{H}$-matrices can be achieved. A more efficient storage and matrix-vector multiplication can be enabled by so-called $\mathcal{H}^{2}$-matrices [129]. The matrix blocks are approximated by low-rank matrices, however, the column and row bases for each element of the cluster tree are fixed. This leads to the fact, that for each block only the coefficients of the basis representation have to be computed. By a successive construction of bases for larger blocks out of the bases for smaller blocks, a significant complexity reduction can be achieved [47]. From the $\mathcal{H}$-matrix structure in Figure 4.9 it can be observed, that blocks exist comprising

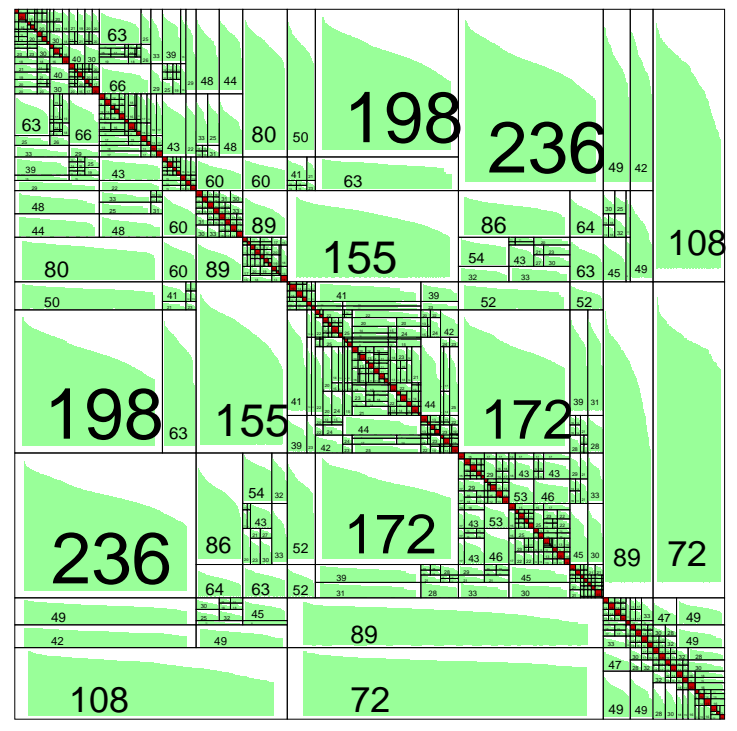

a)

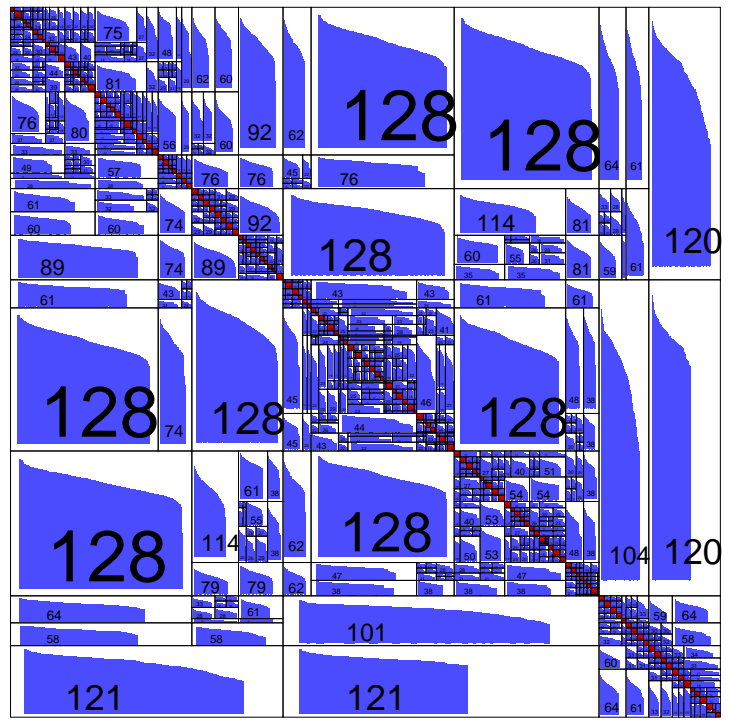

b)

Fig. 4.14: Benchmark PEEC network-matrix with dimension $2024 \times 2024$, density $92.38 \%$ from FD analysis. a) $\mathcal{H}$-matrix approximation; b) $\mathcal{H}^{2}$-matrix approximation.

the same row or column indices. The structure of the matrix can be simplified, if the blocks are approximated not independently from each other. The aim is, to represent the row and column matrices more economically. By exploiting the hierarchy, introduced by the $\mathrm{CT}$ and regarding that each cluster is a disjoint union of its sons, a successive construction of the column matrices from the column matrices of its sons can be realized. For that purpose, polynomials are chosen, enabling a representation in another basis of the polynomial space. It is sufficient to store the matrices for 
such clusters, containing no sons. For all other clusters only a basis transformation is necessary to construct the column matrices from the column matrices of its sons. A graphical illustration of an $\mathcal{H}^{2}$-matrix approximation is depicted in Figure 4.14. 


\section{5 \\ Iterative Methods in Circuit-Level Analysis}

$T$

he conversion of the electromagnetic field problem to the circuit domain (Chapters 2 and 3) allows for solving the problem by means of conventional circuit simulators. Nodal voltages and branch currents can be obtained as solution variables instead of electric or magnetic field strengths. The numerical representation of circuit formulations, however, basically differs from conventional mathematical problems as the solution of partial differential equations. Circuit problems are often described in the nodal analysis (NA) or modified nodal analysis (MNA) formulation as explained in detail in Appendix D. The resulting system of equations contains submatrices of different types and dimensions and possibly different types of solution variables. Parameters as the simulation domain (TD/FD) or the modeling strategy (PEEC/TL) have a great impact to the resulting system matrix appearance. These parameters influence the eigenvalue distribution of the network matrix and consequently the condition number. Simulations in high-frequency ranges require a very fine discretization for achieving accurate results and model validity. Consequently, very large matrix dimensions can be expected. Moreover, various parasitic effects dominating at high frequencies, in general lead to high densities of the resulting system matrices.

Section 5.1 shortly describes the special properties and characteristics of circuit simulation matrices and the challenges for direct as well as for iterative solvers. The basics of classical and Krylov iterative methods are outlined in Section 5.2, to enable a deeper understanding necessary for the following chapters. Due to the ill-conditioning of common circuit system matrices, conventional iterative methods inevitably fail without an adequate preconditioning. Therefore, Section 5.3 presents the mathematical background as well as concrete techniques for an efficient preconditioning with regard to circuit simulation. Implementation issues, modern and efficient sparse matrix storage formats as well as suggestions for further improvements are given in Sections 5.4 to 5.6 to realize an optimal solution process. 


\subsection{Problem Description and Motivation}

Equation systems in circuit analysis of the form

$$
\boldsymbol{A} \vec{x}=\vec{b}
$$

are assumed to contain a real-valued system matrix $\boldsymbol{A} \in \mathbb{R}^{n \times n}$, a right-hand side vector $\vec{b} \in \mathbb{R}^{n}$ and a solution vector $\vec{x} \in \mathbb{R}^{n}$. The equation system arises from a discretization of the EFIE as described in Section 2.5, by applying a domain decomposition to the integration domain (e.g. refer to (3.68)). Depending on the modeling approach and the simulation domain the density of the system matrix can vary tremendously. Due to the increasing complexity of real-life EM field problems it is aspired to develop solution techniques which are able to achieve (almost) linear complexity in time and memory requirements w.r.t. the number of DOF. In PEEC-based circuit analysis the number of DOF corresponds to the number of unknowns. System matrices emanating from differential-based approaches, as resulting e.g. from TL models for cable harnesses, are sparse due to the occuring local interactions, in general. The global interactions emerging in integral-based approaches, however, lead to dense matrices. As shown in Section 3.9, a method exists to sparsify an IE-based PEEC matrix by retaining stability and showing only small degradations in accuracy. Implied by the Green's function a locally assumed current contribution has an effect to the electric field in space. However, the field strength decreases rapidly with increasing distance between observation point and the current source. While the near interactions are placed in the vicinity of the main diagonal, the farfield couplings are placed far away from the main diagonal. As a result, matrices resulting from a BEM approach are dominant in the vicinity of the main diagonal. PEEC matrices in the MNA formulation usually are not diagonally dominant since they are built-up blockwise from BEM matrices and adjacency matrices. Especially w.r.t. the suitability for iterative methods, NA-based PEEC matrices are more convenient for iterative solvers due to their property of being positive definite and symmetric (SPD) ${ }^{1}$ [145]. The symmetry of the matrix entries results from the symmetry of Green's function in combination with the application of real-valued basis functions $[146,3]$. The convergence behavior of iterative methods can be analyzed by means of the condition of the linear equation system. The condition can be quantified by the condition number $\operatorname{cond}(\boldsymbol{A})$ of the system matrix (refer to Appendix A.2). The condition number is a measure how strong a matrix differs from the identity matrix. For simple classical linear iterative methods as the RICHARDSON iteration [147], a mathematical expression for the number of iterations $n_{\text {iter }}(\varepsilon)$, needed to achieve a given accuracy $\varepsilon$ can be quantified as [148]

$$
n_{\text {iter }}(\varepsilon)=\mathcal{O}\left(\operatorname{cond}(\boldsymbol{A}) \ln \varepsilon^{-1}\right) .
$$

Figure 5.1 graphically illustrates (5.2) for varied accuracies $\varepsilon$. Obviously it is desirable to keep the condition number small to achieve the minimum number of required iteration steps. The set of all eigenvalues $\lambda$ of $\boldsymbol{A}$ in the complex plane is called the spectrum $\sigma(\boldsymbol{A})$ of $\boldsymbol{A}$. To analyze the convergence behavior of iterative methods concerning circuit-based system matrices, a fast and efficient method to precisely compute the eigenvalues and their locations is inevitable. While a simple bound for the absolute eigenvalues of a matrix $\boldsymbol{A}$ can easily be derived as

$$
\left|\lambda_{k}\right| \leqslant\|\boldsymbol{A}\|_{M}, \quad \forall k
$$

\footnotetext{
${ }^{1}$ Remark: The property of matrices to be SPD is advantageous for the convergence behavior together with iterative solvers.
} 


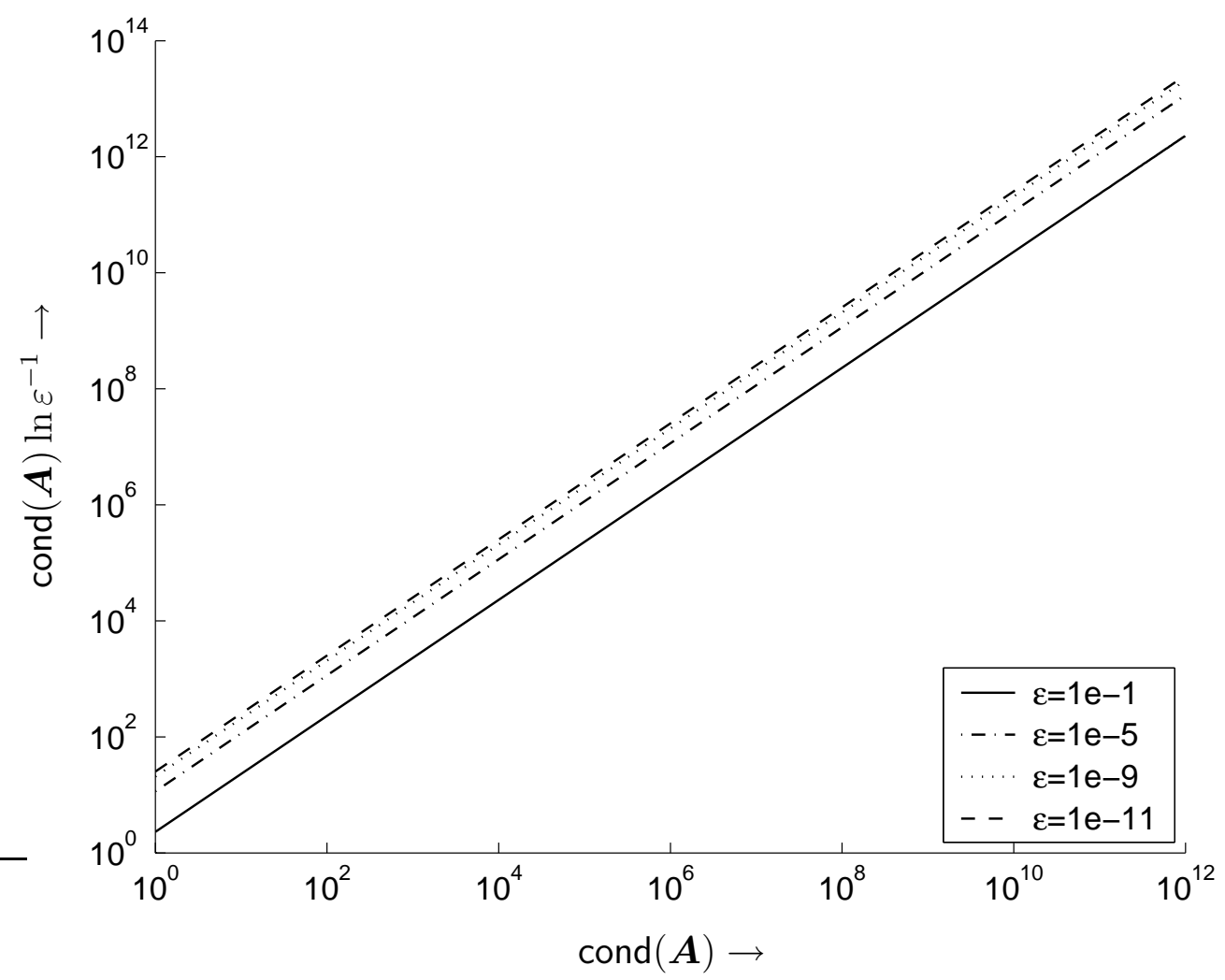

Fig. 5.1: Number of required iteration steps to achieve convergence by applying the Richardson iteration with required accuracy $\varepsilon$ depending on the condition number cond $(\boldsymbol{A})$ of the system matrix, referring to [148]. Remark: The range for the condition number corresponds to the relevant range of condition numbers for PEEC-based circuit simulation matrices in real-life problems.

together with an arbitrary matrix norm $\|\cdot\|_{M}$, these results often are not accurate enough. The diagonal dominance of PEEC matrices can be mapped to the convergence behavior by applying GERSHGORIN's circle theorem [147], as described in detail in Appendix A.4. Gershgorin's theorem is a quite tight bound and enables a fast determination of the locations of the eigenvalues. The union of all GERSHGORIN circles (Gershgorin discs) contains the whole spectrum of the matrix. Particularly the locations and the clustering of the eigenvalues are important to analyze. The clustering effect in circuit-based simulations arises from the different nature of the circuit primitives as inductances, capacitances and ohmic losses. The different natures of the elements lead to a large range between the largest and smallest absolute eigenvalues, which is reflected by a high condition number of the resulting system matrices. The condition numbers for realistic PEEC matrices are in the range $10^{3} \ldots 10^{13}$. In FD analysis the frequency range significantly affects the clustering of the eigenvalues and therefore the characteristic of the matrix. This is obvious from Figure 5.2, illustrating the eigenvalue distributions of a square system matrix with dimension $1024 \times 1024$ at eight discrete frequency points in the complex plane. While for small frequencies the eigenvalues are uniformly distributed along the real axis, a grouping with two clusters can be observed for higher frequencies. In addition, a spreading of the eigenvalues into the negative half plane occurs. The range for the condition number of a system matrix usually can be estimated by means of the meshsize $h$ according to the kind of discretization [138]. For FEM-based matrices a condition number of $\mathcal{O}\left(h^{-2}\right)$ can be achieved, in general. Together with BEM-based matrices and the single layer potential operator (refer to (B.1) in Appendix B) as used for PEEC, however, a complex- 

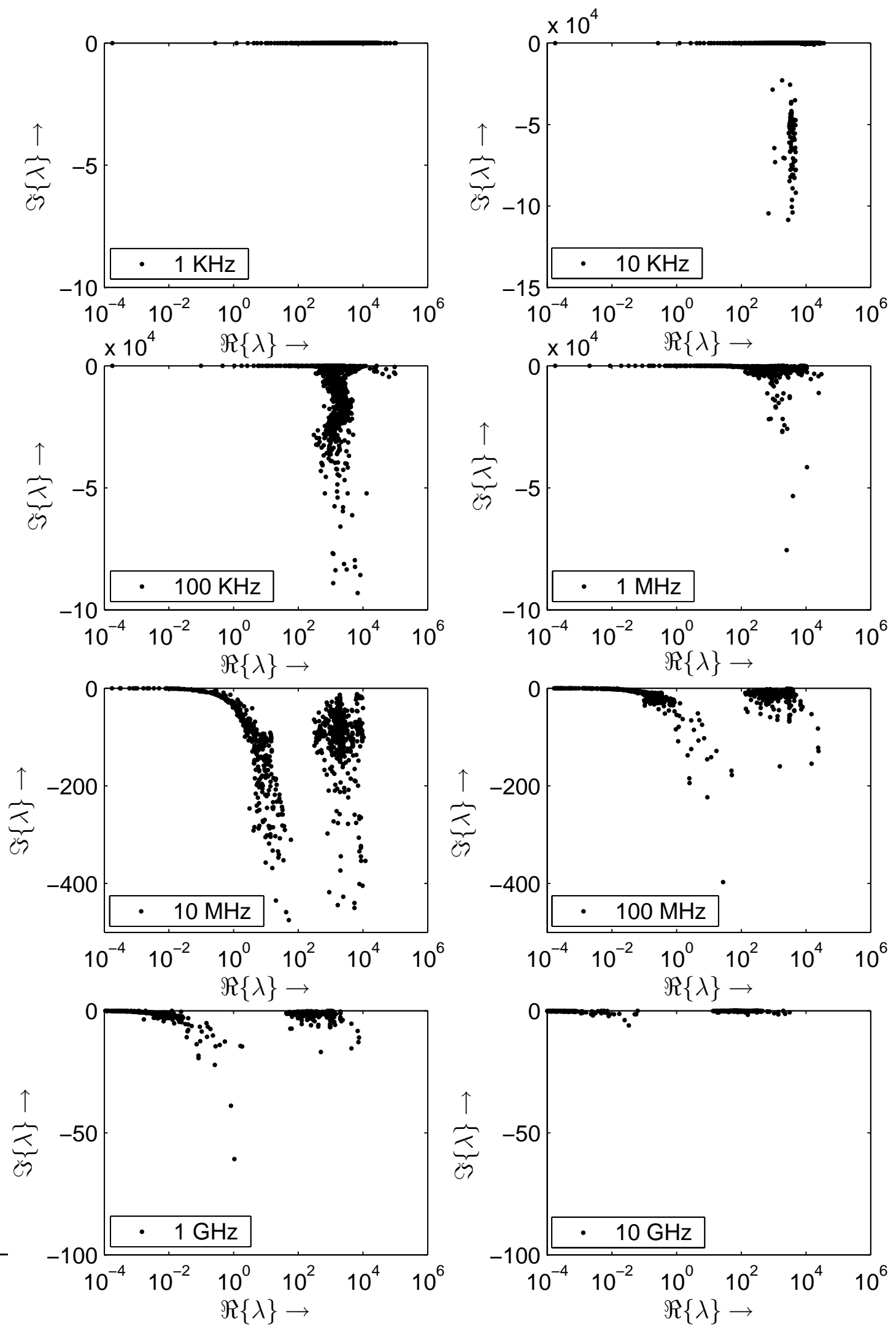

Fig. 5.2: Influence of the frequency range to the eigenvalue distribution of circuit simulation matrices. The clustering effect is illustrated by means of a PEEC benchmark matrix with $\delta_{C}, \delta_{K}=0.2 \%$ and matrix dimension $1024 \times 1024$ at discrete frequency points. 
ity of $\mathcal{O}\left(h^{-1}\right)$ results. At very low frequencies, the capacitive effects will dominate compared to the inductive effects. According to [3], for this reason a difference between largest and smallest absolute eigenvalue by a factor of $\approx 10^{7}$ usually can be observed. Such a strong clustering of very small eigenvalues can cause problems for the iterative solution of circuit-based system matrices. To obtain cognitions about the behavior of the solution process for element matrices resulting from a EFIE formulation, it is very important to analyze the eigenvalues and eigenfunctions of the underlying integral operators. For simple geometries the solution of the EFIE can be taken from [3]. More and more higher eigenfrequencies in the problem occur with decreasing meshsize as can be observed from the eigenvectors. It can be possible that parts of the solution of a given problem in direction of certain frequencies are damped insufficiently. The application of conventional methods is accompanied by a good damping of high eigenfrequencies while the remaining lower eigenfrequencies are damped insufficiently. This fact leads to the idea of algebraic multigrid (AMG) which is described in more details in Chapter 7. Generally, the eigenvectors of the discrete problem approximately correspond to the eigenvectors of the continuous one. The dimension of the underlying grid significantly impacts the eigenfunctions of the system matrix. According to the expected oszillations of the solution with wave length $\lambda$, the grid size has to be chosen in a way so that the resonant modes can be represented accurately enough. In real-life problems, this can be achieved regarding the rule (3.70) from Section 3.7. As described in Section 3.7, a good performance for PEEC models can be achieved using $h \leqslant \lambda / 20$, leading to

$$
\frac{\pi}{h} \geqslant 20 \frac{\pi}{\lambda}=10 \beta
$$

with the wave number $\beta$ as defined in Section 2.1. A grid-refinement leads to an increased condition number for the inductance matrix as well as for the influence matrix, in general. For an infinite plate at low frequencies excluding ohmic losses, the expression

$$
\operatorname{cond}(\boldsymbol{A}) \gtrsim \frac{1}{4} \frac{\lambda^{2}}{h^{2}}
$$

for the condition number can be estimated. For low frequencies the edge effects and the geometry will dictate the element size $h$, leading to a much smaller $h$ than required by (5.4).

\subsubsection{Direct Strategies}

Classical direct methods for the solution of large and dense linear systems of equations as the GAUSS elimination offer a complexity of $\mathcal{O}\left(n^{3}\right)$ w.r.t. CPU time requirements and of $\mathcal{O}\left(n^{2}\right)$ w.r.t. memory requirements for $n$ DOF [149]. Under circumstances the complexity w.r.t. time and memory can be reduced to $\mathcal{O}\left(n^{2}\right)$ and $\mathcal{O}\left(n^{3 / 2}\right)$ by taking specific matrix structures (e.g. banded matrices) into account. An existing sparse structure of the matrix is hardly utilized in the computation and the fill-in increases tremendously. This leads to disproportionate storage requirements which are directly reflected in an increased time complexity. Special techniques as bandwidth optimization and skyline methods only are able to mitigate the problem but they can not solve it. There exists a variety of direct solvers for sparse matrices aiming at a fill-in reduction by applying a suitable pivoting strategy. A matrix generated by a FEM or BEM approach can be associated with a graph representing the non-zero structure of the matrix [147]. In general, the rows are represented by a vertex and every non-zero element by an edge. An ordering of columns and rows 
can be accomplished to minimize the fill-in. To find the best pivoting w.r.t. minimum fill-in, various heuristic ordering strategies as minimum degree ordering, nested dissection, column-count or Cuthill-McKee ordering are possible. METIS [150, 151], for example, is a library based on hypergraph partitioning, suitable for circuit problems. Matrix ordering strategies can help to improve the solution process. Vertices in the matrix graph can be reordered without changing the graph itself. Direct methods are important because of their flexibility and robustness. They can achieve high accuracy and good numerical stability even for ill-conditioned systems. However, it has to be noticed that ill-conditioned system matrices can also influence the performance of direct methods. SPD system matrices, as resulting from many scientific applications, are advantageous for the solution process using direct methods. Matrices are usually not treatable in an efficient way if they do not offer the above mentioned property. This is especially important in the field of parallelization (refer to Chapter 8). As an alternative, a non-SPD matrix can be transformed to a SPD one by applying

$$
\boldsymbol{A}^{\mathrm{H}} \boldsymbol{A} \vec{x}=\boldsymbol{A}^{\mathrm{H}} \vec{b}
$$

which is known as the normal equation [147]. The resulting system of equations usually can be solved equivalently. Although the resulting system matrix $\boldsymbol{A}^{\mathrm{H}} \boldsymbol{A}$ is more dense than $\boldsymbol{A}$ and cond $\left(\boldsymbol{A}^{\mathrm{H}} \boldsymbol{A}\right)=$ cond $(\boldsymbol{A})^{2}$ [152], this technique can exploit certain advantageous for the solution process $^{2}$. The resulting system matrix is SPD and if $\boldsymbol{A}$ is square and non-singular, the solutions of (5.6) and (5.1) are identical. If $\boldsymbol{A}$ is non-square (the system is overdetermined), a unique solution can not be found in principle. Furthermore, system matrices resulting from real-life problems can reach dimensions exceeding the capabilities of sparse direct solvers. This is apparent, looking at Figure 5.3 where time and storage complexities are depicted depending on the DOF for the circuit simulation relevant range. This motivates the application of iterative techniques, intending
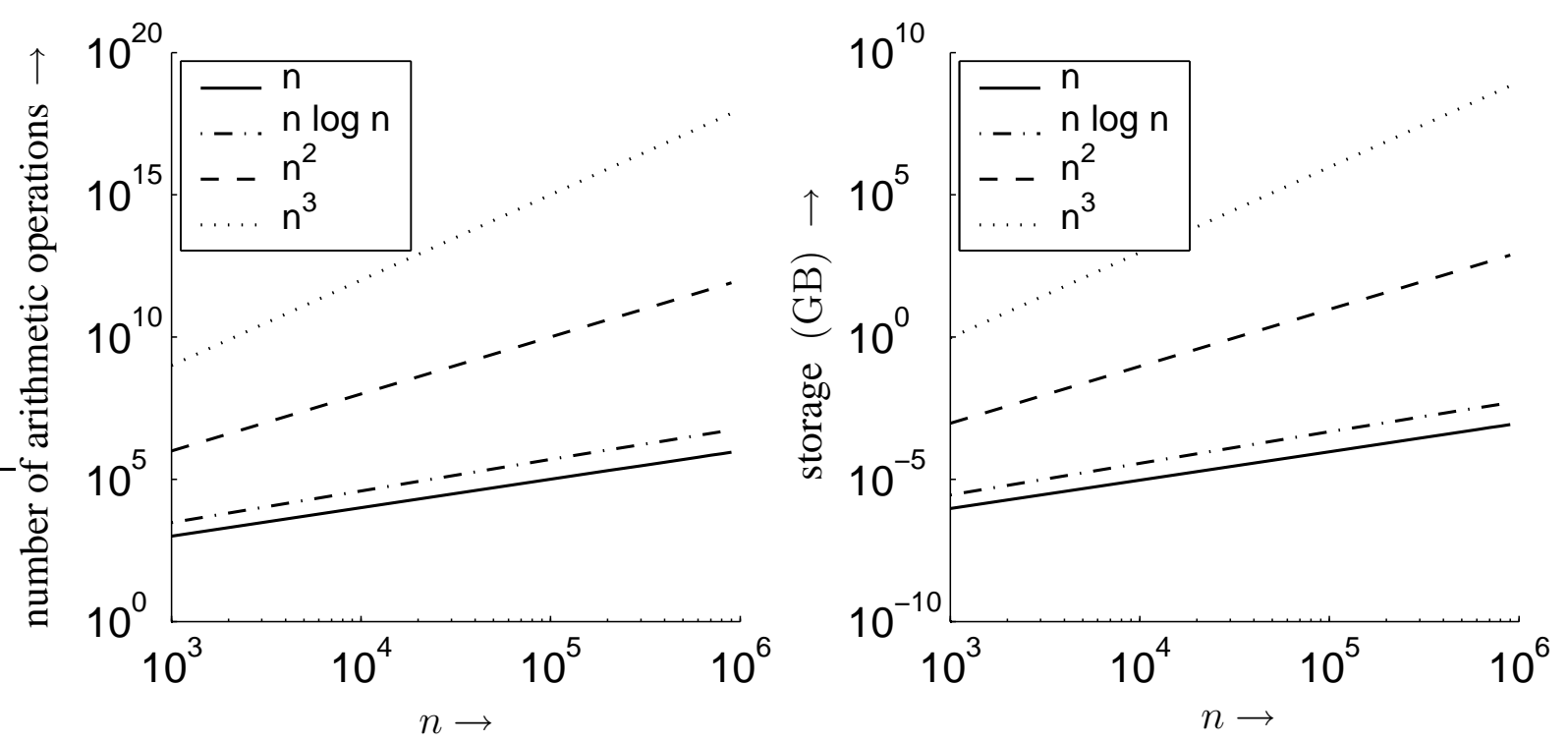

Fig. 5.3: Time and storage complexities of direct and iterative methods. The number of DOF $(n)$ represents the relevant range for circuit simulation purposes.

\footnotetext{
${ }^{2}$ From a geometric point of view, the solution of the normal equation can be interpreted as an extension of the search space, which makes the solving procedure more difficult at first glance.
} 
a completely different approach, compared to direct methods.

\subsubsection{Iterative Strategies}

Direct strategies compute the solution of the system of equations in one processing step. Iterative methods, however, apply several iteration steps to obtain a successive approximation to the solution. By adding a correction term $\vec{\delta}^{(k)}$ to the solution approximant $\vec{x}^{(k)}$ in the $k$ th iteration step, an improved solution $\vec{x}^{(k+1)} \triangleq \vec{x}^{(k)}+\vec{\delta}^{(k)}$ can be obtained for the following step. This procedure has to be applied until a predefined accuracy is reached. It is advantageous to use iterative methods together with sparse matrices, comprising a linear number of non-zero elements $n_{\mathrm{nz}}(\boldsymbol{A})$. Consequently, sparse matrices show storage requirements as well as execution times for one iteration step with a complexity of $\mathcal{O}(n)$. The execution time is substantially dominated by the arithmetic operations - matrix-vector multiplication and matrix-matrix addition. In general, structured sparse matrices and non-structured sparse matrices can be distinguished. Iterative algorithms originally were constructed only for real-valued systems. Each complex-valued system matrix $\boldsymbol{A} \in \mathbb{C}^{n \times n}$, however, can be transformed to an equivalent real-valued matrix $\boldsymbol{A}_{\mathrm{r}}$ by

$$
\boldsymbol{A}_{\mathrm{r}}=\left[\begin{array}{cc}
\Re\{\boldsymbol{A}\} & -\Im\{\boldsymbol{A}\} \\
\Im\{\boldsymbol{A}\} & \Re\{\boldsymbol{A}\}
\end{array}\right] \in \mathbb{R}^{2 n \times 2 n}
$$

with double dimension compared to the original system [126, 147]. At first glance, the systems solution based on the resulting equation system $\boldsymbol{A}_{\mathrm{r}} \vec{x}_{\mathrm{r}}=\vec{b}_{\mathrm{r}}$ is identical to the solution of (5.1). Nevertheless, the convergence behavior of the solution process can differ because of the specific difference in the characters of $\boldsymbol{A}$ and its real-valued equivalent. In circuit simulation, system matrices usually are very ill-conditioned leading to a poor convergence behavior. Therefore, an application of suitable preconditioning techniques is inevitable. The preconditioned system matrix should approximate the identity matrix as closely as possible. Numerical instabilities can limit the performance of direct methods similar to iterative methods. Based on the underlying matrix properties, however, iterative techniques can achieve faster convergence compared to the direct solution process. The spectral properties of the system matrix essentially effects the convergence behavior of iterative methods. A good performance can be obtained if the condition number is small and the eigenvalue distribution in the complex plane shows few clusters, far away from the origin.

\subsection{Iterative Solution of Linear Systems of Equations}

W.r.t. an efficient and accurate simulation process for circuit-level analysis, iterative methods are expected to embody the best choice. The basic principle of iterative methods as well as concrete suggestions and approaches are discussed in the remainder of this chapter. This is necessary to provide the mathematical background for a deeper understanding of advanced solving strategies including AMG or hierachical matrices.

\subsubsection{Basic Iterative Methods}

To obtain an iterative solution of (5.1), a conversion to so-called fixed-point form

$$
\vec{x}=\boldsymbol{Y} \vec{x}+\vec{y}
$$


with a square matrix $\boldsymbol{Y}$ of dimension $n \times n$ and vectors $\vec{x}, \vec{y}$, both of length $n$ is meaningful. Based on the start approximation $\vec{x}^{(0)}$, the iteration rule

$$
\vec{x}^{(k+1)}=\boldsymbol{Y} \vec{x}^{(k)}+\vec{y}, \quad k=0,1,2, \ldots
$$

with discrete iteration steps $k$ can be applied. Compared to the original equation system, a simpler solution of the form $\boldsymbol{Q} \vec{x}=\vec{f}$ can be obtained by introducing a matrix $\boldsymbol{Q}$. Consequently, (5.1) can be interpreted as

$$
(\boldsymbol{Q}-\boldsymbol{Q}+\boldsymbol{A}) \vec{x}=\vec{b}
$$

or

$$
\mathbf{Q} \vec{x}=\mathbf{N} \vec{x}+\vec{b}
$$

respectively, with $N=\boldsymbol{Q}-\boldsymbol{A}$ and $\boldsymbol{Q}$ is assumed to be non-singular. The resulting recursion formula

$$
\boldsymbol{Q} \vec{x}^{(k+1)}=\boldsymbol{N} \vec{x}^{(k)}+\vec{b}
$$

can be converted to fixed-point form (5.9) with the so-called iteration matrix $\boldsymbol{Y}$. Per definition, for the iteration matrix and the right-hand side vector $\vec{y}$

$$
\begin{gathered}
\boldsymbol{Y}=\boldsymbol{Q}^{-1} \boldsymbol{N}=\left(\mathbf{1}-\boldsymbol{Q}^{-1} \boldsymbol{A}\right) \\
\vec{y}=\boldsymbol{Q}^{-1} \vec{b}
\end{gathered}
$$

holds. The iteration method resulting from (5.9) is called consistent, if the exact solution $\vec{x}^{(\infty)}$ is a fixed point [153]. Introducing a damping parameter $\omega$ by

$$
\begin{aligned}
& \hat{\vec{x}} \triangleq \boldsymbol{Y} \vec{x}^{(k)}+\boldsymbol{Q}^{-1} \vec{b} \\
& \vec{x}^{(k+1)} \stackrel{\stackrel{(5.15)}{=}}{\stackrel{\omega}{\hat{x}}+(1-\omega) \vec{x}^{(k)}} \\
&=\hat{\boldsymbol{Y}} \vec{x}^{(k)}+\omega \boldsymbol{Q}^{-1} \vec{b},
\end{aligned}
$$

it is possible to damp the high-frequency error components to obtain a better approximative solution. Starting from (5.12), the error of the iterative algorithm in step $k$ can be computed by

$$
\vec{e}^{(k)}=\vec{x}^{(k)}-\vec{x}^{*}
$$

assuming an exact solution $\vec{x}^{*}$, or

$$
\vec{e}^{(k+1)}=\boldsymbol{Y} \vec{e}^{(k)},
$$

respectively. The so-called residual in step $k$, therefore results in

$$
\vec{r}^{(k)} \triangleq \vec{b}-\boldsymbol{A} \vec{x}^{(k)} \stackrel{(5.18)}{=}-\boldsymbol{A} \vec{e}^{(k)}
$$

so that

$$
\vec{r}^{(k+1)} \stackrel{(5.19)}{=} \boldsymbol{A} \boldsymbol{Y} \boldsymbol{A}^{-1} \vec{r}^{(k)}
$$

follows. The normalized residual

$$
\frac{\left\|\vec{b}-\boldsymbol{A} \vec{x}^{(k)}\right\|}{\|\vec{b}\|} \leqslant \varepsilon
$$


often serves as a criterion for aborting the iteration process. Together with $\vec{e}^{(k)}=\boldsymbol{Y}^{k} \vec{e}^{(0)}$, it follows ${ }^{3}$

$$
\left\|\vec{e}^{(k)}\right\| \leqslant\left\|\boldsymbol{Y}^{k}\right\|\left\|\vec{e}^{(0)}\right\|
$$

for an arbitrary matrix norm $\|\cdot\|$, and

$$
\left\|\boldsymbol{Y}^{k}\right\|=\sup _{\vec{x} \neq \overrightarrow{0}}\left\{\frac{\left\|\boldsymbol{Y}^{k} \vec{x}\right\|}{\|\vec{x}\|}\right\} .
$$

In the case of

$$
\lim _{k \rightarrow \infty}\left\|\boldsymbol{Y}^{k}\right\|=0
$$

the iterative method is called convergent with $\vec{e} \rightarrow \overrightarrow{0}$ for $k \rightarrow \infty$. Reformatting (5.9) w.r.t. $[146,154]$, the iteration rule for a simple iteration method can be written as

$$
\begin{aligned}
\vec{x}^{(k+1)}= & \boldsymbol{Y} \vec{x}^{(k)}+\boldsymbol{Q}^{-1} \vec{b} \\
& \vdots \\
= & \vec{x}^{(k)}-\boldsymbol{Q}^{-1}\left(\vec{b}-\boldsymbol{A} \vec{x}^{(k)}\right), \quad k=1,2, \ldots
\end{aligned}
$$

It is well known that iteration schemes of the form (5.26) with start approximation $\vec{x}^{(0)}$ converge to the exact solution of (5.1), if the spectral radius $\rho(\boldsymbol{Y})$ (refer to (A.15) in Appendix A.2) is smaller than one for the iteration matrix $\boldsymbol{Y}=\mathbf{1}-\boldsymbol{Q}^{-1} \boldsymbol{A}$. Matrix $\boldsymbol{A}$ is assumed to be splitted into the parts $\boldsymbol{A}=\boldsymbol{D}-\boldsymbol{L}-\boldsymbol{U}$ with the lower $(\boldsymbol{L})$ and upper $(\boldsymbol{U})$ triangular parts and diagonal matrix $D$ containing the main diagonal [147]. Variants of simple iterative methods can be defined as for example the Jacobi method $(\boldsymbol{Q} \triangleq \boldsymbol{D})$ or the Gau $\beta$-Seidel method $(\boldsymbol{Q} \triangleq \boldsymbol{D}-\boldsymbol{L})$ simply by modifying matrix $\boldsymbol{Q}$. A frequently applied start approximation is $\vec{x}^{(0)}=\overrightarrow{0}$. An evaluation of such standard iteration methods for circuit-based simulation problems shows, that the solution process converges very slowly or a convergence can not be achieved at all. However, such methods can be advantageous as a part of an algebraic multigrid strategy (refer to Chapter 7). For a deeper understanding of simple iterative methods refer to $[147,153]$.

\subsubsection{Subspace Methods}

Krylov-subspace methods minimize the error over the affine space $\vec{x}^{(0)}+\mathcal{K}^{k}$ in the $k$ th iteration step, with

$$
\mathcal{K}^{k}\left(\boldsymbol{A}, \vec{r}^{(0)}\right)=\operatorname{span}\left\{\vec{r}^{(0)}, \boldsymbol{A} \vec{r}^{(0)}, \boldsymbol{A}^{2} \vec{r}^{(0)}, \ldots, \boldsymbol{A}^{k-1} \vec{r}^{(0)}\right\}
$$

representing the $k$-dimensional Krylov subspace of $\boldsymbol{A}$ and a start residual $\vec{r}^{(0)}=\vec{b}-\boldsymbol{A} \vec{x}^{(0)}$ for an initial guess $\vec{x}^{(0)}$. In each step, an orthogonal basis for this Krylov subspace will be constructed and extended for step $k+1$ to find the optimal solution $\vec{x}^{(k)} \in \mathcal{K}^{k}\left(\boldsymbol{A}, \vec{r}^{(0)}\right)$. For that reason $\mathcal{K}^{k}\left(\boldsymbol{A}, \vec{r}^{(0)}\right)$ is also called the "search space". Krylov-subspace methods fundamentally can be divided into the four variants - Ritz-Galerkin approach, minimum residual approach, Petrov-Galerkin approach and minimum error approach. For further details refer to [152]. The minimal residual Krylov-

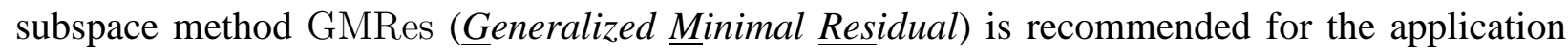

\footnotetext{
${ }^{3}$ Remark: The index with $\boldsymbol{Y}$ represents an exponent!
} 
in circuit simulation approaches. The residual $\vec{r}^{(k)}=\boldsymbol{A} \vec{e}^{(k)}=\vec{r}^{(0)}-\boldsymbol{A} \vec{x}^{(k)}$ can be used as an evaluation criterion for the optimal solution

$$
\vec{x}^{(k)}=\underset{\vec{x}^{(k)} \in \mathcal{K}^{k}\left(\boldsymbol{A}, \vec{r}^{(0)}\right)}{\arg \min }\left\|\vec{r}^{(0)}-\boldsymbol{A} \vec{x}^{(k)}\right\|
$$

since the error $\vec{e}^{(k)}=\vec{x}^{(k)}-\vec{x}^{*}$ is unknown. A successive expansion of the search space will be applied, until a given accuracy $\varepsilon$ is reached. The normalized residual (5.22) in the $k$ th iteration step is a common bound and serves as stopping criterion. While the original CG (Conjugate Gradient) strategy is only efficient for symmetric matrices, its successor BiCG ( $\underline{B} \underline{\text { Conjugate }}$ Gradient), or

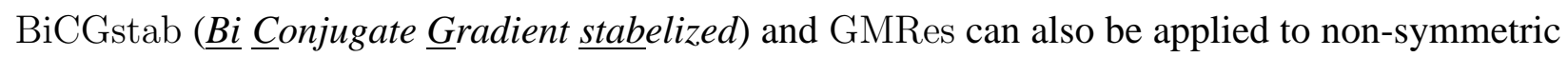
matrices, as arising from the MNA formulation.

\subsection{Preconditioning}

The convergence behavior of iterative methods strongly depends on the spectral properties of the system matrix. Especially matrices resulting from circuit simulation approaches exhibit very large condition numbers. Therefore, an adequate convergence speed and predefined accuracy usually can only be achieved applying suitable preconditioning [3, 155]. The objective of preconditioning is to find a suitable linear operator $\boldsymbol{M}$, well approximating the system matrix $\boldsymbol{A}$ [152]. Using this preconditioner, the system $M \vec{y}=\vec{z}$ should be much simpler to solve than (5.1), while for the setup of $\boldsymbol{M}$ only small cost should be necessary. The application of preconditioning can improve the efficiency of iterative solvers as well as their robustness. Usually, the solutions accuracy depends on the quality of the preconditioner rather than on the iterative solution method itself. By applying the preconditioner $\boldsymbol{M}$, improved spectral properties of the underlying linear system can be obtained. The resulting transformed system

$$
\boldsymbol{M}^{-1} \boldsymbol{A} \vec{x}=\boldsymbol{M}^{-1} \vec{b}
$$

has the identical solution compared to the original system, while showing improved spectral properties of the iteration matrix $\boldsymbol{M}^{-1} \boldsymbol{A}$. In general,

$$
\operatorname{cond}\left(\boldsymbol{M}^{-1} \boldsymbol{A}\right) \ll \operatorname{cond}(\boldsymbol{A})
$$

should hold ${ }^{4}$. Furthermore, preconditioning should also avoid clustering of the eigenvalues of $\boldsymbol{M}^{-1} \boldsymbol{A}$ compared to those of $\boldsymbol{A}$, far away from the origin. Possible variants of preconditioning are the right-preconditioning

$$
\boldsymbol{A} \boldsymbol{M}^{-1} \vec{u}=\vec{b}, \quad \vec{x}=\boldsymbol{M}^{-1} \vec{u}
$$

the left-preconditioning

$$
\boldsymbol{M}^{-1} \boldsymbol{A} \vec{x}=\boldsymbol{M}^{-1} \vec{b}
$$

and the split-preconditioning with $\boldsymbol{M}=\boldsymbol{L} \boldsymbol{U}$ and

$$
\boldsymbol{L}^{-1} \boldsymbol{A} \boldsymbol{U}^{-1} \vec{u}=\boldsymbol{L}^{-1} \vec{b}, \quad \vec{x}=\boldsymbol{U}^{-1} \vec{u} .
$$

\footnotetext{
${ }^{4}$ It is advantageous if the density of $\boldsymbol{M}$ does not exceed the density of $\boldsymbol{A}$ [148].
} 
Where right- and left-preconditioning usually lead to a non-symmetric system matrix, symmetry can be achieved applying factorized preconditioning as shown in (5.33). In the case of symmetric matrices, a Cholesky-decomposition of the form $\boldsymbol{M}=\boldsymbol{L} \boldsymbol{L}^{\top}$ can be applied to reduce the necessary memory requirement by half. The spectral distributions of matrices $\boldsymbol{M}^{-1} \boldsymbol{A}, \boldsymbol{A} \boldsymbol{M}^{-1}$ and $\boldsymbol{L}^{-1} \boldsymbol{A} \boldsymbol{U}^{-1}$ are identical. Generally, the matrix $\boldsymbol{M}^{-1} \boldsymbol{A}$ is not inevitably symmetric or definite, even if $\boldsymbol{M}$ and $\boldsymbol{A}$ are. An evaluation of the effectivity of a preconditioner $\boldsymbol{M}$ is possible, considering the condition number of $\boldsymbol{M}^{-1} \boldsymbol{A}$ and the clustering of the eigenvalues. For efficiency reasons, an explicit computation of $M$ and $M^{-1}$ should be avoided. A tradeoff between the cost of construction (setup) and application compared to the gain in convergence speed has to be taken into account. Furthermore, a good parallelization of the preconditioner's construction and its application is desirable. On parallel machines, the efficiency of the preconditioner in the classical sense and its parallel efficiency have to be considered carefully. A parallel implementation of preconditioning for unstructured matrices can cause problems. It can be advantageous to use the approximate inverse of a given system matrix [126].

\subsection{Implementation Issues}

The mentioned properties of the resulting equation systems (Section 5.1) carefully have to be taken into account for an application of iterative solvers in a circuit simulation context. Direct linear solvers are - especially in the case of ill-conditioned systems - more or less good "approximative LU-decompositions". Consequently, the "solution" might only be a bad approximation, i.e. showing large residuals. Post iteration steps can often be necessary to increase the accuracy. Available iterative solver packages usually are developed for real-valued matrices. In circuit simulation, however, FD analysis leads to complex-valued systems, while the matrices in TD analysis are real-valued. Therefore, a transformation according to (5.7) has to be supported. Iterative solvers, suitable for circuit simulation matrices are ILUT(lfil,droptol)-type GMRes and ILUT(lfil,droptol)-type BiCGstab. The preconditioner construction strongly depends on the appearance and condition of the underlying system matrix. Influencing factors are the simulation domain (TD/FD), the network formulation (NA/MNA) and whether the PEEC model results from an inductance or reluctance approach. It has to be remarked, that MNA-based systems are unsymmetric and therefore need the application of a GMRes or BiCGstab approach. NA-based systems, however, are symmetric and therefore allow the application of a conventional CG approach. Suitable preconditioners are variants of incomplete LU-decompositions or sparse approximate inverse (SAI) preconditioners. It can be shown, that the spectral properties of the system matrices in a simulation process only slightly vary from one simulation step to another. This holds for FD analysis as well as for TD analysis. As a consequence, it is sufficient to recompute only the preconditioner if the convergence behavior becomes too bad. In the case of $\mathcal{H}$-matrix-based iterative solution methods, numerical results for an adaptive preconditioning and the achievable speed-up are given in Section 6.3.2.

\subsection{Sparse Matrix Storage Formats}

It is meaningful to distinguish between dense and sparse matrices depending on how they can be stored effectively. In order to take advantage of the sparsity of system matrices in a linear solver 
framework, an efficient sparse matrix storage format is necessary. All schemes for storing a sparse matrix employ the same basic technique. Linear arrays are used to store non-zero elements and position indices to locate the elements in the original matrix [156]. With all storage schemes one has to pay attention to the fact, that a matrix-vector multiplication can be carried out fast and efficient. The use of symmetric matrices can help to further reduce the storage demands. A very simple and well-known storage scheme for sparse matrices is the so-called coordinate format [147]. Three or four arrays for real- and complex-valued matrices, respectively, store the number of non-zero elements $n_{\mathrm{nz}}$ and row and column indices of the positions in the matrix, which can be ordered arbitrarily. One of the position index arrays may contain redundant information if the elements are listed, sorted by row or column. Another problem is to locate an additional row/column without scanning the whole list. One advantage of the coordinate format is the possibility to extend the list dynamically. Compared to a square real full matrix with storage requirements $\mathcal{N}_{\text {st,full }}=n^{2} \operatorname{sizeof}($ double $)+\operatorname{sizeof}($ int $)$ for the matrix elements and the dimension of the matrix, we only need

$$
\mathcal{N}_{\text {st,cf }}=n_{\text {nz }}(\operatorname{sizeof}(\text { double })+2 \operatorname{sizeof}(\text { int }))+\operatorname{sizeof}(\text { int })
$$

for a matrix stored in the coordinate format. The most general and probably the most popular format for storing general sparse matrices is called the compressed sparse row/column (CS $\underline{\mathrm{R}} / \mathrm{CS} \underline{\mathrm{C}}$ ) storage format ${ }^{5}$. Where in CSR the elements are stored row wise, in CSC the elements are stored column wise ${ }^{6}$. To store a matrix in CSR (CSC) three arrays are needed:

- double a[] real-valued or complex-valued array for storing the non-zero elements row (column) wise;

- int ia[]

column (row) index of each non-zero element in a;

- int ja[] start index with regard to a, ia of first non-zero element in each row (column) ${ }^{7}$.

The formats make absolutely no assumptions about the sparsity structure of the matrix and realize a very efficient storage. The CSR/CSC format is usually more advantageous for iterative methods because it is more efficient for typical arithmetic computations [147]. Using this strategy, the storage requirements from (5.34) can be further reduced to

$$
\mathcal{N}_{\text {st }, \mathrm{CSR} / \mathrm{CSC}}=n_{\mathrm{nz}}(\operatorname{sizeof}(\text { double })+\operatorname{sizeof}(\mathrm{int}))+(n+1) \operatorname{sizeof}(\mathrm{int})
$$

with the dimension $n$ of the considered matrix. If the matrix is symmetric, only the upper (or lower) triangular part of the matrix has to be stored. Example 5.1 illustrates the characteristic properties of the described formats [156].

\footnotetext{
${ }^{5}$ The CSR format is also known as Harwell-Boeing matrix format [157].

${ }^{6}$ Consequently, the CSC format is equivalent to the CSR format for the transposed matrix.

${ }^{7}$ The last element in ja is $n_{\mathrm{nz}}+1$ per definition (refer to Example 5.1).
} 
Example 5.1 Sparse matrix storage formats for iterative methods

Original, real-valued sparse matrix of dimensions $5 \times 5$

$$
\boldsymbol{A}=\left[\begin{array}{ccccc}
1 & -1 & 0 & -3 & 0 \\
-2 & 5 & 0 & 0 & 0 \\
0 & 0 & 4 & 6 & 4 \\
-4 & 0 & 2 & 7 & 0 \\
0 & 8 & 0 & 0 & -5
\end{array}\right]
$$

1. Coordinate sparse matrix format description

$$
\begin{aligned}
\text { values } & =\left[\begin{array}{llllllllllllll}
1 & -1 & -3 & -2 & 5 & 4 & 6 & 4 & -4 & 2 & 7 & 8 & -5
\end{array}\right] \\
\text { row_idx } & =\left[\begin{array}{lllllllllllll}
1 & 1 & 1 & 2 & 2 & 3 & 3 & 3 & 4 & 4 & 4 & 5 & 5
\end{array}\right] \\
\text { col_idx } & =\left[\begin{array}{lllllllllllll}
1 & 2 & 3 & 1 & 2 & 3 & 4 & 5 & 1 & 3 & 4 & 2 & 5
\end{array}\right]
\end{aligned}
$$

2. Compressed sparse row format description

$$
\begin{aligned}
\mathrm{a} & =\left[\begin{array}{llllllllllllll}
1 & -1 & -3 & -2 & 5 & 4 & 6 & 4 & -4 & 2 & 7 & 8 & -5
\end{array}\right] \\
\mathrm{ia} & =\left[\begin{array}{lllllllllllll}
1 & 2 & 4 & 1 & 2 & 3 & 4 & 5 & 1 & 3 & 4 & 2 & 5
\end{array}\right] \\
\mathrm{ja} & =\left[\begin{array}{lllllll}
1 & 4 & 6 & 9 & 12 & 14
\end{array}\right]
\end{aligned}
$$

3. Compressed sparse column format description

$$
\begin{aligned}
\mathrm{a} & =\left[\begin{array}{llllllllllllll}
1 & -2 & -4 & -1 & 5 & 8 & 4 & 2 & -3 & 6 & 7 & 4 & -5
\end{array}\right] \\
\mathrm{i} a & =\left[\begin{array}{lllllllllllll}
1 & 2 & 4 & 1 & 2 & 3 & 4 & 5 & 1 & 3 & 4 & 2 & 5
\end{array}\right] \\
\mathrm{ja} & =\left[\begin{array}{lllllll}
1 & 4 & 7 & 9 & 12 & 14
\end{array}\right]
\end{aligned}
$$

The CSR/CSC sparse matrix format enables a fast row/column oriented access to the matrix entries. Thus, the matrix-vector multiplication as the dominant arithmetic operation in iterative solution algorithms, can significantly be accelerated. Dependent on the individual software library specific variations of the described original storage formats are possible. In the remainder of this section a modification of the CSR format is presented, necessary for the implementation of the software library SAMG in Chapter 7. Storage formats as diagonal storage schemes or skyline storage schemes [147] further can take advantage of special structures of the underlying matrix. However, the general applicability is restricted. For further details refer to [156].

SAMG's CSR For the SAMG library [28], the entries of matrix $\boldsymbol{A} \in \mathbb{R}^{n \times n}$ are assumed to be stored in a modified CSR format consisting of three vectors. Vector a $\left(1: n_{\mathrm{nz}}\right)$ contains $n_{\mathrm{nz}}$ non-zero matrix entries stored row wise, each row starting with the diagonal element. The corresponding column indices are stored in vector $\mathrm{ja}\left(1: n_{\mathrm{nz}}\right)$. The $i$ th element in ia $(1: n)$ stores the beginning of row $i$ in a and ja, defining ia $(n+1)=n_{\mathrm{nz}}+1$. Diagonal elements have to be stored independently of whether they are zero or not and are stored first within a row (i.e. ja $(\mathrm{ia}(i))=i$. The off-diagonal entries can be stored in arbitrary order within a row. With the number of unknowns $n$, the non-zero entries of the $i$ th row $(1 \leqslant i \leqslant n)$ are stored in a $(j)$ with

$$
\mathrm{ia}(i) \leqslant j \leqslant \mathrm{ia}(i+1)-1 \text {. }
$$


Note that $\mathrm{ia}(1)=1$ and $\mathrm{ia}(n+1)=n_{\mathrm{nz}}+1$ [158]. To enable a correct link between elements of array a and index arrays ia and ja, some conventions must be fulfilled. With the diagonal entry $\mathrm{a}(\mathrm{ia}(i))$ of row $i$ and the pointer vector ja the value of $\mathrm{a}(j)$ has to correspond to the solution variable $x(\mathrm{ja}(j))$. Consequently, together with a $(j)=A(i, \mathrm{ja}(j))$ the $i$ th equation of (5.1) reads

$$
\sum_{\mathrm{ia}(i) \leqslant j \leqslant \mathrm{ia}(i+1)-1} \mathrm{a}(j) x(\mathrm{ja}(j))=b(i) .
$$

An example can be found in [158].

\subsection{Further Improvements}

Circuit simulations often suffer from a lack of robustness and efficiency though applying highlysophisticated preconditioned iterative solvers. Small diagonal entries can cause problems in the construction of the ILU factors. This can lead to a drastically decreased convergence behavior and unstable preconditioning [159]. Furthermore, the convergence of preconditioned Krylov-subspace methods can also be affected by off-diagonal values of the coefficient matrix which are several orders of magnitude larger than the diagonal entries as this is often the case in circuit simulation. The robustness of direct as well as iterative solvers can be improved by applying reordering strategies (rCMK, METIS-like reordering, maximizing of diagonal elements) and dynamical pivoting for ILUT decomposition/MILUTP or pre-Gauß elimination steps. The goal in pivoting is, to transform the matrix $\boldsymbol{A}$ with diagonal scaling matrices $\boldsymbol{D}_{r}$ and $\boldsymbol{D}_{c}$ and a permutation matrix $\boldsymbol{P}_{r}$ so as to obtain an equivalent system with a matrix $\boldsymbol{P}_{r} \boldsymbol{D}_{r} \boldsymbol{A} \boldsymbol{D}_{c}$ that is better scaled and more diagonally dominant [159]. The pivoting corresponds to a permutation of rows and columns of the coefficient matrix. A symmetric permutation results in a reordering of the vertices in the matrix graph without changing the graph itself. Especially for FD simulations in higher frequency ranges, clustering effects of matrix eigenvalues lead to a decreased convergence rate which can be avoided by using robust ILU-type preconditioners. In general, matrix factorizations produce fill-ins, leading to enormous memory demands and degraded computational speed. METIS, as mentioned in Section 5.1.1, can help to avoid this drawback. Furthermore, graph partitioning is often used for load balancing in parallel computing [160]. 


\section{6}

\section{Hierarchical Matrices Based Solvers for PEEC}

$\boldsymbol{U}$ tilizing iterative methods from Chapter 5 to obtain an accurate solution of $\boldsymbol{A} \vec{x}=\vec{b}$ inevitably leads to square complexity w.r.t. CPU time requirements even for sparse matrices. In the context of BEM-based PEEC matrices the intention of this chapter is, to realize iterative solutions with almost linear complexity w.r.t. CPU time and memory requirements for dense matrices. This can be accomplished by approximating the PEEC-based system matrix by a data-sparse $\mathcal{H}$-matrix, as described in Section 4.3. The solution of the equation system $\boldsymbol{A}_{\mathcal{H}} \tilde{\vec{x}}=\vec{b}$ must fulfill the accuracy condition ${ }^{1}\|\vec{x}-\tilde{\vec{x}}\| \leqslant \varepsilon$ for a given $\varepsilon>0$. Based on the formatted $\mathcal{H}$ matrix arithmetic [19], efficient preconditioning techniques can be realized. Implementation issues for an appropriate matrix setup and suitable preconditioning for an efficient PEEC-based circuit simulation have to be taken into account.

The following sections combine basics and cognitions from the previous chapters to realize an efficient solution process for circuit-based EMC simulation. Section 6.1 surveys the principle differences between direct and one-level iterative strategies for $\mathcal{H}$-matrices. Concrete implementation details concerning BEM-based circuit simulation are described in Section 6.2. Section 6.3 presents numerical results to validate the proposed strategies. In Section 6.4 some notes are given for the application of $\mathcal{H}$-matrices in the retarded case as a supplementary task.

\subsection{Overview}

To realize an efficient iterative solving process, special techniques are necessary to take the specific properties of dense BEM-based matrices into account. As mentioned in Section 5, direct methods

\footnotetext{
${ }^{1}$ The tilde denoting an approximation is dropped for simplification reasons in the following.
} 
are only appropriate for small problem sizes. Although common Krylov-subspace iterative methods are more effective for sparse matrices, they show a convergence behavior strongly depending on the condition of the system. Consequently, suitable preconditioning techniques are necessary to improve the convergence behavior of the solution process. An efficient preconditioning can be realized utilizing $\mathcal{H}$-matrix techniques. $\mathcal{H}$-matrices can be applied in a black-box manner without the need of an underlying grid hierarchy. The $\mathcal{H}$-matrix arithmetic enables the efficient computation of an approximate inverse for preconditioning purposes ${ }^{2}$. The aim is, to compute, store and apply an approximate inverse with almost linear complexity w.r.t. time and memory requirements. Considering TD analysis, a system with many right-hand sides has to be solved successively with the spectral properties of the system matrices only slightly varying from one step to another. A similar behavior can be observed together with retarded PEEC models in FD analysis where the coefficients of potential have to be recomputed or updated in each frequency step. Using conventional methods, a complexity of $\mathcal{O}\left(n_{s} n^{2}\right)$ for a number of $n_{s}$ simulation steps and dense matrix dimension $n$ [76] can be obtained. The complexity of the matrix-vector multiplication can be reduced to $\mathcal{O}\left(n_{s} n \log ^{2}(n)\right)$ applying a multilevel FMM approach. Where the nearfield interactions have to be computed with complexity $\mathcal{O}\left(n^{2}\right)$, the farfield can effectively be treated using multipole approaches. Hierarchical methods can be well suited also for original sparse matrices since the inverse of a sparse matrix is dense, in general. If the approximate inverse can be calculated with a prescribed accuracy, a bounded number of iterations of Krylov-subspace methods can be obtained [132]. For the following theoretical investigations a circuit simulation in the TD or the FD can be understood as the task to solve a sequence of systems of the form ${ }^{3}$ [132]

$$
\boldsymbol{A}_{N} \vec{x}_{N}=\vec{b}_{N}, \quad N=1, \ldots, n_{s}
$$

with an invertible system matrix $\boldsymbol{A}_{N} \in \mathbb{R}^{n \times n}$ and a number of simulation steps $n_{s}$.

\subsubsection{Direct Strategy}

Applying a specific $\mathcal{H}$ - $L U$-decomposition [144] $\boldsymbol{A} \approx \boldsymbol{L}_{\mathcal{H}} \boldsymbol{U}_{\mathcal{H}}$ of the system matrix, it is possible to realize an efficient direct solution

$$
\vec{x} \triangleq \boldsymbol{U}_{\mathcal{H}}^{-1} \boldsymbol{L}_{\mathcal{H}}^{-1} \vec{b}
$$

of the given equation system. A specific forward/backward substitution optimized for the $\mathcal{H}$-matrix arithmetic is assumed. A time complexity of $\mathcal{O}\left(n|\log \varepsilon|^{4} \log ^{2} n\right)$ for the setup of both factors severely limits the applicability of direct techniques [132]. Once the factors are computed, the system can be solved with complexity $\mathcal{O}\left(n|\log \varepsilon|^{4} \log n\right)$. For the case of varying right-hand sides and a fixed system matrix the total complexity can be reduced compared to $L U$-preconditioned iterative methods. The Cholesky-decomposition $\boldsymbol{A} \approx \boldsymbol{L}_{\mathcal{H}} \boldsymbol{L}_{\mathcal{H}}^{\top}$ with lower triangular $\mathcal{H}$-matrix $\boldsymbol{L}_{\mathcal{H}}$ can be applied if the system matrix is SPD, leading to reduced storage requirements.

\footnotetext{
${ }^{2}$ Conventional sparse approximate inverses are often costly to compute and suffer from a loss in accuracy [126].

${ }^{3}$ Remark: For circuit simulation purposes the system matrix $\boldsymbol{A}$ corresponds to the network matrix $\boldsymbol{Y}$ (refer to Appendix D).
} 


\subsubsection{Iterative Strategy}

The $\mathcal{H}-L U$ - as well as $\mathcal{H}$-Cholesky-decomposition can effectively be applied to realize an efficient $\mathcal{H}$-preconditioned iterative solution approach. Without sacrifying general validity the $\mathcal{H}$ $L U$-decomposition is considered in the following. With a preconditioner $\boldsymbol{M}=\boldsymbol{L}_{\mathcal{H}} \boldsymbol{U}_{\mathcal{H}}$, fulfilling

$$
\left\|\mathbf{1}-\boldsymbol{A} \boldsymbol{M}^{-1}\right\| \leqslant \delta<1
$$

together with $\left\|\boldsymbol{A} \boldsymbol{M}^{-1}\right\| \leqslant\|\mathbf{1}\|+\left\|\mathbf{1}-\boldsymbol{A} \boldsymbol{M}^{-1}\right\| \leqslant 1+\delta$ and applying

$$
\left\|\left(\boldsymbol{A} \boldsymbol{M}^{-1}\right)^{-1}\right\| \leqslant \sum_{j=0}^{\infty}\left\|\mathbf{1}-\boldsymbol{A} \boldsymbol{M}^{-1}\right\|^{j}=\frac{1}{1-\delta},
$$

a bound for the condition number of a Galerkin stiffness matrix can be derived as [132]

$$
\operatorname{cond}\left(\boldsymbol{A} \boldsymbol{M}^{-1}\right) \leqslant \frac{1+\delta}{1-\delta}
$$

Based on practical experience, a value $\delta \approx 0.1$ is sufficient for the construction of a preconditioner. Moreover, the choice of $\delta$ is independent from matrix dimension $n$ [144]. Compared to the computation of the $\mathcal{H}$-approximant for the system matrix with accuracy parameter $\varepsilon_{\mathcal{H}}$, it is adequate to choose $\varepsilon_{\mathcal{H} \text {,pre }} \gg \varepsilon_{\mathcal{H}}$ for the preconditioners accuracy. I.e. the $\mathcal{H}$ - $L U$-decomposition can be applied with much lower precision. For higher efficiency of the iterative solution algorithm the system matrix $\boldsymbol{A}$ should be copied and a recompression (e.g. via $\mathcal{H}^{2}$-matrix techniques) should be applied [144]. The resulting linear equation system in $\mathcal{H}$-notation can be given in the form

$$
\left(\boldsymbol{A}_{\mathcal{H}, \text { pre }}\right)^{-1} \boldsymbol{A}_{\mathcal{H}} \vec{x}=\left(\boldsymbol{A}_{\mathcal{H}, \text { pre }}\right)^{-1} \vec{b}
$$

Consequently, the iteration rule (5.26) can be applied with matrices in the $\mathcal{H}$-matrix format to improve the start approximation $\vec{x}^{(0)}$. The convergence rate is defined by the spectral radius of $1-$ $\left(\boldsymbol{A}_{\mathcal{H}, \text { pre }}\right)^{-1} \boldsymbol{A}_{\mathcal{H}}$. An upper bound for the spectral radius is given by the norm $\left\|\mathbf{1}-\left(\boldsymbol{A}_{\mathcal{H}, \text { pre }}\right)^{-1} \boldsymbol{A}_{\mathcal{H}}\right\|$ which should be $<1$ in accordance with (A.15) in Appendix A.2.2.

\subsection{Implementation Details for PEEC-Based Circuit Simula- tion}

For an efficient implementation basically two aspects are important - the fast and efficient generation of the system matrix in the hierarchical format and the efficient setup and application of the preconditioner. Usually, the construction and application of the preconditioner are the most complex parts in an iterative solving step. The principle structure of a PEEC-based circuit simulator (Figure 6.1) serves as a basis for fundamental investigations concerning the introduction of hierarchical matrix techniques. A conventional stamping procedure including PEEC models and optional subcircuits realizes the setup of the network matrix. The simulation control interpretes the simulation options contained in the circuit file and initiates and supervises the type of simulation. The resulting system matrix can be given in fullmatrix or sparsematrix format. For an $\mathcal{H}-$ compression of the system matrix, position data referring to the DOF is necessary. As mentioned in Section 6.1.2 the preconditioner can be performed with $\varepsilon_{\mathcal{H}, \text { pre }} \gg \varepsilon_{\mathcal{H}}$. A choice $\varepsilon_{\mathcal{H}} \approx 10^{-6}$ and 
$\varepsilon_{\mathcal{H}, \text { pre }} \approx 10^{-2}$ shows good results for industrial relevant problems. An $\mathcal{H}$ - $L U$-preconditioning in combination with a GMRes or BiCGstab iterative solver leads to accurate solutions for circuit analysis. To account for possible convergence problems, a direct solver is provided as a fallback solution. Switching from the iterative to the direct solver is applied, controlled by means of the residual and the number of required iterations. The explicit construction of the equation system (containing the right-hand side vector $\vec{b}$ ) is performed through a hidden setup stage (refer to Section 3.6). The circuit sources specified in the circuit file are implemented in $\vec{b}$. A provided background memory stores the results which are important for future simulation steps.

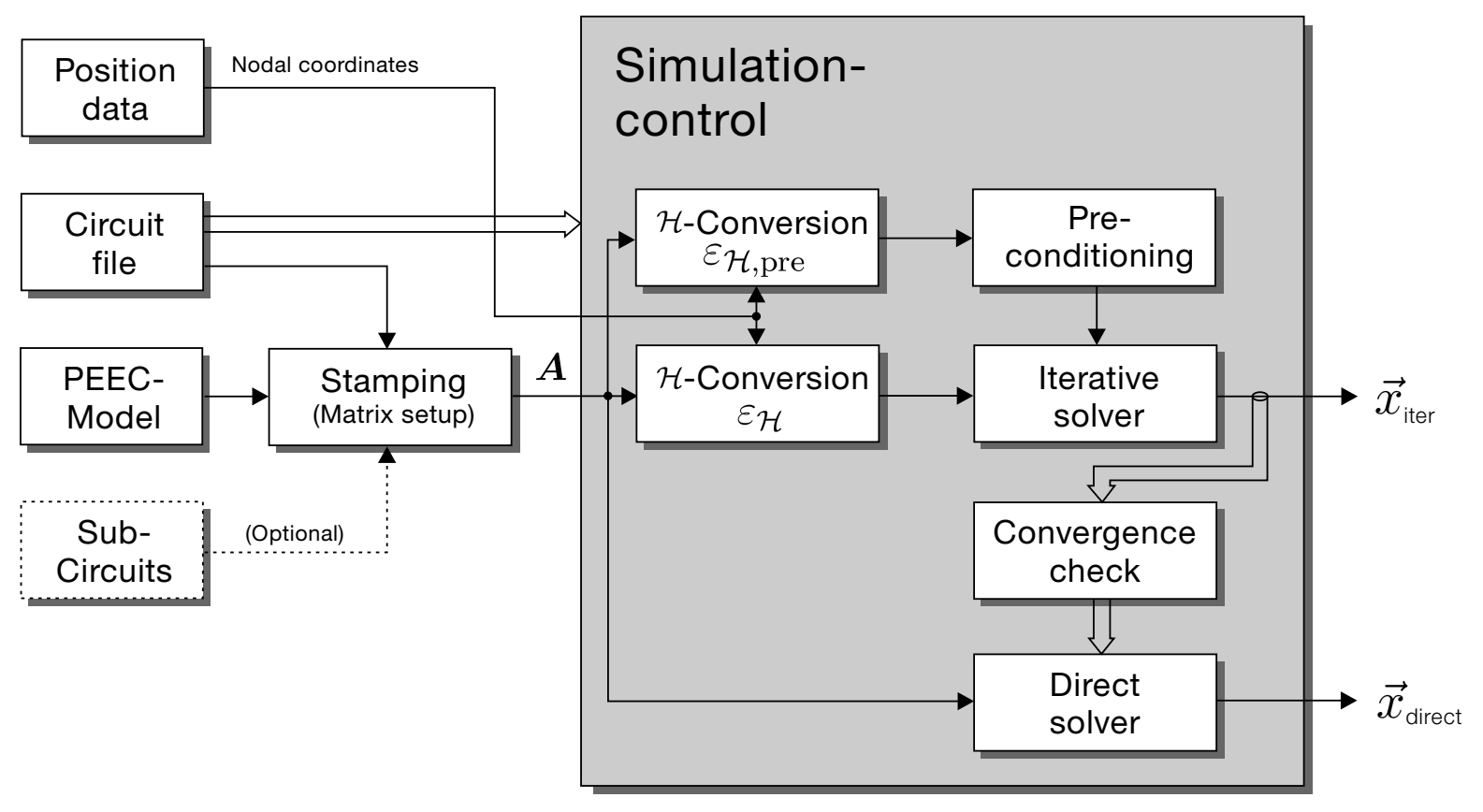

Fig. 6.1: Principle structure of an $\mathcal{H}$-matrix-based circuit simulator with one-level iterative solver. A direct solver is provides as a fallback solution. The approximate solution is denoted by $\vec{x}$.

\subsection{1 $\mathcal{H}$-Stamping}

A "stamping" of circuit elements (refer to Appendix D) has to be enabled for $\mathcal{H}$-matrices to realize an efficient setup of the system matrix. At any time it must be avoided to hold a dense matrix in cache. The parasitics matrices $(\boldsymbol{L}, \boldsymbol{C})$ from PEEC models or optional subcircuits have to be provided in a data-sparse manner. For implementation an empty $\mathcal{H}$-matrix has to be constructed a priori. The structure can be derived from the geometric grid data (position data) already prior to the filling. Each block $\boldsymbol{M}_{b}$ of an $\mathcal{H}$-matrix can be extracted independently from the remaining blocks. The admissible blocks can be assumed to be in low-rank format (refer to Figure 6.2). For inadmissible blocks (full blocks) an introduction of supplementary elements can be accomplished by addition in a straightforward manner since no reallocation is necessary. Inserting single elements in a low-rank block can be realized by an $\mathcal{R}_{1}$-matrix stamp (Figure 6.2). After selection, the memory of the corresponding block in the $\mathcal{H}$-matrix will be reallocated (rank-increment by one) if the actual rank $k t$ in the block is smaller than the maximum rank $k$. In a next step, the element (parasitic value) will be described as an $\mathcal{R}_{1}$-matrix, implying a representation by means of two vectors $\vec{a} \in \mathbb{R}^{n}, \vec{b} \in \mathbb{R}^{m}$. While $\vec{a}$ contains the element 1 at position index $i$, vector $\vec{b}$ 


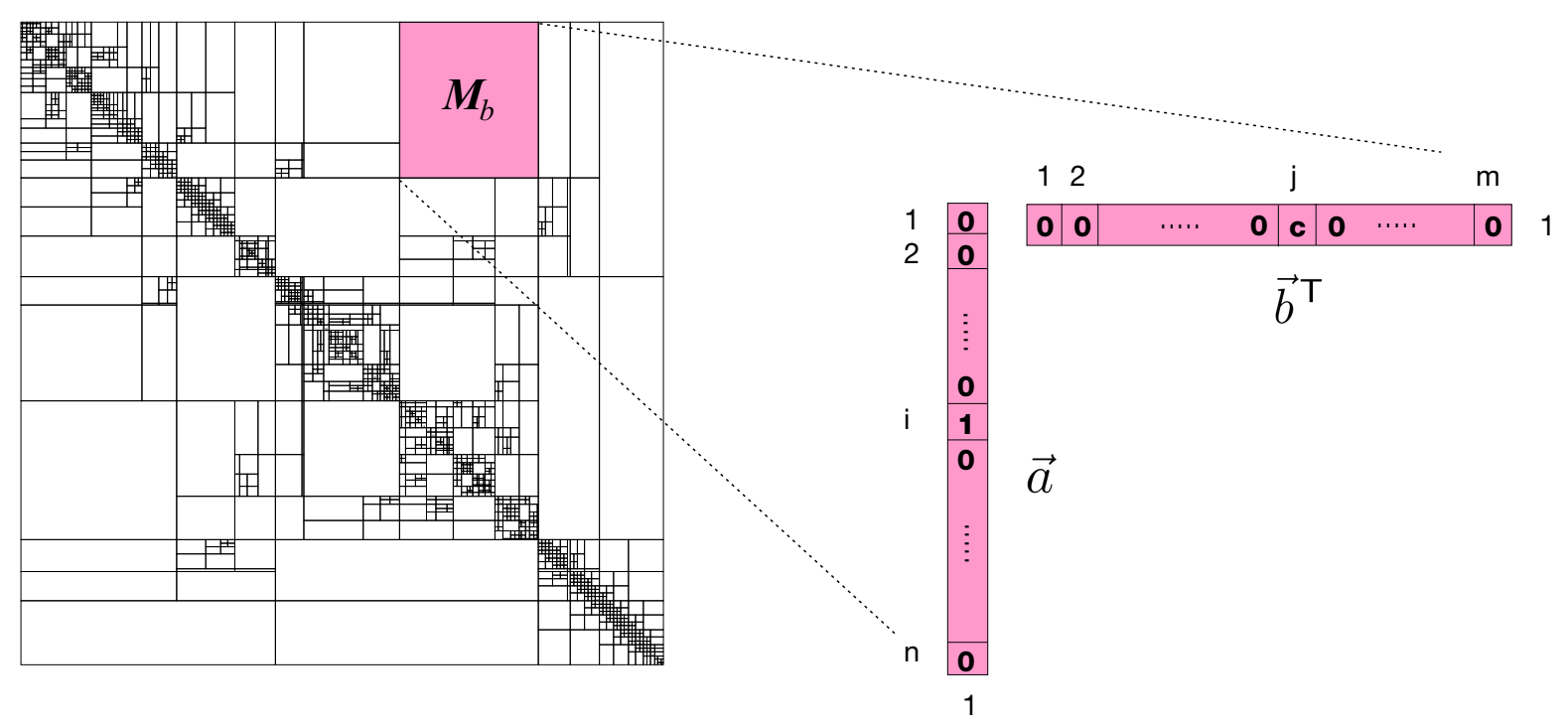

Fig. 6.2: Selected low-rank block $M_{b}$ and the representation of the $\mathcal{R}_{1}$-matrix-stamp for stamping element $c$.

contains the stamp $c$ at position index $j$. The remaining elements in both vectors are zero. An addition of the stamp can be accomplished in low-rank arithmetic (refer to Section 4.3), as depicted in Figure 6.3. The proposed procedure, however, is only efficient if the number of elements to be

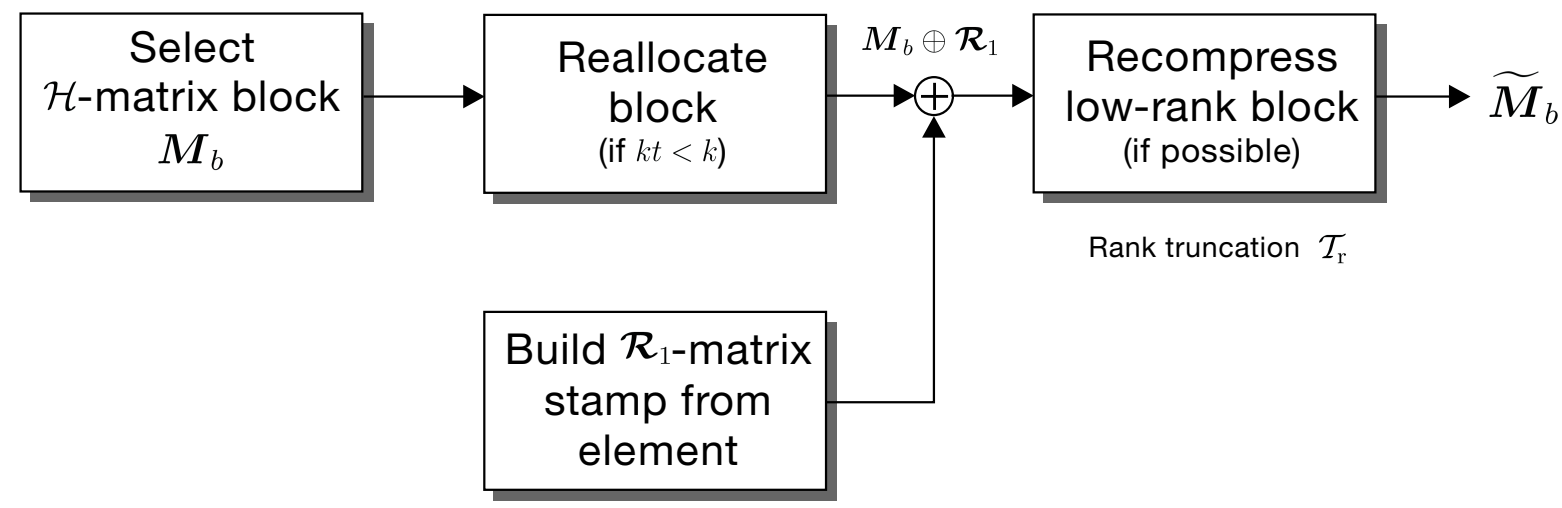

Fig. 6.3: $\mathcal{H}$-stamping procedure for one single stamping element in a low-rank block $M_{b}$.

stamped remains small. It can suitably be applied for terminal nodes or external circuit elements (sources, termination resistances). An $\mathcal{R}_{1}$-stamping of total inductance or capacitance matrices must be avoided. To insert total parasitics matrices in an efficient way, the PEEC proportion of the network matrix can be assembled as depicted in Figure $6.4 \mathrm{a}$ ). The structure is defined by the cluster trees $T_{\mathcal{I}, 1}$ and $T_{\mathcal{I}, 2}$, respectively. The adjacency matrices $\boldsymbol{A}_{1}$ and $\boldsymbol{A}_{2}$ comprise the connectivity of the network and can be constructed as conventional sparse matrices due to their low density. By means of the cluster trees $T_{\mathcal{I}, 1}$ and $T_{\mathcal{I}, 2}$ a construction of a corresponding $\mathcal{H}$-matrix is possible. Additional terminal nodes and external circuit elements can be incorporated into rows and columns of the network matrix by $\mathcal{R}_{1}$-matrix-stamping. Due to their small number and usually ohmic character of the connecting elements the resulting matrix $\Upsilon$ will be extremely sparse. The adjacency matrices can be converted to the hierarchical format based on the cluster trees from the corresponding inductance and capacitance matrices. For matrices without a possible mapping to a corresponding cluster tree (as for matrix $\Upsilon$ ), the application of a so-called black-box clustering 


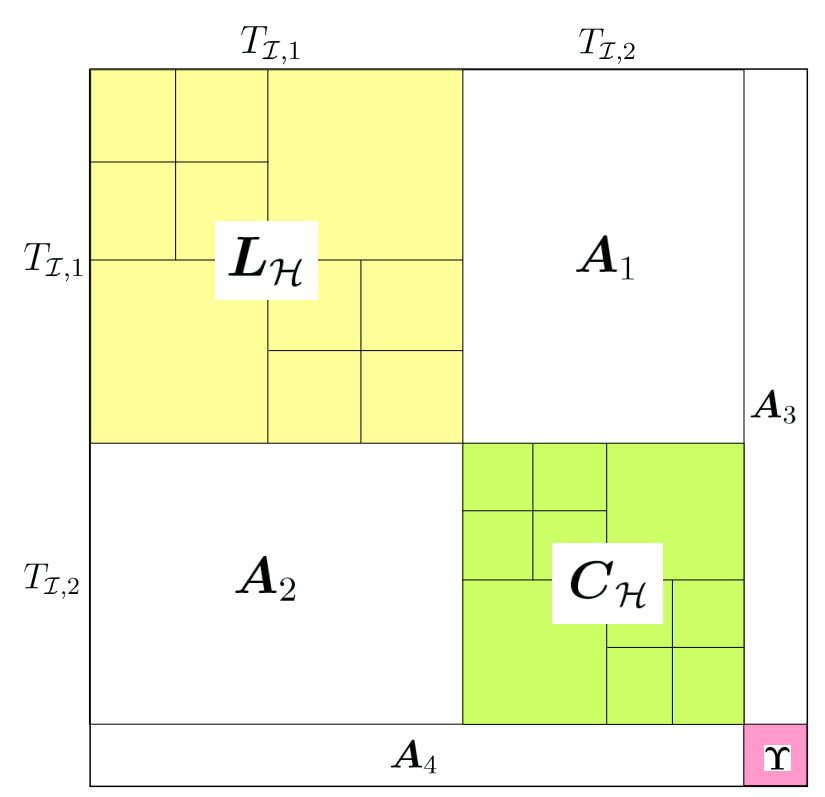

a)

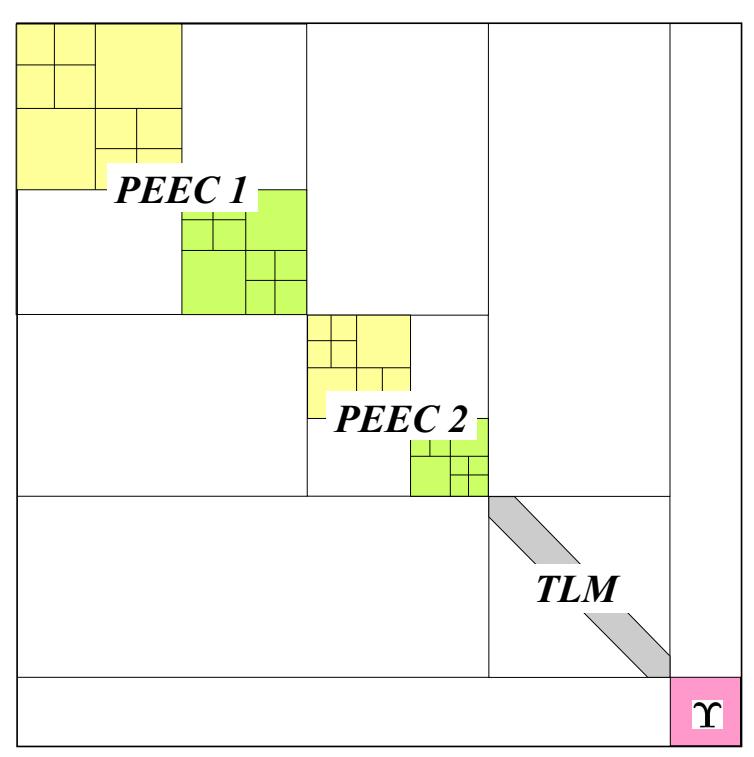

b)

Fig. 6.4: Circuit simulation network matrix constructed by $\mathcal{H}$-stamping. a) Single PEEC model with external circuit elements and terminals, represented by matrix $\Upsilon$; b) Two PEEC models and one TL model, connected via matrix $\Upsilon$. Remark: The main diagonal in $\Upsilon$ exhibits no zero elements, in general. A separate LU-decomposition of $\Upsilon$ is possible.

[161] is necessary. This clustering technique bases on the partitioning of the matrix graph (refer to Section 5.1.1). Geometrical information concerning the DOF of the underlying sparse matrix is not required. A similar implementation of TL models follows in a straightforward manner. TL models usually are more sparse than PEEC models. Due to the different modeling approaches, geometrical data concerning the discretization is not available under circumstances. The black-box clustering, however, can be applied in the same way. The fundamental structure for connecting PEEC models, TL models or common subcircuits is depicted in Figure 6.4 b) for an example comprising two PEEC models and a combining TL model. The proposed representation of an arbitrary network matrix as an $\mathcal{H}$-matrix sets the basis for an iterative scheme.

\subsection{2 $\mathcal{H}$-Preconditioning}

A suitable preconditioner for data-sparse network matrices can effectively be computed in $\mathcal{H}$ matrix arithmetic. Within the context of $\mathcal{H}$-matrices the conventional matrix operations have to be replaced by the formatted or truncated operations, as described in Section 4.3.1.9 [132]. Compared to the $\mathcal{H}$-Inverse-preconditioning the $\mathcal{H}$ - $L U$-preconditioning comprises the same complexity and usually produces less fill-ins. Both approaches, however, are used for practical relevant circuit simulations. For investigations and analysis the $\mathcal{H}$ - $L U$-decomposition is used without sacrifying general validity. The hierarchical $L U$-decomposition is based on a block-decomposition of the matrix and a gradual forward and backward substitution. Summarizing, this approach is more efficient than computing the inverse of the matrix. To realize an efficient $\mathcal{H}$-preconditioner $\boldsymbol{M}$, the approximate decomposition

$$
\boldsymbol{M}^{-1}=\left(\boldsymbol{L}_{\mathcal{H}} \boldsymbol{U}_{\mathcal{H}}\right)^{-1}=\boldsymbol{U}_{\mathcal{H}}^{-1} \boldsymbol{L}_{\mathcal{H}}^{-1}
$$


with the lower and upper triangular $\mathcal{H}$-matrices $\boldsymbol{L}_{\mathcal{H}}$ and $\boldsymbol{U}_{\mathcal{H}}$ can be applied. The $\mathcal{H}$-matrices have to be constructed with $\varepsilon_{\mathcal{H} \text {,pre }} \gg \varepsilon_{\mathcal{H}}$, such that (6.7) represents a decomposition, accurate enough for preconditioning. For circuit simulation purposes w.r.t. efficiency the iterative algorithms GMRes and BiCGstab are recommended, requiring specific forward/backward substitutions and matrix-matrix multiplications to compute the approximate inverse of $M$. The preconditioner computation usually represents the dominating effort in an iterative solving step. Moreover, together with circuit simulations in the TD as well as the FD, the spectral properties of the system matrix only slightly vary during simulation progress. Consequently, monitoring the convergence behavior of the iterative solution process from one step to another can be advantageous to exactly determine the right time for a recomputation or update of the preconditioner instead of recomputing in each step. For a preconditioner update [132],

$$
\widetilde{\boldsymbol{M}}=\boldsymbol{M}+c \vec{e}_{i} \vec{e}_{j}^{\top}
$$

is assumed with the vectors $\vec{e}_{i}, \vec{e}_{j} \in \mathbb{R}^{n}$. Applying the Sherman-Morisson formula for the inverse of $\widetilde{M}$,

$$
\widetilde{\boldsymbol{M}}^{-1}=\left(\boldsymbol{M}+c \vec{e}_{i} \vec{e}_{j}^{\top}\right)^{-1}=\boldsymbol{M}^{-1}-\frac{c}{1+c \vec{e}_{j}^{\top} \boldsymbol{M}^{-1} \vec{e}_{i}} \boldsymbol{M}^{-1} \vec{e}_{i} \vec{e}_{j}^{\top} \boldsymbol{M}^{-1}
$$

holds. From (6.9) it is apparent that matrices $\widetilde{M}^{-1}$ and $M^{-1}$ only differ in a $\mathcal{R}_{1}$-matrix. As a direct result it can be assumed that if $\widetilde{M}$ and $M$ differ in $p$ entries, the update at most exhibits rank $p$. Using the $\mathcal{H}$-matrix addition thus an efficient preconditioner update can be realized. The principle implementation structure into a PEEC-based circuit simulator is depicted in Figure 6.5.

\subsection{Numerical Results}

For our first tests we have restricted ourselves to transient analysis. The numerical experiments were performed on a INTEL(R) Xeon(TM) $2.80 \mathrm{GHz}$ Modell2 CPU with cache size $512 \mathrm{~KB}$ running Linux. Table 6.1 contains the densities of analyzed square PEEC-based benchmark network matrices with matrix dimension $2024 \times 2024$ and the storage requirements for the original sparse matrix, the $\mathcal{H}$ - and $\mathcal{H}^{2}$-approximants of the system matrix and the corresponding preconditioner from a $\mathcal{H}$ - $L U$-decomposition. The $\mathcal{H}$-matrix library HLIB [27] was used to perform the required operations together with parameters $\varepsilon_{\mathcal{H}}=10^{-8}, \varepsilon_{\mathcal{H}^{2}}=10^{-12}, \varepsilon_{\mathcal{H} \text {,pre }}=10^{-6}$. From Table 6.1 it is obvious that $\mathcal{H}$-approximations are most efficient for circuit-level system matrices with densities $\gtrsim 15 \%$. The $\mathcal{H}$-matrix structures for benchmark matrix mb1 and the respective preconditioners are illustrated in Figure 6.6.

\subsubsection{Convergence Behavior Analysis}

The time and memory requirements for iterative solving of the linear systems with stopping criterion $\|\boldsymbol{A} \vec{x}-\vec{b}\| \leqslant 10^{-8}$ are presented in Table 6.2. A comparison was carried out between a standard direct solver (GMATRIX) [73] and a $\mathcal{H}$-matrix-based and preconditioned one-level iterative solver (GMRes) from the HLIB. Results for a sparse, a medium dense and a dense PEEC simulation are compared by varying the coarsening parameter $\varepsilon_{\mathcal{H}}$. The convergence behavior for one timestep in the dense PEEC case and in the sparse PEEC case with varying $\varepsilon_{\mathcal{H}}$ is shown in Figure 6.7. From the investigations and results in $[162,163]$ it is obvious that for medium dense 


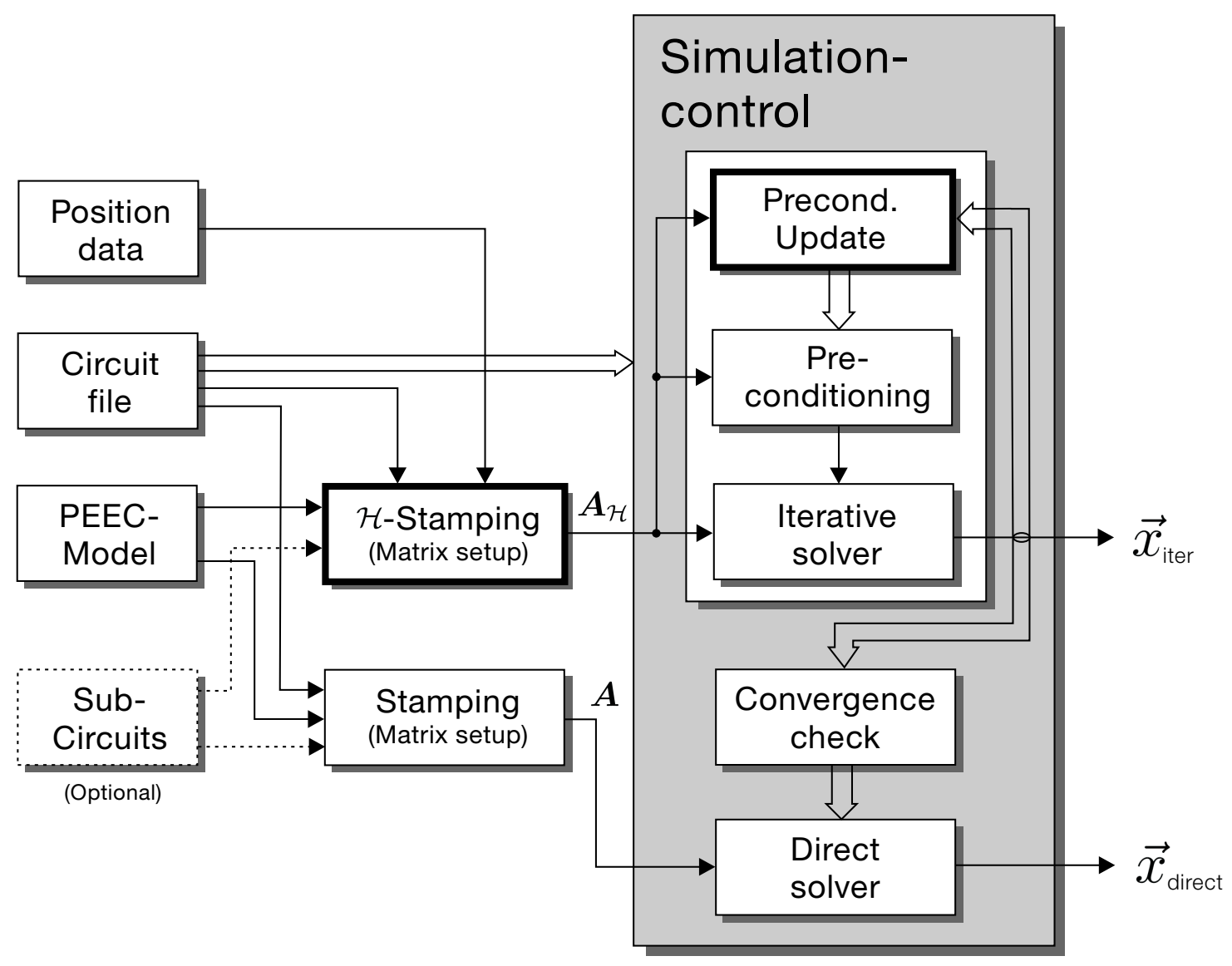

Fig. 6.5: Circuit simulator with $\mathcal{H}$-stamping and adaptive preconditioner update. The adaptive preconditioning is controlled by checking the residual in each iteration step. A conventional matrix stamping is provided for the fallback solver.

\begin{tabular}{|c||c|c|c|c|c|}
\hline \multicolumn{1}{|c||}{} & matrix density & \multicolumn{5}{|c|}{ memory requirements in MB } \\
& in \% & $\boldsymbol{A}$ & $\boldsymbol{A}_{\mathcal{H}}$ & $\boldsymbol{A}_{\mathcal{H}^{2}}$ & $\boldsymbol{M}$ \\
\hline \hline mb1 & 92.38 & 58 & 29 & 9.6 & 24 \\
\hline mb2 & 20.10 & 13 & 22 & 8.5 & 23 \\
\hline mb3 & 12.15 & 7.6 & 17 & 7.5 & 20 \\
\hline mb4 & 6.49 & 4.1 & 13 & 6.7 & 16 \\
\hline mb5 & 4.21 & 2.6 & 10 & 6.0 & 14 \\
\hline mb6 & 3.12 & 2.0 & 8.4 & 5.5 & 12 \\
\hline mb7 & 2.49 & 1.6 & 7.2 & 5.0 & 11 \\
\hline
\end{tabular}

Table 6.1: Memory requirements for PEEC-based benchmark matrices mb1 to $\mathrm{mb} 7(\operatorname{dim}(\boldsymbol{A})=2024 \times 2024)$ and its $\mathcal{H}$ - and $\mathcal{H}^{2}$-approximations. The preconditioner $M$ was computed by $\mathcal{H}$ - $L U$-decomposition.

and dense system matrices $\mathcal{H}$-matrix techniques are most suitable while for sparse system matrices an AMG approach (refer to Chapter 7) represents the best choice. The achievable speed-up by a factor of $\approx 12.7$ for TD simulations and factor of $\approx 1.53$ for FD simulations and memory requirements are presented in Tables 6.3 and 6.4, respectively. 


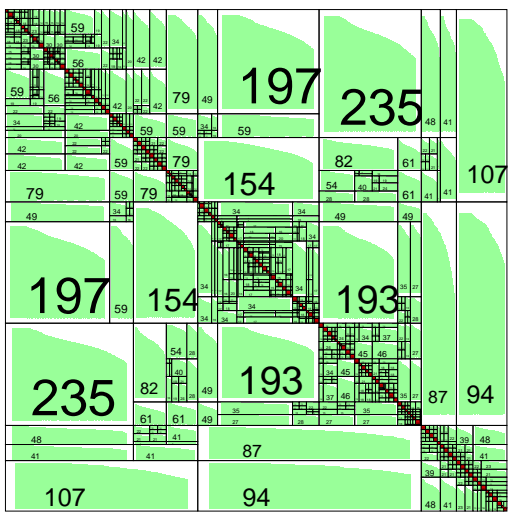

a)

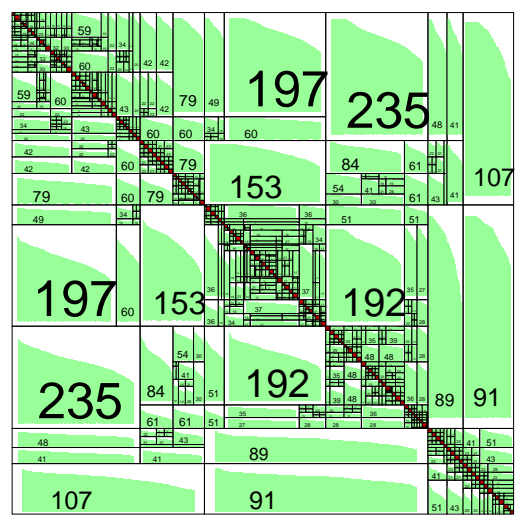

b)

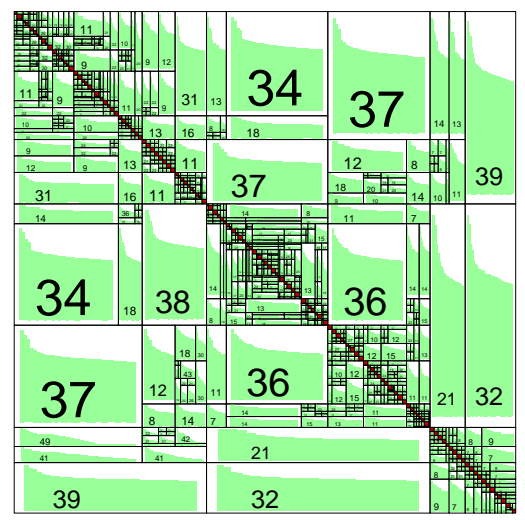

c)

Fig. 6.6: $\mathcal{H}$-matrix structure of the preconditioners. a) original benchmark $\mathcal{H}$-matrix mb 1 from TD analysis $(\operatorname{dim}=2024 \times 2024$, dens $=92 \%)$, b) $\mathcal{H}$ - $L U$-preconditioner, c) $\mathcal{H}$-inverse-preconditioner. Parameters for the $\mathcal{H}$ approximation: $\eta=2.0, \varepsilon=10^{-8}, n_{\min }=20$, adaptive rank allocation.

\begin{tabular}{|c|c||c|c|c|c|c|c|c|c|c|c|c|}
\hline \multicolumn{2}{|c||}{$\begin{array}{c}\text { Circuit } \\
\text { sim. parameters }\end{array}$} & \multicolumn{9}{c|}{ HLIB-GMRes (iterative one-level) } & \multicolumn{3}{c|}{ GMATRIX } \\
$\varepsilon_{\mathcal{H}}=10^{-2}$ & $\varepsilon_{\mathcal{H}}=10^{-4}$ & $\varepsilon_{\mathcal{H}}=10^{-6}$ & $\varepsilon_{\mathcal{H}}=10^{-8}$ & \multicolumn{3}{c|}{ (direct) } \\
\hline . TRAN & density & time & mem & time & mem & time & mem & time & mem & time & mem \\
\hline dense & 92.38 & 8.63 & 342 & 10.88 & 342 & 12.5 & 342 & 13.55 & 342 & 277.88 & 393 \\
\hline medium & 57.81 & 6.88 & 205 & 10.65 & 205 & 11.82 & 205 & 11.67 & 205 & 179.80 & 231 \\
\hline sparse & 0.92 & 2.15 & 45 & 3.02 & 45 & 2.93 & 49 & 3.28 & 50 & 11.67 & 64 \\
\hline
\end{tabular}

Table 6.2: Time and memory requirements for a PEEC-based TD simulation. An $\mathcal{H}$-matrix-based iterative solver GMRes (HLIB) is compared to a standard direct solver (GMATRIX) [162] (CPU times in minutes, memory requirements in MB, matrix densities in \%).

\subsubsection{Adaptive Preconditioning}

As described in Section 6.2.2 (Figure 6.5), an adaptive preconditioner update can help to further improve the simulation efficiency. The following tests were performed on a HP Workstation 850 with $3.8 \mathrm{GHz}$ dual core Intel CPU (SSE2) with cache size $2 \cdot 2 \mathrm{MB}$, system memory $4 \mathrm{~GB}$ ECC DDR RAM running Linux RedHat version 8.0 (Compiler GCC Version 3.3.3). Although the investigated benchmark examples are the same as presented in Table 6.2 with matrix dimension $2024 \times 2024$, a comparison with results in Table 6.2 is not possible due to the deviant hardware. The $\mathcal{H}$-matrix approximation was carried out with $\varepsilon_{\mathcal{H}}=10^{-8}, n_{\min }=20$. The stopping criterion for the iterative method is $\|\boldsymbol{A} \vec{x}-\vec{b}\| \leqslant 10^{-8}$. In FD analysis the equation system was transformed to an equivalent real-valued system according to (5.7) with double dimension representing the reason for the increased storage requirements and decreased speed-up compared to TD analysis and the results for the direct solver (GMATRIX). An interpretation of the results in Tables 6.3 and 6.4 points out some interesting facts. At first glance, the $\mathcal{H}$-preconditioned iterative solvers outperforms the direct solver in any case. As already shown in Section 6.3.1 (Table 6.2), the speed-up increases with increasing matrix density. The peak memory needed in TD analysis is slightly decreased for the $\mathcal{H}$-preconditioned iterative solver compared to the direct solver. In FD analysis the matrix transformation tampers the memory comparison between direct and iterative solver. It can be noticed that for the best case (TD, density $92.19 \%$ ) speed-up with a factor of 66.86 can be 

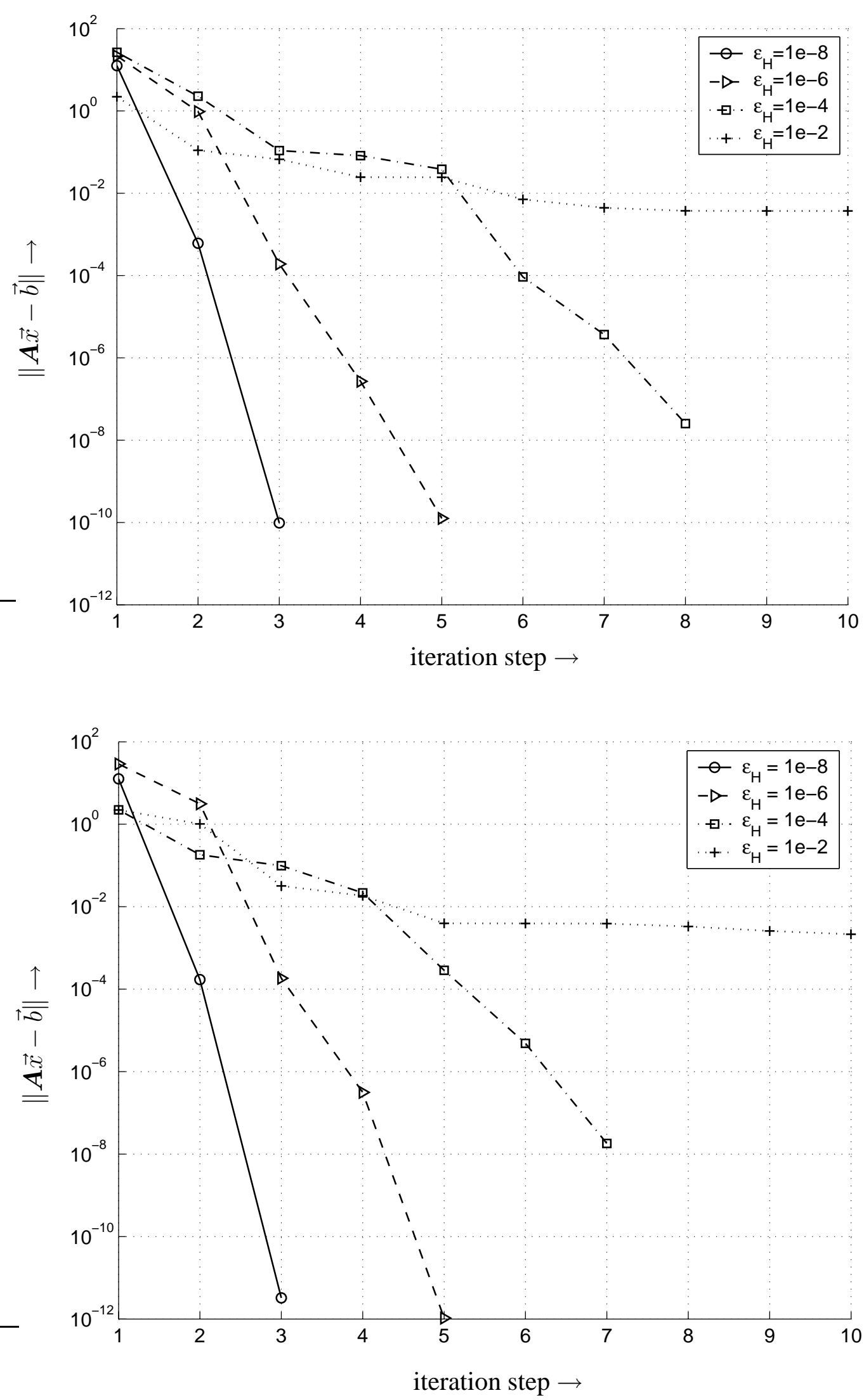

Fig. 6.7: Convergence results for $\mathcal{H}$-preconditioned one-level iterative circuit simulation. The final residuals are given for one-level GMRes iterations in one TD simulation step. The accuracy $\left(\varepsilon_{\mathcal{H}}\right)$ for the $\mathcal{H}$-matrix approximation is varied for the two cases PEEC dense (top) and PEEC sparse (bottom) which is specified in [162]. 
achieved compared to the direct solver. Despite of the overhead in FD analysis still a speed-up with a factor of 9.57 can be obtained using adaptive preconditioning [164]. For investigations

\begin{tabular}{|c||l|r|r|r|}
\hline density & solver & runtime & speed-up & peak memory \\
\hline \hline \multirow{2}{*}{92.19} & GMATRIX & 112927 & - & 392 \\
& $\mathcal{H}$-GMRes & 8897 & 12.69 & 342 \\
& $\mathcal{H}$-GMRes (adapt.) & 1689 & 66.86 & 342 \\
\hline \multirow{2}{*}{57.92} & GMATRIX & 4950 & - & 276 \\
& $\mathcal{H}$-GMRes & 1244 & 3.98 & 231 \\
& $\mathcal{H}$-GMRes (adapt.) & 97 & 51.03 & 231 \\
\hline \multirow{2}{*}{4.21} & GMATRIX & 332 & - & 56 \\
& $\mathcal{H}$-GMRes & 150 & 2.31 & 43 \\
& $\mathcal{H}$-GMRes (adapt.) & 21 & 15.81 & 43 \\
\hline
\end{tabular}

Table 6.3: Conventional and adaptive preconditioning for PEEC matrices in TD analysis. A direct solver (GMATRIX) is compared to an $\mathcal{H}$-matrix-based iterative one-level GMRes solver with preconditioner recomputation in each simulation step and adaptive preconditioning controlled by the actual residuals. (simulation times in seconds, storage requirements in MByte, matrix densities in \%)

\begin{tabular}{|c||l|r|r|r|}
\hline density & solver & runtime & speed-up & peak memory \\
\hline \hline \multirow{2}{*}{92.19} & GMATRIX & 21589 & - & 329 \\
& $\mathcal{H}$-GMRes & 15119 & 1.43 & 611 \\
& $\mathcal{H}$-GMRes (adapt.) & 2618 & 8.25 & 611 \\
\hline \multirow{2}{*}{57.92} & GMATRIX & 19858 & - & 214 \\
& $\mathcal{H}$-GMRes & 12958 & 1.53 & 533 \\
& $\mathcal{H}$-GMRes (adapt.) & 2076 & 9.57 & 533 \\
\hline \multirow{2}{*}{4.21} & GMATRIX & 971 & - & 99 \\
& $\mathcal{H}$-GMRes & 2904 & 0.33 & 126 \\
& $\mathcal{H}$-GMRes (adapt.) & 482 & 2.01 & 126 \\
\hline
\end{tabular}

Table 6.4: Conventional and adaptive preconditioning for PEEC matrices in FD analysis. A direct solver (GMATRIX) is compared to an $\mathcal{H}$-matrix-based iterative one-level GMRes solver with preconditioner recomputation in each simulation step and adaptive preconditioning controlled by the actual residuals. (simulation times in seconds, storage requirements in MByte, matrix densities in \%)

concerning the overall system performance the number of required iterations to achieve convergence using adaptive preconditioning for TD and FD analysis also show some interesting facts. From Figure 6.8 it is obvious that the occurence of preconditioner updates increases with decreasing parameter maxiter. This parameter limits the maximum number of applied iterations. The updating occurence is relatively moderate with lower frequencies. At higher frequencies, however, a decreased convergence behavior can be observed, directly leading to an increased frequency of preconditioner updates. In TD analysis some DC-point analysis are necessary, requiring only few 

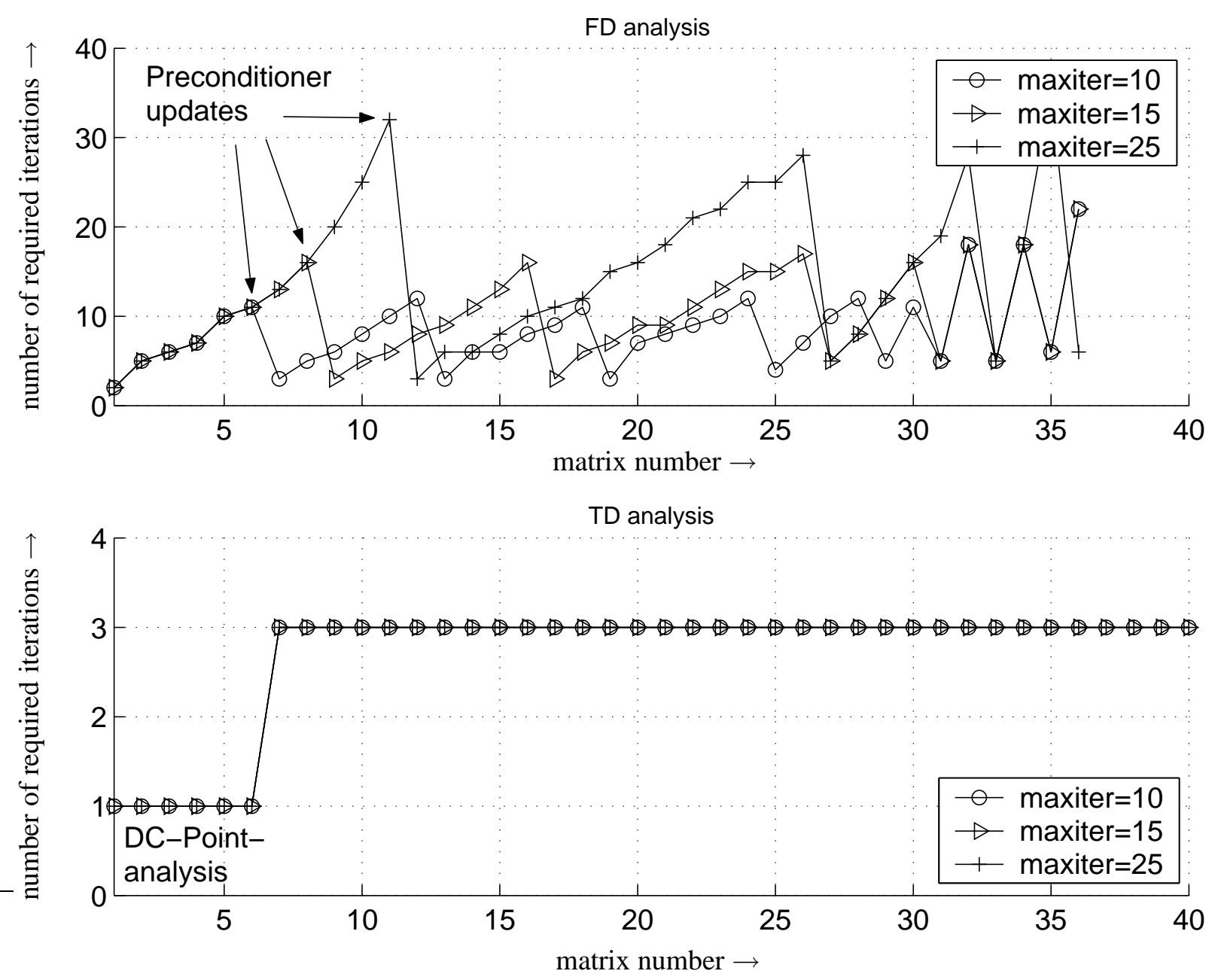

Fig. 6.8: Number of required iteration steps applying adaptive preconditioning for FD and TD analysis. The parameter maxiter denotes the upper bound for the maximum number of iterations until the preconditioner is forced to be updated. In FD analysis an increasing matrix number directly corresponds to an increased frequency. In TD analysis the matrix number can directly be mapped to the simulation progress in time.

numbers of iterations. After the DC-point analysis is finished, a constant number of iterations and a satisfactory convergence behavior can be obtained. This results from the higher sparsity of TD network matrices compared to FD network matrices.

\section{4 $\mathcal{H}$-Matrices for the Retarded Case}

To utilize the benefits from the application of $\mathcal{H}$-matrices also together with retarded PEEC models (refer to Section 3.10), it is necessary to "project" the proposed premises and methods described in detail in Section 4.3 to the retarded case. As shown in Appendix B, Green's function (B.9) in $3 \mathrm{D}$ represents the kernel function originating from the Helmholtz equation. While a separable expansion of the kernel function for the FMM is possible as shown in Section 4.2, the aim is to find a method which is numerically stable and moreover exhibits almost optimal complexity w.r.t. time and memory requirements. From the literature [140] it is well known that for the high-frequency regime in 2D the number of the boundary elements $n$ is proportional to the wave number $\beta$ and in $3 \mathrm{D} n$ is proportional to $\beta^{2}$. With the meshsize $h$ as defined in (3.69), $\beta h \stackrel{!}{=}$ const 
must hold. If a required accuracy is fixed, length $k$ of the kernel functions separable expansion (refer to (4.24)) can be expected to be proportional to $\beta$ and hence strongly dependent on $n$. This length of expansion is constant for a fixed accuracy since for the low-frequency domain $\beta$ is a constant w.r.t. $n$. Most of the FMM techniques comprising a complexity of $\mathcal{O}\left(n \log ^{\kappa} n\right)$ for some small constant $\kappa$, suffer from numerical instabilities at low frequencies in treating retarded Green's functions [140, 23]. The approach proposed by BANJAI and HACKBUSCH [140] approximates the matrices resulting from the low-frequency domain by $\mathcal{H}$-matrices, while for matrices from the high-frequency regime a combination of a $\mathcal{H}$-matrix and a $\mathcal{H}^{2}$-matrix is used. However, while recompression techniques are applicable in a straightforward manner for the low-frequency regime, additional research is necessary for the high-frequency range. In 3D the fundamental solution (B.9) respresents the zero-order spherical Hankel function of the first kind. From basic $\mathcal{H}$-matrix theory it can be observed, that in the high-frequency regime where $\beta h$ is a constant, $\mathcal{H}$-matrix techniques can not be applied. As a remedy, $\mathcal{H}^{2}$-matrices can be used. Starting from a separable expansion of the corresponding "retarded" kernel function (refer to [140]) similar to (4.10) and a truncation as described in (4.11), the $\mathcal{H}^{2}$-matrix construction by construction of the cluster basis, the transfer operator and the corresponding coefficient matrix can be applied in a straightforward manner. 


\section{7 \\ Multigrid Solvers for PEEC}

$\boldsymbol{I}$

n literature it is well known that the number of iteration steps required to achieve convergence, increases with the problem size [165] using conventional preconditioned iterative solvers, as described in Chapter 5. Multilevel approaches, if suitably applied, are "scalable", i.e. the convergence rate is independent of the problem size and the number of iteration steps stays almost constant ${ }^{1}$. Very suited problem classes for multilevel methods are, e.g. discrete elliptic matrices or mixed elliptic-hyperbolic matrices. In general, the maximum efficiency of multilevel methods unfolds with very large problem dimensions. Multilevel methods are often called " multigrid" (MG) methods, which can achieve linear complexity, in principle. The two fundamental principles of multigrid are smoothing and coarse-level correction. To realize a very robust and efficient solution process, the advantages of MG and Krylov one-level methods can be combined in a multigrid preconditioned Krylov method. PEEC matrices from a BEM approach, however, need special treatment in an AMG context [148]. The superiority of AMG particularly results from the robustness and applicability for unstructured grids in 2D and 3D as well and problems with anisotropic or even discontinuous coefficients. Furthermore, AMG allows for an efficient parallel implementation.

After a short motivation in Section 7.1, this Chapter recapitulates the basic multigrid strategies in Section 7.2. W.r.t. circuit simulation purposes, AMG component computation and multigrid preconditioning are explained in Sections 7.3 and 7.4, respectively. The applied standard AMG solver library turns out to be suitable for sparsified reluctance-based PEEC models (Section 7.5). Investigations concerning performance are evaluated in terms of accuracy and complexity. Concrete implementation details as well as numerical results for validation are presented in Sections 7.6 and 7.7.

\footnotetext{
${ }^{1}$ An algorithm is numerically scalable if its complexity in terms of computational time and memory requirements proceeds linearly with the problem size [154].
} 


\subsection{Motivation}

Although one-level iterative solvers exhibit a complexity of $\mathcal{O}\left(n^{\kappa}\right),(\kappa>1)$ w.r.t. computational work [157] for matrix dimension $n$, they can show a bad convergence behavior for large and illconditioned systems of equations. The reason for this lies in the fact that high-frequency error components are much better reduced than low-frequency error components ${ }^{2}$. Furthermore, it is obvious from Figure 7.1 that for the treatment of lower-frequency contributions of the iteration error a coarse grid is sufficient, while highly-oscillating contributions are treated well on a fine grid [148]. From (5.19) it can be observed that the error components are damped by the iteration

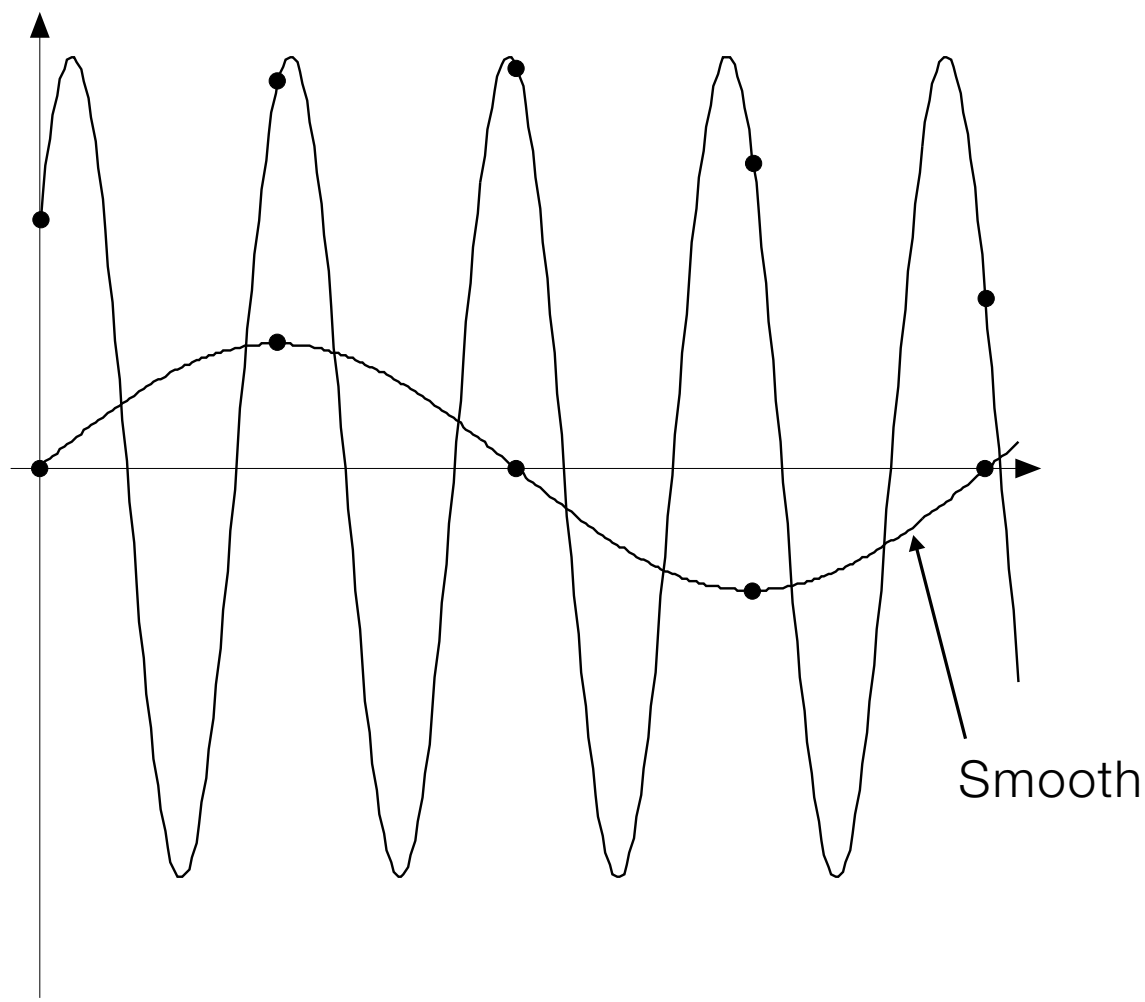

Fig. 7.1: Relation between high- and low-frequency error components for the Poisson equation.

matrix. The spectral radius of the iteration matrix is uniformly bounded by a constant smaller than one, leading to the efficiency of multigrid methods. With decreasing meshsize, more and more high frequencies in the error occur, resulting in a mesh-dependence of the overall efficiency of the iterative method [146]. Due to the capability of better reducing high-frequency error components than low-frequency error components, simple iteration schemes as Jacobi or Gauß-Seidel are also called smoothers. The basic intention of multilevel approaches is, to restrict the remaining relatively smooth error to a coarser grid, where it can be effectively reduced (so-called coarse-grid correction). Consequently, the mesh-dependence of the convergence behavior can be removed and optimal complexity of $\mathcal{O}(n)$, w.r.t both computational work and memory requirements can be achieved [157], in principle.

\footnotetext{
${ }^{2}$ While small eigenvalues exhibit low-frequency eigenfunctions, large eigenvalues correspond to high-frequency eigenfunctions.
} 


\subsection{Basic Multigrid Strategies}

The smoother damps the oscillatory high-frequency error components whereas the coarse-grid correction reduces the smooth low-frequency error components. This strategy represents the basic MG principle. Regarding the conceptional difference between the interplay of fundamental multigrid components, geometric and algebraic multigrid (GMG/AMG) approaches have to be distinguished [157]. The most crucial issue is, how to construct coarse levels. While in GMG the geometry information is used to compute the basic multigrid components, in AMG only the coefficients of the system matrix are available. The development of AMG allows to achieve the asymptotic optimality of GMG ( $h$-independent convergence), together with the advantages of easy-to-use "plug-in" solvers for important classes of problems, posed on large unstructured grids [166].

\subsubsection{Geometric Multigrid}

In geometric multigrid, the problem at hand is formulated as a system of grid-based equations of the form

$$
\mathcal{L}_{h} \vec{v}_{h}=\vec{b}_{h} \quad \text { on } \quad \Omega_{h},
$$

with a differential or integral operator $\mathcal{L}_{h}$ and $\vec{v}_{h}, \vec{b}_{h}$ representing functions, defined on a grid ${ }^{3}$ $\Omega_{h}$. GMG approaches operate on a predefined hierarchy of grids for the underlying mesh. For an optimized overall efficiency, the components smoothing and coarse-grid correction and their interplay is very important [157]. Problems can arise with less-structured grids, in particular in 3D, as usually existing together with PEEC meshes. The definition of suitable coarser grids just by exploiting geometric considerations is more difficult, the more complex the grid. High efficiency can only be obtained by a problem specific adaption of the components, which makes the use of such solvers as a plug-in solver impossible and limits the applicability of GMG in industrial simulation codes.

\subsubsection{Algebraic Multigrid}

The development of algebraic multigrid techniques, based on the observation that for certain matrix classes, suitable coarse levels can be computed solely based on the matrix entries ${ }^{4}$. It should be mentioned that the term "multilevel" would actually express the basic character of AMG because no geometric structure exists. However, due to the historical origins and in accordance with the literature the term "multigrid" is used in the following [167, 157, 149]. Instead of a grid-based formulation as in (7.1), AMG uses a system of linear algebraic equations of the form

$$
\boldsymbol{A} \vec{v}=\vec{b}, \quad \mathcal{V} \triangleq\{1, \ldots, n\}
$$

\footnotetext{
${ }^{3}$ The term "grid" is synonymously used for mesh, where $h$ denotes the meshsize referring to a grid parameter. For a triangulation the meshsize is defined by (3.69) in Section 3.7.

${ }^{4}$ The only formal difference is, that in the context of AMG we are not (necessarily) dealing with grids and subgrids but rather with sets of variables and subsets of variables (represented by their respective indices) [166]. In contrast to geometric multigrid, AMG's coarsening process is fully automated, in the simplest case merely based on algebraic information contained in the matrix such as size and signs of the entries.
} 
with matrix $\boldsymbol{A} \in \mathbb{R}^{n \times n}$ and $\vec{v}, \vec{b} \in \mathbb{R}^{n}$ on the finest level and $\mathcal{V}$ denoting the index set of the matrix. For grid-based problems, each solution variable represents a specific grid point in $\Omega_{h}$. Based on this algebraic foundation, both components - operator-dependent interpolation and coarse-level correction - can be constructed purely algebraically without referring to a grid. The basic idea of the coarse-level correction is to approximate the smooth error $\vec{e}_{h}$ on the coarser level with decreased cost compared to the fine level. For the error on the fine level the residual equation

$$
\boldsymbol{A}_{h} \vec{e}_{h}=\vec{r}_{h}
$$

with the error $\vec{e}_{h}=\vec{v}_{h}^{*}-\vec{v}_{h}$ and the residual $\vec{r}_{h} \triangleq \vec{b}_{h}-\boldsymbol{A}_{h} \vec{v}_{h}$ [22] holds. The exact solution is denoted by $\vec{v}^{*}$. Although the basic error reduction strategies of GMG and AMG are identical, there exist conceptual differences regarding the interplay between the multigrid components. Whereas the grid hierarchy in GMG is predefined and the smoother is adapted, in AMG a relatively simple smoothing process is fixed and a suitable hierarchy of levels is adaptively constructed. The transfer operators and coarse-level operators are computed algebraically, purely by using the information contained in the matrix entries [157]. Referring to [157], it should be noted that AMG as well as GMG provide a whole "methodology" for solving certain matrix equations, not only a simple method. AMG comprises two working-phases. The setup-phase includes a problem analysis, the recursive coarse-level construction and a computation of all operators. In the solution-phase the standard multigrid cycling is performed [167]. For most practical applications AMG can be expected to work most efficiently, when applied as a preconditioner to accelerate Krylov-subspace methods ${ }^{5}$. There exist two general AMG concepts - classical AMG and aggregation-based AMG. For further details, refer to [157]. AMG can be suitable for cases, where only the finest grid level is available or the coarsest grid of a geometric multigrid application is too large for direct solving [148]. AMG should not be regarded as a successor of GMG, but as an efficient alternative to popular one-level methods [166].

\subsection{AMG Components}

In the following classical AMG is used exclusively, referring to [167, 157]. For an efficient implementation of AMG for circuit simulation problems, a precise understanding of the components and the effects to the behavior of the solution process is necessary. This is especially important for the treatment of data-sparse PEEC matrices in Section 7.5.

\subsubsection{The Grid-Coarsening}

A restriction to the two-level process is sufficient to clarify the basic principle of AMG. An extension to the multilevel case is possible in a straightforward manner. Fine-level and coarse-level equation systems can be distinguished using the indices $h$ and $H$, respectively, which are not related to a discretization parameter. The matrix will be interpreted as a description of a "virtual" mesh. To construct the set of coarse-level variables $\mathcal{V}^{H}$, all nodes (unknowns) are classified into

\footnotetext{
${ }^{5}$ Originally, classical AMG has been designed to be used stand-alone. However, in practice AMG's efficiency and robustness can substantially be further enhanced by using it as a preconditioner rather than stand-alone [166].
} 
two categories. The classification can be done by so-called $C / F$-splitting of the whole set of variables $\mathcal{V}^{h}$ on an arbitrary level into two disjoint subsets $C^{h}$ and $F^{h}$, with

$$
\mathcal{V}^{h}=C^{h} \cup F^{h}, \quad C^{h} \cap F^{h}=\emptyset .
$$

Variables contained in the coarse level (C-variables) build the set $C^{h}=: \mathcal{V}^{H}$, while $F^{h}$ represents the complementary set of fine-level variables (F-variables). The set of coarse-level variables usually fulfills two properties [148] in the case of classical AMG:

- coarse-level variables are not connected directly and

- the number of coarse-level variables is as large as possible.

After classification (marking) of each variable, a prolongation is constructed to interpolate the correction value for F-variables from neighbouring $\mathrm{C}$-variables. For a simple selection algorithm, refer to [168]. Based on the theory presented in [157], an algebraic construction of transfer operators as restriction and interpolation is possible. We expect that the remaining error after smoothing on the fine level can be approximated on the coarse level. The crucial point is, how to realize transitions from fine levels to coarse levels, so-called restriction, and transitions from coarse levels to fine levels, so-called prolongation. Missing variables on the fine-level can be computed by interpolation from the neighbouring variables. To restrict a fine-level matrix $\boldsymbol{A}_{h}$ to a coarse-level matrix $\boldsymbol{A}_{H}$, a matrix operator $\boldsymbol{\mathcal { R }}_{h}^{H}$ is necessary. In a similar manner, a prolongation can be realized using a matrix operator $\mathcal{P}_{H}^{h}$. A graphical illustration is presented in Figure 7.2. An algebraic coarse-level
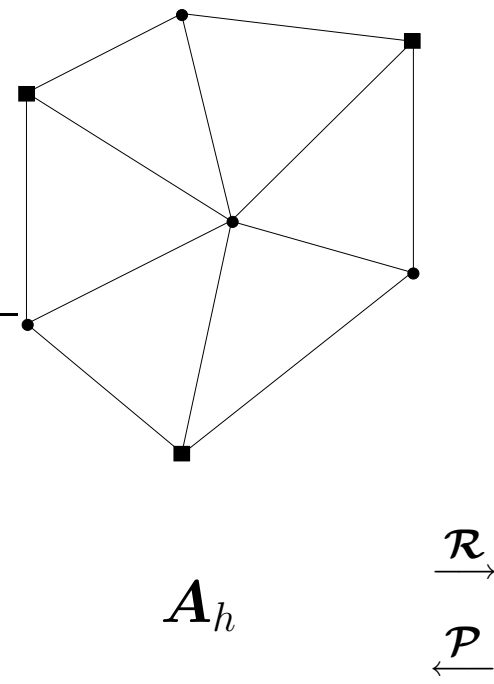

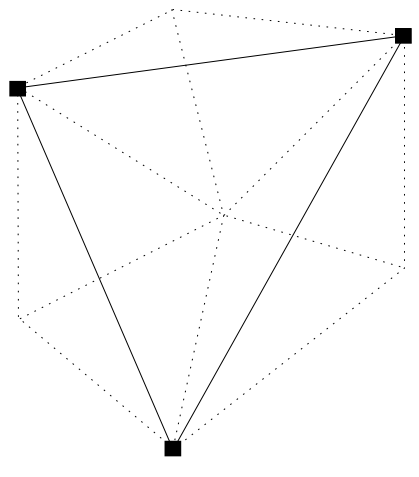

- fine grid

- coarse grid

Fig. 7.2: Interplay between prolongation $(\mathcal{P})$ and restriction $(\mathcal{R})$ in AMG.

operator $\boldsymbol{A}_{H}$, which is known as Galerkin-matrix or Galerkin-operator, can be constructed using the formulation

$$
\boldsymbol{A}_{H} \triangleq \boldsymbol{\mathcal { R }}_{h}^{H} \boldsymbol{A}_{h} \mathcal{P}_{H}^{h}
$$

The corresponding residual vector results in

$$
\vec{r}_{H} \triangleq \mathcal{R}_{h}^{H} \vec{r}_{h},
$$


keeping (7.3) in mind. Successively applying such operations, a hierarchy of systems with a number of coarsening steps ${ }^{6}$ can be obtained. The restriction operator is always defined to be the transpose of the interpolation operator

$$
\mathcal{R}_{h}^{H} \triangleq\left(\mathcal{P}_{H}^{h}\right)^{\top}
$$

and usually the orthogonality condition

$$
\mathcal{R}_{h}^{H} \mathcal{P}_{H}^{h}=\mathbf{1}
$$

holds [155]. The resulting equation system

$$
\boldsymbol{\mathcal { R }}_{h}^{H} \boldsymbol{A}_{h} \mathcal{P}_{H}^{h} \vec{e}_{H}=\boldsymbol{\mathcal { R }}_{h}^{H} \vec{r}_{h}
$$

can be solved iteratively by applying several steps of pre- and post-smoothing and a recursive coarse-level correction process.

\subsubsection{The Smoothing Process}

At each level of the multigrid algorithm, smoothing and coarse-level correction are combined in three steps. The first and the last step represent pre-smoothing and post-smoothing operations to damp the high-frequency modes using a linear smoothing operator $\mathcal{S}_{h}$. A single smoothing step is denoted by

$$
\vec{v}_{h} \longrightarrow \tilde{\vec{v}}_{h} \triangleq \operatorname{SMOOTH}\left(\mathcal{S}_{h}, \boldsymbol{A}_{h}, \vec{b}_{h}, \vec{v}_{h}\right) \triangleq \mathcal{S}_{h} \vec{v}_{h}+\left(\mathbf{1}_{h}-\mathcal{S}_{h}\right) \boldsymbol{A}_{h}^{-1} \vec{b}_{h}
$$

After $\nu$ smoothing steps (which is denoted by $\operatorname{SMOOTH}^{\nu}\left(\mathcal{S}_{h}, \boldsymbol{A}_{h}, \vec{b}_{h}, \vec{v}_{h}\right)$ ), the current error $\vec{e}_{h} \triangleq$ $\vec{v}_{h}^{*}-\vec{v}_{h}$ with exact solution $\vec{v}_{h}^{*}$ writes

$$
\vec{e}_{h} \longrightarrow \tilde{\vec{e}}_{h} \triangleq \mathcal{S}_{h}^{\nu} \vec{e}_{h}
$$

In AMG, usually very simple smoothers are applied. For example, a variable-wise Gauß-Seidel (GS) relaxation is used, i.e. $\mathcal{S}_{h} \triangleq \mathbf{1}_{h}-\boldsymbol{Q}_{h}^{-1} \boldsymbol{A}_{h}$, with $\boldsymbol{Q}$ being the lower triangular part of $\boldsymbol{A}_{h}$ including the diagonal, or $\omega$-Jacobi relaxation, i.e. $\mathcal{S}_{h}=\mathbf{1}_{h}-\omega \boldsymbol{D}_{h}^{-1} \boldsymbol{A}_{h}$ with $\boldsymbol{D}_{h}=\operatorname{diag}\left(\boldsymbol{A}_{h}\right)$ [157].

\subsubsection{The Coarse-Level Correction Process}

Suitable restriction and interpolation operators have to be constructed, fitting to the $C / F$-splitting. We only consider interpolations which are of the form

$$
\vec{e}_{h, i}=\left(\mathcal{P}_{H}^{h} \vec{e}_{H}\right)_{i}=\left\{\begin{array}{cl}
\vec{e}_{H, i} & \text { for } i \in C^{h} \\
\sum_{j \in P_{h, i}} w_{h, i j} \vec{e}_{h, j} & \text { for } i \in F^{h}
\end{array}\right.
$$

where $P_{h, i} \subseteq C^{h}$ is called the set of interpolatory variables (for the $i$ th variable). A restriction operator can be constructed using (7.7). Both interpolation and restriction are full-rank operators [157].

\footnotetext{
${ }^{6}$ The number of coarsening steps is unknown in advance.
} 


\subsubsection{The Multigrid Algorithm}

One coarse-level correction step without smoothing can be described by

$$
\vec{v}_{h}^{\text {new }}=\vec{v}_{h}^{\text {old }}+\mathcal{P}_{H}^{h} \vec{e}_{H},
$$

where $\boldsymbol{A}_{H} \vec{e}_{H}=\boldsymbol{\mathcal { R }}_{h}^{H}\left(\vec{r}_{h}^{\text {old }}\right)=\boldsymbol{\mathcal { R }}_{h}^{H}\left(\vec{b}_{h}-\boldsymbol{A}_{h} \vec{v}_{h}^{\text {old }}\right)$. For the error

$$
\vec{e}_{h}^{\text {new }}=\mathfrak{K}_{h, H} \vec{e}_{h}^{\text {old }}
$$

holds, with the so-called coarse-level correction operator $\mathfrak{K}_{h, H} \triangleq \mathbf{1}_{h}-\mathcal{P}_{H}^{h} \boldsymbol{A}_{H}^{-1} \boldsymbol{\mathcal { R }}_{h}^{H} \boldsymbol{A}_{h}$. An error reduction including $\nu_{1}$ pre-smoothing steps and $\nu_{2}$ post-smoothing steps in a complete twolevel iteration (refer to Algorithm 3), is described by the so-called two-level iteration operator $\mathfrak{M}_{h, H}\left(\nu_{1}, \nu_{2}\right)=\mathcal{S}_{h}^{\nu_{2}} \mathfrak{K}_{h, H} \mathcal{S}_{h}^{\nu_{1}}[167]$, fulfilling

$$
\vec{e}_{h}^{\text {new }}=\mathfrak{M}_{h, H} \vec{e}_{h}^{\text {old }} .
$$

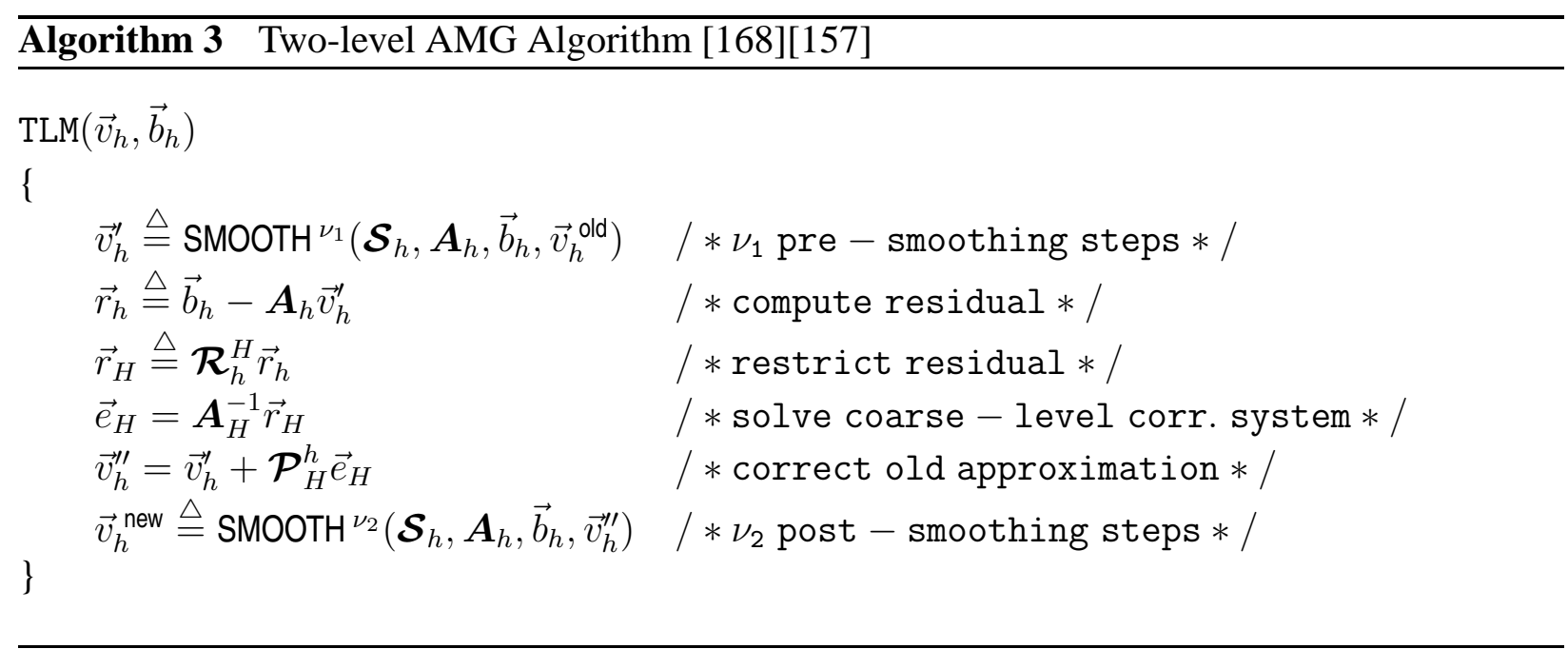

Since a very accurate solution of the coarse system $\boldsymbol{A}_{\ell+1} \vec{e}_{\ell+1}=\vec{r}_{\ell+1}$ usually is too complicated, the exact solving will be realized recursively by applying $\gamma$ steps of a two-level method. This leads to the multilevel AMG algorithm 4 . The method is called $\mathrm{V}\left(\nu_{1}, \nu_{2}\right)$-cycle for $\gamma=1$ and $\mathrm{W}\left(\nu_{1}, \nu_{2}\right)$ cycle for $\gamma=2$, respectively. The size of the $\mathrm{V}$ - or $\mathrm{W}$-cycle depends on the number of levels available to the system, as depicted in Figure 7.3 for 2, 3 and 4 levels (V-cycle, left) and 3 and 4 levels (W-cycle, right). While a dot $(\bullet)$ denotes a smoothing operation, a box stands for the exact solution on the coarsest level. A descending line $(\backslash)$ indicates that a fine level is being restricted down to a coarse one and similarly an ascending line (/) shows that a coarse level is interpolated up to a finer one. Apart from $\mathrm{V}$ - and $\mathrm{W}$-cycles, which belong to the most important cases in practice, other variants as the F-cycle exist. While the $\mathrm{W}$-cycle is more costly, in principle, it needs less iterations. Applying $\nu_{1}$ pre-smoothing steps and $\nu_{2}$ post-smoothing steps, the application of a V-cycle only requires $\nu_{1}+\nu_{2}+1$ matrix-vector multiplications, $\nu_{1}+\nu_{2}$ applications of the preconditioner and one restriction and prolongation on each level [3]. 


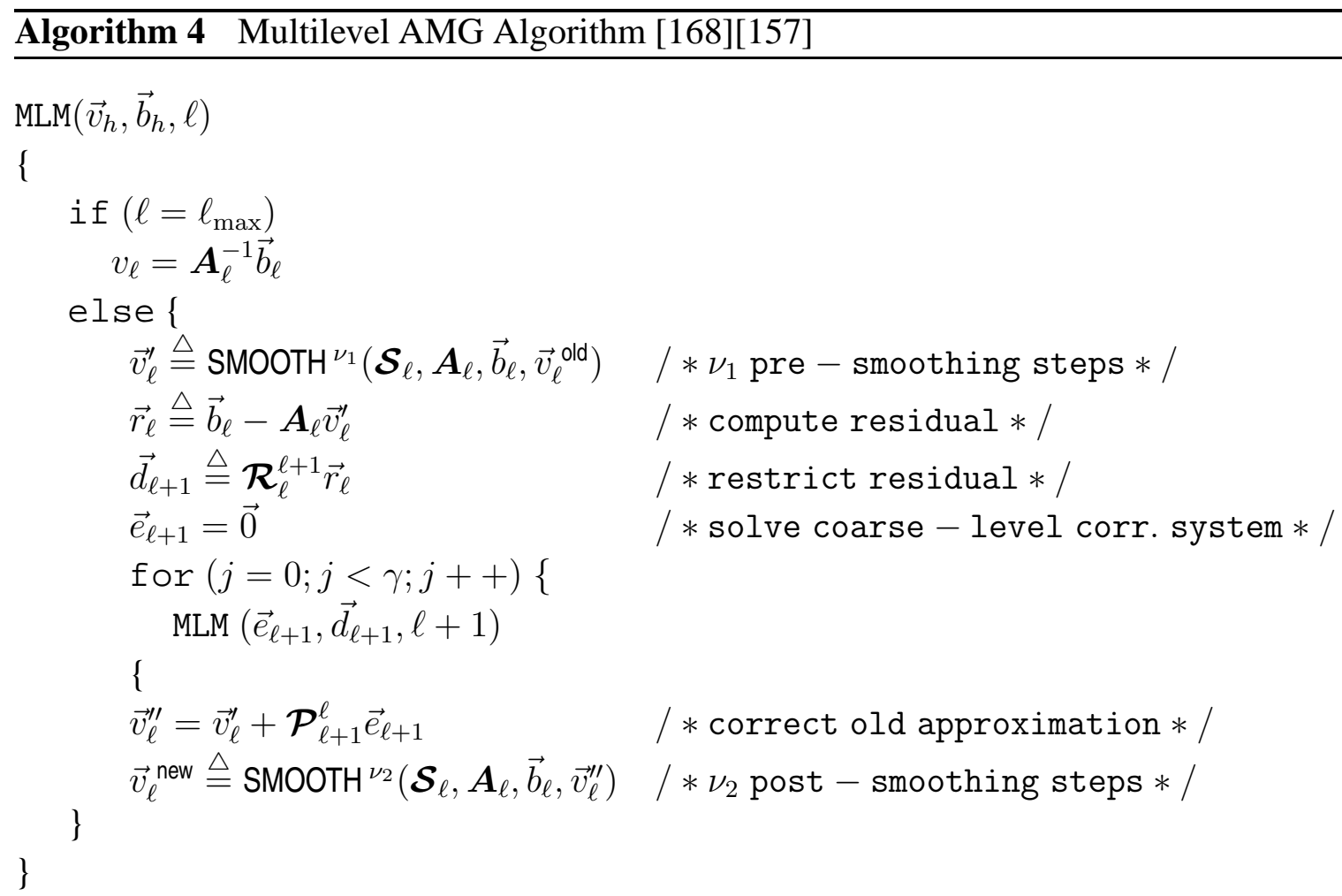

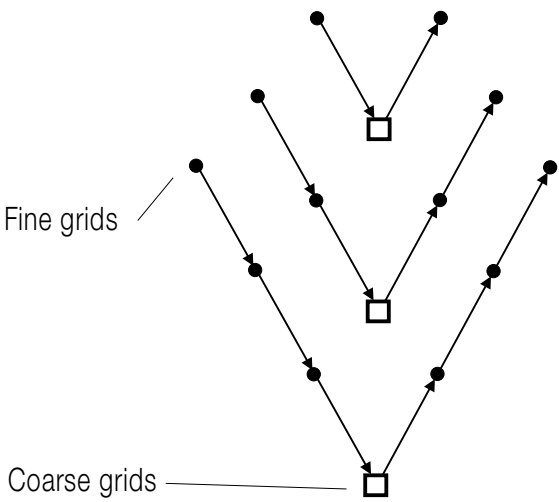

V-cycles

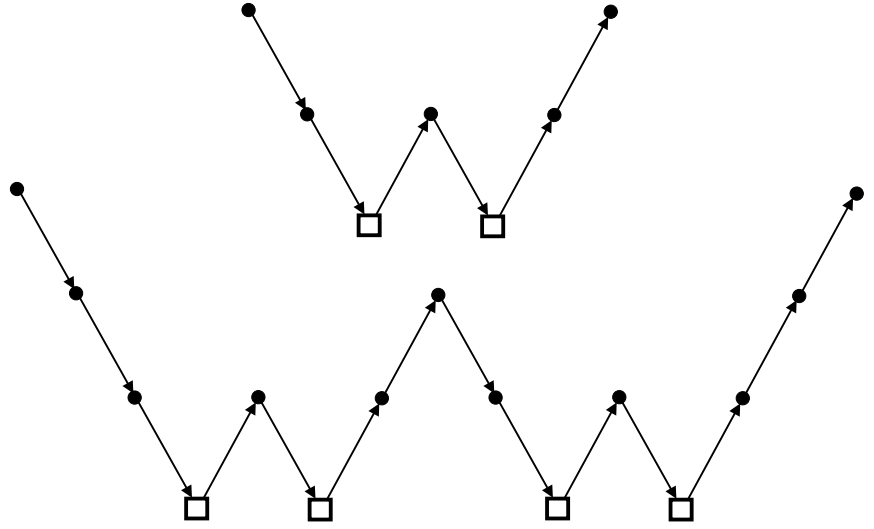

W-cycles

Fig. 7.3: The workflow for V-cycles (left, for 2,3,4 levels) and W-cycles (right, for 3,4 levels) in multigrid methods including smoothing operations and coarse-level correction.

\subsection{Multigrid Preconditioning}

Although by applying multigrid methods one can achieve high efficiency for various problems, slowly converging eigenvectors of the iteration matrix can lead to bad convergence results. Multigrid algorithms tend to be problem specific and less robust than preconditioned Krylov iterative methods. In order to enhance the robustness and efficiency of multigrid methods, Krylov methods can be included. In this context, multigrid is used as a preconditioner in a Krylov-subspace method, often leading to a more robust, efficient and scalable algorithm. As mentioned in the introduction of this chapter, multigrid solvers are scalable if applied suitably, in contrast to conventional preconditioned iterative solvers. Multigrid preconditioning means that the iteration matrix of the multigrid 
method, which is not explicitely needed, plays the role of the preconditioner. The preconditioning steps are substituted by "solving" approximately appropriate systems by the aforementioned multigrid method. Where a classical multigrid solver will repeatedly apply the V- or W-cycle to reduce the error in each step, the multigrid preconditioning will only work if all modes are damped by the multigrid cycle [3]. Since a multigrid cycle is designed to reduce the error, it can also be used as an approximate inverse of the system matrix, and therefore as a preconditioner for an iterative solver. If the multigrid cycle is very effective, classical multigrid already converges very quickly.

\subsection{AMG Preconditioners for PEEC Matrices}

AMG strategies are applicable for various circuit simulation purposes as shown in [25]. In the field of effective power grid reduction [26], sparse system matrices occur, suitable for AMG-based circuit simulation for large-scale power grid transient and FD analysis. The AMG components are constructed directly from the circuit MNA matrices as described in detail in [25]. A significant memory reduction can be obtained, since no geometric information is needed. Assuming smooth voltage variations, experimental results show a significant reduction in problem size without sacrifying the accuracy. Electromagnetic effects (e.g. cross-talking) prevent the resulting system matrices from being M-matrices ${ }^{7}$. Such circuit simulation matrices often exhibit very large condition numbers. To achieve high efficiency of AMG also together with BEM-based PEEC matrices, a special treatment of the fully-populated matrices has to be accomplished. The character of the underlying boundary integral operator has to be taken into account for an efficient component computation. For the single layer potential operator with Green's function as kernel, one has to consider that high-frequency eigenfunctions correspond to small eigenvalues [148]. AMG was originally designed for differential-based approaches. Together with PEEC matrices, therefore, one has to construct a smoother for pseudo-differential operators of order minus one, as mentioned in the context with the SCHWARTZ kernel theorem in Section 3.9.3.1 ${ }^{8}$. To avoid the problem resulting from the conversely behavior of pseudo-differential operators of negative order, a certain inner product can be applied [169]. In fact, the MG reduction process is equivalent to the linear preconditioned iterative scheme. To reduce the algorithmic complexity of a matrix-vector multiplication for BEM-based circuit simulation matrices, two different approaches can be applied truncated reluctance matrix based PEEC and PEEC based on the adaptive cross approximation.

\subsubsection{AMG Preconditioners for Truncated PEEC Matrices}

A truncation process $\mathcal{T}$, applied to reluctance-based PEEC models (refer to Section 3.9.4) leads to sparse fine-grid system matrices $\boldsymbol{A}_{h}$, which behave as originating from a FEM discretization ${ }^{9}$ [163]. A matrix-vector multiplication therefore can be realized with complexity $\mathcal{O}(n)$, as mentioned in Chapter 5. Due to the necessity to compute all $n^{2}$ interactions for reluctances and capacitances prior to the truncation, the memory to hold all values in cache represents a bottleneck

\footnotetext{
${ }^{7}$ M-matrices often occur from discrete elliptic problems. For further details also refer to Appendix A.1.

${ }^{8}$ One reason for the ill-conditioning of BEM matrices can also be found in the character of the single layer potential operator, to be represented as a differential operator of order minus one.
}

${ }^{9} \mathrm{~A}$ truncation causes a distortion of the BEM character of the matrix. 
for the problem size. Furthermore, time complexity for (computation and) truncation amounts to $\mathcal{O}\left(n^{2}\right)$, restricting the achievable efficiency. Although truncation of mutual reluctances and mutual capacitances preserves passivity of the PEEC model, a loss in accuracy due to the truncation error

$$
\varepsilon_{\mathcal{T}} \triangleq\left\|\boldsymbol{A}_{h}-\mathcal{T}\left(\boldsymbol{A}_{h}\right)\right\|_{2}
$$

results. Figure 7.4 illustrates the progression of the truncation error and the corresponding matrix density for an example PEEC-based network matrix of dimension $523 \times 523$ in the FD. The sparser the matrix the higher the loss in accuracy representing a loss of information. Truncation of small off-diagonal inductive and capacitive entries can lead to an increased condition number of the resulting system matrix, which can directly influence the convergence behavior of iterative methods. The construction of the AMG transfer operators as restriction and prolongation and the Galerkin operation, can be accomplished as described in Section 7.3.

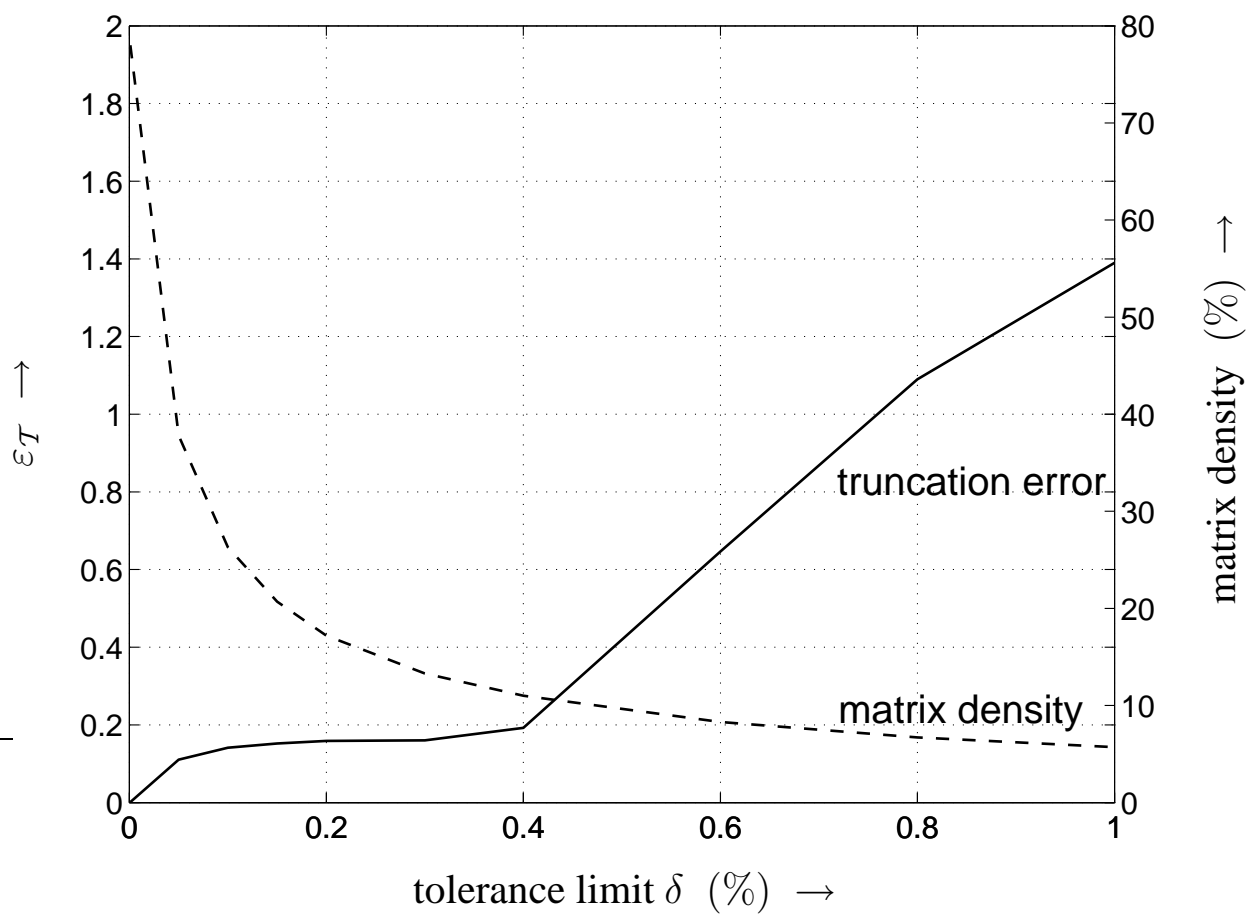

Fig. 7.4: Influence of the tolerance limit to the truncation error $\varepsilon_{\mathcal{T}}$ and the corresponding matrix density for a square PEEC-based network matrix of dimension $523 \times 523$ at $800 \mathrm{MHz}$.

\subsubsection{AMG Preconditioners for Data-Sparse PEEC Matrices}

While truncation applied to PEEC matrices helps to achieve sparse system matrices suitable for iterative MG methods, more accurate and almost optimal solutions need special treatment. Resulting from the contrary behavior of single layer potential's eigenvalues and eigenfunctions conventional smoothers no longer work optimal compared to FEM-based matrices. One possible way to represent dense BEM matrices data-sparse is, to apply hierarchical matrix techniques.

\subsubsection{Restriction and Prolongation Operators}

For BEM-based matrices it proved to be useful to specify an auxiliary matrix $\boldsymbol{B}_{h} \in \mathbb{R}^{n_{h} \times n_{h}}$, containing nodal distances and representing the underlying mesh of the discretization [148, 138]. The 
dimension of $\boldsymbol{B}_{h}$ results from the number of fine-grid nodes, corresponding to the DOF in the PEEC case. Applying the ACA-algorithm from Section 4.3.2 to compute a data-sparse representation of the BEM matrix, the prolongation process can be realized very simple and efficient. In principle, an ACA-compressed BEM matrix $\boldsymbol{A}_{h}$ can be separated into nearfield and farfield part

$$
\boldsymbol{A}_{h}=\boldsymbol{A}_{h}^{\mathrm{far}}+\boldsymbol{A}_{h}^{\text {near }}
$$

as described in Section 4.3.1.6, with the sparse matrix $\boldsymbol{A}_{h}^{\text {near }}$ and the approximate part $\boldsymbol{A}_{h}^{\text {far }}$. The farfield part consists of $n_{l}$ low-rank submatrices

$$
\boldsymbol{A}_{h}^{\mathrm{far}}=\sum_{i=1}^{n_{l}} \sum_{j=1}^{r_{i}} \vec{u}_{j}^{i}\left(\vec{v}_{j}^{i}\right)^{\top}
$$

with rank $r_{i}$ of the $i$ th approximated block. Applying the Galerkin operator (7.5),

$$
\boldsymbol{A}_{H}=\boldsymbol{\mathcal { R }}_{h}^{H}\left(\boldsymbol{A}_{h}^{\text {far }}+\boldsymbol{A}_{h}^{\text {near }}\right) \mathcal{P}_{H}^{h}
$$

follows for the coarse-level matrix. The nearfield matrix can be treated in a similar manner, compared to sparse FE-matrices. Consequently, the coarse nearfield matrix can be computed as

$$
\boldsymbol{A}_{H}^{\text {near }}=\boldsymbol{\mathcal { R }}_{h}^{H} \boldsymbol{A}_{h}^{\text {near }} \mathcal{P}_{H}^{h}
$$

The farfield part results in

$$
\begin{aligned}
\boldsymbol{A}_{H}^{\mathrm{far}} & =\boldsymbol{\mathcal { R }}_{h}^{H} \sum_{i=1}^{n_{l}} \sum_{j=1}^{r_{i}} \vec{u}_{j}^{i}\left(\vec{v}_{j}^{i}\right)^{\top} \mathcal{P}_{H}^{h} \\
\stackrel{(7.7)}{=} & \sum_{i=1}^{n_{l}} \sum_{j=1}^{r_{i}} \boldsymbol{\mathcal { R }}_{h}^{H} \vec{u}_{j}^{i}\left(\boldsymbol{\mathcal { R }}_{h}^{H} \vec{v}_{j}^{i}\right)^{\top} .
\end{aligned}
$$

Applying a substitution $\vec{s}_{j}^{i} \triangleq \boldsymbol{\mathcal { R }}_{h}^{H} \vec{u}_{j}^{i}$ and $\vec{t}_{j}^{i} \triangleq \boldsymbol{\mathcal { R }}_{h}^{H} \vec{v}_{j}^{i}$, we finally obtain

$$
\boldsymbol{A}_{H}^{\mathrm{far}}=\sum_{i=1}^{n_{l}} \sum_{j=1}^{r_{i}} \vec{s}_{j}^{i}\left(\vec{t}_{j}^{i}\right)^{\top},
$$

which gives an analog formulation to (7.18). This result can be advantageous for the numerical realization of the coarsening-process. Especially, the same ACA-datastructure for the fine-grid BEM-matrices $\boldsymbol{A}_{h}$ and the corresponding coarse-level matrix $\boldsymbol{A}_{H}$, can be utilized.

\subsubsection{Coarse-Level Solution}

For an efficient solution of the equation system $\boldsymbol{A}_{H} \vec{v}_{H}=\vec{b}_{H}$ on the coarsest level, a direct solver is sufficient to achieve good accuracy and efficiency. The computation of the inverse $\boldsymbol{A}_{H}^{-1}$ is impossible to realize, if no explicit description of the data-sparse ACA-based BEM matrix is available. Iterative methods can be helpful to increase the performance in computing a good approximation [148]. 


\subsubsection{Complexity Analysis}

As shown in Chapter 5, the matrix-vector multiplication as the dominant arithmetic operation in iterative algorithms, directly bounds the expenditure for the construction of an AMG preconditioner [148]. The main goal for the handling of dense BEM matrices in iterative methods is, to reduce the cost for arithmetic operations and memory requirements. For evaluation purposes, the computational as well as the memory complexity for fine-grid problem dimension $n_{h}$ and coarselevel problem dimension $n_{H}$ are given in Table 7.1 and 7.2, respectively, with the ACA accuracy denoted by $\varepsilon$ and $\kappa \geqslant 0$ (The parameter $\kappa$ depends on the behavior of the kernel function).

\begin{tabular}{|l||c|c|c|}
\hline & classical (full) BEM & reluctance PEEC & ACA-BEM \\
\hline \hline smoothing & $\mathcal{O}\left(n_{h}^{2}\right)$ & $\mathcal{O}\left(n_{h}\right)$ & $\mathcal{O}\left(\varepsilon^{-\kappa} n_{h}^{1+\kappa} \log n_{h}\right)$ \\
\hline prol./restr. & $\mathcal{O}\left(n_{h}\right)$ & $\mathcal{O}\left(n_{h}\right)$ & $\mathcal{O}\left(n_{h}\right)$ \\
\hline Galerkin transf. & $\mathcal{O}\left(n_{h} n_{H}\right)<\mathcal{O}\left(n_{h}^{2}\right)$ & $\mathcal{O}\left(n_{h} n_{H}\right)<\mathcal{O}\left(n_{h}^{2}\right)$ & $\mathcal{O}\left(\varepsilon^{-\kappa} n_{h}^{1+\kappa} \log n_{h}\right)$ \\
\hline \hline total & $\mathcal{O}\left(n_{h}^{2}\right)$ & $\mathcal{O}\left(n_{h} n_{H}\right)$ & $\mathcal{O}\left(\varepsilon^{-\kappa} n_{h}^{1+\kappa} \log n_{h}\right)$ \\
\hline
\end{tabular}

Table 7.1: Computational complexity of AMG component construction for classical, reluctance-based and ACABEM-based system matrices.

\begin{tabular}{|l||c|c|c|}
\hline & classical (full) BEM & reluctance PEEC & ACA-BEM \\
\hline \hline $\boldsymbol{A}_{h}$ & $\mathcal{O}\left(n_{h}^{2}\right)$ & $\mathcal{O}\left(n_{h}\right)$ & $\mathcal{O}\left(\varepsilon^{-\kappa} n_{h}^{1+\kappa} \log n_{h}\right)$ \\
\hline $\boldsymbol{B}_{h}$ & $\mathcal{O}\left(n_{h}\right)$ & $\mathcal{O}\left(n_{h}\right)$ & $\mathcal{O}\left(n_{h}\right)$ \\
\hline \hline total & $\mathcal{O}\left(n_{h}^{2}\right)$ & $\mathcal{O}\left(n_{h}\right)$ & $\mathcal{O}\left(\varepsilon^{-\kappa} n_{h}^{1+\kappa} \log n_{h}\right)$ \\
\hline
\end{tabular}

Table 7.2: $\quad$ Memory complexity for the storage requirements of matrices $\boldsymbol{A}_{h}$ and $\boldsymbol{B}_{h}$ for classical, reluctance-based and ACA-BEM-based system matrices.

It can be observed, that the computational complexity resulting from reluctance-based PEEC matrices, strongly depends on the number of coarse levels, while the ACA-based PEEC matrices closely reach the optimal bound for suitably chosen parameters $\varepsilon$ and $\kappa$. Concerning memory complexity, the storage requirements for matrices $\boldsymbol{A}_{h}$ and $\boldsymbol{B}_{h}$ are optimal or almost optimal in both cases.

\subsection{Implementation Details for PEEC-Based Circuit Simulation}

For an efficient implementation, a multigrid-based solver strategy usually has to be tailored to the specific simulation problem [157]. Indeed, AMG provides a kind of "black-box" solver, since the operators can - in principle - be constructed without referring to a grid. The parameters, however, have to be chosen suitably to achieve acceptable accuracy and good convergence behavior. Moreover, multigrid methods work well for elliptic PDEs in general, but often they can not achieve the same efficiency together with problems of different nature. Grid and relaxation methods as well as restriction and interpolation are highly problem-dependent. A standard interpolation, however, is sufficient in the most cases. The handling of structured grids is unproblematic, in general. Together with unstructured grids as occuring in PEEC, the interpolation matrix can be computed in 
a similar manner. The interplay between relaxation, restriction and interpolation, however, is an important and often difficult part. For circuit-based EMC simulation the standard AMG software library SAMG [28] is used. SAMG is a Fortran90-based multigrid library, containing a variety of coarsening-, interpolation-, smoothing- and acceleration-strategies. Built-up in a modular manner, each of the main components of an AMG algorithm can be applied separately. SAMG can be advised to reuse parts of the setup if the matrix properties only slightly differ in a series of matrix equations, as it usually occurs in TD as well as FD circuit simulation. The matrix data has to be provided to SAMG in the compressed sparse row format, as described in Section 5.5. A PEEC-based circuit simulator, as depicted in Figure 7.5, serves as a platform for fundamental AMG investigation. The setup of the system matrix is performed using a conventional stamping

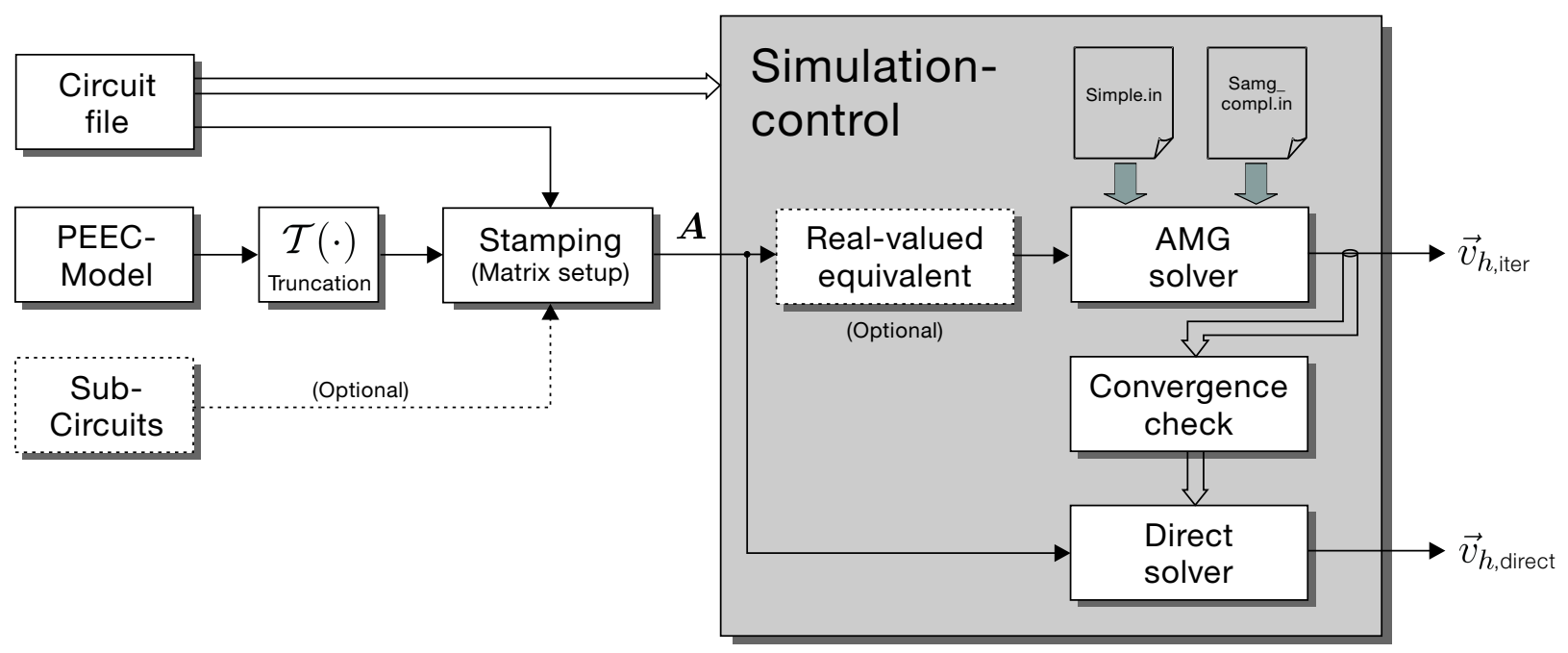

Fig. 7.5: Block diagram for the implementation of SAMG into an existing circuit-based EMC simulator. A direct solver is provided as a fallback-solution.

procedure, including the PEEC model and arbitrary subcircuits. The simulation control interpretes all commands provided in the circuit file and manages the simulation workflow. System matrices resulting in TD analysis are real-valued. The treatment of real-valued matrices is directly supported by the SAMG library. In the case of FD analysis the system matrix is complex-valued, in general, and needs to be transformed to a real-valued equivalent system (refer to (5.7)). In the following, the AMG solver library executes the solution process for each simulation step, including necessary control parameters from the files simple.in and samg_complex.in. As a fallback strategy an automatic switching to a direct solver guarantees the existence of a plausible solution. This can be necessary, especially in the FD for very large frequencies, due to an ill-conditioning of the system. The direct solver can be a Gauß-solver with full/partial pivoting strategy. A reordering strategy for fill-in reduction and the application of an adaptive memory allocation strategy can be meaningful. For PEEC-based matrices a point-based AMG [157] strategy seems suitable at first glance. Together with SPD matrices, as resulting from NA-based circuit simulation (refer to Appendix D), a Gauß-Seidel (GS) smoother usually leads to a good convergence behavior. A block-GS version, moreover, provides good parallelization properties. Parallelization of AMG components is efficient and usually simple to realize. Compared to conventional parallel solvers, however, it requires more communication overhead. To enable an accurate solution in a very high frequency range, an incomplete LU-factorization (ILUT)-based smoother should be prefered. The ILUT level of fill- 
in will be adaptively increased, if the convergence behavior degrades. BiCGstab or GMRes are used as accelerators. In accordance with [3], a frequency-dependence of the convergence behavior can be observed. This effect directly depends on the ratio $\lambda / h$ of wavelength and meshsize. In accordance with Section 3.7, the bound $h \leqslant \lambda / 10$ [3] should be guaranteed. For smaller ratios, however, the discretization is too coarse to accurately represent the oscillations in Green's function. As a result, the linear system does not represent the EFIE any more and the preconditioner fails. In the case of BEM-based system matrices, the bottleneck regarding time and storage can be circumvented by applying matrix compression techniques as the FMM or hierarchical matrices.

\subsection{Numerical Results}

A standard direct solver (GMATRIX) [73] and an iterative, adaptive ILUT(fill-in,droptol)-AMGBiCGstab solver, being part of the SAMG library [28], are compared for two test cases. One test case is a PEEC FD analysis for the range $10^{2}$ to $10^{9} \mathrm{~Hz}, 10$ frequency points per decade. The other one is a PEEC TD analysis with final time $7 \cdot 10^{-7} s$ and 7 time steps of length $10^{-7} s$. More details of these two cases are given in Table 7.3. The numerical experiments were performed on a Intel(R) Xeon(TM) $3.2 \mathrm{GHz}$ CPU with cache size $1024 \mathrm{~KB}$ running Linux. The results for both test cases

\begin{tabular}{|l||c|c|c|c|c|c|}
\hline \multicolumn{1}{|l||}{ type of } & no. & no. & \multicolumn{2}{|c|}{ no. of non-zeros } & \multicolumn{2}{c|}{ matrix density } \\
simulation & steps & rows & min. & max. & min. & max. \\
\hline \hline TD & 8 & 2026 & 6884 & 172562 & 0.17 & 4.20 \\
FD & 61 & 14206 & 55338 & 2532318 & 0.03 & 1.25 \\
\hline
\end{tabular}

Table 7.3: Details (matrix properties) of the two test cases. For FD analysis data refer to matrices obtained from a transformation of the original, complex linear systems into equivalent real-valued linear systems. The matrix densities are given in $\%$.

show that SAMG outperforms the GMATRIX solver in terms of both, memory and computational speed (refer to Table 7.4). In both cases, SAMG is already about 10 times faster than GMATRIX. However, the speed-up for a PEEC FD analysis could be much better if computation times for the high-frequency range would be as small as for the low-frequency range (refer to Figure 7.8). A similar statement holds for memory requirements. The SAMG solver also yields small residuals (refer to Figures 7.7 and 7.10), which indicates that the computed approximations are sufficiently accurate. Figure 7.12 illustrates the influence of the type of multigrid cycle to the convergence behavior for the case of a PEEC matrix with dimension $1024 \times 1024$ and density $\approx 5 \%$. The applied iterative BGS ${ }^{10}$-AMG-GMRes solver, which is also part of the SAMG library, shows good performance for $\mathrm{V}$-, W-, and F-cycles. The best result, however, can be achieved using the V-cycle.

Remark: In FD analysis a degeneration of the convergence behavior can be observed in higher frequency ranges (refer to Figures 7.6 and 7.7 for matrix numbers $>21$ ). An adaptive parameter switching can help to improve the solving process (matrix numbers $\geqslant 33$ ). Higher complexity of the solver, however, directly leads to increased CPU times for a single solving step (refer to Figure 7.8).

\footnotetext{
${ }^{10}$ Block-Gauß-Seidel
} 


\begin{tabular}{|l|l||r|r|r|}
\hline $\begin{array}{l}\text { type of } \\
\text { simulation }\end{array}$ & solver & run time & speed-up & peak memory \\
\hline \hline TD & GMATRIX & 245 & - & 64 \\
& SAMG & 23 & 10.65 & 27 \\
\hline \multirow{2}{*}{ FD } & GMATRIX & 151071 & - & 2100 \\
& SAMG & 15776 & 9.58 & 1200 \\
\hline
\end{tabular}

Table 7.4: Performance of GMATRIX and SAMG summarized for the two test cases. Run times are total simulation run times in seconds. Memory requirements are in MBytes.

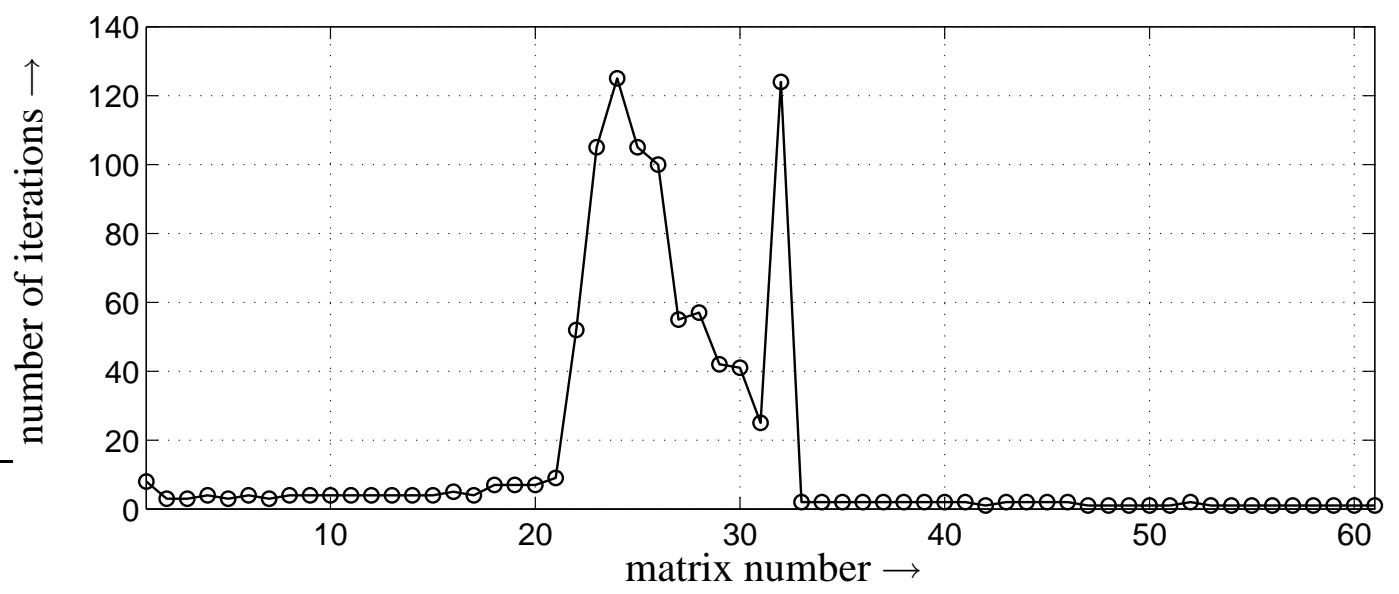

Fig. 7.6: SAMG solver: Required numbers of iterations to reach a residual of $10^{-8}$ for a PEEC FD analysis.

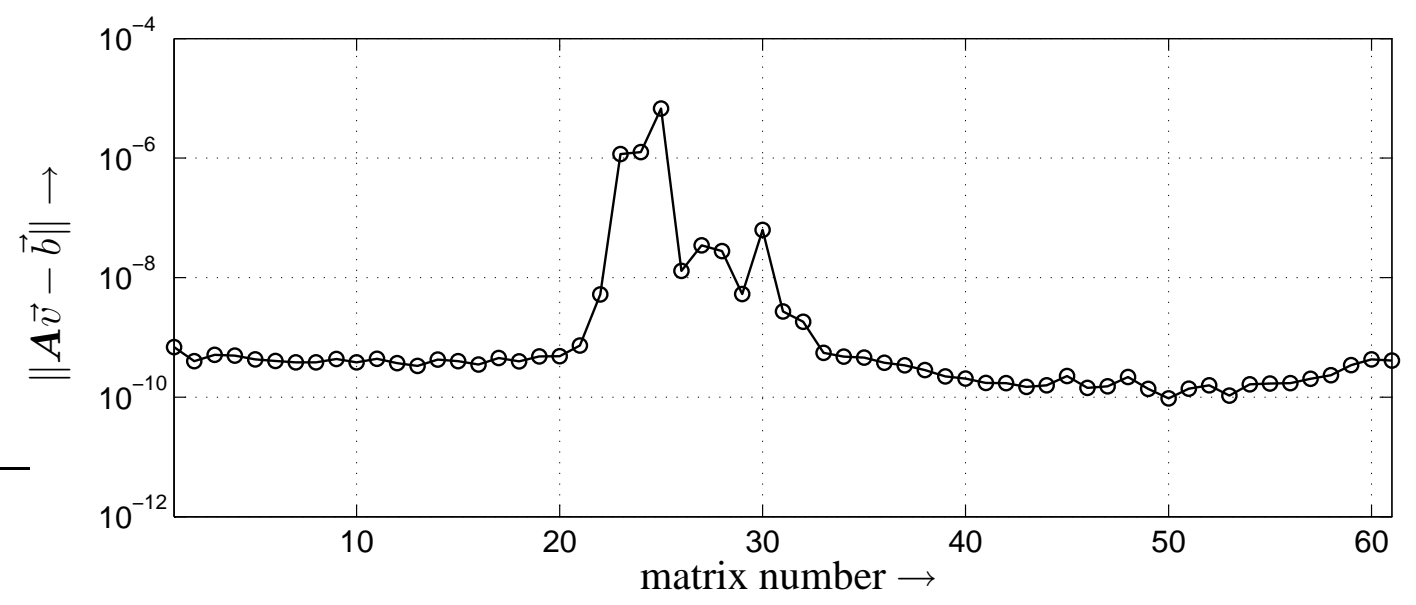

Fig. 7.7: SAMG solver: $L_{2}$-norm of final residual for a PEEC FD analysis. 


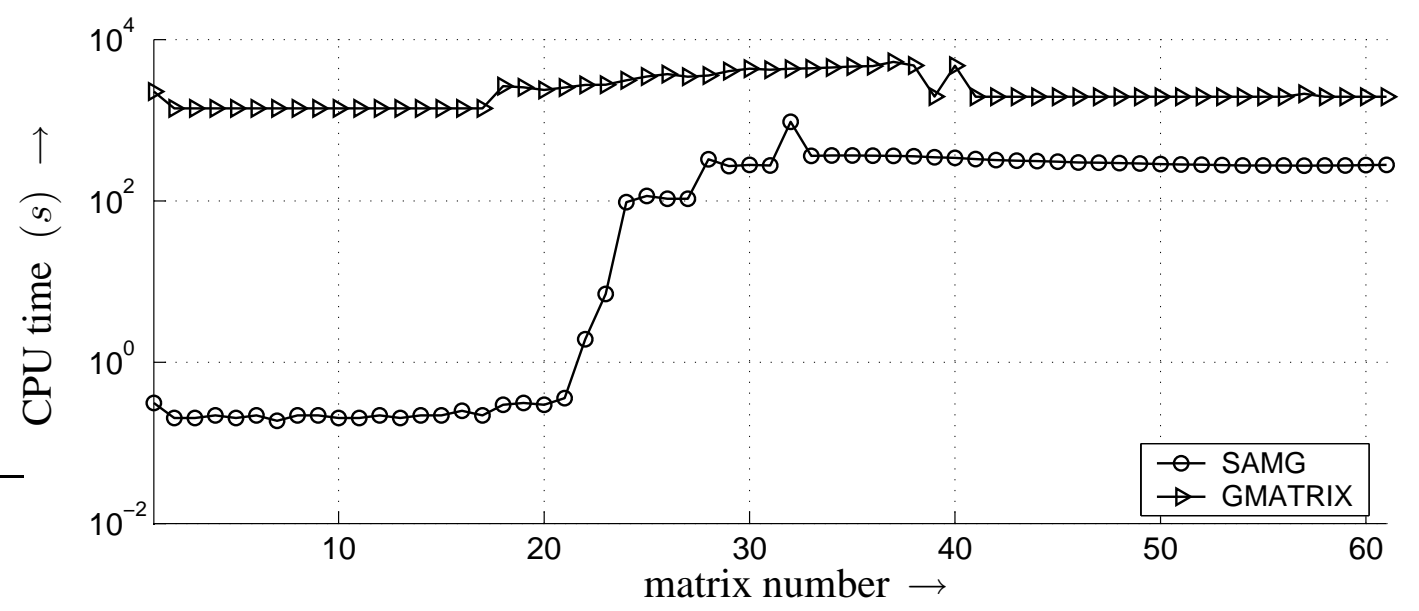

Fig. 7.8: SAMG solver: Total CPU-times for a PEEC FD analysis.

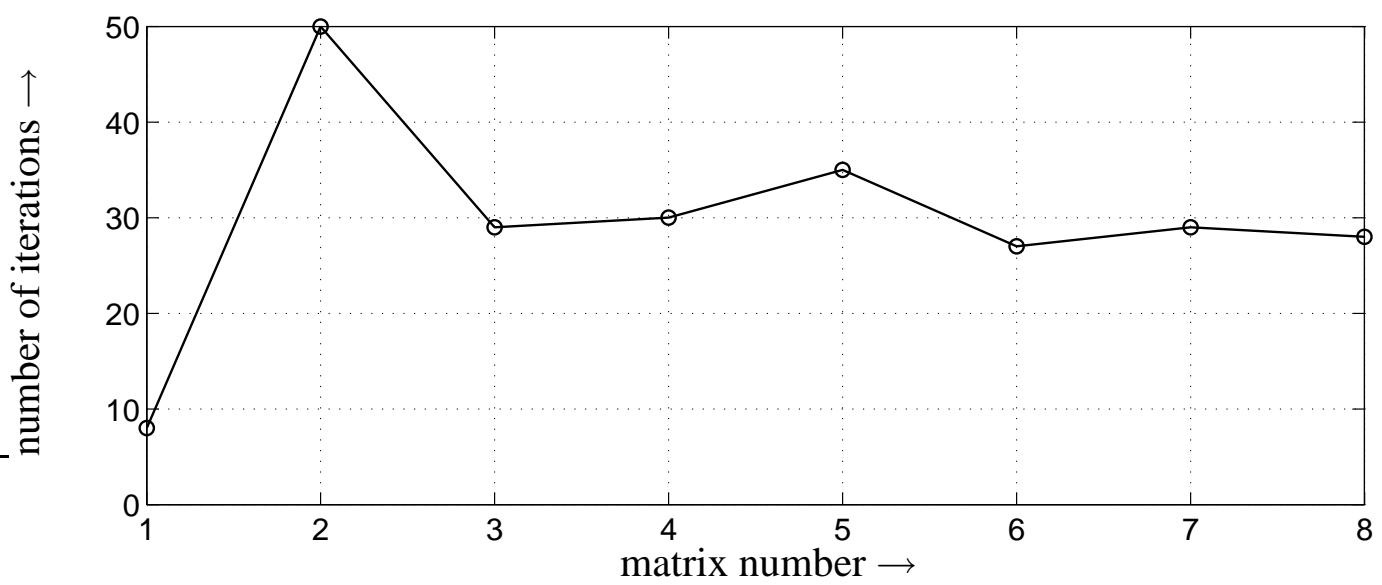

Fig. 7.9: SAMG solver: Required numbers of iterations to reach a residual of $10^{-8}$ for a PEEC TD analysis.

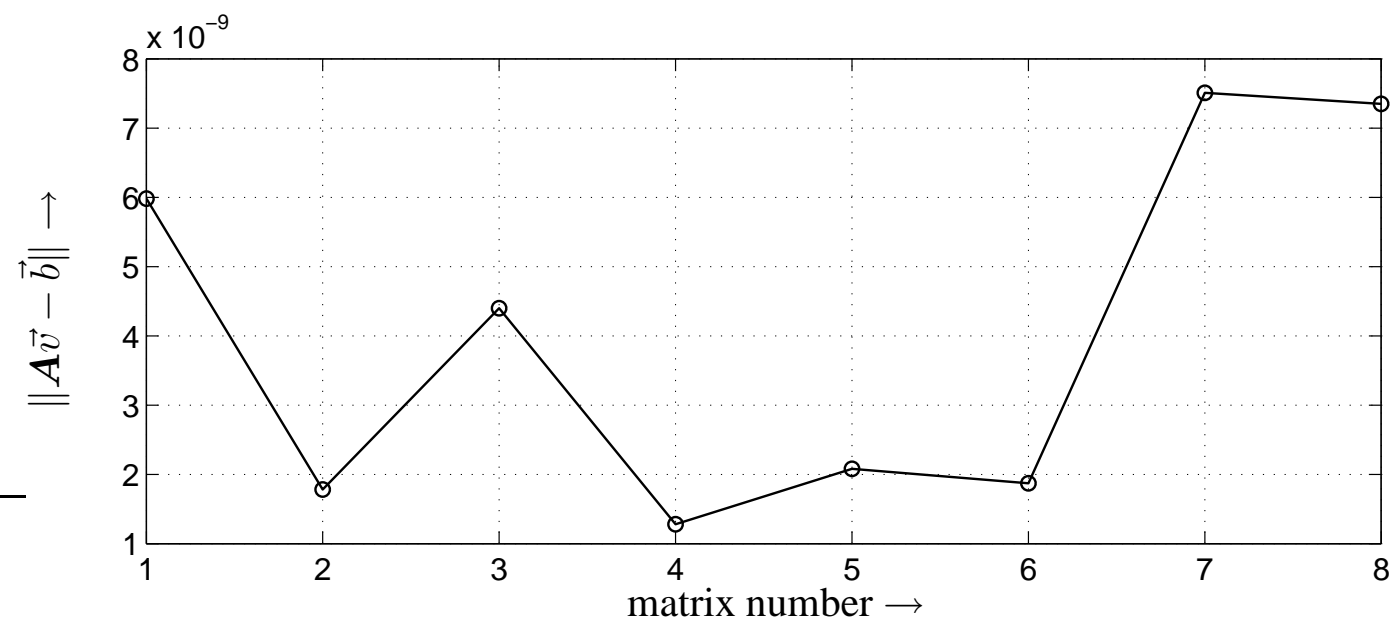

Fig. 7.10: SAMG solver: $L_{2}$-norm of final residual for a PEEC TD analysis. 


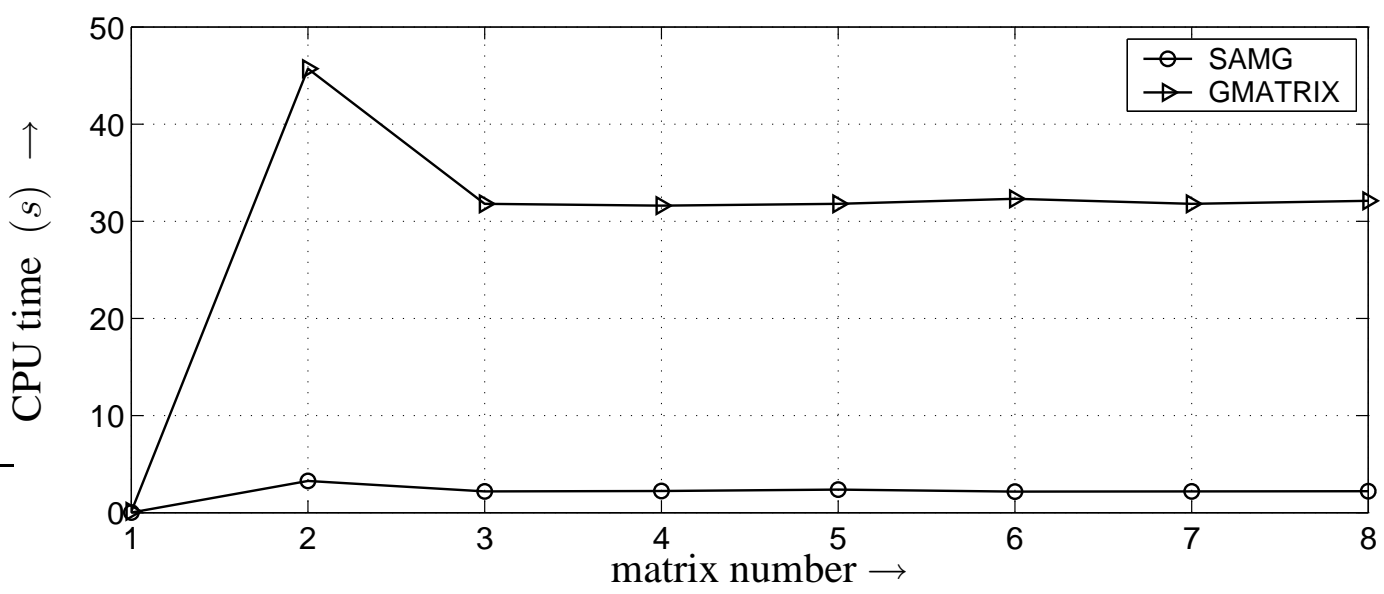

Fig. 7.11: SAMG solver: Total CPU-times for a PEEC TD analysis.

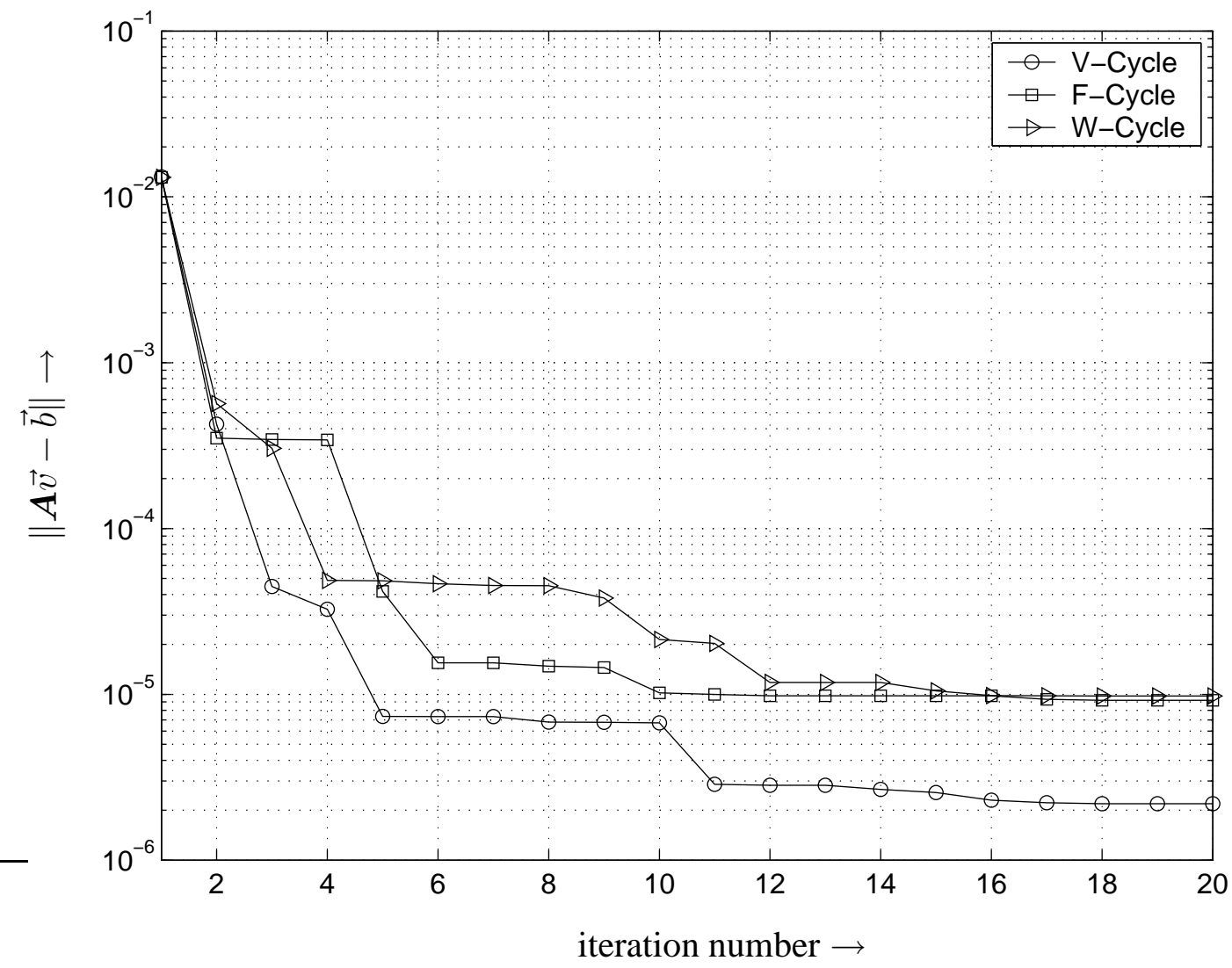

Fig. 7.12: Convergence behavior comparison for a PEEC matrix of dimension 1024 in the FD, applying different multigrid cycles in a GMRes-BGS iterative method. 


\section{8 \\ Parallelization Aspects for Hierarchical Methods}

$M$

ultigrid-based methods as well as $\mathcal{H}$-matrix techniques usually offer high efficiency together with iterative solution methods for circuit simulation purposes. An additional speed-up, however, often can only be achieved by applying parallelization techniques. Only the most powerful and sequentially fastest algorithms can be considered to realize optimal parallel solvers for complex industrial applications. This chapter presents some theoretical basics and concrete practical directives for an efficient parallelization.

This Chapter is organized as follows. After a short overview in Section 8.1, some theoretical performance basics of parallel computations and some specific properties of existing parallelization architectures are described in Sections 8.2 and 8.3. Concrete parallelization details for AMG as well as $\mathcal{H}$-matrices are outlined in Sections 8.4 and 8.5. Section 8.6 reflects parallelization aspects for circuit simulation and some implementation details.

\subsection{Overview}

A decomposition of the domain of interest into subdomains respresents a common parallelization strategy in numerical field computation. Orthogonal (rectangular) meshes easily can be partitioned into boxes or cubes, enabling a balanced workload. For unstructured meshes, however, an equal amount of work has to be assigned to each processor. Furthermore, a minimization of the communication overhead between the processors has to be aspired by essentially minimizing the surface area of the subdomain. The adoption of parallel computing is only efficient if the benefit, resulting from the speed-up of the computations, outperforms the communication overhead between the processors and the work is distributed equally to the processors [170]. As a precondition for an adequate achievable speed-up, the operations to be executed must allow a simultaneous processing in 
addition to an equally distributed workload. The subproblems assigned to each processor are still coupled. This makes a data-transfer between the processors necessary. These data-transfer to be minimized by ensuring uniform distribution of the load, is a high-dimensional discrete optimization problem, a so-called load-balancing problem. Iterative methods are easier to implement on parallel computers than sparse direct methods [147]. In iterative algorithms usually matrix-vector multiplication operations are parallelized since they can be implemented on high-performance computers in an easy manner. Especially the application of the CSR/CSC matrix storage format (refer to Section 5.5) turns out to be advantageous for parallel computations. To implement the computation of the matrix-vector product $\vec{y}=\boldsymbol{A} \vec{x}$ in parallel using the CSR/CSC-format, it has to be taken into account that each component of the resulting vector $\vec{y}$ can be computed independently from the remaining as the dot-product of the $i$ th row of the matrix with vector $\vec{x}$. For a more detailed view refer to [147]. The design and implementation of parallel algorithms requires an exact model of the underlying computer system [171]. The "von Neumann" model is often used togehter with sequential computing and enables the design of algorithms with predictable execution time on almost any computer system. For parallel systems, however, various models (e.g. parallel RAM (PRAM)) exist, trying to emulate the different aspects of real computer systems. Due to the fact that most of the computer languages do not support the parallel computation, a special interface is necessary to utilize the shared memory together with more than one processors. A realization can be achieved by using so-called threads on shared memory systems.

\subsection{Parallel Performance Basics}

An evaluation of parallel algorithms can be performed by considering the parallel speed-up and efficiency. The parallel speed-up $S_{p}$ for the solution of a problem of dimension $n$ on $p$ processing elements is defined by

$$
S_{p} \triangleq \frac{t(1, n)}{t(p, n)}
$$

with the time $t(\ell, n)$ required by a parallel algorithm $\mathcal{A}$ to perform the computation on $\ell$ processing elements. If a very fast but not parallelizable solution method on a single processor exists, the real parallel speed-up can only be achieved if the fastest parallel method will be compared with the fastest serial method. As a result, the solution strategies which are applied to one and $p$ processors can differ. The time $t(p)$, necessary to perform a computation on $p$ processors can be decomposed into

$$
t(p)=t_{s}+t_{p}+t_{0}
$$

with serial operation time $t_{s}$, actual computation time $t_{p}$ on each processor and the communication overhead $t_{0}$. The communication cost is defined as

$$
C \triangleq \frac{t_{0}}{t_{p}}
$$

with the communication overhead significantly be affected by the load-balancing. The parallel efficiency $E_{p}$ of algorithm $\mathcal{A}$ is defined as the speed-up to number of processor ratio

$$
E_{p} \triangleq \frac{S_{p}}{p}=\frac{t(1, n)}{p t(p, n)}
$$


aiming at an optimal workload-balancing. When a task is subdivided between too many processors, the efficiency of the parallelization reaches a limit because the computational task is reduced to an amount where it becomes of the same order as the communication task. The aim is, to realize (almost) optimal hierarchical methods, including strategies for parallel distributed memory implementations. Parallelism can be exploited by assigning disjoint subsets on leaf-boxes (refer to Section 4.3.1.3) to different processors. The optimal behavior would be, to achieve constant solution time when the problem size and the number of processors increase proportionally. Generally spoken, the amount of work on each processor and the number of iterations should remain constant for increasing problem sizes. If a non-optimal method is applied on a parallel system, there always will exist a problem size for which an optimal algorithm will run faster on a serial system. Therefore, it is absolutely necessary to apply optimal algorithms in combination with parallel implementations. For very large problems it is practical to keep the problem size per processor constant instead of the total problem size, while measuring the speed-up. That means, one measures the so-called scaled speed-up

$$
S_{s}=\frac{t(1, p n)}{t(p, p n)}
$$

for a given problem size $p n$ leading to a scaled efficiency of

$$
E_{s}=\frac{S_{s}}{p}
$$

The parallel implementation of algorithms has a great impact to the achievable complexity. Even if the algorithm exhibits adequate parallelism an inadvantageous implementation can degrade the overall performance drastically [172].

\subsection{Parallelization Architectures}

There exist three architecture models [147] for parallel implementation:

- the shared memory model;

- SIMD or data parallel models;

- the distributed memory message passing model.

W.r.t. the importance for AMG and $\mathcal{H}$-matrices only the shared memory and the distributed memory models are explained in more details.

\subsubsection{Shared Memory Systems}

To process parallel computations on a shared memory system one often applies independent and parallel execution paths in a single task, so-called threads. All threads are part of the same task, leading to the fact that all threads share the same address space and therefore no communication between the individual processors is necessary. An implementation can be realized using so-called POSIX-threads (also known as PThreads) as well as Threadpool [118]. Shared memory systems show various advantages [147] for the parallel implementation of hierarchical algorithms. The processors are connected to a large global memory and the address spaces are identical for all processors. Therefore, fast and simple access to data in memory with only minor dependence on the location can be realized. Possible memory- and access-violations respesent a main disadvantage. 


\subsubsection{Distributed Memory Systems}

W.r.t. [147], two different variants of distributed memory systems exist - the message passing architecture (MP) and the distributed memory SIMD computers, containing large numbers of identical processors. These processors possess their own memories and work interconnected in a regular topology. In MP there is no global synchronization of parallel tasks. Instead, computations are data-driven since a processor only carries out a task if the operand needed is available. Each data exchange between processors has to be explicitely programmed. A big advantage is the ability to exploit locality of data in order to keep communication cost to a minimum. Together with strong locality a data exchange between directly neighbouring processors is absolutely sufficient in this case. Two different variants of distributed memory computers exist - SIMD and MIMD. From [166] it is known that parallel approaches on distributed memory computers show good results as long as the number of variables per processor is large enough $(n \approx 20000 \ldots 30000)$.

\subsection{Parallelization of AMG}

For a parallelization of algebraic multigrid algorithms the application of a domain decomposition approach proved to be useful [17]. In contrast to geometric multgrid methods the coarsening hierarchy and the corresponding coarse-grid operators in AMG develop dynamically from one level to another why a parallelization is not trivial, in general [166]. Moreover, a "naive" parallelization would require upredictable and very complicated communication processes. As a consequence, a serious limitation of the achievable scalability can occur. For an efficient parallel implementation a good balance between setup times, convergence rates and cost per iteration is necessary. These features, in turn depend on operator's complexity, coarsening rates and smoother's effectiveness [172]. Applying algorithmic modifications, the communication exchange can be reduced at first glance. The convergence behavior, however, has to be supervised carefully. As a main part, the original sequential coarsening strategy has to be replaced by its parallel equivalent. The parallelization of AMG bases on a partitioning of all variables, similar to the parallelization of GMG. The variables $\Omega^{\ell}$ on each AMG level are distributed on $P$ processors by

$$
\Omega^{\ell}=\bigcup_{p=1}^{P} \Omega_{p}^{\ell},
$$

where $\Omega_{p}^{\ell}$ denotes the subset of variables assigned to processor $p$. Thus, all rows of $\boldsymbol{A}^{(\ell)}$ and $\mathcal{P}_{\ell+1}^{\ell}$ belonging to $i \in \Omega_{p}^{\ell}$ are assigned to processor $p$ [166]. The corresponding sets of matrix rows are denoted by $\boldsymbol{A}^{(\ell)}[p]$ and $\mathcal{P}_{\ell+1}^{\ell}[p]$, repectively. While for $\ell=1$ a suitable load-balancing procedure has to be provided by the user, for $\ell>1$ all variables are assigned to the same processor corresponding to their finest level. During the AMG setup-phase the $C / F$-splitting $\Omega^{\ell}=C^{\ell} \cup F^{\ell}$ has to be recursively applied in parallel, i.e. each processor $p$ constructs

$$
\Omega_{p}^{\ell}=C_{p}^{\ell} \cup F_{p}^{\ell}
$$

independently from the remaining processors. The AMG solution-phase remains as in standard GMG where processor $p$ only requires access to data of its neighbouring processor. For an efficient application of AMG in parallel architectures a load-balance optimization on level $\ell$ is necessary, since the AMG coarse-level strategy can cause load-imbalance problems. This optimization needs a re-mapping of data, causing an additional communication overhead [166]. 


\subsubsection{Implementation Issues}

The standard coarsening algorithms for structured problems in AMG can be applied in parallel in a straightforward manner. For unstructured problems, however, the coarsening algorithms are highly-recursive and therefore not applicable for parallel computing. Traditional smoothers such as Gauß-Seidel are inherently sequential. Smoothers as multicolor Gauß-Seidel smoothers, hybrid Gauß-Seidel with relaxation, weights smoothers, polynomial smoothers or sparse approximate inverse (SAI) smoothers are more suited for parallel implementations. The coarse-grid solver can be realized as a direct or iterative solver. If the problem is small enough, it can be solved completely using one processor. The remaining processors are free for other computations during this time. On the other hand, a parallel computation is necessary if the problem is too large to solve it on one single processor. The communication overhead can be reduced by applying a subset of processors for the solution process.

\subsection{Parallelization of $\mathcal{H}$-Matrices}

For the parallelization of $\mathcal{H}$-matrices, shared as well as distributed memory systems are suitable. To achieve maximum efficiency, both approximating the discrete operator by hierarchical matrices and the realization of the $\mathcal{H}$-matrix-vector multiplication must be realized in parallel. A complexity of

- $\mathcal{O}\left(\frac{1}{p} n \log ^{2 d-1} n\right)$ for parallel $\mathcal{H}$-matrix approximation and

- $\mathcal{O}\left(\frac{1}{p} n \log ^{d} n\right)$ for parallel matrix-vector multiplication

with the number of unknowns $n$ and the number of processors $p$ can be achieved [118]. The $\mathcal{H}$-approximant needs $\mathcal{O}\left(\frac{1}{p} n \log ^{d} n\right)$ units of storage on each processor.

\subsubsection{Parallel $\mathcal{H}$-Matrix Approximation}

The cost for computing an $\mathcal{H}$-matrix-block $\boldsymbol{M}_{b}$ can not be determined in advance. Consequently, online scheduling techniques are preferable for parallelization purposes. The parallelization has to be initiated, starting with the leaves since computing the leaves is one of the most complex operation [171]. The computation of the low-rank approximations can simply be parallelized since no dependence between the individual blocks exists. Varying block sizes, however, lead to varying computational cost for the single blocks. A parallel implementation of the $\mathcal{H}$-matrix-vector multiplication therefore is not obvious at first glance [118]. The sequential Algorithm 5 for $\mathcal{H}$-matrix approximation of matrix $\boldsymbol{A} \in \mathbb{R}^{n \times n}$ constitutes the basis for parallel implementation on shared as well as on distributed memory systems. To achieve optimal parallel speed-up, prior knowledge concerning the amount of work per matrix-block is necessary to realize optimal load-balancing. Such information, however, unfortunately is not available if the low-rank approximation is executed with fixed rank. An adaptively chosen rank for each block can help to avoid these problem. Indeed, the actual cost per matrix-block are unknown. As an alternative to a cost-related balancing algorithm, a list-scheduling scheme assigns the next not yet executed job to the first idle processor, independently of the amount of work associated with the job [118]. It has to be noticed that listscheduling leads to a not optimal processing workflow. The cost for a parallel $\mathcal{H}$-approximation are of order $\mathcal{O}\left(\frac{1}{p} n \log ^{2 d-1} n\right)$. 


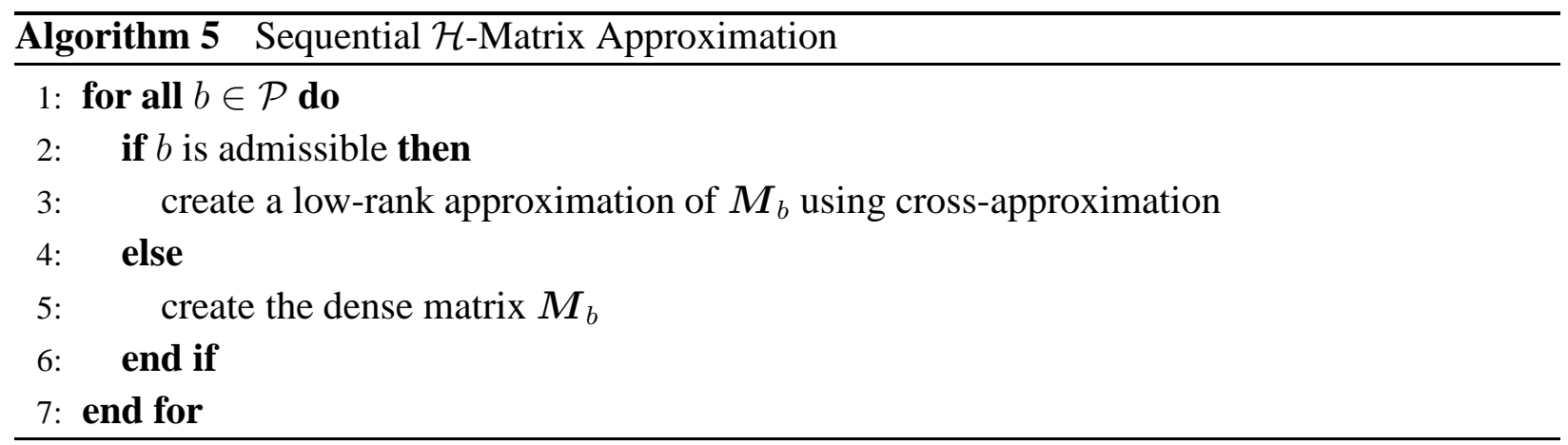

\subsubsection{Parallel $\mathcal{H}$-Matrix-Vector Multiplication}

Access conflicts usually can origin from the application of list-scheduling in a sequential $\mathcal{H}$-matrixvector multiplication algorithm. The reason is, that multiple processors can access to the same part of the solution vector. A required blocking of processors, however, can lead to a reduced parallel efficiency. As an alternative the multiplication can be splitted into two phases. The application of sequence-partitioning and so-called space-filling curves can help to avoid that matrix blocks are assigned independently to processors. Figure 8.1 graphically illustrates the fundamental difference between list-scheduling and sequence-partitioning together with a parallel $\mathcal{H}$-matrix-vector multiplication.

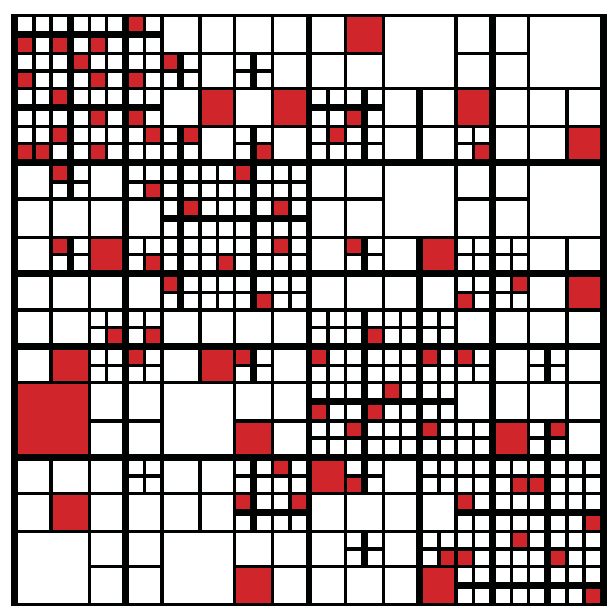

a)

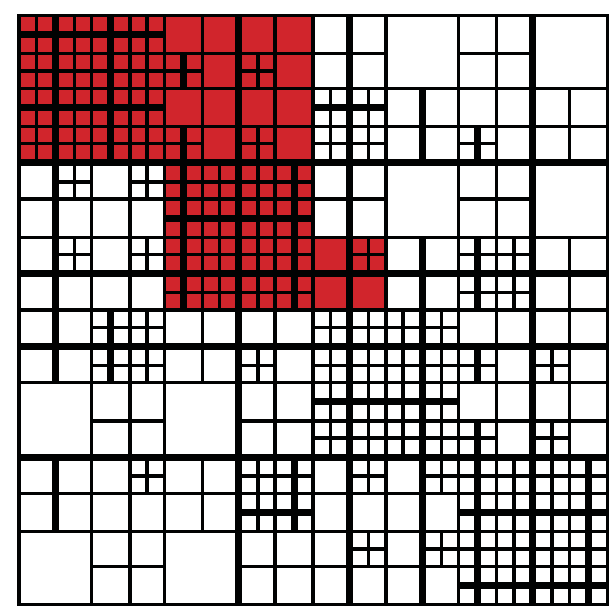

b)

Fig. 8.1: Scheduling for parallel $\mathcal{H}$-matrix-vector multiplication. Matrices are scheduled to the same processor with a) list-scheduling and b) sequence-partitioning, referring to [171].

\subsubsection{Parallel $\mathcal{H}$-Matrix Inversion}

W.r.t. efficient preconditioning, the inverse of a $2 \times 2$ block-matrix of the form

$$
\boldsymbol{A}=\left(\begin{array}{ll}
\boldsymbol{A}_{00} & \boldsymbol{A}_{01} \\
\boldsymbol{A}_{10} & \boldsymbol{A}_{11}
\end{array}\right)
$$

can be written as [171]

$$
\boldsymbol{A}^{-1}=\left(\begin{array}{cc}
\boldsymbol{A}_{00}^{-1}+\boldsymbol{A}_{00}^{-1} \boldsymbol{A}_{01} \boldsymbol{S}^{-1} \boldsymbol{A}_{10} \boldsymbol{A}_{00}^{-1} & -\boldsymbol{A}_{00}^{-1} \boldsymbol{A}_{01} \boldsymbol{S}^{-1} \\
-\boldsymbol{S}^{-1} \boldsymbol{A}_{10} \boldsymbol{A}_{00}^{-1} & \boldsymbol{S}^{-1}
\end{array}\right),
$$


and recursively be accomplished using the SCHUR complement

$$
\boldsymbol{S} \triangleq \boldsymbol{A}_{11}-\boldsymbol{A}_{10} \boldsymbol{A}_{00}^{-1} \boldsymbol{A}_{01}
$$

Resulting from the sequential character of these inversion algorithm, a parallelization can not be realized with optimal efficiency. A distribution of the required $\mathcal{H}$-matrix-vector multiplications to $p$ processors, especially for very small blocks, is not possible in principle. The introduction of a minimal block size can help to define a bound, serving as an indicator for a switching from a parallel to a serial computation strategy.

\subsubsection{Implementation Issues}

Shared memory systems are advantageous for parallel $\mathcal{H}$-matrix implementations for two reasons. On the one hand, existing sequential methods can be reused. Otherwise, an update of the consisting method can be realized with very small effort [171]. In conjuction with simple but efficient load-balancing algorithms for $\mathcal{H}$-matrices as online-scheduling (e.g. list-scheduling, sequence-partitioning) and offline-scheduling, shared memory systems show very good results. While an online-scheduling algorithm assigns a job to an individual processor during execution, an offline-scheduling algorithm computes the load distribution a priori. The online scheduling strategy, therefore needs no information about the cost of an individual task to be executed, whereas offline-scheduling often leads to a more optimal scheduling resulting in minimal execution time. As already mentioned, an optimal parallel speed-up can not be achieved if the load-balancing between the individual processors differs too much. The total execution time will allways be dominated by the most expensive job, independent from the number of processors. If the cost of the individual jobs differ too much, a perfect speed-up can not be guaranteed by an optimal load distribution. $\mathcal{H}$-matrices comprise the advantage that the occuring cost distribution predictable, but anyhow dependent on the hierarchical decomposition of the index set. Generally spoken, almost every practical algorithm in the context of hierarchical matrices can perfectly be distributed on shared memory systems [171]. The optimal parallel complexity of an algorithm $\mathcal{A}$, comprising a sequential complexity of $\mathcal{W}_{\mathcal{A}}^{\text {serial }}$, therefore can be quantified as

$$
\mathcal{W}_{\mathcal{A}, \text { opt }}^{\text {parallel }}(p)=\frac{\mathcal{W}_{\mathcal{A}}^{\text {serial }}}{p}
$$

For further details concerning parallel $\mathcal{H}$-matrix aspects refer to [173].

\subsection{Parallelized Circuit Simulation}

While the "internal" (inner) parallelization of hierarchical approaches, as described in the previous sections, can become difficult to implement and usually requires extensive preparations, an "external" (outer) parallelization of modules on a higher "integration level" can be performed in a straightforward manner. Both modeling as well as simulation processes contain independent parts, suitable for parallel implementation.

\subsubsection{Model Extraction Process}

The calculation of partial coefficients of potential and partial inductances can be performed independently from each other, building the outer parallelization stage. An inner parallelization of the algorithm can be performed in a straightforward manner if it is necessary. 


\subsubsection{Simulation Process}

W.r.t. the selected simulation domain (TD/FD), the solving process contains some possibilities for parallel implementations, too. Similar to the model extraction process in Section 8.6.1, simulation steps in FD can be computed separately from each other. In TD analysis, a parallelization of single time steps is more tricky.

\subsubsection{Implementation Details}

For parallelizing the modeling as well as the simulation process in circuit-level analysis, shared as well as distributed memory systems are suitable. The principle time scheduling for both modules are given in Figures 8.2 and 8.3, respectively. The modeling setup prepares all data from the

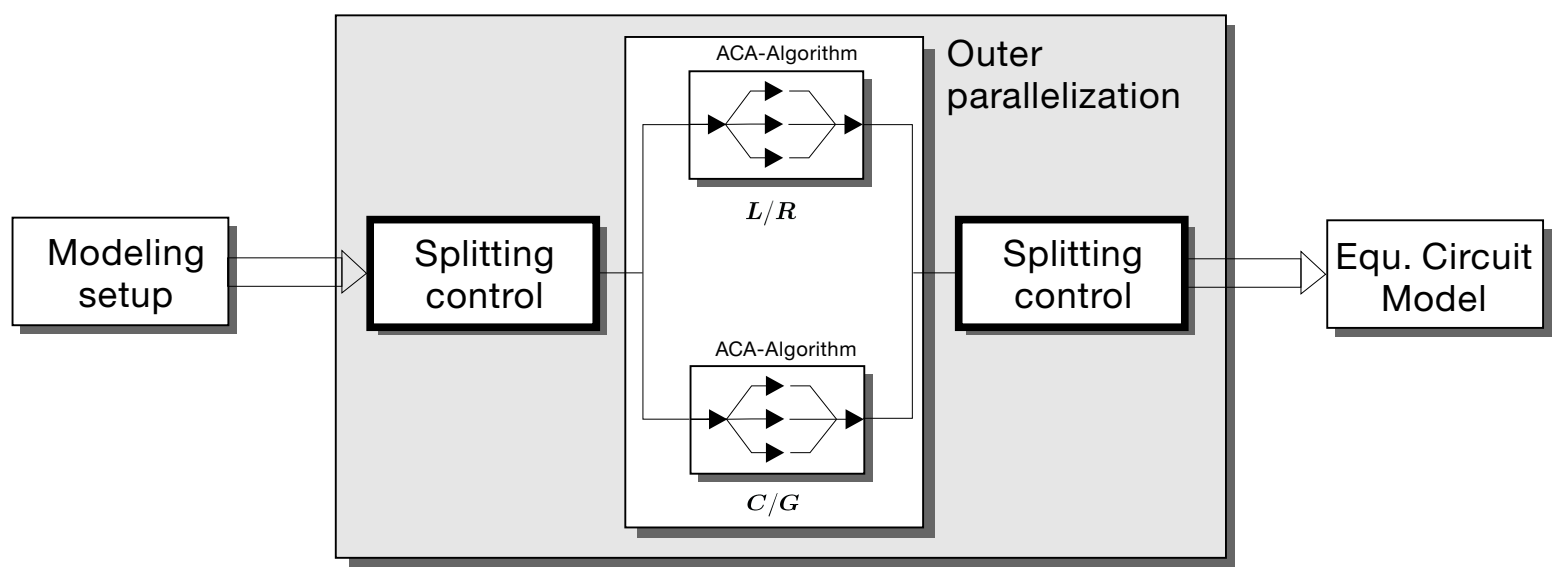

Fig. 8.2: Inner and outer parallelization in model extraction.

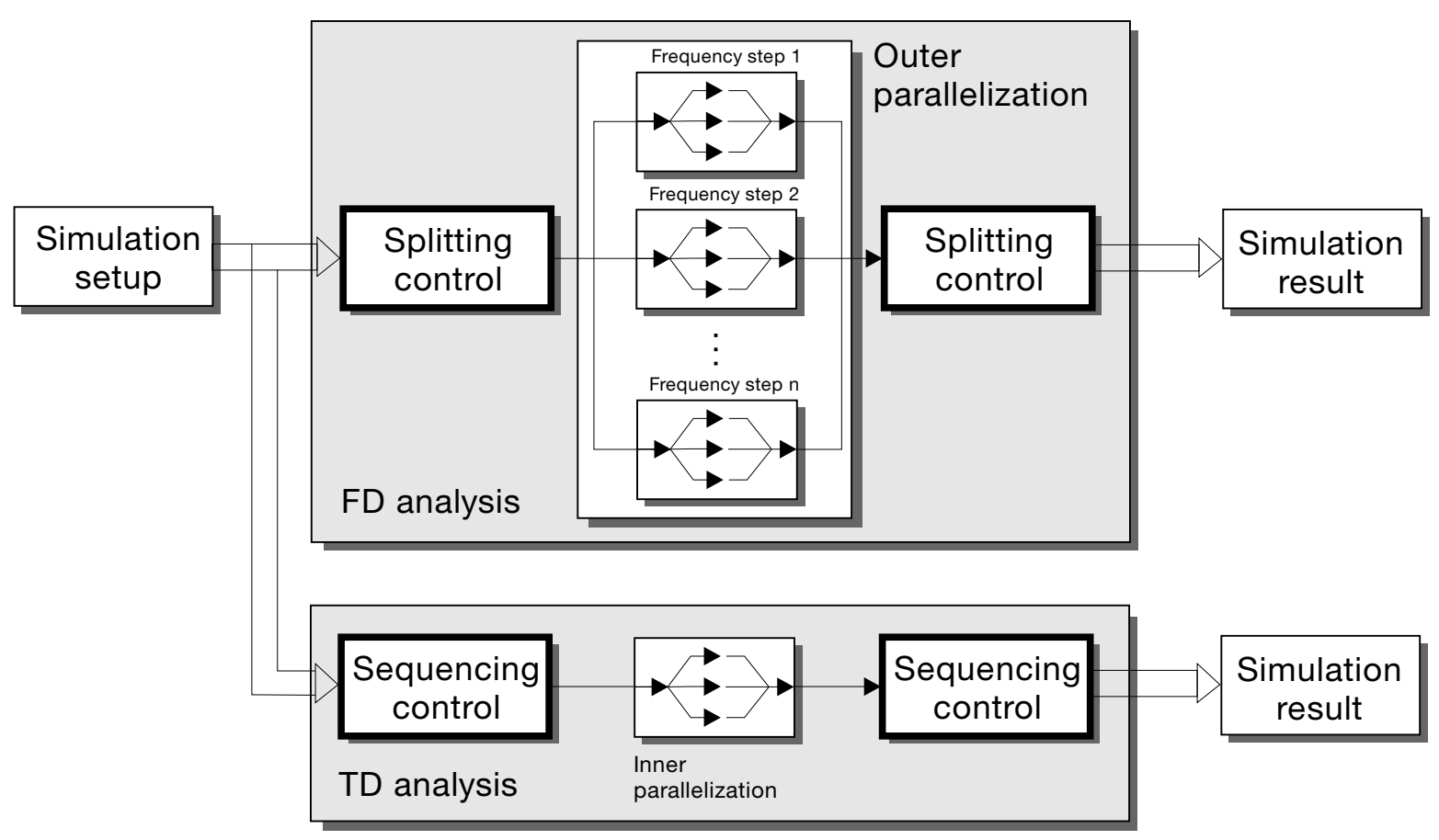

Fig. 8.3: Inner and outer parallelization in FD and TD analysis. 
PCB mesh information together with the modeling parameters, to provide it in a suitable form to a splitting control. The splitting control is necessary for the outer parallelization to assign the different modeling parts to the specific computers. An optional inner parallelization refers to the algorithmic parallelization, in particular matrix-vector multiplications. In parallel circuit simulation, TD and FD simulation have to be handeled separately. In FD analysis a splitting has to be performed w.r.t. the specific frequency steps, building the outer parallelization. Depending on the number of processing nodes $p, p$ frequency calculations can be performed simultaneously and independently from each other. Moreover, each frequency calculation can be parallelized optionally. Different from FD analysis, an outer parallelization is difficult to realize in TD analysis. Supervised by a sequencing control, a sequential data flow is transfered to an inner parallelization. The simlation results can be obtained after a suitable combination of the data resulting from TD or FD processing. 


\section{9}

\section{Industrial Applications}

$F$

or an evaluation of applicability and efficiency of the introduced hierarchical techniques, an automotive EMC relevant simulation example is considered in the following. Fast PEEC-based modeling and circuit-level simulation are accomplished on the basis of an automotive specific demonstrator board. The applicability of $\mathcal{H}$-matrix techniques and AMG is shown by means of simulation and the results are confirmed by experimental verification. Simulation and measurement results are analyzed and gains compared to conventional techniques w.r.t. storage and time requirements are discussed. The structure of a so-called "hybrid solver" is introduced, suitable for circuit simulations on system level [174]. This approach enables to include models from ICs, cable harnesses as well as the chassis and takes specific matrix properties into account. The matrix properties of the resulting network matrices are significantly influenced by the modeling and simulation approaches.

Section 9.1 motivates the need for a continuous and concurrent EMC simulation workflow in modern automotive development processes. Based on the presented demonstrator board (Section 9.2), some experimental investigations are performed (Section 9.3) and compared to the corresponding simulation results (Section 9.4). The framework of a hybrid solver strategy, suitable for EMC simulation on system level, is shortly outlined in Section 9.5.

\subsection{Motivation and Overview}

To ensure a broad EMC coverage in automobile development processes, computer aided engineering (CAE) techniques can help to arrange components and cables in an EMC compliant manner. Consequently, placement and wiring deficiencies can be avoided in an early design stage. While a PEEC-based approach turns out to be advantageous for simulations on component level, a TLbased approach has to be included to realize simulations on system level. Integrated circuits often 
represent origins of potential EMC problems, either as sources of EM emission or sinks. Consequently, simulating EM immunity is assumed to comprise the same importance as the simulation of EM emission. Disturbances originating from switching operations in ICs spread over the whole system via traces and interfaces on the control modules and the vehicle harness system. The positions of the components inside the chassis and the type of connection and wiring are of special importance for the sytem's EMC. Various methods have been developed in the past to map the electromagnetic behavior of harnesses and the surrounding body into suitable simulation models to enable a qualitative and quantitative statement. The influence of different types of models requires the application of an adapted solution process. In general, it should be avoided to use the car chassis as current return path. Nevertheless, existing parasitics have to be regarded in EM analysis to cover relevant electromagnetic properties. Conducted emission as well as irradiation from and to the harnesses can be described using suitable models to evaluate different placement and wiring concepts. To realize a continuous and concurrent EMC simulation process, a research project named MISEA [67], supported by the Bayerische Forschungsstiftung was introduced as a successor of a former research project named COSIME [175]. In the course of the project novel modeling strategies concerning system and parameter identification (e.g. subspace-identification) and modern MOR methods should be applied to achieve an enhanced efficiency. As mentioned in Chapter 1, however, MOR techniques exhibit some serious shortcomings. On the one hand, the bottleneck concerning storage requirements still remains since the reduction process was applied subsequently to the model extraction. Furthermore, MOR directly results in a loss of geometric information which is necessary for irradiation simulations. As a third aspect, an additional modification and extension of a reduced model is not possible after truncation. MOR stand-alone anyhow can not be sufficient to enable extremely large system-level simulations and to achieve accurate results in adequate time. Fast model extraction techniques and efficient and highly-sophisticated solvers are indispensable for an efficient simulation of models developed in the context of MISEA. Usually the storage bottleneck represents the most critical problem. While long simulation times still can be granted in some cases for the analysis of very large models, the available memory often sets the bound for the solvable model size. In the context of the MISEA project, moreover, standardized measurement techniques have to be modeled for simulation purposes, which is indeed subject of further research investigations.

\subsection{Demonstrator Board Description}

For simulation as well as measurement purposes an automotive specific demonstrator board with dimensions $160 \times 150 \mathrm{~mm}$ is considered whose layout is given in Figure 9.1. The board consists of four layers with dielectric medium FR4 $\left(\varepsilon_{r}=4.2\right)$ and stems from a typical application in automotive industry. The fully assembled board contains a microcontroller TC1796 and several power drivers which are driven application-specifically. Diverse operation modes are possible. Measurements are possible via provided connectors. An interface for uC-programming is available. The investigated design is part of the already mentioned MISEA project. A SPICE compatible simulation model of the board is provided as a result of fast model extraction (refer to Section 4.3). It reflects the respective transmission behavior of the power supply traces of the microcontroller as well as for the power drivers and the transmission behavior of the signal traces of the power drivers, respectively. The simulation models have to be valid in the TD as well as in the FD. 


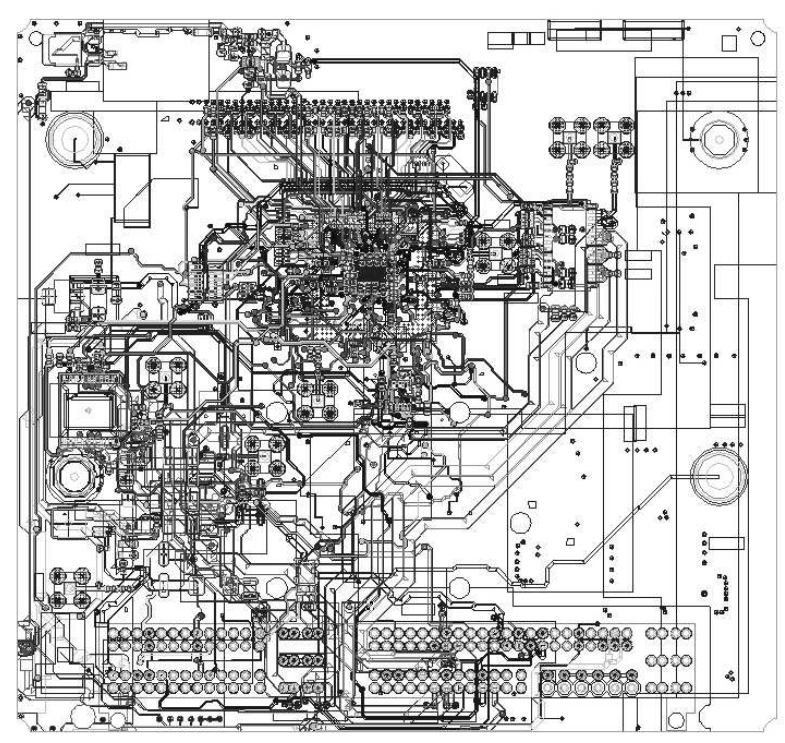

a)

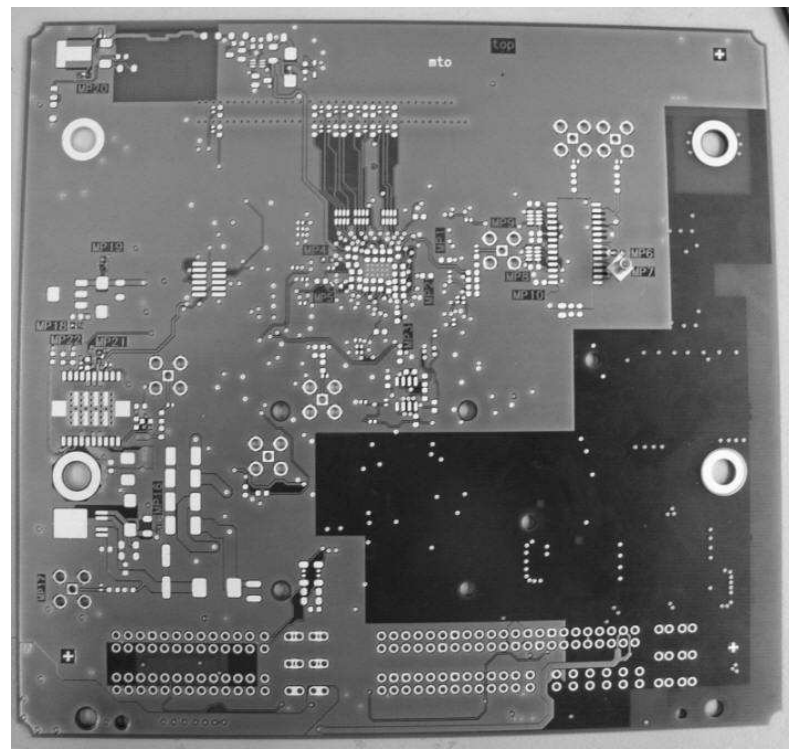

b)

Fig. 9.1: MISEA demonstrator board [67]. a) CAD-layout for simulation purposes; b) PCB for measurement purposes (without components).

\subsection{Experimental Verification}

For an experimental analysis in the FD up to $1 \mathrm{GHz}$ the deviation between simulation and measurement results is aimed to $5 \mathrm{~dB}$, as defined in the specification of the MISEA project. In the high-impedance domain often only less accurate measurement results can be obtained by using an impedance analyzer. Impedance results, however, directly can be mapped to scattering parameters. Scattering parameters express the transition behavior and are used to capture the characteristic properties between the power supply pins on the PCB and the supply pins of the microcontroller and the power drivers. A characterization of the transition behavior between the signal pins of the PCB-connector and the signal pins of the power drivers is also important. The following investigations are performed considering the net $/ \mathrm{N} \$ 45358$ on the MISEA demonstrator board with terminals MP13-2 and MP21-2 as depicted in Figure 9.2. A network analyzer (HP S-parameter network analyzer model $8753 \mathrm{ES}, 30 \mathrm{KHz} \ldots 3 \mathrm{GHz}$ ) was used to perform scattering parameter measurements. Figure 9.3 exemplarily shows the scattering parameters $S_{11}, S_{12}, S_{21}$ and $S_{22}$, measured in the frequency range $30 \mathrm{KHz} \ldots 3 \mathrm{GHz}$ recording 801 measurement points. The equipment under test (EUT) was connected to the network analyzer via cables of the type RG174 $(15 \mathrm{~cm})$ and terminal points. The calibration was realized by specific calibration adapters, regarding the physical conditions to completely cover the properties of the measurement configuration. The HP S-parameter network analyzer was used to acquire the data in real/imaginary description. The simulation parameters (Section 9.4) are chosen w.r.t. a suitable tradeoff between complexity and accuracy to enable an agreement of simulation and measurement. For testing purposes the PCB contains several SMA connectors. The measurement assembly is given in Figures 9.4 and 9.5. 


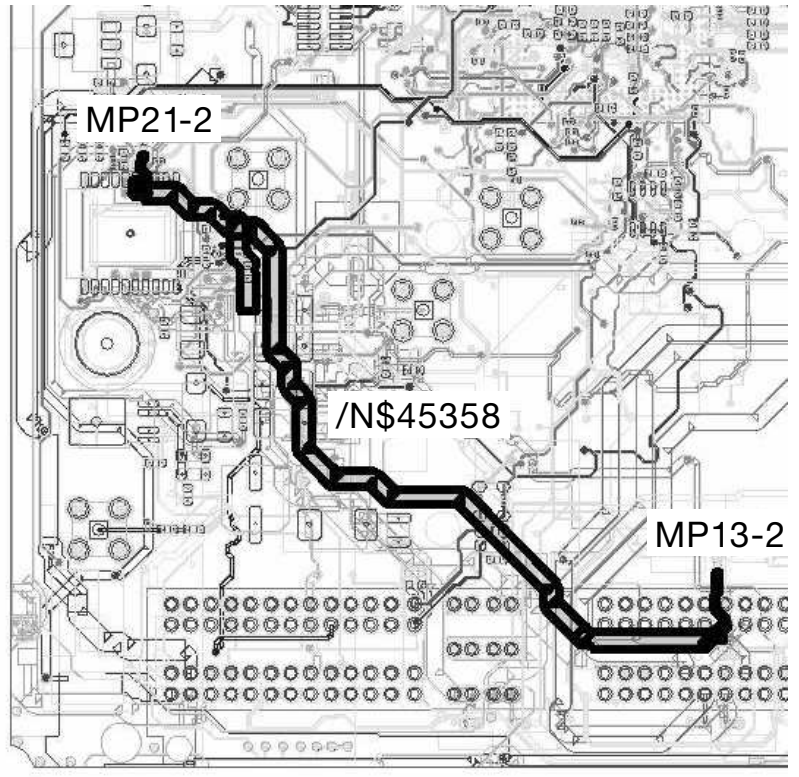

a)

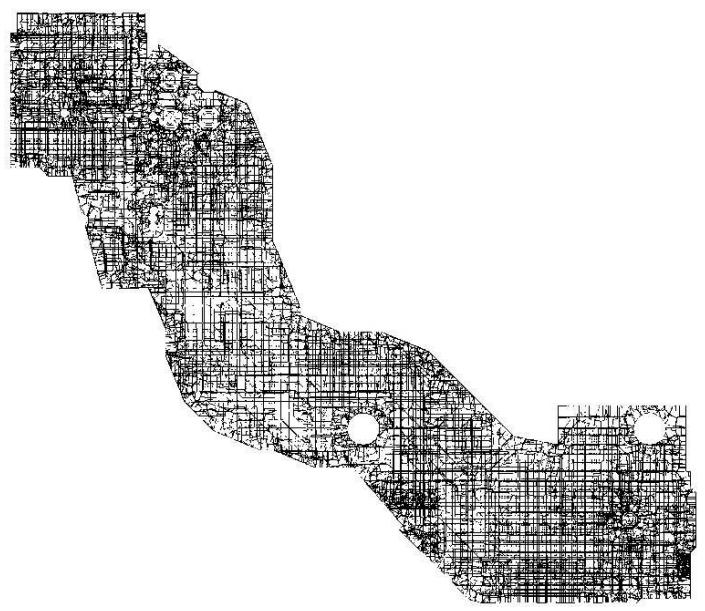

b)

Fig. 9.2: Net $/ \mathrm{N} \$ 45358$ on the MISEA board with a) terminals MP13-2 and MP21-2 and b) the correponding capacitive and inductive PEEC mesh for the signal trace together with the part of the ground plane for a suitably chosen width of the ground discretization.

\subsection{Simulation Verification}

To show the correspondence of measurement and simulation ${ }^{1}$ results a reluctance-based $\left(K_{p}, C_{p}, R\right)$ PEEC model of the net $/ \mathrm{N} \$ 45358$ (refer to Figure 9.2) is considered. A suitably chosen meshsize guarantees the validity of the model in the TD as well as in the FD. Simulations based on a suitably chosen parameter setting agree well with physical measurements. Both model extraction and simulation require a large amount of time. Therefore the total analysis process cannot be carried out in less than one or two days. A simulation acceleration can be enabled by applying simple matrix sparsification by truncation with varying tolerance limits $\delta_{C}, \delta_{K}$ for capacitances and reluctances. Although the complexity of the modeling process remains almost constant or slightly increases, respectively, the simulation can be accelerated by a speed-up factor of $\approx 5$ compared to the full inductance-based approach. The simulation results for $\delta_{C}=\delta_{K}=$ $0.05 \%, 0.08 \%, 0.15 \%, 0.2 \%$ for the parameters $S_{11}$ and $S_{12}$ are given in Figure 9.6 applying a standard direct solver (GMATRIX). The comparison to measurement results shows good accordance for $\delta_{C}=\delta_{K}=0.05 \%, 0.08 \%, 0.15 \%$, while a higher tolerance limit directly results in an unaccaptable loss in accuracy. Although a truncation enables a simple sparsification method, it is not efficient enough to realize accurate simulations for large industrially relevant problems. A further enhancement can be accomplished by accelerating model extraction as well as simulation using hierarchical techniques. Applying $\mathcal{H}$-matrix-based computation of parasitics (refer to Section 4.3),

\footnotetext{
${ }^{1}$ Remark: Good accordance of experimental and simulation results can only be achieved by preserving strictly controlled conditions. Only under these circumstances simulation and measurement results coincide. Already marginal deviations concerning the arrangement, the wiring or the placement of control modules can cause a significant deviation in the numerical results [8].
} 

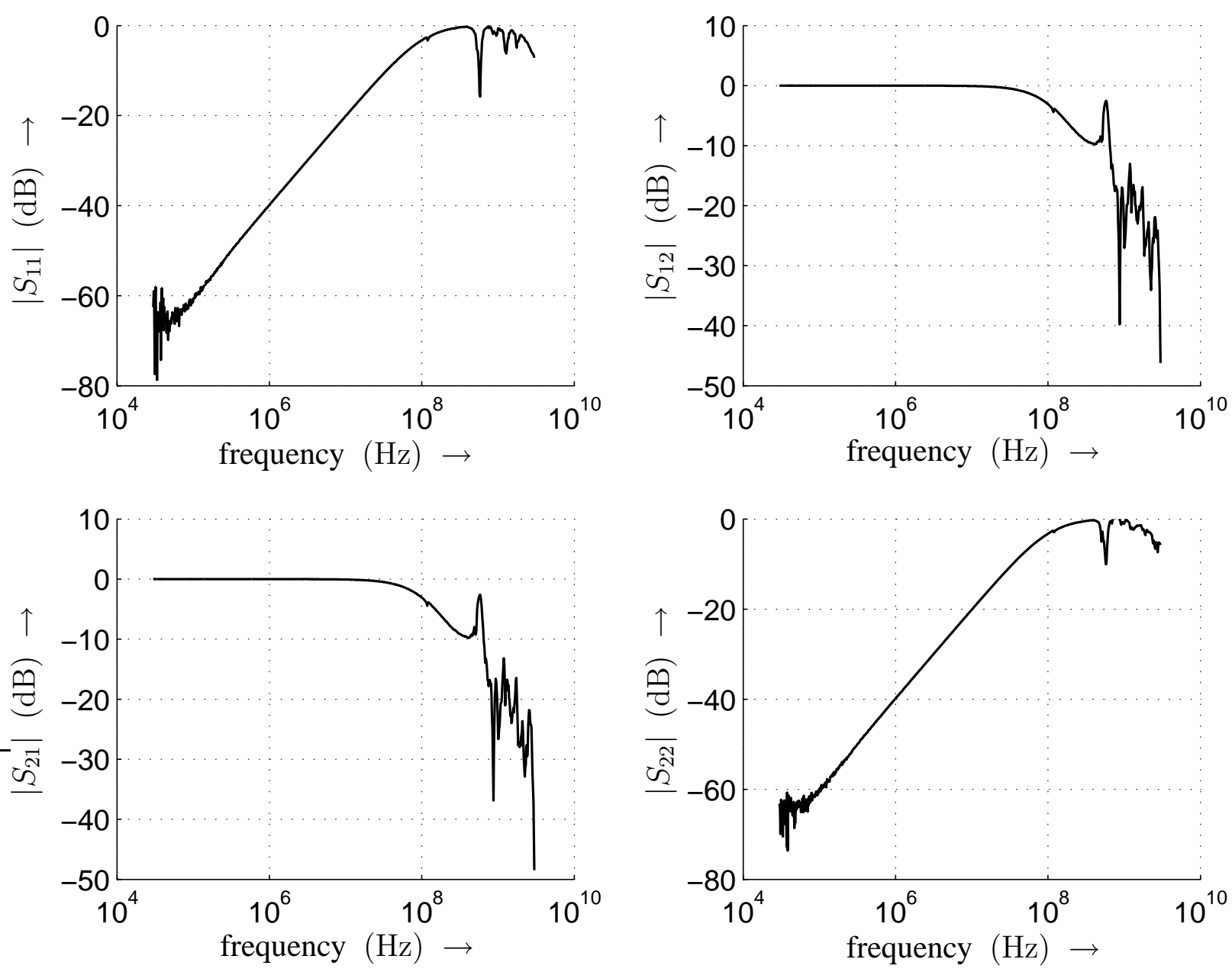

Fig. 9.3: Scattering parameter measurement results in the FD of the net $/ \mathrm{N} \$ 45358$ with terminals MP13-2 and MP21-2 on the MISEA board.

a modeling speed-up by an additional factor of $\approx 7$ can be achieved. Compared to conventional techniques an increased modeling accuracy can be obtained. Therefore, the obtained curves agree much better with the measurement results than in the conventional case with a maximal deviation between measurement and simulation results of $1 \mathrm{~dB}$. An implementation of the proposed algorithms was applied as described in Section 4.3.3. A gain with a factor of $\approx 20$ can be achieved concerning storage requirements by using the $\mathcal{H}$-matrix storage format instead of the conventional coordinate sparse matrix storage format. The simulation process can be accelerated up to a factor of $\approx 10$ by applying an AMG solver, in accordance with the numerical results in Section 7.7. While dense PEEC models represent the most critical case for multigrid solvers, an even higher gain can be achieved for TL models. As mentioned in Section 7.6 the implementation of AMG in existing codes is a crucial task, due to the fact that multigrid-based solvers usually have to be adapted to the specific problem. Using $\mathcal{H}$-matrix techniques the proposed simulation can be carried out with a speed-up factor of $\approx 5$ by applying a one-level iterative solver with $\mathcal{H}$-preconditioning, based on a transformation into an equivalent real-valued system as described in Section 5.1.2 2 . This also explains increased storage requirements compared to the direct solver case. Adaptive

\footnotetext{
${ }^{2}$ An extension of the used $\mathcal{H}$-matrix library is still under work.
} 


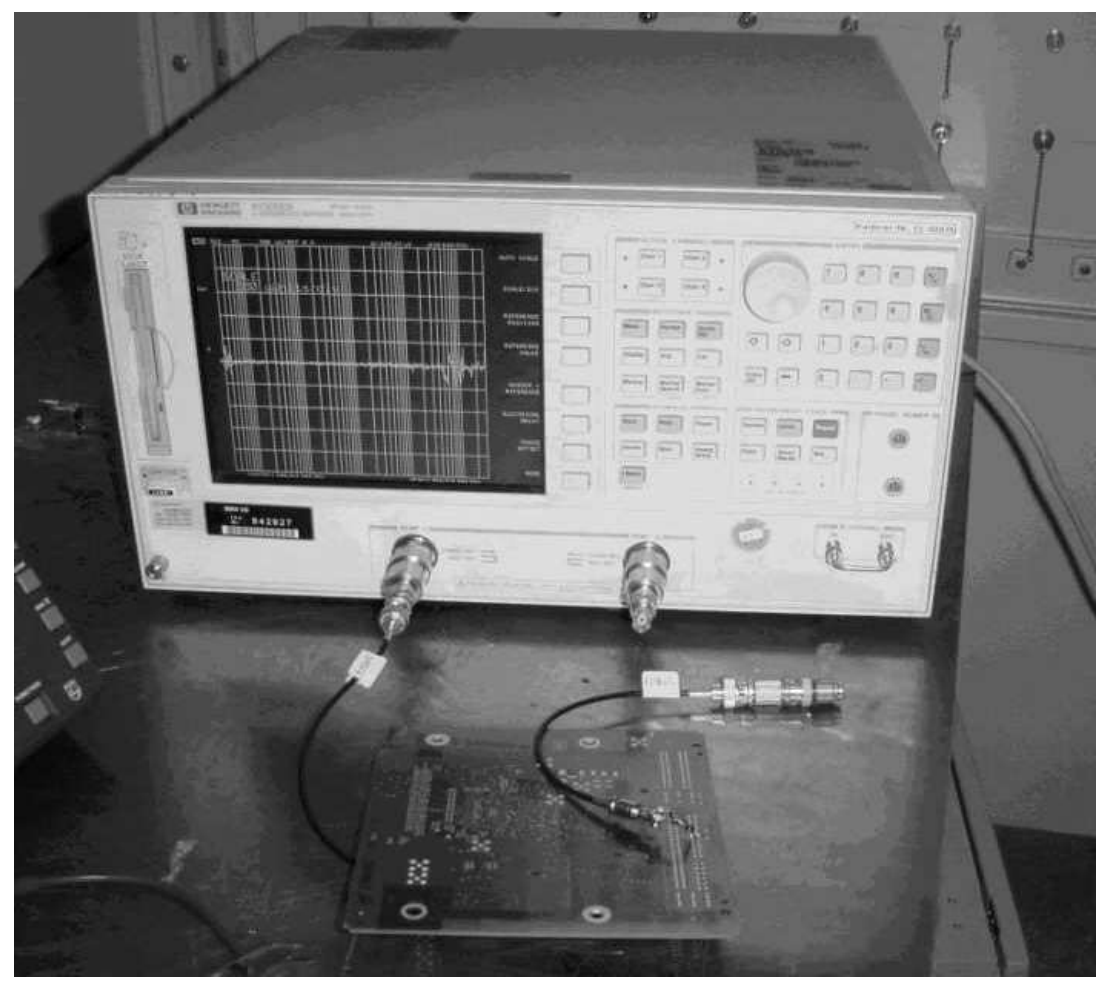

Fig. 9.4: Technical equipment and measurement arrangement for scattering parameter measurements.

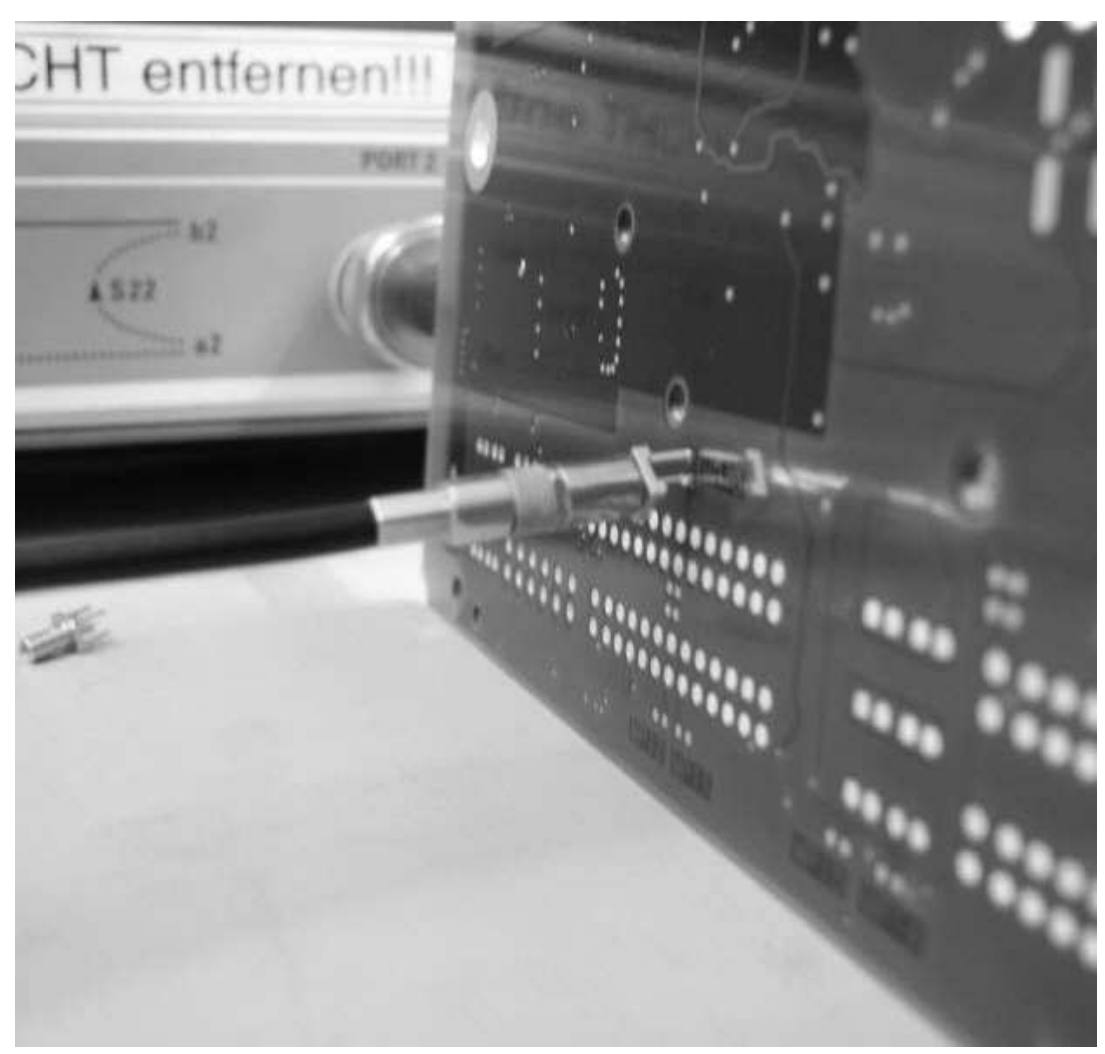

Fig. 9.5: Realization of contacts to measurement points (MP) on the PCB via RG174 cables. 
preconditioning, however, enables a speed-up by a factor of $\approx 10$. A further gain can be predicted using a combination of hierarchical matrix techniques and algebraic multigrid methods. This is the subject of a current research project in joint work together with the Fraunhofer Institute for Algorithms and Scientific Computing (SCAI) and the Max Planck Institute for Mathematics in the Sciences (MPI) Leipzig, Germany.
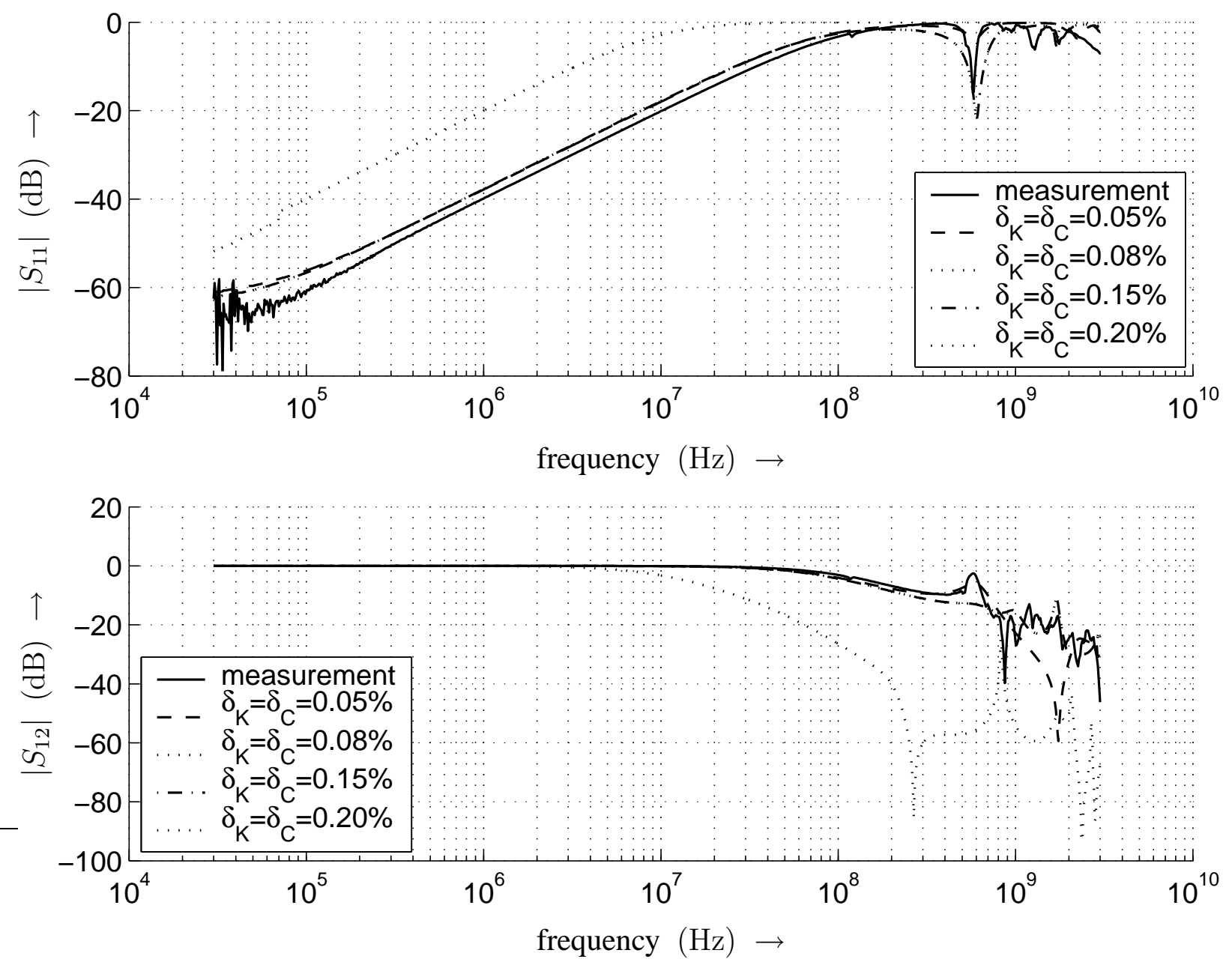

Fig. 9.6: Comparsion between scattering parameter simulation and measurement results. Due to symmetry reasons only $S_{11}$ and $S_{12}$ are given for varying tolerance limits $\delta_{C}, \delta_{K}$.

\subsection{Hybrid Solver Strategy}

To enable a flexible and robust EMC simulation process for complex electronic systems, an extended solver methodology has to be constructed taking all possible matrix properties into account. A first step to realize this project was done within the scope of this thesis. In principle, PEEC-based matrices and TL-based matrices can be distinguished by means of their sparsity. This fundamental separation enables an increased performance by always choosing the most suitable solver. An algebraic multigrid and an $\mathcal{H}$-matrix-based iterative solver are provided in the current library. The solver switching can be performed by means of an a priori density calculation. Preconditioned AMG as well as $\mathcal{H}$-preconditioned one-level iterative solvers show very good convergence behavior in almost any case. A robust direct solver is provided as a fallback solution to guarantee reliable simulation results. 
A comprehensive consideration of all physical effects in a car (refer to Figure 1.1 on page 2) and their influence to the resulting matrix properties are necessary in the future. Electromagnetic effects in automobile applications can be characterized as depicted in principle in Figure 9.7. Electromagnetic disturbances can propagate by radiation as well as galvanically coupled. To cover

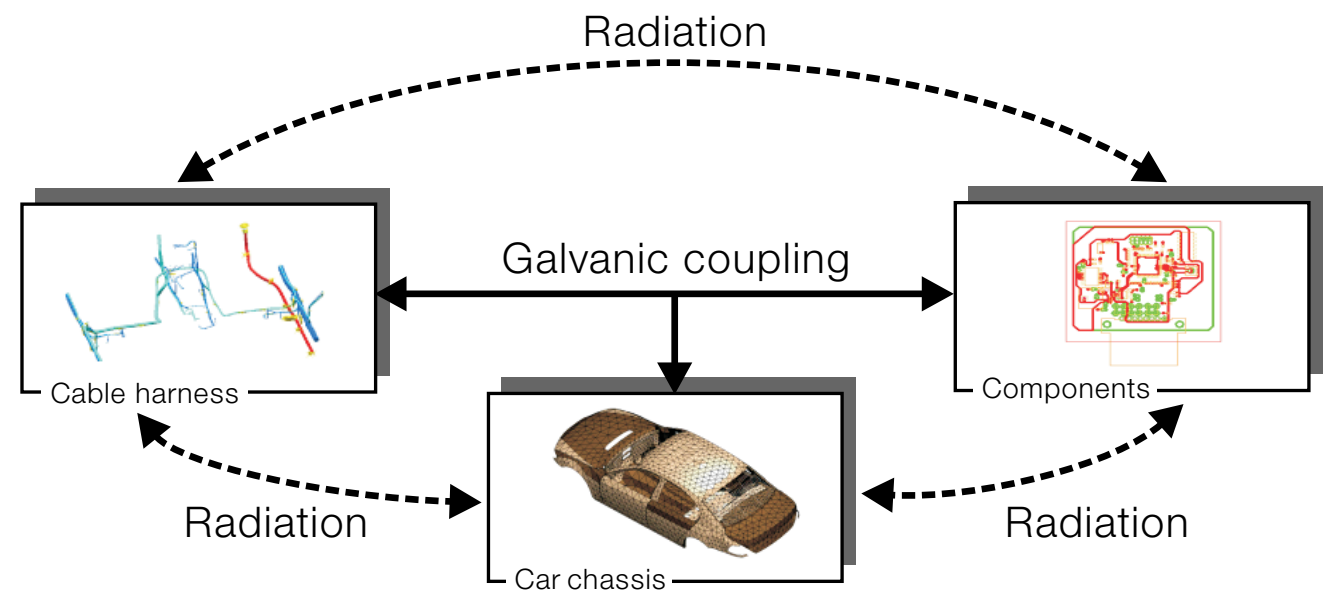

Fig. 9.7: Coupling paths between the main electronic parts in modern cars.

all relevant EM effects between all possible parts, three complementary levels of modeling in a single simulation environment have to be harmonized - full-wave $3 D$ resolution at system level, 2D TL propagation at harness level and 3D circuit formulation at component level. The four main probems in car design are crosstalk (coupling between wires inside a harness), emission (radiation from the harness towards the environment or an antenna), immunity (of equipment against external electromagnetic aggression) and antenna design. In the context of the MISEA project, system simulations usually are applied in the TD to aquire the non-linearities of the integrated circuit models. A mapping to the FD is possible by applying the fast fourier transform (FFT).

Already in the context of the research project AutoEMC [8], a first step was performed to realize a multilevel approach to take different model properties into account (Figure 9.8). After

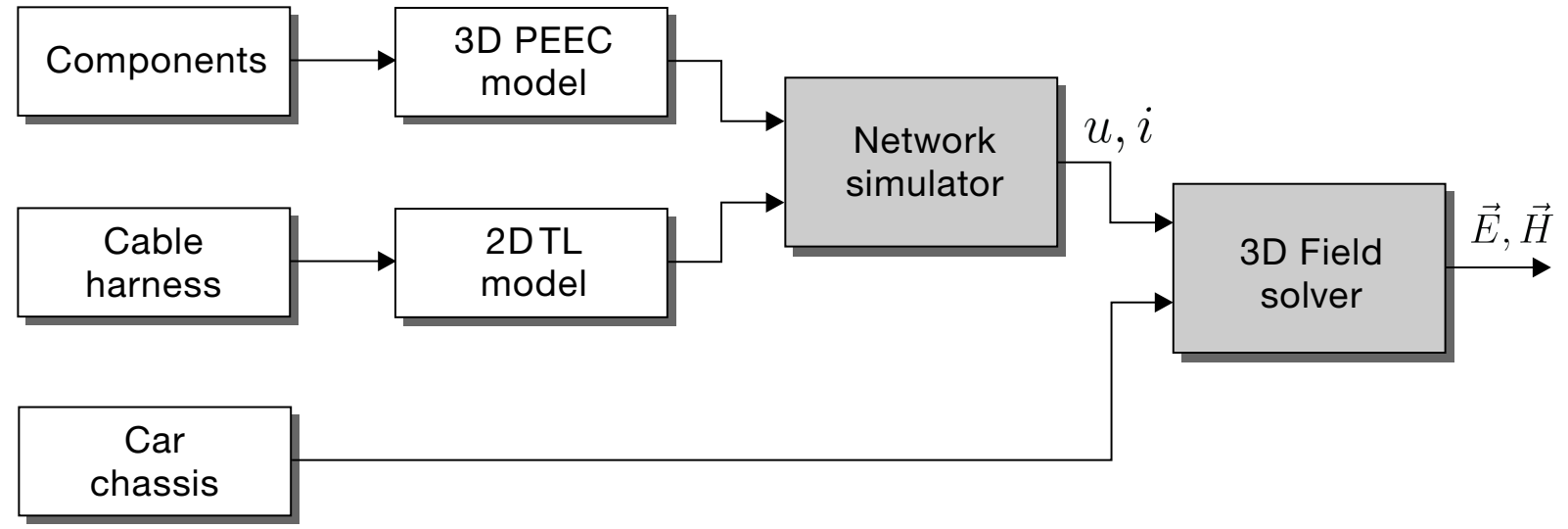

Fig. 9.8: Multilevel modeling and simulation approach for automotive system simulation.

selected components and cable harnesses are modeled using a PEEC or TL modeling approach, a network simulator solves the corresponding network equations to obtain the unknown circuit variables (nodal voltages/branch currents). These variables, together with the geometric data from 
the car body (and/or other conducting parts of the car) can be used to compute the 3D field in space characterized by field variables (electric/magnetic vector fields). The SPICE format enables a tool-independent data exchange between the different levels which simplifies the transfer of models between the partners involved in the design process. Consequently, a simplification of the integration process as well as a bi-directional data workflow can be realized. The analysis of the EM behavior of active components as microcontroller chips $(\mathrm{uC})$ or bus drivers is especially important for an EMC compliant design. Different modeling techniques can be applied to generate an accurate EMC behavioral model of such components, suitable for simulations on component and system level. The common standard modeling formulations as IBIS (input/output buffer information specification) or ICEM (integrated circuit electromagnetic models) allow an electrical circuit description in SPICE syntax. Consequently, an implementation of such models into common network simulators is possible in a straightforward manner. For an introductory view to a multilevel modeling approach and further details refer to [9].

W.r.t. an efficient EMC simulation on system level, however, it has to be noticed that the interconnection of models of different types also has an impact to the properties of the resulting network matrices. The possible impacts and influences in circuit simulations are depicted in Figure 9.9. While for example the modeling of the chassis leads to 3D PDEs and modeling of the harnesses

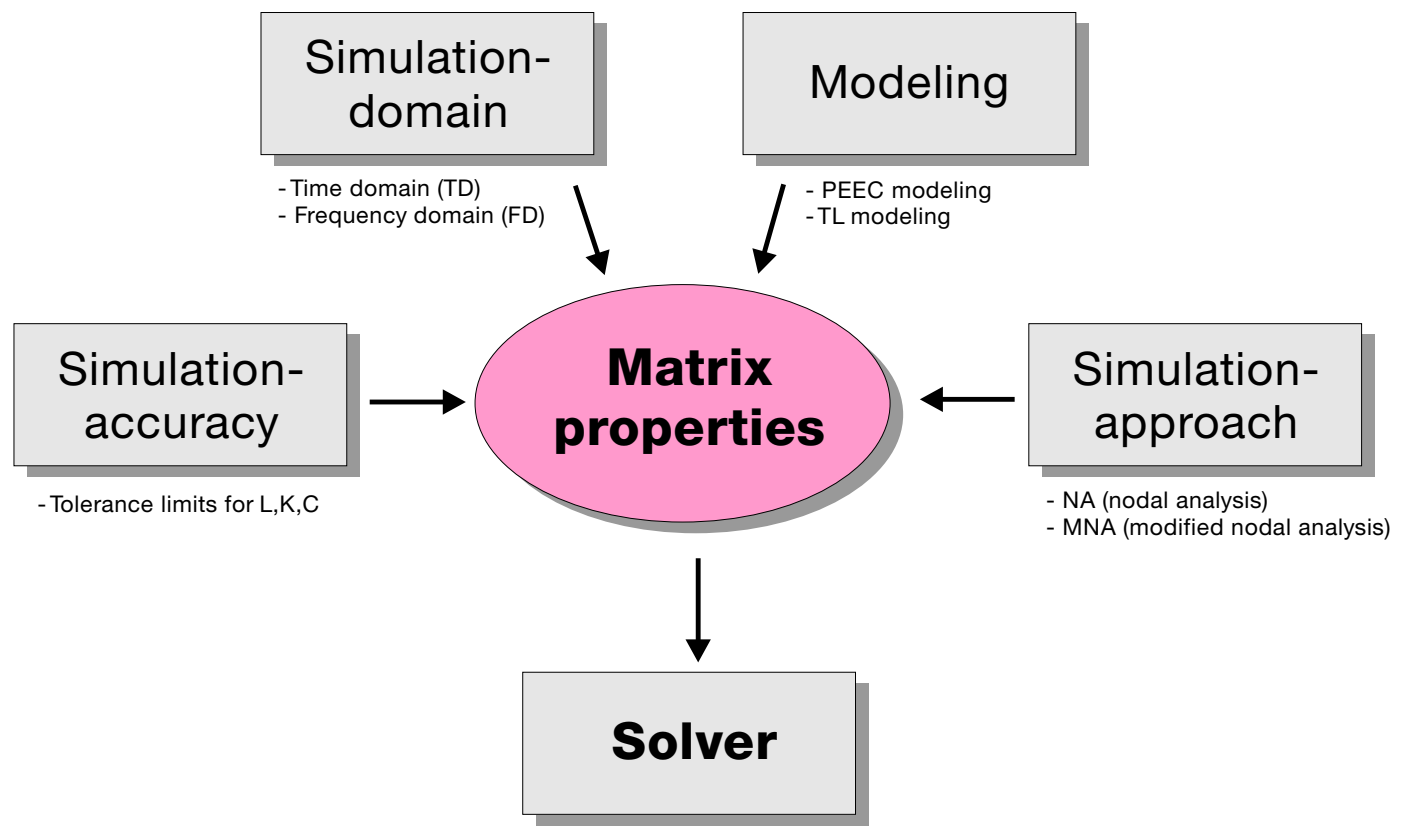

Fig. 9.9: Influencing factors to network matrix properties.

to multiconductor TL equations (MTL), the PCB modeling leads to ODEs. To enhance simulation accuracy as well as efficiency, the hierarchical strategies have to be applied regarding the different modeling approaches as well as the type of simulation. Consequently, the proposed hybrid solver strategy in a first step extensively has to analyze the properties of the resulting system matrix prior to a solver selection stage. Depending on the character of the matrix, AMG and $\mathcal{H}$-matrices offer high potential for circuit simulation. Especially for the field solver (Figure 9.8), highest performance can be achieved by combining $\mathcal{H}$-matrices and AMG. The network simulator represents one of the most crucial parts. A problem specific handling of the resulting network matrices can be necessary. 


\section{0}

\section{Conclusion}

$\boldsymbol{I}$ $\mathrm{n}$ the course of this thesis, a fast and accurate modeling and simulation methodology has been developed, suitable for electromagnetic field problems as they are encountered in automotive applications. The BEM-based PEEC modeling approach (Chapter 3) proved to be useful for the analysis of the electromagnetic behavior of 3D interconnects and traces on PCBs. As the main shortcomings in EMC simulation in the context of a BEM discretization approach, the memory requirements as well as the total simulation times severely limit the performance and therefore the managable problem sizes. The application of direct methods together with dense matrices leads to a time complexity of $\mathcal{O}\left(n^{3}\right)$ and a storage complexity of $\mathcal{O}\left(n^{2}\right)$ with $n$ DOF. Consequently, only small problems could be solved in the past. Even simple iterative solution algorithms usually can only reduce the complexity to $\mathcal{O}\left(n^{1.5}\right)$ by ensuring convergency. MOR techniques often proved to be not efficient enough to enable simulations of real-life problems on system level. The two existing bottlenecks are the modeling as well as the solving processes. Both parts have to be optimized to realize a simulation of industrially relevant problem sizes. This work introduces a complete framework of modern hierarchical techniques for an accelerated model extraction and circuit-level simulation. Hierarchical approaches enable a complexity reduction to $\mathcal{O}(n \log n)$ w.r.t time as well as storage requirements, in principle. The presented methods and algorithms are tested by means of real-life problems.

A precise analysis of the physical properties and laws of the underlying electrodynamic problem has been carried out in Chapter 2, to confirm the suitability and applicability of hierarchical approaches for circuit simulation. Together with the proposed simulation instructions and guidelines given in Chapter 3, conventional PEEC techniques can be improved considerably. By means of a PEEC formulation in curvilinear coordinates, high flexibility and compatibility to orthogonal discretizations can be achieved. The required number of cells can be reduced significantly by applying non-orthogonal discretization. Therefore, this approach enables a kind of model order reduction. In combination with highly-sophisticated quadrature rules for an efficient numerical 
integration and regarding the proposed validity aspects concerning mesh generation in Section 3.7, high demands for simulation accuracy can be met. The proposed reluctance-based PEEC method enables a sparse formulation of element matrices and therefore supports the application of iterative solution methods.

An efficient model extraction approach based on hierarchical matrices is introduced in Chapter 4. This technique overcomes some serious limitations of the FMM and enables a purely mathematical approach to transform dense BEM-based matrices to a so-called data-sparse format. Similar to the FMM a necessary condition for the effectivity of $\mathcal{H}$-matrices represents the smoothness of Green's function far away from the singularity. As one main contribution of this thesis, the functionality of $\mathcal{H}$-matrices has been implemented in an existing industrial PEEC-based circuit simulation environment. Already existing algorithms were modified and improved to accelerate the computation process by simultaneously improving the accuracy. A heuristics speeds up the matrix construction by only requesting required elements to be computed. Realized by means of a callback function, the recursive filling of hierarchical matrices was performed based on circuitsimulation specific modifications of modern algorithms. The introduced data-sparse representation of element matrices as well as complete network matrices enables a very memory-economical description. Consequently, the memory bottleneck can be eliminated. Numerical results, performed by means of relevant examples in the context of a PEEC-based model extraction, show an acceleration of the element evaluation process by a factor of $>100$. In particular, computation times for matrix inversions in the hierarchical format can be drastically reduced compared to a full matrix inversion. The storage requirements can be reduced by a factor of $8 \ldots 50$. Losses in accuracy are vanishing, compared to the accuracy of the conventional PEEC approach. To enable an additional performance improvement, novel methods as adaptive refinement techniques, adaptive recompression and so-called $\mathcal{H}^{2}$-matrices are discussed.

The efficient application of iterative methods for circuit simulation requires an analysis of the matrix properties and influencing factors. Therefore, Chapter 5 discusses the basic properties of circuit simulation matrices and disadvantageous characteristics and establishes the foundation of an iterative solver framework. Apart from being dense, BEM-based circuit simulation network matrices usually exhibit large condition numbers, resulting from the different types of element characteristics involved. Suitable sparse matrix storage formats as well as ordering strategies are discussed. The compressed sparse row (CSR) storage format, as the most common format in modern numerical algorithms, turned out to be suitable for an efficient implementation. Fundamental details are presented to realize efficient preconditioned Krylov-subspace iterative methods w.r.t. the application at hand.

Another special focus of this thesis has been on the application of $\mathcal{H}$-matrices in a PEEC context in combination with iterative solvers as described in Chapter 6 . We introduced $\mathcal{H}$-matrix arithmetics to realize an efficient preconditioning for PEEC-based network matrices for simulations in the TD and the FD. An implementation of iterative one-level solvers such as GMRes and BiCGstab in a $\mathcal{H}$-matrix-based framework pointed out to be straightforward using the HLIB and lead to significant gains w.r.t. time and memory requirements. Numerical results show very robust, fast and accurate simulation behavior for industrially relevant PEEC examples. The proposed algorithms work well together with very large, dense and ill-conditioned PEEC-based network matrices. Although this approach unfolds its highest efficiency together with dense matrices, a significant improvement 
can also be observed for sparse matrices. In combination with different preconditioners, a speedup by a factor of $\approx 32$ in the TD was achieved. The residuals are sufficiently small $\left(\approx 10^{-10}\right)$ to ensure accurate system simulation. The storage requirements can be reduced up to a factor of $\approx 4$ compared to the conventionally used standard direct solver. Especially the proposed adaptive preconditioning as described in Section 6.3.2, enables an additional speed-up by a factor of $\approx 4$. In general, efficiency, robustness and flexibility are drastically increased and the limits for managable problem sizes are extended.

To enable fast and accurate circuit simulations especially together with sparse system matrices, the functionality of an AMG approach was introduced in Chapter 7 as the third main focus of this thesis. This very powerful hierarchical approach enables significant speed-up by a factor of $\approx 10$ in the TD as well as the FD by using the standard AMG library SAMG together with reluctance-based sparsified PEEC models as well as together with TL models. The achievable residuals applying GMRes or BiCGstab are very small, usually leading to very accurate results. Although AMG is well-established for various kinds of numerical problems (e.g. computational fluid dynamics (CFD) or semiconductor simulation) together with circuit simulation approaches it requires some algorithmic modifications to enable accurate and robust system simulations. As a fallback solution a direct solver is provided to guarantee a stable and accurate solution if the iterative solvers fail. We introduced a combination of multigrid solvers and data-sparse PEEC-matrices (Section 7.5), which represents a new and novel approach to realize computational performance with (almost) optimal complexity. Applying the $\mathcal{H}$-matrix stamping procedure, the PEEC model directly can be provided in the $\mathcal{H}$-matrix format, eliminating the memory bottleneck. The computational complexity with $\mathcal{O}(n \log n)$ moreover leads to a fast solution process.

Due to the almost optimal complexity of hierarchical techniques presented so far, a further increase in performance often can only be obtained by applying parallelization techniques. As a completion, Chapter 8 verifies the suitability and applicability of parallelization techniques for hierarchical methods and concludes the theoretical part of the thesis. An acceleration of an existing algorithm by distributing the computational work to several computing nodes should not be considered as a common method for speeding up a workflow. Parallelization alone is not enough to solve large problems efficiently. The main attention of a development of fast algorithms should be on a suitable design of the algorithm itself. Parallelization techniques should be seen as a completion in the application of hierarchical techniques.

To demonstrate the applicability and efficiency of the presented methodology, a comprehensive analysis of an automotive specific subject was accomplished in Chapter 9. W.r.t. the requirements for an automotive EMC simulation on system level, challenges and remedy methods are presented. The applied hierarchical techniques facilitate the inclusion of models of different types in the context of a "hybrid" solver approach. As a basic requirement for real-life simulations, the bottlenecks concerning time and memory consumptions can be avoided.

Possible Extensions and Future Work Although the presented modeling and simulation methodology already allows EMC analysis with high efficiency and accuracy, it comprises some still outstanding scopes for further investigations and improvements. To exploit the total computational power of the proposed hierarchical approaches, a more suitable $\mathcal{H}$-network-matrix construction containing filling and post-processing has to be developed. The inclusion of PEEC models as 
well as TL models must be possible and the interfaces have to be constructed in a way to enable a continuous data exchange between modeling and simulation. As a first step, a simple $\mathcal{H}$-stamping for the elementwise and blockwise addition of matrix entries was presented in Section 6.2.1. A more powerful approach, however, is subject of a current research project together with the MaxPlanck-Institute Leipzig and the Fraunhofer Institute SCAI.

The implementation of $\mathcal{H}^{2}$-matrices for an iterative solution is still under work. Due to the fact, that the preconditioner computation represents the most complex part in the iterative solving process, the application of $\mathcal{H}^{2}$-matrices can help to significantly reduce the complexity w.r.t. time as well as storage requirements. Although modern quadrature rules exhibit powerful techniques to treat complex and singular numerical integrals, problems are inevitable in plate arrangements with very small distances between the plates, as it occurs frequently in PCB analysis. Local mesh refinement techniques can help to overcome these shortcomings. Accuracy problems as well as occuring simulation artifacts can be avoided or at least moderated (refer to Section 3.7.4). The proposed hierarchical techniques proved to be well suited for a parallel implementation. While an "outer" parallelization of the FD circuit analysis is still under work, the "inner" parallelization for the simulator and inner as well as outer parallelizations for the modeling are still open problems. Last but not least additional research work concerning a combination of hierarchical techniques and MOR is supposed to be promising. 


\section{Bibliography}

[1] Directive, 89/336/EEC, "Electromagnetic Compatibility (EMC) Directive, 89/336/EEC," The Council of the European Communities, (will be replaced by Directive 2004/108/EC in 2007).

[2] Directive, 2004/108/EC, "Electromagnetic Compatibility (EMC) Directive, 2004/108/EC," The Council of the European Communities, vol. 47, issue L390, pp. 24-37 of the Official Journal of the European Union (OJ) on December 31, 2004, (will replace the current EMC Directive, 89/336/EEC on July 20, 2007).

[3] M. E. Verbeek, "Iterative solvers and preconditioning for electromagnetic boundary integral equations," Ph.D. dissertation, University of Utrecht, Netherlands, 2001.

[4] A. T. Davis, "Implicit Mixed-Mode Simulation of VLSI Circuits," Ph.D. dissertation, University of Rochester, NY, USA, 1991.

[5] A. Orlandi and J. L. Drewniak, "Electromagnetic Compatibility Issues in Mobile Computing," IEEE Trans. Mobile Comput., vol. 2, no. 4, pp. 273-274, October-December 2003.

[6] ITRS, "International Technology Roadmap for Semiconductors," ITRS2003, 2003 edition. [Online]. Available: http://public.itrs.net/Files/2003ITRS/Home2003.htm

[7] BMW AG, BMW Group Standard GS95002 - Electromagnetic Compatibility (EMC), 2004.

[8] F. Canavero, J.-C. Kedzia, P. Ravier, and B. Scholl, "Numerical Simulation for Early EMC Design of Cars," in Proc. of the CEM 2000 Symposium, Brugge, Belgium, 2000.

[9] R. Neumayer, A. Stelzer, F. Haslinger, G. Steinmair, M. Tröscher, J. Held, B. Unger, and R. Weigel, "Numerical EMC-Simulation for Automotive Applications," in Proc. of the 15th International Zurich Symposium on Electromagnetic Compatibility, Zurich, Switzerland, February 2003, pp. 459-464.

[10] K. Stüben and C.-A. Thole, "Schnellere Simulationen durch neue Lösertechnologien," Der GMD-Spiegel, no. 1/2, pp. 26-28, 2000, (In German).

[11] R. Achar and M. S. Nakhla, "Simulation of High-Speed Interconnects," Proc. IEEE, vol. 89, no. 5, pp. 693-728, May 2001. 
[12] A. E. Ruehli, "Equivalent Circuit Models for Three-Dimensional Multiconductor Systems," IEEE Trans. Microwave Theory Tech., vol. MTT-22, no. 3, pp. 216-221, March 1974.

[13] G. Steinmair, "The Partial Element Equivalent Circuit Method and Model Order Reduction in Automotive EMC Simulation," Ph.D. dissertation, Johannes Kepler University of Linz, Austria, 2003.

[14] J. Cullum, A. E. Ruehli, and T. Zhang, "A Method for Reduced-Order Modeling and Simulation of Large Interconnect Circuits and its Application to PEEC Models with Retardation," IEEE Trans. Circuits Syst. II, vol. 47, no. 4, pp. 261-273, April 2000.

[15] J. Ekman and G. Antonini, "On Characterizing Artifacts Observed in PEEC Based Modeling," in Proc. of the IEEE International Symposium on Electromagnetic Compatibility, Santa Clara, CA, USA, August 2004.

[16] A. E. Ruehli, J. Delsing, J. Ekman, A. Orlandi, and G. Antonini, "PEEC Development Road Map 2005."

[17] G. Haase, M. Kuhn, and S. Reitzinger, "Parallel AMG on Distributed Memory Computers," Johannes Kepler University of Linz, Austria, Special Research Program SFB F013, 2000.

[18] U. Trottenberg, C. W. Oosterlee, and T. Schüller, Multigrid. Academic Press, London, San Diego, 2001.

[19] W. Hackbusch, "A sparse matrix arithmetic based on $\mathcal{H}$-matrices. Part I: Introduction to $\mathcal{H}$-matrices," Computing, vol. 62, pp. 89-108, 1999. [Online]. Available: http: //www.mis.mpg.de/preprints/

[20] —, "A sparse $\mathcal{H}$-matrix arithmetic, Part II: Application to multi-dimensional problems," Computing, vol. 64, pp. 21-47, 2000. [Online]. Available: http: //www.mis.mpg.de/preprints/

[21] G. Alléon, B. Carpentieri, I. S. Duff, L. Giraud, J. Langou, E. Martin, and G. Sylvand, "Efficient parallel iterative solvers for the solution of large dense linear systems arising from the boundary element method in electromagnetism," 2003, CERFACS Technical Report TR/PA/03/65.

[22] M. Bader, "Robuste, parallele Mehrgitterverfahren für die Konvektions-DiffusionsGleichung," Ph.D. dissertation, Technical University of Munich, Germany, 2001, (In German).

[23] W. C. Chew, J. M. Jin, E. Michielssen, and J. Song, Fast and Efficient Algorithms in Computational Electromagnetics. ARTECH House, 2001.

[24] W. C. Chew, J. M. Jin, C. C. Lu, E. Michielssen, and J. M. Song, "Fast Solution Methods in Electromagnetics," IEEE Trans. Antennas Propagat., vol. 45, no. 3, pp. 533-543, March 1997. 
[25] H. Su, E. Acar, and S. R. Nassif, "Power Grid Reduction Based on Algebraic Multigrid Principles," in Proc. of the International Design Automation Conference, Anaheim, CA, USA, June 2003.

[26] S. R. Nassif and J. N. Kozhaya, "Fast Power Grid Simulation," in Proc. of the International Design Automation Conference, Los Angeles, CA, USA, June 2000.

[27] S. Börm and L. Grasedyk, "HLIB - a library for $\mathcal{H}$ - and $\mathcal{H}^{2}$-matrices," Max Planck Institute for Mathematics in the Sciences. [Online]. Available: http://www.hlib.org/

[28] K. Stüben and T. Clees, SAMG User's Manual v. 22c, Fraunhofer Institute SCAI, Schloss Birlinghoven, Sankt Augustin, Germany, May 2005. [Online]. Available: http://www.scai.fhg.de/303.0.html

[29] R. F. Harrington, Field Computation by Moment Methods. Macmillan Publishing Company, 1968, reprint: IEEE Press, New York, 1993.

[30] J. Edlund, "A Parallel, Iterative Method of Moments and Physical Optics Hybrid Solver for Arbitrary Surfaces," Ph.D. dissertation, Uppsala Technical University, Uppsala, Sweden, 2001.

[31] F. M. Tesche, M. V. Ianoz, and T. Karlsson, EMC Analysis Methods and Computational Models. John Wiley \& Sons, Inc., 1997.

[32] F. Schunn, Berechnung elektromagnetischer Felder mit der Momentenmethode im Zeitbereich. Fortschr.-Ber. VDI Reihe 21 Nr. 338, VDI Verlag Düsseldorf, 2003, Ph.D. dissertation, Technical University of Hamburg-Harburg, Germany, (In German).

[33] M. Albach, "Elektromagnetische Felder," Lecture notes, 2001, University of ErlangenNuremberg, Germany, (In German).

[34] C. A. Balanis, Advanced Engineering Electromagnetics. John Wiley \& Sons, 1989.

[35] R. Kröger and R. Unbehauen, Elektrodynamik, 3rd ed. B.G. Teubner Stuttgart, 1993, (In German).

[36] A. Englmaier, "Methoden und Modelle für die EMV-Simulation," Ph.D. dissertation, Technical University of Munich, Germany, 1999, (In German).

[37] G. Lehner, Elektromagnetische Feldtheorie für Ingenieure und Physiker, 2nd ed. SpringerVerlag, 1993, (In German).

[38] S. Blume, Theorie elektromagnetischer Felder, 4th ed. Hüthig, 1994, (In German).

[39] U. Jacobus, Erweiterte Momentenmethode zur Behandlung kompliziert aufgebauter und elektrisch großer elektromagnetischer Streuprobleme. Fortschr.-Ber. VDI Reihe $21 \mathrm{Nr}$. 171, VDI Verlag Düsseldorf, 1995, Ph.D. dissertation, University of Stuttgart, Germany, (In German). 
[40] A. Dietermann, "Numerische Simulation der transienten Strahlungsverkopplung elektrischer Leitungen," Ph.D. dissertation, University of Karlsruhe, Germany, 1997, (In German).

[41] A. F. Peterson, S. L. Ray, and R. Mittra, Computational Methods for Electromagnetics. IEEE Press, 1998.

[42] J. J. H. Wang, Generalized Moment Methods in Electromagnetics. John Wiley \& Sons, Inc., 1991.

[43] J. Kiwitt, "Effiziente Simulation elektrodynamischer Wechselwirkungen und Parameteroptimierung in beliebig geformten Leiterstrukturen," Ph.D. dissertation, University of Karlsruhe, Germany, 1999, (In German).

[44] P. R. Argus, Berechnung von Streuproblemen auf beliebigen leitfähigen Strukturen mit Hilfe der Momentenmethode im Zeitbereich. Fortschr.-Ber. VDI Reihe 21 Nr. 351, VDI Verlag Düsseldorf, 2003, Ph.D. dissertation, Technical University of Hamburg-Harburg, Germany, (In German).

[45] A. Grama, V. Kumar, and A. Sameh, "Parallel Hierarchical Solvers and Preconditioners for Boundary Element Methods," in Proc. of the 1996 ACM/IEEE Conference on Supercomputing, Pittsburgh, Pennsylvania, United States. IEEE Computer Society, 1996, p. 34.

[46] M. Nilsson, “Iterative Solution of Maxwell's Equations in Frequency Domain," Master's thesis, Uppsala University of Technology, Uppsala, Sweden, 2002.

[47] M. Löhndorf, "Effiziente Behandlung von Integraloperatoren mit $\mathcal{H}^{2}$-Matrizen variabler Ordnung,” Ph.D. dissertation, University of Leipzig, Germany, 2003, (In German).

[48] J. Ekman, "Electromagnetic Modeling Using the Partial Element Equivalent Circuit Method," Ph.D. dissertation, Luleå University of Technology, Luleå, Sweden, 2003.

[49] S. M. Rao, T. K. Sarkar, and R. F. Harrington, "The Electrostatic Field of Conducting Bodies in Multiple Dielectric Media," IEEE Trans. Microwave Theory Tech., vol. MTT-32, no. 11, pp. 1441-1448, November 1984.

[50] T. Mader, "Berechnung elektromagnetischer Felderscheinungen in abschnittsweise homogenen Medien mit Oberflächensimulation," Ph.D. dissertation, Technical University of Hamburg-Harburg, Germany, 1992, (In German).

[51] T. H. Hubing, "Survey of numerical electromagnetic modeling techniques," University of Missouri-Rolla, USA, 1991, Report No. TR91-1-001.3.

[52] A. R. Djordjević and T. K. Sarkar, "Computation of Inductance of Simple Vias Between Two Striplines Above a Ground Plane," IEEE Trans. Microwave Theory Tech., vol. MTT33, no. 3, pp. 265-269, March 1985.

[53] A. E. Ruehli and A. C. Cangellaris, "Progress in the Methodologies for the Electrical Modeling of Interconnects and Electronic Packages," Proc. IEEE, vol. 89, no. 5, pp. 740-771, May 2001. 
[54] A. E. Ruehli, "Inductance Calculations in a Complex Integrated Circuit Environment," IBM Journal of Research and Development, vol. 16, no. 5, pp. 470-481, September 1972.

[55] A. E. Ruehli and P. A. Brennan, "Efficient Capacitance Calculations for Three-Dimensional Multiconductor Systems," IEEE Trans. Microwave Theory Tech., vol. MTT-21, no. 2, pp. 76-82, February 1973.

[56] A. Görisch and G. Wollenberg, "Simulation von PEEC Modellen mit SPICE," in Proc. of the EMV, Düsseldorf, Germany, 2000, pp. 181-188, (In German).

[57] A. E. Ruehli, G. Antonini, J. Esch, J. Ekman, A. Orlandi, and A. Mayo, "Nonorthogonal PEEC Formulation for Time- and Frequency Domain EM and Circuit Modeling," IEEE Trans. Electromagn. Compat., vol. 45, no. 2, pp. 167-176, 2003.

[58] M. E. Verbeek, "Partial element equivalent circuit (PEEC) models for on-chip passives and interconnects," International Journal of Numerical Modelling: Electronic Networks, Devices and Fields, vol. 17, no. 1, pp. 61-84, February 2004.

[59] J. Ekman, “Experimental Verification of PEEC Based Electric Field Simulations," Master's thesis, Luleå University of Technology, Luleå, Sweden, 1999.

[60] G. Antonini, A. E. Ruehli, and J. Esch, "Non-Orthogonal PEEC Formulation For Time And Frequency Domain Modeling," in Proc. of the IEEE International Symposium on Electromagnetic Compatibility, Minneapolis, MN, USA, August 2002.

[61] G. Wollenberg and A. Görisch, "Analysis of 3-D Interconnect Structures with PEEC Using SPICE," IEEE Trans. Electromagn. Compat., vol. 41, no. 4, pp. 412-417, November 1999.

[62] J. E. Garrett, "Advancements of the Partial Element Equivalent Circuit Formulation,” Ph.D. dissertation, University of Kentucky, Lexington, Kentucky, USA, 1997.

[63] D. Gope, A. Ruehli, and V. Jandhyala, "Surface-based PEEC Formulation for Modeling Conductors and Dielectrics in Time and Frequency Domain Combined Circuit Electromagnetic Simulation," in Proc. of the EPEP Meeting, Portland, Oregon, USA, 2004, pp. 329332.

[64] V. Jandhyala and C. Yang, "A Time Domain Surface Integral Technique for Mixed Electromagnetic and Circuit Simulation," in Proc. of the IEEE Meeting on Electric. Perf. of Electron. Packaging, San Jose, CA, USA, October 2003, pp. 41-44.

[65] J. D. Rockway, N. J. Champagne, and V. Jandhyala, "Mixed Electromagnetic and Circuit Simulation using Higher-Order Elements and Bases," in Proc. of the International Conference on Electromagnetics in Advanced Applications, Torino, Italy, September 2003.

[66] S. Börm and W. Hackbusch, "Hierarchical Quadrature of Singular Integrals," Max Planck Institute for Mathematics in the Sciences, preprint 50. [Online]. Available: http://www.mis.mpg.de/preprints/

[67] The MISEA Project. [Online]. Available: http://misea.de 
[68] J. Garrett, A. E. Ruehli, and C. R. Paul, "Accuracy and Stability Improvements of Integral Equation Models using the Partial Element Equivalent Circuit (PEEC) Approach," IEEE Trans. Antennas Propagat., vol. 46, no. 12, pp. 1824-1831, December 1998.

[69] SimLab Software GmbH, Para3D User's Manual version 1.8, 2005.

[70] U. Jacobus, "Recent Advances in Electromagnetic Field Modelling Technologies as Applied to Automotive EMC Applications," Talk at the 16th International Zurich Symposium on Electromagnetic Compatibility, Zurich, Switzerland, February 13th - 18th, 2005.

[71] M. J. Hagmann, O. P. Gandhi, and C. H. Durney, "Upper Bound on Cell Size for MomentMethod Solutions," IEEE Trans. Microwave Theory Tech., vol. MTT-25, no. 10, pp. 831832, October 1977.

[72] K. Aygün, B. Shanker, A. A. Ergin, and E. Michielssen, "A Two-Level Plane Wave TimeDomain Algorithm for Fast Analysis of EMC/EMI Problems," IEEE Trans. Electromagn. Compat., vol. 44, no. 1, pp. 152-164, February 2002.

[73] SimLab Software GmbH, SLSim User's Manual version 3.6, 2005.

[74] — PCBMod User's Manual version 3.8, 2005.

[75] M. Tröscher and M. Klamet, "Reduction of Complexity when Simulating Large-Scale PCB Circuits," in Proc. of the International Zurich Symposium on Electromagnetic Compatibility, vol. 13, February 1999, pp. 239-244.

[76] G. Antonini, "Fast Multipole Method for Time Domain PEEC Analysis," IEEE Trans. Mobile Comput., vol. 2, no. 4, pp. 275-287, October-December 2003.

[77] G. Antonini and A. E. Ruehli, "Fast Multipole and Multifunction PEEC Methods," IEEE Trans. Mobile Comput., vol. 2, no. 4, pp. 288-298, October-December 2003.

[78] A. Englmaier, "EMC Modelling Strategy For Automotive Applications," in Proc. of the 29th European Microwave Conference Munich, Germany, November 1999.

[79] J. Ekman, G. Antonini, A. Orlandi, and A. E. Ruehli, "Stability of PEEC Models with Respect to Partial Element Accuracy," in Proc. of the IEEE International Symposium on Electromagnetic Compatibility, Santa Clara, CA, USA, August 2004.

[80] K. L. Shepard and Z. Tian, "Return-limited Inductances: A Practical Approach to On-chip Inductance Extraction," in Proc. of the IEEE Custom Integrated Circuits Conference, 1999, pp. 453-456.

[81] W. H. Press, S. A. Teukolsky, W. T. Vetterling, and B. P. Flannery, Numerical Recipes in C, The Art of Scientific Computing, 2nd ed. Cambridge University Press, 1999.

[82] S. Sauter, Cubature techniques for 3-d Galerkin BEM. in W. Hackbusch, G. Wittum eds, "Boundary Elements: Implementation and Analysis of Advanced Algorithms", NNNFM 54, Vieweg-Verlag, pp. 29-44, 1996. 
[83] S. Sauter and S. Erichsen, "Efficient automatic quadrature in 3-d Galerkin BEM," Computer Methods in Applied Mechanics and Engineering, vol. 157, pp. 215-224, 1998.

[84] W. Hackbusch and Z. P. Nowak, "On the fast matrix multiplication in the boundary element method by panel clustering," Numerische Mathematik, vol. 54, pp. 463-491, 1989.

[85] S. Börm and S. A. Sauter, "Classical BEM with linear complexity," MathComp., vol. 74, pp. 1139-1177, 2005.

[86] B. Krauter and L. Pileggi, "Generating Sparse Partial Inductance Matrices with Guaranteed Stablity," in Proc. of the IEEE International Conference on Computer-Aided Design, San Jose, CA, USA, 1995, pp. 45-52.

[87] A. Devgan, H. Ji, and W. Dai, "How to Efficiently Capture On-Chip Inductance Effects: Introducing a New Circuit Element K," in Proc. of the International Conference on ComputerAided Design, November 2000, pp. 150-155.

[88] Z. He, M. Celik, and L. Pileggi, "SPIE: Sparse Partial Inductance Extraction," in Proc. of the International Design Automation Conference, 1997, pp. 137-140.

[89] Y. Du and W. Dai, "Partial Reluctance $K$ Based Circuit Analysis Is Stable," University of California, Santa Cruz, CA, USA, UCSC-CRL-03-09, Tech. Rep., 2003.

[90] M. Beattie and L. Pileggi, "Efficient Inductance Extraction via Windowing," in Proc. of the International Conference on Design, Automation and Tests in Europe, Munich, Germany, 2001, pp. 430-436.

[91] H. Zheng, B. Krauter, M. Beattie, and L. Pileggi, "Window-Based Susceptance Models for Large-Scale RLC Circuit Analyses," in Proc. of the International Conference on Design, Automation and Test in Europe, 2002, pp. 628-633.

[92] H. Ji, A. Devgan, and W. Dai, "KSPICE: Efficient and Stable RKC Simulation for Capturing On-Chip Inductance Effect," University of California, Santa Cruz, CA, USA, UCSC-CRL00-10, Tech. Rep., April 2000.

[93] Y. Du, H. Ji, and W. Dai, "Locality and Direct Extraction of Partial Reluctance K," University of California, Santa Cruz, CA, USA, UCSC-CRL-03-08, Tech. Rep., 2003.

[94] R. Beals, "Exact fundamental solutions," Journées Équations aux dérivées partielles, June 1998.

[95] S. Börm, L. Grasedyk, and W. Hackbusch, "Introduction to hierarchical matrices with applications," Engineering Analysis with Boundary Elements, Elsevier Science Ltd., vol. 27, pp. 405-422, 2003.

[96] T.-H. Chen, C. Luk, and C. C.-P. Chen, "INDUCTWISE: Inductance-Wise Interconnect Simulator and Extractor," IEEE Trans. Computer-Aided Design, vol. 22, no. 7, pp. 884894, July 2003. 
[97] M. Kamon, S. McCormick, and K. Shepard, "Interconnect Parasitic Extraction in the Digital IC Design Methodology," in Proc. of the IEEE International Conference on ComputerAided Design, San Jose, CA, USA, 1999, pp. 223-231.

[98] H. Ji, Q. Yu, and W. Dai, “Spice Compatible Circuit Model for Reluctance K," University of California, Santa Cruz, CA, USA, UCSC-CRL-03-07, Tech. Rep., April 2003.

[99] J. Jain, C.-K. Koh, and V. Balakrishnan, "Fast Simulation of VLSI Interconnects," in Proc. of the International Conference on Computer Aided Design, San Jose, CA, USA, November 2004, pp. 93-98.

[100] T. Chen and C. C.-P. Chen, "Efficient Large-Scale Power Grid Analysis Based on Preconditioned Krylov-Subspace Iterative Methods," in Proc. of the International Design Automation Conference, June 2001, pp. 559-562.

[101] T.-H. Chen, C. Luk, and C. C.-P. Chen, "SuPREME: Substrate and Power-delivery Reluctance-Enhanced Macromodel Evaluation," in Proc. of the International Conference on Computer Aided Design, 2003, pp. 786-792.

[102] J. Vlach and K. Singhal, Computer Methods for Circuit Analysis and Design, 2nd ed. Van Nostrand Reinhold, New York, 1994.

[103] N. Balabanian, Fundamentals of Circuit Theory. Allyn and Bacon, Inc., Boston, 1961.

[104] E. Chiprout, H. Heeb, M. S. Nakhla, and A. E. Ruehli, "Simulating 3-D Retarded Interconnect Models using Complex Frequency Hopping (CFH)," in Proc. of the IEEE International Conference on Computer-Aided Design, Santa Clara, CA, USA, November 1993, pp. 66-72.

[105] H. Heeb and A. E. Ruehli, "Retarded Models for PC Board Interconnects - or How the Speed of Light Affects Your SPICE Circuit Simulation," in Proc. of the IEEE International Conference on Computer-Aided Design, November 1991, pp. 70-73.

[106] P. J. Restle, A. E. Ruehli, S. G. Walker, and G. Papadopoulos, "Full-Wave PEEC TimeDomain Method for the Modeling of On-Chip Interconnects," IEEE Trans. Computer-Aided Design, vol. 20, no. 7, pp. 877-887, July 2001.

[107] H. Heeb, A. Ruehli, J. E. Bracken, and R. A. Rohrer, "Three Dimensional Circuit Oriented Electromagnetic Modeling for VLSI Interconnects," in Proc. of the IEEE International Conference on Computer-Aided Design, Cambridge, MA, USA, 1992, pp. 218-221.

[108] J. R. Phillips, E. Chiprout, and D. D. Ling, "Efficient Full-wave Electromagnetic Analysis via Model-Order Reduction of fast Integral Transforms," in Proc. of the International Design Automation Conference, Las Vegas, NV, USA, June 1996.

[109] A. Görisch, Netzwerkorientierte Modellierung und Simulation elektrischer Verbindungsstrukturen mit der Methode der partiellen Elemente. Cuvillier Verlag Göttingen, 2002, Ph.D. dissertation, Otto-von-Guericke University of Magdeburg, Germany, (In German). 
[110] F. Schmid, “Optimization of Experimental Computational Electromagnetics Code," Master's thesis, Luleå University of Technology, Luleå, Sweden, 2005.

[111] L. Greengard and V. Rokhlin, "A fast algorithm for particle simulations," Journal of Computational Physics, vol. 73, no. 3, pp. 325-348, December 1987.

[112] R. Coifman, V. Rokhlin, and S. Wandzura, "The Fast Multipole Method for the Wave Equation: A Pedestrian Prescription," IEEE Antennas Propagat. Mag., vol. 35, no. 3, pp. 7-12, 1993.

[113] S. Börm and L. Grasedyk, "Hybrid cross approximation of integral operators," Max Planck Institute for Mathematics in the Sciences, preprint 68/2004. [Online]. Available: http://www.mis.mpg.de/preprints/

[114] — - "Low-rank approximation of integral operators by interpolation," Max Planck Institute for Mathematics in the Sciences, preprint 72/2002. [Online]. Available: http://www.mis.mpg.de/preprints/

[115] C. Lage, G. Schmidlin, and C. Schwab, "Rapid solution of first kind boundary integral equations in $\mathbb{R}^{3}$," Engineering Analysis with Boundary Elements, vol. 27, pp. 469-490, 2003.

[116] K. Giebermann, "Multilevel approximation of boundary integral operators," Computing, vol. 67 , pp. 183-207, 2001.

[117] L. Grasedyk, "Adaptive Recompression of $\mathcal{H}$-Matrices for BEM," Computing, vol. 74, pp. 205-223, 2004.

[118] M. Bebendorf and R. Kriemann, "Fast Parallel Solution of Boundary Integral Equations and Related Problems," Max Planck Institute for Mathematics in the Sciences, preprint 10/2004. [Online]. Available: http://www.mis.mpg.de/preprints/

[119] G. Antonini, "The Fast Multipole Method for PEEC Circuits Analysis," in Proc. of the IEEE International Symposium on Electromagnetic Compatibility, Minneapolis, Minnesota, USA, August 2002, pp. 446-451.

[120] U. Jacobus and J. v. Tonder, "Anwendung einer mehrstufigen schnellen Multipolmethode zur Analyse komplexer EMV Probleme," in Proc. of EMV 2004, Düsseldorf, 2004, pp. 219-226, (In German).

[121] A. T. Ihler, “An Overview of Fast Multipole Methods,” Area Exam, April 2004.

[122] M. Fischer, H. Perfahl, and L. Gaul, "Approximate inverse preconditioning for the fast multipole BEM in accoustics," University of Stuttgart, Germany, 2004, Special Research Program SFB 404, Mehrfeldprobleme in der Kontinuumsmechanik.

[123] J. A. Stratton, Electromagnetic Theory. McGraw-Hill, 1941. 
[124] J. Lee, J. Zhang, and C.-C. Lu, "Sparse Inverse Preconditioning of Multilevel Fast Multipole Algorithm for Hybrid Integral Equations in Electromagnetics," IEEE Trans. Antennas Propagat., vol. 52, no. 9, pp. 2277-2287, September 2004.

[125] J. Song, C.-C. Lu, and W. C. Chew, "Multilevel Fast Multipole Algorithm for Electromagnetic Scattering by Large Complex Objects," IEEE Trans. Antennas Propagat., vol. 45, no. 10, pp. 1488-1493, October 1997.

[126] M. J. Grote and T. Huckle, "Effective Parallel Preconditioning with Sparse Approximate Inverses," in Proc. of the 7th SIAM Conference on Parallel Processing for Scientific Computing, February 1995.

[127] Ö. Ergül and L. Gürel, "Comparison of FMM Implementations Employing Different Formulations and Iterative Solvers," in Proc. of the IEEE AP-S International Symposium and USNC/CNC/URSI National Radio Science Meeting, Columbus, Ohio, USA, June 2003.

[128] M. Lintner, "Lösung der 2D Wellengleichung mittels hierarchischer Matrizen," Ph.D. dissertation, Technical University of Munich, Germany, 2002, (In German).

[129] S. Börm, "Approximation of integral operators by $\mathcal{H}^{2}$-matrices with adaptive bases," Computing, vol. 74, pp. 249-271, 2005.

[130] B. N. Khoromskij, "Data-sparse approximation of integral operators," Max Planck Institute for Mathematics in the Sciences, Lecture notes 17/2003. [Online]. Available: http://www.mis.mpg.de/preprints/

[131] O. Steinbach, "Hierarchische Matrizen," University of Stuttgart, Germany, Lecture notes 16/2004, (In German).

[132] M. Bebendorf, "Approximate Inverse Preconditioning of FE Systems for Elliptic Operators with non-smooth Coefficients," Max Planck Institute for Mathematics in the Sciences, preprint 7/2004. [Online]. Available: http://www.mis.mpg.de/preprints/

[133] O. Steinbach, Lösungsverfahren für lineare Gleichungssysteme. B. G. Teubner Verlag/GWV Fachverlage GmbH, Wiesbaden, 2005, (In German).

[134] L. Grasedyk, “Theorie und Anwendung Hierarchischer Matrizen,” Ph.D. dissertation, University of Kiel, Germany, 2001, (In German).

[135] M. Bebendorf, Effiziente numerische Lösung von Randintegralgleichungen unter Verwendung von Niedrigrang-Matrizen. Ph.D. dissertation, dissertation.de - Verlag im Internet, ISBN 3-89825-183-7, 2001, (In German).

[136] L. Grasedyk, W. Hackbusch, and S. Le Borne, "Adaptive Geometrically Balanced Clustering of $\mathcal{H}$-Matrices," Max Planck Institute for Mathematics in the Sciences, preprint 19/2004. [Online]. Available: http://www.mis.mpg.de/preprints/

[137] M. Bebendorf, "Project homepage." [Online]. Available: http://www.math.uni-leipzig.de/ bebendorf/projekte.html 
[138] U. Langer and D. Pusch, "Sparse Algebraic Multigrid Methods for Large Scale Boundary Element Equations," Johannes Kepler University of Linz, Austria, 2003, Special Research Program SFB F013.

[139] M. Bebendorf, "Approximation of Boundary Element Matrices," Numerical Mathematics, vol. 86, no. 4, pp. 565-589, 2000.

[140] L. Banjai and W. Hackbusch, " $\mathcal{H}$ - and $\mathcal{H}^{2}$-matrices for low and high frequency Helmholtz equation," Max Planck Institute for Mathematics in the Sciences, preprint 17/2005. [Online]. Available: http://www.mis.mpg.de/preprints/

[141] L. Grasedyk, W. Hackbusch, and S. Le Borne, "Adaptive Refinement and Clustering of $\mathcal{H}$-Matrices," Max Planck Institute for Mathematics in the Sciences, preprint 106/2001. [Online]. Available: http://www.mis.mpg.de/preprints/

[142] J. Djokić, "Efficient Update of Hierarchical Matrices in the case of Adaptive Discretisation Schemes," Ph.D. dissertation, University of Leipzig, Germany, 2006.

[143] S. Börm, L. Grasedyk, and W. Hackbusch, "Hierarchical matrices," Max Planck Institute for Mathematics in the Sciences, Lecture notes 21/2003. [Online]. Available: http://www.mis.mpg.de/preprints/

[144] M. Bebendorf, "Hierarchical LU decomposition based preconditioners for BEM," Max Planck Institute for Mathematics in the Sciences, preprint 28/2004. [Online]. Available: http://www.mis.mpg.de/preprints/

[145] A. George and J. W.-H. Liu, Computer Solution of Large Sparse Positive Definite Systems. Prentice-Hall, Inc., 1981.

[146] M. Bollhöfer, "Geometrische Mehrgitterverfahren und Algebraische Mehrgitterverfahren Eine Einführung," Lecture notes, Technical University of Berlin, Germany, 2001, (In German).

[147] Y. Saad, Iterative Methods for Sparse Linear Systems. PWS Publishing Company, 1996.

[148] D. Pusch, "Efficient Algebraic Multigrid Preconditioners for Boundary Element Methods," Master's thesis, Johannes Kepler University of Linz, Austria, 2003.

[149] C. Wrobel, "Die Parallelisierung des filternden algebraischen Mehrgitterverfahrens zum Lösen partieller Differentialgleichungen," Ph.D. dissertation, Ruprecht-Karls-University of Heidelberg, Germany, 2003, (In German).

[150] G. Karypis and V. Kumar, "A Fast and High Quality Multilevel Scheme for Partitioning Irregular Graphs," TR 95-035, Tech. Rep., 1995. [Online]. Available: http://citeseer.ifi.unizh.ch/karypis98fast.html

[151] G. Karypis, R. Aggarwal, V. Kumar, and S. Shekhar, "Multilevel Hypergraph Partitioning: Application in VLSI Domain," in Proc. of the IEEE International Conference on ComputerAided Design, Anaheim, CA, USA, June 1997. 
[152] H. A. van der Vorst, Iterative Krylov Methods for Large Linear Systems. Cambridge University Press, 2003.

[153] P. Wesseling, An Introduction to Multigrid Methods. John Wiley \& Sons, 1992.

[154] V. E. Henson, "An Algebraic Multigrid Tutorial," Center of Applied Scientific Computing, Lawrence Livermore National Laboratory, 1999.

[155] T. Brandtner, "Hierarchical Simulation of Substrate Coupling with Precalculated Macromodels," Ph.D. dissertation, Johannes Kepler University of Linz, Austria, 2003.

[156] Intel Corporation, "Intel MKL Reference Manual 8.0," Intel Corporation, linear Solver Basics. [Online]. Available: http://developer.intel.com

[157] T. Clees, "AMG Strategies for PDE Systems with Applications in Industrial Semiconductor Simulation,” Ph.D. dissertation, University of Köln, Germany, 2005.

[158] K. Stüben, SAMG - Data Structure and File Format Specification, Fraunhofer Institute SCAI, Sankt Augustin, Germany, February 2005.

[159] O. Schenk, S. Röllin, and A. Gupta, "The Effects of Unsymmetric Matrix Permutations and Scalings in Semiconductor Device and Circuit Simulation," IEEE Trans. Computer-Aided Design of Integrated Circuits and Systems, vol. 23, no. 3, pp. 400-411, March 2004.

[160] K. D. Devine, E. G. Boman, R. T. Heaphy, R. H. Bisseling, and U. V. Catalyurek, "Parallel Hypergraph Partitioning for Scientific Computing," in Proc. of the IEEE International Parallel \& Distributed Processing Symposium, 2006, to appear.

[161] L. Grasedyk, R. Kriemann, and S. Le Borne, "Parallel Black Box Domain Decomposition Based $\mathcal{H}-L U$ Preconditioning," Mathematics of Computation, 2005, submitted to.

[162] M. L. Zitzmann, R. Grillmair, T. Clees, and R. Weigel, "Hybrid Solver Strategies in Automotive EMC Simulation," in Proc. of the 17th International Zurich Symposium on Electromagnetic Compatibility, Singapore 2006, February 27th - March 3rd, 2006, pp. 340-343.

[163] M. L. Zitzmann, T. Clees, and R. Weigel, "Iterative Methods for Reluctance based PEEC Models," in Proc. of the 17th International Zurich Symposium on Electromagnetic Compatibility, Singapore 2006, February 27th - March 3rd, 2006, pp. 81-84.

[164] R. Grillmair, "Iterative Gleichungslösung mittels hierarchischer Matrizen für die Schaltkreissimulation," Master's thesis, Munich University of Applied Sciences, Munich, Germany, 2006, (In German).

[165] W. Hackbusch, "Multi-Grid Methods for FEM and BEM Applications," Max Planck Institute for Mathematics in the Sciences, preprint 72, 2003. [Online]. Available: http://www.mis.mpg.de/preprints/

[166] A. Krechel and K. Stüben, "GMD Report 71: Parallel Algebraic Multigrid Based on Subdomain Blocking," GMD - Forschungszentrum Informationstechnik GmbH, Germany, Tech. Rep., 1999. 
[167] K. Stüben, An Introduction to Algebraic Multigrid. in U. Trottenberg, C. W. Oosterlee and T. Schüller, "Multigrid”, Academic Press, London, San Diego, pp. 413-532, 2001.

[168] C. Wagner, "Ein algebraisches Mehrgitterverfahren - Entwicklung und Anwendung auf einen Sanierungsfall," Habilitationsschrift - Ruprechts-Karls-University of Heidelberg, Germany, 2000, (In German).

[169] J. H. Bramble, Z. Leyk, and J. E. Pasciak, "The Analysis of Multigrid Algorithms for Pseudo-Differential Operators of Order Minus One," Math. Comp., vol. 63, no. 208, pp. 461-478, 1994.

[170] A. Schüller, K. Stüben, C.-A. Thole, and U. Trottenberg, "Paralleles Rechnen und schnelle Simulation," Der GMD-Spiegel, no. 1/2, pp. 9-11, 2000, (In German).

[171] R. Kriemann, "Parallel $\mathcal{H}$-Matrix Arithmetics on Shared Memory Systems," Max Planck Institute for Mathematics in the Sciences, preprint 29/2004. [Online]. Available: http://www.mis.mpg.de/preprints/

[172] E. Chow, R. D. Falgout, J. J. Hu, R. S. Tuminaro, and U. M. Yang, "A Survey of Parallelization Techniques for Multigrid Solvers," University of California, Lawrence Livermore National Laborator, USA, UCRL-BOOK-205864, 2005.

[173] R. Kriemann, "Parallele Algorithmen für $\mathcal{H}$-Matrizen," Ph.D. dissertation, ChristianAlbrechts-University of Kiel, Germany, 2004, (In German).

[174] R. H. Neumayer, "Network-Parameter-Based Modeling and Simulation of System-Level Automotive Electromagnetic Compatibility," Ph.D. dissertation, Johannes Kepler University of Linz, Austria, 2003.

[175] The COSIME Project. [Online]. Available: http://cosime.icie.uni-linz.ac.at

[176] G. M. A. Sessler, "Schnell Konvergierender Polynomial Expansion Multiuser Detektor mit niedriger Komplexität," Ph.D. dissertation, University of Karlsruhe, Germany, 2003, (In German).

[177] N. J. Higham, “The Matrix Computation Toolbox for MATLAB (Version 1.0),” Numerical Analysis Report No. 410, University of Manchester, GB, August 2002.

[178] M. M. Ney, "Method of Moments as Applied to Electromagnetic Problems," IEEE Trans. Microwave Theory Tech., vol. MTT-33, no. 10, pp. 972-980, October 1985.

[179] A. H. Stroud and D. Secrest, Gaussian Quadrature Formulas. Prentice-Hall, Inc., 1966.

[180] W. Hackbusch, "Panel clustering technique and hierarchical matrices for BEM and FEM," Max Planck Institute for Mathematics in the Sciences, preprint 71/2003. [Online]. Available: http://www.mis.mpg.de/preprints/

[181] M. Bebendorf, "Effektive algebraische Realisierung von Randelementmethoden in 3D," Master's thesis, University of Kaiserslautern, Germany, 1997, (In German). 
[182] C.-T. Tai, Generalized Vector and Dyadic Analysis: Applied Mathematics in Field Theory. IEEE Press, 1999.

[183] H. J. Korsch, Mathematische Ergänzungen zur Einführung in die Physik. Binomi Verlag, Springe, 1999, Lecture notes, University of Kaiserslautern, Germany, (In German).

[184] W. J. McCalla and D. O. Pederson, "Elements of Computer-Aided Circuit Analysis," IEEE Trans. Circuit Theory, vol. CT-18, no. 1, pp. 14-26, January 1971.

[185] T. Bashkow, “The A Matrix, New Network Description," IEEE Trans. Circuit Theory, vol. CT-4, no. 3, pp. 117-119, September 1957.

[186] C.-W. Ho, A. E. Ruehli, and P. A. Brennan, "The Modified Nodal Approach to Network Analysis," IEEE Trans. Circuits Syst., vol. CAS-22, no. 6, pp. 504-509, June 1975.

[187] E. E. Höfer and H. Nielinger, SPICE. Springer-Verlag, 1985.

[188] B. Johnson, T. Quarles, A. R. Newton, D. O. Pederson, and A. Sangiovanni-Vicentelli, SPICE3 Version 3e User's manual, Department of Electrical Engineering and Computer Sciences, University of California, Berkley, USA, 1991.

[189] A. Vladimirescu, "SPICE - The Fourth Decade - Analog and Mixed-Signal Simulation - A State of the Art," pp. 39-44, University of California, Berkley, USA, 1999.

[190] D. O. Pederson, "A Historical Review of Circuit Simulation," IEEE Trans. Circuits Syst., vol. CAS-31, no. 1, pp. 103-111, January 1984.

[191] J. Siegl, "Simulationsmethoden für die Schaltungsverifikation," University of Applied Sciences, Nuremberg, Germany, Lecture notes 1999, (In German). 


\section{List of Figures}

1.1 Car body with cable harnesses and control systems. . . . . . . . . . . . . . 2

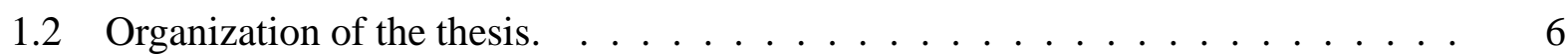

2.1 The electromagnetic scattering problem. . . . . . . . . . . . . . . 12

3.1 PEEC volume and surface discretization principle for cartesian coordinates. . . . 20

3.2 Hexahedral shaped volume cell with quadrilateral shaped surface cells. . . . . . . 22

3.3 PEEC volume and surface discretization principle. . . . . . . . . . . . . . . 23

3.4 Equivalent circuit representation of PEEC models. . . . . . . . . . . . . . . . 26

3.5 Volume equivalence principle for dielectric objects. . . . . . . . . . . . 28

3.6 Equivalent circuit representation of a dielectric cell. . . . . . . . . . . . . . . 29

3.7 Equivalent circuit representation of a dielectric-to-conductor coupling. . . . . . . 30

3.8 Original problem, surface equivalence principle and electric/magnetic separation of a dielectric-conductor problem. . . . . . . . . . . . . . . 31

3.9 (S)PEEC equivalent circuit representation for a single dielectric cell. . . . . . . . 33

3.10 Workflow of a PEEC-based modeling and circuit simulation. . . . . . . . . . 33

3.11 Surface triangulation for the extraction of partial coefficients of potential. . . . . 35

3.12 Calculation domains for PEEC modeling. . . . . . . . . . . . . . 36

3.13 Frequency domain simulation artifacts. . . . . . . . . . . . . . . 39

3.14 Distribution of Sauter quadrature points in the reference triangle. . . . . . . . . 40

3.15 Convergence behavior and accuracy of the Sauter quadrature. . . . . . . . . . . . 41

3.16 Quasi-stationary PEEC model with three nodes and two branches. . . . . . . . . 47

3.17 Sparsity pattern of the resulting network matrices. . . . . . . . . . . . 48

3.18 Impedance analysis comparison at different degrees of sparsification. . . . . . . . 49

4.1 Influence of the integral operators kernel function. . . . . . . . . . . . . . 55

4.2 Grouping of basis functions in FMM. . . . . . . . . . . . . . . . 56

4.3 Index set mapping between nodal points (DOF) and matrix indices. . . . . . . . . 62

4.4 The cluster tree $T_{\mathcal{I}}$ for index set $\mathcal{I} \ldots \ldots \ldots \ldots$ 
4.5 Decomposition of a given triangular discretization (bisection) into clusters. . . . 64

4.6 Correspondence between the geom. arrangement and the matrix-block. . . . . . . 64

4.7 Hierarchical admissible partitioning of a matrix. . . . . . . . . . . . . . . 65

$4.8 \mathcal{H}$-matrix approximation for a PEEC-based circuit simulation matrix. . . . . . . . 67

4.9 Admissible partitioning for an empty influence matrix . . . . . . . . . . . . . . 69

4.10 Construction of an empty $\mathcal{H}$-influence-matrix . . . . . . . . . . . . . . . 70

4.11 Recursive filling of the $\mathcal{H}$-influence-matrix. . . . . . . . . . . . . 71

4.12 Power supply net VB on the MISEA demonstrator board. . . . . . . . . . . . . 72

4.13 Benchmark example PEEC21. $\mathcal{H}$-influence-matrix structure. . . . . . . . . . . 74

4.14 Benchmark PEEC network matrix. $\mathcal{H}$-matrix and $\mathcal{H}^{2}$-matrix approximation. . . . 75

5.1 Number of required iteration steps by applying the Richardson iteration. . . . . . 79

5.2 Influence of the frequency range to circuit simulation matrices. . . . . . . . . . . 80

5.3 Time and storage complexities of direct and iterative methods. . . . . . . . . 82

6.1 Principle structure of an $\mathcal{H}$-matrix-based circuit simulator. . . . . . . . . . . . . 94

6.2 Selected low-rank block $M_{b}$ and the representation of the $\mathcal{R}_{1}$-matrix-stamp. . . . 95

$6.3 \mathcal{H}$-stamping procedure for one single stamping element. . . . . . . . . . . . 95

6.4 Circuit simulation network matrix constructed by $\mathcal{H}$-stamping. . . . . . . . . . . 96

6.5 Circuit simulator with $\mathcal{H}$-stamping and adaptive preconditioner update. . . . . . 98

$6.6 \mathcal{H}$-matrix structure of the preconditioner. . . . . . . . . . . . . . . . 99

6.7 Convergence results for $\mathcal{H}$-precond. one-level iterative circuit simulation. . . . . 100

6.8 Number of required iteration steps applying adaptive preconditioning. . . . . . . 102

7.1 Relation between high- and low-frequency error components (Poisson equation). 106

7.2 Interplay between prolongation $(\mathcal{P})$ and restriction $(\mathcal{R})$ in AMG. . . . . . . . . 109

7.3 The workflow for V-cycles and W-cycles in multigrid methods. . . . . . . . . . 112

7.4 Influence of the tolerance limit to the truncation error $\varepsilon_{\mathcal{T}} \ldots \ldots \ldots \ldots$

7.5 Block diagram for the implementation of SAMG. . . . . . . . . . . . . . . 117

7.6 SAMG solver: Required numbers of iterations in FD analysis. . . . . . . . . . . 119

7.7 S AMG solver: $L_{2}$-norm of final residual in FD analysis. . . . . . . . . . . . . . 119

7.8 SAMG solver: Total CPU-times for PEEC (FD-analysis) . . . . . . . . . . . . . 120

7.9 SAMG solver: Required numbers of iterations (.Trans) . . . . . . . . . . . . . 120

7.10 SAMG solver: $L_{2}$-norm of final residual for a PEEC TD analysis. . . . . . . . . . 120

7.11 SAMG solver: Total CPU-times for a PEEC TD analysis. . . . . . . . . . . . . 121

7.12 Convergence behavior comparison for a PEEC matrix. . . . . . . . . . . 121

8.1 Scheduling for parallel $\mathcal{H}$-matrix-vector multiplication. . . . . . . . . . . . . 128

8.2 Inner and outer parallelization in model extraction. . . . . . . . . . . . . . . . 130 
8.3 Inner and outer parallelization in TD and FD analysis. . . . . . . . . . . . . 130

9.1 MISEA demonstrator board . . . . . . . . . . . . . . . . . . . 135

9.2 Net $/ \mathrm{N} \$ 45358$ on the MISEA board with terminals. . . . . . . . . . . . . 136

9.3 Scattering parameter measurement results in the FD of the net $/ \mathrm{N} \$ 45358$. . . . 137

9.4 Technical equipment and measurement arrangement. . . . . . . . . . . . 138

9.5 Realization of contacts to measurement points (MP) on the PCB. . . . . . . . . . 138

9.6 Comparsion between scattering parameter simulation and measurement results. . 139

9.7 Coupling paths between the main electronic parts in modern cars. . . . . . . . . 140

9.8 Multilevel approach for automotive system simulation. . . . . . . . . . . . . . 140

9.9 Influencing factors to network matrix properties. . . . . . . . . . . . . . 141

A.1 Gershgorin discs for reluctance PEEC based network matrices. . . . . . . . . . . 173

A.2 Bijective mapping from reference triangle to an arbitrary triangle. . . . . . . . 177

B.1 Green's function as a fundamental solution of the Laplace equation in 3D. . . . . 180

C.1 Illustration of cartesian coordinates. . . . . . . . . . . . . . . . . . . . 184

C.2 Illustration of curvilinear coordinates. . . . . . . . . . . . . . . . 185

C.3 Parametrization of the surface via two variables $u$ and $v \ldots \ldots \ldots$

C.4 Differential surface element. . . . . . . . . . . . . . . . . . . . 188 


\section{List of Tables}

3.1 Stamp for partial self-reluctance and mutual reluctance couplings. . . . . . . . . 47

3.2 Total simulation times for a FD impedance analysis. . . . . . . . . . . . . . 50

4.1 Complexities of formatted (truncated) $\mathcal{H}$-matrix operations. . . . . . . . . . . . 68

4.2 Parameter settings for ACA-based recursive influence extraction. . . . . . . . . . 73

4.3 Performance of conventional and recursive ACA-based influence extraction. . . . 73

6.1 Memory requirements for PEEC-based benchmark matrices. . . . . . . . . . . 98

6.2 Time and memory requirements for a PEEC-based TD simulation. . . . . . . . . 99

6.3 Conventional and adaptive preconditioning for PEEC matrices in TD analysis. . . 101

6.4 Conventional and adaptive preconditioning for PEEC matrices in FD analysis. . . 101

7.1 Computational complexity of AMG component construction. . . . . . . . . . 116

7.2 Memory complexity for the storage requirements of matrices $\boldsymbol{A}_{h}$ and $\boldsymbol{B}_{h} \ldots \ldots$

7.3 Details (matrix properties) of the two test cases. . . . . . . . . . . . 118

7.4 Performance of GMATRIX and SAMG summarized for the two test cases. . . . . . 119 


\section{Appendix $\boldsymbol{A}$}

\section{Mathematical Basics, Definitions and Notations}

$\boldsymbol{T}$

his appendix reviews some basic definitions and notations from linear algebra, which are used in this thesis. Elementary matrix notations and operations are assumed to be known. If $\boldsymbol{A}$ is a non-negative matrix, it has no negative entries. This may also be written as $\boldsymbol{A} \geqslant 0$. If $\boldsymbol{A}$ is real and symmetric, $\boldsymbol{A}=\boldsymbol{A}^{\top}$. If $\boldsymbol{A}$ is complex and Hermitian, $\boldsymbol{A}=\boldsymbol{A}^{\mathrm{H}}$ holds. If $\boldsymbol{A}$ is structurally symmetric, $\boldsymbol{A}$ and $\boldsymbol{A}^{\top}$ have non-zeros in the same locations, though the values may differ. Finally, $|\boldsymbol{A}|$ is shorthand for the matrix whose entries are $\left|A_{i j}\right|$.

\section{A.1 Basic Matrix Types}

With regard to circuit simulation purposes and iterative methods, the most important matrix types are listed in the following. Then an arbitrary square matrix $\boldsymbol{A}=\left(A_{i j}\right)$ of dimension $n \times n$ is called

- symmetric if $A_{i j}=A_{j i}, \forall i, j$.

- weakly diagonally dominant if

$$
\forall i: A_{i i} \geqslant \sum_{j \neq i}\left|A_{i j}\right|
$$

- strongly diagonally dominant if

$$
\forall i: A_{i i}>\sum_{j \neq i}\left|A_{i j}\right|
$$

- positive definite if for any non-zero vector $\vec{x} \in \mathbb{C}^{n}$

$$
\mathfrak{R}\left(\vec{x}^{\mathrm{H}} \boldsymbol{A} \vec{x}\right)>0
$$

holds. In the case of a real matrix $\boldsymbol{A}$ the expression reduces to $\vec{x}^{\top} \boldsymbol{A} \vec{x}>0$. 
- positive semi-definite if for any non-zero vector $\vec{x} \in \mathbb{C}^{n}$

$$
\mathfrak{R}\left(\vec{x}^{\mathrm{H}} \boldsymbol{A} \vec{x}\right) \geqslant 0
$$

holds. In the case of a real matrix $\boldsymbol{A}$ the expression reduces to $\vec{x}^{\top} \boldsymbol{A} \vec{x} \geqslant 0$.

- an M-Matrix (MINKOWSKI-Matrix) if its eigenvalues are positive.

For further details, refer to [157].

\section{A.2 Inner Products and Norms}

We use norms to measure the "size" of vectors and matrices, and to derive important information about the behavior in iterative solution methods. An arbitrary vector or matrix norm is denoted by $\|\cdot\|$ in the following.

\section{A.2.1 Vector Norms}

If $\vec{x}$ is a length $n$ vector, some of the vector norms we use are defined as follows:

$$
\begin{aligned}
& L^{1} \text {-norm }:\|\vec{x}\|_{1} \equiv \sum_{i=1}^{n}\left|x_{i}\right| \\
& L^{2} \text {-norm }:\|\vec{x}\|_{2} \equiv \sqrt{\sum_{i=1}^{n}\left|x_{i}\right|^{2}} \\
& L^{\infty} \text {-norm }:\|\vec{x}\|_{\infty} \equiv \max _{\forall i}\left|x_{i}\right|
\end{aligned}
$$

\section{A.2.2 Matrix Norms}

If $\boldsymbol{A}$ is a $n \times n$ matrix, some of the matrix norms we use are defined as follows:

$$
\begin{aligned}
L^{1} \text {-norm } & :\|\boldsymbol{A}\|_{1} \equiv \max _{\forall j} \sum_{i=1}^{n}\left|A_{i j}\right| \\
L^{2} \text {-norm }: & \|\boldsymbol{A}\|_{2} \equiv \max _{\forall j}\left\{\sqrt{\lambda_{j}}: \lambda_{j} \text { is an eigenvalue of } \boldsymbol{A}^{\mathrm{H}} \boldsymbol{A}\right\} \\
L^{\infty} \text {-norm } & :\|\boldsymbol{A}\|_{\infty} \equiv \max _{\forall i} \sum_{j=1}^{n}\left|A_{i j}\right| \\
\text { Frobenius norm } & :\|\boldsymbol{A}\|_{F} \equiv \sqrt{\sum_{i=1}^{n} \sum_{j=1}^{n}\left|A_{i j}\right|^{2}}
\end{aligned}
$$

Remark: The Frobenius norm is sometimes also called the Euclidean norm, which may cause confusion with the Euclidean norm for vectors ( $L^{2}$-norm).

The square roots of the non-negative eigenvalues of $\boldsymbol{A}^{\mathrm{H}} \boldsymbol{A}$ are called singular values of $\boldsymbol{A}$ and are denoted by $\sigma_{i}, i=1, \ldots, m$. Therefore, together with (A.20), $\|\boldsymbol{A}\|_{2}$ is equal to $\sigma_{1}$, which represents the largest singular value of $\boldsymbol{A}$. 
The Frobenius-, $L^{1}-, L^{2}$ - and $L^{\infty}$-norms satisfy the sub-multiplicative property

$$
\left\|\boldsymbol{A}_{1} \boldsymbol{A}_{2}\right\| \leqslant\left\|\boldsymbol{A}_{1}\right\|\left\|\boldsymbol{A}_{2}\right\| \quad \forall \boldsymbol{A}_{1} \in \mathbb{C}^{n \times m}, \forall \boldsymbol{A}_{2} \in \mathbb{C}^{m \times p} .
$$

Furthermore, the relation

$$
\|\boldsymbol{A}\|_{2} \leqslant\|\boldsymbol{A}\|_{F}
$$

between $L^{2}$ - and Frobenius norm holds.

The condition number ${ }^{1} \operatorname{cond}(\boldsymbol{A})$ of a matrix $\boldsymbol{A}$ is a measure of how sensitive the solution to that problem is to perturbations in $\boldsymbol{A}$. The condition number of $\boldsymbol{A}$ w.r.t. matrix inversion is defined as

$$
\operatorname{cond}(\boldsymbol{A}) \triangleq\|\boldsymbol{A}\| \cdot\left\|\boldsymbol{A}^{-1}\right\|
$$

if $\boldsymbol{A}$ is non-singular. If $\boldsymbol{A}$ is singular, cond $(\boldsymbol{A})=\infty$ holds. For further details, e.g. refer to [147]. The spectral radius $\rho(\boldsymbol{A})$ of an arbitrary matrix $\boldsymbol{A} \in \mathbb{R}^{n \times n}$ is defined by

$$
\rho(\boldsymbol{A}) \triangleq \max _{\forall i}\left|\lambda_{i}(\boldsymbol{A})\right|=\lambda_{\max }(\boldsymbol{A}) .
$$

For all matrices $\boldsymbol{A}_{1}, \boldsymbol{A}_{2}$, the relation

$$
\rho\left(\boldsymbol{A}_{1} \boldsymbol{A}_{2}\right)=\rho\left(\boldsymbol{A}_{2} \boldsymbol{A}_{1}\right)
$$

holds. Keeping this in mind, the Euclidean norm results in

$$
\|\boldsymbol{A}\|_{E} \triangleq \sqrt{\rho\left(\boldsymbol{A}^{\top} \boldsymbol{A}\right)} .
$$

If $\boldsymbol{A}$ is symmetric,

$$
\|\boldsymbol{A}\|_{E}=\rho(\boldsymbol{A})
$$

holds, and if $\boldsymbol{A}>0$, we obtain

$$
\left\|\boldsymbol{A}^{-1}\right\|_{E}=\rho\left(\boldsymbol{A}^{-1}\right)=\frac{1}{\lambda_{\min }(\boldsymbol{A})}
$$

Within the context of iterative solution methods, the following equalities lead to alternative definitions

$$
\begin{aligned}
\|\boldsymbol{A}\|_{2} & =\sqrt{\rho\left(\boldsymbol{A}^{\mathrm{H}} \boldsymbol{A}\right)}=\sqrt{\rho\left(\boldsymbol{A} \boldsymbol{A}^{\mathrm{H}}\right)} \\
\|\boldsymbol{A}\|_{F} & =\sqrt{\operatorname{trace}\left(\boldsymbol{A}^{\mathrm{H}} \boldsymbol{A}\right)}=\sqrt{\operatorname{trace}\left(\boldsymbol{A} \boldsymbol{A}^{\mathrm{H}}\right)},
\end{aligned}
$$

that are often easier to work with, where the trace of a matrix is equal to the sum of all its diagonal elements

$$
\operatorname{trace}(\boldsymbol{A}) \triangleq \sum_{i=1}^{n} A_{i i}
$$

\footnotetext{
${ }^{1}$ The condition number can be illustrated as the degree of distortion of a standardized sphere, applying the mapping of $\boldsymbol{A}$.
} 
and the spectral radius $\rho$.

For a regular matrix $\boldsymbol{A}$, its condition number w.r.t. $\|\cdot\|_{E}$ is defined by

$$
\operatorname{cond}_{E}(\boldsymbol{A}) \triangleq\|\boldsymbol{A}\|_{E}\left\|\boldsymbol{A}^{-1}\right\|_{E} \geqslant 1
$$

If $\boldsymbol{A}>0$, we obtain

$$
\operatorname{cond}_{E}(\boldsymbol{A})=\frac{\lambda_{\max }(\boldsymbol{A})}{\lambda_{\min }(\boldsymbol{A})}
$$

with $\lambda_{\max }, \lambda_{\min }$ the maximum and minimum eigenvalues of $\boldsymbol{A}$, respectively.

\section{A.3 Truncated Singular Value Decomposition}

Each matrix $\boldsymbol{A} \in \mathbb{C}^{m \times n}$ can be expressed as [174]

$$
\boldsymbol{A}=\boldsymbol{U} \Sigma \boldsymbol{V}^{\mathrm{H}}
$$

where $\boldsymbol{U} \in \mathbb{C}^{m \times m}$ and $\boldsymbol{V} \in \mathbb{C}^{n \times n}$ are unitary and $\boldsymbol{\Sigma} \in \mathbb{R}^{m \times n}$ is unique, non-negative and diagonal with its diagonal elements arranged in non-increasing order. The diagonal elements of $\Sigma$ are the singular values ${ }^{2}$ of $\boldsymbol{A}$. If the fundamental solution is an asymptotically smooth function, it can be shown that the singular values of $\boldsymbol{A}$ decay exponentially. To find a low-rank approximation the truncated singular value decomposition (truncated SVD) [131] proved to provide a best-approximation in the Frobenius norm as well as in the spectral norm. Starting point is a matrix $\boldsymbol{A} \in \mathbb{C}^{m \times n}$ of rank $\ell$, whose singular value decomposition can be given as

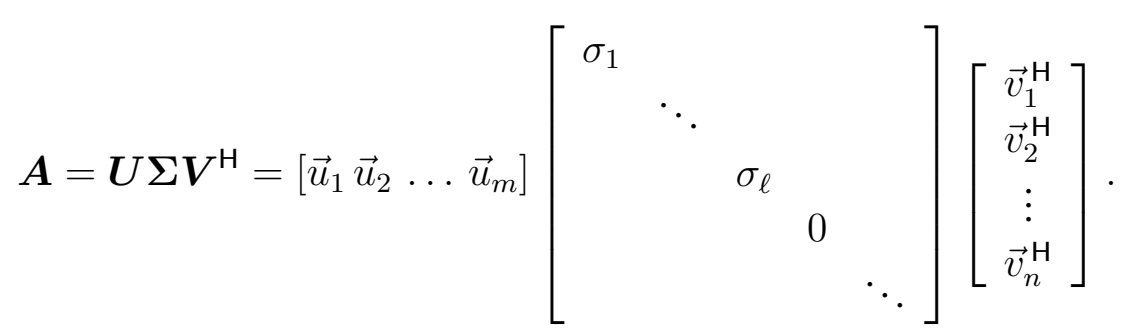

with singular values ordered so that $\sigma_{1} \geqslant \sigma_{2} \geqslant \ldots \geqslant \sigma_{\ell} \geqslant 0$. Then for any $\varepsilon>0$ there exists a $k \geqslant 1$ such that $\sigma_{k+1} \leqslant \varepsilon$. For asymptotically smooth functions $k=\mathcal{O}\left(\log ^{d-1}(1 / \varepsilon)\right)$ holds. Applying a rank truncation with $k<\ell$ by setting all singular values $\sigma_{i}, i>k$ to zero results in an approximation

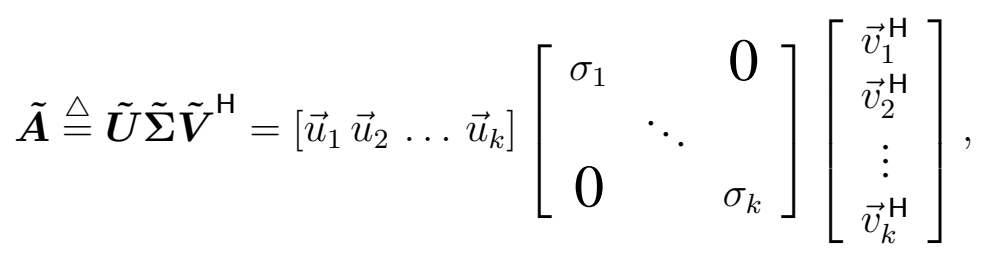

with $\tilde{\boldsymbol{\Sigma}} \triangleq \operatorname{diag}\left(\sigma_{1}, \sigma_{2}, \ldots, \sigma_{k}\right)$ and $\tilde{\boldsymbol{U}} \in \mathbb{C}^{m \times k}$ and $\tilde{\boldsymbol{V}}^{\mathrm{H}} \in \mathbb{C}^{k \times n}$ consist of the first $k$ columns of matrices $\boldsymbol{U}$ and $\boldsymbol{V}$, respectively. The error of the approximation in the spectral norm is bounded by

$$
\left\|\boldsymbol{A}-\tilde{\boldsymbol{U}} \tilde{\boldsymbol{\Sigma}} \tilde{\boldsymbol{V}}^{\mathrm{H}}\right\|_{2} \leqslant \varepsilon
$$

\footnotetext{
${ }^{2}$ Remark: The non-zero singular values $\sigma_{i}$ of $\boldsymbol{A}$ are the positive square roots of the non-zero eigenvalues of $\boldsymbol{A}^{\mathrm{H}} \boldsymbol{A}: \sigma_{i}=\sqrt{\lambda_{i}\left(\boldsymbol{A}^{\mathrm{H}} \boldsymbol{A}\right)}$.
} 
The calculation of a singular value decomposition of a matrix of dimension $m \times n$ requires $\mathcal{O}\left(\mathrm{m}^{3}+\right.$ $\left.n^{3}\right)$ operations [134]. The aim is, to find a method which enables a direct computation of the rank$k$ truncation, comprising a lower complexity compared to the original SVD. In principle, a method exists to determine the SVD of a $\mathcal{R}_{k}$-matrix with complexity $\mathcal{O}\left(k^{3}\right)+\mathcal{O}\left(k^{2}(m+n)\right)$. Therefore, $k \ll n, m$ is assumed, so that the dominating complexity is $\mathcal{O}\left(k^{2}(m+n)\right)$ [134].

\section{A.4 Gershgorin's Circle Theorem}

An accurate knowledge concerning the eigenvalues of a given matrix and their distribution in the complex plane is very important for stability considerations of extracted parasitic elements and for convergence analysis for iterative methods. An exact mathematical computation of the eigenvalues is not meaningful in the most cases, since the complexity behaves as $\mathcal{O}\left(n^{3}\right)$ [176] with matrix dimension $n$. Coarse approximations of the eigenvalue distribution are usually sufficient to identify interesting properties. A low-cost estimation of the locations of the eigenvalues can be realized using GERSHGORIN's circle theorem ${ }^{3}$. This theorem provides a precise localization bound for the placement of eigenvalues of an arbitrary square matrix by directly exploiting some knowledge on the matrix entries [147].

Assume an arbitrary square matrix $\boldsymbol{S} \in \mathbb{C}^{m \times m}$ with eigenvalues $\left\{\lambda_{k}(\boldsymbol{S})\right\}, k=1, \ldots, m$. Then, an arbitrary eigenvalue $\lambda_{k^{\prime}}(\boldsymbol{S})$ exists, for which the following equations hold in row- and columnnotation, respectively:

$$
\begin{aligned}
& \left|s_{k, k}-\lambda_{k^{\prime}}(\boldsymbol{S})\right| \leqslant \sum_{\substack{\ell=1 \\
\ell \neq k}}^{m}\left|s_{k, \ell}\right| \\
& \left|s_{k, k}-\lambda_{k^{\prime}}(\boldsymbol{S})\right| \leqslant \sum_{\substack{\ell=1 \\
\ell \neq k}}^{m}\left|s_{\ell, k}\right|
\end{aligned}
$$

\section{Proof:}

With $\vec{x}=\left(x_{1}, x_{2}, \ldots, x_{m}\right)^{\top}$ being the eigenvector corresponding to eigenvalue $\lambda_{k^{\prime}}(\boldsymbol{S})$, the expression

$$
\boldsymbol{S} \vec{x}=\lambda_{k^{\prime}}(\boldsymbol{S}) \vec{x} \quad \text { or } \quad\left(\boldsymbol{S}-\lambda_{k^{\prime}}(\boldsymbol{S}) \mathbf{1}\right) \vec{x}=0
$$

holds with $x_{k}$ representing the component of $\vec{x}$ with the largest absolute value, and $\left|x_{\ell} / x_{k}\right| \leqslant 1, \forall \ell$ holds. Equation (A.31) corresponds to an equation system with $m$ equations for the $m$ components of the vector. Then, the $k$ th equation writes

$$
s_{k, 1} x_{1}+\ldots+s_{k, k-1} x_{k-1}+\left(s_{k, k}-\lambda_{k^{\prime}}(\boldsymbol{S})\right) x_{k}+s_{k, k+1} x_{k+1}+\ldots+s_{k, m} x_{m}=0 .
$$

Transforming (A.32) results in

$$
s_{k, k}-\lambda_{k^{\prime}}(\boldsymbol{S})=-s_{k, 1} \frac{x_{1}}{x_{k}}-\ldots-s_{k, k-1} \frac{x_{k-1}}{x_{k}}-s_{k, k+1} \frac{x_{k+1}}{x_{k}}-\ldots-s_{k, m} \frac{x_{m}}{x_{k}}
$$

and applying the generalized triangle inequality together with the absolute values leads to

$$
\left|s_{k, k}-\lambda_{k^{\prime}}(\boldsymbol{S})\right| \leqslant\left|s_{k, 1} \frac{x_{1}}{x_{k}}\right|+\ldots+\left|s_{k, k-1} \frac{x_{k-1}}{x_{k}}\right|+\left|s_{k, k+1} \frac{x_{k+1}}{x_{k}}\right|+\ldots+\left|s_{k, m} \frac{x_{m}}{x_{k}}\right| .
$$

\footnotetext{
${ }^{3}$ Semyon Aranovich Gershgorin, Belarusian mathematician, 1901-1933.
} 
Together with $\left|x_{\ell} / x_{k}\right| \leqslant 1 \forall k$, Gershgorin's theorem in row-notation

$$
\left|s_{k, k}-\lambda_{k^{\prime}}(\boldsymbol{S})\right| \leqslant\left|s_{k, 1}\right|+\ldots+\left|s_{k, k-1}\right|+\left|s_{k, k+1}\right|+\ldots+\left|s_{k, m}\right|=\sum_{\substack{\ell=1 \\ \ell \neq k}}^{m}\left|s_{k, \ell}\right|
$$

can be obtained. Due to the fact that the eigenvalues of $S$ and $\boldsymbol{S}^{\mathrm{H}}$ are identical, the derivation and the corresponding proof for column notation

$$
\left|s_{k, k}-\lambda_{k^{\prime}}(\boldsymbol{S})\right| \leqslant \sum_{\substack{\ell=1 \\ \ell \neq k}}^{m}\left|s_{\ell, k}\right|
$$

can be achieved in a straightforward manner.

q.e.d.

From (A.35) and (A.36) it can be concluded, that each eigenvalue of $\boldsymbol{S}$ is localized in one of the $m$ Gershgorin discs in the complex plane. The center of each disc corresponds to the main diagonal entry $s_{k, k}$. The radius of the discs can be computed as the sum of the absolute values of the remaining row sum or column sum, respectively. As a consequence, all eigenvalues of a square matrix are placed in the set union of all $m$ Gershgorin discs. In row and column notation, therefore, the disc centers are identical while the radii vary. Therefore, a better estimation usually is possible by considering a combination of row and column formulation. The combination of the discs for row and column formulation contains the whole spectrum of $\boldsymbol{S}$. For a hermitian matrix, the spectrum is localized on the real axis. Otherwise, the spectrum is spread over the complex plane. The diameters of the discs specify, how close a matrix is to a diagonal matrix. The placement of the discs represents the location of the eigenvalues in the complex plane [177].

Gershgorin's Theorem for Reluctance-based Matrices From (3.81) it is well known, that reluctance matrices are strict diagonally dominant. By help of Gershgorin's circle theorem it is obvious that all eigenvalues of a square matrix are located in the set of discs, with the center points corresponding to the main diagonal entries $K_{i i}$. The respective radius corresponds to the sum of absolute values of all off-diagonal elements of the according row $i=1,2, \ldots, n$. If the matrix is strict diagonally dominant and its main diagonal entries are non-zero, it is known that all discs of the matrix and therefore the eigenvalues are placed in the right half section of the complex plane. Therefore, a matrix is positive definite if the matrix is strict diagonally dominant and all diagonal elements are positive. Consequently, a reluctance matrix is strict diagonally dominant for arbitrary degrees of truncation and for diverse conductor arrangements [92]. The property of being positive definite for arbitrary reluctance matrices can be guaranteed together with the property $K_{i i}>0$. The network matrix can proven to be SPD in NA formulation. For an impedance simulation of the MISEA benchmark HBUS(0) in the FD, the Gershgorin discs for the network matrices (dimensions $334 \times 334$ ) at discrete frequency points $1 \mathrm{KHz}$ and $1 \mathrm{GHz}$ are depicted in Figure A.1 in the complex plane. While it can be observed that the discs are located in the right half section of the complex plane, an increasing concentration to the origin and a decreasing diameter of the discs can be noticed with increasing frequency. 

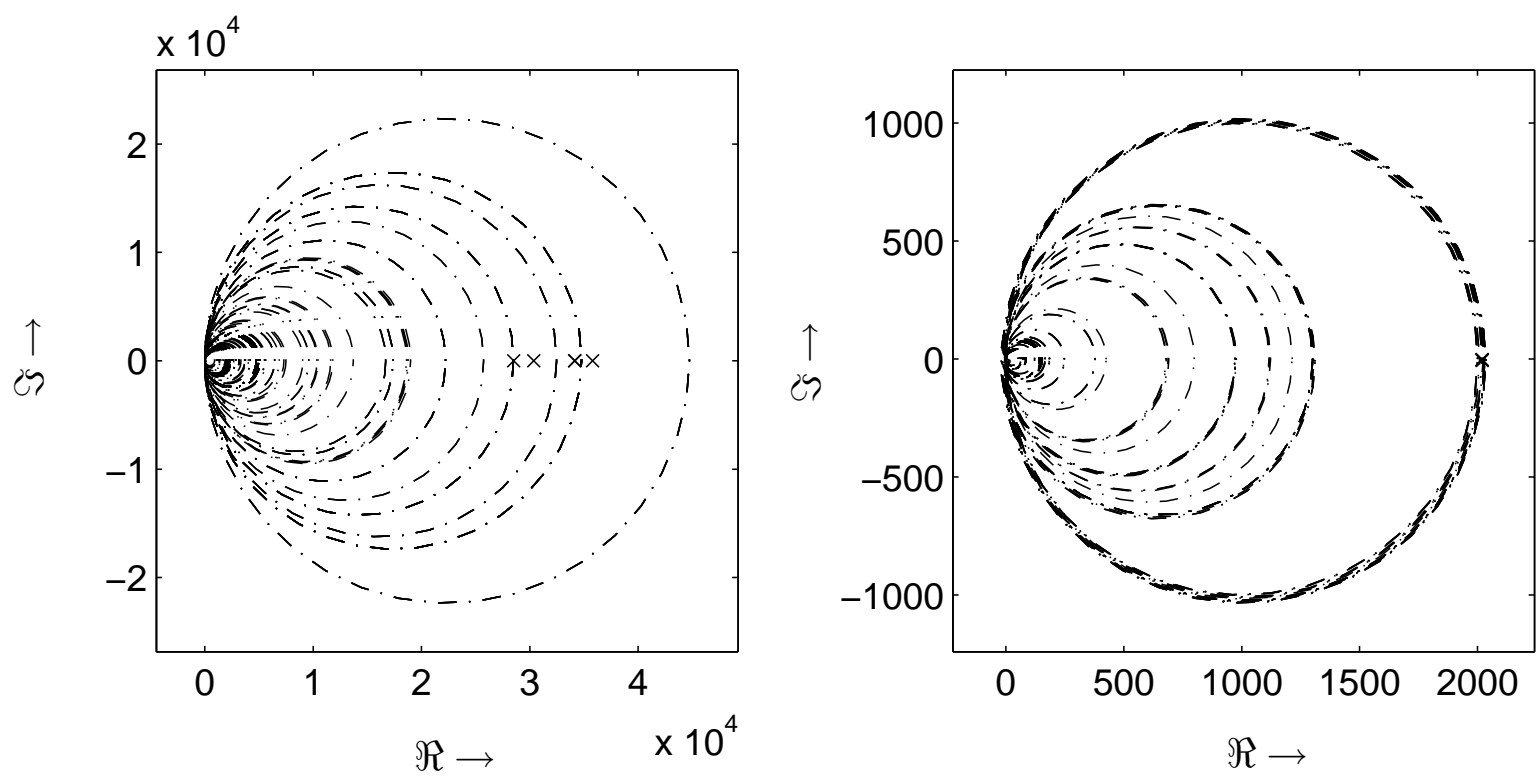

Fig. A.1: Gershgorin discs for reluctance PEEC based network matrices in FD simulation at $1 \mathrm{KHz}$ (left) and $1 \mathrm{GHz}$ (right).

\section{A.5 Method of Moments}

Using elementary basis functions $f_{b}$ from the functional ("ansatz") space $\mathcal{F}_{b} \triangleq \operatorname{span}\left\{f_{b 1}\right.$, $\left.\ldots, f_{b N}\right\}$, a general current or charge density distribution $f$ can be approximated as

$$
f(\vec{r})=\sum_{n=1}^{N} \alpha_{n} f_{b n}(\vec{r})
$$

with $\alpha_{n}$ representing the current and charge density amplitudes and $f_{b n}$ the corresponding elementary basis functions. The determination of the unknowns $\alpha_{n}$ represents the problem to be solved. An application of the method of moments for common linear equations implies, that the linear inhomogeneous functional equation

$$
\mathcal{L}\{f\}=b
$$

with a general linear operator $\mathcal{L}$ and unknown vector $b$ can be solved by series expansion of the unknown function $f$ by

$$
f=\sum_{\forall n} \alpha_{n} f_{b n}
$$

using basis functions $\left\{f_{b 1}, f_{b 2}, f_{b 3}, \ldots\right\}$ in the range of definition of $\mathcal{L}$ and unknown coefficients $\alpha_{n}$ [29]. For $n \rightarrow \infty$ the approximation becomes the exact solution and the functions $f_{b n}$ form a complete system of basis functions. Exploiting the linearity of the operator $\mathcal{L}$ and by inserting (A.39) into (A.38), the expression

$$
\sum_{\forall n} \alpha_{n} \mathcal{L}\left\{f_{b n}\right\}=b
$$

can be obtained. Regarding the relationship between (A.39) and (A.37) using the expression (2.21), for an arbitrarily located point in space the context

$$
\sum_{n=1}^{N} \alpha_{n} \vec{Z}_{n}\left(\vec{r}_{p}, \vec{r}_{q}\right)=\vec{E}^{i}\left(\vec{r}_{p}, t\right)
$$


with

$$
\begin{aligned}
\vec{Z}_{n}\left(\vec{r}_{p}, \vec{r}_{q}\right)=\frac{\vec{f}_{b n}\left(\vec{r}_{p}\right)}{\sigma} & +\frac{\partial}{\partial t} \iiint_{V_{n}} \mu G\left(\vec{r}_{p}, \vec{r}_{q}\right) \vec{f}_{b n}\left(\vec{r}_{q}, t_{r}\right) \mathrm{d} V \\
& +\iint_{S_{n}} \frac{1}{\varepsilon} \operatorname{grad} G\left(\vec{r}_{p}, \vec{r}_{q}\right) \int_{t} \operatorname{div} \vec{f}_{b n}\left(\vec{r}_{q}, t_{r}\right) \mathrm{d} t \mathrm{~d} S
\end{aligned}
$$

results, directly leading to the formulation of the EFIE (2.22) as

$$
\vec{n}\left(\vec{r}_{p}\right) \times \vec{E}^{i}\left(\vec{r}_{p}, t\right)=\sum_{n=1}^{N} \alpha_{n}\left(\vec{n}\left(\vec{r}_{p}\right) \times \vec{Z}\left(\vec{r}_{p}\right)\right), \quad \forall \vec{r}_{p} \in \Gamma
$$

with the observation point $\vec{r}_{p}$ located on the objects surface $\Gamma$. To transform a functional equation into a matrix equation as mentioned at the beginning of this section, it is necessary to introduce a suitable inner product, which has to take into consideration special properties and the necessary boundary conditions of the electromagnetic scattering problem [32, 40, 178]. The inner product

$$
\left\langle f_{t}, \mathcal{L}\{f\}\right\rangle=\left\langle f_{t}, b\right\rangle=\int f_{t} \mathcal{L}\{f\} \mathrm{d} x
$$

represents a projection of $f$ in the direction of $b$ [178]. Defining suitable test functions from the functional space $\mathcal{F}_{t} \triangleq \operatorname{span}\left\{f_{t 1}, f_{t 2}, f_{t 3}, \ldots\right\}$ also in the range of defintion of the operator $\mathcal{L}$, a weighting of the basis functions as well as the excitation can be achieved. This results in an equation system

$$
\sum_{\forall n} \alpha_{n}\left\langle f_{t m}, \mathcal{L}\left\{f_{b n}\right\}\right\rangle=\left\langle f_{t m}, b\right\rangle \quad m=1,2,3, \ldots
$$

which can be written in matrix form

$$
\mathcal{L}^{(m, n)} \cdot \vec{\alpha}^{(n)}=\vec{b}^{(m)}
$$

with

$$
\begin{gathered}
\mathcal{L}^{(m, n)}=\left[\begin{array}{cccc}
\left\langle f_{t 1}, \mathcal{L}\left\{f_{b 1}\right\}\right\rangle & \left\langle f_{t 1}, \mathcal{L}\left\{f_{b 2}\right\}\right\rangle & \ldots & \left\langle f_{t 1}, \mathcal{L}\left\{f_{b n}\right\}\right\rangle \\
\left\langle f_{t 2}, \mathcal{L}\left\{f_{b 1}\right\}\right\rangle & \left\langle f_{t 2}, \mathcal{L}\left\{f_{b 2}\right\}\right\rangle & \ldots & \left\langle f_{t 2}, \mathcal{L}\left\{f_{b n}\right\}\right\rangle \\
\vdots & \vdots & & \vdots \\
\left\langle f_{t m}, \mathcal{L}\left\{f_{b 1}\right\}\right\rangle & \left\langle f_{t m}, \mathcal{L}\left\{f_{b 2}\right\}\right\rangle & \ldots & \left\langle f_{t m}, \mathcal{L}\left\{f_{b n}\right\}\right\rangle
\end{array}\right] \\
\vec{\alpha}^{(n)}=\left[\begin{array}{c}
\alpha_{1} \\
\alpha_{2} \\
\vdots \\
\alpha_{n}
\end{array}\right]
\end{gathered}
$$

If matrix $\mathcal{L}$ is not singular (i.e. the inverse $\mathcal{L}^{-1}$ exists) a clear solution of the equation system can be found as

$$
\vec{\alpha}^{(n)}=\mathcal{L}^{-1} \cdot \vec{b}^{(m)}
$$


regarding (A.39) and the determined coefficients. Applying suitable test functions $\vec{f}_{t}\left(\vec{r}_{p}\right)$, along with the application of the inner product to the EFIE (A.43), a linear equation system of the form

$$
\begin{aligned}
\iint_{\mathbb{D}_{j}}\left(\vec{n}\left(\vec{r}_{p}\right) \times \vec{E}^{i}\left(\vec{r}_{p}, t\right)\right) \vec{f}_{t j}\left(\vec{r}_{p}\right) \mathrm{d} S & =\sum_{n=1}^{N} \alpha_{n} \iint_{\mathbb{D}_{j}}\left(\vec{n}\left(\vec{r}_{p}\right) \times \vec{Z}_{n}\left(\vec{r}_{p}, t\right)\right) \vec{f}_{t j}\left(\vec{r}_{p}\right) \mathrm{d} S \\
& \mathbb{1} \\
M_{j} & =\sum_{n=1}^{N} \alpha_{n} \xi_{n j}\left(\vec{r}_{p}, t\right) \quad j=1 \ldots N
\end{aligned}
$$

can be obtained, with $\vec{r}_{p} \in \Gamma$ and the range of definition $\mathbb{D}_{j}$ of the $j$ th segment. The unknown current amplitudes $\alpha_{n}$ can be determined using well known techniques. In this context, the method of moments can be interpreted as a technique of error minimization. For a graphical illustration of the method of weighted residuals in the functional space the reader is referred to [178]. To achieve optimal results for a given problem, a suitable choice of basis and test functions is inevitable. With regard to its importance concerning performance, accuracy and complexity of the process, the following two techniques are important.

Galerkin method The Galerkin method, which was developed by the Russian engineer BORIS GRIGORIEVICH GALERKIN (1871-1945) in 1920, is a special case of the method of moments, which comprises high relevance for the PEEC Method in Chapter 3. Its major characteristics are identical basis and test functions $\mathcal{F}_{b} \equiv \mathcal{F}_{t}$. Although implementation complexities for the Galerkin method are comparatively high, high accuracy and fast convergence can be achieved $[32,178]$.

Collocation method For higher order solutions requiring larger numbers of basis functions for an approximation of the unknowns, the inner product and thus the elements of the matrix $\mathcal{L}$ are more complicated to calculate. This can be simplified by determining the approximations exactly, solely at discrete points using DIRAC distributions $\delta\left(\vec{x}-\vec{x}_{m}\right)$ as test functions. The sifting property $^{4}$ of the dirac impulse leads to the fact, that the integral degenerates to an evaluation of the integrand at discrete points. This specialization of the method of moments therefore is called point-matching. The matrix entries result in

$$
\begin{gathered}
L_{i j}=\left.\mathcal{L}\left\{f_{b j}\right\}\right|_{\vec{r}=\vec{r}_{i}} \\
b_{i}=f_{b j}\left(\vec{r}_{i}\right) .
\end{gathered}
$$

A degradation in accuracy and usually a worse convergence behavior can be observed. Especially for lower order solutions, these properties strongly depend on the location of the matching points [178]. Whereas the collocation method together with a BEM discretization leads to dense and non-symmetric matrices, matrices from a Galerkin-BEM approach are dense and symmetric. The Galerkin method is more advantageous than the collocation of NYSTRÖM discretizations due to improved convergence and stability aspects [82].

\footnotetext{
${ }^{4}$ In the literature often the term "sampling property" is used equivalently.
} 


\section{A.6 Numerical Integration by Quadrature}

A direct numerical computation of elements from Section 3.2 and 3.3 basically suffers from two bottlenecks [85]. On the one hand the evaluation of the matrix entries requires the computation of proper singular, nearly singular and regular surface integrals over pairs of discretization panels. On the other hand the system matrix from a BEM approach is fully populated leading to computational as well as storage cost of $\mathcal{O}\left(n^{2}\right)$. While the kernel function in the proper singular case is singular in the domain of integration, for the nearly singular case the kernel function is analytic, but its derivatives blow up as the distance of the panels tend to zero. While analytic integration techniques can only be used efficiently for special cases, numerical techniques are universally applicable. The aim of quadrature techniques, which are called cubature techniques in more than one dimension [82], is, to render all integrands analytically by using regularizing coordinate transforms. The advantage of such numerical integration techniques is, that they do not depend on the explicit form of the kernel function, on the trial and test space or on the surface parametrization [83]. Gaussian formulas [179] are interpolatory quadrature formulas. Compared to conventional quadrature rules for the numerical solution of integrals, the weighting coefficients as well as locations of quadrature points in Gaussian quadrature can be chosen arbitrarily. Thus, two degrees of freedom can be achieved, supporting an optimization concerning the tradeoff between accuracy and computational effort. Note that higher quadrature orders not inevitably lead to higher accuracy [81]. Generally, it can be observed that higher orders only lead to higher accuracy if the integrand behaves smooth in a sense, that it can be well approximated by polynomials. With the Gaussian quadrature, quadrature points and weights are normally determined w.r.t. a specific integrand, regarding the best tradeoff between accuracy and complexity. For the applications considered in this thesis, the approximation rule

$$
\int_{a}^{b} g(x) \mathrm{d} x \approx \sum_{j=1}^{N} v_{j} g\left(x_{j}\right)
$$

is appropriate. Function $g(x)$ can be described by the weight function $W(x)$ as $g(x) \equiv W(x) f(x)$, with a polynomial $f(x)$. Furthermore, $v_{j} \equiv w_{j} / W\left(x_{j}\right)$ with the set of weights $w_{j}$ and quadrature points $x_{j}$ holds. The weight function has to fulfill certain contraints [81]. Approximation methods for multidimensional integrals are called cubature techniques. Based on composite cubature formulas, the problem can be described as

$$
I=\int_{\mathcal{B}} f(x, y) \mathrm{d} x \mathrm{~d} y=\sum_{i=1}^{N} \int_{\mathfrak{T}_{i}} f(x, y) \mathrm{d} x \mathrm{~d} y
$$

with the bounded polygonal fringed area $\mathcal{B} \subset \mathbb{R}^{2}$ and $\left\{\mathfrak{T}_{i}\right\}_{i=1}^{N}$ a triangulation of $\mathcal{B}$ as stated in (3.68). For PEEC based integrals, as (3.11) or (3.12), inductive and capacitive areas are assumed to be subdiscretized into triangles without violating general validity. The linear transformation

$$
\varphi:\left[\begin{array}{c}
\zeta \\
\eta
\end{array}\right] \mapsto\left[\begin{array}{l}
x(\zeta, \eta) \\
y(\zeta, \eta)
\end{array}\right]=\left[\begin{array}{l}
x_{1} \\
y_{1}
\end{array}\right]+\left[\begin{array}{ll}
x_{2}-x_{1} & x_{3}-x_{1} \\
y_{2}-y_{1} & y_{3}-y_{1}
\end{array}\right] \cdot\left[\begin{array}{l}
\zeta \\
\eta
\end{array}\right]
$$

realizes a bijective mapping of an isosceles, rectangular reference triangle $\mathfrak{T}_{\text {ref }}$ to an arbitrary triangle $\mathfrak{T}$, where $(0,0) \mapsto P_{1}=\left(x_{1}, y_{1}\right),(1,0) \mapsto P_{2}=\left(x_{2}, y_{2}\right),(0,1) \mapsto P_{3}=\left(x_{3}, y_{3}\right)$, as depicted in Figure A.2. 

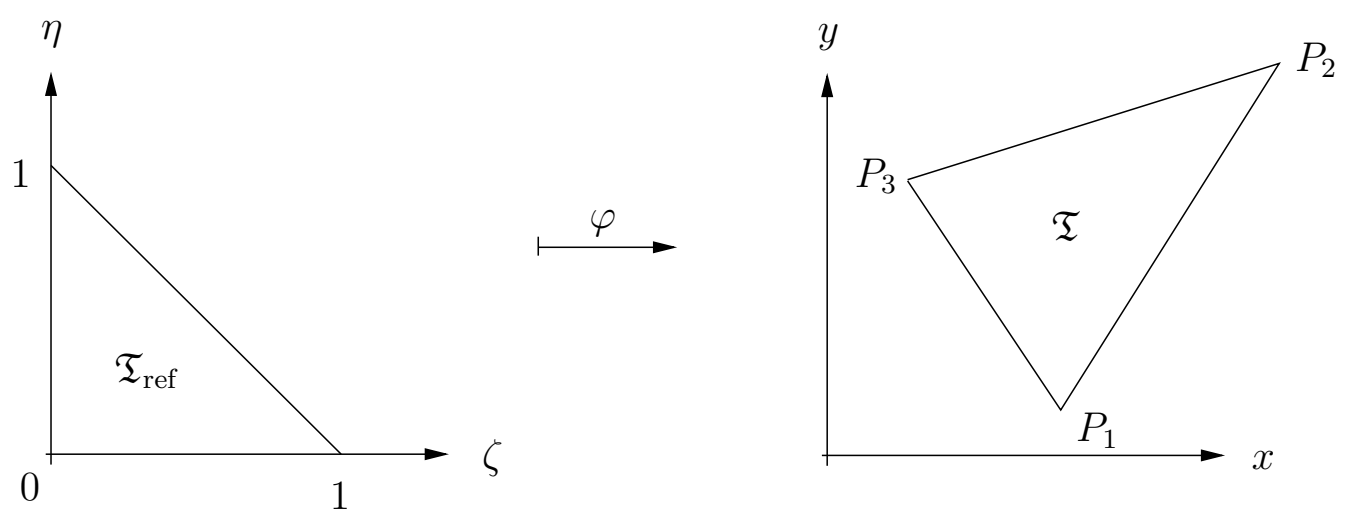

Fig. A.2: Bijective mapping from reference triangle $\mathfrak{T}_{\text {ref }}$ to an arbitrary triangle $\mathfrak{T}$.

By means of the substitution rule

$$
\int_{\mathfrak{T}} f(x, y) \mathrm{d} x \mathrm{~d} y=\int_{\mathfrak{T}_{\text {ref }}} f(\varphi(\zeta, \eta))\left|\operatorname{det} \varphi^{\prime}\right| \mathrm{d} \zeta \mathrm{d} \eta=D \int_{\mathfrak{T}_{\text {ref }}} f(\varphi(\zeta, \eta)) \mathrm{d} \zeta \mathrm{d} \eta
$$

with the fundamental determinant

$$
D=\operatorname{det} \varphi^{\prime} \triangleq \operatorname{det}\left[\begin{array}{cc}
x_{2}-x_{1} & x_{3}-x_{1} \\
y_{2}-y_{1} & y_{3}-y_{1}
\end{array}\right]
$$

of $\varphi$, all single integrals $\int_{\mathfrak{T}_{i}} f(x, y) \mathrm{d} x \mathrm{~d} y$ can be ascribed in terms of integrals over $\mathfrak{T}_{\text {ref }}$. Thus, it is efficient to approximate integrals of the form

$$
\int_{\mathfrak{T}_{\text {ref }}} g(\zeta, \eta) \mathrm{d} \zeta \mathrm{d} \eta .
$$




\section{Appendix \\ $B$}

\section{Green's Function}

$\boldsymbol{T}$ he specific properties of the integral operator's kernel function enormously influence the achievable performance of fast hierarchical techniques. For this reason, the possible variants of GREEN's function, as the most important kernel function for electromagnetic problems, are described in the following. In a boundary problem representation as described in Section 2.2, an object $\mathcal{X} \subset \mathbb{R}^{d}$ with space dimension $d \in \mathbb{N}_{>1}$ can be considered as a bounded, connected LIPSCHITZ domain with smooth boundary $\Gamma=\partial \mathcal{X}$. Any integral operator of such problems originates from a so-called kernel function $g: \Gamma \times \Gamma \rightarrow \mathbb{K}$, with $\mathbb{K}=\mathbb{R}, \mathbb{C}$ [47]. This kernel function generates a linear integral operator $\mathcal{L}$ by the rule

$$
\mathcal{L}: X(\Gamma) \rightarrow Y(\Gamma), f \mapsto\left(x \mapsto \int_{\Gamma} g(x, y) f(y) d x\right),
$$

with $X(\Gamma), Y(\Gamma)$ describing function spaces over $\Gamma$. An integral operator of kind (B.1) is called single layer potential operator [148]. For all integral operators $\mathcal{L}$ with constant coefficients, the so called fundamental solution $s(\vec{x}, \vec{y})$ fulfills the condition

$$
\mathcal{L} s(\vec{x}, \vec{y})=-\delta(\vec{x}-\vec{y}),
$$

with the DIRAC distribution $\delta[180,130,47]$. Green's function, occuring in (2.16) and (2.17), represents a fundamental solution of various electromagnetic problems. Maxwell's equations can be written as a set of wave equations of the form

$$
\begin{aligned}
& \triangle \Phi-\frac{1}{c^{2}} \frac{\partial^{2}}{\partial t^{2}} \Phi=-\frac{1}{\varepsilon} \rho \\
& \triangle \vec{A}-\frac{1}{c^{2}} \frac{\partial^{2}}{\partial t^{2}} \vec{A}=-\mu \vec{J},
\end{aligned}
$$

with the electrodynamic potentials as unknowns [58]. This shows, that potentials and correspondingly the fields, propagate with the speed of light. Both equations (B.3) and (B.4) are coupled by the continuity equation (2.12). Green's function for the scalar HELMHOLTZ equation $\triangle \Psi(\vec{x})+\beta^{2} \Psi(\vec{x})=-f(\vec{x})$ fulfills the condition

$$
\Delta G\left(\vec{r}_{p}, \vec{r}_{q}\right)+\beta^{2} G\left(\vec{r}_{p}, \vec{r}_{q}\right)=-\delta\left(\vec{r}_{p}-\vec{r}_{q}\right) .
$$


The solution of the HELMHOLTZ equation can be expressed in accordance with HUYGEN's principle, as a superposition of waves, propagating from an individual source point $\vec{r}_{q}$. For this reason, it can be written as [46]

$$
\Psi\left(\vec{r}_{p}\right)=\int_{\mathbb{R}^{3}} G\left(\vec{r}_{p}, \vec{r}_{q}\right) f\left(\vec{r}_{q}\right) \mathrm{d} \vec{r}_{q}
$$

or for the electrodynamic potentials as

$$
\vec{A}\left(\vec{r}_{p}, t\right)=\mu \iiint_{V_{q}^{\prime}} G\left(\vec{r}_{p}, \vec{r}_{q}\right) \vec{J}\left(\vec{r}_{q}, t_{r}\right) \mathrm{d} V^{\prime}
$$

and

$$
\Phi\left(\vec{r}_{p}, t\right)=\frac{1}{\varepsilon} \iiint_{V_{q}^{\prime}} G\left(\vec{r}_{p}, \vec{r}_{q}\right) \rho\left(\vec{r}_{q}, t_{r}\right) \mathrm{d} V^{\prime}
$$

respectively ${ }^{1}$. Consequently, Green's function in 2D and 3D result in

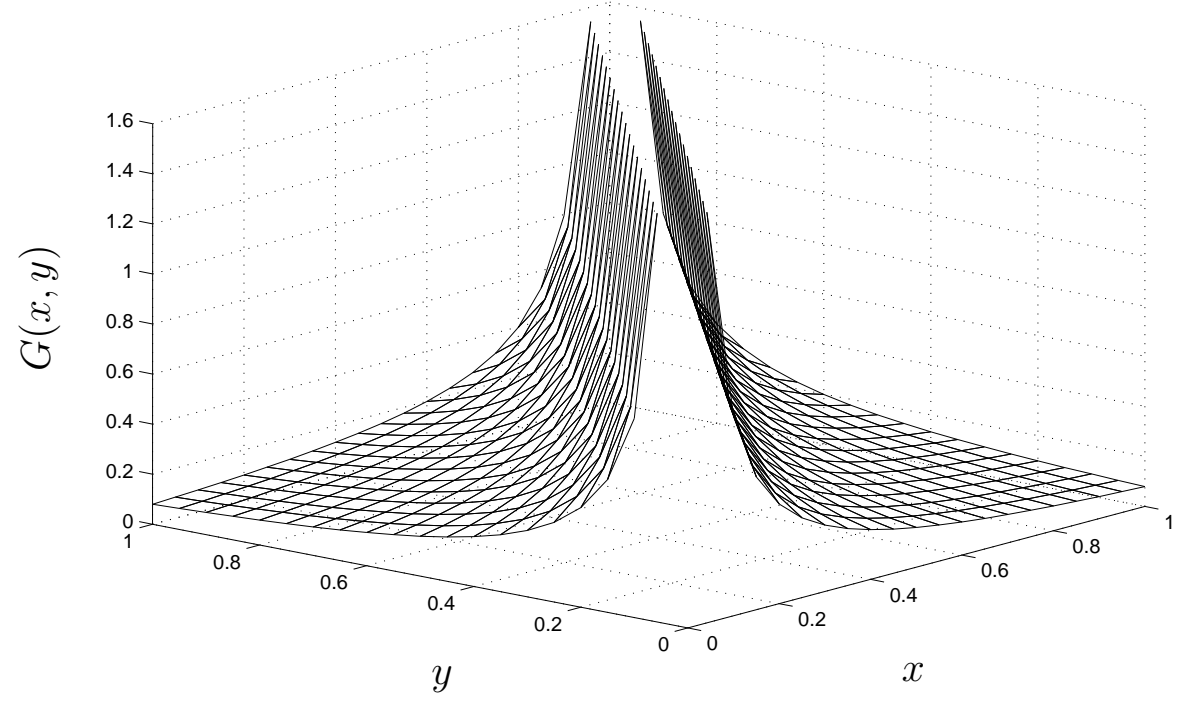

Fig. B.1: Green's function $G(x, y)=1 /(4 \pi\|x-y\|), x, y \in[0,1]$ as a fundamental solution of the LAPLACE equation in 3D (quasi-stationary case).

$$
G\left(\vec{r}_{p}, \vec{r}_{q}\right)=\left\{\begin{array}{cl}
\frac{e^{\mathrm{j} \beta\left\|\vec{r}_{p}-\vec{r}_{q}\right\|}}{4 \pi\left\|\vec{r}_{p}-\vec{r}_{q}\right\|} & \text { for } d=3\left(\text { i.e., } \vec{r}_{p}, \vec{r}_{q} \in \mathbb{R}^{3}\right) \\
\frac{\mathrm{j}}{4} H_{0}^{(1)}\left(\beta\left\|\vec{r}_{p}-\vec{r}_{q}\right\|\right) & \text { for } d=2\left(\text { i.e., } \vec{r}_{p}, \vec{r}_{q} \in \mathbb{R}^{2}\right)
\end{array},\right.
$$

with the HANKEL function $H_{0}^{(1)}$ of the first kind and order 0 , containing the retardation effects of the electromagnetic waves. For the LAPLACE equation the condition

$$
\Delta G\left(\vec{r}_{p}, \vec{r}_{q}\right)=-\delta\left(\vec{r}_{p}-\vec{r}_{q}\right)
$$

\footnotetext{
${ }^{1}$ The retardation time is denoted by $t_{r}$ (refer to (2.18) in Chapter 2).
} 
must hold, leading to Green's function

$$
G\left(\vec{r}_{p}, \vec{r}_{q}\right)=\left\{\begin{array}{cl}
\frac{1}{4 \pi\left\|\vec{r}_{p}-\vec{r}_{q}\right\|} & \text { for } d=3\left(\text { i.e., } \vec{r}_{p}, \vec{r}_{q} \in \mathbb{R}^{3}\right) \\
\frac{1}{2 \pi} \ln \left(\left\|\vec{r}_{p}-\vec{r}_{q}\right\|\right) & \text { for } d=2\left(\text { i.e., } \vec{r}_{p}, \vec{r}_{q} \in \mathbb{R}^{2}\right) .
\end{array}\right.
$$

Kernel functions of this kind showing the properties $G\left(\vec{r}_{p}, \vec{r}_{q}\right)=G\left(\vec{r}_{p}-\vec{r}_{q}\right)$ and $G\left(\vec{r}_{p}, \vec{r}_{q}\right)=$ $G\left(\vec{r}_{q}, \vec{r}_{p}\right)$ have a singularity for $\vec{r}_{p}=\vec{r}_{q}$ (refer to Figure B.1). By means of the fundamental solution of integral operator $\mathcal{L}$, an electromagnetic boundary value problem with DIRICLET or NEUMANN conditions can be transformed to an integral equation over the boundary $\Gamma$ of the domain $\mathcal{X}$. In this case, integral and differential formulations are equivalent. Instead of a 3D differential equation in space, an integral equation for a 2D surface occurs. Green's function enables a formulation of a boundary integral equation (BIE), whose solution and its derivative can be determined. Applying Green's formula together with the knowledge about the boundary values the solution inside the domain can be calculated [181]. 


\section{Appendix}

\section{Coordinate Systems}

$\boldsymbol{E}$ lectromagnetic field problems can only be treated in an efficient way if a suitable coordinate system is applied which allows an accurate and simple formulation of boundary conditions. The well known cartesian coordinate system often turns out to be not suitable enough. Curvilinear coordinates, however, enable a flexible and efficient numerical surface or volume integration for complex geometrical structures and non-orthogonal discretizations.

Starting from the basics of orthogonal right-handed coordinate systems in Section C.1 and the well-known cartesian coordinate system in Section C.2, the theory of curvilinear coordinates is described in Section C.3. W.r.t. non-orthogonal surface and volume discretizations for the PEEC modeling, the application of curvilinear coordinates can lead to a simpler geometrical description of the cells and therefore to a simplified surface and volume integration as described in Section C.4.

\section{C.1 Orthogonal Right-Handed Coordinate Systems}

Orthogonal right-handed coordinate systems fulfill the condition

$$
\vec{e}_{1} \cdot \vec{e}_{2}=\vec{e}_{2} \cdot \vec{e}_{3}=\vec{e}_{3} \cdot \vec{e}_{1}=0
$$

implying that the unit vectors $\vec{e}_{1}, \vec{e}_{2}$ and $\vec{e}_{3}$ in direction of increasing coordinate values are orthogonal. In addition, the vector products of two successive unit vectors

$$
\vec{e}_{1} \times \vec{e}_{2}=\vec{e}_{3}, \quad \vec{e}_{2} \times \vec{e}_{3}=\vec{e}_{1}, \quad \vec{e}_{3} \times \vec{e}_{1}=\vec{e}_{2}
$$

provide the remaining unit vector. Consequently, an arbitrary vector $\vec{f}=\left(f_{1}, f_{2}, f_{3}\right)^{\top}$ can be described in terms of its individual components as

$$
\vec{f}=\vec{e}_{1} \cdot f_{1}+\vec{e}_{2} \cdot f_{2}+\vec{e}_{3} \cdot f_{3}=\vec{e}_{1} \cdot\left(\vec{e}_{1} \cdot \vec{f}\right)+\vec{e}_{2} \cdot\left(\vec{e}_{2} \cdot \vec{f}\right)+\vec{e}_{3} \cdot\left(\overrightarrow{e_{3}} \cdot \vec{f}\right) .
$$




\section{C.2 Cartesian Coordinates}

The directions of the unit vectors $\vec{e}_{x}, \vec{e}_{y}, \vec{e}_{z}$ of the cartesian coordinate system are position independent (location invariant). An arbitrary point in space $P(x, y, z)$ is defined by its position vector

$$
\vec{r}=\vec{e}_{x} x+\vec{e}_{y} y+\vec{e}_{z} z, \quad r=|\vec{r}|=\sqrt{x^{2}+y^{2}+z^{2}}
$$

from the origin with length $r=|\vec{r}|$. An infinitesimal variation of the position vector by $\mathrm{d} \vec{r}$ to a point $Q(x+\mathrm{d} x, y+\mathrm{d} y, z+\mathrm{d} z)$ is also called vectorial path element and can be defined by $[33,182]$

$$
\mathrm{d} \vec{r}=\vec{e}_{x} \mathrm{~d} x+\vec{e}_{y} \mathrm{~d} y+\vec{e}_{z} \mathrm{~d} z, \quad \mathrm{~d} r=|\mathrm{d} \vec{r}|=\sqrt{(\mathrm{d} x)^{2}+(\mathrm{d} y)^{2}+(\mathrm{d} z)^{2}}
$$

as is graphically visualized in Figure C.1.
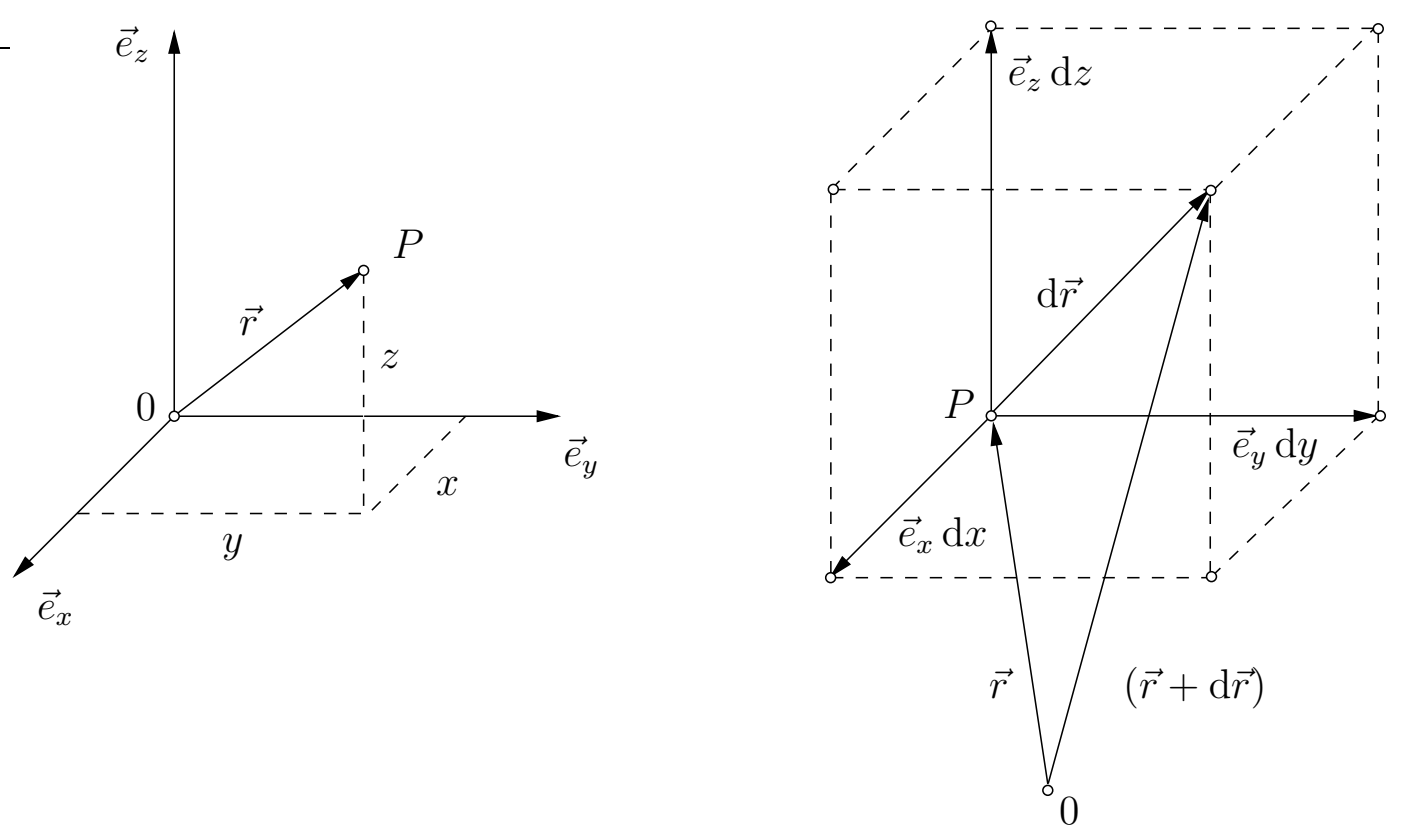

Fig. C.1: Illustration of cartesian coordinates and the infinitesimal vectorial path element $\mathrm{d} \vec{r}$.

\section{C.3 Curvilinear Coordinates}

Applying a transformation to cartesian coordinates $(x, y, z)$, arbitrary coordinates $\left(v_{1}, v_{2}, v_{3}\right)$ can be obtained. Consequently, an arbitrary point in space becomes dependent on the new coordinates, i.e.

$$
\vec{r}=\left(\begin{array}{l}
x\left(v_{1}, v_{2}, v_{3}\right) \\
y\left(v_{1}, v_{2}, v_{3}\right) \\
z\left(v_{1}, v_{2}, v_{3}\right)
\end{array}\right) .
$$

A graphical visualization of an arbitrary point in space in the new coordinates and a shift by an arbitrary infinitesimal path element $\mathrm{d} \vec{r}$ is depicted in Figure C.2. The unit vectors $\vec{e}_{i}, i=1,2,3$ at $P\left(v_{1}, v_{2}, v_{3}\right)$ point in tangential direction to the coordinates $v_{i}, i=1,2,3$ in $P$. The direction of the tangent can be computed via a variation of the position vector $\partial \vec{r} / \partial v_{i}$ w.r.t. the corresponding 

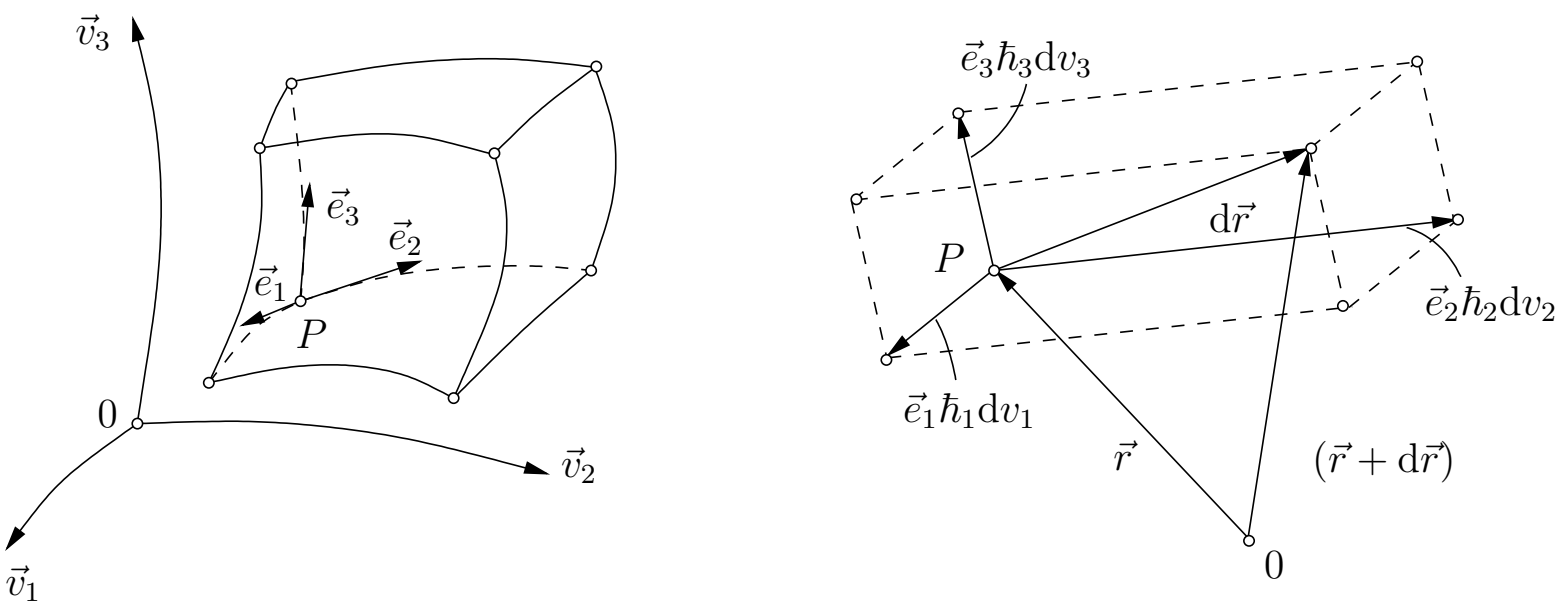

Fig. C.2: Illustration of curvilinear coordinates and the infinitesimal vectorial path element $\mathrm{d} \vec{r}$.

coordinate $v_{i}$ [33]. Thus, again the shift of $P$ to $Q\left(v_{1}+\mathrm{d} v_{1}, v_{2}+\mathrm{d} v_{2}, v_{3}+\mathrm{d} v_{3}\right)$ by an infinitesimal path element can be described as

$$
\begin{aligned}
\mathrm{d} \vec{r} & =(\mathrm{d} x, \mathrm{~d} y, \mathrm{~d} z) \\
& =\vec{e}_{x} \mathrm{~d} x+\vec{e}_{y} \mathrm{~d} y+\vec{e}_{z} \mathrm{~d} z \\
& =\frac{\partial \vec{r}}{\partial v_{1}} \mathrm{~d} v_{1}+\frac{\partial \vec{r}}{\partial v_{2}} \mathrm{~d} v_{2}+\frac{\partial \vec{r}}{\partial v_{3}} \mathrm{~d} v_{3}
\end{aligned}
$$

with

$$
\frac{\partial \vec{r}}{\partial v_{i}}=\left(\frac{\partial x}{\partial v_{i}}+\frac{\partial y}{\partial v_{i}}+\frac{\partial z}{\partial v_{i}}\right), \quad i=1,2,3 .
$$

The corresponding coefficients $\partial \vec{r} / \partial v_{i}$ represent the variation of $\vec{r}$ as a result of a shift of the corresponding coordinate $v_{i}$ and thus are placed in direction of $\vec{e}_{i}$. The unit vectors can be computed by applying the normalization

$$
\vec{e}_{i}=\frac{1}{\left|\frac{\partial \vec{r}}{\partial v_{i}}\right|} \frac{\partial \vec{r}}{\partial v_{i}}=\frac{1}{\hbar_{i}} \frac{\partial \vec{r}}{\partial v_{i}}, \quad i=1,2,3 \quad \text { with } \quad \hbar_{i}=\left|\frac{\partial \vec{r}}{\partial v_{i}}\right|
$$

with the so-called metric coefficients $\hbar_{i}$. If the coordinate variables show the dimension "length", these metric coefficients are dimensionless. In general, the directions of the unit vectors depend on the coordinates $\left(v_{1}, v_{2}, v_{3}\right)$ and thus on the position of $P$, which directly follows from (C.9).

Moreover, the vectorial path element can be written as

$$
\begin{aligned}
\mathrm{d} \vec{r} & =\frac{\partial \vec{r}}{\partial v_{1}} \mathrm{~d} v_{1}+\frac{\partial \vec{r}}{\partial v_{2}} \mathrm{~d} v_{2}+\frac{\partial \vec{r}}{\partial v_{3}} \mathrm{~d} v_{3} \\
& =\vec{e}_{1} \hbar_{1} \mathrm{~d} v_{1}+\vec{e}_{2} \hbar_{2} \mathrm{~d} v_{2}+\vec{e}_{3} \hbar_{3} \mathrm{~d} v_{3} \\
& =\sum_{i=1}^{3} \vec{e}_{i} \hbar_{i} \mathrm{~d} v_{i} .
\end{aligned}
$$

An analog definition can be given for the vectorial surface element of the boundary surface

$$
\mathrm{d} \vec{F}_{i}=\hbar_{j} \mathrm{~d} v_{j} \vec{e}_{j} \times \hbar_{k} \mathrm{~d} v_{k} \vec{e}_{k}
$$


and the infinitesimal volume element of the cell

$$
\mathrm{d} V=\hbar_{i} \mathrm{~d} v_{i} \vec{e}_{i} \cdot\left(\hbar_{j} \mathrm{~d} v_{j} \vec{e}_{j} \times \hbar_{k} \mathrm{~d} v_{k} \vec{e}_{k}\right)
$$

\section{C.4 Integration in Curvilinear Coordinates}

We consider vectorial functions with many variables, so-called vector fields $\vec{F}: \mathbb{R}^{n} \rightarrow \mathbb{R}^{m}$, with

$$
\vec{F}=\vec{F}(\vec{r})=\left(\begin{array}{c}
F_{1}\left(v_{1}, v_{2}, \ldots, v_{n}\right) \\
F_{2}\left(v_{1}, v_{2}, \ldots, v_{n}\right) \\
\vdots \\
F_{m}\left(v_{1}, v_{2}, \ldots, v_{n}\right)
\end{array}\right), \quad \vec{r}=\left(v_{1}, v_{2}, \ldots, v_{n}\right)
$$

based on the coordinates $\left(v_{1}, v_{2}, \ldots, v_{n}\right)[183]$.

\section{C.4.1 The Functional Determinant}

If the partial first derivatives of all $F_{i}, i=1, \ldots, m$ exist, they can be written in form of a functional matrix

$$
\boldsymbol{\Delta} \triangleq \frac{\partial \vec{F}}{\partial \vec{u}}=\left[\frac{\partial\left(F_{1}, F_{2}, \ldots, F_{m}\right)}{\partial\left(u_{1}, u_{2}, \ldots, u_{n}\right)}\right]=\left(\frac{\partial \vec{F}}{\partial u_{1}}, \frac{\partial \vec{F}}{\partial u_{2}}, \ldots, \frac{\partial \vec{F}}{\partial u_{n}}\right)=\left(\begin{array}{ccc}
\frac{\partial F_{1}}{\partial u_{1}} & \ldots & \frac{\partial F_{1}}{\partial u_{n}} \\
\vdots & \ddots & \vdots \\
\frac{\partial F_{m}}{\partial u_{1}} & \ldots & \frac{\partial F_{m}}{\partial u_{n}}
\end{array}\right)
$$

which is also known as Jacobian matrix. Transforming a problem to curvilinear coordinates $\left(v_{1}, v_{2}, \ldots, v_{n}\right)$, a valid coordinate transformation $\vec{u}=\vec{u}\left(v_{1}, v_{2}, \ldots, v_{n}\right) \in \mathbb{R}^{n}$ can be defined, if the Jacobian matrix $\Delta$ exists and for the Jacobian ${ }^{1}$

$$
\operatorname{det} \Delta \neq 0
$$

holds. Consequently, the derivatives of $\vec{F}$ w.r.t. the original coordinates $u_{i}, i=1, \ldots, n$ can be written as functions of the new coordinates $v_{i}, i=1, \ldots, n$. If $\vec{F}=\vec{F}\left(v_{1}, v_{2}, \ldots, v_{n}\right)$ is known and applying the chain rule it results

$$
\frac{\partial \vec{F}}{\partial u_{i}}=\sum_{k=1}^{n} \frac{\partial \vec{F}}{\partial v_{k}} \frac{\partial v_{k}}{\partial u_{i}}=\sum_{k=1}^{n} \frac{\partial \vec{F}}{\partial v_{k}} \boldsymbol{W} \quad i=1, \ldots, n
$$

where matrix $\boldsymbol{W}\left(v_{1}, v_{2}, \ldots, v_{n}\right)$ can be computed as

$$
\boldsymbol{W}=\left[\left(\frac{\partial u_{i}}{\partial v_{k}}\right)\right]^{-1} \quad i, k=1, \ldots, n,
$$

regarding the aforementioned considerations.

\footnotetext{
${ }^{1}$ Remark: If only the phrase Jacobian is given, the functional determinant is denoted.
} 


\section{Example C.1 Polar coordinates}

Considering circular geometric objects, it often turns out to be advantageous, to apply polar coordinates with the norm $r$ and angle $\varphi$. The mapping

$$
\left(\begin{array}{l}
x(r, \varphi) \\
y(r, \varphi)
\end{array}\right)=\left(\begin{array}{c}
r \cos \varphi \\
r \sin \varphi
\end{array}\right)
$$

from cartesian coordinates to polar coordinates by assuming a continuous and differentiable function $\vec{F}(r, \varphi)$, the Jacobian matrix and its determinant can be written as

$$
\boldsymbol{\Delta}=\left[\frac{\partial(x, y)}{\partial(r, \varphi)}\right]=\left(\begin{array}{cc}
\cos \varphi & -r \sin \varphi \\
\sin \varphi & r \cos \varphi
\end{array}\right), \quad \operatorname{det} \boldsymbol{\Delta}=r \cos ^{2} \varphi+r \sin ^{2} \varphi=r
$$

and matrix $\boldsymbol{W}$ resulting in

$$
\boldsymbol{W}=\left[\frac{\partial(x, y)}{\partial(r, \varphi)}\right]^{-1}=\left(\begin{array}{cc}
\cos \varphi & \sin \varphi \\
-\frac{1}{r} \sin \varphi & \frac{1}{r} \cos \varphi
\end{array}\right) .
$$

The corresponding partial first derivatives result in

$$
\begin{aligned}
& \frac{\partial \vec{F}}{\partial x}=\frac{\partial \vec{F}}{\partial r} \cos \varphi-\frac{1}{r} \frac{\partial \vec{F}}{\partial \varphi} \sin \varphi \\
& \frac{\partial \vec{F}}{\partial y}=\frac{\partial \vec{F}}{\partial r} \sin \varphi+\frac{1}{r} \frac{\partial \vec{F}}{\partial \varphi} \cos \varphi
\end{aligned}
$$

and all higher derivates result in an analog manner.

\section{Example C.2 Cylindrical coordinates}

Applying the mapping rule

$$
\vec{r}=\left(\begin{array}{l}
x \\
y \\
z
\end{array}\right)=\left(\begin{array}{c}
r \cos \varphi \\
r \sin \varphi \\
z
\end{array}\right)
$$

from cartesian into sherical coordinates, the metric factors can be computed as

$$
\hbar_{r}=\left|\frac{\partial \vec{r}}{\partial r}\right|=1 \quad \hbar_{\varphi}=\left|\frac{\partial \vec{r}}{\partial \varphi}\right|=r \quad \hbar_{z}=\left|\frac{\partial \vec{r}}{\partial z}\right|=1 .
$$

A comparison between the determinant of the Jacobian matrix

$$
\operatorname{det} \Delta=\operatorname{det}\left(\begin{array}{ccc}
\cos \varphi & -r \sin \varphi & 0 \\
\sin \varphi & r \cos \varphi & 0 \\
0 & 0 & 1
\end{array}\right)=r
$$

and the metric factors results in

$$
\operatorname{det} \boldsymbol{\Delta}=\prod_{k=1}^{n} \hbar_{k}=\hbar_{r} \hbar_{\varphi} \hbar_{z} .
$$

The unit vectors, together with (C.9), therefore result in

$$
\vec{e}_{r}=\left(\begin{array}{c}
\cos \varphi \\
\sin \varphi \\
0
\end{array}\right) \quad \vec{e}_{\varphi}=\left(\begin{array}{c}
-\sin \varphi \\
\cos \varphi \\
0
\end{array}\right) \quad \vec{e}_{z}=\left(\begin{array}{l}
0 \\
0 \\
1
\end{array}\right)
$$




\section{C.4.2 Surface Integration}

We consider a given, arbitrary shaped surface $\mathcal{F}$, which can be parametrized by means of two variables $u$ and $v$ from the range of definition $\mathbb{D}$, formally defined as

$$
\mathcal{F}=\{\vec{r}=\vec{r}(u, v) \mid u, v \in \mathbb{D}\} .
$$

By varying one variable while keeping the remaining fixed, a grid corresponding to the individual coordinate lines can be obained as depicted in Figure C.3. Although the resulting grid lines run

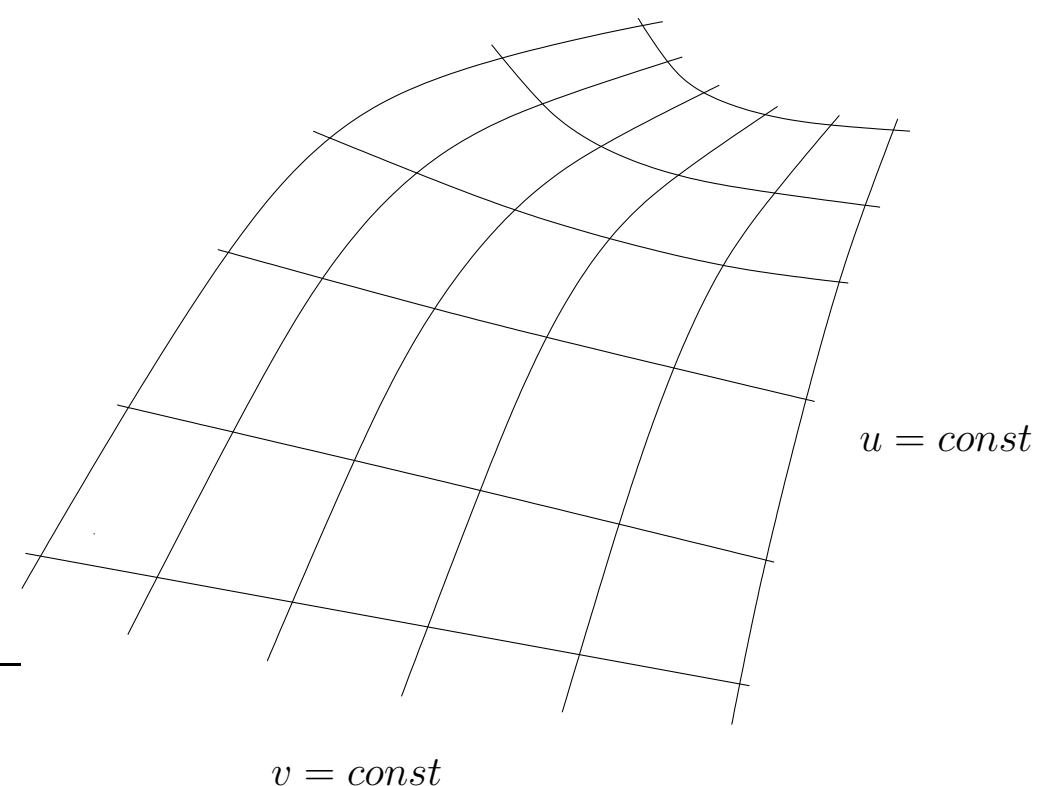

Fig. C.3: Parametrization of the surface via two variables $u$ and $v$.

non-orthogonal in general, a suitably chosen parametrization can lead to a local and orthogonal grid. A differential surface element is depicted in Figure C.4. Such an infinitesimal grid element

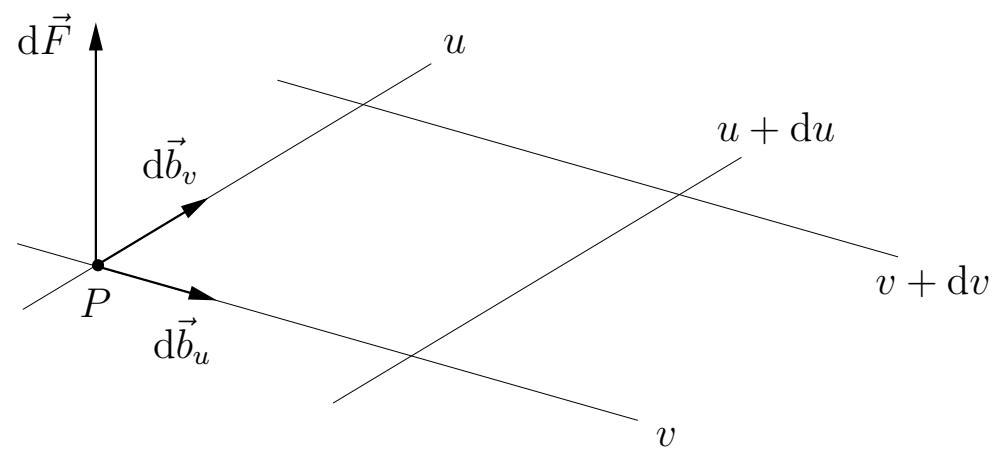

Fig. C.4: Differential surface element.

can be approximated by a parallelogram, defined by its vertices $\vec{r}(u, v), \vec{r}(u+\mathrm{d} u, v), \vec{r}(u, v+\mathrm{d} v)$ and $\vec{r}(u+\mathrm{d} u, v+\mathrm{d} v)$ with infinitesimally small $\mathrm{d} u$ and $\mathrm{d} v$. The base vectors of the parallelogram result in

$$
\begin{aligned}
\mathrm{d} \vec{b}_{u} & =\vec{r}(u+\mathrm{d} u, v)-\vec{r}(u, v) \\
\mathrm{d} \vec{b}_{v} & =\vec{r}(u, v+\mathrm{d} v)-\vec{r}(u, v),
\end{aligned}
$$


which can be written as

$$
\begin{aligned}
& \mathrm{d} \vec{b}_{u}=\frac{\partial \vec{r}}{\partial u} \mathrm{~d} u \triangleq \vec{r}_{u} \mathrm{~d} u \\
& \mathrm{~d} \vec{b}_{v}=\frac{\partial \vec{r}}{\partial v} \mathrm{~d} v \triangleq \vec{r}_{v} \mathrm{~d} v
\end{aligned}
$$

using the formal definition of partial derivatives. The vectorial surface element

$$
\mathrm{d} \vec{F}=\mathrm{d} \vec{b}_{u} \times \mathrm{d} \vec{b}_{v}=\left(\vec{r}_{u} \times \vec{r}_{v}\right) \mathrm{d} u \mathrm{~d} v
$$

can be represented by a vector normal to the surface, spanned by the base vectors with its norm equal to the area of the element. Together with the surface normal vector

$$
\vec{n}=\frac{\vec{r}_{u} \times \vec{r}_{v}}{\left|\vec{r}_{u} \times \vec{r}_{v}\right|}
$$

the vectorial surface element can be written as

$$
\mathrm{d} \vec{F}=\vec{n} \mathrm{~d} F \quad \text { with } \quad \mathrm{d} F=\left|\vec{r}_{u} \times \vec{r}_{v}\right| \mathrm{d} u \mathrm{~d} v .
$$

For a surface representation, curvilinear coordinates $(u, v)$ in the $x-y$-plane are assumed to be given in the following. Applying the well-known relation $V=(\vec{a} \times \vec{b}) \cdot \vec{c}$ in the context of the spatproduct $[183,182]$, the vectorial surface element can be given as

$$
\mathrm{d} \vec{F}=\vec{e}_{z} \mathrm{~d} F=\left(\vec{r}_{u} \times \vec{r}_{v}\right) \cdot \vec{e}_{z} \mathrm{~d} u \mathrm{~d} v
$$

with the unit vector $\vec{e}_{z}$ in $z$-direction. Together with the Jacobian

$$
\operatorname{det} \boldsymbol{\Delta}=\left|\begin{array}{ccc}
\frac{\partial x}{\partial u} & \frac{\partial y}{\partial u} & 0 \\
\frac{\partial x}{\partial v} & \frac{\partial y}{\partial v} & 0 \\
0 & 0 & 1
\end{array}\right|=\left|\begin{array}{ll}
\frac{\partial x}{\partial u} & \frac{\partial y}{\partial u} \\
\frac{\partial x}{\partial v} & \frac{\partial y}{\partial v}
\end{array}\right|=: \frac{\partial(x, y)}{\partial(u, v)}
$$

(C.36) can be represented as

$$
\mathrm{d} \vec{F}=\left(\vec{r}_{u} \times \vec{r}_{v}\right) \cdot \vec{e}_{z} \mathrm{~d} u \mathrm{~d} v=\operatorname{det} \Delta \mathrm{d} u \mathrm{~d} v
$$

Consequently, the Jacobian describes a transformation between surface elements by

$$
\mathrm{d} x \mathrm{~d} y=|\operatorname{det} \Delta| \mathrm{d} u \mathrm{~d} v=\left|\frac{\partial(x, y)}{\partial(u, v)}\right| \mathrm{d} u \mathrm{~d} v
$$

showing that an infinitesimal shift in the original coordinate system directly causes a variation in the new coordinates. Since the coordinates $(x, y, z)$ now represent functions in $\left(v_{1}, v_{2}, v_{3}\right)$ the integration region has to be transformed in a similar manner. As evident in (C.39), the Jacobian matrix distorts the differential surface element as well. Assuming the scalar function $F(x, y)$ and the vectorial function $\vec{F}(x, y)$ to represent functions in the conventional sense, depending on the coordinates $\left(v_{1}, v_{2}\right)$ and describing the considered surface $\mathcal{F}$ by an equation of the form

$$
z=g(x, y)
$$


the integration results in

$$
\iint_{\mathcal{F}(x, y)} F(x, y) \mathrm{d} x \mathrm{~d} y=\iint_{\tilde{\mathcal{F}}\left(v_{1}, v_{2}\right)} \tilde{F}\left(v_{1}, v_{2}\right) \operatorname{det} \Delta \mathrm{d} v_{1} \mathrm{~d} v_{2}
$$

or

$$
\begin{aligned}
\iint_{\mathcal{F}(x, y)} \vec{F}(x, y) \mathrm{d} x \mathrm{~d} y & =\vec{e}_{x} \iint_{\tilde{\mathcal{F}}\left(v_{1}, v_{2}\right)} \tilde{F}_{x}\left(v_{1}, v_{2}\right) \operatorname{det} \Delta \mathrm{d} v_{1} \mathrm{~d} v_{2} \\
& +\vec{e}_{y} \iint_{\tilde{\mathcal{F}}\left(v_{1}, v_{2}\right)} \tilde{F}_{y}\left(v_{1}, v_{2}\right) \operatorname{det} \Delta \mathrm{d} v_{1} \mathrm{~d} v_{2},
\end{aligned}
$$

respectively.

\section{C.4.3 Volume Integration}

An extension of the Jacobian to 3D by means of

$$
\operatorname{det} \boldsymbol{\Delta}=\frac{\partial(x, y, z)}{\partial(u, v, w)}=\left|\begin{array}{lll}
\frac{\partial x}{\partial u} & \frac{\partial y}{\partial u} & \frac{\partial z}{\partial u} \\
\frac{\partial x}{\partial v} & \frac{\partial y}{\partial v} & \frac{\partial z}{\partial v} \\
\frac{\partial x}{\partial w} & \frac{\partial y}{\partial w} & \frac{\partial z}{\partial w}
\end{array}\right|
$$

is possible in a straightforward manner, leading to a transformation

$$
\mathrm{d} V=\mathrm{d} x \mathrm{~d} y \mathrm{~d} z=\left(\vec{r}_{u} \times \vec{r}_{v}\right) \cdot \vec{r}_{w} \mathrm{~d} u \mathrm{~d} v \mathrm{~d} w=\operatorname{det} \Delta \mathrm{d} u \mathrm{~d} v \mathrm{~d} w
$$

between the differential volume elements. In volume integration the scalar function $F(x, y, z)$ and the vectorial function $\vec{F}(x, y, z)$ can be regarded in a similar manner to the surface integration. Now functions in $\left(v_{1}, v_{2}, v_{3}\right)$ the resulting integration for a domain $\mathcal{X}$ can be formulated as

$$
\iiint_{\mathcal{X}(x, y, z)} F(x, y, z) \mathrm{d} x \mathrm{~d} y \mathrm{~d} z=\iiint_{\tilde{\mathcal{X}}\left(v_{1}, v_{2}, v_{3}\right)} \tilde{F}\left(v_{1}, v_{2}, v_{3}\right) \operatorname{det} \Delta \mathrm{d} v_{1} \mathrm{~d} v_{2} \mathrm{~d} v_{3}
$$

or

$$
\begin{aligned}
\iiint_{\mathcal{X}(x, y, z)} \vec{F}(x, y, z) \mathrm{d} x \mathrm{~d} y \mathrm{~d} z & =\vec{e}_{x} \iiint_{\tilde{\mathcal{X}}\left(v_{1}, v_{2}, v_{3}\right)} \tilde{F}_{x}\left(v_{1}, v_{2}, v_{3}\right) \operatorname{det} \Delta \mathrm{d} v_{1} \mathrm{~d} v_{2} \mathrm{~d} v_{3} \\
& +\vec{e}_{y} \iiint \int_{\tilde{\mathcal{X}}\left(v_{1}, v_{2}, v_{3}\right)} \tilde{F}_{y}\left(v_{1}, v_{2}, v_{3}\right) \operatorname{det} \Delta \mathrm{d} v_{1} \mathrm{~d} v_{2} \mathrm{~d} v_{3} \\
& +\vec{e}_{z} \iiint_{\tilde{\mathcal{X}}\left(v_{1}, v_{2}, v_{3}\right)} \tilde{F}_{z}\left(v_{1}, v_{2}, v_{3}\right) \operatorname{det} \Delta \mathrm{d} v_{1} \mathrm{~d} v_{2} \mathrm{~d} v_{3} .
\end{aligned}
$$




\section{Appendix $D$ \\ Circuit Simulation Basics}

$\boldsymbol{E}$

lectrical networks as resulting from PEEC or TL approaches inherently contain the geometrical mesh data included in the parasitic elements. This enables a simplified mathematical problem description. The system of Maxwell's equations turns over into a system of ordinary differential equations with derivaties only existing w.r.t. time. Furthermore, constant terms without time dependence can be obtained by restricting the analysis to harmonic functions. Consequently, the system of ODEs changes to a system of algebraic equations. These equations can be solved by means of conventional solving strategies [36, 184]. The efficiency and flexibility of a computer aided circuit simulation is strongly influenced by the formulation of network equations. A formulation of electrical circuits suitable for computational analysis was introduced by BASHKOW in 1957 [185]. The introduction of the MNA formulation was accomplished by IBM in 1975 [186], suitable for the computational analysis of large circuit networks. It proved to be useful in the past to apply the well-established SPICE [187, 188, 189] syntax, as it is done in many industrial circuit simulators [190].

Starting from the description of Kirchhoff's laws in Section D.1, the basics of nodal analysis (NA) for electrical circuits are given in Section D.2. The modified nodal analysis (MNA) formulation as a modification of the NA approach is described in Section D.3. Implemented in SPICE these approaches enable a circuit analysis in the TD and the FD, which is shortly recapitulated in Sections D.4 and D.5.

\section{D.1 Fundamental Network Analysis}

As it is well-established in graph theory, the connectivity structure of a linear network can be described by means of a so-called adjacency matrix $\boldsymbol{A}^{N_{b} \times N_{n}}$. Such a matrix enables an interpretation of the network as a directed graph, containing $N_{n}$ nodes and $N_{b}$ branches. Each row of $\boldsymbol{A}$ contains only two non-zero entries

$$
A_{l k}=\left\{\begin{array}{cl}
+1 & \text { if node } k \text { is source of branch } l \\
-1 & \text { if node } k \text { is sink of branch } l \\
0 & \text { else }
\end{array}\right.
$$


representing the connectivity information concerning the direction of the branches and the nodal sources and sinks with row sum zero. By means of the adjacency matrix, KIRCHHOFF's laws can be formulated in an elegant manner. KIRCHHOFF's current law (KCL) results in

$$
\boldsymbol{A}^{\top} \overrightarrow{i_{b}}=\overrightarrow{0}
$$

and KIRCHHOFF's voltage law (KVL) can be represented by

$$
\boldsymbol{A} \vec{u}_{n}=\vec{u}_{b}
$$

with $\vec{i}_{b}, \vec{u}_{b}$, the vectors of branch currents and branch voltages and the vector of nodal voltages $\vec{u}_{n}$, referring to a reference node with potential $0 \mathrm{~V}$. The transposed of the adjacency matrix is denoted by $\boldsymbol{A}^{\top}$. The relations between the integral field quantities at the discrete circuit elements voltages and currents are called branch constitutive relations ${ }^{1}$ (BCR)

$$
\vec{i}_{b}-\vec{i}_{q}=\boldsymbol{Y}_{b}\left(\vec{u}_{b}-\vec{u}_{q}\right)
$$

with the sparse matrix $\boldsymbol{Y}_{b}$ containing the branch admittances. Optional current and voltages sources are denoted by $\vec{i}_{q}$ and $\vec{u}_{q}$, respectively.

\section{D.2 The Nodal Analysis Formulation}

Formulating the element equations by (D.4), the physical relations in a linear electrical network can be described by an equation system of the form $[36,184,191]$

$$
\underbrace{\left(\boldsymbol{A}^{\top} \boldsymbol{Y}_{b} \boldsymbol{A}\right)}_{\boldsymbol{Y}_{n}} \vec{u}_{n}=\underbrace{\boldsymbol{A}^{\top}\left(\boldsymbol{Y}_{b} \vec{u}_{q}-\vec{i}_{q}\right)}_{\vec{i}_{n}},
$$

with a so-called nodal admittance matrix $\boldsymbol{Y}_{n}$ of dimension $n \times n$. Matrix $\boldsymbol{Y}_{n}$ can efficiently be generated by applying an element stamping procedure. For further details refer to [102]. Regarding all capacitive and inductive couplings, the nodal admittance matrix is of the form

$$
\boldsymbol{Y}_{n}=\boldsymbol{A}^{\top} \boldsymbol{Y}_{b} \boldsymbol{A}-\boldsymbol{A}^{\top} \boldsymbol{Y}_{b} \boldsymbol{Q} \boldsymbol{Y}_{b}^{\top} \boldsymbol{A}
$$

with matrix $Q$ containing current controlled voltages sources representing the inductive couplings between the branches [36]. The inductive couplings exhibit a much lower decay with increasing distance from the main diagonal compared to the capacitive couplings. Therefore, the density of matrix $\boldsymbol{Y}_{n}$ is dominated by the second term on the right-hand side of (D.6). An integration of inductances, independent voltage sources or controlled voltage sources can cause problems in circuit simulation, as it is well known from the literature (refer to (D.6) and [191]). To enable a flexible and universal circuit simulation, a modification is necessary to achieve a more suitable network formulation. A linearization of non-linear elements is necessary. For a detailed view refer to [184].

\footnotetext{
${ }^{1}$ In accordance with the literature, the BCRs often are also called branch constitutive equations (BCE), representing the mathematical models of circuit primitives via the connection between currents, voltages, charges and fluxes.
} 


\section{D.3 The Modified Nodal Analysis Formulation}

The MNA approach avoids the aforementioned disadvantages of the NA formulation and extends the network equations by additional branch current equations [186, 191]. For this purpose Kirchhoff's laws (D.2) and (D.3) are combined with (D.4). According to [96] the corresponding adjacency matrix and the branch current vector as well as the branch voltage vector for $R L C$-networks with current sources can be partitioned as

$$
\boldsymbol{A}=\left[\begin{array}{c}
\boldsymbol{A}_{s} \\
\boldsymbol{A}_{g} \\
\boldsymbol{A}_{c} \\
\boldsymbol{A}_{l}
\end{array}\right] \quad \vec{u}_{b}=\left[\begin{array}{c}
\vec{u}_{s} \\
\vec{u}_{g} \\
\vec{u}_{c} \\
\vec{u}_{l}
\end{array}\right] \quad \vec{i}_{b}=\left[\begin{array}{c}
\vec{i}_{s} \\
\vec{i}_{g} \\
\vec{i}_{c} \\
\vec{i}_{l}
\end{array}\right],
$$

where the indices represent branches with independent current sources $(s)$, ohmic resistances $(g)$, capacitances $(c)$ and inductances $(l)$. Between the branch currents and voltages the relations

$$
\begin{gathered}
\vec{i}_{s}=-\vec{i}_{q}(t) \\
\vec{i}_{g}=G^{\prime} \cdot \vec{u}_{g} \\
\vec{i}_{c}=\boldsymbol{C}^{\prime} \cdot \frac{\mathrm{d}}{\mathrm{d} t} \vec{u}_{c} \\
\vec{u}_{l}=\boldsymbol{L} \cdot \frac{\mathrm{d}}{\mathrm{d} t} \vec{i}_{l}
\end{gathered}
$$

must hold, with $\vec{i}_{q}(t)$ representing the vector of current sources, the diagonal admittance matrix $G^{\prime}$, the diagonal capacitance matrix $C^{\prime}$ and the partial inductance matrix $L$. Consequently, from KCL (D.2) it follows

$$
\begin{aligned}
{\left[\begin{array}{c}
\boldsymbol{A}_{s} \\
\boldsymbol{A}_{g} \\
\boldsymbol{A}_{c} \\
\boldsymbol{A}_{l}
\end{array}\right]^{\top} \cdot \vec{i}_{b} } & =\left[\begin{array}{llll}
\boldsymbol{A}_{s}^{\top} & \boldsymbol{A}_{g}^{\top} & \boldsymbol{A}_{c}^{\top} & \boldsymbol{A}_{l}^{\top}
\end{array}\right] \cdot\left[\begin{array}{c}
\vec{i}_{s} \\
\overrightarrow{i_{g}} \\
\overrightarrow{\vec{i}_{c}} \\
\overrightarrow{\vec{i}_{l}}
\end{array}\right] \\
& =\boldsymbol{A}_{s}^{\top} \cdot \vec{i}_{s}+\boldsymbol{A}_{g}^{\top} \cdot \vec{i}_{g}+\boldsymbol{A}_{c}^{\top} \cdot \vec{i}_{c}+\boldsymbol{A}_{l}^{\top} \cdot \vec{i}_{l} \\
& \stackrel{!}{=} \overrightarrow{0 .}
\end{aligned}
$$

Inserting (D.8) till (D.10) into (D.12) results in

$$
\boldsymbol{A}_{s}^{\top} \cdot\left(-\vec{i}_{q}\right)+\boldsymbol{A}_{g}^{\top} \cdot \boldsymbol{G}^{\prime} \cdot \vec{u}_{g}+\boldsymbol{A}_{c}^{\top} \cdot \boldsymbol{C}^{\prime} \cdot \frac{\mathrm{d}}{\mathrm{d} t} \vec{u}_{c}+\boldsymbol{A}_{l}^{\top} \cdot \vec{i}_{l}=\overrightarrow{0} .
$$

From KVL (D.3), it follows

$$
\left[\begin{array}{l}
\boldsymbol{A}_{s} \\
\boldsymbol{A}_{g} \\
\boldsymbol{A}_{c} \\
\boldsymbol{A}_{l}
\end{array}\right] \cdot \vec{u}_{n}=\left[\begin{array}{l}
\vec{u}_{s} \\
\vec{u}_{g} \\
\vec{u}_{c} \\
\vec{u}_{l}
\end{array}\right]
$$

Combining (D.14) and (D.13) results in

$$
\boldsymbol{A}_{g}^{\top} \cdot \boldsymbol{G}^{\prime} \cdot \boldsymbol{A}_{g} \cdot \vec{u}_{n}+\boldsymbol{A}_{c}^{\top} \cdot \boldsymbol{C}^{\prime} \cdot \boldsymbol{A}_{c} \cdot \dot{\vec{u}}_{n}+\boldsymbol{A}_{l}^{\top} \cdot \vec{i}_{l}=\boldsymbol{A}_{s}^{\top} \cdot \vec{i}_{q}
$$


and furthermore from the fourth equation of (D.14) it follows

$$
-\boldsymbol{A}_{l} \cdot \vec{u}_{n}+\boldsymbol{L} \cdot \dot{\overrightarrow{i_{l}}}=\overrightarrow{0}
$$

From a combination of (D.15) and (D.16) the equation system

$$
M \cdot \vec{x}+N \cdot \dot{\vec{x}}=\vec{b}
$$

can be obtained. In detail, this equation system can be written as

$$
\left[\begin{array}{cc}
\boldsymbol{G} & \boldsymbol{A}_{l}^{\top} \\
-\boldsymbol{A}_{l} & \mathbf{0}
\end{array}\right] \cdot\left[\begin{array}{c}
\vec{u}_{n} \\
\vec{i}_{l}
\end{array}\right]+\left[\begin{array}{cc}
\boldsymbol{C} & \mathbf{0} \\
\mathbf{0} & \boldsymbol{L}
\end{array}\right] \cdot\left[\begin{array}{c}
\dot{\vec{u}}_{n} \\
\dot{\vec{i}}_{l}
\end{array}\right]=\left[\begin{array}{c}
-\boldsymbol{A}_{s}^{\top} \cdot \vec{i}_{q} \\
\overrightarrow{0}
\end{array}\right]
$$

with the SPD matrices $\boldsymbol{G}=\boldsymbol{A}_{g}^{\top} \boldsymbol{G}^{\prime} \boldsymbol{A}_{g}$ and $\boldsymbol{C}=\boldsymbol{A}_{c}^{\top} \boldsymbol{C}^{\prime} \boldsymbol{A}_{c}$. Each row of (D.18) represents the KCL at the corresponding node. In cases where the element current can not be represented by means of nodal voltages, the current has to be regarded by an extra variable. Furthermore, the output equations result in

$$
\vec{u}_{p}=\boldsymbol{B}^{\top} \cdot \vec{x}
$$

with the binary matrix $\boldsymbol{B}$, connecting the terminal voltages $\vec{u}_{p}$ and the solution vector $\vec{x}$.

\section{D.4 Time Domain Analysis}

In the TD (D.17) and (D.19) can be written as

$$
\begin{gathered}
\boldsymbol{M} \cdot \vec{x}(t)+\boldsymbol{N} \cdot \dot{\vec{x}}(t)=\boldsymbol{B} \cdot \vec{i}_{p}(t) \\
\vec{u}_{p}(t)=\boldsymbol{B}^{\top} \cdot \vec{x}(t),
\end{gathered}
$$

providing the basis for a transient circuit simulation. Applying the backward-Euler method

$$
\left.\frac{\mathrm{d} x}{\mathrm{~d} t}\right|_{t=(n+1) h}=\frac{x((n+1) h)-x(n h)}{(n+1) h-n h}=\frac{x((n+1) h)-x(n h)}{h}
$$

to the time derivatives with constant time step $h$, (D.20) results in

$$
\boldsymbol{N} \cdot \frac{\vec{x}((n+1) h)-\vec{x}(n h)}{h}+\boldsymbol{M} \cdot \vec{x}((n+1) h)=\boldsymbol{B} \cdot \vec{i}_{p}((n+1) h) .
$$

A transformation of (D.23) offers the iteration rule

$$
\vec{x}((n+1) h)=\left(\frac{\boldsymbol{N}}{h}+\boldsymbol{M}\right)^{-1} \cdot\left[\boldsymbol{B} \cdot \vec{i}_{p}((n+1) h)+\frac{\boldsymbol{N}}{h} \cdot \vec{x}(n h)\right]
$$

for TD simulation. 


\section{D.5 Frequency Domain Analysis}

Applying the LAPLACE transform to (D.17) and (D.19)

$$
\begin{gathered}
(\boldsymbol{M}+s \cdot \boldsymbol{N}) \vec{x}(s)=\boldsymbol{B} \cdot \vec{i}_{p}(s) \\
\vec{u}_{p}(s)=\boldsymbol{B}^{\top} \cdot \vec{x}(s)
\end{gathered}
$$

with the complex frequency variable $s=\mathrm{j} \omega=\mathrm{j} 2 \pi f$ and frequency $f$, a simulation can be accomplished in the FD. The solution of the equation system results from

$$
\begin{gathered}
\vec{x}(s)=\underbrace{(\boldsymbol{M}+s \cdot \boldsymbol{N})}_{\triangleq \boldsymbol{T}(s)}{ }^{-1} \cdot \boldsymbol{B} \cdot \vec{i}_{p}(s) \\
\vec{u}_{p}(s)=\boldsymbol{B}^{\boldsymbol{\top}} \cdot \vec{x}(s),
\end{gathered}
$$

for varying frequency steps. From (D.27) the frequency dependence of $\boldsymbol{T}$ is obvious. 


\section{Appendix $\bar{H}$}

\section{List of Symbols, Variables and Acronyms}

he present Appendix summarizes the most important notations, relevant variables, constants, transforms and major acronyms. We generally use bold letters for representing matrices, arrowed letters for vectors and normal letters for constants. For a systematic listing the used symbols and abbreviations are presented according to their field of major applications.

\section{E.1 Important Sets of Numbers and Constants}

$\begin{array}{ll}\mathbb{N} & \text { set of natural numbers (including } 0) \\ \mathbb{Z} & \text { set of integer numbers } \\ \mathbb{R} & \text { set of real numbers } \\ \mathbb{C} & \text { set of complex numbers } \\ \mathbb{T} & \text { set of triangles } \\ \mathrm{e} & \text { Euler number }(\mathrm{e}=2.718281828 \ldots) \\ \mathrm{j} & \text { imaginary number }\left(\mathrm{j}^{2}=-1\right) \\ \pi & \pi=3.14159265358979 \ldots \\ \infty & \text { infinity } \\ c_{0} & \text { velocity (speed of light }) \text { in vacuum }\left(2.998 \cdot 10^{8} \mathrm{~m} / \mathrm{s}\right) \\ \varepsilon_{0} & \text { vacuum permittivity }\left(8.854 \cdot 10^{-12} \mathrm{As} / \mathrm{Vm}\right) \\ \mu_{0} & \text { vacuum permeability }\left(4 \pi \cdot 10^{-7} \mathrm{Vs} / \mathrm{Am}\right)\end{array}$




\section{E.2 Important Variables}

\section{E.2.1 Electromagnetic Basics}

\begin{tabular}{|c|c|}
\hline & time $(\mathrm{s})$ \\
\hline & retardation time $(\mathrm{s})$ \\
\hline & frequency $(\mathrm{Hz})$ \\
\hline & velocity in medium $(\mathrm{m} / \mathrm{s})$ \\
\hline & angular frequency $(\mathrm{rad} / \mathrm{s})$ \\
\hline & wavelength (m) \\
\hline & permittivity (As/Vm) \\
\hline & relative permittivity \\
\hline & permeability (Vs/Am) \\
\hline & relative permeability \\
\hline & electric conductivity $(\mathrm{S} / \mathrm{m}=1 /(\Omega \mathrm{m}))$ \\
\hline & voltage (V) \\
\hline & current $(\mathrm{A})$ \\
\hline & electric charge (As) \\
\hline & electric charge density $\left(\mathrm{As} / \mathrm{m}^{3}\right)$ \\
\hline & current density vector $\left(\mathrm{A} / \mathrm{m}^{2}\right)$ \\
\hline & wave number (rad/m) \\
\hline & volume $\left(\mathrm{m}^{3}\right)$ \\
\hline & surface area $\left(\mathrm{m}^{2}\right)$ \\
\hline & cross section $\left(\mathrm{m}^{2}\right)$ \\
\hline & vector of electric field intensity $(\mathrm{V} / \mathrm{m})$ \\
\hline & vector of magnetic field intensity $(\mathrm{A} / \mathrm{m})$ \\
\hline & vector of electric flux density $\left(\mathrm{As} / \mathrm{m}^{2}\right)$ \\
\hline & vector of magnetic flux density $\left(\mathrm{Vs} / \mathrm{m}^{2}\right)$ \\
\hline & magnetic vector potential $(\mathrm{Vs} / \mathrm{m})$ \\
\hline & Poynting vector \\
\hline & electric scalar potential (V) \\
\hline & electric potential (V) \\
\hline & arbitrary scattering object \\
\hline & boundary (surface) of scattering object $\mathcal{X}(\Gamma \triangleq \partial \mathcal{X})$ \\
\hline & (surface) normal vector on $\Gamma$ \\
\hline & kernel function (of an integral operator) \\
\hline & Green's function $(1 / \mathrm{m})$ \\
\hline & fundamental solution \\
\hline
\end{tabular}

\section{E.2.2 PEEC Modeling}

$l \quad$ length $(\mathrm{m})$

$V_{k} \quad$ volume of volume cell $k\left(\mathrm{~m}^{3}\right)$

$S_{l} \quad$ surface area of surface cell $l\left(\mathrm{~m}^{2}\right)$ 


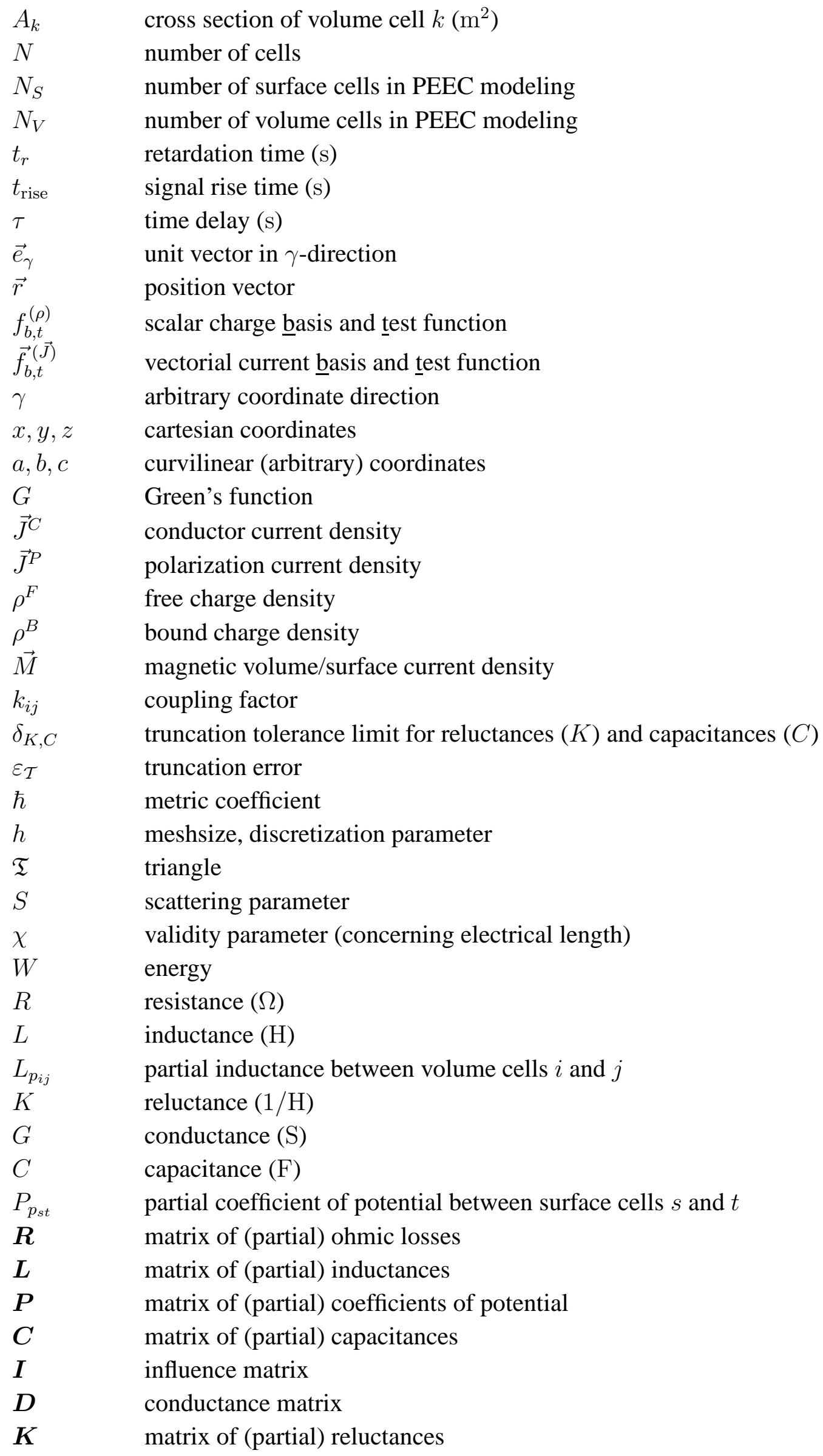




$\begin{array}{ll}\boldsymbol{A} & \text { adjacency matrix } \\ \boldsymbol{Y} & \text { admittance matrix } \\ \boldsymbol{\Delta} & \text { Jacobian matrix (functional matrix) }\end{array}$

\section{E.2.3 Hierarchical/Multilevel Methods}

$\begin{array}{ll}d & \text { space dimension } \\ n & \text { matrix dimension } \\ k & \text { rank of a matrix (block) } \\ \kappa & \text { influence parameter dependent on the kernel function } \\ \sigma & \text { singular value } \\ J_{\ell} & \text { spherical Bessel function of the first kind } \\ H_{\ell}^{(1)} & \text { spherical Hankel function of the first kind } \\ P_{\ell} & \text { Legendre polynomial } \\ \mathcal{F} & \text { ansatz space } \\ \boldsymbol{A} & \text { system matrix }\end{array}$

\section{FMM}

$\mathcal{G} \quad$ group of basis functions

$\Lambda \quad$ transfer operator

\section{$\mathcal{H}$-Matrices}

$k \quad$ rank of matrix (block)

kt actual rank

$\varepsilon_{\mathcal{H}} \quad$ accuracy in $\mathcal{H}$-matrix computation

$\eta \quad$ admissibility parameter

$\mathcal{B} \quad$ bounding box

$\mathfrak{S}$ support of basis functions

$\mathcal{I} \quad$ index set

$\mathcal{P} \quad$ index set partitioning

$T_{\mathcal{I}} \quad$ cluster tree $(\mathrm{CT})$ for index set $\mathcal{I}$

$T_{\mathcal{I} \times \mathcal{I}} \quad$ block cluster tree (BCT) for index set $\mathcal{I}$

$\mathcal{N}_{\text {st }} \quad$ storage requirements

$C_{\mathrm{sp}} \quad$ sparsity of a CT (BCT)

$\mathcal{R}_{k} \quad$ set of rank- $k$-matrices

$\mathcal{M}$ set of hierarchical matrices

$M_{b} \quad$ block in an $\mathcal{H}$-matrix

$\mathcal{T}_{\text {r }} \quad$ rank-truncation process

$\operatorname{depth}(T) \quad$ depth of tree $T$

$\Upsilon$ Connectivity matrix

\section{AMG}

$h \quad$ fine-level variable

$H \quad$ coarse-level variable 


$\begin{array}{ll}\Omega & \text { grid } \\ \mathcal{V} & \text { index set of the matrix } \\ C^{h} & \text { subset of coarse-level variables } \\ F^{h} & \text { subset of fine-level variables } \\ \mathcal{R} & \text { restriction operator } \\ \mathcal{P} & \text { prolongation operator } \\ \mathcal{S} & \text { smoothing step } \\ \mathfrak{K} & \text { coarse-level correction operator } \\ \mathfrak{M} & \text { two-level iteration operator } \\ \nu & \text { number of pre- or post-smoothing steps }\end{array}$

\section{E.2.4 Iterative Methods}

$\vec{x} \quad$ solution vector

$\vec{x}^{*} \quad$ exact solution vector

$\vec{e} \quad$ error vector

$\vec{r} \quad$ residual vector

$\vec{b} \quad$ right-hand side vector

$\omega \quad$ damping parameter

$\varepsilon \quad$ accuracy

$W \quad$ energy

$n \quad$ matrix dimension

$n_{s} \quad$ number of simulation steps

$n_{\text {iter }} \quad$ number of iteration steps required for convergence

$n_{\mathrm{nz}}(\boldsymbol{A}) \quad$ number of non-zero elements of matrix $\boldsymbol{A}$

$\boldsymbol{A} \quad$ system matrix

$\boldsymbol{Y} \quad$ iteration matrix

$\hat{\boldsymbol{Y}} \quad$ damped iteration matrix

$M \quad$ preconditioner matrix

$\mathcal{K}^{k} \quad$ Krylov subspace of order $k$

$\mathcal{N}_{\text {st }} \quad$ storage requirements

\section{E.2.5 Parallelization}

$S_{p} \quad$ parallel speed-up on $p$ processing elements

$\mathcal{A} \quad$ arbitrary serial or parallel algorithm

E parallel efficiency

$\mathcal{W} \quad$ parallel complexity

\section{E.3 Mathematical Notations, Transforms and Operators}

$\Re\{z\} \quad$ real part of complex variable $z=a+\mathrm{j} b$

$\Im\{z\} \quad$ imaginary part of complex variable $z=a+\mathrm{j} b$ 


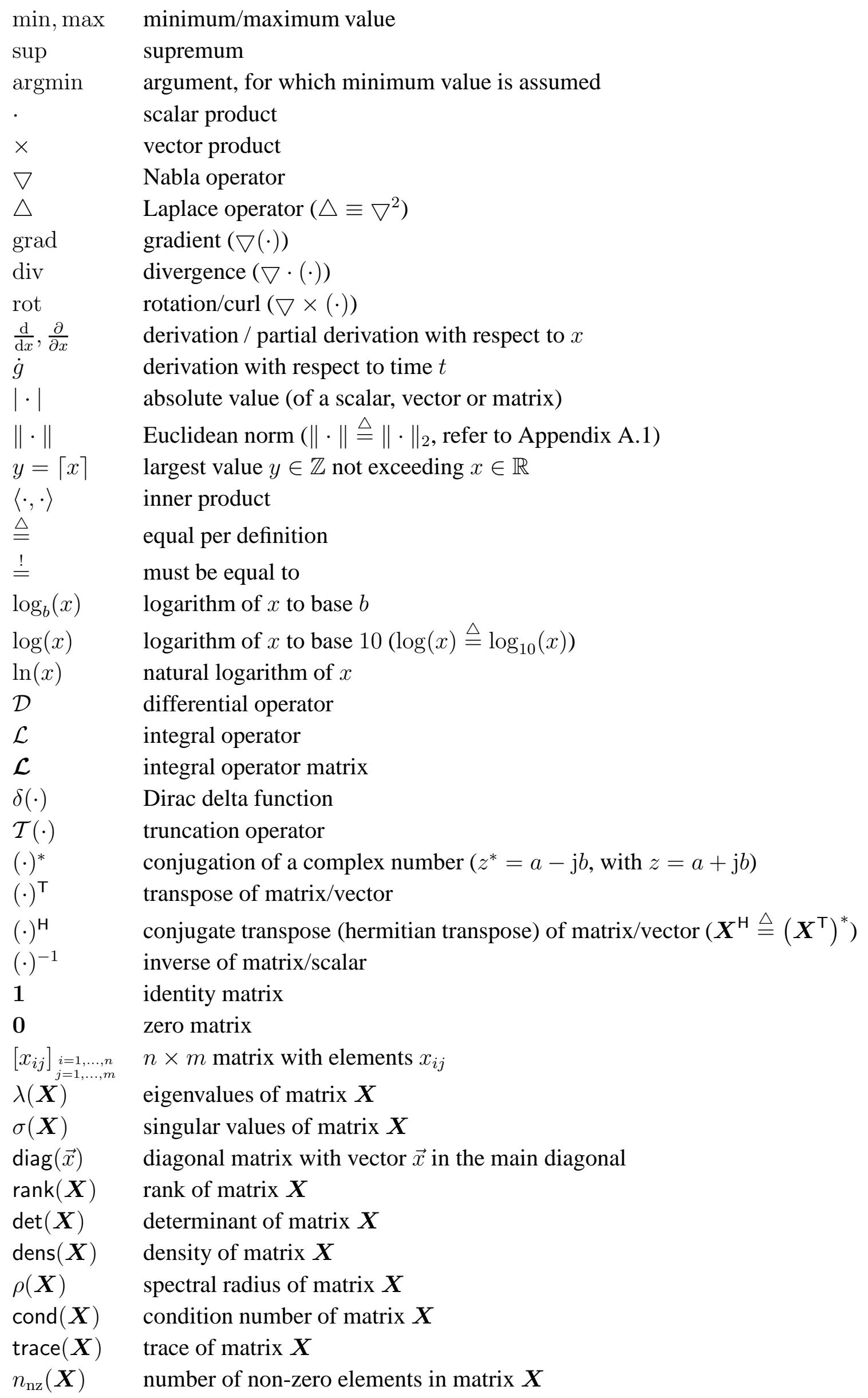




$\begin{array}{ll}\operatorname{dist}(\cdot) & \text { distance (between supports of basis functions) } \\ \operatorname{diam}(\cdot) & \text { diameter (of a bounding box) } \\ \operatorname{span}(\cdot) & \text { space spanned by the basis functions in brackets } \\ \mathcal{O}(\cdot) & \text { order/complexity (performance, } \mathcal{O} \text {-notation) } \\ \Pi(\cdot) & \text { mapping operation }\end{array}$

\section{E.4 Acronyms}

2D two-dimensional

3D three-dimensional

$\mathrm{AC} \quad$ alternating current

ACA adaptive cross approximation

ACA+ modified adaptive cross approximation

AMG algebraic multigrid

ANSI American National Standards Institute

BCE branch constitutive equation

BCR branch constitutive relation

BCT block cluster tree

BEM boundary element method

BGS block Gauss-Seidel

BiCGstab bi conjugate gradient stabalized

BIE boundary integral equation

CAD computer aided design

CAE computer aided engineering

CCCS current controlled current source

CCVS current controlled voltage source

CFD computational fluid dynamics

CFIE combined field integral equation

$\mathrm{CG} \quad$ conjugate gradient

$\mathrm{CPU} \quad$ central processing unit

CSR/CSC compressed sparse row/column matrix storage format

CT cluster tree

DAE differential algebraic equations

DC direct current

DOF degree of freedom

EFIE electric field integral equation

EM electromagnetic

EMC electromagnetic compatibility

EMI electromagnetic interference

EMS electromagnetic susceptibility

ESD electrostatic discharge

EUT equipment under test

FD frequency domain

FDTD finite difference time domain 


\begin{tabular}{|c|c|}
\hline FEM & finite element method \\
\hline FFT & fast fourier transform \\
\hline FMM & fast multipole method \\
\hline GB & giga byte \\
\hline GCC & GNU compiler collection \\
\hline GMG & geometric multigrid \\
\hline GMRes & generalized minimal residual \\
\hline GS & Gauß-Seidel \\
\hline $\mathrm{HCA}$ & hybrid cross approximation \\
\hline HLIB & hierarchical matrix library \\
\hline IBIS & input/output buffer information specification \\
\hline IBM & International Business Machines \\
\hline ICEM & integrated circuit electromagnetic models \\
\hline IE & integral equation \\
\hline IC & integrated circuit \\
\hline ILU & incomplete lower-upper-decomposition \\
\hline ILUT & incomplete lower-upper-decomposition with dropping tolerance \\
\hline KB & kilo byte \\
\hline KCL & Kirchhoff's current law \\
\hline KVL & Kirchhoff's voltage law \\
\hline LU & lower-upper-decomposition \\
\hline MB & mega byte \\
\hline METIS & hypergraph partitioning package \\
\hline MFIE & magnetic field integral equation \\
\hline MG & multigrid \\
\hline MIMD & multiple instruction multiple data \\
\hline MISEA & $\begin{array}{l}\text { Modellierung integrierter Schaltungen für die EMV-Simulation in der } \\
\text { Automobilindustrie }\end{array}$ \\
\hline MLFMA & multilevel fast multipole algorithm \\
\hline MLFMM & multilevel fast multipole method \\
\hline MNA & modified nodal approach \\
\hline MoM & method of moments \\
\hline MOR & model order reduction \\
\hline MP & message passing \\
\hline MPIE & mixed potential integral equation \\
\hline MTL & multiconductor transmission line \\
\hline MV & matrix-vector multiplication \\
\hline NA & nodal analysis approach \\
\hline ODE & ordinary differential equation \\
\hline PCB & printed circuit board \\
\hline PDE & partial differential equation \\
\hline PEEC & partial element equivalent circuit \\
\hline POSIX & portable operating system interface, Unix heritage of the API \\
\hline PRAM & parallel random access memory \\
\hline
\end{tabular}




$\begin{array}{ll}\text { RAM } & \text { random access memory } \\ \text { rPEEC } & \text { retarded partial element equivalent circuit } \\ \text { SAMG } & \text { standard algebraic multigrid library } \\ \text { SI } & \text { signal integrity } \\ \text { SIMD } & \text { single instruction, multiple data } \\ \text { SAI } & \text { sparse approximate inverse } \\ \text { sPEEC } & \text { partial element equivalent circuit including scattering } \\ \text { SPICE } & \text { simulation program with integrated circuit emphasis } \\ \text { SPD } & \text { symmetric positive definite } \\ \text { SSE } & \text { streaming SIMD extensions (originally called ISSE, Internet SSE) } \\ \text { SSN } & \text { simultaneous switching noise } \\ \text { SVD } & \text { singular value decomposition } \\ \text { TD } & \text { time domain } \\ \text { TL } & \text { transmission line } \\ \text { TLM } & \text { transmission line modeling } \\ \text { TRAN } & \text { transient analysis } \\ \text { uC } & \text { microcontroller } \\ \text { VCVS } & \text { voltage controlled voltage source } \\ \text { VLSI } & \text { very large scale integration } \\ \text { W.r.t. } & \text { with respect to }\end{array}$




\section{Index}

Access violation, 125, 128

Active frequency range, 37

Actual rank, 94

Adaptive

cross approximation (ACA), 68, 71, 113, see also $\mathrm{HCA}$

$\mathrm{ACA}+, 71$

recompression, $74-75$

refinement, 73-74

Address space, 125

Adjacency matrix, 47, 78, 95, 191

Admissibility condition, 64-65, 68, 70 weak, 67

Affine space, 85

Aggressor, 44

Algebraic equation, 191

Algebraic multigrid (AMG), 5, 81, 85, 98, 141

aggregation-based, 108

classical, 108, 113

cycle, 111, 113, 118

Krylov-subspace method, 112

multilevel algorithm, 111

PEEC, 113-118

point-based, 117

preconditioning, 112-113

setup/solution-phase, 108, 126

two-level iteration operator, 111

Algorithmic complexity, 65

Ansatz space, 18, 62, 173

Antenna design, 140

Anterpolation, 59

Approximate inverse, 87, 92, 97, 113, see also Sparse approximate inverse (SAI)

Artifacts, 3, 38, 146

AutoEMC, 140

Automotive design process, 2

development process, 133

EMC, 1

simulation process, 2

Background memory, 51

Backward substitution, 68, 92, 96

Backward-Euler method, 194

Banded matrices, 81

Bandwidth optimization, 81

Basis function, 173

Behavioral model, 3, 141

Bessel function, 57

Best-approximation, 66, 170

BiCG, 86

BiCGstab, 86, 94, 97, 118, 144

Bijective mapping, 176

Binary tree, 63, 64

Bisection, 63

Black-box clustering, 95

Block cluster tree (BCT), 63

Block-Gauß-Seidel, 118

Boundary

condition, 11

Diriclet, 181

Neumann, 181

element method (BEM), 13, 52, 53, 91, 113,175

discretization, 3

integral equation (BIE), 53, 181

integral operator, 60

value problem, 11,181

Bounding box, 64, 65, 70

Branch constitutive relations (BCR), 192

C-variables, 109

C/F-splitting, 109, 110, 126

Calibration adapter, 135

Callback-function, 71, 144 
Capacitance matrix, 21, 44, 50, 71 short circuit, 21

CG, 86

Chain rule, 186

Charge

conservation, 10

density, 11

density distribution, 173

Cholesky-decomposition, 87, 92

Circuit

-level modeling, 4

model

distributed, 3, 36

lumped, 2, 36, 45

simulation, 32

reliability, 139

simulator, 97

Cluster

basis, 103

factory, 70

size, 55

tree (CT), 63, 74, 95

Clustering, 55

black-box, 95

geometrically-balanced, 70

Coarse

-grid correction, 106

-level correction, 108

operator, 111

process, 110

-level matrix, 115

level, 107

Coarsening process, 107, 115, 126

Collocation

method, 175

scheme, 61

Column-count ordering, 82

Combined field integral equation (CFIE), 14, 59

Common mode, 50

Communication overhead, 126

Compressed sparse row/column storage format (CSR/CSC), 88, 124, 144 modified, 89

Computational fluid dynamics (CFD), 145

Computer aided circuit simulation, 191

Computer aided engineering (CAE), 133

Condition number, 4, 78, 144, 169 ill-conditioning, 59, 106

Continuity equation, 10, 179

discretization, 18

Convergence

analysis, 171

behavior, 74, 78, 83, 86, 90, 92, 97, 118, $121,139,175$

rate, 93

speed, 65

Coordinate

storage format, 88

system

cartesian, 19, 184

curvilinear, 21, 184-186

global, 22

integration, 186

Jacobi determinant, 22, 186

local, 22

mapping, 22

Polar coordinates, 39, 187

right-handed, 19, 183

spherical, 187

transformation, 21

COSIME, 134

Coupling

factor, 45

galvanic, 140

paths, 140

Cross-approximation principle, 69, see also Adaptive cross approximation (ACA)

Crosstalk, 2, 113, 140

Cubature techniques, 176

Sauter, 39

splitting tree, 42

Current density, 11

Cuthill-McKee ordering, 82

Damping parameter, 84

Data-sparse, 3, 52, 59, 68, 91, 108, 114, 144

DC point analysis, 34

Degenerate kernel, 56

Degree of freedom (DOF), 13, 53, 62, 63, $70,78,93$

Diagonal storage scheme, 89

Dielectric modeling

curvilinear coordinates, 29, 32

excess capacitance, 29

free/bound charges, 28 
Maxwell's equations, 27

PEEC formulation, 28

relative permittivity, 27

surface formulation, 30-32

volume formulation, 27-30

Differential mode, 50

Differential operator, 70

Dirac distribution, 175, 179

Direct method, 4, 81-83, 108, 127

accuracy, 4

Direct solver, 48, 115, 117, 118, 145

Discrete elliptic matrices, 105

Discretization, see also Grid

error, 74

hexahedrals, 21

Manhattan, 18

mesh conformity, 34

meshsize, 34, 53, 118, 136

non-orthogonal, 143

orthogonal, 143

quadrilaterals, 21

refinement, 34

triangular, 34

Displacement current density, 10

Distributed

memory

system, 126

memory message passing model, 125

Domain decomposition, 78, 123, 126

Dot-product, 124

Eddy current effect, 11

Effective rank, 56

Eigenfrequency, 81

Eigenfunction, 113

Eigenvalue, 106

bound, 171

clustering, 86, 90

Eigenvector, 112

Electric field integral equation (EFIE), 2, $13-14,78,81,118,174$

Electrical length, 34

Electrodynamic potentials, 12-13

Electromagnetic

compatibility, 1

emission, 1, 140

environment, 1

immunity, 1, 134, 140

interference, 1, 17, 50 scattering problem, 11, 27, 174

source-field-relation, 13

superposition, 11

susceptibility, 1

total field, 11, 14

Electrostatic discharge (ESD), 1, 17

Elliptic PDE, 116

Elliptic-hyperbolic matrices, 105

Equipment under test (EUT), 135

Equivalence principle, 12, 14

boundary conditions, 31

magnetic surface current, 30

surface, 30

volume, 27

Euclidean

distance, 63

norm, 168

Extended frequency range, 37

F-variables, 109

Fallback solution, 94, 117, 139, 145

Fast fourier transform (FFT), 60, 140

Fast multipole method (FMM), 3, 53, 68, 74, $92,102,118,144$

Fill-in, 81, 90, 96

Finite difference time domain (FDTD), 50

Finite element method (FEM), 13, 79, 113 discretization, 13

Fixed-point form, 83

Formatted arithmetic operation, 96

Fortran, 117

Forward substitution, 68, 92, 96

Fraunhofer SCAI, 139, 146

Free-space problem, 27

Frobenius norm, 66, 168

Full-spectrum method, 17

Full-wave analysis, 2, 17, 36, 50, 52, 140

PEEC approach, 3

Functional

determinant, see also Jacobian, 186

equation, 174

matrix, 186

space, 174

Fundamental solution, 179

Galerkin

discretization, 70

matrix/operator, 109 
method, 175

operator, 114

Gauß elimination, 81

Gauß-Seidel

method, 85, 106, 127

relaxation, 110

smoother, 117

Gegenbauer's addition theorem, 57

Generalized triangle inequality, 171

Generator function, 70

Geometrically-balanced clustering, 70

Geometry-based splitting, 63

Gershgorin's circle theorem, 44, 79, 171-172 discs, 172

GMATRIX, 97, 99, 118

GMRes, 85, 94, 97, 118, 144

Graph, 81, 96

theory, 191

vertices, 90

Green's function, 13, 44, 51, 60, 78, 118, 144, 179-181

integral operator, 179

kernel function, 113, 179

retarded, 56

smoothness, 60

Grid, 81, see also Discretization

coarsening, 108-110

data, 94

hierarchy, 92

hybrid, 34

quasi-uniform, 63, 67

refinement, 81, 146

structured/unstructured, 34, 107, 116

Ground plane, 136

Group theory, 55

Grouping, 55, 63

Hankel function, 57, 103, 180

Hardware architecture, 59

Harwell-Boeing matrix format, 88

Helmholtz equation, 68, 102, 179

Heuristics, 68, 144

Hierarchical decomposition, 61

matrices, 3, 52, 59-76, 114, 118, 141

$L U$-preconditioning, 96

$\mathcal{H}$-matrix arithmetic, 52, 54, 60, 67-68, 144

$\mathcal{H}$-stamping, 94, 146
$\mathcal{H}^{2}$-matrices, 60, 75, 93, 144, 146

$\mathcal{R}_{1}$-matrix stamp, 94

$\mathcal{R}_{k}$-matrix, 66, 171

inverse-preconditioning, 96

partitioning, 55, 59, 60, 62

cardinality, 63

tree, 62

HLIB, 70, 97, 144

Huygen's principle, 180

Hybrid

cross approximation (HCA), 69, 71

Gauß-Seidel, 127

solver strategy, 139-141, 145

Hypergraph partitioning, 82

IBIS, 141

ICEM, 141

ILU, 90

ILUT, 87, 90, 117, 118

Impedance analysis, 50

Index set, 62, 70

partitioning, 62

Influence matrix, 70

Inner product, 113, 174

Instabilities, 51

high-frequency, 38

inductance, 42

late-time, 38

Integral operator, 179

Integration level, 129

Integro-differential equation, 13

Interpolation, 54, 109

formulae, 61

operator-dependent, 108

polynomial, 66

Interpolatory variables, 110

Irradiation, 134

Isosceles, 176

Iteration matrix, 84, 112

Iterative

method

consistent, 84

convergence behavior, 4

efficiency, 4

robustness, 86

solver, 48

strategy, 83

Jacobi method, 85, 106 
relaxation, 110

Kernel function, 179

asymptotic smoothness, 69, 170

degenerate, 70

separable expansion, 102

smoothness, 53, 60, 65, 67

Kirchhoff's laws, 191, 193

Krylov

AMG-preconditioned, 108

method, 59, 144

subspace, $85,90,92$

Laplace

equation, 180

transform, 195

Legendre polynomial, 57

Level

hierarchy, 108

logic, 1, 36

modeling, 140

of abstraction, 1

partitioning, 62

Lipschitz domain, 179

List-scheduling, 127, 129

Load balancing, 90

Load-balancing, 124, 127, 129

Locality data, 126

Low-rank approximation, 52, 54, 55, 61, 64, $65,127,170$

LU-decomposition, 92 approximative, 87

M-matrix, 21, 45, 113, 168

Magnetic field integral equation (MFIE), 14, 59

Magnetic sources, 11

Mapping, 70

Marking, 109

Matching points, 175

Material equations, 10

Matrix

compression, 42

equation, 174

graph, 96

norms, 168

structured, 83

unstructured, 87
Maxwell's equations, 9-11, 191

Ampere's law, 10

differential/integral formulation, 9

Faraday's law, 11

Gauß's law, 11

impulse response, 12

linearity, 11

partial differential equations, 12

quasi-stationary approximation, 11

solution, 12

transfer function, 12

Mesh, 70, see also Grid

orthogonal, 123

refinement, 146

virtual, 108

Meshsize, 34, 53, 72, 79, 102, 106, 107, 136, see also Discretization

Message passing architecture, 126

Method of moments (MoM), 15, 17, 173-175

matrix equation, 15

METIS, 82, 90

Metric coefficients, 185

Microcontroller, 134, 141

MILUTP, 90

MIMD, 126

Minimum

degree ordering, 82

error approach, 85

residual approach, 85

Minkowski-matrix, see M-matrix

MISEA project, 48, 71, 134, 140

Mixed potential integral equation, 14

Mixed-signal design, 1

MNA formulation, 45, 51, 77, 86, 87, 113, 191, 193-194

Model exchange, 2

Model order reduction (MOR), 3, 38, 71, $134,143,146$

Modes, 113

MPI Leipzig, 139, 146

Multicolor Gauß-Seidel smoother, 127

Multiconductor TL (MTL), 141

Multigrid, 105

algebraic, 107-108

geometric, 107, 126

methods, 60, 74

Multilevel 
approach, 140

FMM, 59

modeling, 141

Multipole expansion, 54

N-body problem, 55

NA formulation, 46, 77, 87, 172, 191-192

Nearfield/farfield separation, 54, 55, 115

Nested dissection, 82

Netlist description, 3

Network

analyzer, 135

matrix, 43,48

simulator, 140

Neumann computer model, 124

Nodal admittance matrix, 192

Non-linearity, 140

Normal equation, 82

Normalized residual, 84

Norton model, 26

Numerical integration, 38, 144, 176

Nyström discretization, 175

Offline-scheduling, 129

Ohm's law, 10, 12, 24, 29

Online-scheduling, 127, 129

Ordering, 81

Ordinary differential equation (ODE), 17, 34, 141, 191

Orthogonality condition, 110

Orthonormality, 57

Overdetermined system, 82

Panel clustering, 40, 54, 60

Parallelization, 4, 59, 82, 145

$\mathcal{H}$-matrices, 127-129

AMG, 117, 126-127

architectures, 125

blocking, 128

communication cost, 124

complexity, 129

efficiency, 87, 124

inner/outer, 129

model extraction, 129

preconditioner, 87

scaled speed-up,efficiency, 125

simulation process, 130

speed-up, 124, 127

Partial differential operator, 44
Partial elements

capacitances, 21

coefficients of capacitance, 21

coefficients of electrostatic induction, 21

coefficients of potential, 21, 25, 58

inductance, 20, 24, 58

mutual capacitive couplings, 21

mutual inductive couplings, 42

ohmic loss, 24

self-capacitance, 21

Partitioning, 64, 66

admissible, 40, 61, 64

binary, 63

block, 74

conductor, 19

graph, 90, 96

hierarchical, 55, 62

cardinality, 63

hypergraph, 82

index set, 62

sequence, 128

tensor-product, 62

PEEC, 61

(S)PEEC, 30

+PEEC model, 37

accuracy, 36

dielectric effects, 26

hexahedral cell, 21

Manhattan discretization, 18-21

non-orthogonal discretization, 21-25

non-orthogonal formulation, 5

orthogonal elements, 19

orthogonal formulation, 5

quadrilateral cell, 21

stamping, 117

surface discretization, 18

validity, 36-38, 136, 144

volume discretization, 18

Perfect electric conductor, 11

Permutation matrix, 90

Petrov-Galerkin approach, 18-19, 85

Pivoting, 117

dynamical, 90

strategy, 81

PMCHWT formulation, 30

Point-matching, 175

Poisson equation, 106

Polarization current density, 27 
Polynomial smoother, 127

POSIX, 125, see also PThreads

Post iterations, 87

Power density, 10

Power driver, 134

Power grid reduction, 113

Poynting vector, 10

PRAM, 124

Pre-/postsmoothing, 110, see also Smoothing

Pre-Gauß elimination steps, 90

Preconditioner, 86

factorized, 87

setup, 87

update, 97

Preconditioning, 55, 59, 86-87

$\mathcal{H}$-matrix, 96-97

adaptive, 87, 99-102, 145

Printed circuit board (PCB), 17, 21, 131, $135,141,143$

Processing nodes, 131

Projection, 174

Prolongation, 109

Proximity effect, 43

Pseudo-differential operator, 113

PThreads, 125

Quad-tree, 64

Quadrature, 70, 143, 176-177

admissible partitioning, 40

Gaussian formulas, 176

hierarchical, 40

order, 176

theory, 38

Quasi-stationary, 46, 69

condition, 35

model, 3

Real-valued equivalent, 83, 99, 117

Reallocation, 94

Recompression, 4, 60, 103

adaptive, 54, 144

rank, 74

Recursion formula, 84

Reference triangle, 176

Refinement, 40

Regular matrices, 170

Regulizing coordinate transform, 176

Reliability, 139

Reluctance, 42-50, 144
AMG, 113

Gershgorin's theorem, 172

Locality, 44

matrix properties, 44

model, 136

model passivity, 114

partial reluctance method, 43

screening effect, 44

stamping, 46

Reordering, 90

Residual, 84

equation, 108

Restriction, 109

operator, 110

Retardation, 50-52

$\mathcal{H}$-matrix, 102-103

effects, 3, 37, 180

rPEEC, 50, 92

time, 13

Return-limited loop inductance method, 43

Richardson iteration, 78

Ritz-Galerkin

approach, 18, 85

discretization, 62

matrix, 62

SAMG, 89, 117, 118, 145

Scalability, 126

Scattered field, 11

Scattering

object, 9

parameter measurements, 135

problem, 174

Schur complement, 67, 129

Schwartz kernel theorem, 44, 113

Search space, 82, 85

Separable expansion, 60

Sequence-partitioning, 128, 129

Sequential complexity, 129

Series expansion, 53, 56, 60

Shared memory

model, 125

system, 124, 125, 129

Sherman-Morisson formula, 97

Shielding effect, 44

Shift-truncate potential method, 43

Shifting, 59

Sifting property, 175

Signal 
integrity (SI), 1

analysis, 17

Signal rise time, 37

SIMD, 126

model, 125

Simulation

control, 93

methodology, 7, 143

Single layer potential operator, 79, 113, 179

Singular

integrals, 38

value, 168,170

value decomposition (SVD), 171

Singularity, 53, 60, 68, 144, 181

Skin effect, 11, 27, 43

Skyline

method, 81

storage scheme, 89

Slew-rate

behavior, 36

time, 37

Smoother, 106

Smoothing, 110

step, 110

Space-filling curves, 128

Sparse approximate inverse (SAI), 59, 87,

127, see also Approximate inverse

Sparse matrix storage formats, 87-90

Sparsification, 45, 48

Sparsity, 139

pattern, 48

Spatproduct, 189

Spectral

norm, 66, 170

properties, 86,87

radius, $85,93,106,169$

Spectrum, 78

SPICE, 3, 17, 26, 32, 45, 134, 141, 191

Splitting control, 131

Splitting tree, 42

Stability, 36, 39, 44, 48, 78, 82

Stamping, 93, 117, 192

$\mathcal{H}$-stamping, 94, 146

$\mathcal{R}_{1}$-stamp, 94

reluctance, 46

Standardization institutes, 1

Stiffness matrix, 60, 62, 93

Stopping criterion, 86
Storage sparse, 3

Strict diagonal dominance, 44

Strongly diagonally dominant, 167

Structurally symmetric, 167

Subcircuit, 93, 117

Subgrid, 107

Subspace identification, 134

method, 85-86

Supermatrix, 71

Support, 62, 63, 67

Surface

impedance approximation, 30

parametrization, 176

Susceptance, 43

Switching-induced noise, 2

System

-level simulation, 5

level, 140, 145

level simulation, 2, 133

multilevel modeling approach, 3

Taylor series expansion, 54

Telegraphers equations, 3

Tensor-product partitioning, 62

Test function, 174

Thevenin model, 26

Thread, 124, 125

Threadpool, 125

Time-of-flight, 50

Tolerance limit, 48, 136

Trace, 169

Transfer operator, 108

Transformer equations, 47

Translation, 59

Transmission

behavior, 134

line (TL) theory, 2

line model, 50, 139

Triangulation, 73, 176

Truncated

arithmetic operation, 96

matrix operations, 68

singular value decomposition (SVD), 56, $66,68,170-171$

Truncation, 42-44, 136

error, 114

process, 45,113

rank, 170 
series expansion, 53

Taylor expansion, 61

Vector

field, 186

norms, 168

Vectorial

path element, 184

surface element, 185

Vertex, 81

Very large scale integration (VLSI), 2

systems, 17

Wave

equation, 179

field, 9, 11

number, 11, 81, 102

Wavelets, 4, 60

Weakly diagonally dominant, 167

Weight smoother, 127

Weighted residual method, 15, 18

Weighting coefficients, 176

Workload, 123 


\section{Curriculum Vitae}

Personal Information

Name
Date of birth
Place of birth
Nationality
EDUCATION

02/2004 - 08/2006

$03 / 2001-10 / 2003$

10/1996 - 02/1997 and

$02 / 1998-02 / 2001$

$09 / 1995-08 / 1996$

$09 / 1991-02 / 1995$

S

Martin Ludwig Zitzmann

1975, June 17th

Pegnitz, Germany

German

Ph.D. studies at the Institute for Electronics Engineering, University of Erlangen-Nuremberg, Germany together with the BMW Group Munich, Germany

Specialization: Fast EMC modeling and simulation techniques

Diploma studies in electrical engineering at the University of Erlangen-Nuremberg, Germany

Specialization: Communications engineering

Diploma studies in electrical engineering at the University of Applied Sciences Nuremberg, Germany

Specialization: Communications engineering

Technical College Bayreuth, Germany

Graduation: Fachabitur

Vocational School Bayreuth, Germany

Specialization: Information and communication technology

WORK

09/1991 - 02/1995

Vocational training at German Telecom, Bayreuth, Germany

MiLitARY SERVICE

03/1997- 12/1997

Staff services

German air force, Freising, Germany 



\section{Zusatz/Supplement (in deutsch/in german)}





\title{
Schnelle und effiziente Methoden für die schaltkreisbasierte EMV-Simulation im Automobilbereich
}

\author{
Der Technischen Fakultät der \\ Universität Erlangen-Nürnberg \\ zur Erlangung des Grades \\ D O K T O R - I N G E N I E U R
}

vorgelegt von

Martin Ludwig Zitzmann

Erlangen - 2007 

1 Einleitung 1

1.1 EMV-Simulation im Automobilbereich $\ldots \ldots \ldots \ldots \ldots \ldots \ldots \ldots \ldots \ldots$

1.2 Stand der Technik . . . . . . . . . . . . . . . . . . . . 3

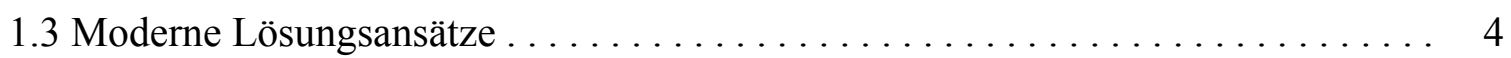

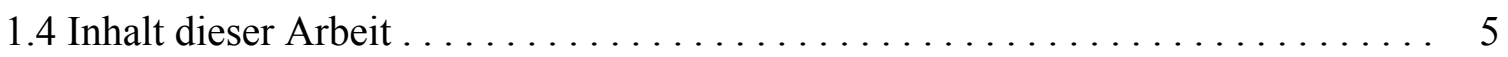

2 Grundlagen der Simulation elektromagnetischer Effekte 9

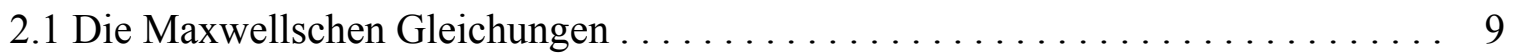

2.2 Das elektromagnetische Streuproblem . . . . . . . . . . . . . . . 11

2.3 Die retardierten elektrodynamischen Potenziale . . . . . . . . . . . . . . 12

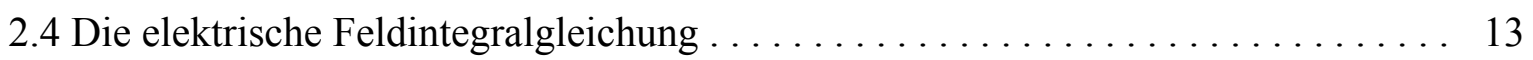

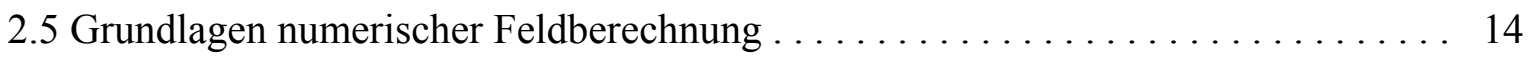

3 Äquivalente Schaltkreismodellierung 17

3.1 Der Petrov-Galerkin Ansatz . . . . . . . . . . . . . . . . . . . . 18

3.2 Partielle Elemente für eine Manhattan-Disktretisierung . . . . . . . . . . . . 19

3.2.1 Diskretisierung der EFIE . . . . . . . . . . . . . . . . . . . . . 19

3.2.2 Extraktion partieller Elemente . . . . . . . . . . . . . . . 20

3.3 Partielle Elemente für eine nichtorthogonale Diskretisierung . . . . . . . . 21

3.3.1 Diskretisierung der EFIE . . . . . . . . . . . . . . . . 22

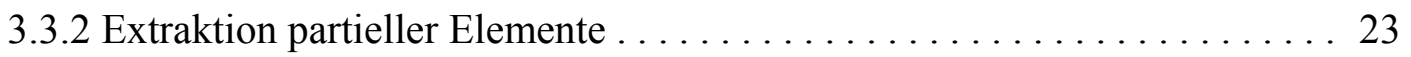

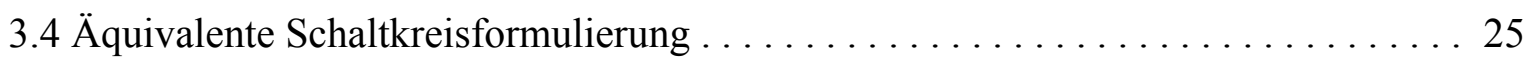

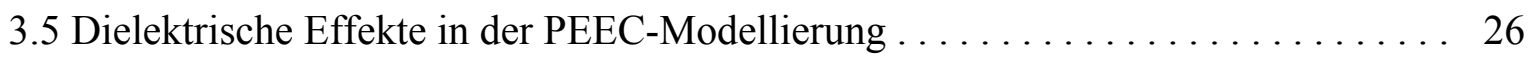

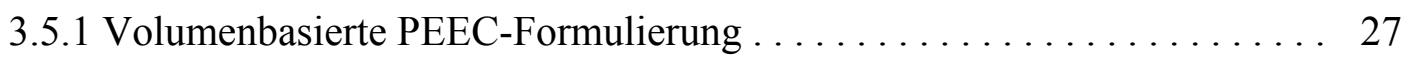

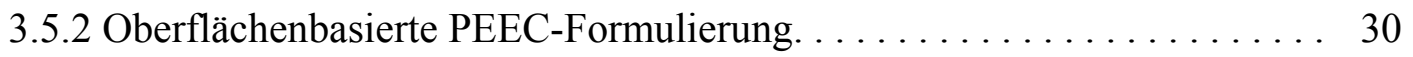

3.6 Struktur einer PEEC-basierten Schaltkreissimulation . . . . . . . . . . . . . 32

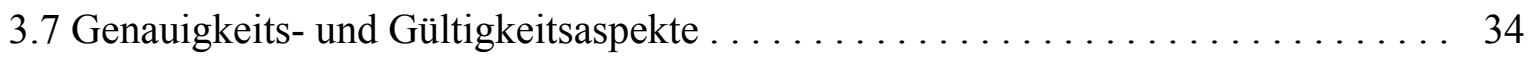

3.7.1 Elektrische Länge von Verbindungsstrukturen . . . . . . . . . . . . 34

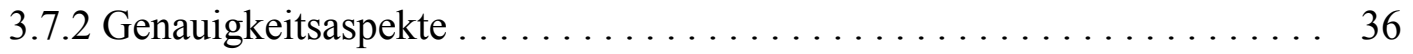

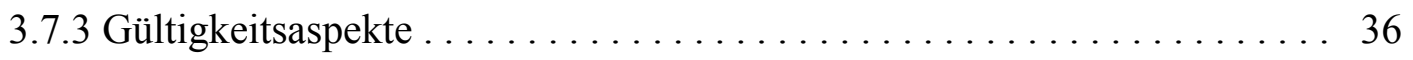

3.7.4 Simulationsartefakte . . . . . . . . . . . . . . . . . . . . . 38

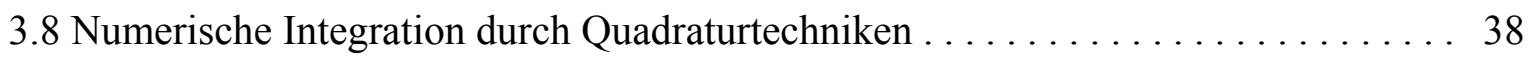

3.8.1 Quadraturtechniken nach Sauter . . . . . . . . . . . . . . . . 38

3.8.2 Hierarchische Quadratur . . . . . . . . . . . . . . . . . . 40

3.9 Formulierung dünn besetzter Matrizen durch partielle Reluktanzen . . . . . . . . . . 42

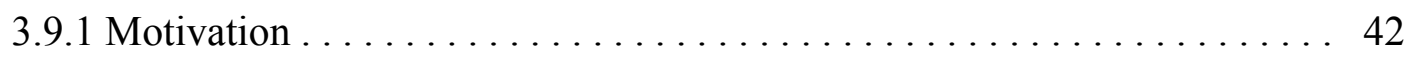

3.9.2 Partielle Reluktanz K . . . . . . . . . . . . . . . . . . . . 43

3.9.3 Eigenschaften von Reluktanzmatrizen . . . . . . . . . . . . . . . . 44 
3.9.4 Der Ausdünnprozess . . . . . . . . . . . . . . . . . . . . 45

3.9.5 Reluktanzbasierte Schaltkreisrepräsentation .............. 45

3.9.6 Numerische Beispiele ......................... 48

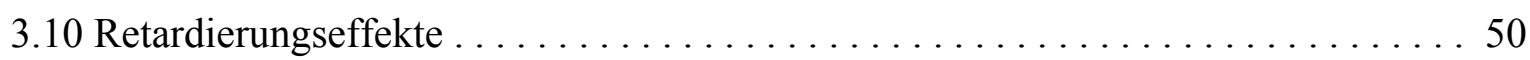

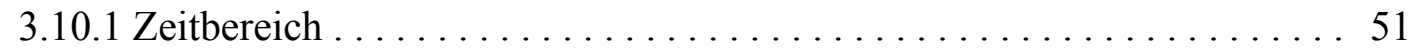

3.10 .2 Frequenzbereich .............................. 51

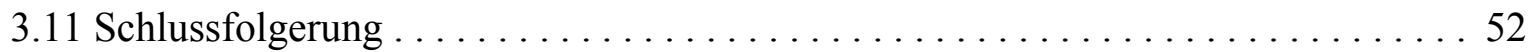

4 Schnelle und effiziente Extraktion parasitärer Effekte 53

4.1 Historischer Hintergrund und Motivation $\ldots \ldots \ldots \ldots \ldots \ldots \ldots \ldots \ldots \ldots$

4.2 Die schnelle Multipolmethode (Fast Multipole Method) . . . . . . . . . . . 54

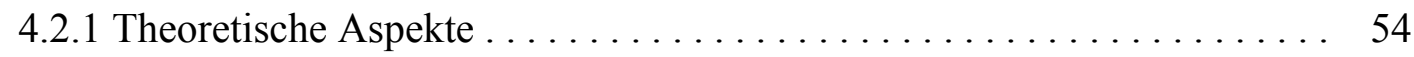

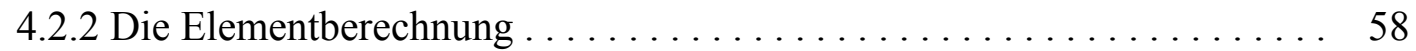

4.2.3 Die Multilevel Fast Multipole Methode . . . . . . . . . . . . . . . . . 59

4.2.4 Implementierungsaspekte . . . . . . . . . . . . . . . . . . . . . . 59

4.3 Hierarchische Matrizen . . . . . . . . . . . . . . . . . . . . . 59

4.3.1 Theoretische Aspekte . . . . . . . . . . . . . . . . . . . . . . 60

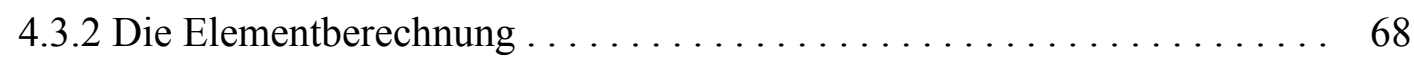

4.3.3 Implementierungsaspekte . . . . . . . . . . . . . . . . . . . 70

4.3.4 Numerische Beispiele . . . . . . . . . . . . . . . . . . 71

4.3.5 Weitere Verbesserungen . . . . . . . . . . . . . . . . . . . . . . . 73

5 Iterative Methoden in der schaltkreisbasierten Analyse

5.1 Problembeschreibung und Motivation . . . . . . . . . . . . . . . 78

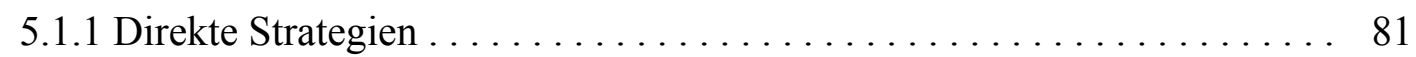

5.1 .2 Iterative Strategien $\ldots \ldots \ldots \ldots \ldots \ldots \ldots \ldots \ldots \ldots \ldots \ldots \ldots$

5.2 Die iterative Lösung linearer Gleichungssysteme $\ldots \ldots \ldots \ldots \ldots \ldots \ldots \ldots$

5.2 .1 Grundlagen iterativer Methoden $\ldots \ldots \ldots \ldots \ldots \ldots \ldots \ldots . \ldots . \ldots . \ldots 3$

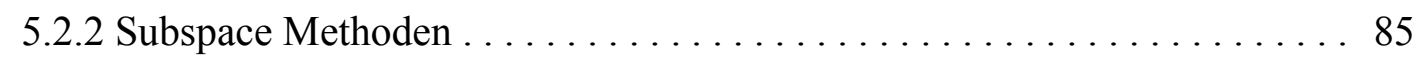

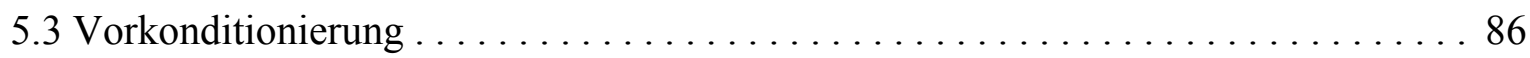

5.4 Implementierungsaspekte $\ldots \ldots \ldots \ldots \ldots \ldots \ldots \ldots \ldots \ldots \ldots \ldots \ldots$

5.5 Speicherformate für dünn besetzte Matrizen $\ldots \ldots \ldots \ldots \ldots \ldots \ldots \ldots \ldots$

5.6 Weitere Verbesserungen . . . . . . . . . . . . . . . . . . . . 90

6 Löser für PEEC basierend auf hierarchischen Matrizen 91

6.1 Überblick . . . . . . . . . . . . . . . . . . . . . . . . . . . . 91

6.1.1 Direkte Strategien . . . . . . . . . . . . . . . . . . . 92

6.1 .2 Iterative Strategien . . . . . . . . . . . . . . . . . . 93

6.2 Implementierungsaspekte für PEEC-basierte Schaltkreissimulation . . . . . . . . . 93

6.2.1 Das H-Stempelverfahren . . . . . . . . . . . . . . . . . . . . 94

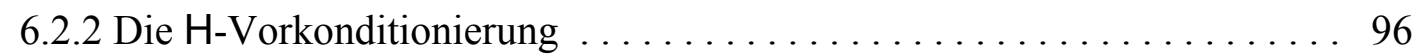

6.3 Numerische Ergebnisse $\ldots \ldots \ldots \ldots \ldots \ldots \ldots \ldots \ldots \ldots \ldots \ldots \ldots \ldots \ldots$

6.3.1 Analyse des Konvergenzverhaltens . . . . . . . . . . . . . . . 97 
6.3.2 Adaptive Vorkonditionierung . . . . . . . . . . . . . . . . . . . . . . 99

$6.4 \mathrm{H}$-Matrizen für den retardierten Fall . . . . . . . . . . . . . . . . 102

7 Löser für PEEC basierend auf Mehrgitterverfahren 105

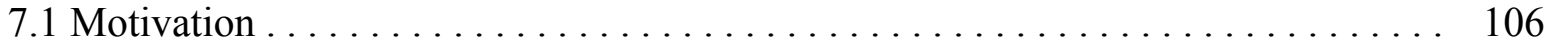

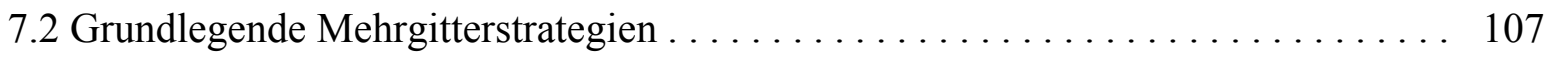

7.2.1 Geometrisches Mehrgitter . . . . . . . . . . . . . . . . . . . 107

7.2.2 Algebraisches Mehrgitter . . . . . . . . . . . . . . . . . . 107

7.3 AMG Komponenten . . . . . . . . . . . . . . . . . . . . . . . . . . . 108

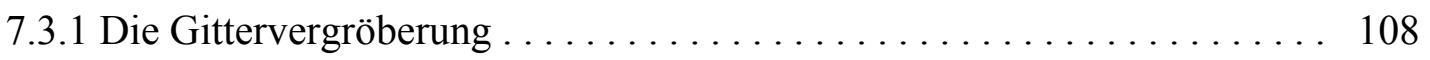

7.3.2 Der Glättungsprozess . . . . . . . . . . . . . . . . . . . . . . . 110

7.3.3 Der Coarse-Level Correction Prozess. . . . . . . . . . . . . . . . . . . 110

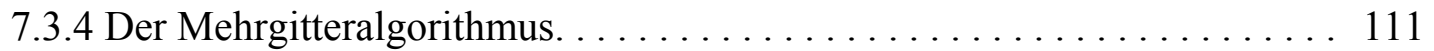

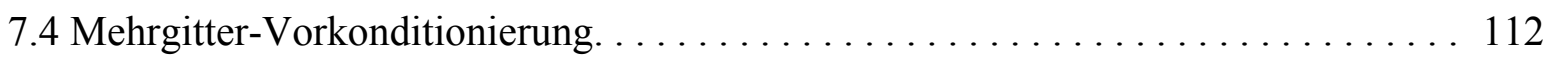

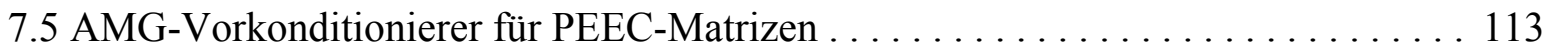

7.5.1 AMG-Vorkonditionierer für ausgedünnte PEEC-Matrizen . . . . . . . . . . 113

7.5.2 AMG-Vorkonditionierer für Daten-sparse PEEC-Matrizen. . . . . . . . . . . . 114

7.5.3 Komplexitätsanalyse. . . . . . . . . . . . . . . . . . . . 116

7.6 Implementierungsaspekte für die PEEC-basierte Schaltkreissimulation . . . . . . . . 116

7.7 Numerische Ergebnisse . . . . . . . . . . . . . . . . . . . 118

8 Parallelisierungsaspekte für Hierarchische Methoden 123

8.1 Überblick . . . . . . . . . . . . . . . . . . . . . . . . . . . . . . . 123

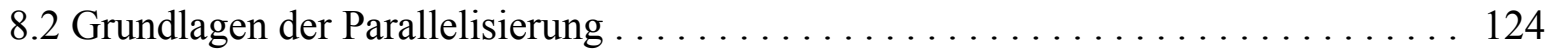

8.3 Parallelisierungsarchitekturen . . . . . . . . . . . . . . . . . . . 125

8.3.1 Shared Memory Systeme . . . . . . . . . . . . . . . . . . . . 125

8.3.2 Distributed Memory Systeme . . . . . . . . . . . . . . . . 126

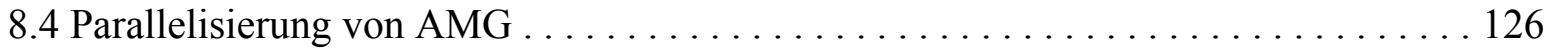

8.4.1 Implementierungsaspekte . . . . . . . . . . . . . . . . . . 127

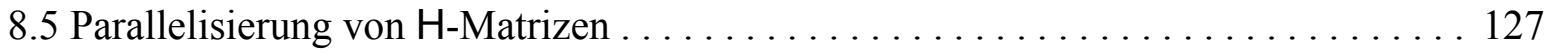

8.5.1 Parallele $\mathrm{H}$-Matrix Approximation . . . . . . . . . . . . . . . . . 127

8.5.2 Parallele H-Matrix-Vector Multiplikation . . . . . . . . . . . . . . . . . 128

8.5.3 Parallele H-Matrix Inversion . . . . . . . . . . . . . . . . . . . 128

8.5.4 Implementierungsaspekte . . . . . . . . . . . . . . . . . . . . . . . . 129

8.6 Parallelisierte Schaltkreissimulation . . . . . . . . . . . . . . . . . . . . . 129

8.6.1 Der Modellextraktionsprozess . . . . . . . . . . . . . . . . . . . . . . . 129

8.6.2 Der Simulationsprozess . . . . . . . . . . . . . . . . . . 130

8.6 .3 Implementierungsaspekte . . . . . . . . . . . . . . . . 130

9 Industrielle Anwendungen 133

9.1 Motivation und Überblick . . . . . . . . . . . . . . . . . . . . . . . . 133

9.2 Beschreibung des Demonstratorboards . . . . . . . . . . . . . . . . . . . . . . . . 134

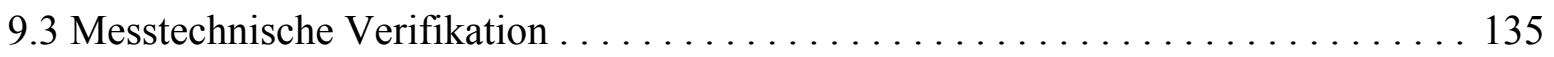


9.4 Simulationstechnische Verifikation. ......................... 137

9.5 Hybride Löserstrategie . . . . . . . . . . . . . . . . . . . . . . . . . . . . 139

10 Schlussfolgerung $\quad 143$

$\begin{array}{ll}\text { Literaturverzeichnis } & 147\end{array}$

$\begin{array}{ll}\text { Abbildungsverzeichnis } & 163\end{array}$

$\begin{array}{ll}\text { Tabellenverzeichnis } & 165\end{array}$

$\begin{array}{ll}\text { A Mathematische Grundlagen, Definitionen und Bezeichnungen } & 167\end{array}$

A.1 Elementare Matrizentypen ................................ 167

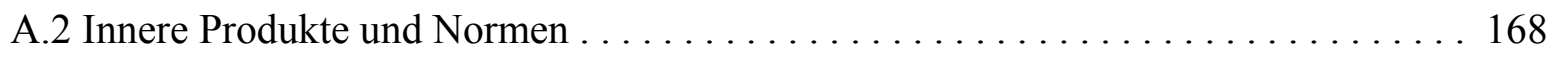

A.2.1 Vektornormen ................................... 168

A.2.2 Matrixnormen .................................... 168

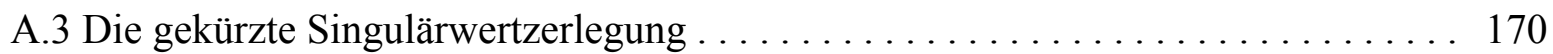

A.4 Das Kreistheorem nach Gershgorin .......................... 171

A.5 Die Momentenmethode ..................................... 173

A.6 Numerische Integration durch Quadratur ...................... 175

B Die Greensche Funktion $\quad 179$

$\begin{array}{lr}\text { C Koordinatensysteme } & 183\end{array}$

C.1 Orthogonale rechtshändige Koordinatensysteme................... 183

C.2 Kartesische Koordinaten ................................... 184

C.3 Krummlinige Koordinaten ................................. 184

C.4 Integration in krummlinigen Koordinaten ...................... 186

C.4.1 Die Funktionaldeterminante . . . . . . . . . . . . . . . . . . . . . 186

C.4.2 Oberflächenintegration ............................. 188

C.4.3 Volumenintegration ............................. 190

D Grundlagen der Schaltkreissimulation $\quad 191$

D.1 Grundlagen der Netzwerkanalyse . . . . . . . . . . . . . . . . . . . . . . . . 191

D.2 Das Knotenpotentialverfahren ................................. 192

D.3 Das modifizierte Knotenpotentialverfahren ........................... 193

D.4 Zeitbereichssimulation ..................................... 194

D.5 Frequenzbereichssimulation ............................. 195

$\begin{array}{ll}\text { E Verzeichnis der Symbole, Variablen und Abkürzungen } & 197\end{array}$

E.1 Wichtige Mengen und Konstanten . . . . . . . . . . . . . . . . . . . . . . . . 197

E.2 Wichtige Variablen ...................................... 198

E.2.1 Elektromagnetische Grundlagen ...................... 198 
E.2.2 PEEC-Modellierung. . . . . . . . . . . . . . . . . . . . . . . . . . . . 198

E.2.3 Hierarchische Methoden . . . . . . . . . . . . . . . . . . . . 200

E.2.4 Iterative Methoden . . . . . . . . . . . . . . . . . . . . . . . . . . . . . 201

E.2.5 Parallelisierung . . . . . . . . . . . . . . . . . . . 201

E.3 Mathematische Bezeichnungen, Transformationen und Operatoren . . . . . . . . 201

E.4 Abkürzungen . . . . . . . . . . . . . . . . . . . . . . 203

$\begin{array}{lr}\text { Stichwortverzeichnis } & 207\end{array}$

$\begin{array}{lr}\text { Lebenslauf } & 217\end{array}$ 



\section{Einleitung}

$\boldsymbol{D}$

ie Elektromagnetische Verträglichkeit (EMV) befasst sich mit den Wechselwirkungen zwischen elektronischen Systemen und ihrer elektromagnetischen (EM) Umgebung. Bei der Entwicklung elektronischer Komponenten sind einige EMVAspekte zu berücksichtigen, um die Gesamtfunktionalität in einem komplexen elektromagnetischen Umfeld zu gewährleisten. Die beiden in den gängigen Normen enthaltenen $[1,2]$ grundlegenden EMV-Anforderungen, umfassen Aspekte bezüglich der elektromagnetischen Störaussendung (engl. electromagnetic interference, EMI) ebenso wie die elektromagnetische Störfestigkeit (engl. electromagnetic susceptibility, EMS). Die Sensibilität eines Gerätes gegenüber EMI wird durch die Störfestigkeit oder Unempfindlichkeit (engl. immunity) beschrieben. Im Allgemeinen unterscheiden Normen zwischen privatem und industriellem Einsatz [3]. Diese basieren auf internationalen oder nationalen Empfehlungen von Normungsgremien.

Stetig zunehmende Datenraten in Kommunikationssystemen, verringerte Spannungspegel in logischen Schaltungen, sich stetig verringernde Packungsdichten, gemischte analog/digitalSchaltungsdesigns [4] und hochfrequente Schaltnetzteile stellen Herausforderungen für moderne Schaltungsentwicklungen dar. Aus diesen Gründen ist es zunehmend von Bedeutung, EMV-Anforderungen zu berücksichtigen [5]. Für den Entwurf von schnellen digitalen und gemischt analogen/digitalen Systemen ist es notwendig, EMI, Störfestigkeitsaspekte (einschließlich elektrostatische Entladung, engl. electrostatic discharge (ESD)) und die Signalintegrität (SI) zu berücksichtigen. Diese Aspekte müssen durch eine geeignete Modellierung parasitärer Effekte berücksichtigt werden. Viele Signalintegritätsprobleme sind elektromagnetischen Ursprungs und somit verwandt zur EMV. Die Bezeichnung Signalintegrität bei der Entwicklung elektronischer Systeme umfasst zwei Aspekte - das Timing und die Qualität des Signals. Ein digitales System kann auf drei verschiedenen Ebenen abstrahiert werden - auf der Logikebene, auf der Schaltkreisebene und auf der Feldebene. Numerische Simulationstechniken können eingesetzt werden, um das elektromagnetische Systemverhalten bereits in einem sehr frühen Entwicklungsstadium zu bewerten [3]. Die Forderung nach Modellierungsmethoden für den Bereich hochfrequenter elektromagnetischer Felder in 3D und ihre zunehmende Bedeutung für die kommenden Jahrzehnte wird in [6] herausgestellt.

\subsection{EMV-Simulation im Automobilbereich}

Die zunehmende Anzahl elektronischer Einrichtungen (elektronische Komponenten für Sicherheit, Zuverlässigkeit und Komfort) in modernen Automobilen führt zu einem erhöhten Grad an elektromagnetischen Emissionen und Störungen. Deshalb sind die vorgenannten Aspekte ebenso für den Automobilbereich von Bedeutung. Empfehlungen zu Emissions- und Immunitätsgrenzwerten sind in [7] $\mathrm{zu}$ finden. Ein Automobil ist ein sehr komplexes 
dreidimensionales, aus einer Vielzahl von Teilsystemen bestehendes elektronisches Gesamtsystem, wie in Abbildung 1.1 prinzipiell dargestellt.

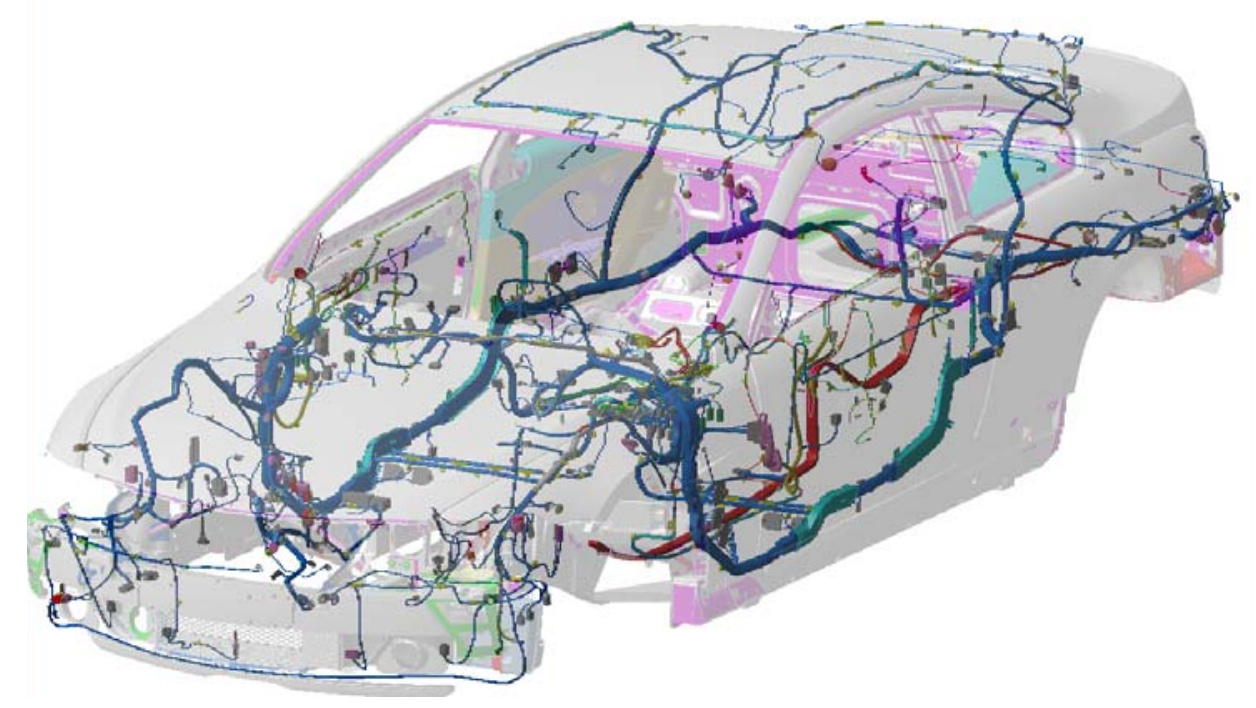

Abb. 1.1: Karosserie mit Kabelbäumen und Steuergeräten als elektronische Hauptkomponenten eines modernen Automobils.

Die Kombination aller Teilsysteme kann potentielle Gefahrenquellen erzeugen, die sich negativ auf das elektromagnetische Verhalten auswirken kann [8], auch wenn alle Teilsysteme unter Berücksichtigung der EMV-Anforderungen entwickelt wurden. Die bedeutendsten elektromagnetischen Phänomene in automobilen Applikationen sind das Nebensprechen (engl. crosstalk) und Störungen durch Schaltvorgänge in integrierten Schaltkreisen (engl. switching-induced noise). Für den Entwurf von elektronischen Hochleistungsgeräten müssen diese Effekte genauestens bekannt sein. Numerische Simulationstechniken stellen eine wichtige Maßnahme dar, EMV-verwandte Probleme bereits in einem frühen Stadium des automobilen Entwicklungsprozesses beherrschen zu können [9]. Kostenintensive Überarbeitungszyklen in einem späten Entwicklungsstadium können somit vermieden werden. Für eine genaue Analyse ist ein kontinuierlicher und stimmiger EMVSimulationsprozess notwendig, der auf einem Informationsaustausch zwischen Automobilherstellern, Zulieferern von Elektronikteilen und Entwicklern integrierter Schaltungen basiert $[8,9]$. Während das elektromagnetische Verhalten einzelner Signalleitungen auf Leiterplatten (engl. printed circuit board, PCB) bereits effizient simuliert werden kann, stellen Systemsimulationen auf Gesamtfahrzeugebene derzeit eine Herausforderung dar. Der Hauptgrund dafür liegt in der Schwierigkeit, sehr unterschiedliche geometrische Objekte zu handhaben, die sich auf die drei elektronischen Hauptkomponenten des Systems beziehen - die Karosserie, die Kabelbäume und die Steuergeräte. Unter Zuhilfenahme von Vollwellen 3D-, Transmission line (TL) 2D- oder äquivalenten Schaltkreisansätzen können die verschiedenen Teilkomponenten modelliert und analysiert werden. Dies führt zu hohen Anforderungen an moderne Simulationstechniken. Eine genaue Simulation elektromagnetischer Effekte bis in hohe Frequenzbereiche erfordert eine sehr feine Diskretisierung im Hinblick auf die geometrischen Abmessungen [10]. Dies kann zu sehr großen Dimensionen der resultierenden Gleichungssysteme führen. 
Eine integralgleichungsbasierte Methode, die sich gut für die Modellierung von Leiterplatten, VLSI (engl. very large scale integration) Verbindungsstrukturen [11] und für Leistungssysteme eignet, stellt die Methode der partiellen Elemente (engl. partial element equivalent circuit, PEEC) dar. Diese Methode ermöglicht eine Schaltkreisrepräsentation der elektrischen Feldintegralgleichung (engl. electric field integral equation, EFIE) und stellt somit eine Verbindung zwischen dem Bereich der elektromagnetischen Felder und dem Bereich der Schaltkreisbeschreibung her [12]. Schaltkreissimulatoren berechnen Spannungen und Ströme in Schaltungselementen wie Widerständen, Kapazitäten und Induktivitäten. Eine quasistatische Betrachtung ist für Signalintegritätsuntersuchungen häufig ausreichend. Verbindungsstrukturen können deshalb meist durch Schaltkreisprimitive modelliert werden. Bewegt sich die Signalanstiegszeit jedoch im Bereich der Gesamtsignallaufzeit im Objekt, so ist ein Modell auf der Basis diskreter Schaltkreiselemente nicht mehr ausreichend. Werden so genannte Retardierungseffekte berücksichtigt, müssen 2D- und 3D-Effekte unterschieden werden. Während ein ,verteiltes“ Schaltkreismodell in 2D auf der Leitungstheorie beruht (Telegraphengleichungen), können 3D-Effekte nur durch Anwendung eines Vollwellen PEEC-Ansatzes analysiert werden. Das SPICE-Format ist ein etablierter Quasi-Standard, um Netzwerkgleichungen aus einer Netzlistenbeschreibung zu formulieren. Es ermöglicht einen programmunabhängigen Austausch von EMV-Modellen verschiedener Modellierungsebenen zwischen den Partnern im Entwicklungsprozess. Die Schaltkreisbeschreibung von Verhaltensmodellen für Steuergeräte und ICs, zusammen mit Leitungsmodellen für die Kabelbäume vereinfacht den Integrationsprozess und macht zusätzliche Erweiterungen möglich. $\mathrm{Zu}$ weiteren Einzelheiten siehe [9].

\subsection{Stand der Technik}

Aus den Informationen des vorangegangenen Abschnittes wird deutlich, dass ein einzelnes Modellierungs-Werkzeug nicht in der Lage ist, die Effekte der verschiedenen Elektronikkomponenten im Automobil zufrieden stellend abzubilden. Eine funktionale Einteilung kann helfen, die charakteristischen Eigenschaften zu berücksichtigen und ermöglicht eine genaue Vorhersage der EMV [9,13]. Der in [8] vorgestellte Multilevel-Modellierungsansatz berücksichtigt alle relevanten Aspekte um eine Simulation auf Systemebene zu realisieren. Matrizen aus schaltkreisbasierten Ansätzen können in der Regel sehr groß, dicht besetzt und schlecht konditioniert sein. Der Speicherbedarf ebenso wie die Gesamtsimulationszeit, begrenzen somit stark die Leistungsfähigkeit und die lösbare Problemgröße bei Verwendung konventioneller Techniken. Unter Einsatz von direkten Methoden können in der Regel lediglich kleine Probleme effizient gelöst werden. Die genannten Gründe machen die numerische EMV-Analyse zu einer Herausforderung und erklären die derzeitigen Forschungsaktivitäten auf diesem Gebiet. Die beiden Hauptengpässe in der numerischen EMV-Analyse stellen der Modellierungs- und der Lösungsprozess dar. Für die Realisierung einer sehr leistungsstarken Simulation sind beide Teile zu optimieren. Techniken zur Modellordnungsreduktion (engl. model order reduction, MOR) stellen eine etablierte Methodik dar. Diese kann auf große lineare elektrische Netzwerke angewandt werden. Eine Komplexitätsreduktion für die Simulation kann in Form von Modellen mit reduzierten Dimensionen und vergleichbarem Ein-/Ausgangsverhalten zum Originalmodell erreicht werden [14,13]. Techniken zur Modellordnungsreduktion weisen jedoch einige gravierende Schwachstellen 
auf. Als Hauptnachteil wird der Speicherengpass angesehen, der vor der Modellordnungsreduktion beim Aufbau der vollbesetzten Matrizen mit den parasitären Elementen entsteht. Wichtige elektrische und magnetische Effekte sowie geometrische Bezugsdaten können darüber hinaus während der Reduktion verloren gehen und bestehende Simulationsartefakte im Simulationsergebnis können verstärkt werden [15]. Eine Optimierung der Modellierungsund Lösungsprozesse im Simulator ist deshalb zu bevorzugen [16]. Die PEEC-basierte elektromagnetische Modellierung kann in Verbindung mit hoch entwickelten mathematischen Techniken stark verbessert werden, um einen äußerst effizienten und genauen Gesamtsimulationsprozess zu gewährleisten.

Die PEEC-Methode ermöglicht die Extraktion von Schaltkreiselementen aus einem physikalischen Design. Ähnlich zu anderen integralgleichungsbasierten Techniken formen die parasitären Elemente dicht besetzte Matrizen. Diese resultieren aus einem Randelementeansatz, der auf die EFIE angewandt wird. Die Komplexität ist quadratisch mit der Matrizendimension sowohl in Bezug auf die Extraktionszeit als auch in Bezug auf den Speicherbedarf. Um Analysen großer und dicht besetzter Systeme zu ermöglichen, ist es wünschenswert, Matrizen in einem speichersparenden Format zu erhalten.

Hierarchische Techniken erlauben es, den Simulationsprozess enorm zu beschleunigen. Die schnelle Multipolmethode (engl. fast multipole method, FMM) und ihre eng verwandte Technik der hierarchischen Matrizen (H-Matrizen) ermöglichen eine sehr effiziente Parameterextraktion. Speziell H-Matrizen liefern eine ,black-box“-artige Methodik, um so genannte ,data-sparse“ Matrizen zu erzeugen. Diese stellen eine Approximation der dicht besetzten Matrizen dar. Eine speziell entwickelte H-Matrix-Arithmetik ermöglicht Matrizenoperationen mit annähernd linearer Komplexität. Dies ist für eine effiziente Anwendung zusammen mit iterativen Methoden von besonderer Bedeutung. H-Matrizen erlauben darüber hinaus eine spätere Matrix-Rekompression.

\subsection{Moderne Lösungsansätze}

In der numerischen schaltkreisbasierten EMV-Simulation ist es notwendig, eine effiziente aufeinander folgende Lösung großer linearer Gleichungssysteme zu realisieren [17]. Eine Effizienzsteigerung kann unter Umständen bereits dadurch erzielt werden, dass Faktorisierungen der Systemmatrix für nachfolgende Simulationsschritte wieder verwendet werden können (z.B. Problem mehrerer rechter Seiten). Für die Realisierung einer sehr leistungsstarken Simulation ist dennoch der Einsatz hoch entwickelter numerischer Lösungsalgorithmen absolut notwendig. Neben Mehrgitterverfahren [18] wurden auch hierarchische Matrizen [19,20] zu diesem Zwecke untersucht. Im Automobilbereich stellen verschiedene Modellierungsarten eine zusätzliche Herausforderung für den Löser dar. Die verschiedenen Modellcharakteristiken beeinflussen direkt die Eigenschaften der resultierenden Systemmatrix. Sowohl die Besetzungsmuster als auch die Dimensionen der Matrizen können enorm variieren. Während die Modellierung von Kabelbäumen in der Regel zu dünn besetzten Matrizen führt, sind Matrizen, die aus einer Modellierung von Teilen einer Leiterplatte auf Schaltkreisebene basieren dicht besetzt. 
Direkte Lösungsmethoden sind zuverlässig und robust und ihre Genauigkeit und Komplexität sind abschätzbar [21]. Aus diesen Gründen werden sie häufig für die Lösung von Systemen im industriellen Umfeld eingesetzt. Solche Methoden weisen kubische Komplexität mit der Matrizendimension in Bezug auf CPU Zeit und im schlechtesten Fall quadratische Komplexität in Bezug auf den Speicherbedarf für die Matrix auf. Obwohl eine Vielzahl von Neuerungen auf diesem Gebiet bereits zu weit reichenden Verbesserungen geführt haben, haben sich direkte Methoden für große Systeme als nicht effizient genug erwiesen. Der Speicherbedarf wird bei EMV-Simulationen im Automobilbereich oft kritischer angesehen, als der notwendige Zeitbedarf. Während lange Simulationszeiten unter Umständen toleriert werden können, begrenzt der Mangel an Speicher schnell die lösbare Problemgröße und als direkte Folge die erreichbare Genauigkeit [22]. Gewöhnlich sind iterative Standard one-levelLöser nicht in der Lage, Lösungen mit optimaler Komplexität zu erzielen. Dennoch weisen sie im Vergleich zu direkten Lösern bessere Leistungsfähigkeit auf. Für typische Problemstellungen kann die Berechnung der Iterationsschritte mit linearer Komplexität erfolgen. Das Konvergenzverhalten wird mit zunehmender Problemgröße jedoch schlechter. Die Anzahl der benötigten Iterationsschritte, um eine vorgegebene Genauigkeit zu erreichen, wächst linear mit der Anzahl der Unbekannten. Dies kann besonders im Bereich der Schaltkreissimulation beobachtet werden, wo Matrizen sehr große Konditionszahlen aufweisen. Obwohl eine Vielzahl von Verbesserungen durchgeführt wurden, um die Leistungsfähigkeit von iterativen Methoden zu steigern, können große Probleme unter Einsatz direkter oder vorkonditionierter iterativer Löser für dünn besetzte Matrizen nicht effizient genug gelöst werden. Durch die Einführung hierarchischer Lösungsalgorithmen wurde ein Durchbruch erzielt, um eine effiziente Lösung großer industriell relevanter Probleme aus dem Bereich der rechentechnischen EMV-Analyse zu ermöglichen. Für weitere Informationen siehe [23,24,25,26,18]. Zumindest für spezielle Problemstellungen können solche hierarchische Methoden optimale Komplexität mit linearer Ordnung mit der Matrizendimension sowohl in Bezug auf Berechnungszeit als auch in Bezug auf Speicherbedarf erzielen. Eine weitere Verbesserung solcher Techniken kann häufig nur durch die Anwendung von Parallelisierung erreicht werden. Diese ist in der Regel jedoch schwierig zu realisieren. Um die volle Leistungsfähigkeit auf parallelen Systemen zu erzielen, müssen die Algorithmen in Bezug auf ihre Eignung für parallele Verarbeitung getestet und entsprechend angepasst werden. Hierarchische Algorithmen wie die FMM, H-Matrizen oder Wavelet-basierte Ansätze sind gut parallelisierbar. Die vorgestellten hierarchischen Techniken sind viel versprechend um eine effiziente numerische Simulation auf Systemebene zu ermöglichen. In [8] werden solche Simulationstechniken angewandt, um die verschiedenen Abstraktionsebenen zu berücksichtigen. Die Modellgenauigkeiten auf individuellen Abstraktionsebenen haben jedoch einen enormen Einfluss auf die Genauigkeit der Systemsimulation.

\subsection{Inhalt dieser Arbeit}

Diese Arbeit befasst sich mit den Unzulänglichkeiten und Hauptengpässen in der PEECbasierten Schaltkreissimulation. Sie stellt eine Methodologie für eine umfassend anwendbare Simulationsstrategie vor, die sich für EMV-Simulationen im Automobilbereich auf Systemebene eignet. Eine Bewertung der Eigenschaften spezifischer Modellierungs- sowie auch Simulationstechniken und Einschränkungen im Simulationsprozess werden aufgezeigt und 
Abhilfemaßnahmen diskutiert. Obwohl die dargestellten Ausführungen sehr allgemeingültig gehalten sind, wird die Verbindung zum Automobilbereich stets hergestellt. Die vorliegende Arbeit verbindet Themen aus der Mathematik mit Themen aus der Elektrotechnik. Dies ist für die Entwicklung von Hochleistungsalgorithmen und ihren Einsatz in industriellen Programmen unabdingbar. Die vorgestellten Algorithmen sollen mit universellen schaltkreisbasierten Ansätzen und speziell mit PEEC-basierten Ansätzen für kartesische sowie für krummlinige Koordinaten und mit TL-Modellen gut kompatibel sein. Wie in den folgenden Kapiteln beschrieben wird, haben sich das algebraische Mehrgitterverfahren sowie hierarchische Matrizen für die Anwendung zusammen mit schaltkreisbasierter Simulation als geeignet erwiesen. Aus historischen Gründen wird darüber hinaus ein Ausdünnungsverfahren betrachtet, das auf partiellen Reluktanzen beruht und robuste und stabile Simulationen gewährleistet. Die Kombination von H-Matrizen, AMG und PEEC-Modellen stellt einen neuartigen Ansatz dar, um Systemsimulationen mit deutlich reduzierter Komplexität zu ermöglichen. Im Verlauf dieser Arbeit wird der vollständige Umfang der genannten modernen hierarchischen Techniken detailliert vorgestellt um die Modellierung ebenso wie den Lösungsprozess zu beschleunigen. Mehrgitteransätze wurden in Verbindung mit der PEECModellierung bereits in der Vergangenheit diskutiert [3]. Um eine effiziente Vorkonditionierung für geringe Frequenzen zu ermöglichen, stellte M. Verbeek eine Basistransformation vor, die kapazitive und induktive Effekte separiert. Ein flexiblerer Ansatz für die Behandlung von PEEC-basierten Matrizen aus der Schaltkreissimulation, der darüberhinaus auch eine gesteigerte Effizienz und eine verbesserte Genauigkeit erwarten lässt, wird im Rahmen dieser Arbeit zusammen mit einem Standard AMG-Löser vorgestellt. Aufgrund der spezifischen Eigenschaften von Matrizen aus der Schaltkreissimulation, ist eine effiziente Implementierung jedoch mit Sorgfalt durchzuführen. Die in dieser Arbeit vorgestellten Untersuchungen veranschaulichen die Erfordernisse sowie auftretende Probleme, konkrete Implementierungsrichtlinien und numerische Ergebnisse. Es kommen effiziente Matrix-Speicherformate zum Einsatz, die in professionellen industriellen Programmen bereits etabliert sind. Unter Einbeziehung der geforderten Genauigkeit wird jede Methode anhand einer Komplexitätsbetrachtung bewertet. Praktische Implementierungsaspekte runden jedes Kapitel ab. Die Arbeit schließt mit einer ausführlichen Analyse eines industriell relevanten EMV-Problems aus dem automobilen Umfeld. Um die Übereinstimmung mit der Wirklichkeit zu verdeutlichen, werden für dieses Beispiel Simulationsergebnisse mit Messergebnissen verglichen. Die vorgestellten hierarchischen Strategien werden bereits in anderen Anwendungsfeldern erfolgreich eingesetzt.

Die Arbeit ist wie in Abbildung 1.2 dargestellt aufgebaut. Im Anschluß an diese Einleitung werden in Kapitel 2 einige physikalische Grundlagen zusammengefasst, die für die numerische Analyse elektromagnetischer Effekte von Bedeutung sind. Kapitel 3 enthält Informationen zur PEEC-Methode für orthogonale ebenso wie für nicht-orthogonale Diskretisierungen. Die prinzipielle Struktur eines Schaltkreissimulators wird im Hinblick auf seine Bedeutung für die nachfolgenden Kapitel kurz erläutert. Zur Vermeidung von Simulationsartefakten und zur Gewährleistung von Ergebnissen mit hoher Genauigkeit, werden einige Richtlinien bezüglich Diskretisierung und numerischer Integration dargestellt. Durch Verwendung eines Reluktanzansatzes zusammen mit einer nachfolgenden Ausdünnung kann eine für iterative Methoden geeignete dünn besetzte und positiv definite Systemmatrix 
erzeugt werden. Dies ermöglicht stabile Simulationen im Zeitbereich wie auch im Frequenzbereich mit sehr genauen Ergebnissen. Mit Bezug auf die Schaltkreisrealisierung werden grundlegende Details präsentiert, um Dielektrika und Retardierungseffekte einzubeziehen.

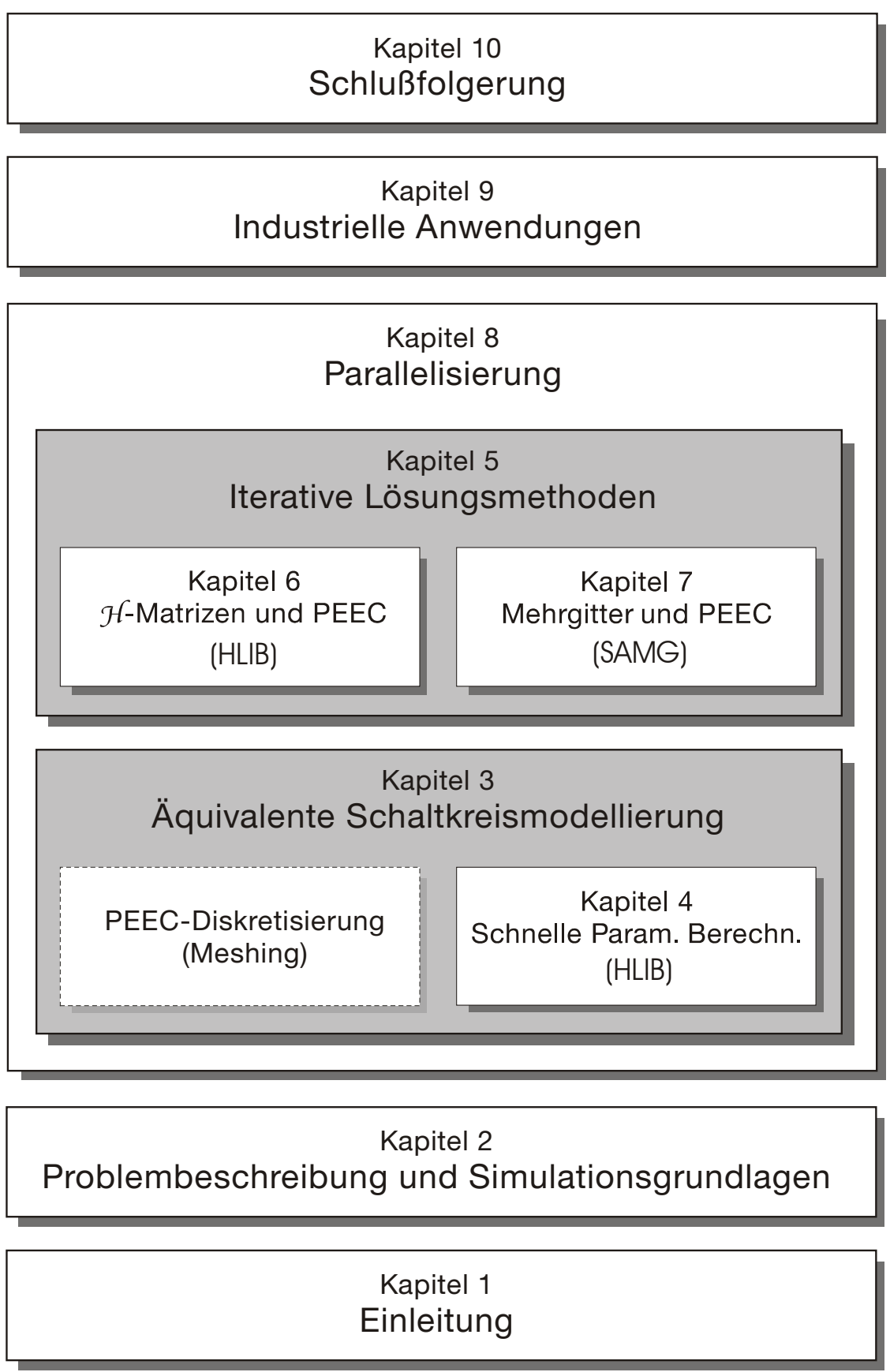

Abb. 1.2: Gliederung der Arbeit.

$\mathrm{Zu}$ Beginn von Kapitel 4 wird das Grundprinzip der schnellen Multipolmethode kurz wiederholt, um die Vorteile hierarchischer Matrizen für eine schnelle Modellextraktion deutlich zu machen. Nach einer detaillierten Erläuterung der Grundlagen von H-Matrizen und Komplexitätsbetrachtungen, wird der Elementberechnungsprozess basierend auf dem crossapproximation-Prinzip beschrieben. Die vorgestellten Strategien gründen auf Techniken aus 
der H-Matrix-Bibliothek HLIB [27]. Die Funktionsfähigkeit von H-Matrizen wird verwendet, um die Effizienz im Hinblick auf Zeit- und Speicherbedarf ebenso wie die Modellierungsgenauigkeit $\mathrm{zu}$ steigern. Implementierungsaspekte und numerische Beispiele werden vorgestellt, um den Arbeitsablauf zu verdeutlichen und die Funktionalität anhand konkreter Problemstellungen $\mathrm{zu}$ verifizieren. $\mathrm{Zu}$ einer weiteren Steigerung der Leistungsfähigkeit werden zusätzliche Verbesserungsansätze präsentiert. Die Realisierung eines effizienten Gesamtsimulationsprozesses bedarf einer Analyse der Matrizeneigenschaften aus dem Schaltkreisansatz. Grundsätzliche Untersuchungen im Hinblick auf geeignete Löserstrategien werden in Kapitel 5 vorgestellt. Sowohl grundlegende iterative Methoden für die Lösung linearer Gleichungssysteme aus einem Schaltkreisansatz als auch Techniken der Vorkonditionierung werden erklärt. Um die Vorteile der geringen Besetzungsdichte der Systemmatrizen ausnutzen zu können, werden effiziente und für die vorgestellten Methoden geeignete Matrixspeicherformate erläutert. Speziell für Systemmatrizen aus der Schaltkreisanalyse, die einem BEM-basierten Ansatz entstammen, kann eine data-sparse Repräsentation von Vorteil sein. Dies wird in Kapitel 6 dargestellt. Obwohl H-Matrizen durch direkte Strategien unter Zuhilfenahme der H-Matrix-Arithmetik effizient gelöst werden können, kann die Anwendung von iterativen one-level Methoden zu einer gesteigerten Leistungsfähigkeit führen. Implementierungsaspekte für ein spezielles $\mathrm{H}$-Stempelverfahren für Matrizen aus der Schaltkreissimulation und eine geeignete $\mathrm{H}$-Vorkonditionierung werden erläutert. Ebenso wie numerische Ergebnisse industriell relevanter Problemstellungen bei Anwendung adaptiver $\mathrm{H}$ vorkonditionierter Krylov-subspace Simulationen. Kapitel 7 stellt den Umfang eines algebraischen Mehrgitteransatzes für allgemeine schaltkreisbasierte Simulationen vor. Nach einer kurzen Zusammenfassung der grundlegenden Mehrgittertheorie und der mehrgitterbasierten Vorkonditionierung werden Details zur Behandlung von ausgedünnten PEECMatrizen und data-sparse PEEC-Matrizen aufgezeigt. Die verwendeten Strategien basieren auf Techniken der standard AMG Bibliothek SAMG [28]. Zu einer Steigerung der Gesamteffizienz und Genauigkeit verbinden die vorgestellten Funktionen Strategien eines Mehrgitteransatzes mit $\mathrm{H}$-Matrizen und lokaler $\mathrm{H}$-Matrix-basierter Gitterverfeinerung. Anhand ihrer Komplexität im Hinblick auf Zeit- und Speicherbedarf werden die einzelnen Ansätze verglichen. Implementierungsaspekte zur Softwarebibliothek SAMG werden für reluktanzbasierte Schaltkreismodelle im Zeit- sowie im Frequenzbereich vorgestellt. Eine Überprüfung der Effizienz der aufgezeigten Methodik wird anhand industriell relevanter Problemstellungen verdeutlicht. Vergleichsergebnisse $\mathrm{zu}$ einem direkten Löser sind vorhanden. Aufgrund der annähernd optimalen Komplexität hierarchischer Ansätze kann eine zusätzliche Effizienzsteigerung meist nur durch den Einsatz von Parallelisierungstechniken erzielt werden. Kapitel 8 macht einige wichtige Aspekte von Parallelisierungsstrategien für AMG sowie auch für H-Matrizen deutlich. Dieses Kapitel soll die Arbeit vervollständigen und abrunden. Implementierungsaspekte für die Integration in eine bestehende EMVSimulationsumgebung werden diskutiert. Die Effizienz, Robustheit und Genauigkeit der vorgestellten Methodik wird in Kapitel 9 demonstriert. Die Übereinstimmung der gewonnenen Resultate wird anhand von Vergleichsmessungen an bestehender Hardware bestätigt. Kapitel 10 rundet die Arbeit mit abschließenden Bemerkungen und einem Ausblick auf zukünftige Forschungsaktivitäten ab. 